Prepared in cooperation with the Georgia Department of Natural Resources, Environmental Protection Division

\title{
Estimated Use of Water in Georgia for 2015 and Water-Use Trends, 1985-2015
}
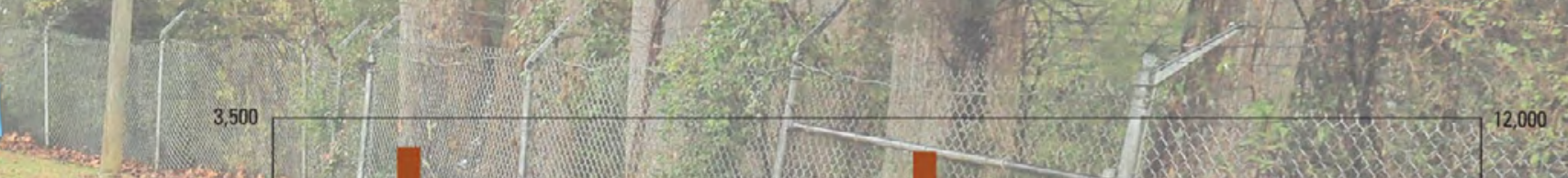
Cover. Burton Trout Hatchery, Lake Burton, Georgia Department of Natural Resources, Rabun County, Georgia. Photograph by Alan M. Cressler, U.S. Geological Survey. 


\section{Estimated Use of Water in Georgia for 2015 and Water-Use Trends, 1985-2015}

By Jaime A. Painter

Prepared in cooperation with the Georgia Department of Natural Resources,

Environmental Protection Division

Open-File Report 2019-1086 


\title{
U.S. Department of the Interior DAVID BERNHARDT, Secretary
}

\author{
U.S. Geological Survey \\ James F. Reilly II, Director
}

U.S. Geological Survey, Reston, Virginia: 2019

For more information on the USGS - the Federal source for science about the Earth, its natural and living resources, natural hazards, and the environment-visit https://www.usgs.gov or call 1-888-ASK-USGS (1-888-275-8747).

For an overview of USGS information products, including maps, imagery, and publications, visit https://store.usgs.gov.

Any use of trade, firm, or product names is for descriptive purposes only and does not imply endorsement by the U.S. Government.

Although this information product, for the most part, is in the public domain, it also may contain copyrighted materials as noted in the text. Permission to reproduce copyrighted items must be secured from the copyright owner.

Suggested citation:

Painter, J.A., 2019, Estimated use of water in Georgia for 2015 and water-use trends, 1985-2015: U.S. Geological Survey Open-File Report 2019-1086, 216 p., https://doi.org/10.3133/ofr20191086.

ISSN 2331-1258 (online) 


\section{Contents}

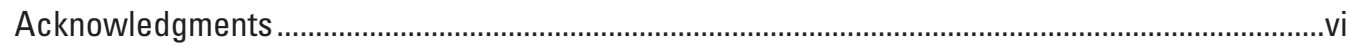

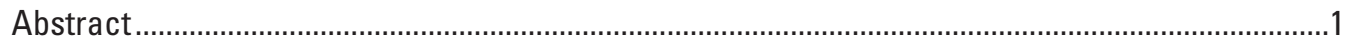

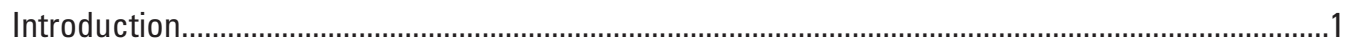

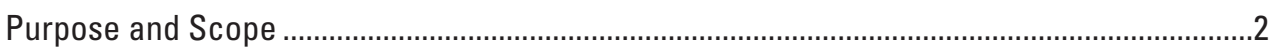

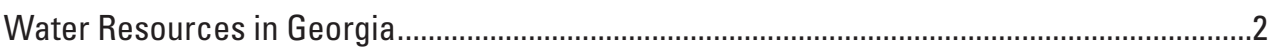

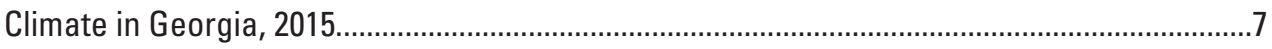

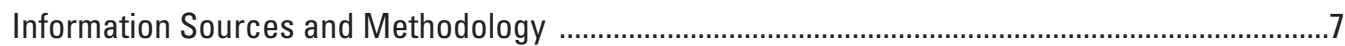

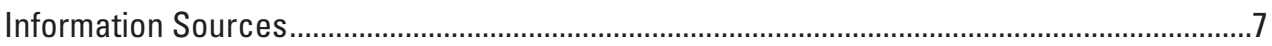

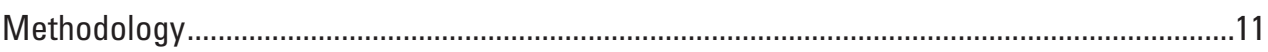

Self-Supplied Water ......................................................................................................

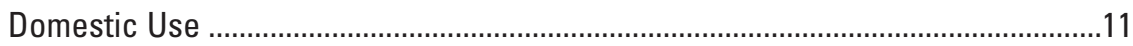

Commercial and Industrial Use ...............................................................................

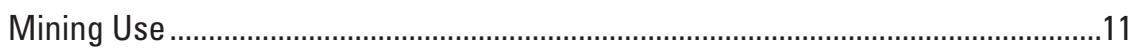

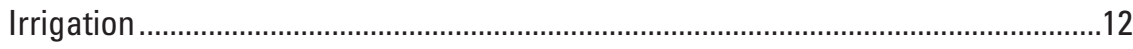

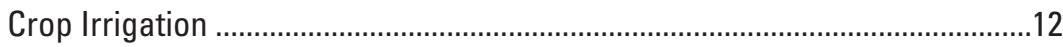

Golf Course Irrigation .....................................................................................12

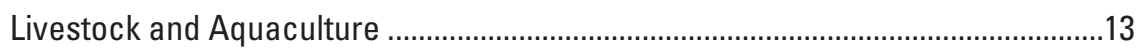

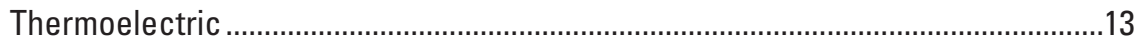

Public-Supplied Water ........................................................................................... 14

System Losses ..............................................................................................

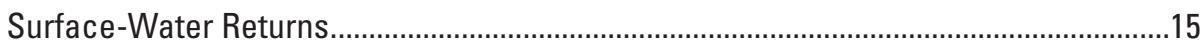

Water Withdrawals, Estimated Water Use, and Surface-Water Returns ......................................15

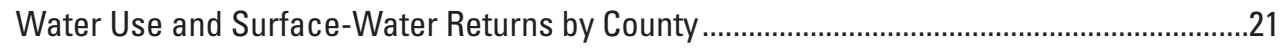

Water Use and Surface-Water Returns by Water-Planning Region.......................................24

Water Use and Surface-Water Returns by Major River Basin ................................................24

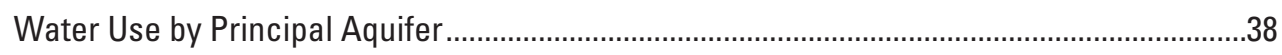

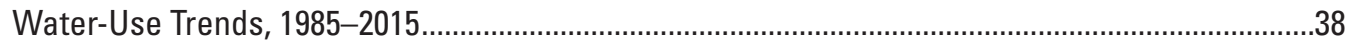

Discussion and Conclusions ......................................................................................................

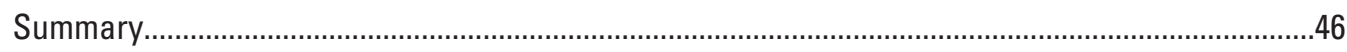

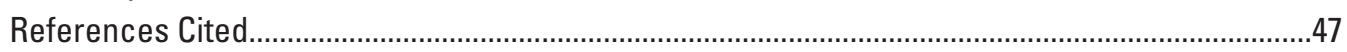

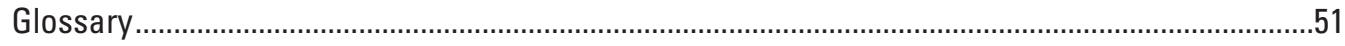

Appendix 1. North American Industrial Classification System Codes .......................................55

Appendix 2. Population, Water Withdrawals, and Water Use by Source of Water for Each County in Georgia, 2015........................................................................................57

\section{Figures}

1. Map of the State of Georgia showing all 159 counties and select cities...........................3

2. Physiographic provinces and area of principal aquifers in Georgia ..................................4

3. Major river basins and streams in Georgia .................................................................

4. Water-planning regions in Georgia .......................................................................6

5. Georgia statewide average annual precipitation, average growing-season precipitation for $1980-2015$, and periods of drought........................................................... 
6. Schematic diagram of public and self-supplied water withdrawals and components of total water use for a typical county in Georgia.

7. Water withdrawals in Georgia, by source and county, for 2015 ....................................16

8. Water withdrawals in Georgia by source and water-use category for 2015,

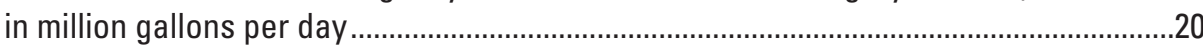

9. Water withdrawals in Georgia by water-use category, by county, for 2015 ...................22

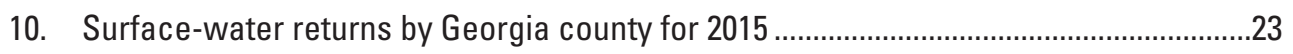

11. Surface-water and groundwater withdrawals for counties within each water-planning region. Ordered by smallest withdrawals to largest withdrawals

12. Water withdrawals in Georgia water-use regions for 2015 , by water-use category, in million gallons per day.....

13. Water withdrawals in Georgia by water-use category for major river basins in 2015, in million gallons per day. 32

14. Water withdrawals in Georgia, by major river basin, for 2015......................................34

15. Water-withdrawal trends by Georgia water source for 1985-2015 .................................39

16. Statewide water-use trends in Georgia, by category, for 1985-2015 ….........................40

17. Water-use trends in Georgia water-planning regions by category and population trend for 1985-2015.....................................................................................

18. Water-use trends in Georgia water-planning regions for 1985-2015 ..............................45

\section{Tables}

1. Water-withdrawal, surface-water return flows, and water-use information sources for Georgia, 2015.

2. 2015 total population, and water withdrawals by source and water-use category, in Georgia

3. Permitted commercial and industrial water withdrawals by major North American Industrial Classification code in Georgia, 2015

4. Water withdrawals by water-use category for water-planning regions in Georgia, 2015.

5. Surface-water returns by water-use category for water-planning regions in Georgia, 2015

6. Water withdrawals by water-use category, for major river basins in Georgia, 2015 ...

7. Surface-water returns by water-use category for major river basins in Georgia, 2015

8. Groundwater withdrawals by use category for the principal aquifers in Georgia, 2015 


\section{Conversion Factors}

U.S. customary units to International System of Units

\begin{tabular}{|c|c|c|}
\hline Multiply & By & To obtain \\
\hline \multicolumn{3}{|c|}{ Length } \\
\hline inch (in.) & 2.54 & centimeter $(\mathrm{cm})$ \\
\hline inch (in.) & 25.4 & millimeter $(\mathrm{mm})$ \\
\hline \multicolumn{3}{|c|}{ Volume } \\
\hline gallon (gal.) & 3.785 & liter (L) \\
\hline gallon (gal.) & 0.003785 & cubic meter $\left(\mathrm{m}^{3}\right)$ \\
\hline \multicolumn{3}{|c|}{ Flow rate } \\
\hline gallon per day (gal/d) & 0.003785 & cubic meter per day $\left(\mathrm{m}^{3} / \mathrm{d}\right)$ \\
\hline million gallons per day (Mgal/d) & 0.04381 & cubic meter per second $\left(\mathrm{m}^{3} / \mathrm{s}\right)$ \\
\hline
\end{tabular}

\section{Abbreviations}

$\begin{array}{ll}\text { CAFR } & \text { Comprehensive annual financial report } \\ \text { GaEPD } & \text { Georgia Department of Natural Resources, Environmental Protection Division } \\ \text { gal/d } & \text { gallons per day } \\ \text { GGCSA } & \text { Georgia Golf Course Superintendents Association } \\ \text { GIS } & \text { geographic information system } \\ \text { GWSA } & \text { Georgia Water Stewardship Act } \\ \text { Mgal/d } & \text { million gallons per day } \\ \text { NAICS } & \text { National American Industrial Classification System } \\ \text { NPDES } & \text { National Pollutant Discharge Elimination System } \\ \text { NWIS } & \text { National Water Information System } \\ \text { NWUSP } & \text { National Water-Use Science Project } \\ \text { USGS } & \text { U.S. Geological Survey }\end{array}$




\section{Acknowledgments}

The compilation of water-use information for Georgia in 2015 would not be possible without the assistance and data provided by the Georgia Environmental Protection Division, local water supply (public and private) and water management agencies in Georgia, and the Georgia Power Company. The author acknowledges the Georgia Soil and Water Conservation Commission for providing crop-irrigation metered water-use information and the Georgia Water Planning \& Policy Center for assisting with revision of the livestock water-use estimates methodology for 2015. The author thanks U.S. Geological Survey hydrologists Gerard Gonthier, Debbie Gordon, and Lynn Torak for assisting in the compilation of surface-water returns, livestock, and crop irrigation information, respectively. 


\title{
Estimated Use of Water in Georgia for 2015 and Water-Use Trends, 1985-2015
}

\author{
By Jaime A. Painter
}

\section{Abstract}

Water-withdrawal, water-use, and water-return information have been collected and compiled for each county in Georgia every 5 years since 1980 using data obtained from various Federal, State, and private agencies, as well as additional online sources. For 2015, water use, water withdrawal, and water returns were estimated for each county, water-planning region, major river basin, and principal aquifer in Georgia. Offstream water use in 2015 is estimated for the categories of domestic, commercial, industrial processing, mining, irrigation (subdivided into crop and golf course irrigation), livestock, aquaculture, and thermoelectric power cooling.

According to the U.S. Census Bureau, approximately 10.2 million people in Georgia needed water resources to meet their personal, commercial, and recreational needs in 2015. Public water suppliers provided water to about 85 percent of the population of Georgia. Estimated total water withdrawals from both surface-water and groundwater sources were about 3,384 million gallons per day (Mgal/d) in 2015, which is a 27-percent reduction from 2010, a 48.1-percent reduction from 2000, and a 49.7-percent reduction from 1980. In 2015, surface-water withdrawals were greatest for thermoelectric power cooling (839.8 Mgal/d), and groundwater withdrawals were greatest for irrigating crops $(547.9 \mathrm{Mgal} / \mathrm{d})$. Water needs in northern Georgia are typically met by withdrawing a larger percentage of water from surface-water than groundwater sources; conversely, counties in southern Georgia withdraw more water from groundwater sources. About 1,571 Mgal/d of water were returned to Georgia streams and lakes in 2015, which represents about 46 percent of the total water withdrawn from all sources in 2015.

Water users in the Apalachicola River Basin, in 2015, withdrew the highest percentage of water (35 percent) and returned the highest percentage of water to surface-water bodies (almost 40 percent) compared to other major river basins in Georgia. Withdrawals in the Apalachicola River Basin are primarily extracted by public-supply systems (43 percent) and irrigation (34 percent). The aquifer from which 68 percent of statewide groundwater withdrawals were extracted was the Floridan aquifer system, and the majority of the water was used for irrigation (57 percent).
Historically, statewide water use in Georgia was highest in $1980(6,735 \mathrm{Mgal} / \mathrm{d})$, decreased to 5,353 Mgal/d in 1990, peaked at $6,531 \mathrm{Mgal} / \mathrm{d}$ in 2000, and has been declining since that time. The reduction in water use between 2000 and 2015 came primarily from surface-water withdrawals (90 percent of total reduction) and thermoelectric power cooling use (78 percent of total reduction). Water use for livestock and aquaculture increased between 1985 and 2015, and this increase correlates with the growth of agriculture in Georgia during that period. The driving forces behind the observed water-use changes include (1) shifts in population numbers and locations, (2) five periods of major drought, (3) water conservation efforts and education programs initiated by State and local governments and water utilities, and (4) changing water needs for thermoelectric power cooling, industry, and agricultural activities.

\section{Introduction}

Understanding the movement of water from its natural source, through its interaction with human activity and the quantities of water consumed or returned to the natural water system, is paramount to resource sustainability and societal prosperity. Knowledge of the amounts withdrawn by source - surface water and groundwater - and the amounts consumed or returned for further use is necessary to manage the water resources of Georgia effectively and ensure that all water users have a water supply sufficient to meet current and future needs. Water withdrawals provide information on the quantity of water removed from the natural source; however, knowledge about where water goes, how it is used, and whether it returns to a surface-water source is described as "water use." Water information-including water withdrawals, distribution and deliveries, consumptive use, return flows and losses - have been collected and compiled in Georgia since 1980 as part of an ongoing cooperative agreement between the U.S. Geological Survey (USGS) South Atlantic Water Science Center and the Environmental Protection Division of the Georgia Department of Natural Resources. Statewide, annual water withdrawal totals have averaged 4.3 billion gallons per day since 1980, with fluctuations in total withdrawal 
through 2015 (U.S. Geological Survey, 2018). During the 35 -year history of the Georgia water-use program (Fanning, 1985), natural and human-induced alterations have resulted in observed changes in water use.

Water-use estimates for the State of Georgia were first made during the 1950s as part of an effort to compile water-use information for the Nation (MacKichan, 1951). This report on 2015 is the ninth in a bi-decadal series of reports that describe water use by county in Georgia. Previous reports from the Georgia Water-Use Program were published as companions to the USGS series "Estimated Use of Water in the United States" and include summaries for 1980 (Pierce and others, 1982), 1985 (Turlington and others, 1987), 1987 (Trent and others, 1990), 1990 (Fanning and others, 1992), 1995 (Fanning, 1997), 2000 (Fanning, 2003), 2005 (Fanning and Trent, 2009) and 2010 (Lawrence, 2016). From 1950 to 2000 , however, the categories used for water-use estimates were not always comparable (Fanning and Trent, 2009). Category definitions and estimation techniques have been the most consistent since 1985 because of the efforts of the USGS National Water-Use Science Project (NWUSP) (formerly the National Water-Use Information Program, https://water. usgs.gov/watuse/wunwup.html). Due to inconsistent category definitions and estimation techniques, the 1980 category data are not presented in this report when water-use trends are described. This report is the first report in the series to provide water-use trends by subregions of the State (known as waterplanning regions) in addition to the statewide water-use trends historically provided.

\section{Purpose and Scope}

This report provides information on compiled water withdrawals, surface-water returns (treated and untreated water), and water-use estimates for nine categories of use in Georgia during 2015. The categories for which water use was estimated are domestic, commercial, industrial processing, mining, irrigation (subdivided into crop and golf course irrigation), livestock, aquaculture, and thermoelectric power cooling. Water withdrawals and water-use estimates for each use category and surface-water returns are reported by county, water-planning region, major river basin, and principal aquifer in Georgia. Water-use trends for 1985-2015 are provided at the State scale as well as for each water-planning region. These estimates are presented in tabular, map, and graphical form.

\section{Water Resources in Georgia}

Georgia's water resources are grouped into 11 major river basins, eight principal aquifers, 11 water-planning regions, and 159 counties for estimating and describing water use, withdrawals, and returns (figs.1-4). Contrasting geologic features and landforms of the physiographic provinces of Georgia (fig. 2) affect the amount, distribution, and availability of Georgia's water resources throughout the State. Surface water from reservoirs or reservoir-augmented streamflow is the primary source of water in northern Georgia; groundwater availability is only limited in northern Georgia because the storage capacity is typically low in the underlying fractured-crystalline and Paleozoic rock aquifers. Most of the groundwater use is in southern Georgia where aquifers are highly productive.

Georgia is considered a "headwaters" State, as most rivers begin as springs or small streams in northern Georgia and flow southward into southern Georgia, Alabama, South Carolina, and Florida. The two exceptions are in north-central Georgia, where streams in the Tennessee River Basin flow northward into southern Tennessee, and streams in the Coosa and Tallapoosa Basins flow southwest into northeastern Alabama (fig. 3). These stream networks flow through many political jurisdictions, some extending outside the State boundary of Georgia, thereby affecting the distribution and use of water throughout the State. Surface water available for distribution and use can also be constrained by instream flow requirements for ecological needs and wastewater assimilative capacity.

The major river basins originating in Georgia are the Savannah, Ogeechee, Oconee, Ocmulgee, Altamaha, Satilla and St. Marys, Suwanee and Aucilla, Ochlockonee, Apalachicola, Coosa and Tallapoosa, and Tennessee (fig. 3). This sharing of waters across State boundaries has, in part, resulted in nearly 30 years of litigation between Alabama, Florida, and Georgia about the amount and distribution of surface-water withdrawals from the ApalachicolaChattahoochee-Flint River Basin and the Alabama-CoosaTallapoosa River Basin.

The principal aquifer systems in Georgia are the surficial aquifer system, Floridan aquifer system, Southeastern Coastal Plain aquifers (Claiborne and Clayton aquifers and the Cretaceous aquifer system), Brunswick aquifer system, crystalline-rock aquifers, and Paleozoic-rock aquifers (fig. 2). The surficial aquifer system is present in each of the physiographic provinces in Georgia and is semiconfined to confined locally in the coastal area. The most productive aquifers in Georgia are in the Coastal Plain province in the southern half of the State. Coastal Plain aquifers (those south of the Fall Line) are typically confined, except in recharge zones near their northern limits, where the aquifers crop out or are near land surface. Aquifers in the Coastal Plain include the surficial aquifer system, Brunswick aquifer system, Floridan aquifer system, Claiborne aquifer, Clayton aquifer, and Cretaceous aquifer system. Small quantities of water are withdrawn for use in the Valley and Ridge province, where groundwater is transmitted through primary and secondary openings in folded and faulted sedimentary and metasedimentary rocks of Paleozoic age, which are referred to as Paleozoic rock aquifers. In the Piedmont and Blue Ridge provinces, the geology is complex and consists of structurally deformed metamorphic and igneous 


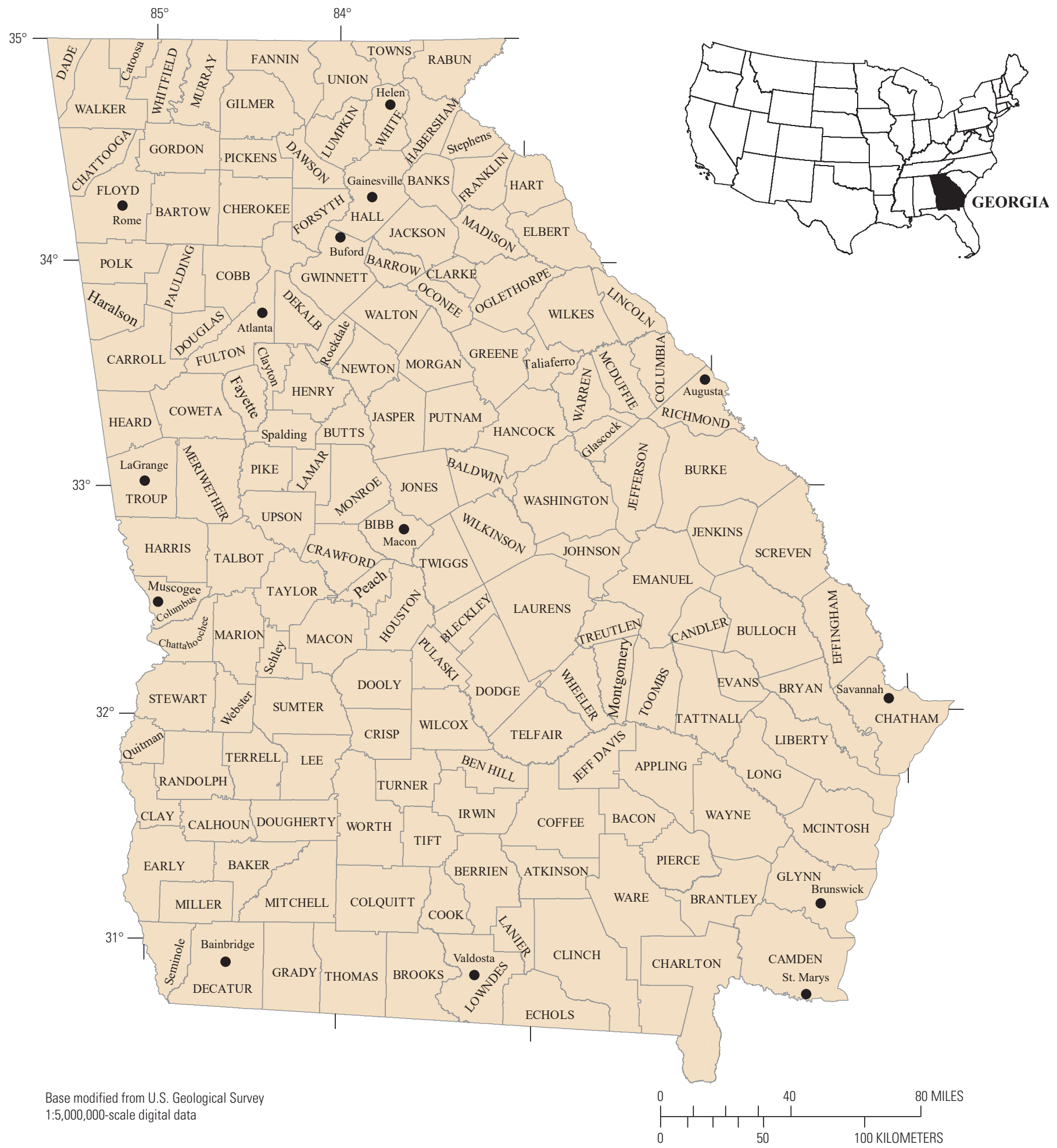

Figure 1. Map of the State of Georgia showing all 159 counties and select cities. 


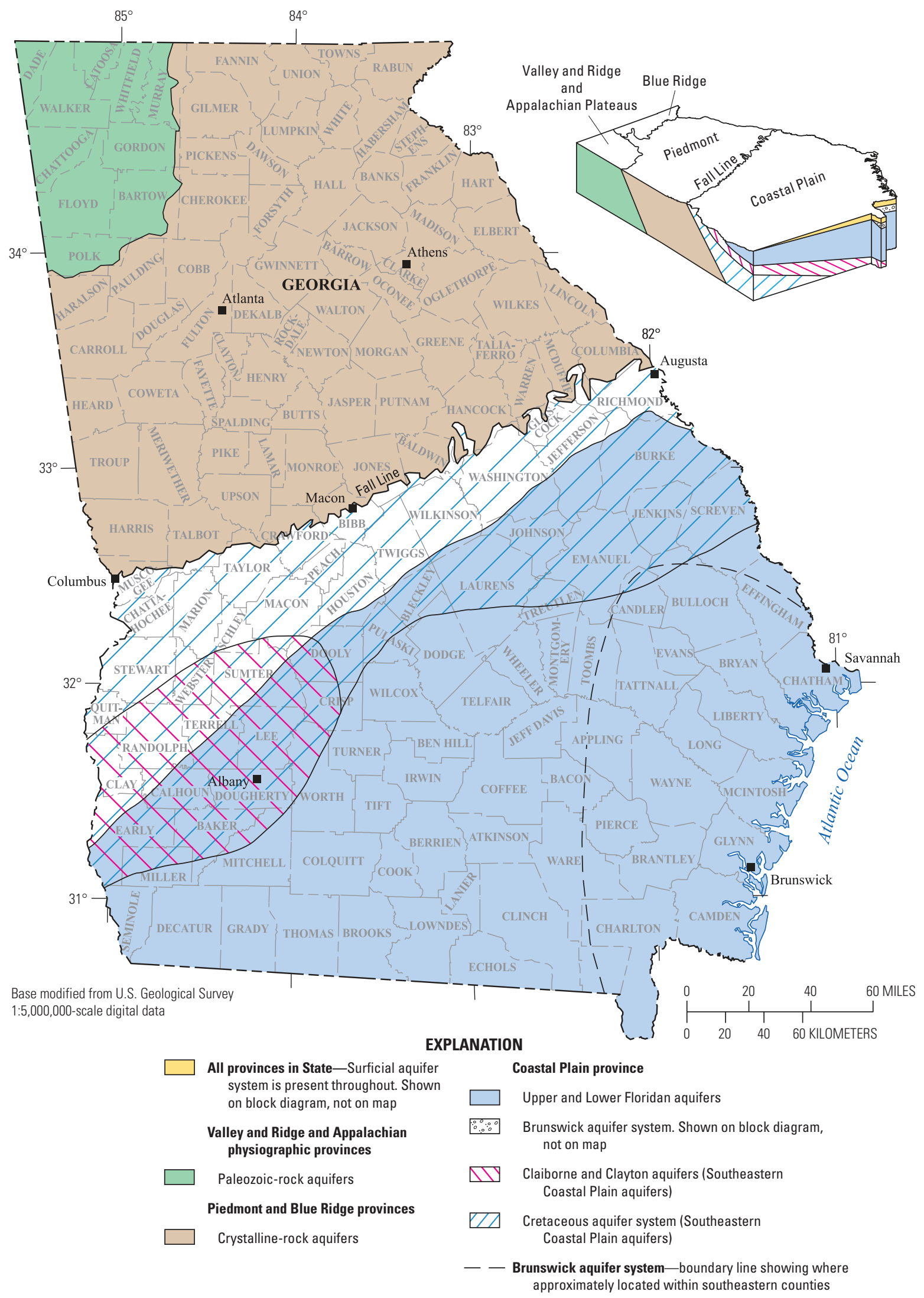

Figure 2. Physiographic provinces and area of principal aquifers in Georgia. 


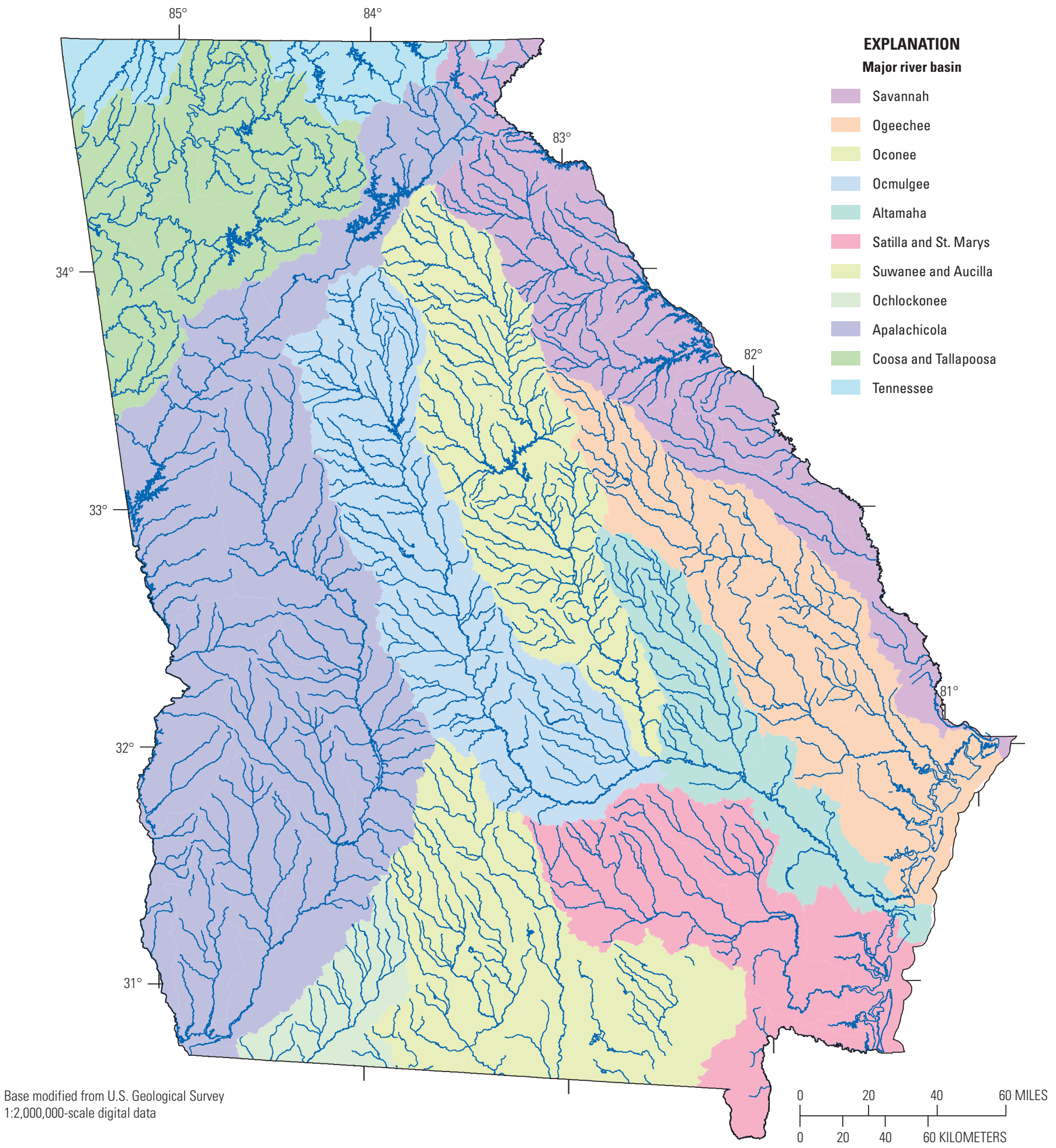

Figure 3. Major river basins and streams in Georgia. 


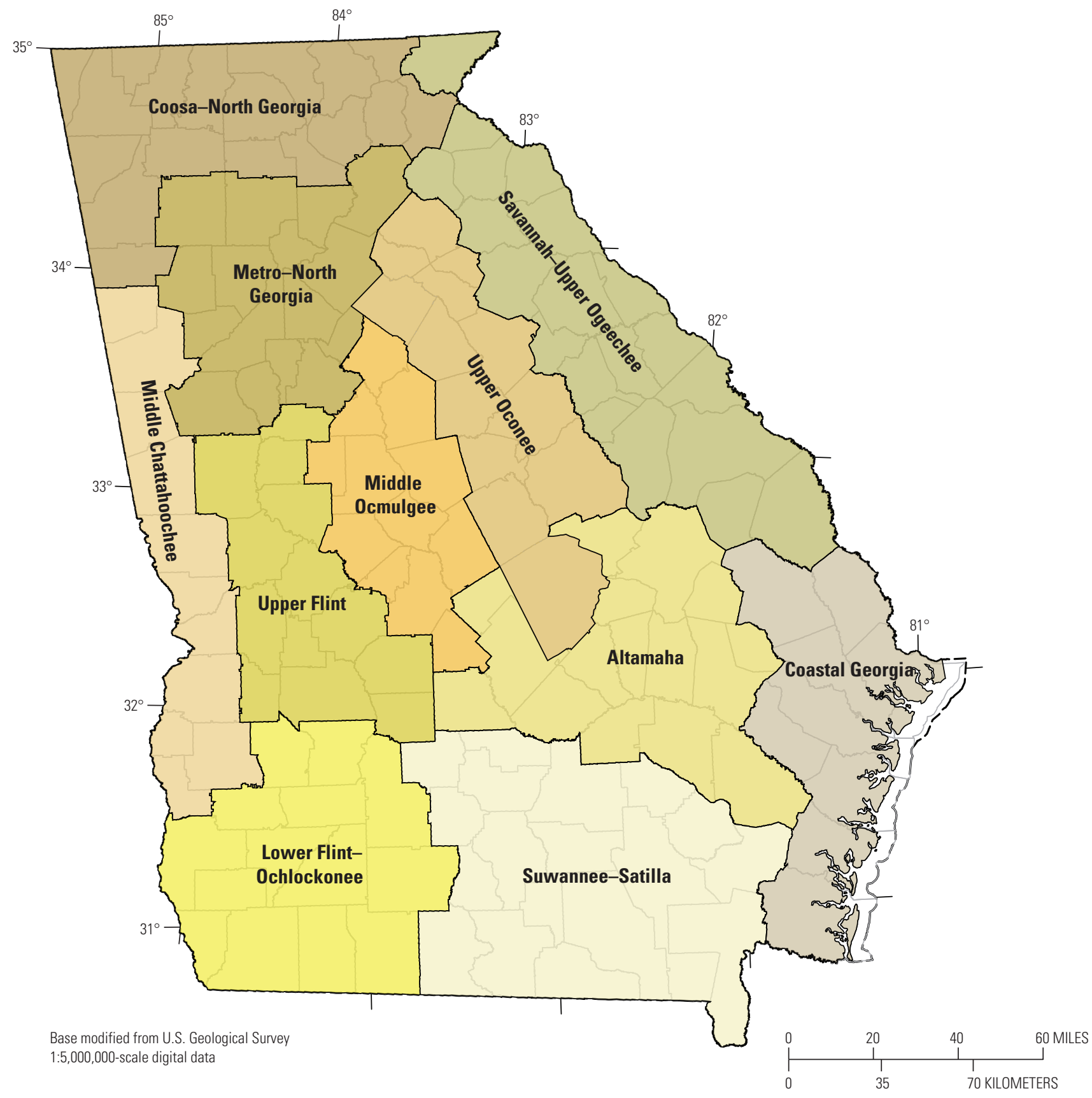

Figure 4. Water-planning regions in Georgia. 
rocks. Groundwater is transmitted through secondary openings along fractures, foliation, joints, contacts, or other features in the crystalline bedrock, which are referred to as crystalline-rock aquifers. A thorough discussion of the State's groundwater resources is provided in Clarke and Pierce (1985).

Georgia passed legislation between the years 2000 and 2015 to mandate water management strategies that "create and support a culture of conservation throughout the State by improving how efficiently water is used" (Georgia Environmental Protection Division, 2015). Georgia's water resources are managed through a Comprehensive Statewide Water Management Plan, adopted by the Georgia General Assembly in January 2008, which "provides a framework to measure water resources, to forecast how much water supply and assimilative capacity will be needed to support future growth, and to identify regional solutions to water needs" (Georgia Environmental Protection Division, 2008). Eleven water-planning regions were identified in the Georgia Statewide Water Management Plan for water-resource planning (fig. 4). In 2010, the State Legislature passed the Georgia Water Stewardship Act of 2010 ([GWSA], Official Code of Georgia Annotated [OCGA] § 12-5-4) "to create a culture of water conservation in the State of Georgia" (Georgia General Assembly, 2010). This Act requires local governments to use water conservation practices consisting of comprehensive planning, water loss abatement, public outreach programs, water efficient fixtures and equipment, water efficient landscaping and irrigation, rainwater and gray water use, submetering, conservation pricing, permitting, and public water-system financial management. Specifically, the GWSA requires water providers serving a population of 3,300 or more to conduct an annual water-system audit and implement a water-loss detection program. These management strategies affect the quantity and quality of the data provided for estimating water use in the State of Georgia.

\section{Climate in Georgia, 2015}

Georgia has a humid, subtropical climate characterized by short, mild winters and long, hot summers. Northeastern Georgia is typically wetter than southern Georgia. Data from the Southeast Regional Climate Center indicate a long-term average annual rainfall (1985-2015) for Georgia of about 50 inches (in.) (Southeast Regional Climate Center, 2018) (fig. 5). However, the rainfall totals vary geographically within the State and temporally throughout the growing season, affecting withdrawals for some categories of water use. For example, the long growing season in Georgia lasts about two-thirds of the year (March-October) and the average rainfall during this period is 34 in. (Southeast Regional Climate Center, 2018). Georgia's climate is also marked with periods of drought. Five drought periods occurred between 1980 and 2015 , specifically $1980-82,1985-89,1998-2003,2006-2007$, and 2012 (fig. 5). Drought affects the amount of water used to irrigate crops, golf courses, lawns, gardens, and trees.

\section{Information Sources and Methodology}

A generalized view of water use for a typical county in Georgia and the types of data needed to summarize water use in Georgia for 2015 are shown in figure 6. A variety of Federal, State, local, private, and online sources were used to gather surface-water and groundwater withdrawal information, surface-water return flow data, and water-use information sources for 2015 (table 1). All water-use categories are described individually because each requires unique methods for estimating water use. The movement of water from the point of withdrawal to the location of use, and then farther to the location of return to the surface-water network, can occur through multiple counties or river basins and result in some portion of the water being lost before consumption by an intended user. Therefore, data are collected and reported in this document geopolitically (by aggregate estimates per county and water-planning region) and hydrologically (by major river basin and aquifer). This reporting schema is crucial for computing public-supply deliveries to domestic, commercial, and industrial customers, and for self-supplied domestic use, mining, crop irrigation, and livestock use. A geographic information system (GIS) was used to spatially locate water intakes (surface-water withdrawal points) and outfalls (surface-water return points), groundwater wells, public-supply water service areas, irrigated acres, and land permitted for mining operation within a county and major river basin. The percentage of water withdrawals from the various aquifers in Georgia was determined using location information on withdrawal permits and knowledge of the hydrologic properties of aquifers throughout the State of Georgia.

\section{Information Sources}

Information used to estimate 2015 water withdrawal, water use, and surface-water returns was gathered from a variety of Federal, State, local, private, and online sources. Some of the estimates are based on reported data required by Georgia and Federal legislation, whereas other estimates were made using coefficients and geospatial techniques. Confidence in the accuracy of the estimates derived for this report depends upon the reliability of the source material and the completeness of the parent data. There is greater confidence for categories that used reported withdrawals and less confidence where coefficients were needed to estimate water use.

Permitted withdrawal data for public-supplied and self-supplied water for commercial, industrial, and mining use; golf course irrigation; and thermoelectric power use were obtained from the Georgia Department of Natural Resources, Environmental Protection Division (GaEPD) Drinking Water and Water Withdrawal Program (table 1). Georgia law (the Georgia Groundwater Use Act of 1972, [OCGA § 12-5-90] and the Georgia Water Supply Act [OCGA § 12-5-170]; https://epd. georgia.gov/existing-rules-and-corresponding-laws) requires a withdrawal permit for any city, industry, or other water user 
that withdraws more than 100,000 gallons per day (gal/d; monthly average) and a monthly report of withdrawals to the GaEPD each year. Water withdrawals for most crop-irrigation operations are provided through the State Agricultural Water Conservation and Metering Program established by House Bill 579 in 2003 (OCGA § 2-6-27). By 2015, over 13,000 water meters were installed on wells, surface-water intakes, and irrigation systems to record cumulative water withdrawals (Georgia General Assembly, 2003). These meter data for the 2015 growing season were collected and made available by the Georgia Soil and Water Conservation Commission. Water withdrawal estimates for the remaining self-supplied use categories - meaning self-supplied domestic, livestock, aquaculture, non-reported mining, crop, and golf course irrigation - were derived using coefficients, geospatial techniques, and location information.
Additionally, estimates of publicly supplied water deliveries and losses are made using information from the private sector and online sources. Surface-water return flow data were obtained from discharge monitoring reports supplied by GaEPD, which administers the National Pollutant Discharge Elimination System (NPDES) program created by The Clean Water Act of 1972 (33 U.S.C. 1251 et seq.). The program requires a permit for all wastewater and other water discharges with the potential to carry pollutants to surface water, regardless of the volume discharged (U.S. Environmental Protection Agency, 2014). All NPDES permit holders are required to report monthly surface-water discharges to the GaEPD.
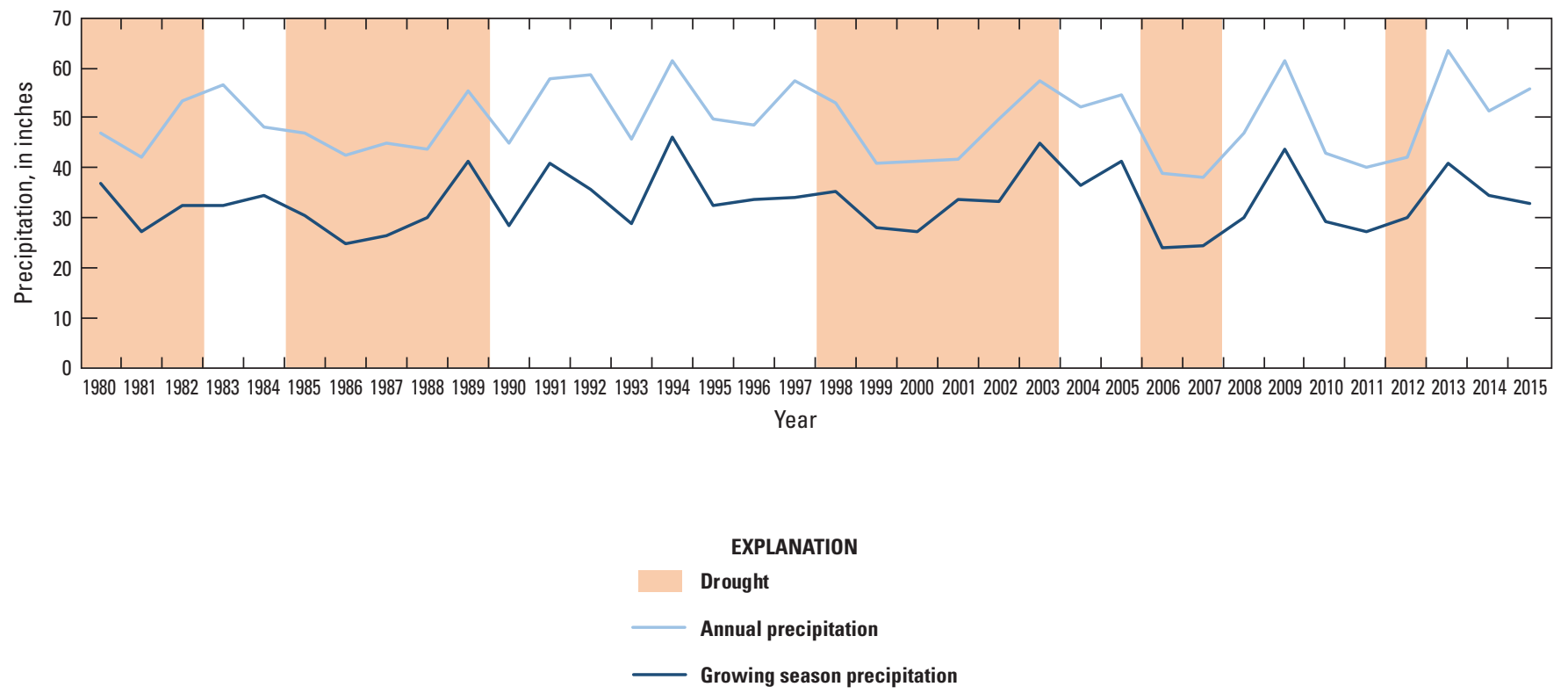

Figure 5. Georgia statewide average annual precipitation, average growing-season precipitation (March through 0 ctober) for 1980-2015, and periods of drought. 


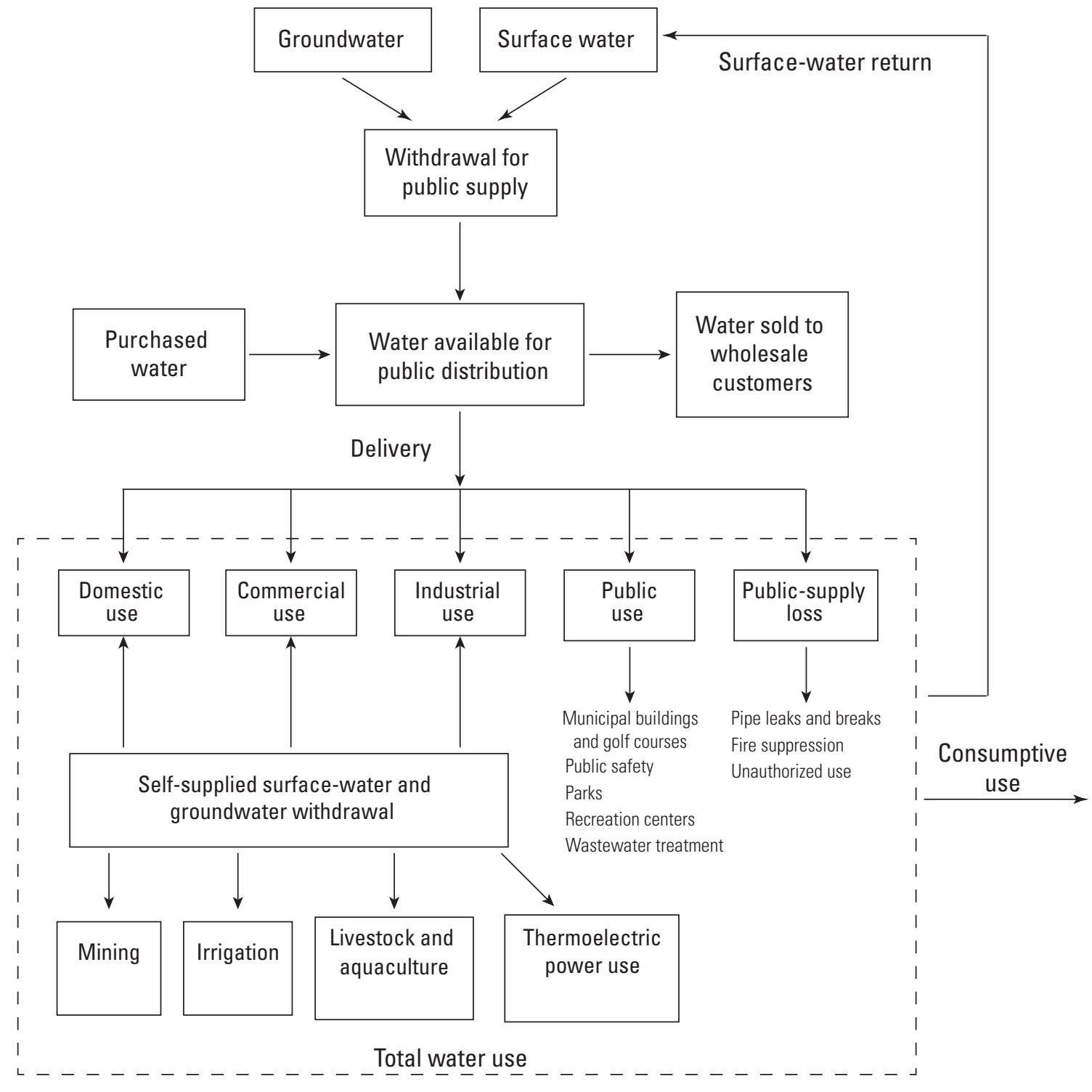

Figure 6. Schematic diagram of public and self-supplied water withdrawals and components of total water use for a typical county in Georgia. 
Table 1. Water-withdrawal, surface-water return flows, and water-use information sources for Georgia, 2015.

[GaEPD, Georgia Environmental Protection Division, Watershed Protection Branch; USGS, U.S. Geological Survey]

\begin{tabular}{|c|c|c|}
\hline Water-use category & Type of data & Data source \\
\hline \multirow[t]{2}{*}{ Public supply } & $\begin{array}{l}\text { Reported groundwater and surface-water } \\
\text { withdrawals by permitted users }\end{array}$ & $\begin{array}{l}\text { GaEPD Drinking Water and Water Withdrawal Program, } \\
\text { groundwater and surface-water permitting units. }\end{array}$ \\
\hline & $\begin{array}{l}\text { Population served by small public suppliers } \\
\text { (subdivisions and mobile home parks) }\end{array}$ & $\begin{array}{l}\text { GaEPD Drinking Water and Water Withdrawal Program, } \\
\text { Drinking Water permit unit. }\end{array}$ \\
\hline Commercial use & $\begin{array}{l}\text { Reported groundwater and surface-water } \\
\text { withdrawals by permitted users }\end{array}$ & $\begin{array}{l}\text { GaEPD Drinking Water and Water Withdrawal Program, } \\
\text { groundwater and surface-water permitting units. }\end{array}$ \\
\hline Industrial use & $\begin{array}{l}\text { Reported groundwater and surface-water } \\
\text { withdrawals by permitted users }\end{array}$ & $\begin{array}{l}\text { GaEPD Drinking Water and Water Withdrawal Program, } \\
\text { groundwater and surface-water permitting units. }\end{array}$ \\
\hline Livestock and aquaculture & $\begin{array}{l}\text { Livestock water-use estimates by county, } \\
\text { water-planning region, and 8-digit } \\
\text { hydrologic unit }\end{array}$ & $\begin{array}{l}\text { Marke Masters and Kristin Rowles, Georgia Water Planning } \\
\text { and Policy Center, written commun., } 2017 \text { (estimates } \\
\text { based on } 2013 \text { Georgia Farm Gate Value Report } \\
\text { (AR-14-01, October 2014)). }\end{array}$ \\
\hline \multirow[t]{2}{*}{ Irrigation-Crops } & $\begin{array}{l}\text { Withdrawal estimates and irrigated acreage } \\
\text { by county }\end{array}$ & $\begin{array}{l}\text { Georgia State-Wide Water Plan (Georgia Environmental } \\
\text { Protection Division, 2008); }\end{array}$ \\
\hline & $\begin{array}{l}\text { Metered agricultural withdrawals and } \\
\text { estimated withdrawals in non-metered } \\
\text { counties in south Georgia }\end{array}$ & $\begin{array}{l}\text { Georgia Soil and Water Conservation Commission; USGS; } \\
\text { Torak and Painter }(2011,2013) \text {. }\end{array}$ \\
\hline Surface-water return flows & $\begin{array}{l}\text { Reported surface-water discharges by } \\
\text { permitted users }\end{array}$ & $\begin{array}{l}\text { GaEPD Drinking and Wastewater Regulatory Program, } \\
\text { Municipal and Industrial permitting units. }\end{array}$ \\
\hline
\end{tabular}




\section{Methodology}

The methods used to estimate withdrawal, water use, and returns are grouped into three sections: self-supplied water, public-supplied water, and surface-water returns. Each use category has a unique methodology to derive estimates from parent information for each county, major river basin, and aquifer in Georgia.

\section{Self-Supplied Water}

Self-supplied water is surface water or groundwater that is withdrawn, treated (for drinking water use), and used onsite within a facility. Many self-supplied commercial, industrial, mining, golf course, and thermoelectric power facilities hold surface-water or groundwater (or both) withdrawal permits and report withdrawals monthly to the GaEPD. Self-supplied domestic water use was estimated using a per capita rate or coefficient.

\section{Domestic Use}

Self-supplied water withdrawals for domestic use are commonly less than the Georgia reporting threshold of $100,000 \mathrm{gal} / \mathrm{d}$ and typically occur at individual dwellings in rural or semirural areas where access to public-supplied water is limited or non-existent. Water for self-supplied domestic use is typically withdrawn from a private source such as a well or captured as rainwater in a cistern. All self-supplied domestic withdrawals compiled for 2015 were from groundwater sources. The amount of water withdrawn and used for domestic purposes was calculated as the selfsupplied population for each county multiplied by a per capita coefficient of 75 gallons per day (gal/d) per person (Fanning, 1997; Trent and others, 1990). The self-supplied domestic population was computed as the difference between the county's total population and total public-supplied population.

Estimates of self-supplied domestic water use for each major river basin were derived using geospatial techniques. The location of populations served by public water systems are not usually known; therefore, the percentage of county area within each river basin was used to distribute the countylevel, self-supplied water-use estimates among the river basins within each county. Withdrawals were also estimated by aquifer. The distribution of the self-supplied water use among aquifers required the compilation of construction information on wells inventoried in the USGS National Water Information System (NWIS) database (U.S. Geological Survey, 2018). Percentages were developed for each county based on the likelihood that a domestic well was in an aquifer.

\section{Commercial and Industrial Use}

Large, self-supplied commercial and industrial establishments - those withdrawing more than $100,000 \mathrm{gal} / \mathrm{d}$ reported annual water withdrawals to GaEPD (table 1), and these data were used to calculate county-level, major river-basin level, and aquifer-level withdrawals. Small, self-supplied commercial establishments (specifically those facilities that withdraw $<100,000 \mathrm{gal} / \mathrm{d}$ and have drinking water permits) such as campgrounds, marinas, State or county parks, restaurants, convenience stores, recreation areas, and recreational vehicle parks, were included in estimates of withdrawal for 2015. Water withdrawal estimates were not made for small, self-supplied commercial establishments without a drinking water permit for 2015 .

Compilations of commercial and industrial withdrawal locations (meaning wells or intakes) previously prepared for the Georgia Water-Use Program were used to estimate withdrawals by county, major river basin, and aquifer. Each location has a county, river basin, and aquifer assignment (the latter for wells only) allowing all withdrawals to be aggregated to the various summarizing zones. Industrial withdrawals were also compiled by major National American Industrial Classification System code (NAICS; appendix 1). Industrial withdrawal permits were associated with an NAICS as part of the Georgia Water-Use Program.

\section{Mining Use}

Most water withdrawn during mining operations is used for extracting minerals, quarrying, milling, and other standard preparations at mine sites. Granite, kaolin, fuller's earth, marble, and sand and gravel are mined extensively in the State, but these mining operations require relatively minor quantities of water. Although large amounts of water can be pumped to dewater mine pits and quarries, this water is not withdrawn for a beneficial use and is not included in the mining water-use estimates. However, this water is included as a surface-water return if it is discharged into a surface-water source. Kaolin and fuller's earth operations use large amounts of water to transport and process clay after extraction. Although some mining operations (especially kaolin and fuller's earth) had water withdrawal permits that indicated they withdrew more than $100,000 \mathrm{gal} / \mathrm{d}$, these withdrawals were included in the industrial-use category rather than the mining category because the water is not used in the extraction of the clay but in its processing.

For mining operations that withdrew less than $100,000 \mathrm{gal} / \mathrm{d}$, which is the threshold for a withdrawal permit, 2015 water-withdrawal estimates were provided for each Georgia county by the USGS NWUSP. A detailed description of the methods used for estimating water use during mining operations are given in Lovelace (2009b). Mine production data were used with a water-use coefficient to compute water use for mining in Georgia. Water-use coefficients for specific minerals are not presented in this 2015 water-use compilation to avoid disclosure of proprietary data provided by mining operations to the USGS.

Distributing county-level mining withdrawals to major river basins and aquifers within each county involved a geospatial assessment of the location information found 
on mining land-use permits and the review of construction information on wells inventoried in the USGS NWIS database (U.S. Geological Survey, 2018). Percentages were developed for each county based on the likelihood that a mining well was located in each major river basin and each aquifer.

\section{Irrigation}

Surface-water and groundwater withdrawals for crop and golf course irrigation in 2015 were estimated using multiple methods. These methods are related to different reporting requirements and the State-established collection methods used for each subcategory and subregion of Georgia.

\section{Crop Irrigation}

Crop irrigation estimates were calculated using three methods:

1. metered data from the State Agricultural Water Conservation and Metering Program, which provided per meter withdrawals for much of the crop irrigation in southern Georgia, and an agricultural lands polygon GIS dataset allowed that assignment to counties, major river basins, and aquifers;

2. unmetered irrigation in southern Georgia, which was estimated using the geostatistical techniques described in Torak and Painter $(2011,2013)$;

3. and for north Georgia, withdrawals were estimated using the water-use forecasts in the Georgia Statewide Water Plan.

In Georgia, crop irrigation is used primarily in the Coastal Plain province, where many irrigation systems are equipped with flowmeters that record cumulative withdrawals. The location of these meters, the number of acres they irrigate, and the groundwater aquifer source (for wells only) were provided with the withdrawal values. The distributions of metered and unmetered agricultural acres compiled for 2014 for southern Georgia were provided by the Georgia Department of Natural Resources (GaDNR) and termed "GaDNR Agricultural Lands" (Vicki Trent, Georgia Department of Natural Resources, written commun., April 26, 2016). These agricultural acreages are associated with farm water-use permits and were used to identify acres where there was the potential for water that could be used for crop irrigation in 2015. The GaDNR Agricultural Lands dataset is a static representation of acreages and was developed from previous growing season data.

Estimates of crop-irrigation withdrawals at unmetered locations were derived from metered data using geostatistical techniques (Torak and Painter, 2011, 2013). The geostatistical techniques of structural analysis, variogram development, kriging, and conditional simulation were applied to metered withdrawal values. Variogram models of irrigation depth, or rate of applied irrigation, were used at the metered sites in a coupled kriging-conditional simulation process to estimate the irrigation depth at unmetered agriculture fields. The derived irrigation-depth estimates were applied to the GaDNR Agricultural Lands using a GIS. The depths were multiplied by the unmetered, irrigated acreage to calculate a per field volume of irrigation withdrawal. Metered withdrawals and estimated withdrawals at unmetered fields were aggregated by county, major river basin, and aquifer to derive total withdrawals for crop irrigation.

North Georgia crop irrigation-water withdrawals were not included in the data provided by the State Agricultural Water Conservation and Metering Program; therefore, water withdrawals were estimated for 2015. Agricultural irrigation-water demand was forecast for groundwater and surface-water sources for the years 2011, 2020, 2030, 2040, and 2050 for each county in the Georgia Statewide Water Plan. Estimates were computed for average, above-average, and below-average precipitation during the growing season. These withdrawal projections are a product of the projected irrigation area for a crop (in acres), predicted monthly irrigation application depth (in inches), and the proportion of irrigation water derived from a water source (as a fraction for groundwater and surface water). Precipitation for the average growing season in Georgia was 34.1 inches between 1895 and 2014. In 2015, the growing season precipitation totaled 32.8 inches, but because most of the 2015 growing season in Georgia was normal when compared with the long-term average, the forecast for an average precipitation year was used for north Georgia counties when estimating withdrawals per county (fig. 5). Since there was not a forecast for 2015, an interpolated estimate for the period between the 2011 and 2020 forecasts was used for total withdrawals for the north Georgia counties. Water withdrawal estimates for each major river basin in north Georgia were calculated using a ratio of cropland from the 2015 Cropland Data Layer (U.S. Department of Agriculture, 2015) within each county and its associated major river basin (hereafter referred to as "county-major river basin"). That ratio was multiplied by the county withdrawal total to estimate withdrawals for each major river basin within each county. All estimates for each county-major river basin were summed together to get one total withdrawal per major river basin within the State. Aquifer spatial extents were used to determine aquifer assignments to groundwater withdrawals.

\section{Golf Course Irrigation}

Surface-water and groundwater withdrawals for golf course irrigation in 2015 were estimated using data from two sources: (1) farm water withdrawal permits and (2) nonfarm withdrawal permits. Both resources are managed by the GaEPD. The Georgia Groundwater Use Act (OCGA § 12-5-90) states that farm use shall include "the irrigation of recreational turf, except in Chatham, Effingham, Bryan, and Glynn counties, where irrigation of recreational turf shall not be considered farm use." Also, the 1988 amendments to the Georgia Water Quality Control Act (OCGA § 12-5-20) state that the irrigation of recreational turf "in the Chattahoochee 
River watershed upstream from [the] Peachtree Creek [confluence] where irrigation of recreational turf shall not be considered a farm use." Thus, in the areas where the irrigation of recreational turf is considered a nonfarm use, water withdrawals greater than $100,000 \mathrm{gal} / \mathrm{d}$ are permitted under Georgia's nonfarm water-withdrawal permit program. Under this permit program, according to the Georgia Water Quality Control Act, 42 golf courses received permits and were required to report water withdrawn in 2015. These data were summarized by county, major river basin, and aquifer for 2015 .

Estimated water withdrawals were calculated for golf courses with farm withdrawal permits (meaning those golf courses not required to report withdrawals) using irrigation demand computations developed from a partnership established with the GaEPD, Georgia Golf Course Superintendents Association (GGCSA), and the University of Georgia for the Georgia Statewide Water Plan (Waltz, 2008). Irrigation demand was estimated for above-average, average, and below-average rainfall conditions (Lewis, 2010). The recommended irrigation depth for turfgrass is 1 in. per week without rainfall (Waltz, 2008). Because Georgia has a 30-week growing season (April 1-October 31), 30 in. of water is the recommended golf course irrigation rate in Georgia for a dry year (Waltz, 2008). The average irrigation depth reported in the GGCSA best management practices program was 14.06 in. per year for 2004-2007 (Waltz, 2008). This average depth of $14.06 \mathrm{in}$. was considered the amount of water needed to irrigate golf courses in an average rainfall year and this value was used in 2015. For each GaEPD farm-permitted golf course, 14.06 in. was multiplied by the permitted acreage listed on each farm water-withdrawal permit to determine the amount of water needed to irrigate golf courses in an average rainfall year. Golf course locations were geocoded to attribute each course with a county-major river basin, which allowed for aggregating water withdrawals for each county-major river basin. For groundwater-sourced irrigation, an aquifer was usually included on the farm water-withdrawal permit. If the aquifer associated with a golf course was not known, the spatial extents of aquifers and an investigation of construction information on wells inventoried in the USGS NWIS database (U.S. Geological Survey, 2018) were used to assign an aquifer to a golf course irrigation-water source. Total groundwater withdrawals were aggregated by aquifer.

\section{Livestock and Aquaculture}

County and major river basin estimates of water use (cumulative for both groundwater and surface water) for livestock were supplied by Mark Masters, Albany State University, Georgia Water Planning \& Policy Center, in a format similar to the one used in the Statewide Water Plan (Georgia Environmental Protection Division, 2008; Masters and Rowles, 2016). The Georgia Water Planning \& Policy Center used data on the amount of water used for each animal group (in gallons per day per head) for 2015 and animal census data by county from the 2014 Farm Gate report (University of Georgia, 2015). The total daily water use for each animal group (goats, sheep, horses, beef cattle, dairy cattle, swine, and broilers) was computed by multiplying the estimated daily water use per animal group by the number of animals. This daily water use was multiplied by 365 to annualize the water use and then converted to million gallons per day. Water withdrawals by water source (either groundwater or surface water) were not explicitly provided by the Georgia Water Planning \& Policy Center and were estimated using the same county percentage of groundwater and surface-water withdrawals for crop irrigation.

The USGS NWUSP computed the 2015 water-use estimates for aquaculture in each Georgia county. A detailed description of the methods used for commercial and noncommercial aquaculture operations are given in Lovelace (2009a). In that report, county-level data on the number of farms using particular sources of water (meaning groundwater and surface water), pond acreage, the number of raceways and raceway flow rates, and the number of recirculating and non-recirculating tanks and their volumes were obtained from the National Agricultural Statistics Service (U.S. Department of Agriculture, 2014). In 2015, catfish and trout production were the predominant aquaculture enterprises in Georgia (University of Georgia, 2015).

Livestock and aquaculture water-withdrawal estimates for each county were disaggregated and reaggregated for each major river basin based on the percentage of cropland in the 2015 Cropland Data Layer (U.S. Department of Agriculture, 2015 ) within each river basin compared to total cropland in the county; exact locations of livestock and aquaculture operations were unknown. Aquifer assignments for groundwater withdrawals were identified by the same percentage of crop irrigation, by aquifer, within each county.

\section{Thermoelectric}

The 2015 water withdrawal data for thermoelectric facilities in Georgia were obtained from three sources (table 1): (1) Georgia Power Company monthly surface-water withdrawals, water discharge, and consumptive-use data for the thermoelectric facilities they own or operate; (2) permitted groundwater withdrawals from the GaEPD groundwaterwithdrawal permit program; and (3) USGS thermoelectric power model estimates. The methodology for the USGS model is described in Diehl and Harris (2014). Twenty-one thermoelectric power facilities (19 in Georgia; one each in Alabama and South Carolina, which have intakes located in Georgia) were permitted to withdraw water in 2015. Of the 21 facilities, 15 use fossil fuels (such as oil, coal, or natural gas), four use biomass (specifically wood based), and two use nuclear energy to generate electricity. Most of the water withdrawn for thermoelectric power generation is used for cooling.

Intake and well locations for these facilities, previously compiled for the Georgia Water-Use Program, were used to estimate withdrawals by county, major river basin, and aquifer. Each location has a county, river basin, and aquifer assignment (the latter for wells only) allowing all withdrawals within a zone to be summarized. 


\section{Public-Supplied Water}

For this report, public-supplied water is defined as water withdrawn, treated, and delivered to domestic (residential), commercial, and industrial customers in Georgia and adjacent States. Estimates of water deliveries to customer groups, as wholesale sales and system losses for 2015, were made using a methodology and use-category percentages identical to those reported in Lawrence (2016) and are provided in table 1 of the USGS data release by Painter (2019). Any water supplier (either a municipal or private entity) that regularly serves at least 25 people or has at least 15 water connections is considered a public water system under Georgia law (OCGA $\S 12-5-172-11)$. This definition includes large municipal, private, and county water systems, and small community water systems. Small community water systems commonly withdraw less than 100,000 gal/d (monthly average) of water and typically are not required to obtain a withdrawal permit, but they may be required to obtain a permit or certificate of use to deliver drinking water to customers under the Georgia Safe Drinking Water Act of 1977 (OCGA § 12-5-70-12-5170). Small community water systems include incorporated and unincorporated towns and cities, subdivisions, mobile home parks, and apartment or condominium complexes.

Compiling public-supplied water use for 2015 was a multi-tiered effort. Surface-water and groundwater withdrawals per withdrawal permit were obtained from GaEPD (table 1), which accounted for the large public supply systems in Georgia. A list of entities with a drinking water permit under the GaEPD Drinking Water Program was used to identify small community water systems and the water source used for withdrawal (whether it was from groundwater, surface water, or surface-water purchases). The drinking water permit information also provided information about the population served for systems that distribute water for domestic use (both large and small systems). This information was verified utilizing the U.S. Census Bureau's 2015 Estimates of Resident Populations for Incorporated Places (U.S. Census Bureau, 2016, 2017). For some communities, the data about populations served were adjusted to align with Census Bureau data if substantial differences were found. An assumption was made that new community residents were likely served water from a public water system, but the drinking water permit information had not been updated and, therefore, did not reflect the population increase. Water withdrawals were estimated for small-community water systems - the size of the population served was multiplied by a water-use coefficient of $75 \mathrm{gal} / \mathrm{d}$ per person. This water-use coefficient, or per capita water use, was derived from a survey of subdivisions in the Athens, Georgia, area in 1983, which showed that each person, on average, used 75 gallons of water per day (Fanning, 1997; Trent and others, 1990).

Public-supply water is not always withdrawn from the same county or major river basin in which the water is used. Defining the location and direction of surface-water withdrawals has been a component of the Georgia Water-Use
Program, which is cooperatively coordinated by the USGS and the GaEPD. In some instances, water is withdrawn from a river or stream in one county and conveyed to a reservoir in a different county. In those instances, if the amount of water withdrawn from the reservoir was less than the amount conveyed from the river or stream for a given month, then the amount withdrawn from the reservoir was attributed to the county where the withdrawal point was located on the river or stream. For example, if $5 \mathrm{Mgal} / \mathrm{d}$ of water were withdrawn from the Ocmulgee River in Bibb County and conveyed to Town Creek Reservoir in Jones County from which $2 \mathrm{Mgal} / \mathrm{d}$ were withdrawn for treatment and distribution, then the $5 \mathrm{Mgal} / \mathrm{d}$ were counted as a withdrawal from the Ocmulgee River in Bibb County. If the water withdrawn from the reservoir exceeded the amount conveyed from the river or stream, then the difference was attributed to the county where the reservoir was located, and the remainder attributed to the county where the conveyance from the river or stream occurred. For example, if $5 \mathrm{Mgal} / \mathrm{d}$ of water were withdrawn from the Ocmulgee River in Bibb County and conveyed to Town Creek Reservoir in Jones County, from which $8 \mathrm{Mgal} / \mathrm{d}$ were withdrawn for treatment and distribution, then $3 \mathrm{Mgal} / \mathrm{d}$ were counted as a withdrawal from Jones County, and $5 \mathrm{Mgal} / \mathrm{d}$ were counted as a withdrawal from Bibb County.

Water withdrawn by public water systems is distributed to domestic, industrial, and commercial customers. Domestic water use is water used for ordinary household purposes, such as drinking, food preparation, bathing, washing clothes and dishes, flushing toilets, washing cars, and watering lawns and gardens. Commercial establishments include motels, hotels, restaurants, medical centers, hospitals, military installations, public safety facilities (such as police stations and firehouses), correctional institutions, retail stores, (public and private) educational facilities, campgrounds, and recreational vehicle parks. Industrial establishments include those involved with the manufacturing and processing of chemicals, food, textiles, paper, wood, petroleum and allied products, fabricated metals, and machinery. The percentage of total county water withdrawals delivered to each customer group (domestic, commercial, and industrial) shown in this report is identical to the percentage reported in Lawrence (2016) (table 1 in Painter, 2019). For the 2010 report, these county delivery percentages were determined by results summarized from a 2010 water-use questionnaire, from 2010-11 comprehensive annual financial reports (CAFRs), and from the proportion stated in a 2005 water-use questionnaire, or they were estimated using water-use coefficients (Lawrence, 2016). Since deliveries were estimated using county percentages of withdrawals, delivery information in this report is not categorized by major river basin or aquifer.

\section{System Losses}

Nonrevenue deliveries include unbilled, authorized water use; unauthorized water use; meter inaccuracies and data errors; and storage and system losses (also known 
as distribution or conveyance losses) due to leakage (U.S. Environmental Protection Agency, 2010, p. 2-2). Unbilled, authorized deliveries include water deliveries to churches, charitable organizations, schools, and fire suppression; public connections, such as government office buildings, vehicle garages, and maintenance buildings; and public recreation areas such as parks, recreation complexes, athletic fields, and public golf courses. Unauthorized water deliveries often represent a theft of water. Nonrevenue deliveries by privately owned water suppliers are usually restricted to system losses and unauthorized water use. The percentage of system losses for 2015 were the same percentage per county as reported in the 2010 report, which were in turn estimated from information on the 2010 water-use questionnaires or in the 2010-11 CAFRs (Lawrence, 2016; table 1 in Painter, 2019). System losses ranged from about 4 to 32 percent of the total water entering the distribution system. If the actual system-loss values were unavailable, then the average system loss was estimated at 15 percent for public water suppliers and 5 percent for subdivisions. The 15-percent loss was the average for all water losses reported on the 2010 questionnaires or in the 2010-11 CAFRs. A 15-percent loss is similar to the numbers reported nationally by the U.S. Environmental Protection Agency (2010, p. A-3) and statewide by a consulting firm (CH2M HILL, 2007). The GWSA requires water providers serving 3,300 or more in population to conduct an annual water system audit and submit the audits to GaEPD (Georgia General Assembly, 2010). These data were not used for this report but could be used for system loss information in the future.

\section{Surface-Water Returns}

The amount of treated and untreated water discharged into rivers, streams, and reservoirs in Georgia (surface-water returns) during 2015 were provided by the GaEPD. Each entity with a discharge permit under Georgia's NPDES wastewater permit program is required to report average monthly discharges to the GaEPD. These discharge monitoring reports were the source of surface-water return data. These data required extensive quality assurance to ensure reported wastewater returns were accurate and consistent. The reported wastewater returns were summarized and compiled for each county, major river basin, and water-planning region in Georgia.

\section{Water Withdrawals, Estimated Water Use, and Surface-Water Returns}

Total statewide water use for 2015 was estimated at 3,384 million gallons per day (Mgal/d). Of that amount, 2,231 Mgal/d were withdrawn from surface water and 1,153 Mgal/d from groundwater (fig. 7; table 2). Withdrawals made by public-water suppliers, thermoelectric power operations, and crop irrigation equate to 78 percent of total statewide withdrawals in 2015 (fig. 8). Thermoelectric power operations primarily withdraw from surface water (more than 99 percent) as do public water suppliers (78 percent), while crop-irrigation water is primarily withdrawn from groundwater (78 percent) (fig. 8; table 2). Of the $2,540 \mathrm{Mgal} / \mathrm{d}$ of water withdrawn for non-thermoelectric uses in 2015, about 62 percent was self-supplied water (1,570 Mgal/d; table 2). Although surface water is used throughout the State, surface water was sourced more frequently in north Georgia and around major cities such as Atlanta, Savannah, and Augusta (figs. 1, 7). Similarly, groundwater had a distinctly higher use in south Georgia (below the Fall Line) and was used primarily for irrigation (figs. 2, 7).

According to the U.S. Census Bureau (U.S. Census Bureau, 2016; table 2), over 10 million people lived in Georgia in 2015. Public water suppliers served the water for 85 percent of the population. Water withdrawals provide information on the quantity of water removed from the natural source; however, knowledge on where water goes, how it is used, and if it returns to a surface-water source is a description of water use. Therefore, water use is the amount of self-supplied water plus the amount delivered by public water systems. In 2015, water used for domestic purposes was $765.2 \mathrm{Mgal} / \mathrm{d}$, equating to 74.91 gallons per day per person; commercial uses accounted for $214.8 \mathrm{Mgal} / \mathrm{d}$, and industrial uses totaled 576.2 Mgal/d (table 2).

Self-supplied industrial withdrawals (475.5 Mgal/d) are organized by NAICS category (table 3 ). Statewide, paper and pulp facilities withdrew 77 percent of the total withdrawals by self-supplied industries (367.7 Mgal/d). Kaolin and ball clay withdrawals ranked second among NAICS classified industries (53.31 Mgal/d), followed by chemical products (30.25 Mgal/d) (table 3). Kaolin and ball clay facilities are primarily located in middle Georgia (south of the Fall Line) and withdraw more groundwater (35.49 Mgal/d) than surface water $(17.82 \mathrm{Mgal} / \mathrm{d})$. In contrast, 66 percent of withdrawals from paper and pulp facilities come from surface-water sources.

About 1,571 Mgal/d of water was returned to surfacewater bodies in Georgia, representing about 46 percent of the total water withdrawn in 2015 (table 2). Public water-supply facilities accounted for 63 percent of surface-water returns. Industrial facilities discharged $380.6 \mathrm{Mgal} / \mathrm{d}$ (24 percent of total returns). Thermoelectric power facilities discharged 109.8 $\mathrm{Mgal} / \mathrm{d}$ (7 percent of total returns). 


\section{$\boldsymbol{A}$}

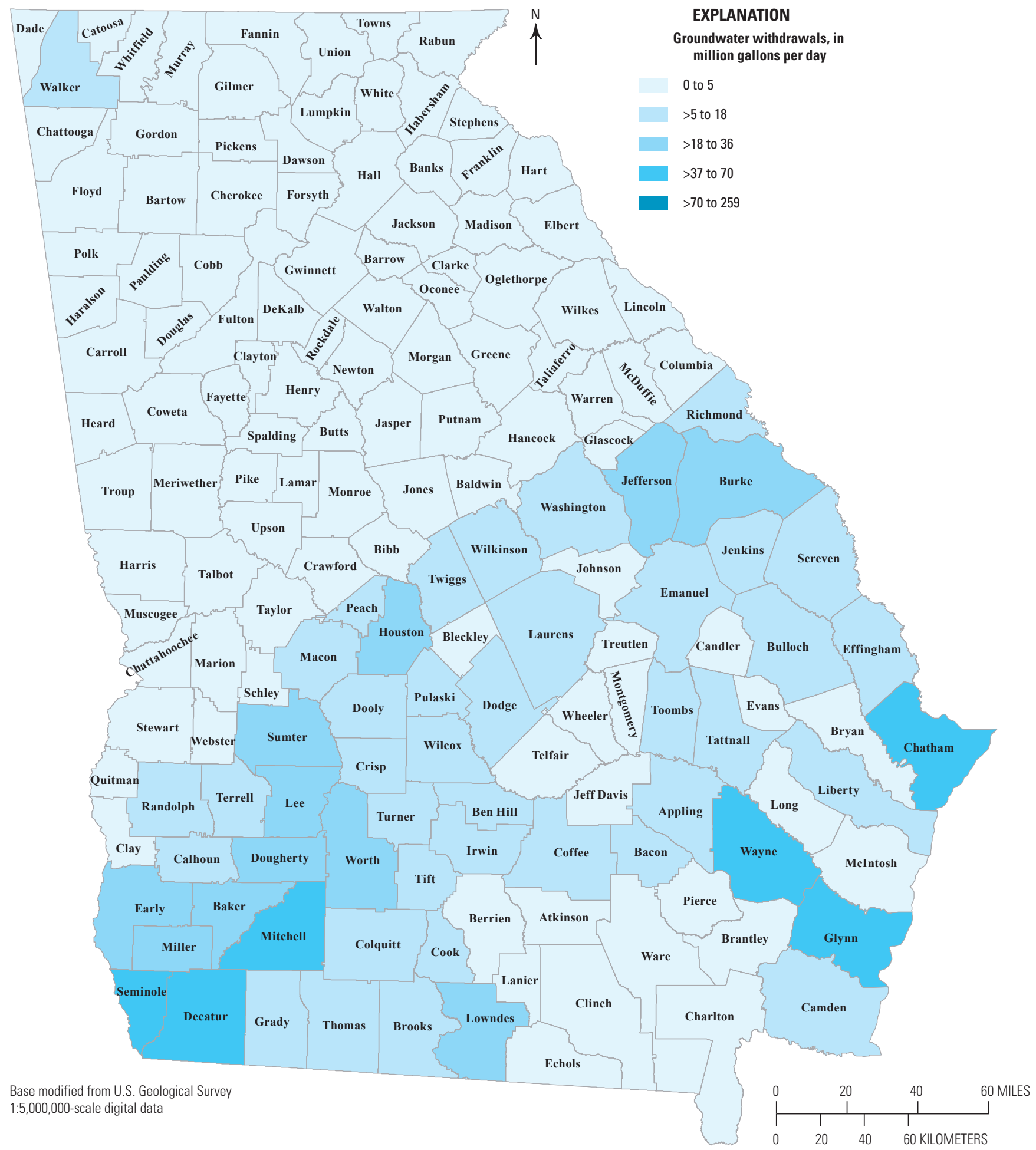

Figure 7. Maps of Georgia showing, by county for $2015,(A)$ groundwater withdrawals, $(B)$ surface-water withdrawals, and (C) groundwater and surface-water withdrawals. 


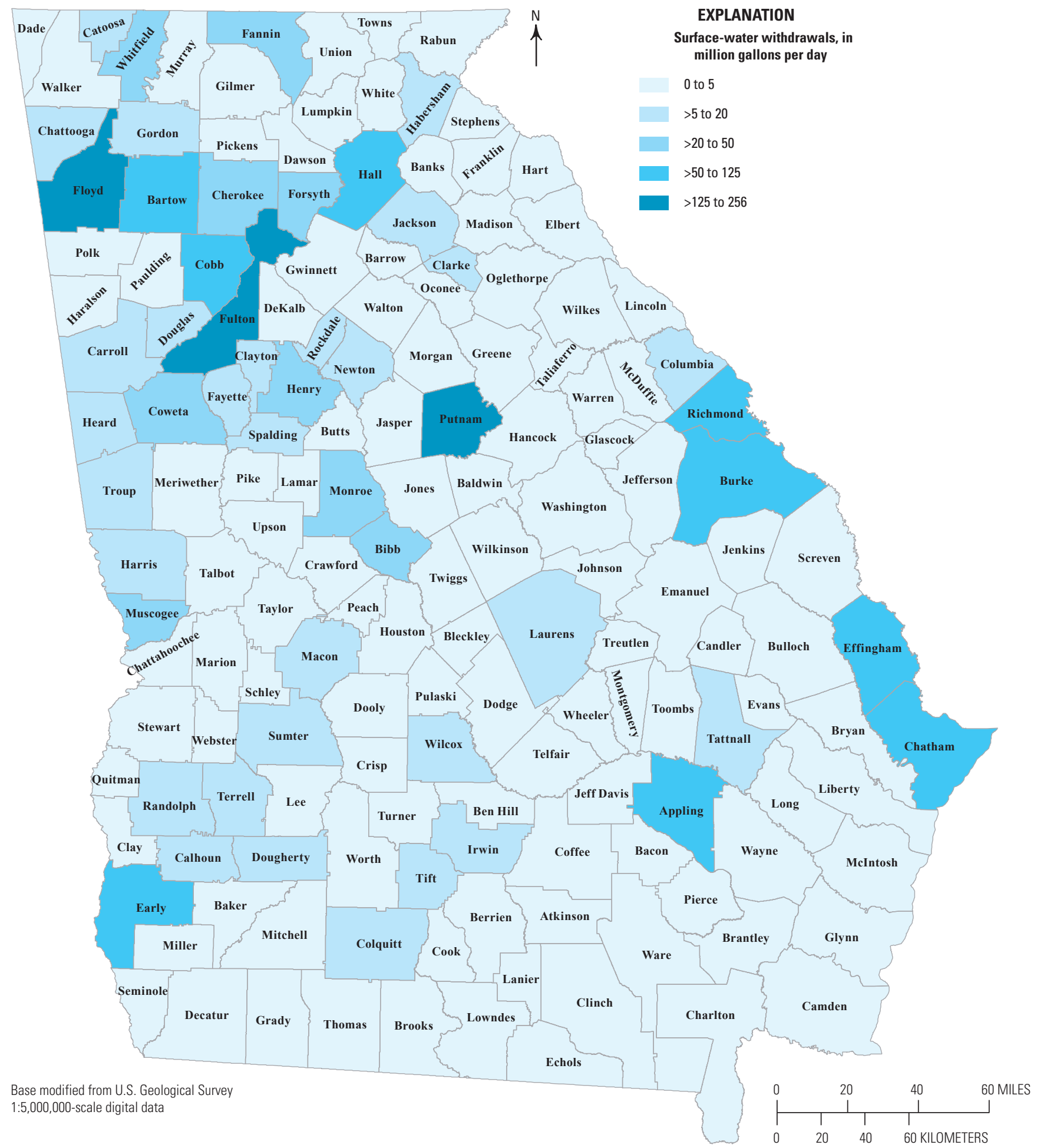

Figure 7. - Continued 
C

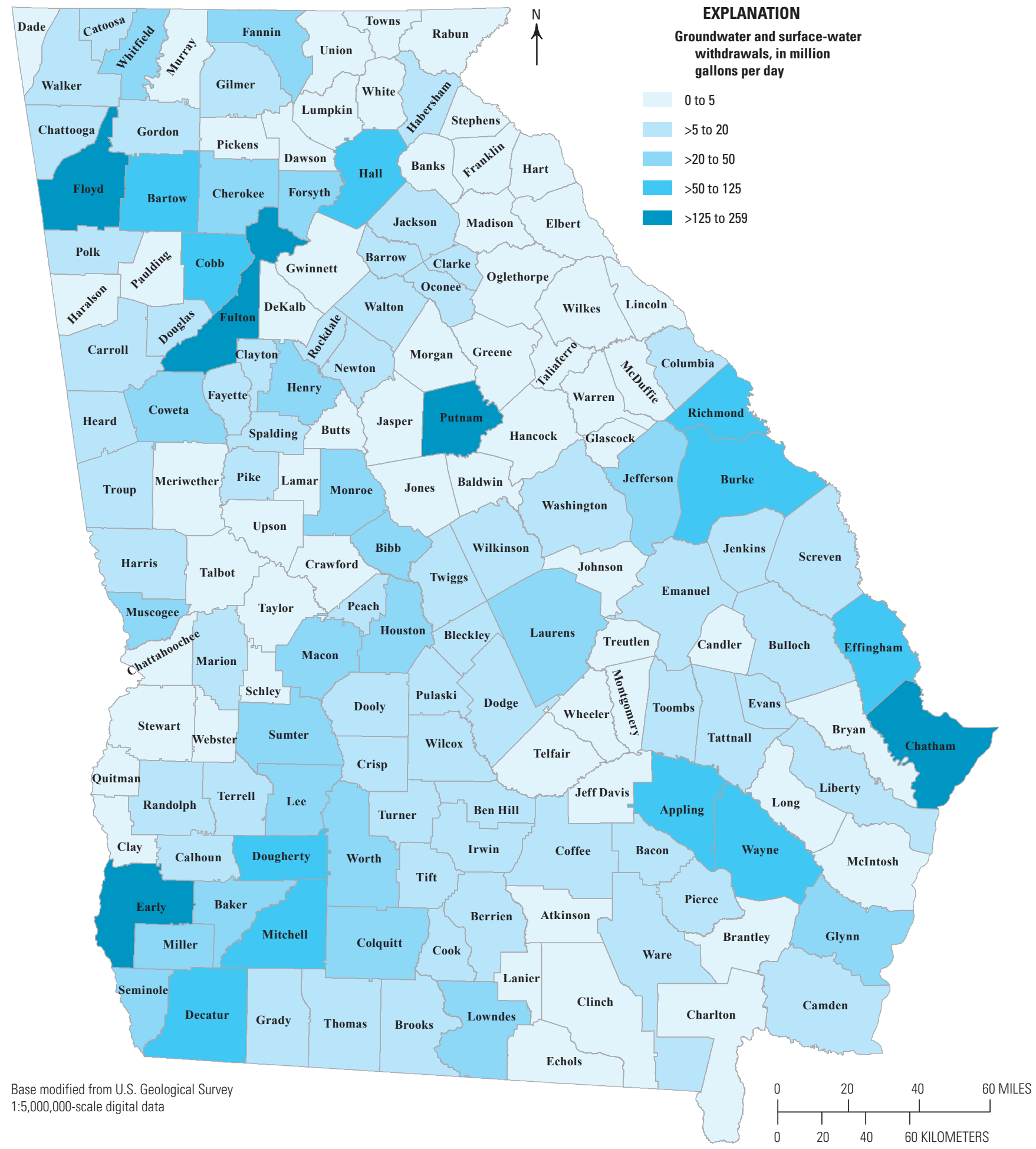

Figure 7. - Continued 
Table 2. 2015 total population, and water withdrawals by source and water-use category, in Georgia.

[For 2015, the total population of Georgia was 10,214,860. Of the total population, 8,700,463 were served by public supply, which includes $1,753,976$ using groundwater and 6,946,487 using surface water. The population using self-supplied groundwater was 1,514,397. The total per capita water use was 331 gallons per person per day ${ }^{1}$ For 2015, irrigation covered 1,445,880 acres. Mgal/d, million gallons per day; -, not applicable]

\begin{tabular}{|c|c|c|c|c|c|c|}
\hline \multirow{2}{*}{ Category } & \multicolumn{3}{|c|}{ Water withdrawals, in Mgal/d } & \multirow{2}{*}{$\begin{array}{c}\text { Deliveries } \\
\text { from public } \\
\text { supply, in Mgal/d² }\end{array}$} & \multirow{2}{*}{$\begin{array}{l}\text { Total use, in } \\
{\mathrm{Mgal} / \mathrm{d}^{3}}^{3}\end{array}$} & \multirow{2}{*}{$\begin{array}{l}\text { Surface-water } \\
\text { returns, in Mgal/d }\end{array}$} \\
\hline & Groundwater & Surface water & Total & & & \\
\hline Public supply & 230.9 & 839.1 & 1,070 & - & - & - \\
\hline Domestic use & 104.5 & 0 & 104.5 & 660.7 & 765.2 & - \\
\hline $\begin{array}{l}\text { Commercial/public } \\
\text { used }^{4}\end{array}$ & 2.5 & 3.21 & 5.71 & 209.1 & 214.8 & 10.61 \\
\hline $\begin{array}{l}\text { Public supply system } \\
\text { losses }^{5}\end{array}$ & - & - & - & 99.52 & 99.50 & - \\
\hline Inter-county deliveries & - & - & - & - & 96.47 & - \\
\hline $\begin{array}{l}\text { Public wastewater } \\
\text { treatment }\end{array}$ & - & - & - & - & - & 993.7 \\
\hline $\begin{array}{l}\text { Livestock and } \\
\text { aquaculture }\end{array}$ & 42.08 & 87.92 & 130 & - & 130.0 & 1.16 \\
\hline $\begin{array}{l}\text { Thermoelectric power } \\
\text { use }\end{array}$ & 3.43 & 839.8 & 843.2 & - & 843.2 & 109.7 \\
\hline TOTAL & 1,153 & 2,231 & 3,384 & 970.5 & 3,481 & 1,571 \\
\hline
\end{tabular}

${ }^{1}$ Sum of withdrawals by all categories divided by the total 2015 population

${ }^{2} \mathrm{~A}$ net transfer of about $6.7 \mathrm{Mgal} / \mathrm{d}$ of public-supplied water occurred from Alabama and Tennessee to Georgia in 2015.

${ }^{3}$ Total use is total withdrawal plus public supply deliveries.

${ }^{4}$ Public use includes self-supplied withdrawals and unbilled public water delivered to municipal buildings, parks, golf courses, schools, churches, and other public facilities.

${ }^{5}$ Public-supply system losses consist of water lost from the water distribution system and includes pipe leaks and breaks, fire suppression, and unauthorized use. 


\section{A. Groundwater withdrawals (1,153 Mgal/d)}

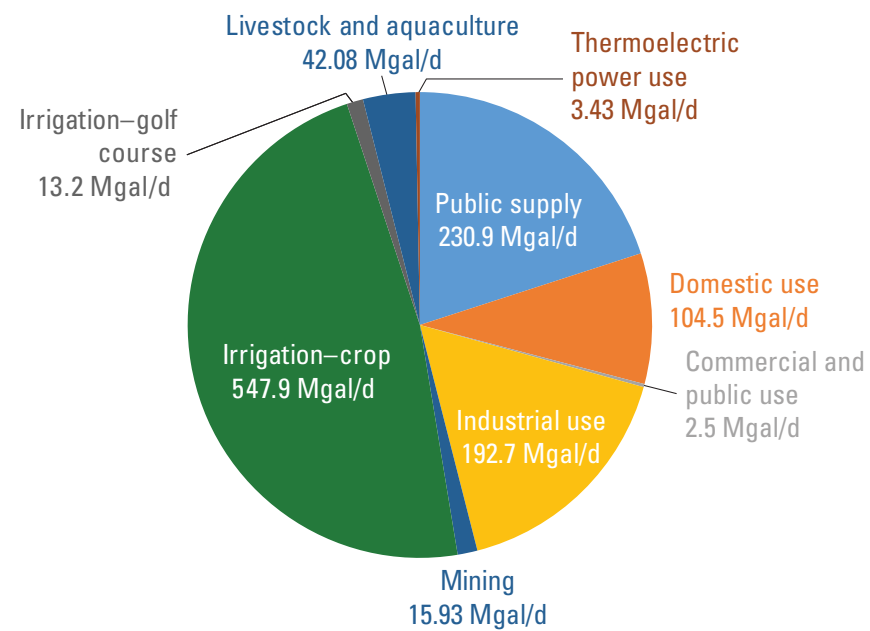

C. Total withdrawals $(3,384 \mathrm{Mgal} / \mathrm{d})$

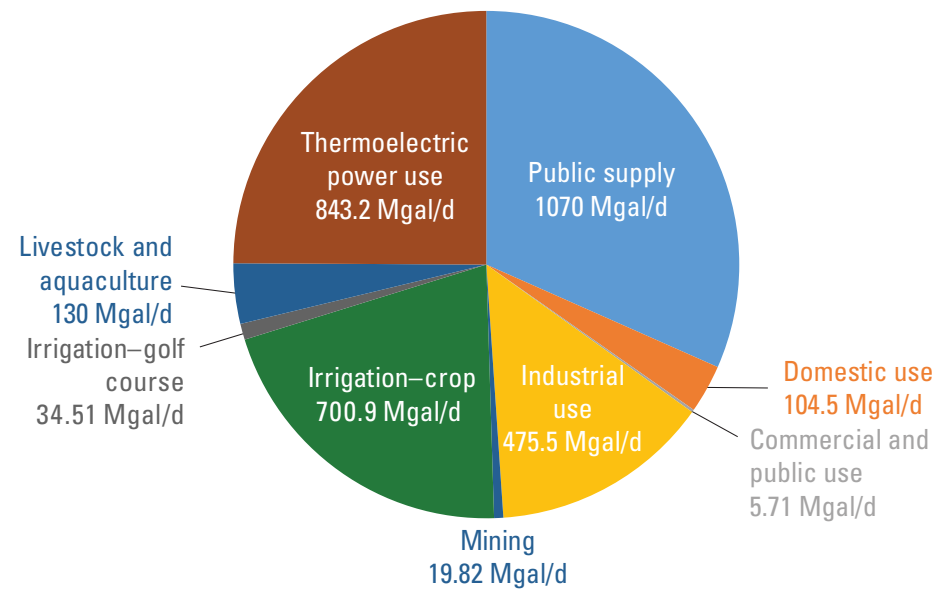

B. Surface water withdrawals $(2,231 \mathrm{Mgal} / \mathrm{d})$

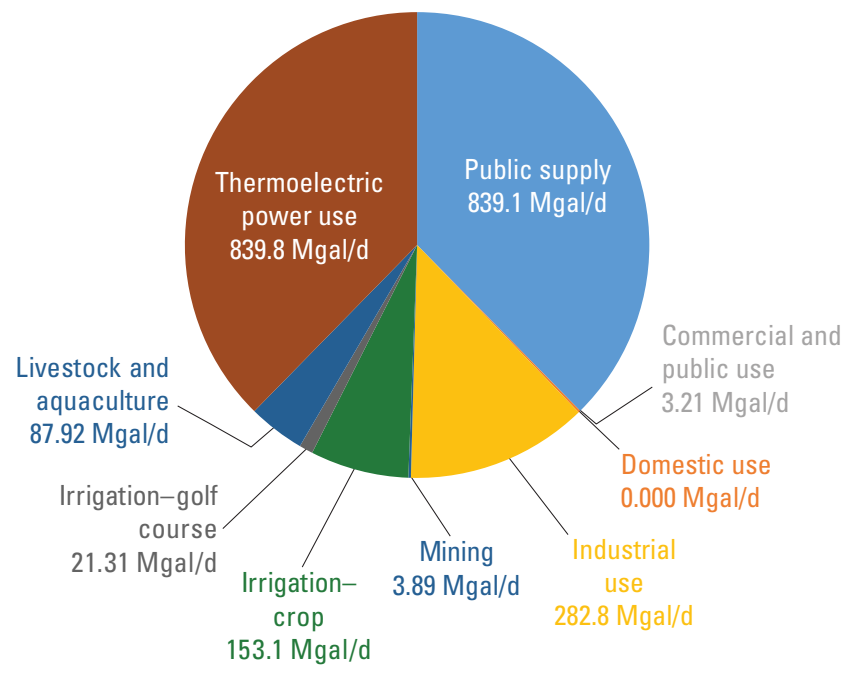

Figure 8. Water withdrawals in Georgia by source and water-use category for 2015, in million gallons per day (Mgal/d). 
Table 3. Permitted commercial and industrial water withdrawals by major North American Industrial Classification code (NAICS, appendix 1) in Georgia, 2015.

\begin{tabular}{|c|c|c|c|}
\hline \multirow{2}{*}{$\begin{array}{c}\text { NAICS } \\
\text { (Appendix 1) }\end{array}$} & \multicolumn{3}{|c|}{ Withdrawals, in million gallons per day } \\
\hline & Groundwater & Surface water & Total \\
\hline 322-Paper, pulp & 126.5 & 241.2 & 367.7 \\
\hline 212-Kaolin and ball clay & 35.49 & 17.82 & 53.31 \\
\hline 325 - Chemical products & 11.54 & 18.71 & 30.25 \\
\hline 311-Food & 6.67 & 0.00 & 6.67 \\
\hline 313,314-Textiles & 1.90 & 6.81 & 8.71 \\
\hline 221-Electrical utility & 0.07 & 0.00 & 0.07 \\
\hline 327-Stone, clay & 2.45 & 0.11 & 2.56 \\
\hline 312-Beverage manufacturing & 2.16 & 0.00 & 2.16 \\
\hline 321-Lumber and wood products & 2.49 & 0.00 & 2.49 \\
\hline 331-Primary metals & 0.40 & 0.09 & 0.49 \\
\hline 339-Miscellaneous manufacturing & 0.49 & 0.00 & 0.49 \\
\hline 326-Rubber, plastic & 0.29 & 0.00 & 0.29 \\
\hline Total & 190.5 & 284.7 & 475.2 \\
\hline All other industries & 0.31 & 0.00 & 0.31 \\
\hline
\end{tabular}

\section{Water Use and Surface-Water Returns by County}

The primary reporting unit for water-use information is found at the county level so that each county can be compared with other counties in the United States as part of the USGS National Water-Use Science Project and for historical comparisons in Georgia since 1980 (fig. 1). On a county basis, in 2015, total water withdrawals for all use categories ranged from less than $1 \mathrm{Mgal} / \mathrm{d}$ in Taliaferro County to $258 \mathrm{Mgal} / \mathrm{d}$ in Floyd County (table 2 in Painter, 2019). The spatial distributions of water use, by category, are shown in figure 9, and surfacewater returns are shown in figure 10. Domestic, commercial, industrial, and thermoelectric power water-use distribution maps, by county, reflect the surface-water return map because collectively, the higher water-use areas (greater than $5 \mathrm{Mgal} / \mathrm{d}$ ) in each of these use categories are located around large population centers, industrial facilities, or thermoelectric power operations. Of the $8,700,463$ persons receiving public-supplied water in 2015, Fulton, Gwinnett, DeKalb, Cobb, Chatham, Clayton, Cherokee, Muscogee, and Forsyth Counties provided water to 51 percent of the people (table 2) via public-supplied sources. Seven of the nine counties are in metropolitan Atlanta, and the eighth highest by use county, Chatham County, represents the Savannah metropolitan area. Water withdrawal, water use, and surface-water returns for each of the 159 Georgia counties are provided graphically in appendix 2 in individual report pages. Each entry in appendix 2 details water-use categories, major industrial-sector withdrawals, top water-supply systems in the county, the division of withdrawal by water source (groundwater and surface water), the division of groundwater withdrawal by principal aquifer, and water-withdrawal trends by water source for 1985-2015. 
A

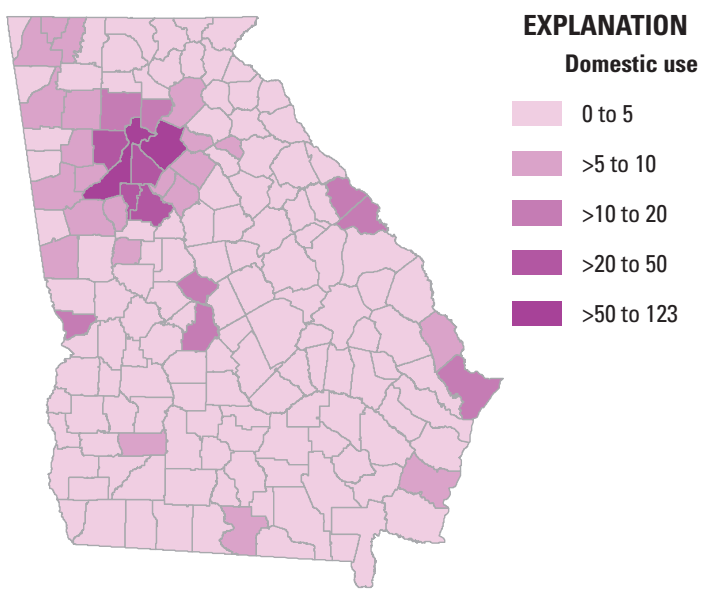

C

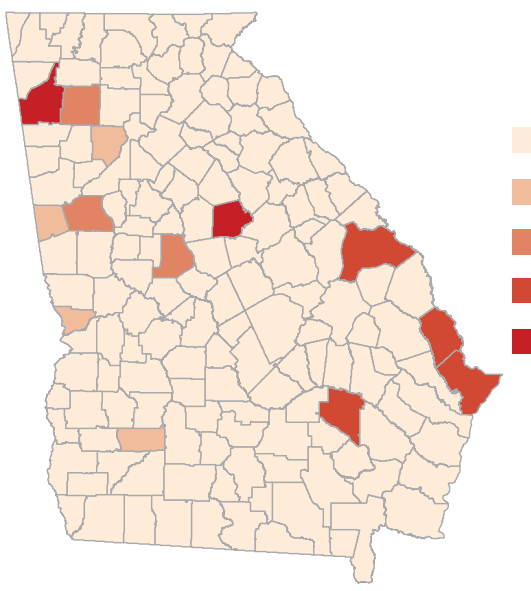

E

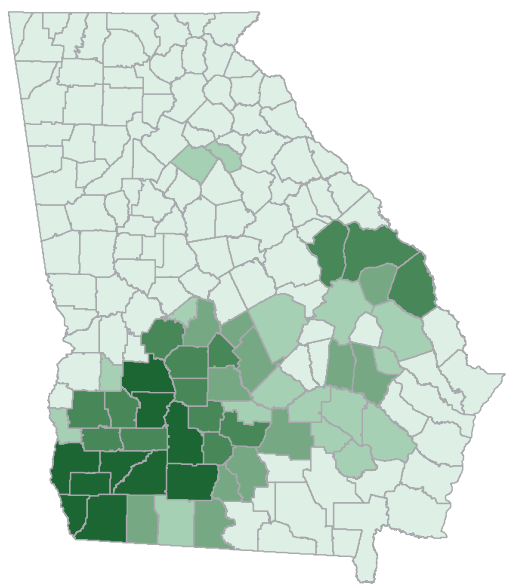

EXPLANATION

Irrigation-crop

0 to 2

$>2$ to 5

$>5$ to 10

$>10$ to 20

$>20$ to 56
EXPLANATION

Thermoelectric

power use

0 to 5

$>6$ to 20

$>20$ to 50

$>50$ to 125

$>125$ to 211
B

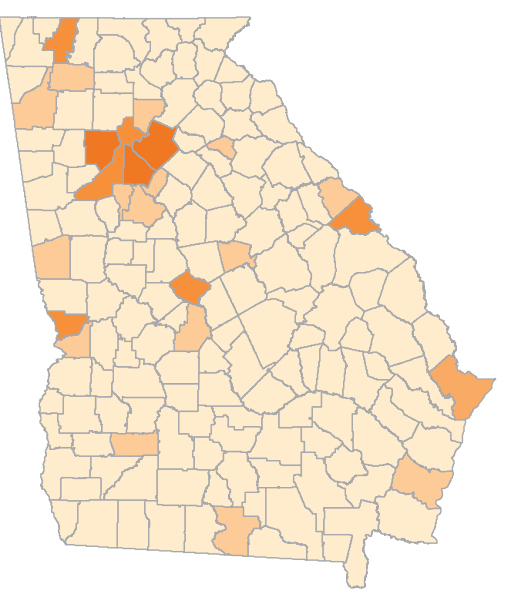

D
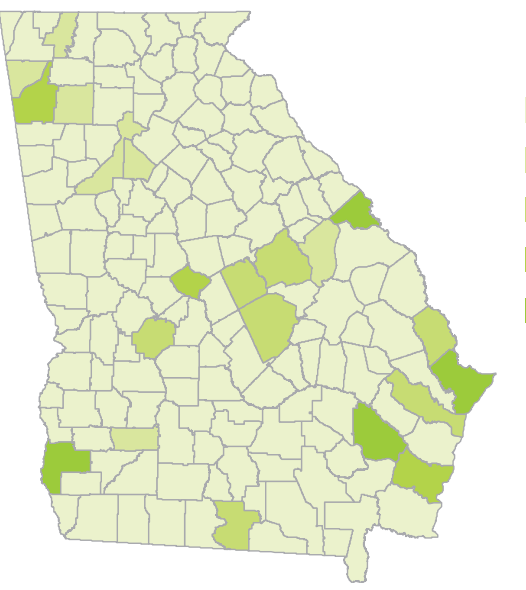

EXPLANATION

Commercial use

0 to 2

$>2$ to 4

$>4$ to 6

$>6$ to 15

$>15$ to 23
EXPLANATION

Industrial use and mining

0 to 2

$>2$ to 4

$>4$ to 6

$>6$ to 15

$>15$ to 23
$\boldsymbol{F}$

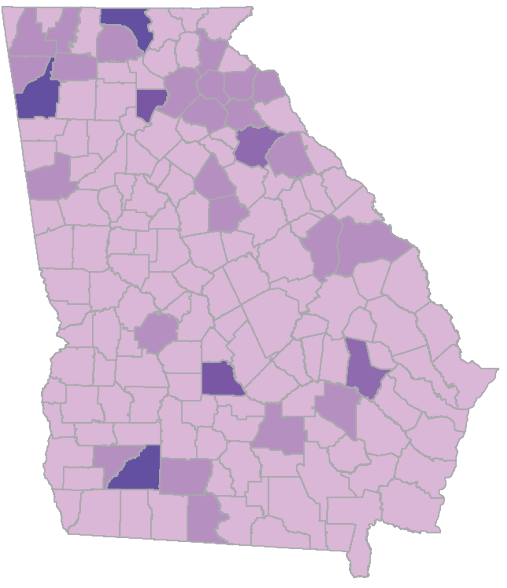

EXPLANATION

Livestock and aquaculture

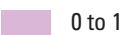

2

$>2$ to 5

$>5$ to 10

$>10$ to 44
G

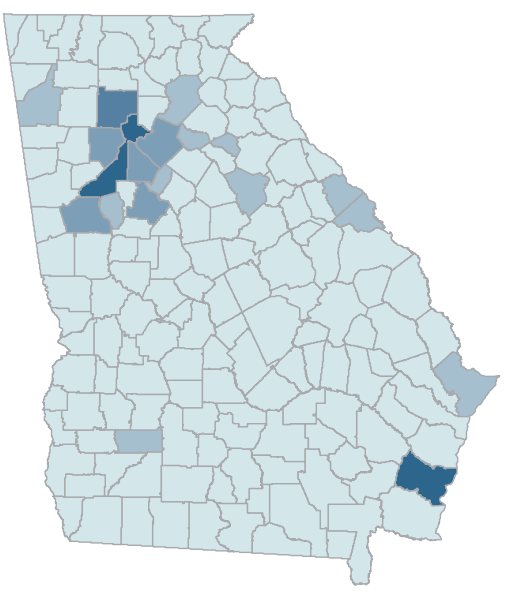

EXPLANATION

Irrigation-golf course

0.0 to 0.5

$>0.05$ to 1.0

$>1.0$ to 1.5

$>1.5$ to 2.0

$>2.0$ to 2.5

Figure 9. Water withdrawals in Georgia by water-use category, by county, for 2015 . 


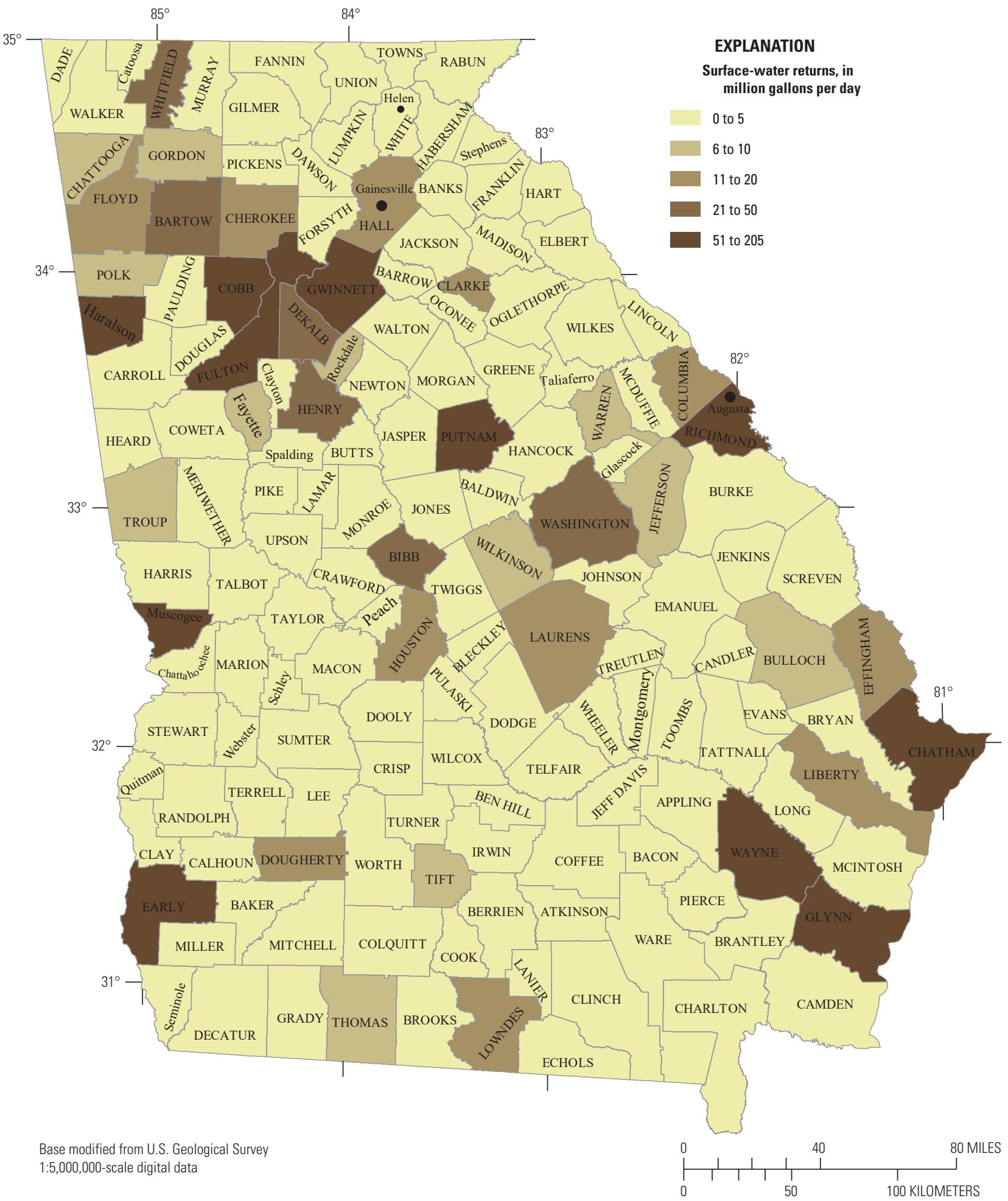

Figure 10. Surface-water returns by Georgia county for 2015. 


\section{Water Use and Surface-Water Returns by Water-Planning Region}

Water-planning regions are units in which water-use forecasts were developed for Georgia and regions where conservation and other management strategies are implemented (Georgia Environmental Protection Division, 2008); therefore, water withdrawals, water use, and surfacewater returns are compiled for each of those regions (fig. 4). Each of the 11 water-planning regions contains a unique set of counties, so compiling water withdrawals, water use, and surface-water return information by water-planning region is the task of aggregating the county data into each water-planning region (table 4). Compiling these data for the different regions of Georgia aids in visualizing how water is used in different parts of the State. Figure 11 shows the counties in each water-planning region, ranked by both total water withdrawal and how the withdrawals are subdivided by water source (groundwater and surface water). This distribution illustrates that north Georgia regions (CoosaNorth Georgia, Metro-North Georgia, Middle Chattahoochee, and Upper Oconee) use more surface water than south Georgia regions (Suwannee-Satilla, Upper Flint, Lower Flint-Ochlockonee, and Altamaha), where groundwater is the primary source.

Across the 11 water-planning regions in Georgia, the highest percentage of use, by water-use category, varies widely. Total water withdrawals from public-supply systems for each planning region range from 6 percent in the Lower Flint-Ochlockonee (35.13 Mgal/d) and Altamaha (14.20 Mgal/d) regions to 82 percent in the Metro-North Georgia (510.9 Mgal/d) region (fig. 12). The Middle Chattahoochee region withdraws the lowest percentage of water $(0.14$ percent; $0.17 \mathrm{Mgal} / \mathrm{d})$ for industrial use, while the Altamaha (59.73 Mgal/d), Coastal Georgia (95.29 Mgal/d), and Savannah-Upper Ogeechee $(75.53 \mathrm{Mgal} / \mathrm{d})$ regions have the highest percentage (about 25 percent) of water withdrawn for industrial use. The Lower Flint (359.2 Mgal/d), Upper Flint (68.60 Mgal/d), and Suwanee-Satilla (91.23 Mgal/d) regions use more than half of their entire water withdrawals for irrigation (crop and golf course). The Upper Oconee region uses 60 percent $(180.9 \mathrm{Mgal} / \mathrm{d})$ of its total withdrawals, followed by the Coosa-North Georgia region, which uses 51 percent $(211.1 \mathrm{Mgal} / \mathrm{d})$ of its withdrawals for thermoelectric power cooling. Water withdrawals for mining activities are relatively small in Georgia, representing less than 1 percent of water withdrawals in each region except for in the Upper Flint (3.6 percent; $4.32 \mathrm{Mgal} / \mathrm{d}$ ), Middle Ocmulgee (1.6 percent; $3.05 \mathrm{Mgal} / \mathrm{d}$ ) and Savannah-Upper Ogeechee (1.1 percent; $3.24 \mathrm{Mgal} / \mathrm{d}$ ). The Coosa-North Georgia region has the highest percentage of livestock and aquaculture withdrawals, compared to other regions, with $61.38 \mathrm{Mgal} / \mathrm{d}$ (or nearly 15 percent) of total withdrawals.

Surface-water dischargers return the most water to surface-water bodies in the Metro-North Georgia waterplanning region with $476.7 \mathrm{Mgal} / \mathrm{d}$, followed by the Upper
Oconee (189.6 Mgal/d) and then the Coastal Georgia (176.9 Mgal/d) regions (table 5). Collectively, these three water-planning regions return 38 percent of all surface-water withdrawals $(2,231 \mathrm{Mgal} / \mathrm{d})$ in the State.

\section{Water Use and Surface-Water Returns by Major River Basin}

There are 11 major river basins for which statewide withdrawals were compiled in 2015 (fig. 3). Reporting water withdrawals and return data by river basin aligns water use with other water-budget parameters (meaning precipitation, streamflow, evapotranspiration, and changes in groundwater storage) often compiled in the same units. Water withdrawals were highest in the Apalachicola Basin (1,173 Mgal/d), which had the highest use category of public-supply withdrawals (509.6 Mgal/d), followed by irrigation (398.7 Mgal/d) and industry (128.7 Mgal/d) (figs. 13, 14; table 6). The basin with the least volume of water withdrawn is the Ochlocknee Basin (32.78 Mgal/d), where withdrawals are primarily used for irrigation. The Savannah Basin ranks second in total withdrawals (518.2 Mgal/d), with the highest use categories being thermoelectric power cooling (238.5 Mgal/d) and industry (126.8 Mgal/d). The Coosa and Tallapoosa Basins has more water withdrawn for thermoelectric power cooling $(244.9 \mathrm{Mgal} / \mathrm{d})$ than any other basin, and yet thermoelectric power cooling use in the Oconee Basin accounts for 65 percent of total withdrawals for that basin. The Suwanee and Aucilla Basins rank second in total irrigation withdrawal (100.9 Mgal/d) behind the Apalachicola Basin. The basin with the largest withdrawals for the livestock and aquaculture category is the Tennessee Basin (44.62 Mgal/d). Mining water use is greatest in the Apalachicola Basin, which accounts for 37 percent of total statewide withdrawals for mining operations, and second highest in the Oconee Basin, which accounts for 18 percent statewide.

Surface-water withdrawals for thermoelectric power cooling accounted for around 38 percent of all surface-water withdrawals in 2015 (table 2). The thermoelectric power cooling from the Branch, Hatch, and Scherer plants - in the Oconee River Basin-withdrew 225.3 Mgal/d in 2015. Within the Coosa and Tallapoosa River Basins, the Bowen and Hammond plants withdrew $244.9 \mathrm{Mgal} / \mathrm{d}$ of surface water. In the Savannah River Basin, the McIntosh, Vogtle, and Kraft plants withdrew 238.5 Mgal/d (table 6).

The river basins in which the greatest amount of water was returned to surface water are the Apalachicola (625.6 Mgal/d), followed by the Savannah (220.7 Mgal/d), the Coosa and Tallapoosa (183.5 Mgal/d), and the Ocmulgee (142.4 Mgal/d) (table 7). The largest use category in the Apalachicola (80 percent), Ocmulgee (93 percent), and Coosa and Tallapoosa (72 percent) River Basins is water returned by public wastewater facilities, while the largest use category for discharging water into the Savannah River Basin is industry (49 percent; table 7). 
Table 4. Water withdrawals by water-use category for water-planning regions in Georgia, 2015.

[Mgal/d, million gallons per day]

\begin{tabular}{|c|c|c|c|c|c|c|c|c|c|c|c|c|c|c|c|c|}
\hline \multirow[b]{2}{*}{$\begin{array}{l}\text { Water-planning } \\
\text { region } \\
\text { (fig. 4) }\end{array}$} & \multirow{2}{*}{$\begin{array}{l}\text { Public- } \\
\text { supplied } \\
\text { opulation } \\
\text { (thousands) }\end{array}$} & \multirow{2}{*}{$\begin{array}{c}\text { Self- } \\
\text { supplied } \\
\text { population } \\
\text { (thousands) }\end{array}$} & \multicolumn{9}{|c|}{ Withdrawals, in Mgal/d } & \multicolumn{5}{|c|}{ Public-supply deliveries, in Mgal/d } \\
\hline & & & $\begin{array}{l}\text { Public } \\
\text { supply }\end{array}$ & $\begin{array}{c}\text { Domestic } \\
\text { use }\end{array}$ & $\begin{array}{l}\text { Commercial } \\
\text { use }\end{array}$ & $\begin{array}{c}\text { Industrial } \\
\text { use }\end{array}$ & Mining & Irrigation & $\begin{array}{l}\text { Livestock } \\
\text { and } \\
\text { aquaculture }\end{array}$ & Thermoelectric & $\begin{array}{c}\text { Total } \\
\text { withdrawal }\end{array}$ & $\begin{array}{c}\text { Domestic } \\
\text { use }\end{array}$ & $\begin{array}{c}\text { Commercial } \\
\text { and public } \\
\text { use }\end{array}$ & $\begin{array}{l}\text { Industrial } \\
\text { use }\end{array}$ & $\begin{array}{l}\text { System } \\
\text { losses' }\end{array}$ & $\begin{array}{c}\text { Total } \\
\text { deliveries }^{2}\end{array}$ \\
\hline $\begin{array}{l}\text { Coosa-North } \\
\text { Georgia }\end{array}$ & 635.9 & 122.3 & 90.09 & 9.19 & 0.29 & 34.64 & 0.74 & 5.10 & 61.38 & 211.1 & 412.5 & 47.36 & 18.78 & 16.80 & 7.15 & 82.94 \\
\hline $\begin{array}{l}\text { Middle } \\
\text { Chattahoochee }\end{array}$ & 423.3 & 65.24 & 69.03 & 4.90 & 2.68 & 0.17 & 0.30 & 23.36 & 1.92 & 20.50 & 122.9 & 33.11 & 11.29 & 9.72 & 14.91 & 54.12 \\
\hline $\begin{array}{l}\text { Metro-North } \\
\text { Georgia }^{3}\end{array}$ & $4,936.3$ & 313.6 & 510.9 & 14.90 & 0.01 & 3.99 & 2.33 & 16.85 & 8.31 & 66.17 & 623.4 & 368.7 & 101.1 & 27.86 & 13.13 & 497.7 \\
\hline Upper Flint & 158.7 & 83.40 & 24.84 & 6.25 & 0.23 & 13.38 & 4.32 & 68.60 & 3.71 & 0.00 & 121.3 & 18.84 & 4.81 & 1.19 & 0.00 & 24.84 \\
\hline Middle Ocmulgee & 459.2 & 119.9 & 75.53 & 8.99 & 0.0 & 26.46 & 3.05 & 26.39 & 1.67 & 44.39 & 186.5 & 45.21 & 12.73 & 4.09 & 13.50 & 62.03 \\
\hline Upper Oconee & 436.2 & 138.6 & 46.56 & 10.41 & 0.01 & 37.77 & 2.99 & 16.36 & 4.67 & 180.9 & 299.7 & 28.57 & 12.80 & 4.71 & 0.48 & 46.08 \\
\hline $\begin{array}{l}\text { Savannah-Upper } \\
\text { Ogeechee }\end{array}$ & 468.8 & 159.1 & 75.88 & 11.93 & 0.24 & 75.53 & 3.24 & 58.23 & 11.21 & 66.60 & 302.9 & 34.31 & 19.93 & 7.31 & 14.33 & 61.55 \\
\hline $\begin{array}{l}\text { Lower Flint- } \\
\text { Ochlockonee }\end{array}$ & 229.2 & 121.8 & 35.13 & 9.14 & 0.40 & 115.4 & 1.65 & 359.2 & 18.07 & 18.95 & 557.9 & 18.40 & 7.23 & 3.01 & 6.49 & 28.64 \\
\hline Suwannee-Satilla & 250.8 & 159.4 & 35.34 & 11.95 & 0.22 & 13.14 & 0.51 & 91.23 & 4.20 & 0.00 & 156.6 & 20.73 & 7.00 & 2.64 & 4.97 & 30.37 \\
\hline Altamaha & 125.9 & 125.5 & 14.20 & 9.40 & 0.99 & 59.73 & 0.15 & 60.37 & 14.26 & 60.78 & 219.9 & 8.25 & 2.73 & 1.01 & 2.21 & 11.99 \\
\hline Coastal Georgia & 576.2 & 105.5 & 92.53 & 7.42 & 0.64 & 95.3 & 0.54 & 9.73 & 0.60 & 173.8 & 380.6 & 37.18 & 10.64 & 22.36 & 22.35 & 70.18 \\
\hline
\end{tabular}

Public-supply system losses is water lost from the water distribution system and includes pipe leaks and breaks, fire suppression, and unauthorized use.

${ }^{2}$ Public-supply withdrawals may not equal public-supply deliveries because of interregion and interstate transfers.

${ }^{3}$ Metropolitan North Georgia Water Planning Region. 

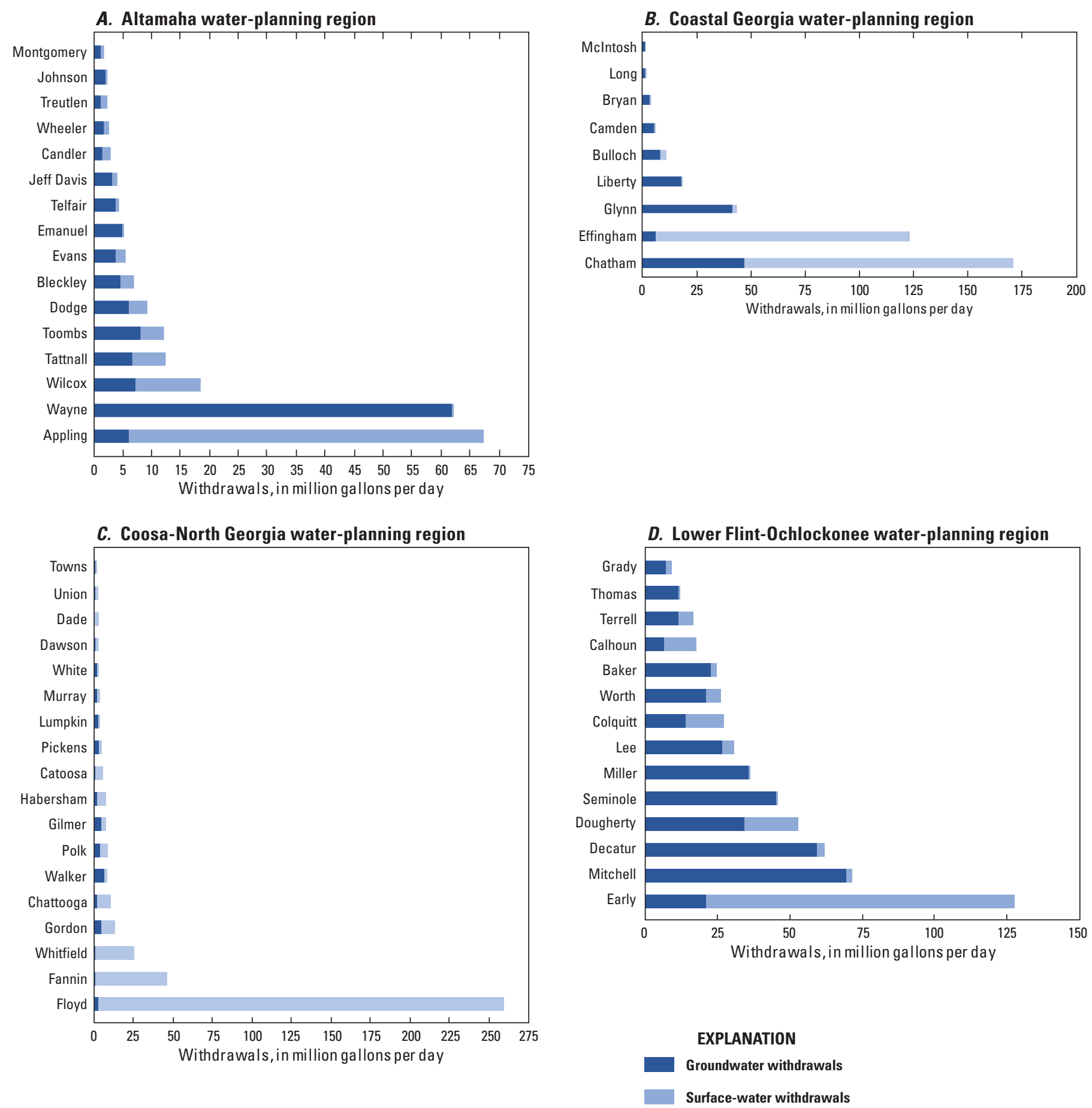

Figure 11. Surface-water and groundwater withdrawals for counties within each water-planning region. Ordered by smallest withdrawals to largest withdrawals. 

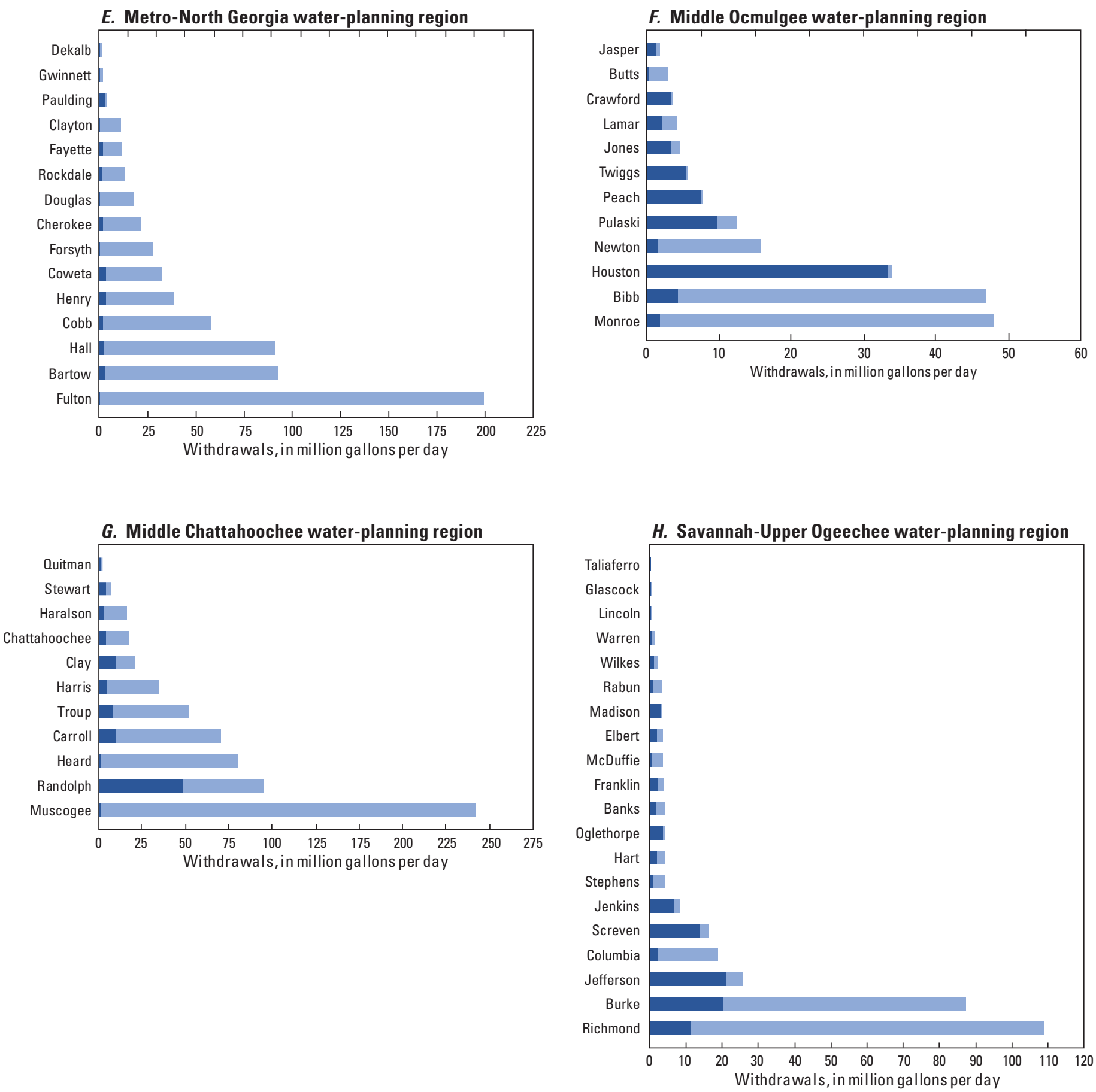

EXPLANATION

Groundwater withdrawals

Surface-water withdrawals

Figure 11. Surface-water and groundwater withdrawals for counties within each water-planning region. Ordered by smallest withdrawals to largest withdrawals. - Continued 

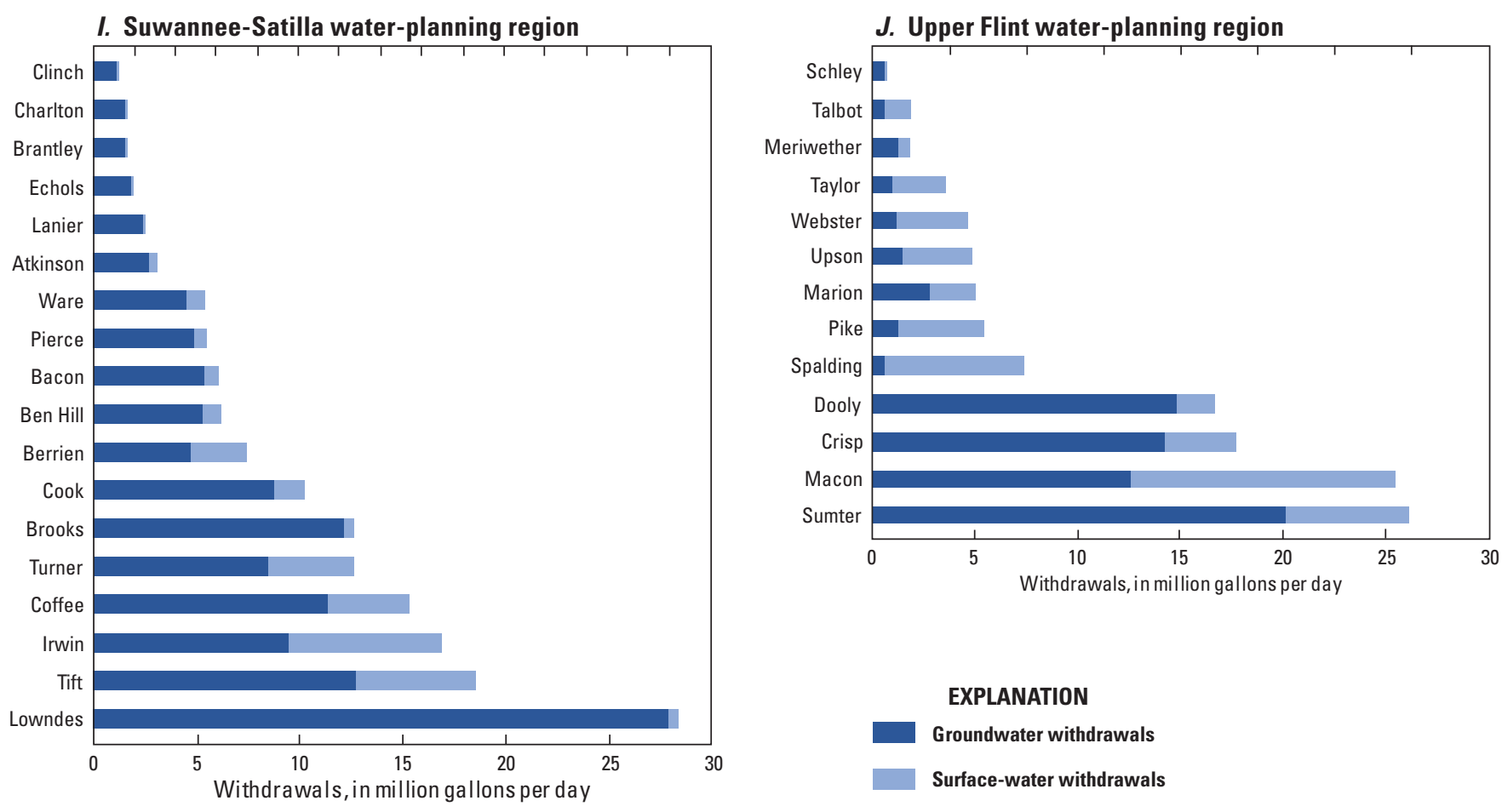

$K$. Upper Oconee water-planning region

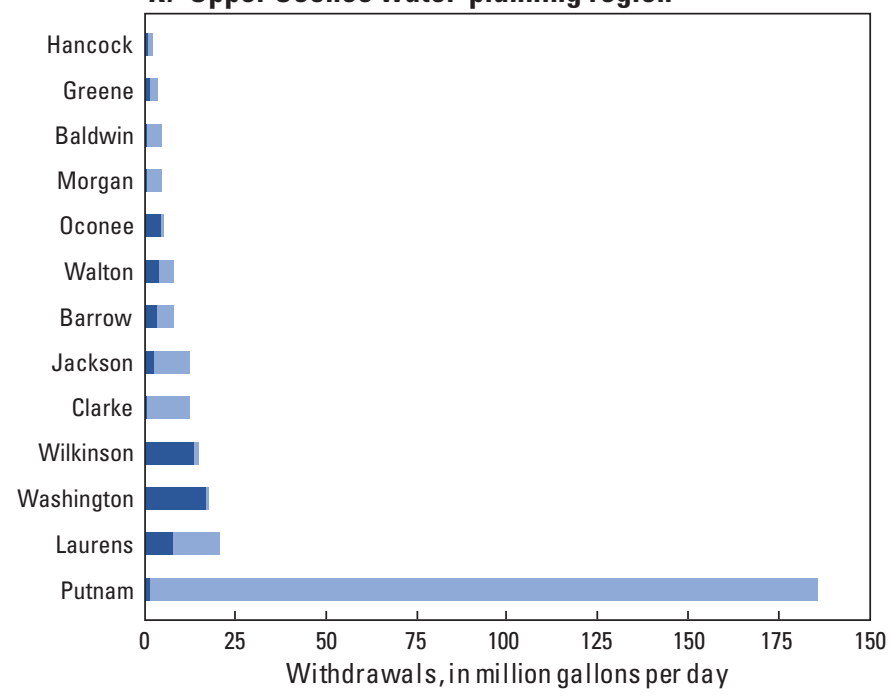

EXPLANATION

Groundwater withdrawals

Surface-water withdrawals

Figure 11. Surface-water and groundwater withdrawals for counties within each water-planning region. Ordered by smallest withdrawals to largest withdrawals.-Continued 


\section{A. Altamaha water-planning region}

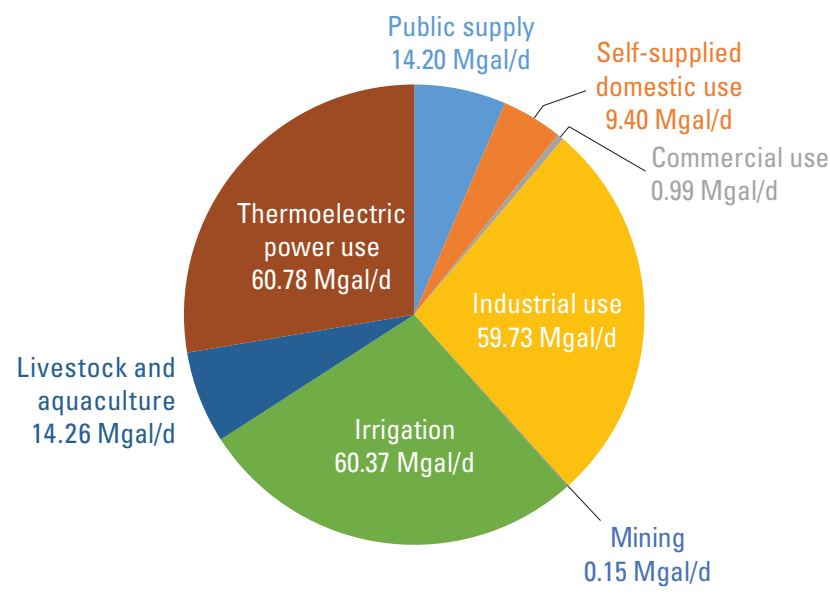

C. Coosa North Georgia water-planning region

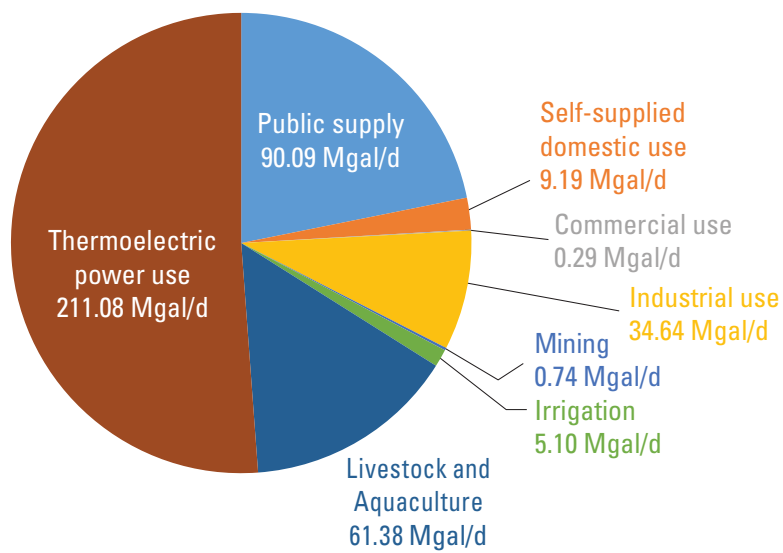

E. Metro-North Georgia water-planning region

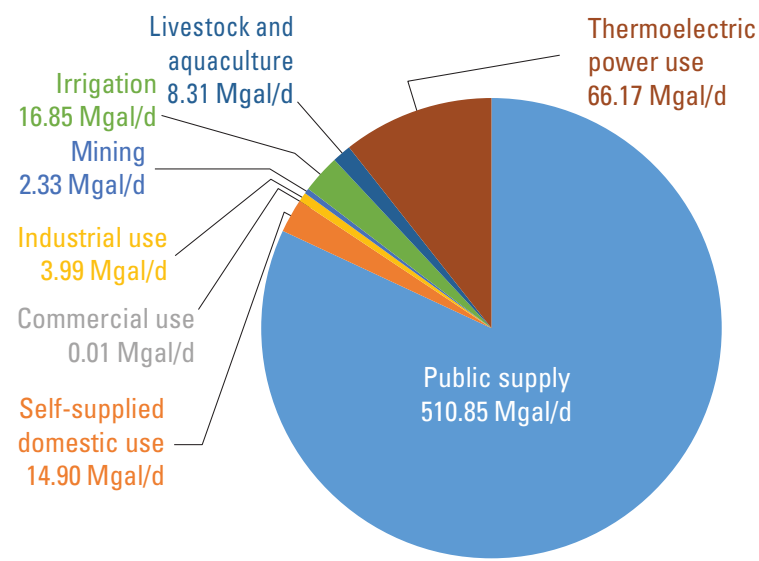

\section{B. Coastal Georgia water-planning region}

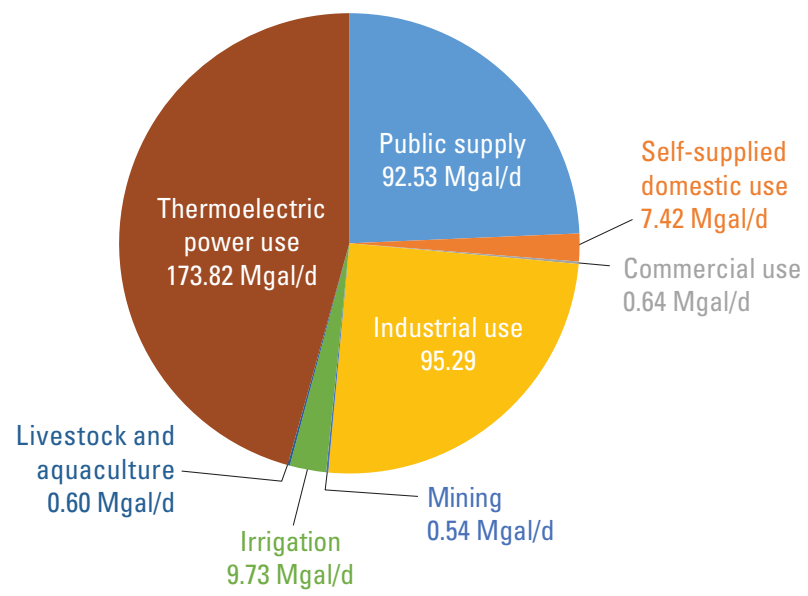

D. Lower Flint-Ochlockonee water-planning region

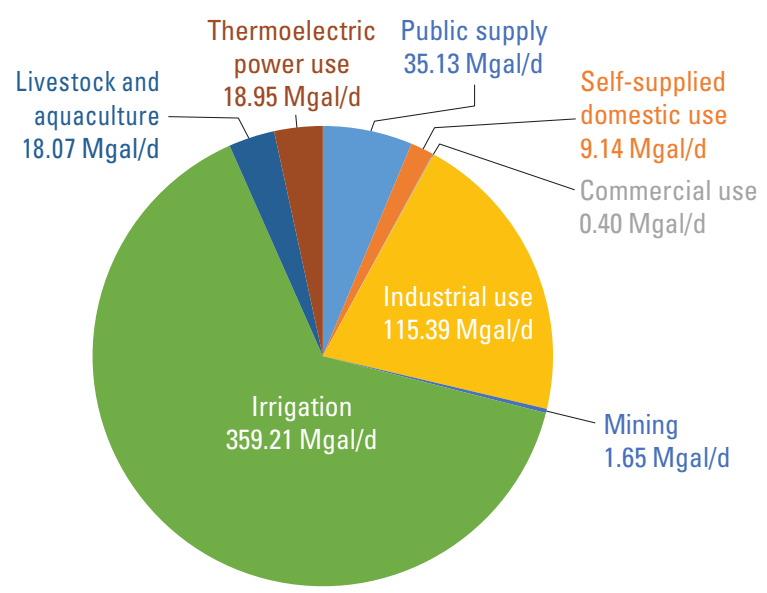

F. Lower Flint-Ochlockonee water-planning region

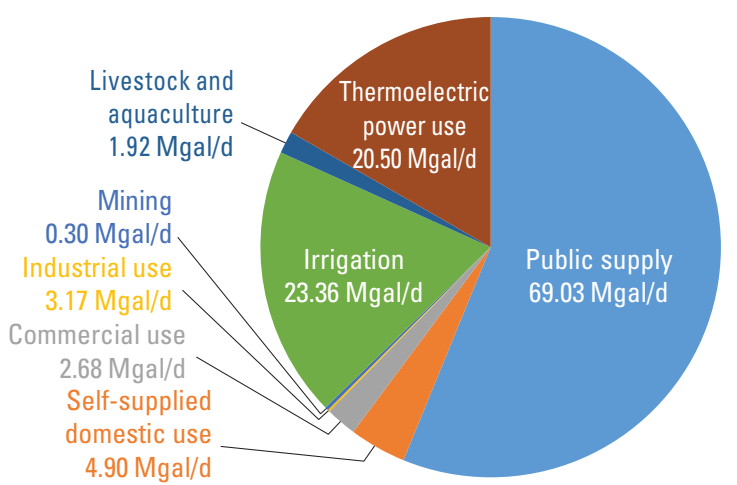

Figure 12. Water withdrawals in Georgia water-planning regions for 2015, by water-use category, in million gallons per day (Mgal/d). 


\section{G. Middle Ocmulgee water-planning region}

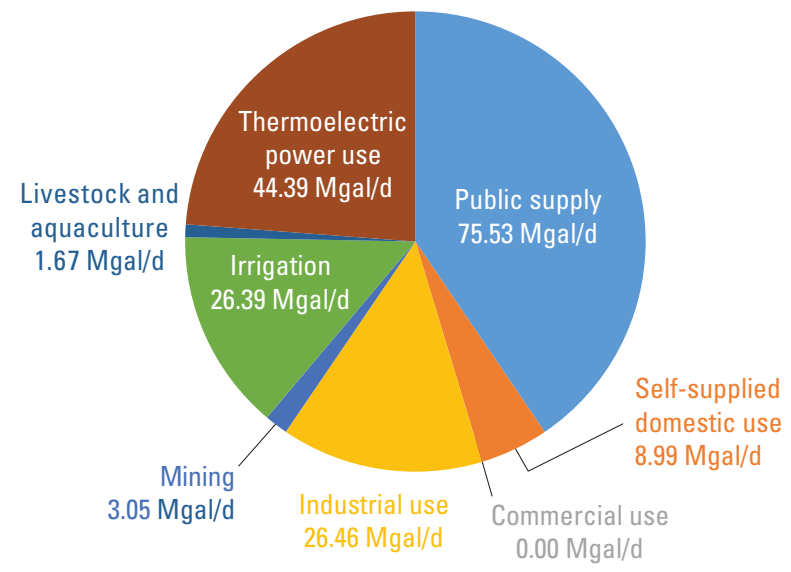

\section{Suwannee-Satilla water-planning region}

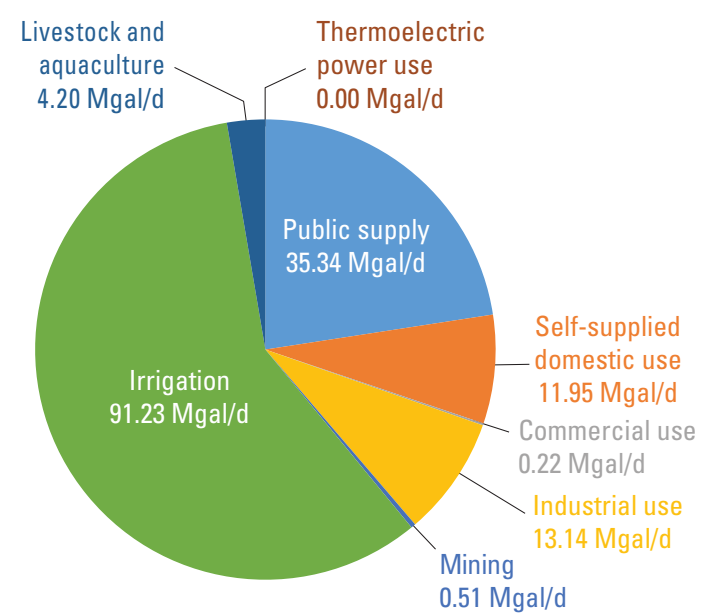

\section{$K$. Upper Oconee water-planning region}

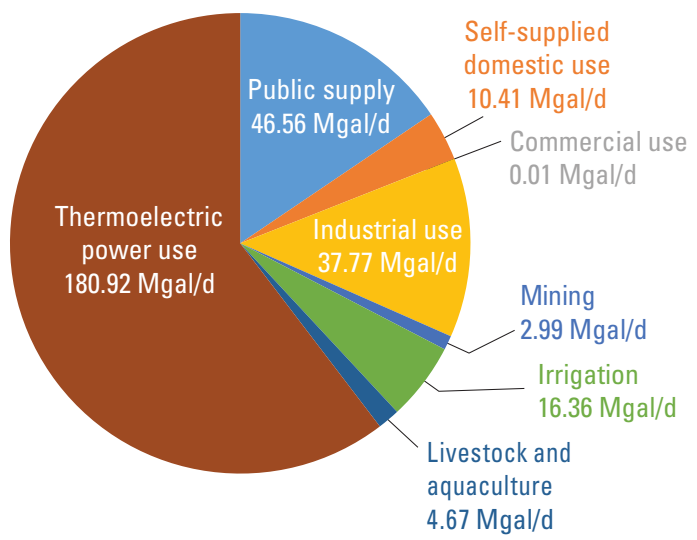

\section{H. Savannah-Upper Ogeechee water-planning region}

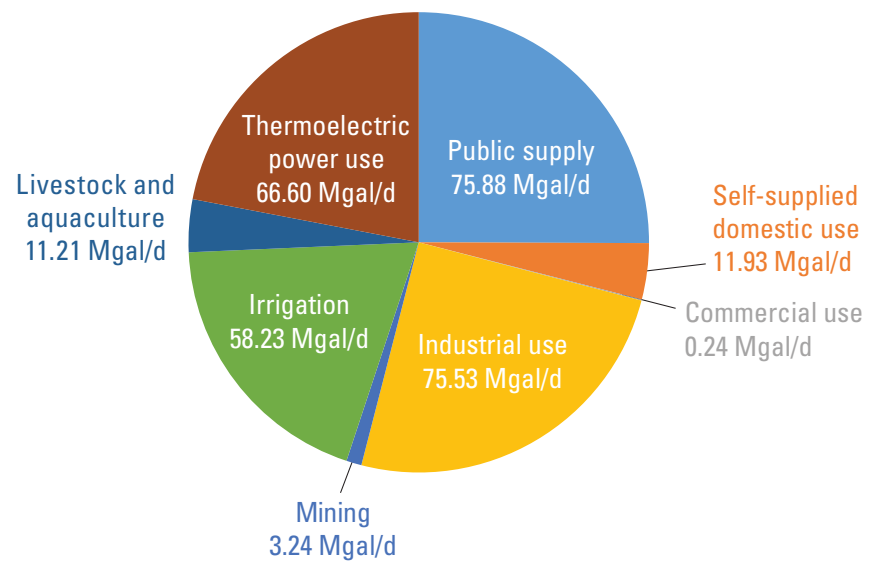

\section{J. Upper Flint water-planning region}

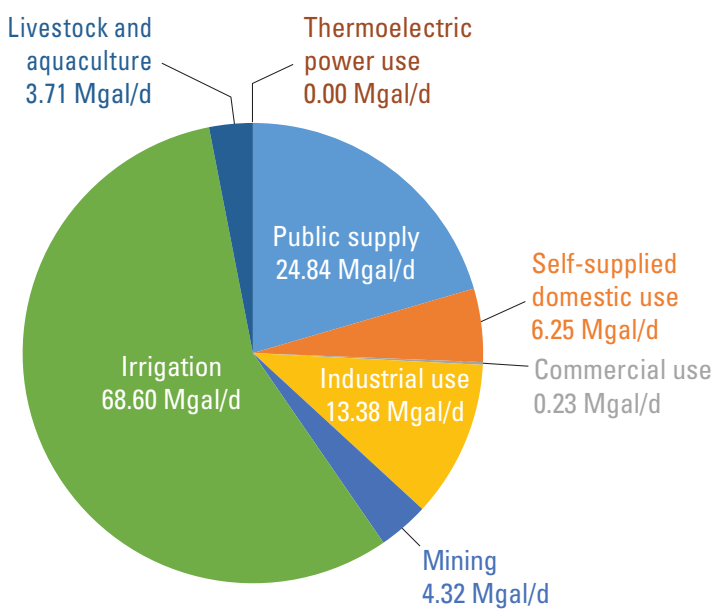

Figure 12. Water withdrawals in Georgia water-planning regions for 2015, by water-use category, in million gallons per day (Mgal/d).Continued 
Table 5. Surface-water returns by water-use category for water-planning regions in Georgia, 2015.

[Mgal/d, million gallons per day]

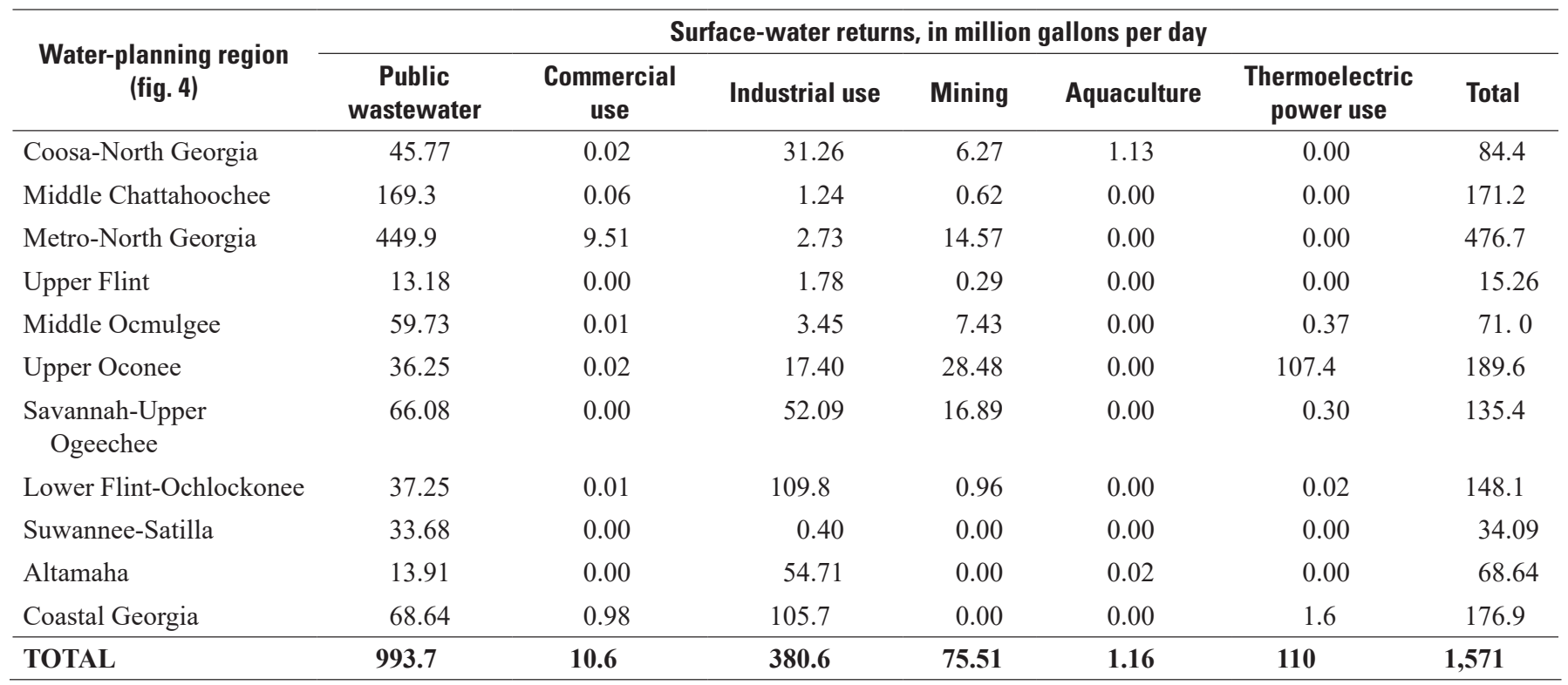

Table 6. Water withdrawals by water-use category, for major river basins in Georgia, 2015.

[Mgal/d, million gallons per day]

\begin{tabular}{|c|c|c|c|c|c|c|c|c|c|}
\hline \multirow[b]{2}{*}{$\begin{array}{l}\text { Major river basin } \\
\text { (fig. 3) }\end{array}$} & \multicolumn{9}{|c|}{ Withdrawals, in Mgal/d } \\
\hline & $\begin{array}{l}\text { Public } \\
\text { supply' }\end{array}$ & $\begin{array}{l}\text { Domestic } \\
\text { use }\end{array}$ & $\begin{array}{c}\text { Commercial } \\
\text { use }\end{array}$ & $\begin{array}{l}\text { Industrial } \\
\text { use }\end{array}$ & Mining & Irrigation & $\begin{array}{c}\text { Livestock } \\
\text { and } \\
\text { aquaculture }\end{array}$ & $\begin{array}{l}\text { Thermo- } \\
\text { electric } \\
\text { power use }\end{array}$ & Total \\
\hline Savannah & 111.4 & 11.24 & 0.23 & 126.8 & 3.11 & 17.80 & 9.10 & 238.5 & 518.2 \\
\hline Ogeechee & 47.27 & 7.06 & 0.58 & 16.26 & 0.37 & 56.74 & 3.21 & 0.00 & 131.5 \\
\hline Oconee & 43.26 & 10.91 & 0.01 & 37.77 & 3.66 & 17.98 & 5.55 & 225.3 & 344 \\
\hline Altamaha & 8.63 & 4.93 & 0.90 & 57.81 & 0.23 & 18.23 & 2.70 & 60.78 & 154.2 \\
\hline Satilla and St. Marys & 23.54 & 7.76 & 0.06 & 30.90 & 0.21 & 33.60 & 2.39 & 1.63 & 100.1 \\
\hline $\begin{array}{l}\text { Suwannee and } \\
\text { Aucilla }\end{array}$ & 32.51 & 8.89 & 0.24 & 12.92 & 0.30 & 100.9 & 3.44 & 0.00 & 159.2 \\
\hline Tennessee & 16.62 & 1.42 & 0.00 & 0.47 & 0.10 & 1.23 & 44.62 & 0.28 & 64.74 \\
\hline TOTAL & 1,069 & 104.5 & 5.71 & 475.2 & 19.82 & 735.2 & 130.0 & 843 & 3,383 \\
\hline
\end{tabular}

${ }^{1}$ About $6.7 \mathrm{Mgal} / \mathrm{d}$ of water was delivered into and used in Georgia by public water suppliers in Alabama and Tennessee in 2015. 


\section{A. Altamaha withdrawals by category}

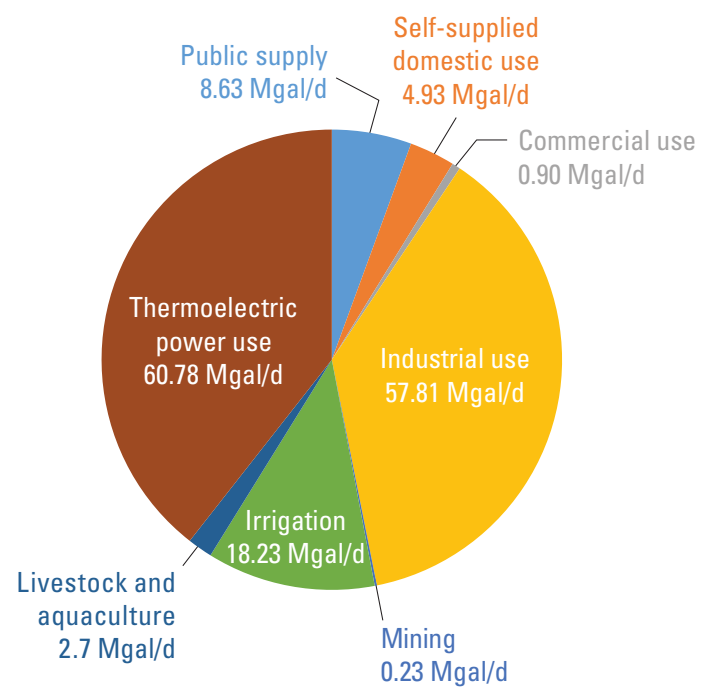

C. Coosa and Tallapoosa withdrawals by category

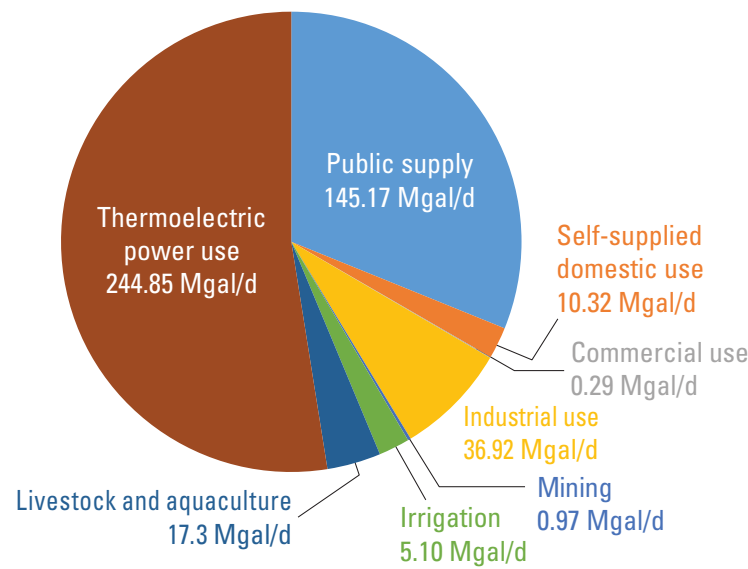

E. Ocmulgee withdrawals by category

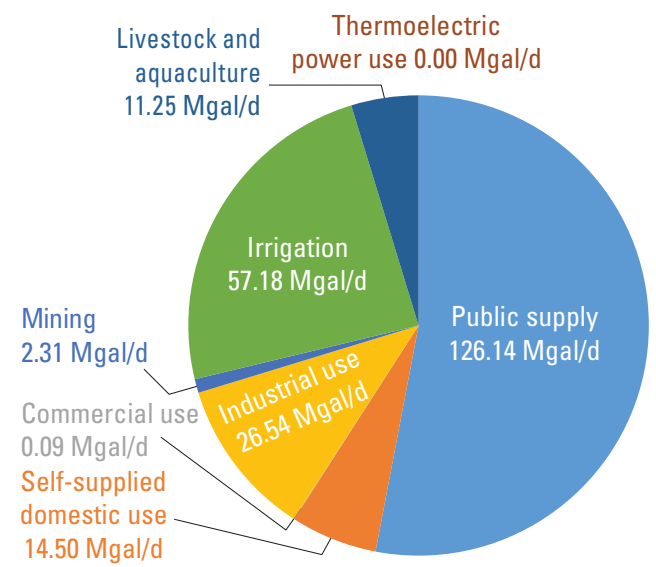

\section{B. Apalachicola withdrawals by category}

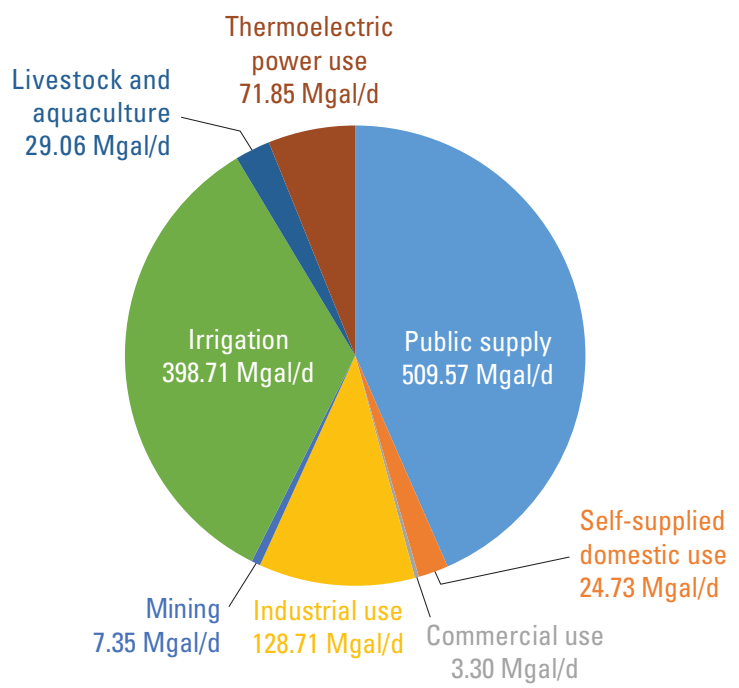

\section{Ochlockonee withdrawals by category}

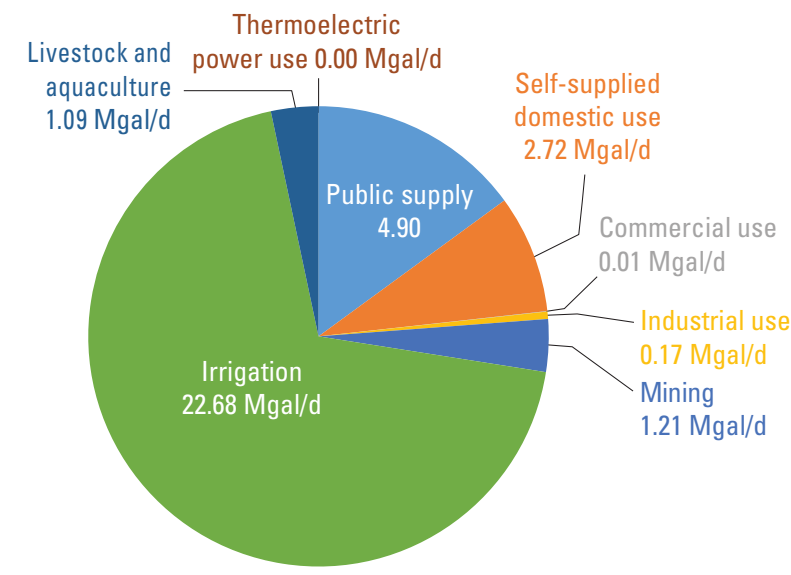

F. Oconee withdrawals by category

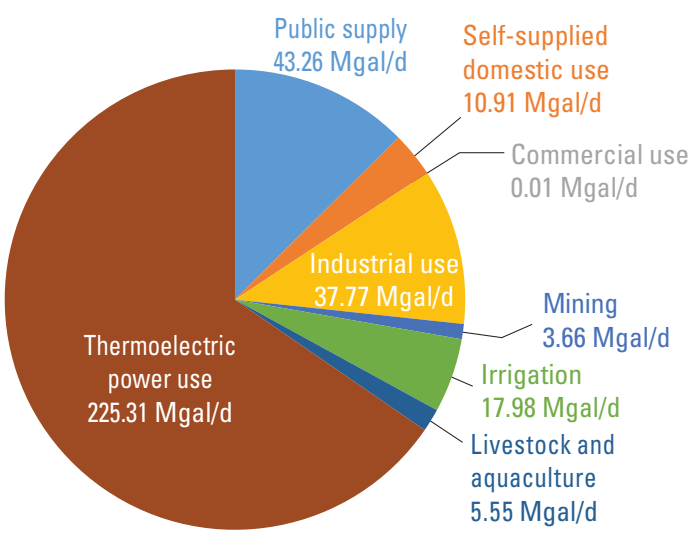

Figure 13. Water withdrawals in Georgia by water-use category for major river basins in 2015 , in million gallons per day (Mgal/d). 


\section{G. Ogeechee withdrawals by category}

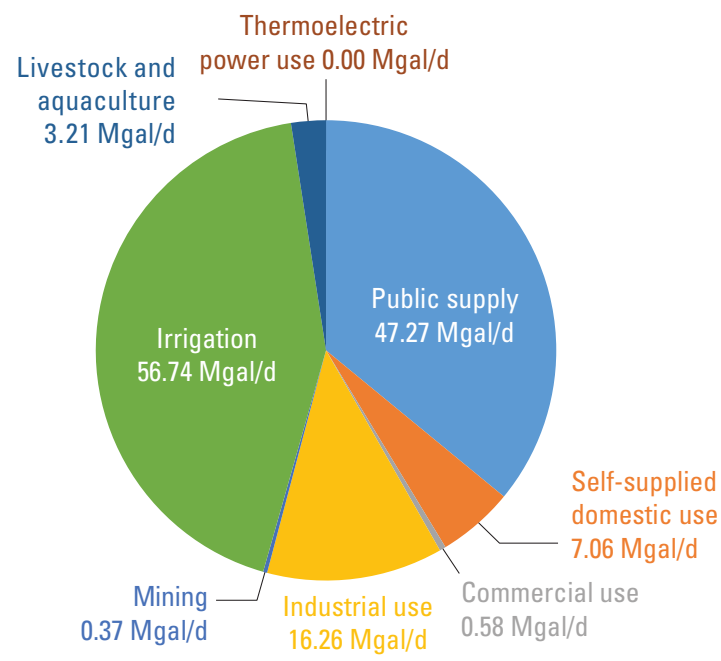

\section{Savannah withdrawals by category}

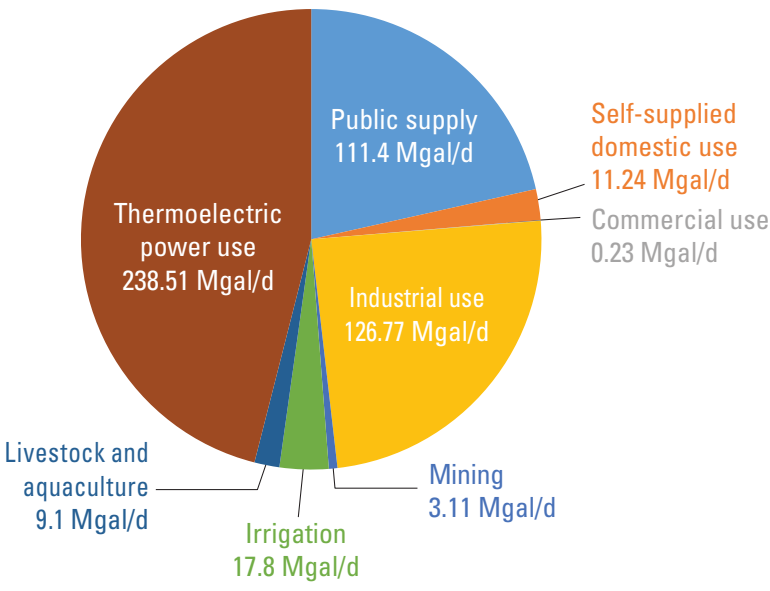

\section{K. Tennessee withdrawals by category}

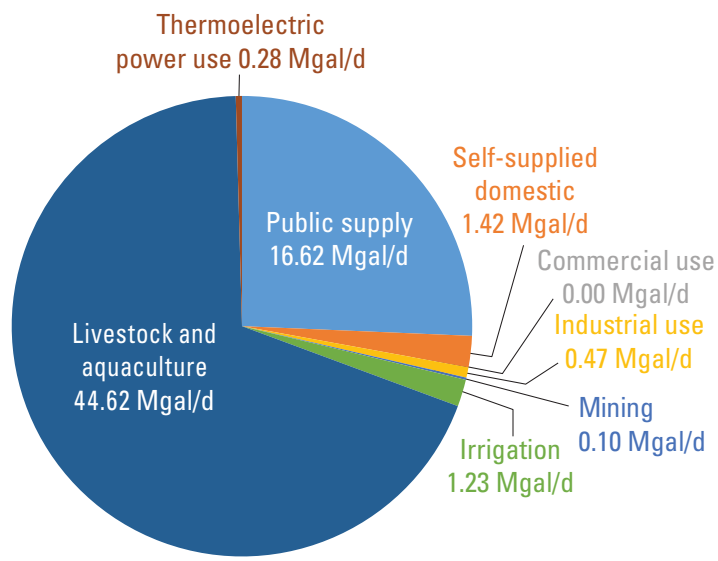

\section{H. Satilla and St. Marys withdrawals by category}

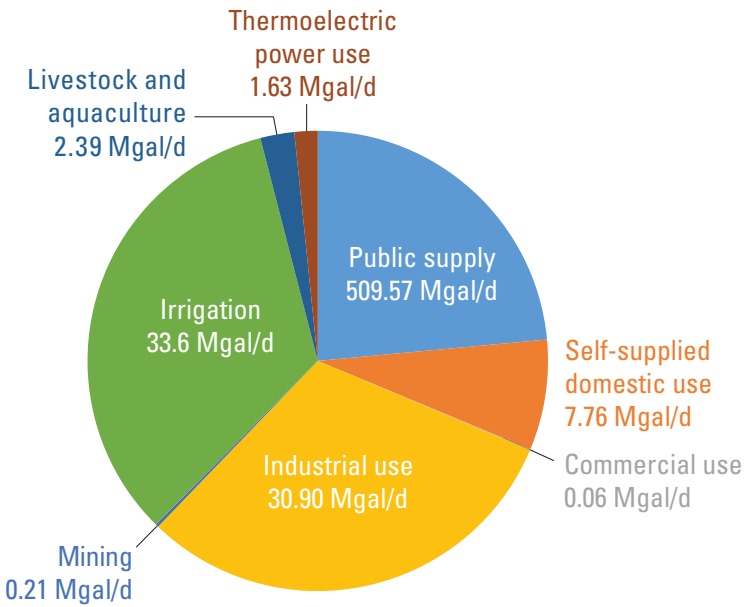

\section{J. Suwanee Aucilla withdrawals by category}

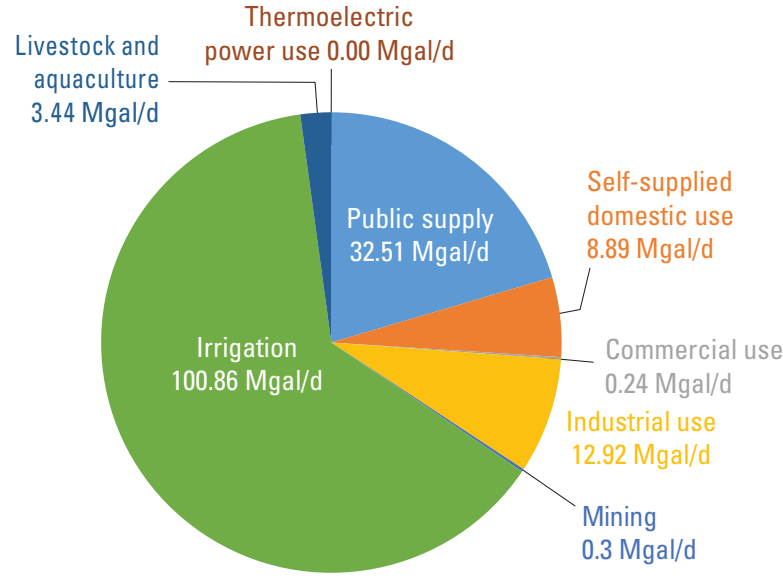

Figure 13. Water withdrawals in Georgia by water-use category for major river basins in 2015, in million gallons per day (Mgal/d).Continued 


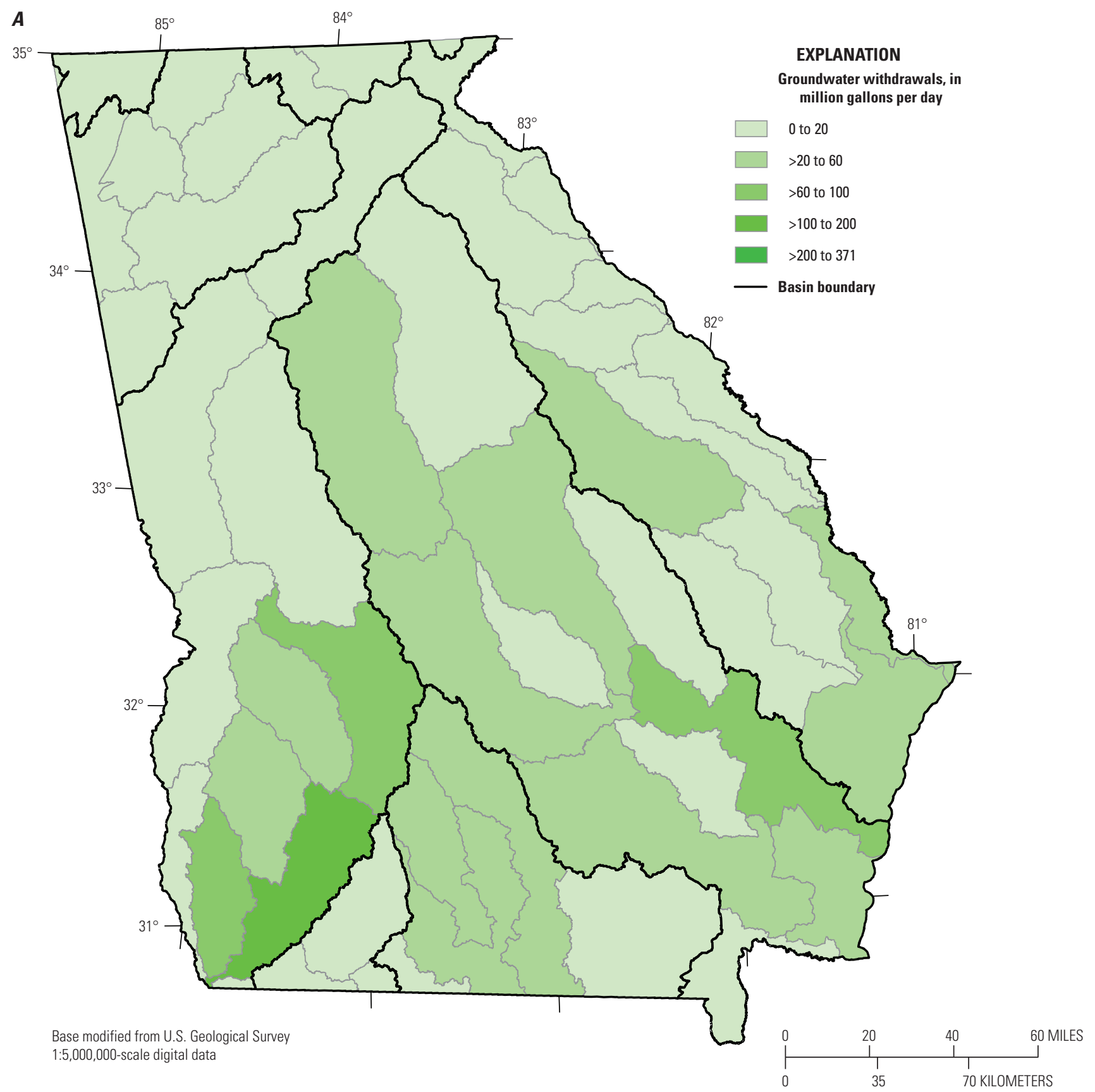

Figure 14. Map of Georgia showing, by major river basin in 2015, $(A)$ groundwater withdrawals, $(B)$ surface-water withdrawals, and (C) groundwater and surface-water withdrawals, in million gallons per day. 


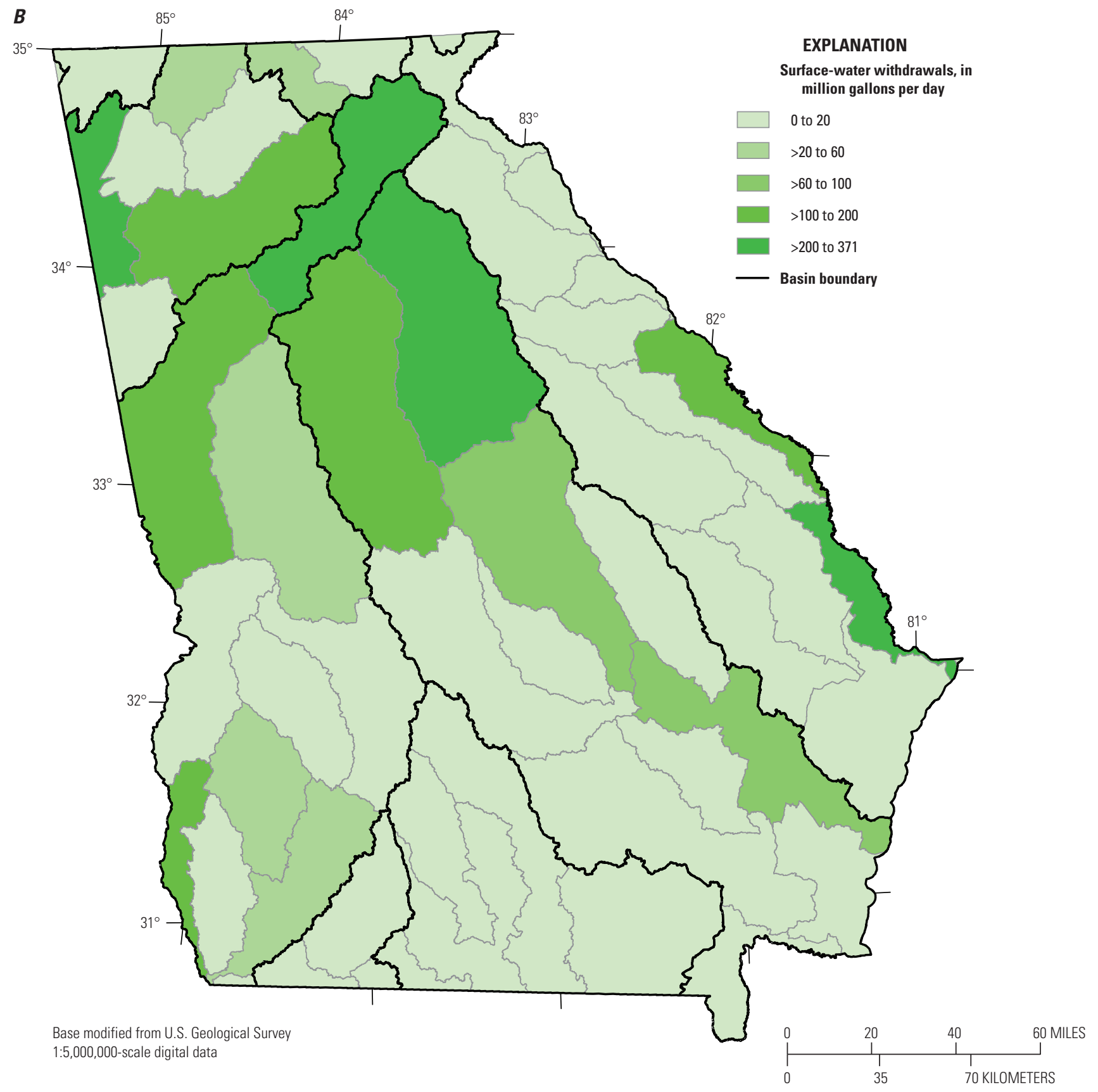

Figure 14. - Continued 
C $35^{\circ}$

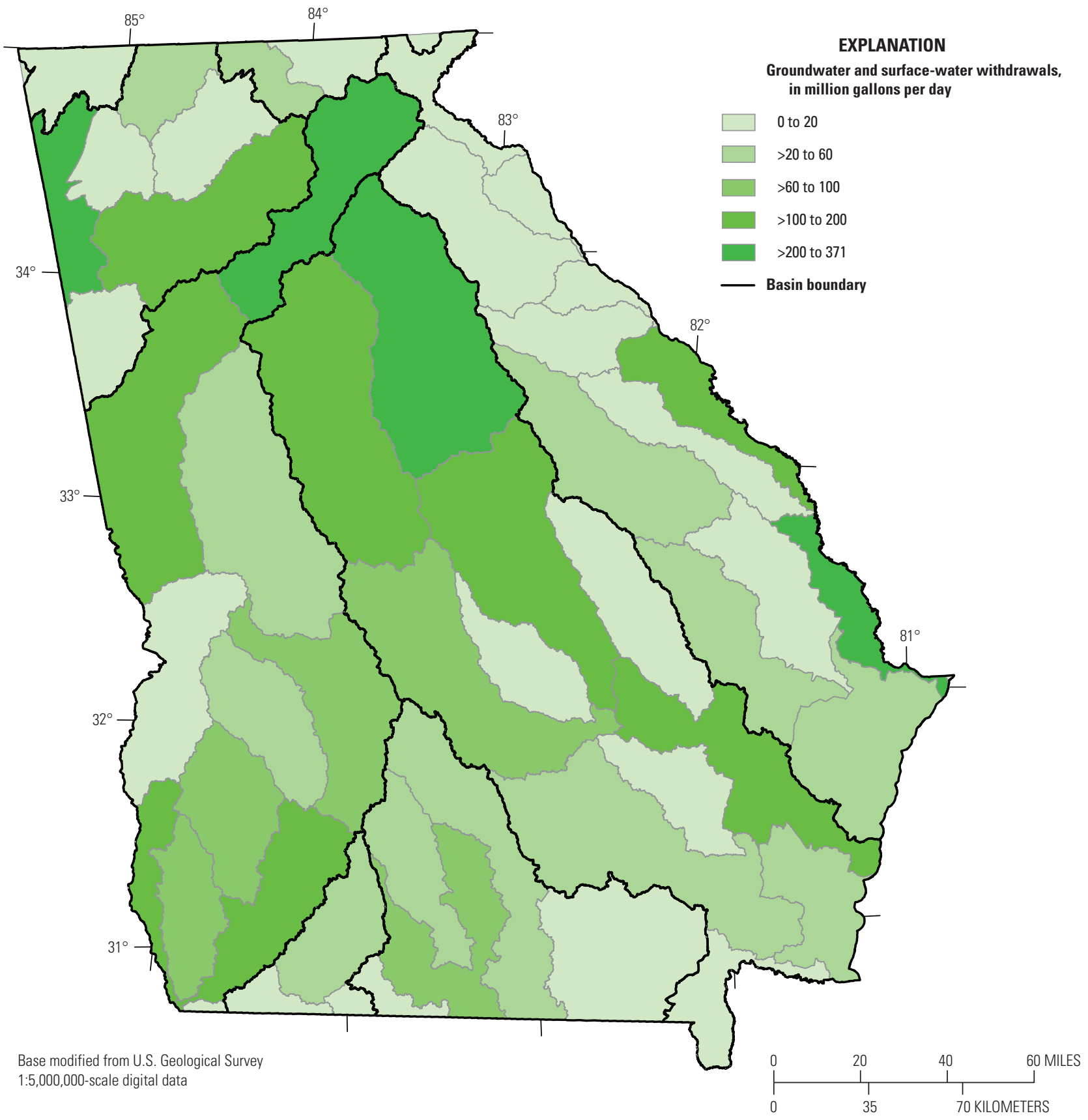

Figure 14. - Continued 
Table 7. Surface-water returns by water-use category for major river basins in Georgia, 2015.

[Mgal/d, million gallons per day]

\begin{tabular}{|c|c|c|c|c|c|c|c|}
\hline \multirow{2}{*}{$\begin{array}{l}\text { Major river basin } \\
\text { (fig. 3) }\end{array}$} & \multicolumn{7}{|c|}{ Surface-water returns, in million gallons per day } \\
\hline & $\begin{array}{c}\text { Public } \\
\text { wastewater }\end{array}$ & $\begin{array}{c}\text { Commercial } \\
\text { use }\end{array}$ & $\begin{array}{c}\text { Industrial } \\
\text { use }\end{array}$ & Mining & Aquaculture & $\begin{array}{c}\text { Thermoelectric } \\
\text { power use }\end{array}$ & Total \\
\hline Savannah & 94.36 & 0.49 & 108.9 & 16.58 & 0.0 & 0.30 & 220.7 \\
\hline Ogeechee & 24.68 & 0.37 & 12.16 & 0.31 & 0.00 & 0.00 & 37.52 \\
\hline Oconee & 35.2 & 0.02 & 17.40 & 31.38 & 0.00 & 107.4 & 191.4 \\
\hline Ocmulgee & 132.3 & 0.01 & 3.86 & 5.80 & 0.00 & 0.37 & 142.4 \\
\hline Altamaha & 8.39 & 0.00 & 54.35 & 0.0 & 0.02 & 0.00 & 62.77 \\
\hline Satilla and St. Marys & 27.94 & 0.12 & 37.24 & 0.0 & 0.00 & 1.6 & 66.93 \\
\hline Suwannee and Aucilla & 22.96 & 0.00 & 0.20 & 0.0 & 0.00 & 0.00 & 23.16 \\
\hline Ochlockonee & 10.01 & 0.01 & 1.57 & 0.96 & 0.00 & 0.00 & 12.55 \\
\hline Apalachicola & 501.5 & 9.52 & 112.0 & 2.61 & 0.00 & 0.02 & 625.6 \\
\hline Coosa and Tallapoosa & 132.9 & 0.07 & 32.82 & 17.76 & 0.00 & 0.00 & 183.5 \\
\hline Tennessee & 3.48 & 0.00 & 0.05 & 0.10 & 1.13 & 0.00 & 4.76 \\
\hline TOTAL & 993.7 & 10.6 & 380.6 & 75.51 & 1.16 & 110 & 1,571 \\
\hline
\end{tabular}

Table 8. Groundwater withdrawals by use category for the principal aquifers in Georgia, 2015.

[Mgal/d, million gallons per day; (S400FLORDN), National Aquifer code]

\begin{tabular}{|c|c|c|c|c|c|c|c|c|c|c|}
\hline \multirow[b]{2}{*}{$\begin{array}{l}\text { Aquifer name } \\
\text { (fig. 2) }\end{array}$} & \multirow[b]{2}{*}{$\begin{array}{l}\text { Population, } \\
\text { in } \\
\text { thousands }\end{array}$} & \multicolumn{9}{|c|}{ Withdrawals, in Mgal/d } \\
\hline & & $\begin{array}{l}\text { Public } \\
\text { supply }\end{array}$ & $\begin{array}{c}\text { Domestic } \\
\text { use }\end{array}$ & $\begin{array}{c}\text { Commercial } \\
\text { use }\end{array}$ & $\begin{array}{l}\text { Industrial } \\
\text { use }\end{array}$ & Mining & Irrigation & $\begin{array}{c}\text { Livestock } \\
\text { and } \\
\text { aquaculture }\end{array}$ & $\begin{array}{l}\text { Thermo- } \\
\text { electric } \\
\text { power } \\
\text { use }\end{array}$ & Total \\
\hline \multicolumn{11}{|c|}{ Floridan aquifer system (S400FLORDN) } \\
\hline Floridan system & 1,457 & 129.1 & 38.03 & 2.10 & 141.1 & 1.44 & 449.5 & 25.02 & 1.24 & 787.5 \\
\hline \multicolumn{11}{|c|}{ Brunswick aquifer system (N9990THER) } \\
\hline Brunswick & 14.54 & 1.35 & 0.0 & 0.04 & 0.0 & 0.49 & 1.20 & 0.02 & 0.00 & 3.10 \\
\hline \multicolumn{11}{|c|}{ Surficial aquifer system (S100SURFCL) } \\
\hline Surficial system & 27.9 & 0.69 & 0.74 & 0.00 & 0.21 & 0.06 & 2.10 & 0.07 & 0.91 & 4.78 \\
\hline \multicolumn{11}{|c|}{ Southeastern Coastal Plain aquifer system (S100SECSLP) } \\
\hline Claiborne & 95.69 & 10.31 & 1.51 & 0.0 & 0.82 & 0.0 & 29.71 & 0.35 & 0.00 & 42.70 \\
\hline Clayton & 59.77 & 7.18 & 0.85 & 0.02 & 0.56 & 0.08 & 15.98 & 0.11 & 0.00 & 24.78 \\
\hline $\begin{array}{l}\text { Cretaceous aquifer } \\
\text { system }\end{array}$ & 505.1 & 52.77 & 13.14 & 0.08 & 41.58 & 7.65 & 47.27 & 2.72 & 1.21 & 166.4 \\
\hline \multicolumn{11}{|c|}{ Piedmont and Blue Ridge crystalline rock aquifers (N400PDMBRX) } \\
\hline Crystalline rock & 963.8 & 15.55 & 47.03 & 0.26 & 5.25 & 5.77 & 14.07 & 12.50 & 0.06 & 100.5 \\
\hline \multicolumn{11}{|c|}{ Valley and ridge aquifer (N500VLYRDG) } \\
\hline Paleozoic rock & 145.0 & 13.99 & 3.19 & 0.00 & 3.16 & 0.44 & 1.24 & 1.29 & 0.00 & 23.31 \\
\hline Total & 3,268 & 230.9 & 104.5 & 2.50 & 192.7 & 15.93 & 561.0 & 42.08 & 3.42 & 1,153 \\
\hline
\end{tabular}

\footnotetext{
aPublic-supplied population and self-supplied domestic population using groundwater.
} 


\section{Water Use by Principal Aquifer}

Groundwater withdrawals were compiled for each of the eight principal aquifers in Georgia (fig. 2). Nearly $1,160 \mathrm{Mgal} / \mathrm{d}$ of groundwater were withdrawn from the principal aquifers in Georgia during 2015 (tables 2, 8). Coastal Plain aquifers (Floridan aquifer system and Southeastern Coastal Plain aquifers [Claiborne and Clayton aquifers and Cretaceous aquifer system]) account for nearly 90 percent of all the groundwater withdrawn. The Floridan aquifer system is the dominant aquifer for 2015 withdrawals (68.3 percent), followed by the Cretaceous aquifer system (14.4 percent), the crystalline-rock aquifers ( 8.7 percent), the Claiborne aquifer (3.7 percent), the Clayton aquifer (2.1 percent), Paleozoic rock aquifers (2.0 percent), the surficial aquifer system ( 0.4 percent), and the Brunswick aquifer system ( 0.3 percent).

In 2015, more than 3.2 million people in Georgia (32 percent of the population) used groundwater (self-supplied and public supplied) for domestic purposes (tables 2,8). Of that population, about 1.5 million people relied on small domestic wells for their water supply (table 2). The Floridan aquifer system supplied about 44.6 percent of the population with groundwater for domestic purposes, followed by the crystalline-rock aquifers (29.5 percent) and the Cretaceous aquifer system (15.4 percent), with the remaining aquifers supplying 10.5 percent of the population (table 8 ).

\section{Water-Use Trends, 1985-2015}

Water-use estimates have been compiled and compared temporally from 1985-2015 and spatially at a statewide scale as well as at a water-planning region scale. Historically, the highest estimated statewide water use was in 1980 at $6,735 \mathrm{Mgal} / \mathrm{d}$; however, water-use estimates at the time were based primarily on permitted volumes and not on reported data (Pierce and others, 1982). Water use, beginning in 1985, was estimated using reported information from most water users and public-water distributors, which makes 1985 the first reliable year for an accurate evaluation of water-use trends through 2015. Population totals and trends are included in relation to domestic and commercial water-use categories. There are several driving forces behind the observed water-use changes that are also described.

Statewide, water-use estimates for 2015 continued a trend in decreasing water use, first identified in 2005, when compared with 2000 estimates. Estimated statewide water use for 2000 was $6,532 \mathrm{Mgal} / \mathrm{d}$, which decreased to 3,384 Mgal/d in 2015 (fig. 15); this is a 48-percent reduction (3,147 Mgal/d) in 15 years (fig. 15). A reduction of withdrawals from surface-water bodies accounts for 90 percent of this difference (fig. 15). The categories of use primarily responsible for these reductions are thermoelectric power cooling (75-percent decrease) and industrial and mining (26-percent decrease) (fig. 16). Water-use estimates for irrigation - a weather- and economy-driven category-also decreased between the years $2000(1,142 \mathrm{Mgal} / \mathrm{d})$ and $2015(735.4 \mathrm{Mgal} / \mathrm{d})$; however, $2010(802 \mathrm{Mgal} / \mathrm{d})$ showed greater water use than 2005 (751.6 Mgal/d) (fig. 16). The minor-use categories of aquaculture and livestock are combined for this report and counter the statewide trend of decreasing water use, with a 273-percent increase in water use between the years 2000 and 2015 (fig. 16).

Before 2000, there was variability in statewide water use, yet the overall trend was an increase in water use, with a total increase of 20 percent between 1985 and 2000 (fig. 15). The increases in withdrawals from 1990 to 2000 were split by water source: 60 percent came from surface water $(712 \mathrm{Mgal} / \mathrm{d})$, and 40 percent came from groundwater $(466.4 \mathrm{Mgal} / \mathrm{d})$ (fig. 15). The water-use categories primarily responsible for these increases are domestic and commercial use (15-percent increase) and irrigation (159-percent increase) (fig. 16).

Water-use trends were compiled for each Georgia water-planning region to highlight different use trends among regions of the State and identify where the water-use increases and decreases statewide, by category, are recorded (figs. 17, 18). The declining statewide water-use trend identified between 2000 and 2015 persists for all waterplanning regions; however, the Metro-North Georgia and the Upper Oconee water-planning regions account for 58 percent of the reduction (table 3 in Painter, 2019). The Coosa-North Georgia (342.8 Mgal/d) and Lower Flint-Ochlockonee (328.95 Mgal/d) regions account for another 21 percent of the reductions in use between 2000 and 2015. The Coastal Georgia (219.9 Mgal/d), Middle Ocmulgee (209.8 Mgal/d), Middle Chattahoochee (66.85 Mgal/d), Suwanee-Satilla (46.18 Mgal/d), Upper Flint (46.62 Mgal/d), Savannah-Upper Ogeechee (39.40 Mgal/d), and Altamaha (29.11 Mgal/d) regions account for the remaining reductions in water use.

Water-use trends between 2000 and 2015 were compared by use category for each water-planning region (fig. 17; table 3 in Painter, 2019). Thermoelectric power cooling was the primary category for reductions in water use for the Metro-North Georgia (883.3 Mgal/d), Coosa-North Georgia (325.8 Mgal/d), Middle Ocmulgee (177.92 Mgal/d), and Upper Oconee (803.4 Mgal/d) regions. Irrigation (crop and golf course) was the dominant category for decreases in water use for the Lower Flint-Ochlockonee (178.9 Mgal/d), Suwanee-Satilla (45.65 Mgal/d), Upper Flint (44.09 Mgal/d), Altamaha (41.74 Mgal/d/), and Savannah-Upper Ogeechee (33.42 Mgal/d) regions. The Coastal Georgia region saw the greatest water-use decrease (106.5 Mgal/d) from the industry and mining categories. Increases in water use occurred between 2000 and 2015:

- for domestic and commercial uses in the Metro-North Georgia (27 Mgal/d), Upper Flint (5.12 Mgal/d), Upper Oconee (2.18 Mgal/d), and Suwanee-Satilla (0.47 Mgal/d) regions;

- for industry and mining in the Upper Flint (4.74 Mgal/d) region; 
- for thermoelectric cooling in the Altamaha (2.96 Mgal/d) and Savannah-Upper Ogeechee (2.49 Mgal/d) regions;

- for livestock and aquaculture in all regions (except the Middle Ocmulgee and Upper Flint regions) with the most notable increase in the Coosa-North Georgia region, which had a 58.4-Mgal/d increase in withdrawals; and,

- for irrigation in the Middle Chattahoochee region (0.67 Mgal/d).

Population changes between 1985 and 2015 were also compiled and reported to compare with estimated domestic and commercial water use. The total population in Georgia increased from 5.98 million people in 1985 to 10.21 million people in 2015-a 71-percent increase. The statewide trend in domestic and commercial water use increased from 1985 to 2005 ; conversely, between 2005 and 2015 , the quantity of water used in this category declined (10 percent) despite a population growth of about 13 percent during this period. The increase in population does not exist equally throughout the State (fig. 17; table 3 in Painter, 2019). The Metro-North Georgia region had a 6-percent decrease in water use for domestic and commercial categories relative to a 16-percent increase in population between 2005 and 2015. The MetroNorth Georgia region experienced a population growth, from 2005 (4,516,958 people) to 2015 (5,249,872 people), that equates to 64 percent of the total increase in the statewide population (about 1.1 million people). The Coastal Georgia region had the highest percentage (19 percent increase) of population growth between 2005 (572,665 people) and 2015 (681,698 people), and yet there was a 31-percent decline in domestic and commercial water use during this period. Population totals in 2015 for the Altamaha, Upper Flint, and Lower Flint regions decreased compared with 2010 totals, although population totals in 2015 are greater than the 2005 totals for the Altamaha and Upper Flint regions. The Upper Flint region estimates for domestic and commercial water use were greater for 2015 (30.13 Mgal/d) than the 2010 estimates $(23.80 \mathrm{Mgal} / \mathrm{d})$, which encompasses a period of population decline (at almost 3 percent).

Conversely, the Lower Flint region had a 24-percent decrease in domestic and commercial water use from 2005 to 2015, which corresponded to a 3-percent decrease in population for this same period. Self-supplied domestic withdrawals underwent a notable increase in 2015 from previous water-use estimates for the crystalline-rock aquifers, indicating a possible over, under, or simply "error in" estimation due to the uncertainty in the served population size reported by public water-supply systems in the region. This uncertainty resulted in a historically higher percentage of the population reported as requiring self-supplied water, but this uncertainty could not be quantified with available information.

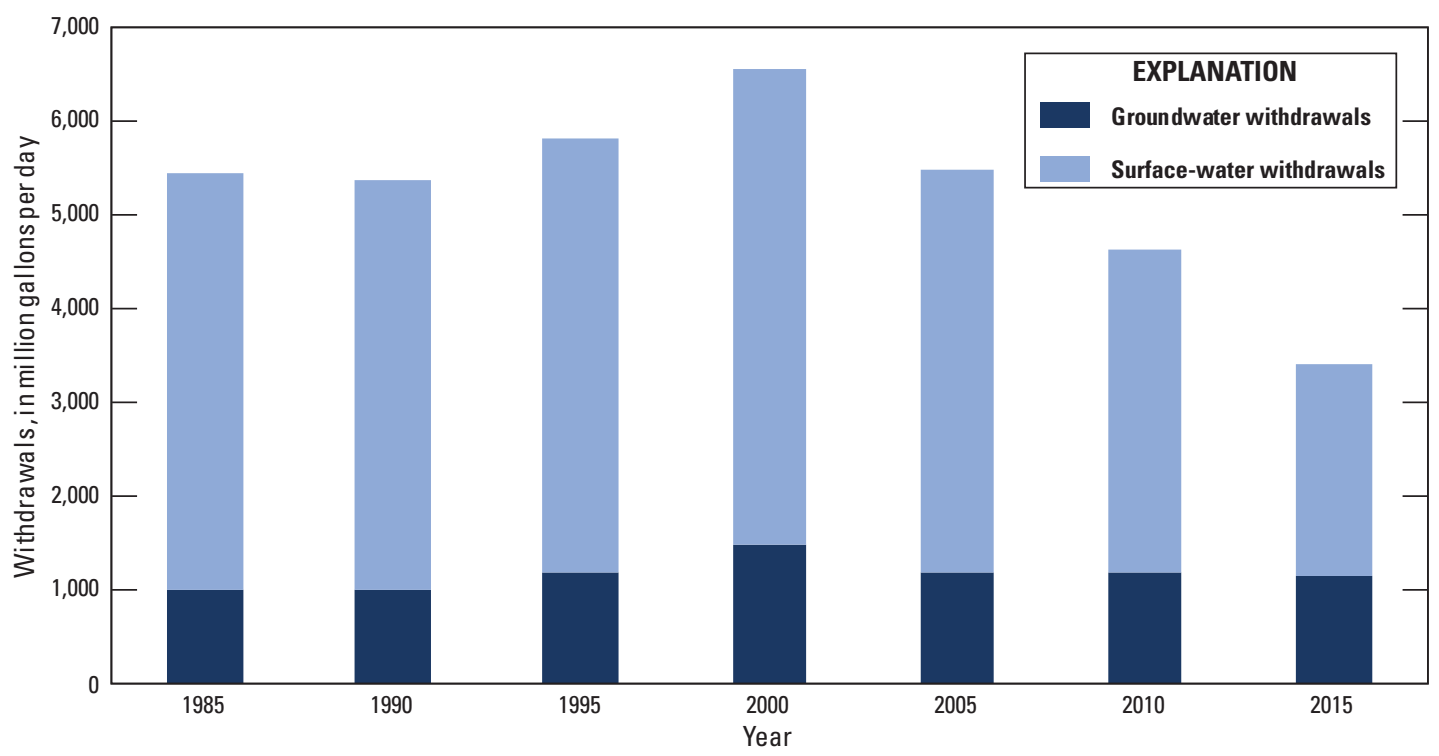

Figure 15. Water-withdrawal trends by Georgia water source for 1985-2015. 


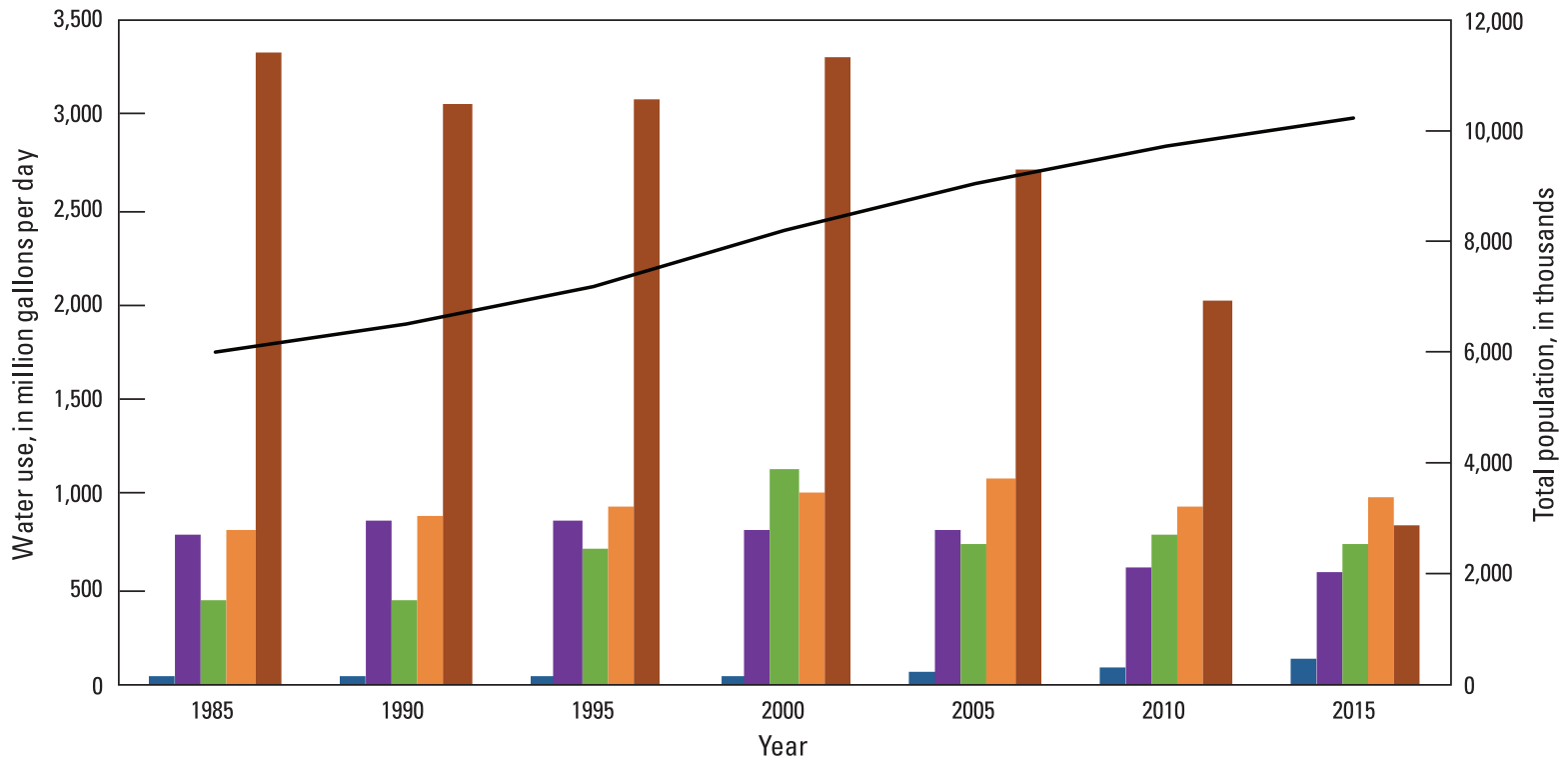

EXPLANATION

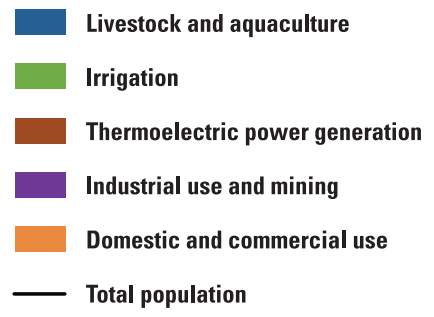

Figure 16. Statewide water-use trends in Georgia, by category, for 1985-2015. 
A. Altamaha water-planning region

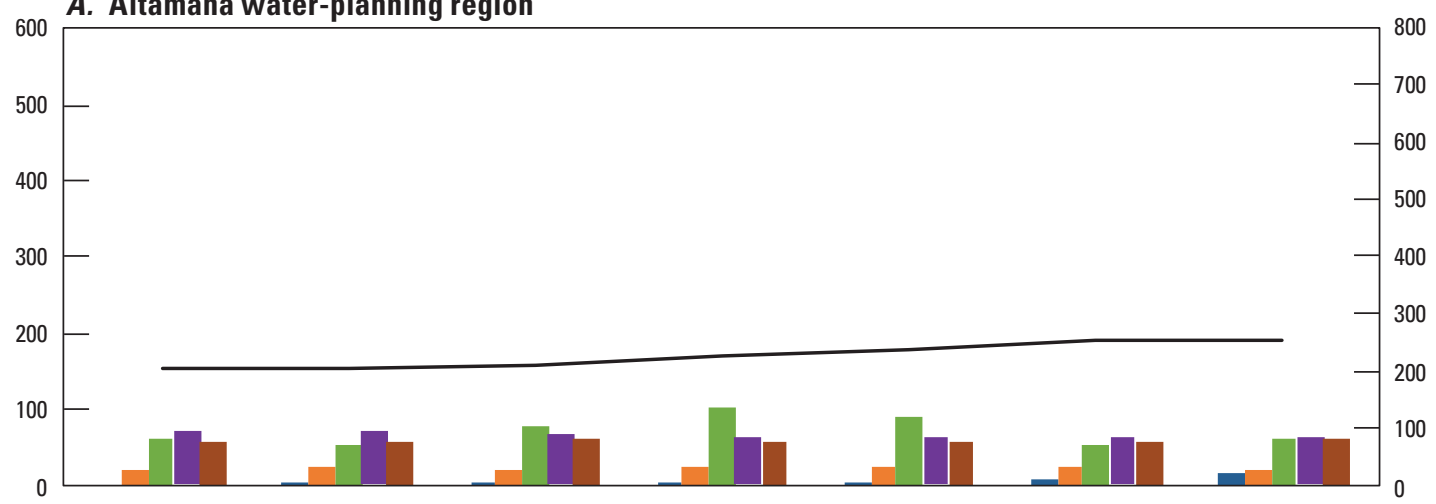

B. Coastal Georgia water-planning region

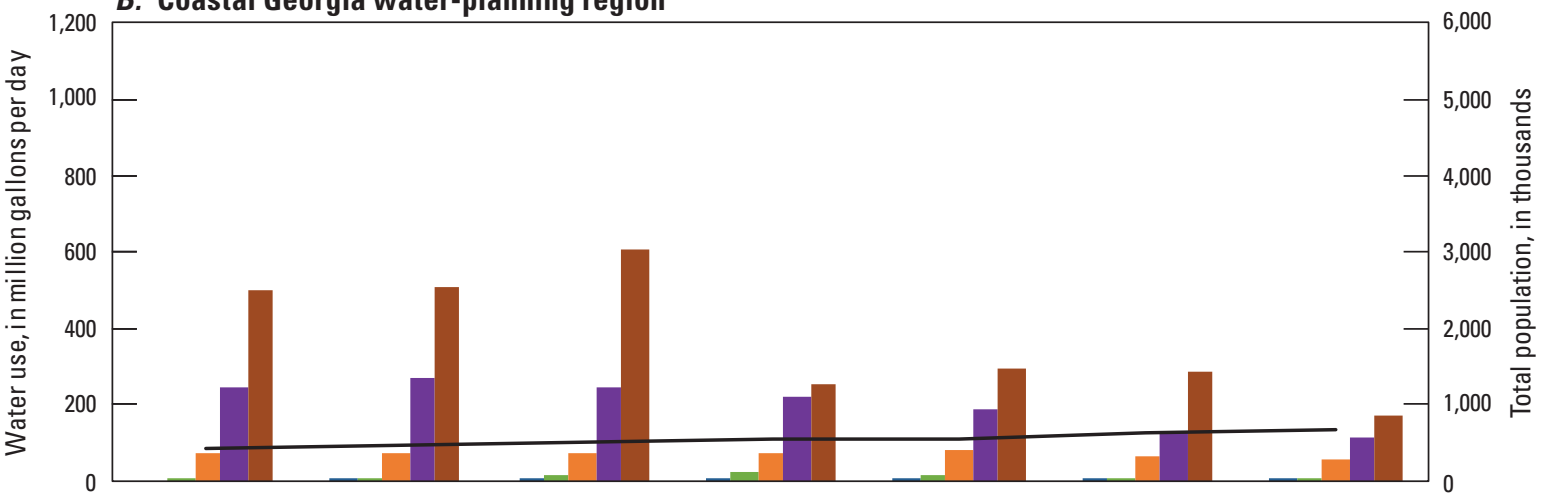

C. Coosa-North Georgia water-planning region

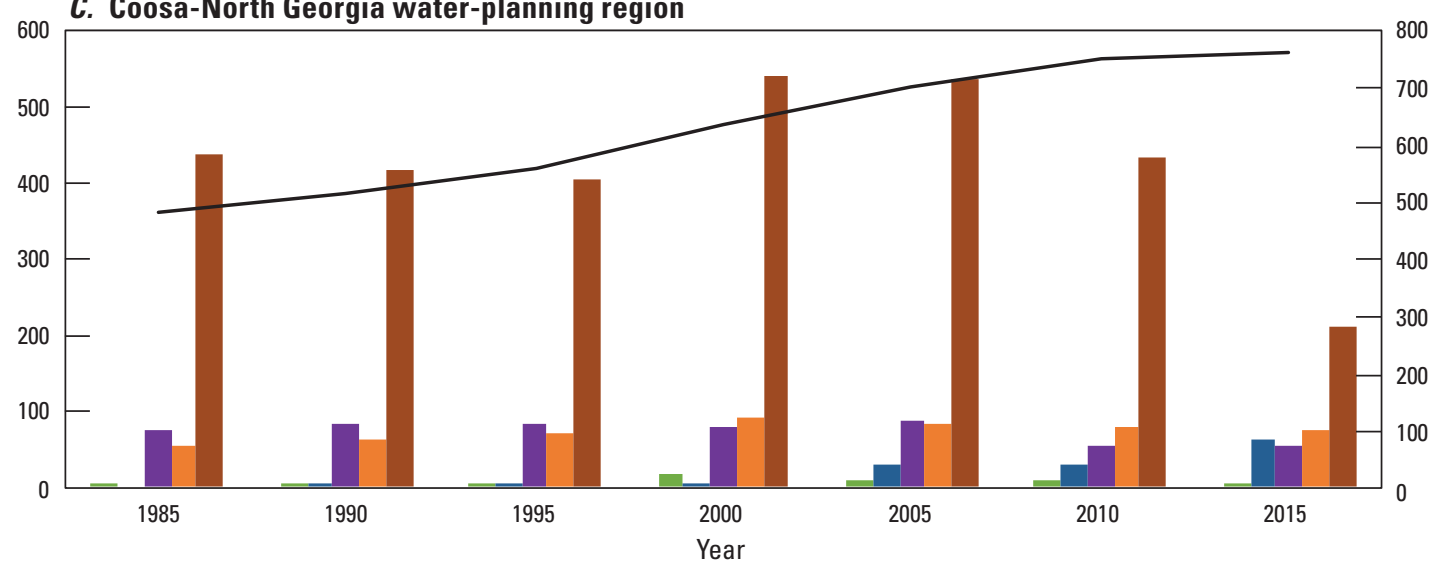

EXPLANATION

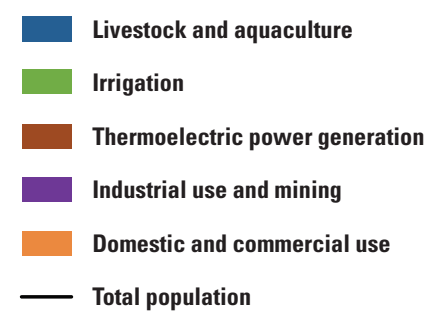

Figure 17. Water-use trends in Georgia water-planning regions by category and population trend for 1985-2015. 

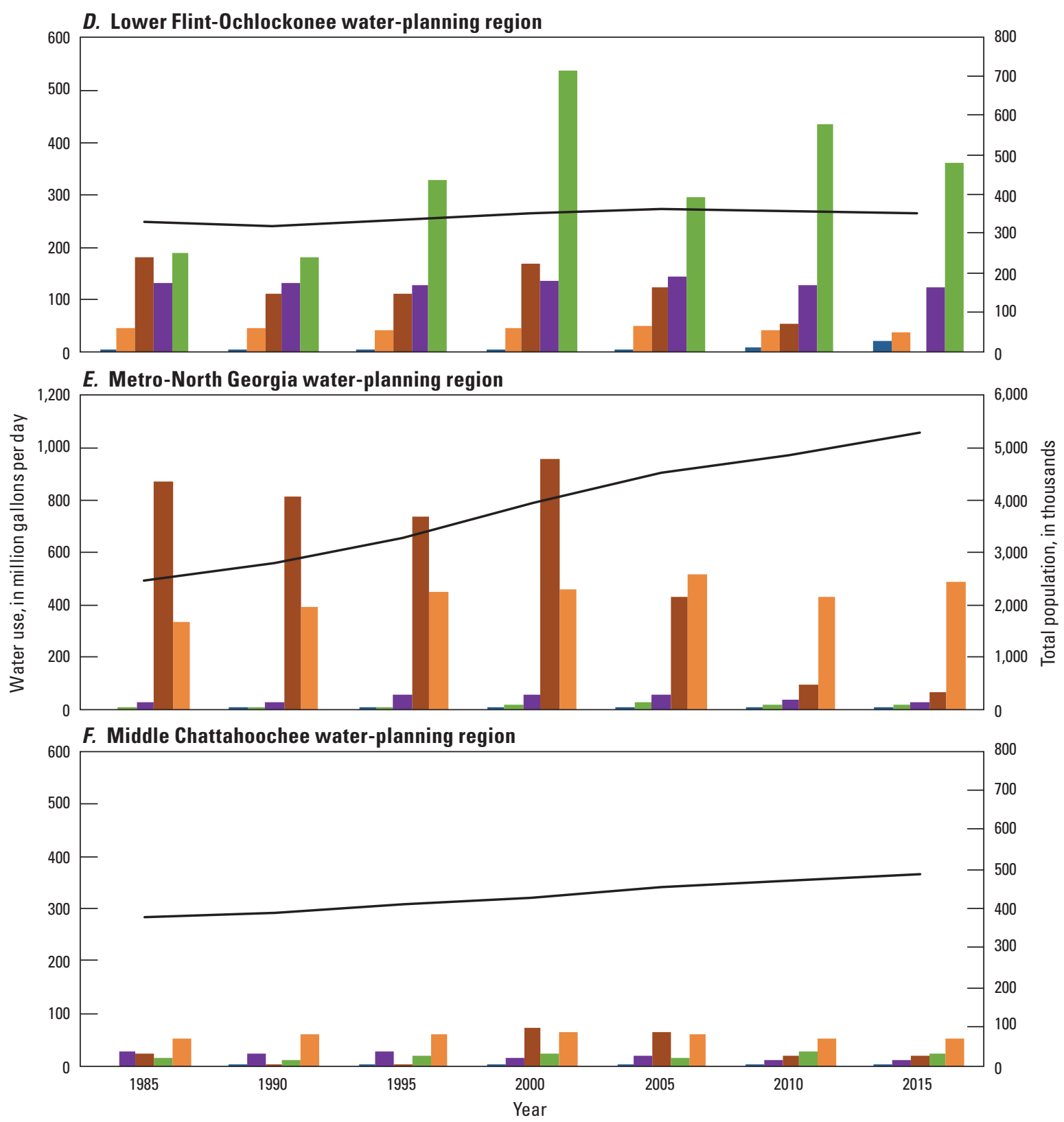

EXPLANATION

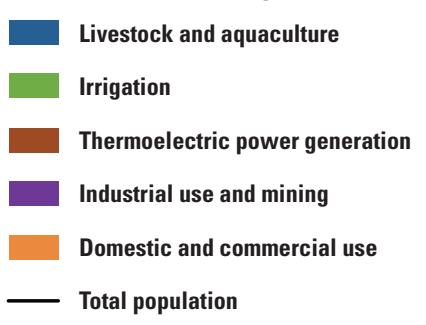

Figure 17. Water-use trends in Georgia water-planning regions by category and population trend for 1985-2015. Continued 
G. Middle Ocmulgee water-planning region
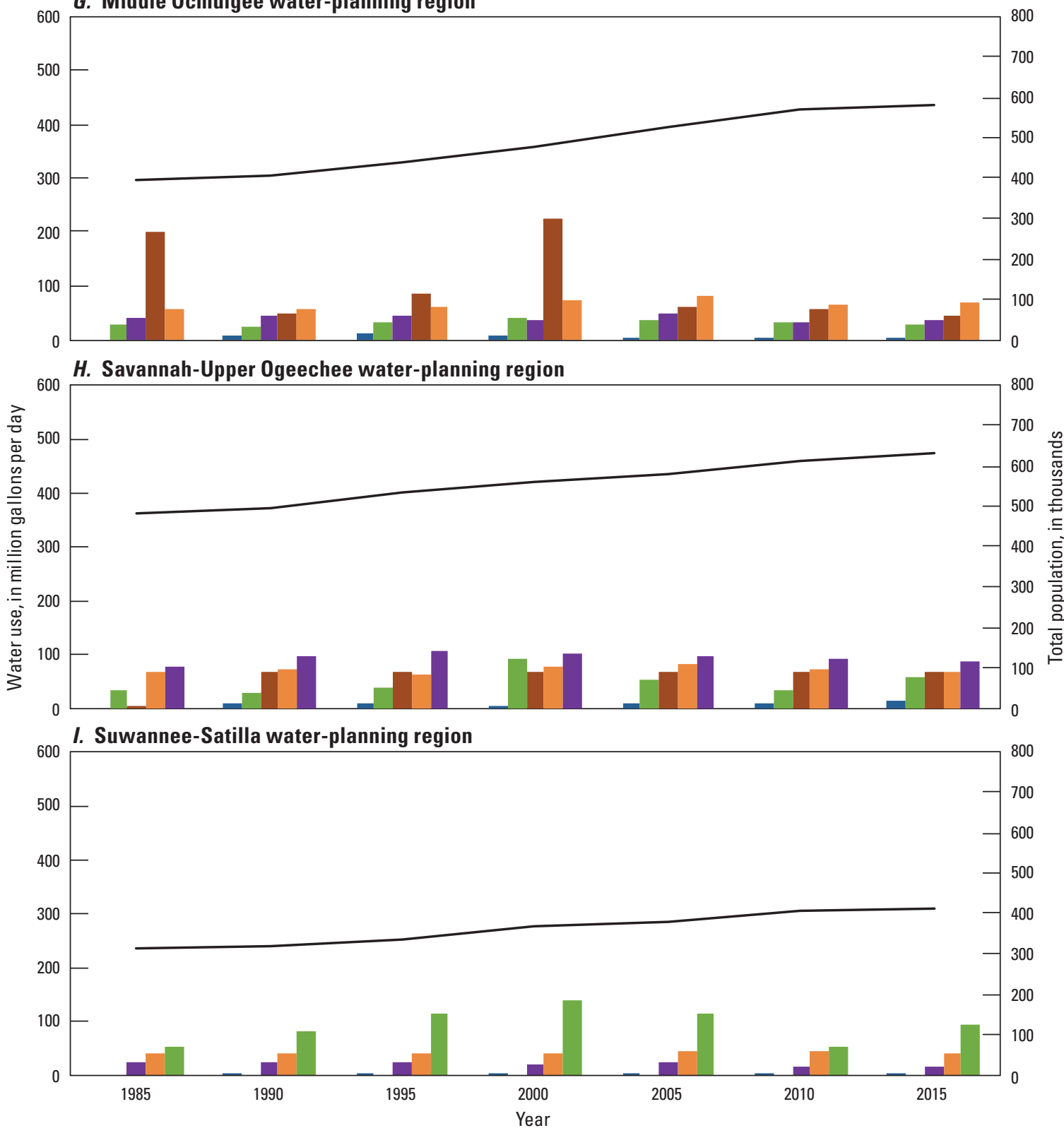

EXPLANATION

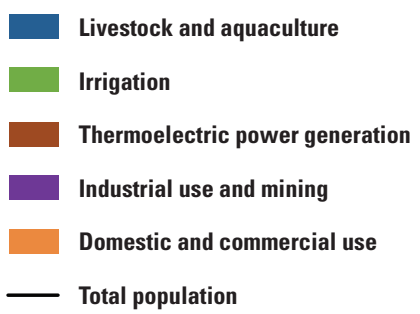

Figure 17. Water-use trends in Georgia water-planning regions by category and population trend for 1985-2015.Continued 


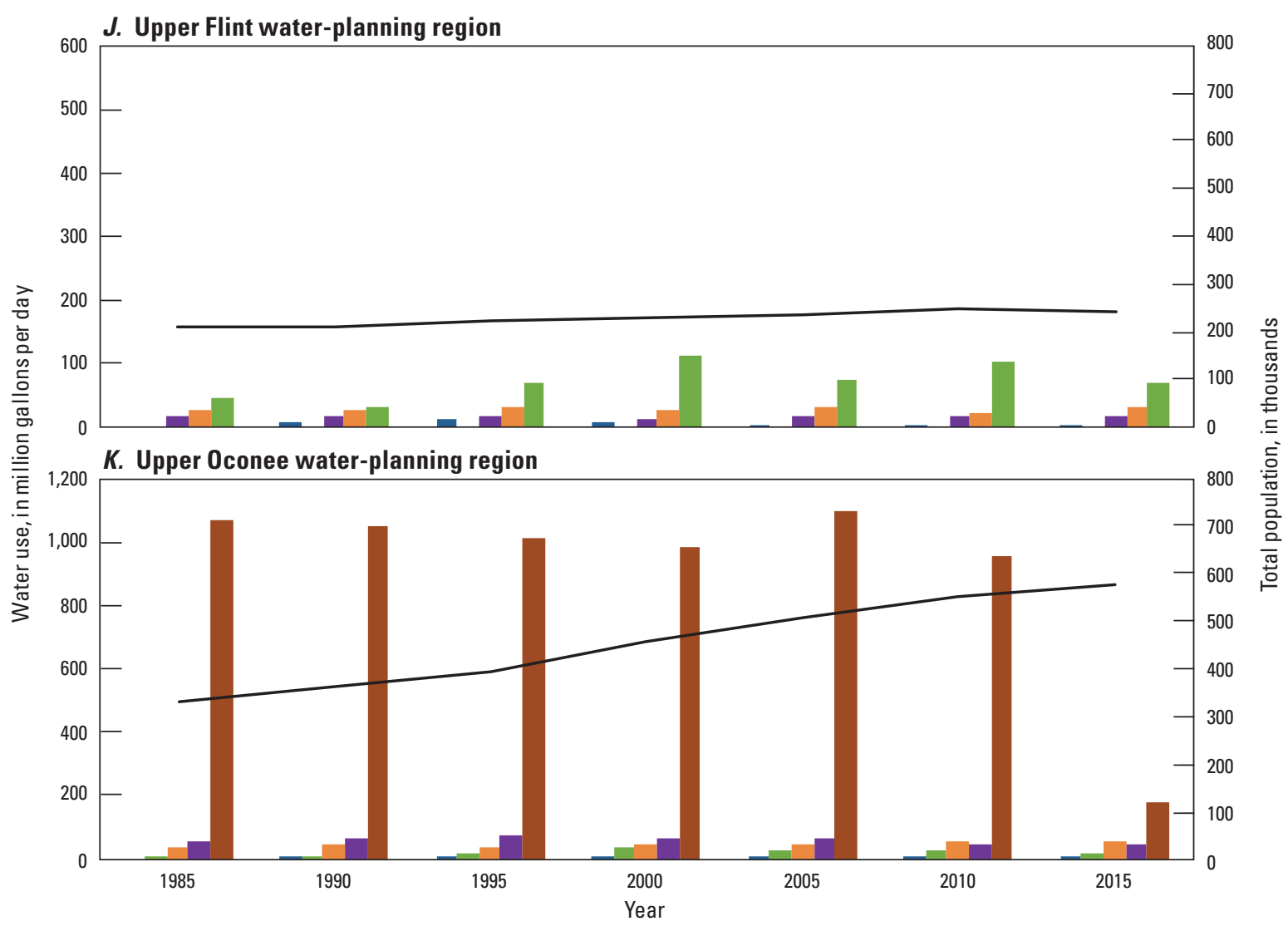

EXPLANATION

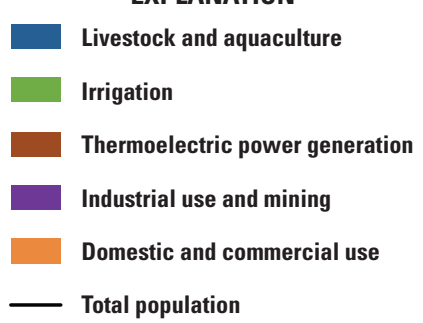

Figure 17. Water-use trends in Georgia water-planning regions by category and population trend for 1985-2015.Continued 


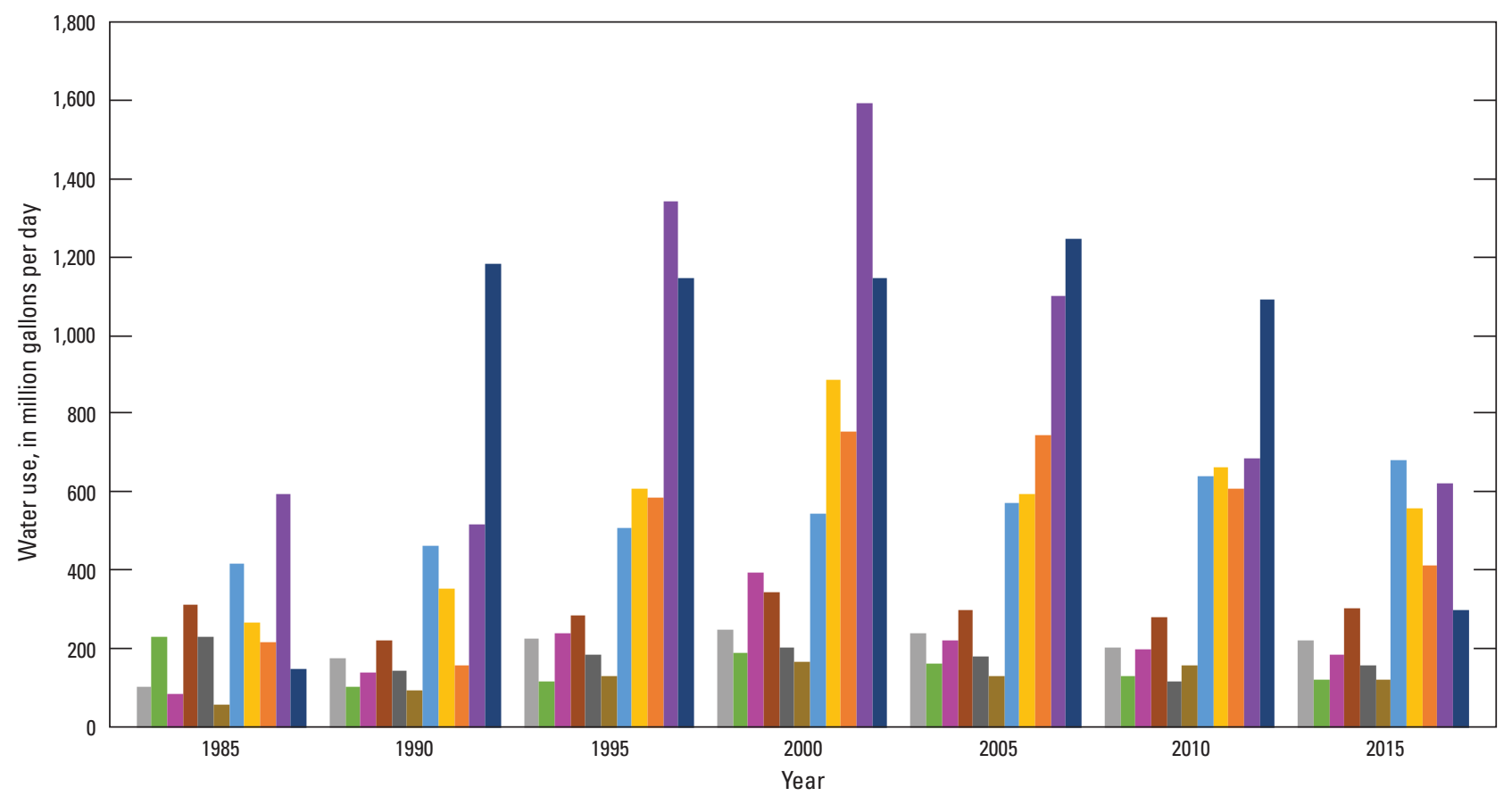

EXPLANATION

Georgia water-planning region

\begin{tabular}{|l|l|}
\hline Altamaha & Middle Ocmulgee \\
\hline Suwanee-Satilla & Coastal Georgia \\
\hline Coosa-North Georgia & Upper Oconee \\
\hline Middle Chattahoochee & Savannah-Upper Ogeechee \\
\hline Upper Flint & Lower Flint \\
\hline Metro-North Georgia & \\
\hline
\end{tabular}

Figure 18. Water-use trends in Georgia water-planning regions for 1985-2015. 


\section{Discussion and Conclusions}

Several of the factors driving changes in estimated water use in Georgia from 1985 to 2015 include (1) population changes in number and location; (2) five periods of major drought; (3) water conservation efforts and education programs initiated by State and local governments and water utilities; (4) and changing water needs for thermoelectric power cooling, industry, and agricultural activities. One or more of these factors are evident in trends for each of the water use categories compiled in this report.

Population changes have long been a predictor of water needs. From 1985 to 2000, estimates of water use from domestic and commercial applications increased, as did the statewide population; however, after the year 2000, statewide water withdrawals declined while the population of the State continued to increase. This declining water-use trend assumes other factors are directing the quantity of water withdrawn for personal, commercial, and recreational uses in Georgia as opposed to population alone. For this reason, three other driving factors are discussed to help explain the trends identified in this report.

Climatic variations in the short-term (yearly or monthly) and long-term (decadal) affect the amount of water used in specific water-use categories. Georgia has experienced five drought periods between 1980 and 2015, specifically 1980-82, 1985-89, 1998-2003, 2006-2007, and 2012. Drought affects the amount of water available to irrigate crops, golf courses, lawns, gardens, and trees. If there is insufficient precipitation during each growth phase of a crop, farmers are likely to increase the amount of water used for irrigation to meet the desired seasonal crop yield and avoid adverse economic effects. Long-term droughts require prioritization of water resources to the effected water-use areas (commercial, personal, or recreational) most essential to societal and economic health without jeopardizing the future availability of water resources while meeting regulatory requirements. The small variations identified in the compiled water withdrawals for irrigation are most likely related to the climatic conditions during those years, as expected. The longer-term (decadal) decreases likely represent changes in agricultural practices, such as the use of more efficient irrigation systems or changes in crop types and seasonal growing patterns. It is important to note that because the water-use compilation series for Georgia provides temporal snapshots of water use in 5-year cycles, it is likely that the full variation of water use during drought periods is not fully documented.

Changes in water availability during drought periods and the need to provide water for all use categories has prompted the establishment of legislation and best management practices by State and local governments and water utilities. One example of this type of legislation is GWSA 2010 (OCGA $\S 12-5-4)$, which identifies drought intensity levels and institutes specific procedures that must be followed at each level. For instance, when the State is in a "level 2" drought, the outdoor watering of lawns, gardens, and trees is limited to certain hours, twice a week, while other outdoor watering activities are prohibited. The Metro-North Georgia waterplanning region has encouraged the population to make water conservation a priority (https://northgeorgiawater.org/currentwater-stats/drought-status/). In turn, the water conservation efforts and education programs initiated by State and local governments, as well as by water utilities, has affected the quantity of water used within specific water-use categories.

A final factor influencing the trends in water withdrawals discussed in this report is the changing water needs for thermoelectric power cooling and agriculture activities. Power plants have historically been the largest water-use category, but the quantity of water needed to produce the power necessary for the population is declining because of decommissioned plants and the restructuring of other plants for the use of less water-intensive technology. For example, the Harllee Branch plant power station in Putnam County closed in April of 2015, which led to reduced withdrawals for 2015 (180.92 Mgal/d) compared with 2010 (952.42 Mgal/d). Changing water requirements for agriculture activities throughout the State, namely crop irrigation, livestock, and aquaculture, affect estimated water use for these use categories. Agriculture is a top industry in Georgia, contributing over $\$ 73$ billion annually to the State's economy (https://www.gfb.org/education-and-outreach/aboutga-agriculture.cms). The U.S. Department of Agriculture, National Agricultural Statistics Service 2012 Census of Agriculture ranked Georgia first in the United States in the production of broiler chickens and peanuts and second in cotton (U.S. Department of Agriculture, 2014). These rankings show that the health of the agricultural industry is paramount to the economy of Georgia and likely encourages producers to use the water needed to meet the desired production. Efforts have been made, through the 40 soil and water conservation districts in the State, to provide citizens with information about the best management practices and conservation technologies, some of which are water related.

\section{Summary}

The quantity of water estimated to meet the personal, commercial, and recreational needs of the 10.2 million people residing in Georgia for 2015 is the smallest quantity since the compilation of water-use data began in 1980. Water-use, withdrawal, and surface-water return data were compiled for 2015 in nine use-categories: domestic, commercial, industry, mining, thermoelectric power cooling, livestock, aquaculture, and irrigation (crops and golf courses). An estimated $3,384 \mathrm{Mgal} / \mathrm{d}$ of water were withdrawn in 2015, continuing a decreasing trend of withdrawals that was first identified when the compilation estimates for 2000 and 2005 were compared. The withdrawals from surface water are greatest in the Apalachicola River Basin, and most groundwater withdrawals are from the Floridan aquifer system. The total population 
of Georgia continues to increase, and the 2015 population is 71-percent greater than the population was in 1985.

The categories of use that underwent the greatest change or variation in water quantity are thermoelectric power cooling, irrigation (crop and golf course), domestic and commercial, and livestock and aquaculture, respectively. Although livestock and aquaculture have shown an increase as irrigation and domestic and commercial water use has varied, these changes are greatly outweighed by major statewide decreases in thermoelectric power cooling use. Statewide water-withdrawal totals for thermoelectric power cooling have decreased due to the decommissioning of plants and the restructuring of others to less water-intensive technology. The variability of water withdrawals for the irrigation and livestock and aquaculture use categories can likely be tied to climate conditions during the growing season and changes in agricultural practices, such as the implementation of more efficient irrigation systems or changes in crop types and seasonal growing patterns. Domestic and commercial water needs have historically close ties to population changes. However, since 2005, water withdrawals decreased for these two categories even though the population continued to increase. This trend is likely connected to the numerous water conservation efforts and educational programs initiated by State and local governments and water utilities throughout Georgia since 2000.

\section{References Cited}

CH2M HILL, 2007, Georgia water use and conservation profiles, TM-2 data analysis: Technical memorandum no. 2, prepared for Georgia Environmental Protection Division, $40 \mathrm{p}$.

Clarke, J.S., and Pierce, R.R., 1985, Georgia groundwater resources, in National Water Summary 1984-Hydrologic events, selected water-quality trends, and ground-water resources: U.S. Geological Survey Water-Supply Paper 2275, p. 179-184, [Also available at https://doi.org/10.3133/ wsp2275.]

Diehl, T.H., and Harris, M.A., 2014, Withdrawal and consumption of water by thermoelectric power plants in the United States, 2010: U.S. Geological Survey Scientific Investigations Report 2014-5184, 28 p., accessed March 15, 2018 at https://doi.org/10.3133/sir20145184.

Fanning, J.L., 1985, The Georgia water-use program: U.S. Geological Survey Open-File Report 85-481, 1 [foldable] sheet. [Also available at https://doi.org/10.3133/ ofr85481.].
Fanning, J.L., 1997, Water use in Georgia by county for 1995: Georgia Geologic Survey Information Circular 101, 95 p., [Also available at https://epd.georgia.gov/sites/epd.georgia. gov/files/related_files/site_page/IC-101.pdf.]

Fanning, J.L., 2003, Water use in Georgia, 2000; and trends, 1950-2000, in Kathryn J. Hatcher, ed., Proceedings of the 2003 Georgia Water Resources Conference-April 23-24, 2003, Athens, Georgia: Athens, Ga., Institute of Ecology, University of Georgia, 5 p., accessed October 29, 2014, at http://ga.water.usgs.gov/publications/other/gwrc2003/pdf/ Fanning-GWRC2003.pdf.

Fanning, J.L., Doonan, G.A., and Montgomery, L.T., 1992, Water use in Georgia by county for 1990: Georgia Geologic Survey Information Circular 90, 98 p., [Also available at https://epd.georgia.gov/sites/epd.georgia.gov/files/related files/site_page/IC-90.pdf.]

Fanning, J.L. and Trent, V.P., 2009, Water use in Georgia by county for 2005; and water-use trends, 1980-2005: U.S. Geological Survey Scientific Investigations Report 2009-5002, 186 p., accessed June 25, 2014, at https:/doi. org/10.3133/sir20095002.

Georgia Environmental Protection Division, 2008, Georgia comprehensive state-wide water management plan: Atlanta, Ga., Georgia Water Council, 39 p. [Also available at https://waterplanning.georgia.gov/state-water-plan.]

Georgia Environmental Protection Division, 2015, Georgia water systems audits and water loss control manual, (3d ed. [ver. 1.2, rev. January]): Marietta, Ga., Georgia Department of Natural Resources, prepared by the Georgia Association of Water Professionals, 45 p. [Also available at https://epd.georgia.gov/sites/epd.georgia.gov/files/ GAWaterLossManual_V1.2.pdf.]

Georgia General Assembly, 2003, House Bill 579 (as passed House and Senate): Georgia General Assembly website, accessed April 9, 2018, at http://www.legis.ga.gov/ Legislation/20032004/27094.pdf.

Georgia General Assembly, 2010, Senate Bill 370 Water Stewardship Act, accessed April 13, 2018, at https://epd. georgia.gov/sites/epd.georgia.gov/files/related_files/site page/SB370\%20rpt\%20review\%20Final.pdf

Lawrence, S.J., 2016, Water use in Georgia by county for 2010 and water-use trends, 1985-2010 (ver. 1.1, January 2016): U.S. Geological Survey Open-File Report 2015-1230, 206 p., [Also available at https://doi.org/10.3133/ofr20151230.

Lewis, Clifford, 2010, Water use for Georgia agricultural withdrawal permitted golf courses: Atlanta, Ga., Georgia Department of Natural Resources, Environmental Protection Division, accessed October 30, 2014, at http://www.nespal.org/sirp/waterinfo/State/awd/ AgWaterDemand_GolfCourses.htm. 
Lovelace, J.K., 2009a, Methods for estimating water withdrawals for aquaculture in the United States, 2005: U.S. Geological Survey Scientific Investigations Report 2009-5042, 13 p., accessed June 26, 2014, at https://doi.org/10.3133/sir20095042.

Lovelace, J.K., 2009b, Methods for estimating water withdrawals for mining in the United States, 2005: U.S. Geological Survey Scientific Investigations Report 2009-5053, 7 p., [Also available at https://doi.org/10.3133/ sir20095053.

MacKichan, K.A., 1951, Estimated use of water in the United States-1950: U.S. Geological Survey Circular 115, 13 p., [Also available at https://doi.org/10.3133/cir115.]

Masters, Mark and Rowles, Kristin, 2016, Agriculture water use for Georgia's livestock and green industry production: Albany, Ga., Georgia Water Planning and Policy Center, Albany State University, 6p.

Painter, J.A., 2019, 2015 Georgia water-use information by county and water-use trends by water-planning region: U.S. Geological Survey data release, https://doi.org/10.5066/P9T6P5SM.

Pierce, R.R., Barber, N.L., and Stiles, H.R., 1982, Water use in Georgia by county for 1980: Georgia Geologic Survey Information Circular 59, $180 \mathrm{p}$. [Also available at https://epd.georgia.gov/sites/epd.georgia.gov/files/related_ files/site_page/IC-59.pdf.]

Southeast Regional Climate Center, 2018, Monthly and seasonal climate information: Chapel Hill, N.C., University of North Carolina at Chapel Hill, accessed May 23, 2018, at http://www.sercc.com/climateinfo_files/monthly/Georgia_ prcp_DivNew.htm.

Trent, V.P., Fanning, J.L., and Doonan, G.A., 1990, Water use in Georgia by county for 1987: Atlanta, Georgia Geological Survey Information Circular 85, 112p. [Also available at https://epd.georgia.gov/sites/epd.georgia.gov/files/related_ files/site_page/IC-85.pdf.]

Torak, L.J., and Painter, J.A., 2011, Summary of the Georgia Agricultural Water Conservation and Metering Program and evaluation of methods used to collect and analyze irrigation data in the middle and lower Chattahoochee and Flint River basins, 2004-2010: U.S. Geological Survey Scientific Investigations Report 2011-5126, 35p. [Also available at https://doi.org/10.3133/sir20115126.]
Torak, L.J., and Painter, J.A., 2013, Geostatistical estimation of growing season irrigation rates using monthly metered data, middle-to-lower Chattahoochee-Flint River Basin, Southwestern Georgia., in Clarke, J.S., and Dalton, M.J., comp.), Hydrologic monitoring and selected hydrologic and environmental studies by the U.S. Geological Survey in Georgia, 2011-2013: U.S. Geological Survey OpenFile Report 2013-1278, p. 63-68. [Also available at https://doi.org/10.3133/ofr20131278.]

Turlington, M.C., Fanning, J.L., and Doonan, G.A., 1987, Water use in Georgia by county for 1985: Georgia Geological Survey Information Circular 81, 100 p. [Also available at https://epd.georgia.gov/sites/epd.georgia.gov/ files/related_files/site_page/IC-81.pdf.]

University of Georgia, 2015, 2014 Georgia farm gate value report: Athens, Ga., The Center for Agribusiness and Economic Development, AR-15-01, 174 p., accessed May 23, 2018, at http://caes2.caes.uga.edu/center/caed/ documents/2014UGACAEDFGVR_FINAL.pdf.

U.S. Census Bureau, 2017, American estimates of resident population-April 1,2010 to July 1, 2015; 2015 population estimates: U.S. Census Bureau web page, accessed April 12,2018, at https://factfinder.census.gov/faces/ tableservices/jsf/pages/productview.xhtml? $\mathrm{src}=\mathrm{bkmk}$

U.S. Census Bureau, 2016, Population, population change and estimated components of population change-April 1, 2010 to July 1, 2015: U.S. Census Bureau web page, accessed April 12,2018, at https://factfinder.census.gov/faces/ tableservices/jsf/pages/productview.xhtml?pid=PEP_2017 PEPANNRES\&src $=$ pt.

U.S. Department of Agriculture, 2014, 2012 Census of Agriculture, United States summary and State data, v. 1, Geographic area series, pt. 51, chap. 2, County level dataGeorgia: Washington, D.C., National Agricultural Statistics Service, AC-12-A-51 accessed May 23, 2018, at https:// www.agcensus.usda.gov/Publications/2012/\#full_report.

U.S. Department of Agriculture, 2015, Cropland data layer [CDL], National CDL's for year 2015: Washington, D.C., National Agricultural Statistics Service, U.S. Department of Agriculture, accessed May 9, 2018, at https://www.nass. usda.gov/Research_and_Science/Cropland/SARS1a.php.

U.S. Environmental Protection Agency, 2010, Control and mitigation of drinking water losses in distribution systems: Office of Water, U.S. Environmental Protection Agency EPA 816-R-10-019, 176 p. [Also available at https://nepis.epa. gov/Exe/ZyPDF.cgi/P1009VCZ.PDF?Dockey=P1009VCZ. PDF.] 
U.S. Environmental Protection Agency, 2014, National Pollutant Discharge Elimination System (NPDES) NPDES permit basics: Environmental Protection Agency web page, accessed September 24, 2018, at https://www.epa.gov/npdes/npdes-permit-basics.

U.S. Geological Survey, 2018, USGS water data for the Nation: National Water Information System database, accessed May 9, 2018, at https://doi.org/10.5066/ F7P55KJN.
Waltz, Clint, 2008, Research update-Evaluation of the GGCSA BMPs: Athens, Ga., University of Georgia, 4 p., accessed March 30, 2018, at http://cdn.cybergolf.com/ images/994/Research-Update---BMPs-_01-08_.pdf 



\section{Glossary}

aquaculture water use Water use associated with the farming of organisms that live in water (such as finfish and shellfish) and offstream water use associated with fish hatcheries. See also fish-farm water use, fishhatchery water use, animal-specialties water use, and livestock water use.

census block U.S. census blocks are statistical areas bounded by visible features such as roads, streets, water bodies, railroad tracks, and city, town, county, township, or school district boundaries.

commercial water use Water for motels, hotels, restaurants, office buildings, other commercial facilities, military and nonmilitary institutions, and (for 1990 and 1995) offstream fish hatcheries. Water may be obtained from a public-supply system or may be self-supplied.

cooling system An equipment system that provides water for cooling purposes, such as to condensers at power plants or factories. May include water intakes, outlets, cooling towers, ponds, canals, pumps, and pipes. See also cooling-system type, industrial water use, and thermoelectric-power water use.

cooling-system type Defined as either a once-through or recirculating cooling system. See also industrial water use, once-through cooling system, recirculating cooling system, and thermoelectric-power water use.

domestic water use Water used for indoor household purposes such as drinking, food preparation, bathing, washing clothes and dishes, flushing toilets, and outdoor purposes such as watering lawns and gardens. Domestic water use includes water provided to households by a public water supply (domestic deliveries from public suppliers) and self-supplied water. See also publicsupply deliveries, public-supply water use, rural water use, and self-supplied water use.

freshwater Water that contains less than 1,000 milligrams per liter $(\mathrm{mg} / \mathrm{L})$ of dissolved solids. Generally, water with more than 500 $\mathrm{mg} / \mathrm{L}$ of dissolved solids is undesirable for drinking. groundwater All subsurface water, distinct from surface water. Specifically, the part of the subsurface water in the saturated zone, which is a zone where all voids are filled with water.

industrial water use Water used for fabrication, processing, washing, and cooling. Includes industries such as chemical and allied products, food, mining, paper and allied products, petroleum refining, and steel.

irrigation water use Water that is applied by an irrigation system to assist crop and pasture growth, or to maintain vegetation on recreational lands such as parks and golf courses. Irrigation includes water that is applied for pre-irrigation, frost protection, chemical application, weed control, field preparation, crop cooling, harvesting, dust suppression, leaching of salts from the root zone, and conveyance losses. Irrigation water use includes self-supplied water and reclaimed wastewater.

livestock water use Water used for livestock watering, feedlots, dairy operations, and other on-farm needs. Types of livestock include dairy cows and heifers, beef cattle and calves, sheep and lambs, goats, hogs and pigs, horses, and poultry.

micro-irrigation system An irrigation system that wets only a discrete portion of the soil surface in the vicinity of a plant using applicators (such as orifices, emitters, porous tubing, or perforated pipe) and operated under low pressure. The applicators may be placed on or below the surface of the ground or suspended from supports. See also irrigation water use, sprinkler irrigation system, and surface irrigation system

mining water use Water used for the extraction of naturally occurring minerals including solids (such as coal, sand, gravel, and other ores), liquids (such as crude petroleum), and gases (such as natural gas). Also includes uses associated with quarrying, milling of mined materials, injection of water for secondary oil recovery or unconventional oil and gas recovery (such as hydraulic 
fracturing), and other operations associated with mining activity. Does not include water associated with dewatering of the aquifer that is not put to beneficial use. Also does not include water used in processing, such as smelting, refining petroleum, or slurry pipeline operations. These processing uses are included in industrial water use

offstream water use Water withdrawn or diverted from a groundwater or surfacewater source for aquaculture, commercial, self-supplied domestic, industrial, irrigation, livestock, mining, public supply, thermoelectric power, and other uses.

once-through cooling system Also known as an open-loop cooling system. A cooling system in which the water is withdrawn from a source, circulated through the heat exchangers, and then returned to a body of water at a higher temperature. See also cooling system, cooling-system type, and thermoelectric-power water use.

per capita water use The average amount of water used per person during a standard time period, generally per day. Per capita use may be calculated based on total water use, public-supply water use, self-supplied domestic water use, or domestic deliveries from a public supply.

public-supply water use Water withdrawn by public and private water suppliers that furnish water to at least 25 people or have a minimum of 15 connections. Public suppliers provide water for a variety of uses, such as domestic, commercial, industrial, thermoelectric-power, and public water use. See also commercial water use, domestic water use, industrial water use, publicsupply deliveries, public water use, and thermoelectric-power water use.

public-supply deliveries Amount of water delivered from a public supplier to users for domestic, commercial, industrial, thermoelectric power, or public-use purposes

public water supplier Any water supplier (municipal or private entity) that regularly serves at least 25 people or has at least 15 water connections is considered a public water system under Georgia Law (OCGA § 12-5-172-11). public water use Water supplied from a public supplier and used for such purposes as firefighting, street washing, flushing of water lines, and maintaining municipal parks and swimming pools. Public-use water is not usually billed by the public supplier.

recirculating cooling system Also known as closed-loop cooling system or recirculation cooling system. Water is withdrawn from a source, circulated through heat exchangers, cooled, and then reused in the same process. Recirculating cooling systems may use induced draft cooling towers, forced draft cooling towers, cooling ponds, or canals.

reclaimed wastewater Wastewatertreatment plant effluent that has been diverted for beneficial uses such as irrigation, industrial use, or thermoelectric power cooling instead of being released to a natural waterway or aquifer.

raw water Water that has not been filtered or treated before use.

saline water Water that contains more than 1,000 milligrams per liter of dissolved solids.

self-supplied domestic Surface water or groundwater withdrawn from a private intake or well at a single residence for domestic use.

self-supplied water Water withdrawn from a groundwater or surface-water source by a user rather than being obtained from a publicsupply source.

sprinkler irrigation system An irrigation system in which water is applied using perforated pipes or nozzles operated under pressure to form a spray pattern.

surface water An open body of water, such as a creek, canal, river, stream, lake, pond, or reservoir.

surface-water return flows Raw or treated water discharge to a river, stream, pond, lake, or reservoir.

thermoelectric power use Water used in the process of generating electricity with steamdriven turbine generators. Thermoelectric power water use includes water provided by a public water supply (deliveries from public suppliers), self-supplied water, and reclaimed wastewater. 
water use Water use for the domestic, commercial, industrial, and thermoelectric categories included both self-supplied withdrawals and deliveries from a public supply, and some categories included reclaimed wastewater use. More broadly, water use pertains to the interaction of humans with and influence on the hydrologic cycle, and includes elements such as water withdrawal, delivery, consumptive use, wastewater release, reclaimed wastewater, return flow, and instream use. water-use coefficient A factor or ratio used to estimate a quantity of water used based on a related quantity. Examples of water-use coefficients include daily per capita water use, consumptive crop irrigation requirements, livestock water requirements, per employee water use, and per unit of product water use.

water withdrawal Water removed from a groundwater or surface-water source for use. 


\section{Appendix 1. North American Industrial Classification System Codes}

Industrial water use is given by industry type, classified by a North American Industrial Classification System code. The following is a brief description of the codes used in this publication (U.S. Census Bureau, 2012).

212-Mining and processing of kaolin and fuller's earth. This major group includes establishments engaged in the mining and processing of kaolin and fuller's clay. Water washing is a processing method in which water and chemical dispersants are added to the mined clay to produce a slurry. The slurry is then transported through pipes to the processing facility.

311 - Food and kindred products. This major group includes establishments that manufacture or process foods and beverages for human consumption, and certain related products, such as manufactured ice, chewing gum, vegetable oils and animal fats, and prepared feeds for animals and fowls.

312-Beverage manufacturing. The industrial group includes establishments such as breweries, distilleries, wineries, and those that produce soft drinks and bottled water. 313, 314-Textile mills and textile product mills. This major group includes establishments engaged in performing any of the following operations: (1) preparation of fiber and subsequent manufacturing of yarn, thread, braids, twine, and cordage; (2) manufacturing broad woven fabric, narrow woven fabric, knit fabric, and carpets and rugs from yarn; (3) dyeing and finishing fiber, yarn, fabric, and knit apparel; (4) coating, waterproofing, or otherwise treating fabric; (5) the integrated manufacture of knit apparel and other finished articles from yarn; and (6) the manufacture of felt goods, lace goods, nonwoven fabrics, and miscellaneous textiles. 321-Wood product manufacturing, except furniture. This major group includes sawmills, lathe mills, shingle mills, cooperage stock mills, planing mills, and pulpwood mills and veneer mills engaged in producing veneers, plywood, engineered wood products, millwork, and wood door and window manufacturing.
322 - Paper, pulp, and allied Products. This major group includes the manufacture of pulps from wood and other cellulose fibers and rags; the manufacture of paper and paperboard; and the manufacture of paper and paperboard into converted products such as paper coated off the paper machine, paper bags, paper boxes, and envelopes.

325-Chemicals and allied products.

This major group includes establishments producing basic chemicals and those manufacturing products by predominantly chemical processes. Establishments classified in this major group manufacture three general classes of products: (1) basic chemicals such as acids, alkalis, salts, and organic chemicals; (2) chemical products to be used in further manufacture such as synthetic fibers, plastics materials, dry colors, and pigments; and (3) finished chemical products to be used for ultimate consumption such as drugs, cosmetics, and soaps, or to be used as materials or supplies in other industries such as paints, fertilizers, and explosives. 326-Rubber and miscellaneous plastics products. This major group includes establishments manufacturing from natural, synthetic, or reclaimed rubber; rubber products such as tires, rubber footwear, mechanical rubber goods, heels and soles, flooring, and rubber sundries; plastics such as pipes, pipe fittings, plastic bags, sheets, packaging material, polystyrene, and urethane foam products.

327-Stone, clay, glass, and concrete products. This major group includes establishments engaged in manufacturing flat glass and other glass products, cement, structural clay products, pottery, ceramics, concrete and gypsum products, cut stone, abrasive and asbestos products, and so forth, from materials taken principally from the earth in the form of stone, clay, and sand. 
331-Primary metal industries. This major group includes establishments engaged in the smelting and refining of ferrous and nonferrous metals from ore, pig, or scrap; in the rolling, drawing, and alloying of ferrous and non-ferrous metals; in the manufacture of castings and other basic products of ferrous and nonferrous metals; and in the manufacture of aluminum sheet, foil, extruded products, pipes, tubes, nails, spikes, and insulated wire and cable.

332-Fabricated metal products, except machinery and transportation equipment. This major group includes establishments engaged in fabricating ferrous and nonferrous metal products such as metal cans, tinware, hand tools, cutlery, general hardware, non-electric heating apparatus, fabricated structural metal products, metal forging, metal stamping, ordnance (except vehicles and guided missiles), and a variety of metal and wire products not elsewhere classified. 339_Miscellaneous manufacturing. This major group includes establishments engaged in manufacturing medical equipment such as surgical, dental, and medical instruments and supplies; laboratory apparatus and furniture; manufacturing of jewelry and silverware; and a variety of other goods such as toys, sporting goods and athletic equipment, office supplies, musical instruments, signs, fasteners, needles, buttons, and caskets. 


\section{Appendix 2. Population, Water Withdrawals, and Water Use by Source of Water for Each County in Georgia, 2015}

\section{Terms}

Total use is total withdrawal plus public supply deliveries and losses.

Net transfers/losses for a county is the sum of all water purchased by public suppliers in a county from public-water suppliers in a different county minus the sum of all publicsupplied water sold to public suppliers or delivered to customers in a different county and public-supply losses. 


\section{APPLING COUNTY}

Population

Population served by public supply-groundwater

Population served by public supply-surface water

0

Acres irrigated

2015 WATER WITHDRAWALS AND ESTIMATED USE, IN MILLION GALLONS PER DAY

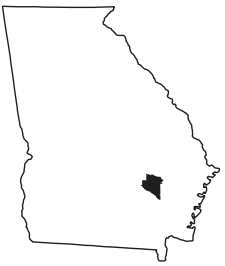

[--, not applicable; Mgal/d, million gallons per day]

\begin{tabular}{|c|c|c|c|c|c|c|c|c|}
\hline \multirow{3}{*}{ Category } & \multicolumn{3}{|c|}{ Withdrawals } & \multirow{3}{*}{$\begin{array}{c}\text { Total } \\
\text { use }\end{array}$} & \multirow{3}{*}{$\begin{array}{l}\text { Returns to } \\
\text { Surface }\end{array}$} & \multirow{2}{*}{\multicolumn{3}{|c|}{$\begin{array}{l}\text { Withdrawals by Major Industrial Groups } \\
\text { [NAICS, North American Industrial Classification System code] }\end{array}$}} \\
\hline & \multirow{2}{*}{$\begin{array}{c}\text { Ground - } \\
\text { water }\end{array}$} & \multirow{2}{*}{$\begin{array}{c}\text { Surface } \\
\text { water }\end{array}$} & \multirow[b]{2}{*}{ Total } & & & & & \\
\hline & & & & & & NAICS & Groundwater & Surface water \\
\hline Public supply & 0.76 & 0.00 & 0.76 & -- & -- & & & \\
\hline Domestic use & 0.92 & 0.00 & 0.92 & 1.45 & 0.00 & None & -- & -- \\
\hline Commercial use & 0.00 & 0.00 & 0.00 & 0.03 & 0.00 & & & \\
\hline Industrial use & 0.00 & 0.00 & 0.00 & 0.04 & 0.00 & & & \\
\hline Public-supply loss & -- & -- & -- & 0.16 & -- & & & \\
\hline Inter-county delivery & -- & -- & -- & 0 & -- & & & \\
\hline Public wastewater treatment & -- & - & -- & -- & 1.18 & & & \\
\hline Mining use & 0.00 & 0.00 & 0.00 & 0.00 & 0.00 & Withdrawa & jor Public Supp & \\
\hline Irrigation (crop) & 3.76 & 0.43 & 4.19 & 4.19 & 0.00 & Name & Groundwater & Surface water \\
\hline Irrigation (golf) & 0.00 & 0.00 & 0.00 & 0.00 & 0.00 & City of Baxley & 0.69 & 0.00 \\
\hline Livestock-aquaculture & 0.63 & 0.07 & 0.70 & 0.70 & 0.00 & & & \\
\hline Thermoelectric power & 0.19 & 60.59 & 60.78 & 60.78 & 0.00 & & & \\
\hline TOTAL & 6.26 & 61.09 & 67.35 & 67.35 & 1.18 & & & \\
\hline
\end{tabular}

Total use is total withdrawal plus public supply deliveries and losses.

Withdrawals by water source

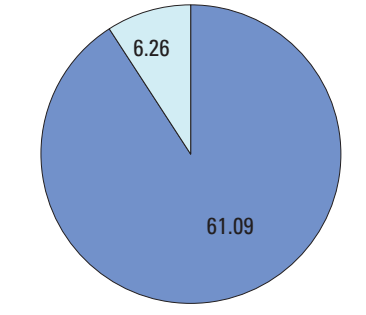

Withdrawals, in million

gallons per day

Surface water

Groundwater
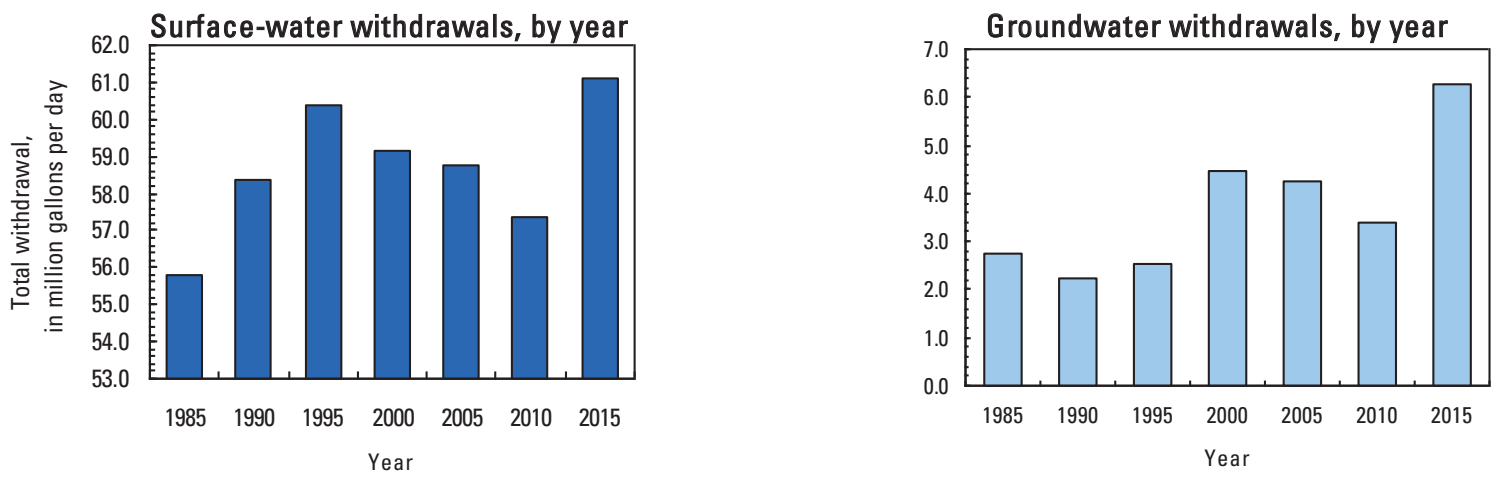


\section{ATKINSON COUNTY}

Population 8,398

Population served by public supply-groundwater $\quad 4,409$

Population served by public supply-surface water 0

Acres irrigated

10,310

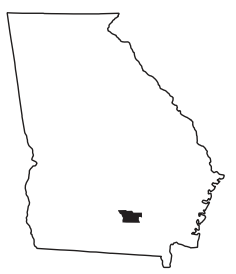

2015 WATER WITHDRAWALS AND ESTIMATED USE, IN MILLION GALLONS PER DAY

$[--$, not applicable; Mgal/d, million gallons per day]

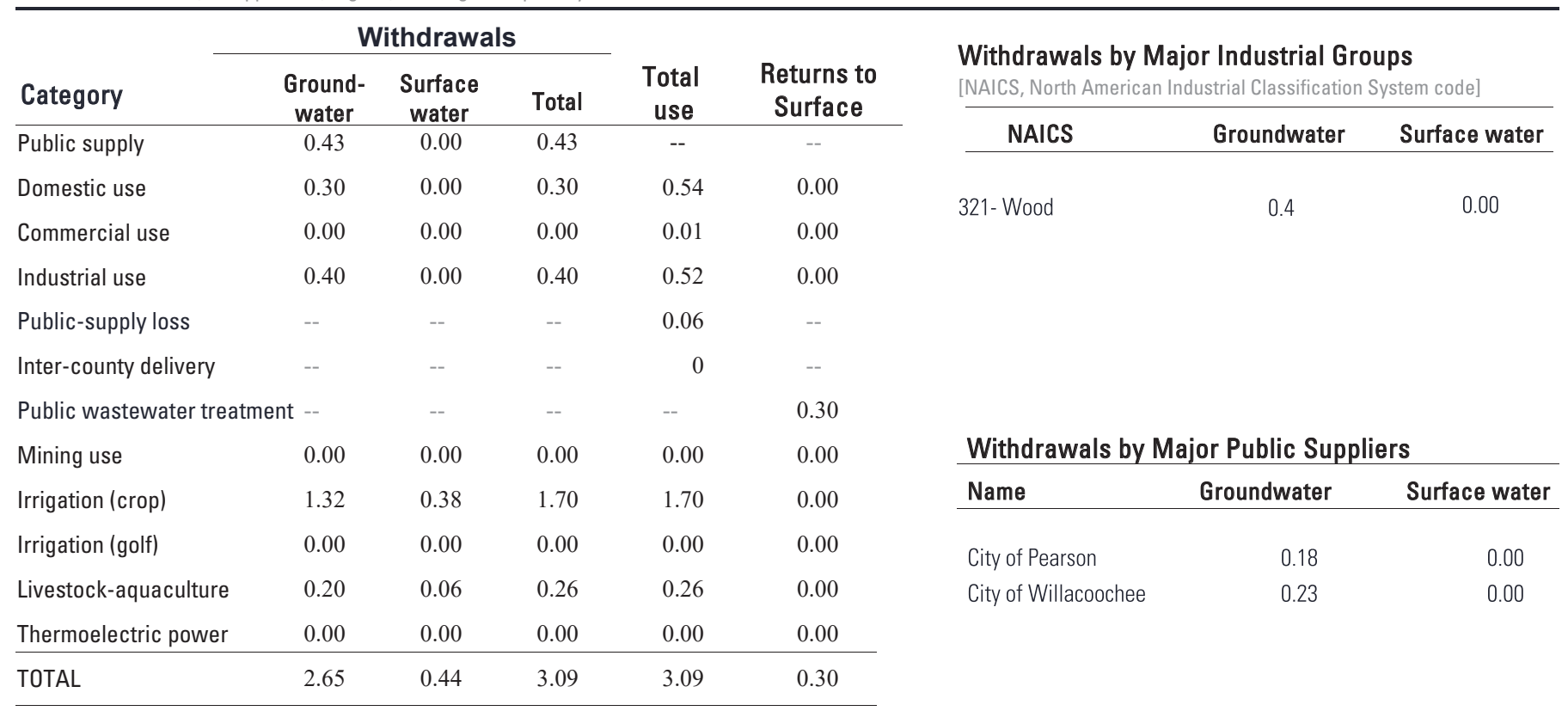

Total use is total withdrawal plus public supply deliveries and losses.

Withdrawals by water source

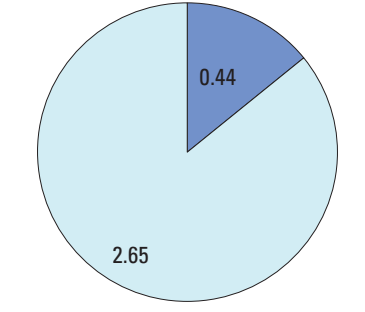

Withdrawals, in million

gallons per day

Surface water

Groundwater

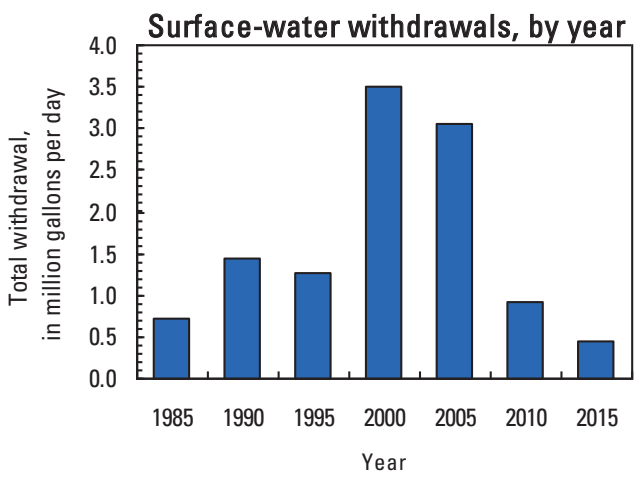

Groundwater withdrawals by aquifer

\begin{tabular}{|l|l}
\hline & Crystalline-rock aquifers \\
\hline & Floridan aquifer system \\
\hline & Southeastern Coastal Plain aquifers \\
& Surficial aquifer system \\
\hline$\quad$ Brunswick aquifer system \\
Valley and Ridge aquifer
\end{tabular}

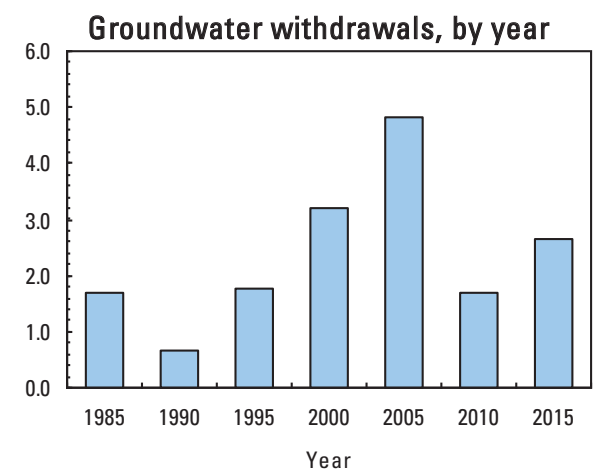




\section{BACON COUNTY}

Population

Population served by public supply-groundwater

Population served by public supply-surface water

Acres irrigated

2015 WATER WITHDRAWALS AND ESTIMATED USE, IN MILLION GALLONS PER DAY

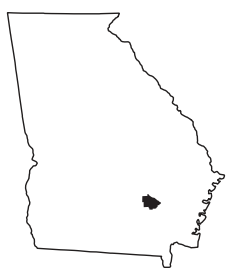

[--,not applicable; Mgal/d, million gallons per day]

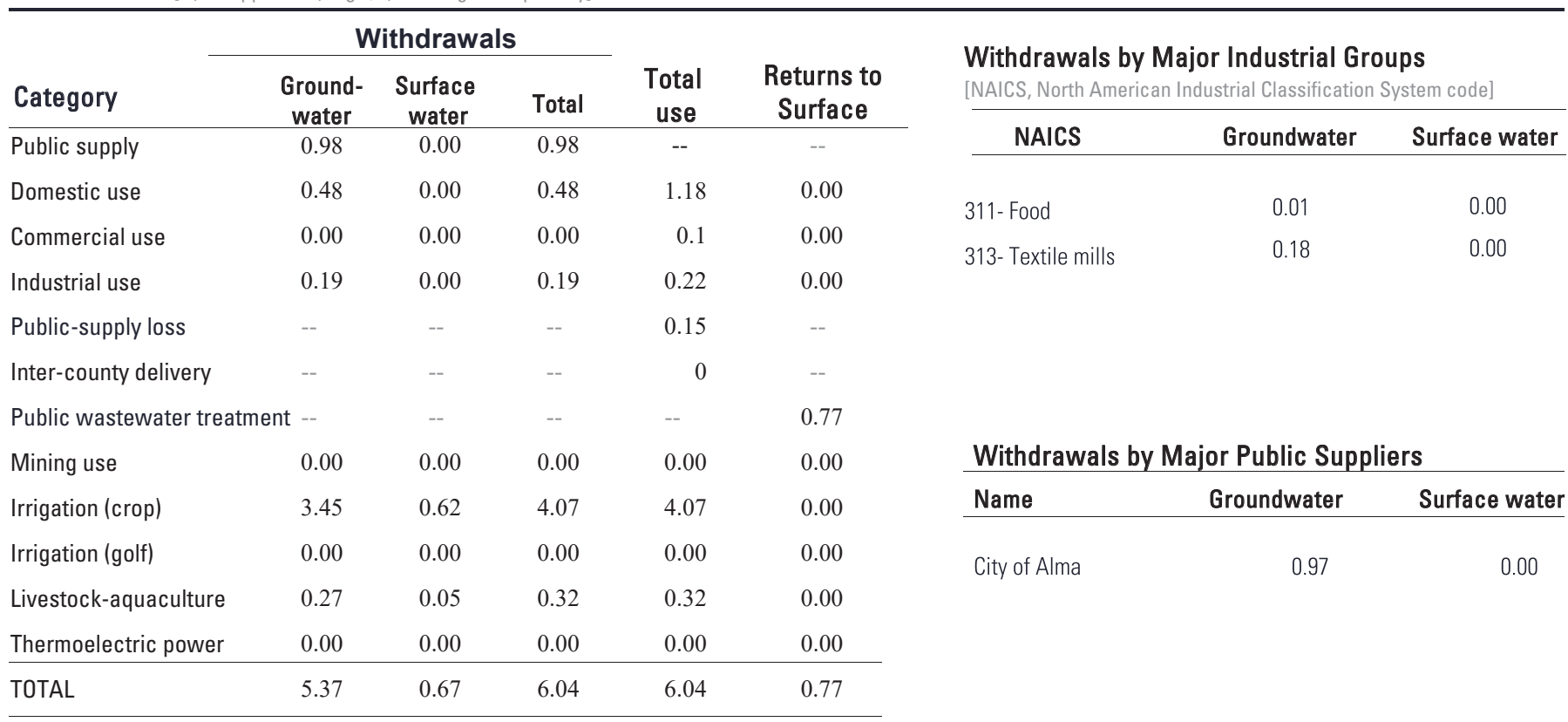

Total use is total withdrawal plus public supply deliveries and losses.

Withdrawals by water source

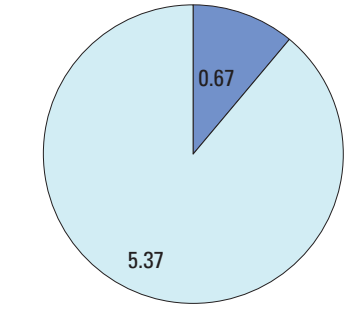

Withdrawals, in million gallons per day

Surface water

Groundwater

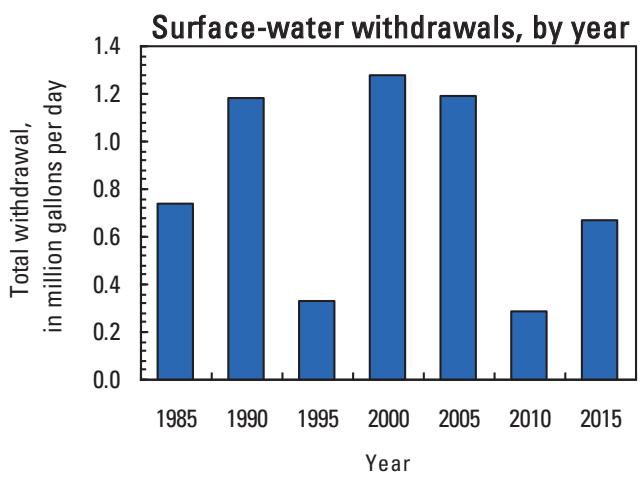

Groundwater withdrawals by aquifer

\begin{tabular}{ll}
\hline & Crystalline-rock aquifers \\
\hline & Floridan aquifer system \\
\hline & Southeastern Coastal Plain aquifers \\
& Surficial aquifer system \\
& Brunswick aquifer system \\
& Valley and Ridge aquifer
\end{tabular}

Groundwater withdrawals, by year

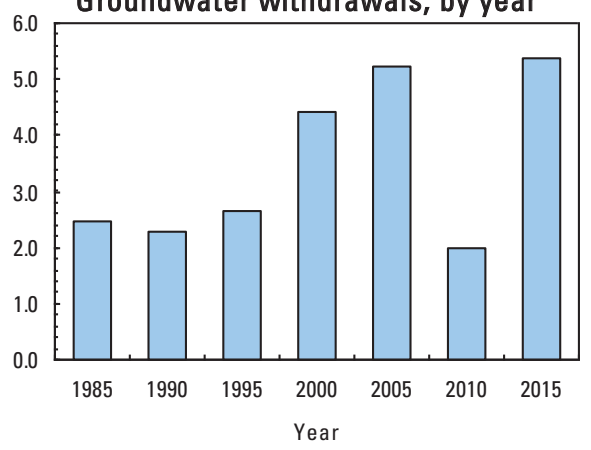




\section{BAKER COUNTY}

Population

Population served by public supply-groundwater

Population served by public supply-surface water

Acres irrigated
3,180

681

0

40,340

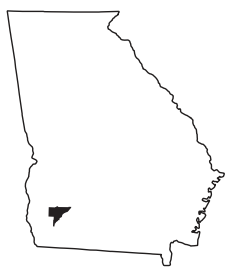

2015 WATER WITHDRAWALS AND ESTIMATED USE, IN MILLION GALLONS PER DAY

[--,not applicable; Mgal/d, million gallons per day]

\begin{tabular}{|c|c|c|c|c|c|c|c|c|}
\hline \multirow{3}{*}{ Category } & \multicolumn{3}{|c|}{ Withdrawals } & \multirow{3}{*}{$\begin{array}{c}\text { Total } \\
\text { use }\end{array}$} & \multirow{3}{*}{$\begin{array}{l}\text { Returns to } \\
\text { Surface }\end{array}$} & \multirow{2}{*}{\multicolumn{3}{|c|}{$\begin{array}{l}\text { Withdrawals by Major Industrial Groups } \\
\text { [NAICS, North American Industrial Classification System code] }\end{array}$}} \\
\hline & \multirow{2}{*}{$\begin{array}{c}\text { Ground - } \\
\text { water }\end{array}$} & \multirow{2}{*}{$\begin{array}{c}\text { Surface } \\
\text { water }\end{array}$} & \multirow[b]{2}{*}{ Total } & & & & & \\
\hline & & & & & & NAICS & Groundwater & Surface water \\
\hline Public supply & 0.15 & 0.00 & 0.15 & -- & -- & & & \\
\hline Domestic use & 0.19 & 0.00 & 0.19 & 0.31 & 0.00 & None & -- & -- \\
\hline Commercial use & 0.00 & 0.00 & 0.00 & 0 & 0.00 & & & \\
\hline Industrial use & 0.00 & 0.00 & 0.00 & 0 & 0.00 & & & \\
\hline Public-supply loss & -- & -- & -- & 0.03 & -- & & & \\
\hline Inter-county delivery & -- & -- & -- & 0 & -- & & & \\
\hline Public wastewater treatment & t -- & -- & -- & -- & 0.00 & & & \\
\hline Mining use & 0.00 & 0.00 & 0.00 & 0.00 & 0.00 & Withdrawals & jor Public Supp & \\
\hline Irrigation (crop) & 21.80 & 1.75 & 23.55 & 23.55 & 0.00 & Name & Groundwater & Surface water \\
\hline Irrigation (golf) & 0.00 & 0.00 & 0.00 & 0.00 & 0.00 & City of Newton & 0.15 & 0.00 \\
\hline Livestock-aquaculture & 0.37 & 0.28 & 0.65 & 0.65 & 0.00 & & & \\
\hline Thermoelectric power & 0.00 & 0.00 & 0.00 & 0.00 & 0.00 & & & \\
\hline TOTAL & 22.51 & 2.03 & 24.54 & 24.54 & 0.00 & & & \\
\hline
\end{tabular}

Total use is total withdrawal plus public supply deliveries and losses.

Withdrawals by water source

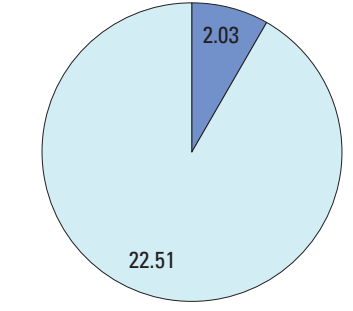

Withdrawals, in million gallons per day

Surface water

Groundwater

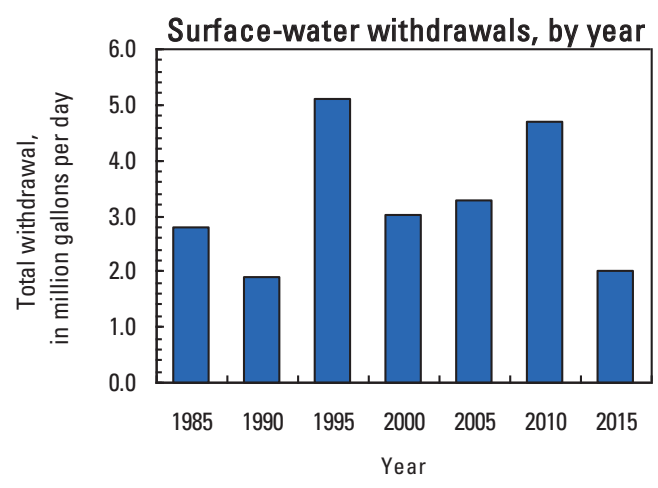

Groundwater withdrawals by aquifer
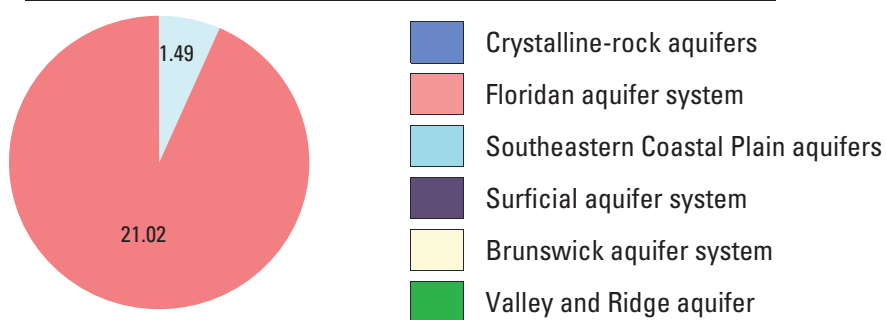

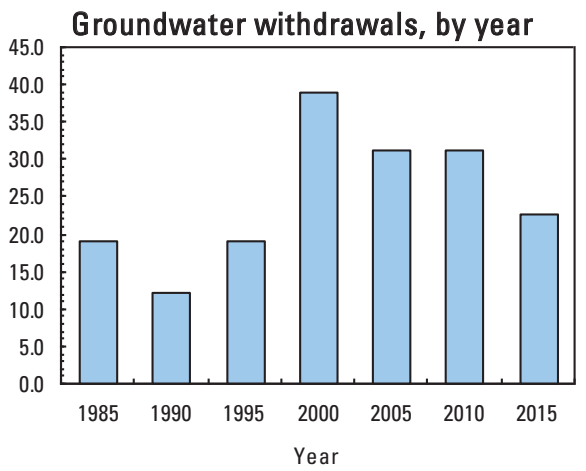




\section{BALDWIN COUNTY}

Population

Population served by public supply-groundwater

Population served by public supply-surface water $\quad 41,296$

Acres irrigated

2015 WATER WITHDRAWALS AND ESTIMATED USE, IN MILLION GALLONS PER DAY

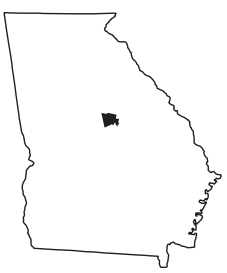

[--,not applicable; Mgal/d, million gallons per day]

\begin{tabular}{|c|c|c|c|c|c|}
\hline \multirow[b]{2}{*}{ Category } & \multicolumn{3}{|c|}{ Withdrawals } & \multirow[b]{2}{*}{$\begin{array}{c}\text { Total } \\
\text { use }\end{array}$} & \multirow[b]{2}{*}{$\begin{array}{c}\text { Returns to } \\
\text { Surface }\end{array}$} \\
\hline & $\begin{array}{l}\text { Ground- } \\
\text { water }\end{array}$ & $\begin{array}{c}\text { Surface } \\
\text { water }\end{array}$ & Total & & \\
\hline Public supply & 0.02 & 3.80 & 3.82 & -- & -- \\
\hline Domestic use & 0.29 & 0.00 & 0.29 & 2.08 & 0.00 \\
\hline Commercial use & 0.00 & 0.00 & 0.00 & 2.75 & 0.00 \\
\hline Industrial use & 0.02 & 0.00 & 0.02 & 0.55 & 0.00 \\
\hline Public-supply loss & -- & -- & -- & -1.25 & -- \\
\hline Inter-county delivery & -- & -- & -- & 2.11 & -- \\
\hline Public wastewater treatment & t -- & -- & -- & -- & 3.93 \\
\hline Mining use & 0.15 & 0.00 & 0.15 & 0.15 & 0.00 \\
\hline Irrigation (crop) & 0.00 & 0.00 & 0.00 & 0.00 & 0.00 \\
\hline Irrigation (golf) & 0.00 & 0.05 & 0.05 & 0.05 & 0.00 \\
\hline Livestock-aquaculture & 0.05 & 0.01 & 0.06 & 0.06 & 0.00 \\
\hline Thermoelectric power & 0.00 & 0.00 & 0.00 & 0.00 & 0.00 \\
\hline TOTAL & 0.53 & 3.86 & 4.39 & 6.50 & 3.93 \\
\hline
\end{tabular}

Withdrawals by Major Industrial Groups

[NAICS, North American Industrial Classification System code]

NAICS Groundwater Surface water

321- Wood

0.02

0.00

Withdrawals by Major Public Suppliers

Name Groundwater Surface water

City of Milledgeville

0.00

3.80

Total use is total withdrawal plus public supply deliveries and losses. An estimate of $2.11 \mathrm{Mgal} / \mathrm{d}$ was delivered from neighboring counties in 2015.

Withdrawals by water source

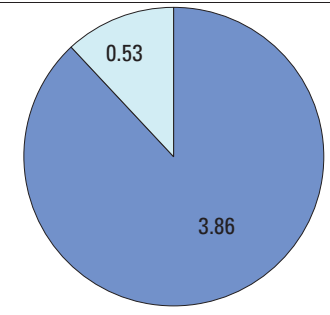

Withdrawals, in million gallons per day

Surface water

Groundwater
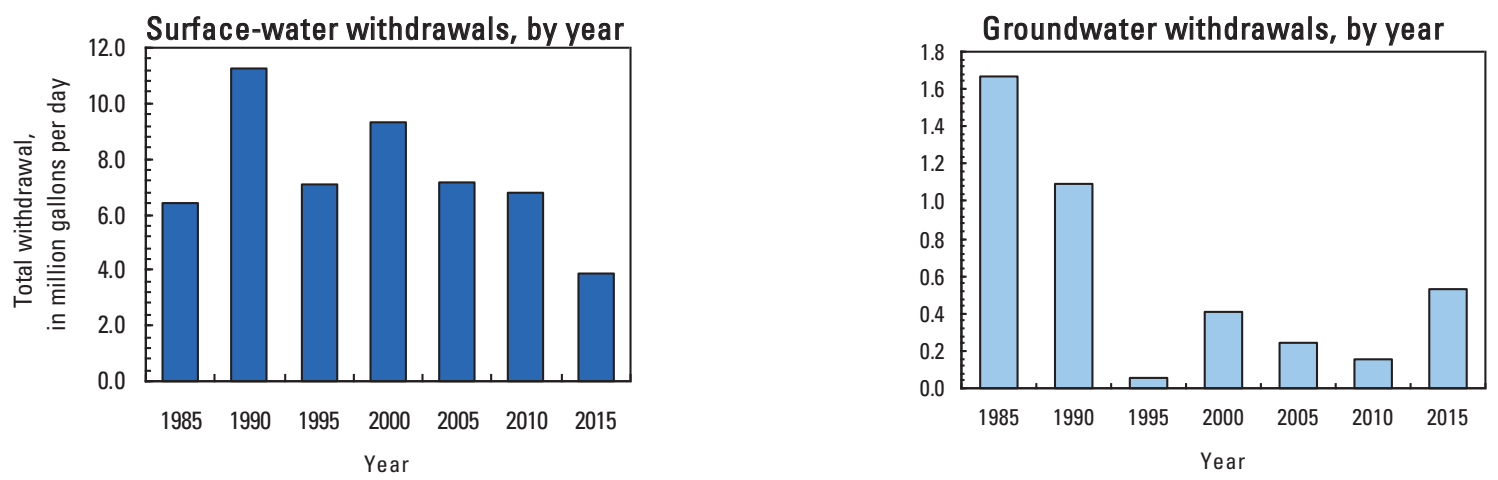


\section{BANKS COUNTY}

Population

Population served by public supply-groundwater $\quad 2,009$

Population served by public supply-surface water $\quad 7,451$

Acres irrigated

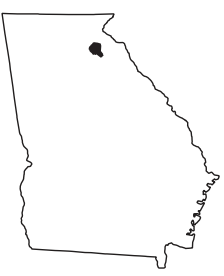

2015 WATER WITHDRAWALS AND ESTIMATED USE, IN MILLION GALLONS PER DAY

[--,not applicable; Mgal/d, million gallons per day]

\begin{tabular}{|c|c|c|c|c|c|}
\hline \multirow[b]{2}{*}{ Category } & \multicolumn{3}{|c|}{ Withdrawals } & \multirow[b]{2}{*}{$\begin{array}{c}\text { Total } \\
\text { use }\end{array}$} & \multirow[b]{2}{*}{$\begin{array}{c}\text { Returns to } \\
\text { Surface }\end{array}$} \\
\hline & $\begin{array}{l}\text { Ground- } \\
\text { water }\end{array}$ & $\begin{array}{c}\text { Surface } \\
\text { water }\end{array}$ & Total & & \\
\hline Public supply & 0.25 & 2.36 & 2.61 & -- & -- \\
\hline Domestic use & 0.68 & 0.00 & 0.68 & 1.17 & 0.00 \\
\hline Commercial use & 0.00 & 0.00 & 0.00 & 0.04 & 0.00 \\
\hline Industrial use & 0.00 & 0.00 & 0.00 & 0.01 & 0.00 \\
\hline Public-supply loss & -- & -- & -- & 2.07 & -- \\
\hline Inter-county delivery & -- & -- & -- & -1.73 & -- \\
\hline Public wastewater treatment & it -- & -- & -- & -- & 0.01 \\
\hline Mining use & 0.00 & 0.00 & 0.00 & 0.00 & 0.00 \\
\hline Irrigation (crop) & 0.00 & 0.00 & 0.00 & 0.00 & 0.00 \\
\hline Irrigation (golf) & 0.00 & 0.13 & 0.13 & 0.13 & 0.00 \\
\hline Livestock-aquaculture & 0.55 & 0.18 & 0.73 & 0.73 & 0.00 \\
\hline Thermoelectric power & 0.00 & 0.00 & 0.00 & 0.00 & 0.00 \\
\hline TOTAL & 1.48 & 2.67 & 4.15 & 2.42 & 0.01 \\
\hline
\end{tabular}

Withdrawals by Major Industrial Groups

[NAICS, North American Industrial Classification System code]

NAICS Groundwater Surface water

None

Withdrawals by Major Public Suppliers

$\begin{array}{lcc}\text { Name } & \text { Groundwater } & \text { Surface water } \\ \begin{array}{l}\text { Banks County, GA Board of } \\ \text { Commissioners }\end{array} & 0.00 & 0.79 \\ \text { City of Commerce } & 0.00 & 11.57\end{array}$

${ }^{1}$ Water withdrawan was used in Jackson County, Georgia

Total use is total withdrawal plus public supply deliveries and losses. An estimate of $1.73 \mathrm{Mgal} / \mathrm{d}$ was delivered from Banks County to neighboring counties in 2015

Withdrawals by water source

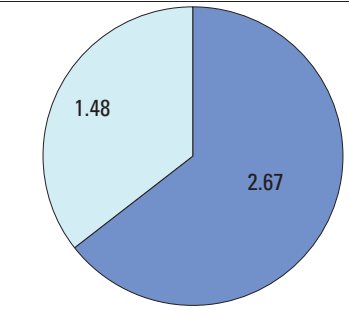

Withdrawals, in million gallons per day

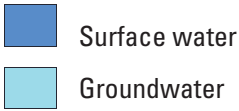

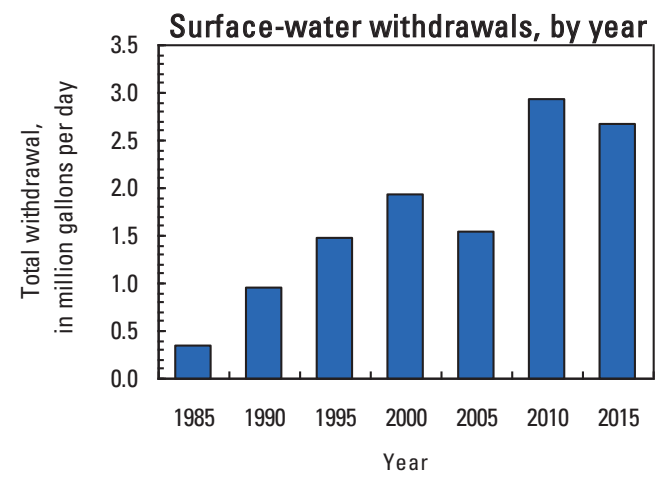

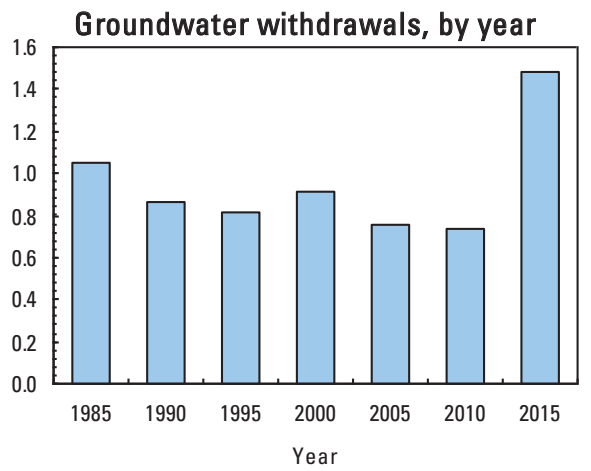

Groundwater withdrawals by aquifer

\begin{tabular}{ll}
\hline & Crystalline-rock aquifers \\
\hline & Floridan aquifer system \\
\hline & Southeastern Coastal Plain aquifers \\
& Surficial aquifer system \\
\hline$\quad$ Brunswick aquifer system \\
& Valley and Ridge aquifer
\end{tabular}




\section{BARROW COUNTY}

Population

Population served by public supply-groundwater

Population served by public supply-surface water $\quad 43,861$

Acres irrigated

480

2015 WATER WITHDRAWALS AND ESTIMATED USE, IN MILLION GALLONS PER DAY

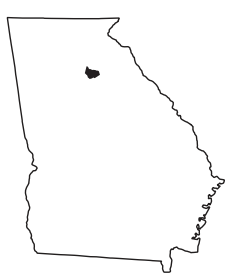

[--,not applicable; Mgal/d, million gallons per day]

\begin{tabular}{|c|c|c|c|c|c|c|c|c|}
\hline \multirow[b]{2}{*}{ Category } & \multicolumn{3}{|c|}{ Withdrawals } & \multirow{2}{*}{$\begin{array}{c}\text { Total } \\
\text { use }\end{array}$} & \multirow{2}{*}{$\begin{array}{l}\text { Returns to } \\
\text { Surface }\end{array}$} & \multirow{2}{*}{\multicolumn{3}{|c|}{$\begin{array}{l}\text { Withdrawals by Major Industrial Groups } \\
\text { [NAICS, North American Industrial Classification System code] }\end{array}$}} \\
\hline & \multirow{2}{*}{$\begin{array}{c}\begin{array}{c}\text { Ground } \\
\text { water }\end{array} \\
0.03\end{array}$} & $\begin{array}{c}\text { Surface } \\
\text { water }\end{array}$ & Total & & & & & \\
\hline Public supply & & $\begin{array}{c}\text { water } \\
4.43\end{array}$ & 4.46 & $\begin{array}{c}\text { use } \\
--\end{array}$ & surtace & NAICS & Groundwater & Surface water \\
\hline Domestic use & 2.34 & 0.00 & 2.34 & 6.4 & 0.00 & 211 [nod & 041 & ח \\
\hline Commercial use & 0.00 & 0.00 & 0.00 & 0.74 & 0.00 & $311-$ Food & 0.41 & U.UU \\
\hline Industrial use & 0.41 & 0.00 & 0.41 & 1.08 & 0.00 & & & \\
\hline Public-supply loss & -- & -- & -- & -1.01 & -- & & & \\
\hline Inter-county delivery & -- & -- & - & 2.07 & -- & & & \\
\hline Public wastewater treatment & t -- & -- & -- & -- & 2.18 & & & \\
\hline Mining use & 0.03 & 0.00 & 0.03 & 0.03 & 0.00 & Withdrawals & jor Public Sup & \\
\hline Irrigation (crop) & 0.00 & 0.00 & 0.00 & 0.00 & 0.00 & Name & Groundwater & Surface water \\
\hline Irrigation (golf) & 0.16 & 0.35 & 0.51 & 0.51 & 0.00 & City of Statham & 0.00 & 0.28 \\
\hline Livestock-aquaculture & 0.14 & 0.03 & 0.17 & 0.17 & 0.00 & City of Winder & 0.00 & 4.15 \\
\hline Thermoelectric power & 0.00 & 0.00 & 0.00 & 0.00 & 0.00 & & & \\
\hline TOTAL & 3.11 & 4.81 & 7.92 & 9.99 & 2.18 & & & \\
\hline
\end{tabular}

Total use is total withdrawal plus public supply deliveries and losses. An estimate of $2.17 \mathrm{Mgal} / \mathrm{d}$ was delivered from neighboring counties in 2015. An estimate of $0.1 \mathrm{Mgal} / \mathrm{d}$ was delivered from Barrow County to neighboring counties in 2015.

Withdrawals by water source

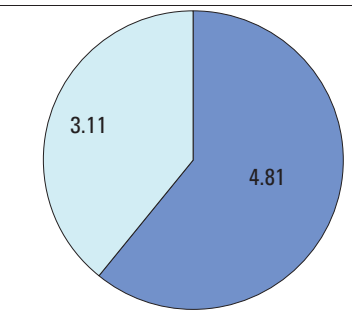

Withdrawals, in million gallons per day

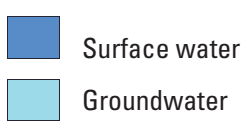

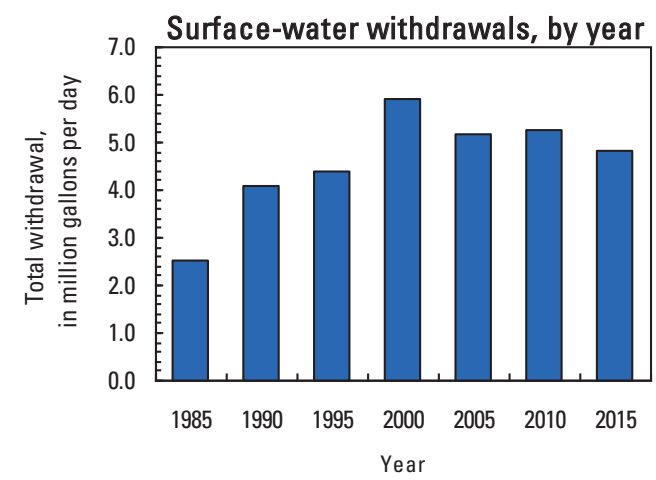

Groundwater withdrawals by aquifer

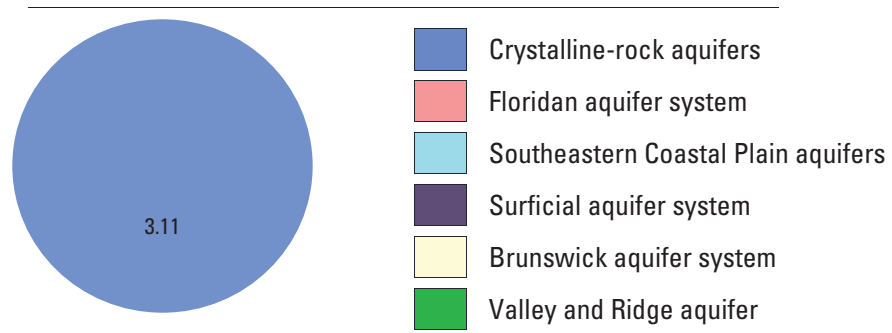

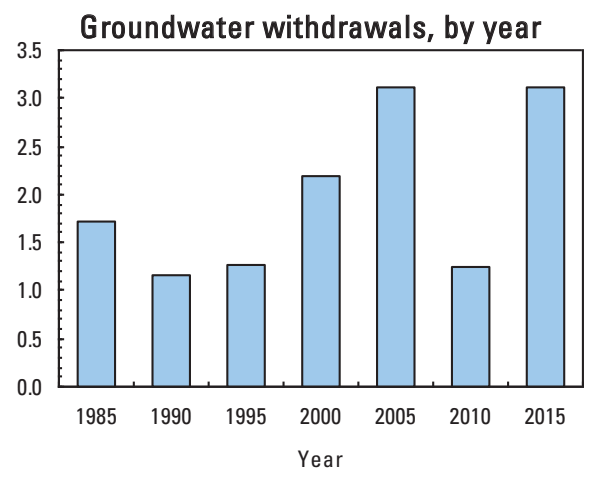




\section{BARTOW COUNTY}

Population

Population served by public supply-groundwater

Population served by public supply-surface water $\quad 83,153$

Acres irrigated

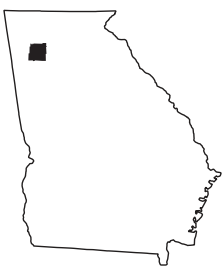

2015 WATER WITHDRAWALS AND ESTIMATED USE, IN MILLION GALLONS PER DAY

$[--$,not applicable; Mgal/d, million gallons per day]

\begin{tabular}{|c|c|c|c|c|c|}
\hline \multirow[b]{2}{*}{ Category } & \multicolumn{3}{|c|}{ Withdrawals } & \multirow[b]{2}{*}{$\begin{array}{c}\text { Total } \\
\text { use }\end{array}$} & \multirow[b]{2}{*}{$\begin{array}{c}\text { Returns to } \\
\text { Surface }\end{array}$} \\
\hline & $\begin{array}{c}\text { Ground } \\
\text { water }\end{array}$ & $\begin{array}{c}\text { Surface } \\
\text { water }\end{array}$ & Total & & \\
\hline Public supply & 0.19 & 52.22 & 52.41 & -- & -- \\
\hline Domestic use & 1.31 & 0.00 & 1.31 & 7.71 & 0.00 \\
\hline Commercial use & 0.00 & 0.00 & 0.00 & 1.5 & 0.00 \\
\hline Industrial use & 1.33 & 1.57 & 2.90 & 4.84 & 0.00 \\
\hline Public-supply loss & -- & -- & -- & 42.57 & -- \\
\hline Inter-county delivery & -- & -- & -- & -40.14 & -- \\
\hline Public wastewater treatment & nt -- & -- & -- & -- & 21.85 \\
\hline Mining use & 0.18 & 0.00 & 0.18 & 0.18 & 0.00 \\
\hline Irrigation (crop) & 0.11 & 1.71 & 1.82 & 1.82 & 0.00 \\
\hline Irrigation (golf) & 0.00 & 0.23 & 0.23 & 0.23 & 0.00 \\
\hline Livestock-aquaculture & 0.03 & 0.44 & 0.47 & 0.47 & 0.00 \\
\hline Thermoelectric power & 0.00 & 33.77 & 33.77 & 33.77 & 0.00 \\
\hline TOTAL & 3.15 & 89.94 & 93.09 & 52.95 & 21.85 \\
\hline
\end{tabular}

Total use is total withdrawal plus public supply deliveries and losses. An estimate of $0.47 \mathrm{Mgal} / \mathrm{d}$ was delivered from neighboring counties in 2015. An estimate of $40.61 \mathrm{Mgal} / \mathrm{d}$ was delivered from Bartow County to neighboring counties in 2015.

Withdrawals by Major Industrial Groups

[NAICS, North American Industrial Classification System code]

NAICS Groundwater Surface water

$\begin{array}{lll}\text { 212- Mining } & 0.00 & 1.48 \\ \text { 325-Chemicals } & 0.00 & 1.22 \\ \text { 331-Primary metals } & 0.11 & 0.09\end{array}$

Withdrawals by Major Public Suppliers

\begin{tabular}{lcc} 
Name & Groundwater & Surface water \\
\hline City of Adairsville & 0.00 & 2.45 \\
City of Cartersville & 0.00 & 10.77 \\
$\begin{array}{l}\text { Cobb County.-Marietta } \\
\quad \text { Water Authority }\end{array}$ & 0.00 & 138.30 \\
City of Kingston & 0.10 & 0.00 \\
City of White & 0.09 & 0.00 \\
Bartow County & 0.51 & 0.00
\end{tabular}

'Delivered and used in Cobb County, Georgia

\section{Withdrawals by water source}

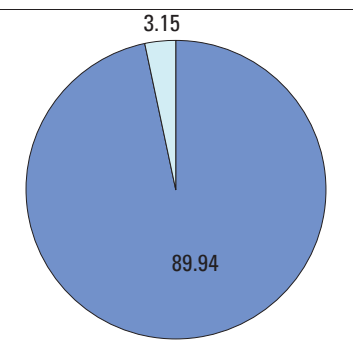

Withdrawals, in million

gallons per day

Surface water

Groundwater
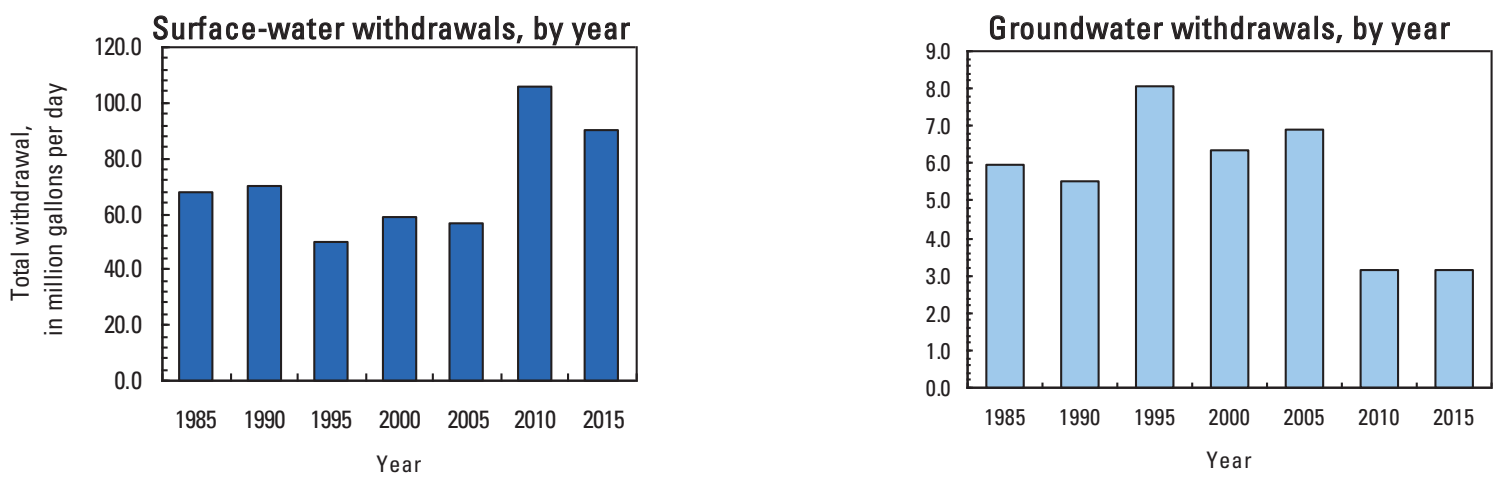


\section{BEN HILL COUNTY}

Population

Population served by public supply-groundwater $\quad 12,961$

Population served by public supply-surface water 0

Acres irrigated

8,320

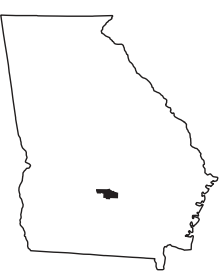

2015 WATER WITHDRAWALS AND ESTIMATED USE, IN MILLION GALLONS PER DAY

[--,not applicable; Mgal/d, million gallons per day]

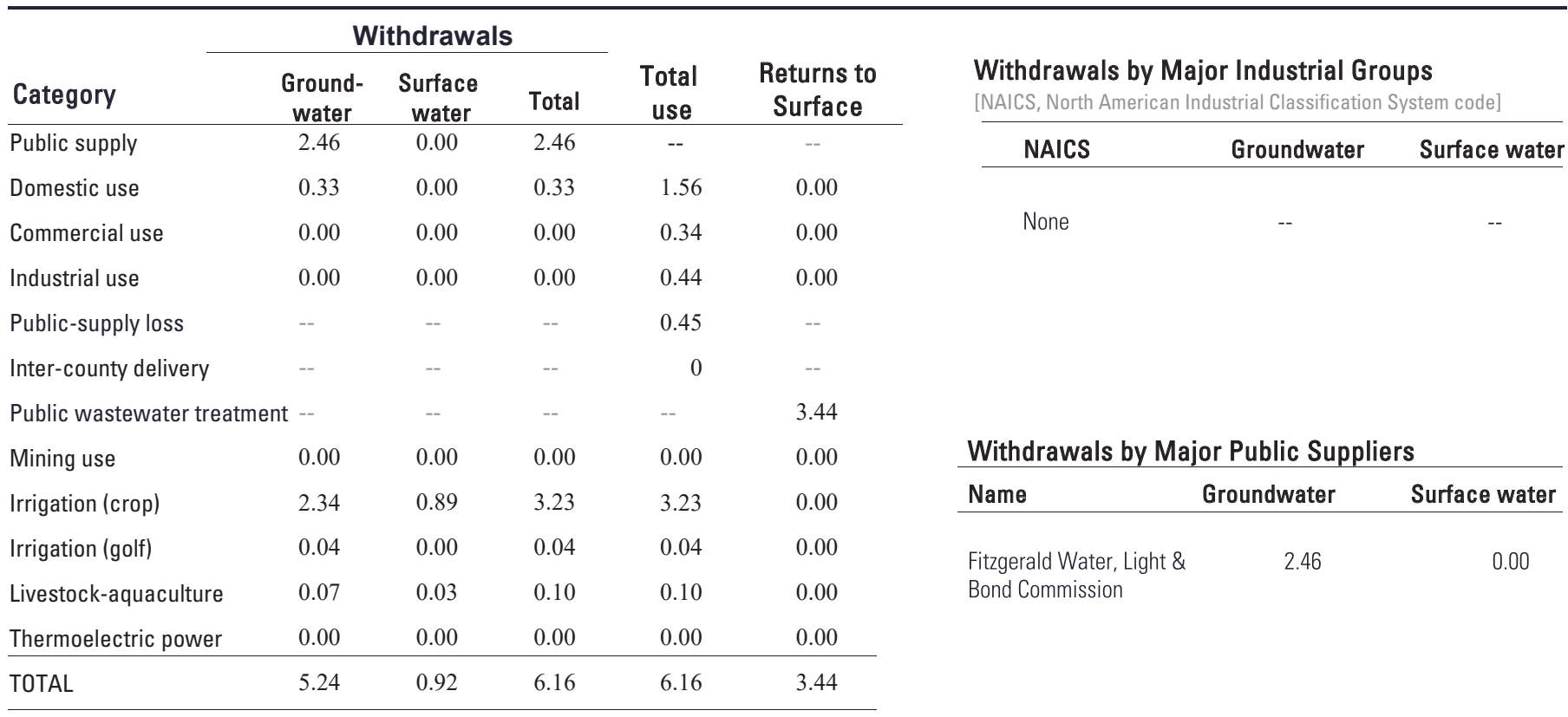

Total use is total withdrawal plus public supply deliveries and losses.

Withdrawals by water source

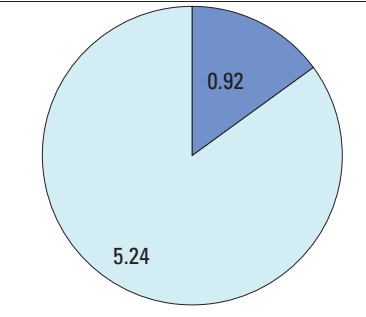

Withdrawals, in million gallons per day

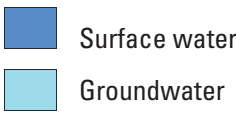

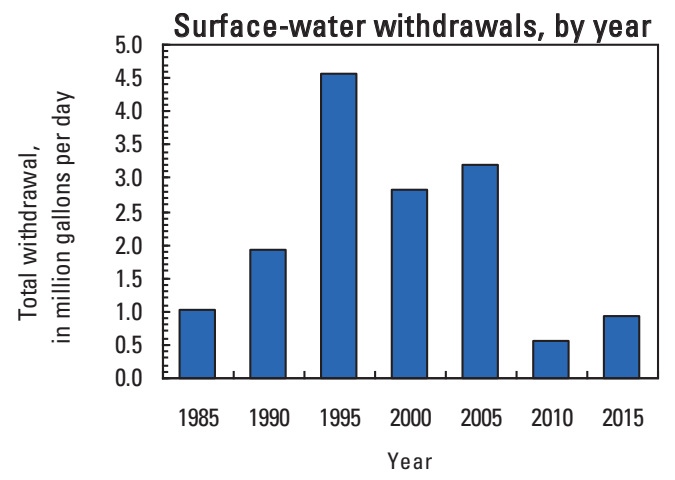

Groundwater withdrawals by aquifer

\begin{tabular}{ll}
\hline 0.30 & Crystalline-rock aquifers \\
& Floridan aquifer system \\
\hline & Southeastern Coastal Plain aquifers \\
& Surficial aquifer system \\
$\square .94$ & Brunswick aquifer system \\
& Valley and Ridge aquifer
\end{tabular}

Groundwater withdrawals, by year

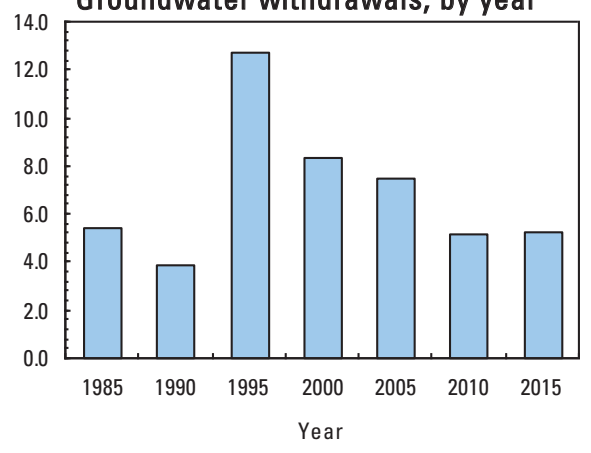




\section{BERRIEN COUNTY}

Population

Population served by public supply-groundwater

7,496

Population served by public supply-surface water

0

Acres irrigated

19,360

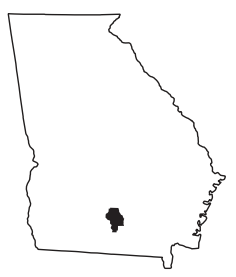

2015 WATER WITHDRAWALS AND ESTIMATED USE, IN MILLION GALLONS PER DAY

$[--$,not applicable; Mgal/d, million gallons per day]

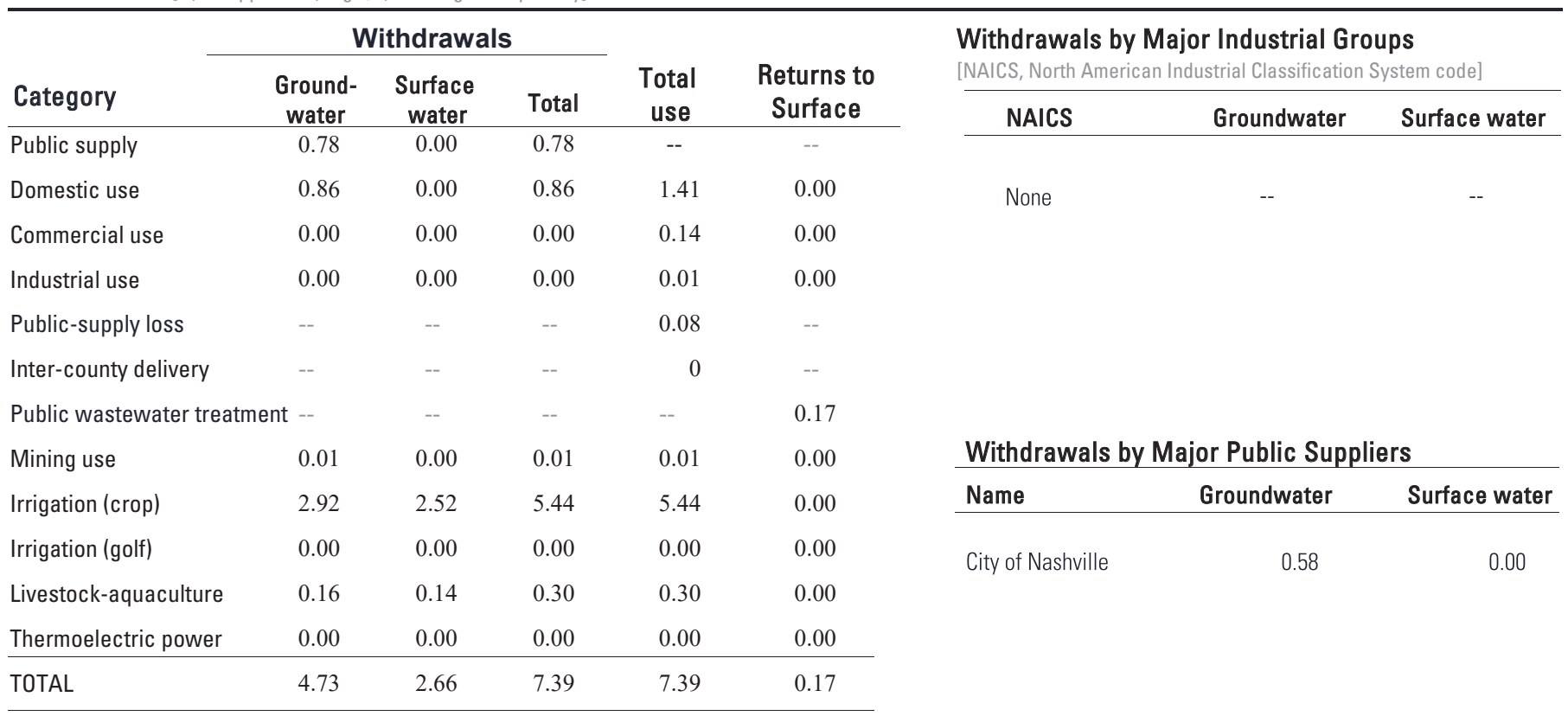

Total use is total withdrawal plus public supply deliveries and losses.

Withdrawals by water source

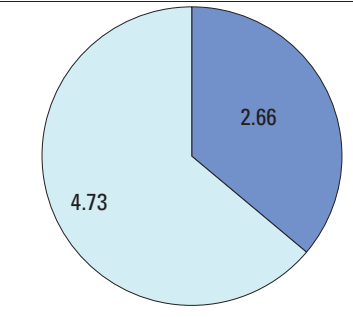

Withdrawals, in million

gallons per day

Surface water

Groundwater
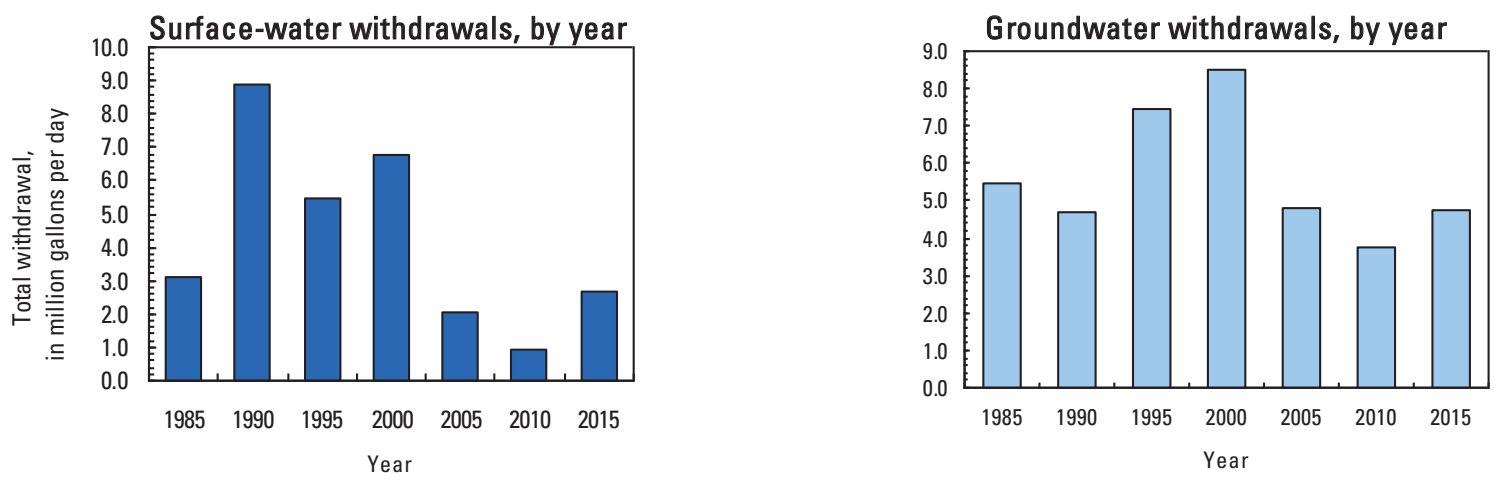


\section{BIBB COUNTY}

Population

153,721

Population served by public supply-groundwater

Population served by public supply-surface water 130,024

Acres irrigated

2015 WATER WITHDRAWALS AND ESTIMATED USE, IN MILLION GALLONS PER DAY

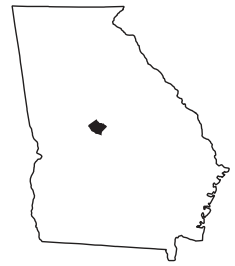

[--,not applicable; Mgal/d, million gallons per day]

\begin{tabular}{|c|c|c|c|c|c|c|c|c|}
\hline \multirow[b]{2}{*}{ Category } & \multicolumn{3}{|c|}{ Withdrawals } & \multirow{2}{*}{$\begin{array}{c}\text { Total } \\
\text { use }\end{array}$} & \multirow{2}{*}{$\begin{array}{c}\text { Returns to } \\
\text { Surface }\end{array}$} & \multirow{2}{*}{\multicolumn{3}{|c|}{$\begin{array}{l}\text { Withdrawals by Major Industrial Groups } \\
\text { [NAICS, North American Industrial Classification System code] }\end{array}$}} \\
\hline & \multirow{2}{*}{$\begin{array}{c}\text { Ground- } \\
\text { water }\end{array}$} & \multirow{2}{*}{$\begin{array}{c}\text { Surface } \\
\text { water } \\
23.99\end{array}$} & Total & & & & & \\
\hline Public supply & & & 24.13 & $\begin{array}{c}\text { use } \\
--\end{array}$ & surtace & NAICS & Groundwater & Surface water \\
\hline Domestic use & 1.65 & 0.00 & 1.65 & 15.95 & 0.00 & \multirow[b]{2}{*}{ 212- Mining } & \multirow[b]{2}{*}{0.00} & \multirow[b]{2}{*}{7.53} \\
\hline & & & & & & & & \\
\hline Commercial use & 0.00 & 0.00 & 0.00 & 6.87 & 0.00 & 321-Wood & 1.12 & 0.00 \\
\hline Industrial use & 1.72 & 18.20 & 19.92 & 21.9 & 0.00 & 322-Paper, pulp & 0.04 & 10.69 \\
\hline Public-supply loss & -- & -- & -- & 0.98 & -- & \multirow[t]{2}{*}{ 326- Rubber, plastics } & \multirow[t]{2}{*}{0.2} & \multirow[t]{2}{*}{0.00} \\
\hline Inter-county delivery & -- & -- & -- & 3.01 & -- & & & \\
\hline Public wastewater treatment & tt -- & -- & -- & -- & 40.88 & & & \\
\hline Mining use & 0.52 & 0.01 & 0.53 & 0.53 & 0.00 & \multicolumn{3}{|c|}{ Withdrawals by Major Public Suppliers } \\
\hline Irrigation (crop) & 0.03 & 0.30 & 0.33 & 0.33 & 0.00 & \multirow{3}{*}{$\begin{array}{l}\text { Name } \\
\text { Macon Water Authority }\end{array}$} & Groundwater & Surface water \\
\hline Irrigation (golf) & 0.13 & 0.05 & 0.18 & 0.18 & 0.00 & & \multirow[t]{2}{*}{0.00} & \multirow[t]{3}{*}{25.02} \\
\hline Livestock-aquaculture & 0.07 & 0.01 & 0.08 & 0.08 & 0.00 & & & \\
\hline Thermoelectric power & 0.00 & 0.00 & 0.00 & 0.00 & 0.00 & & & \\
\hline TOTAL & 4.26 & 42.56 & 46.82 & 49.83 & 40.88 & & & \\
\hline
\end{tabular}

Total use is total withdrawal plus public supply deliveries and losses. An estimate of $3.01 \mathrm{Mgal} / \mathrm{d}$ was delivered from neighboring counties in 2015.

Withdrawals by water source

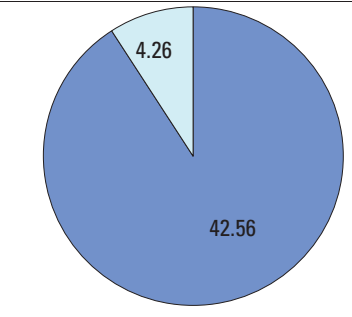

Withdrawals, in million gallons per day
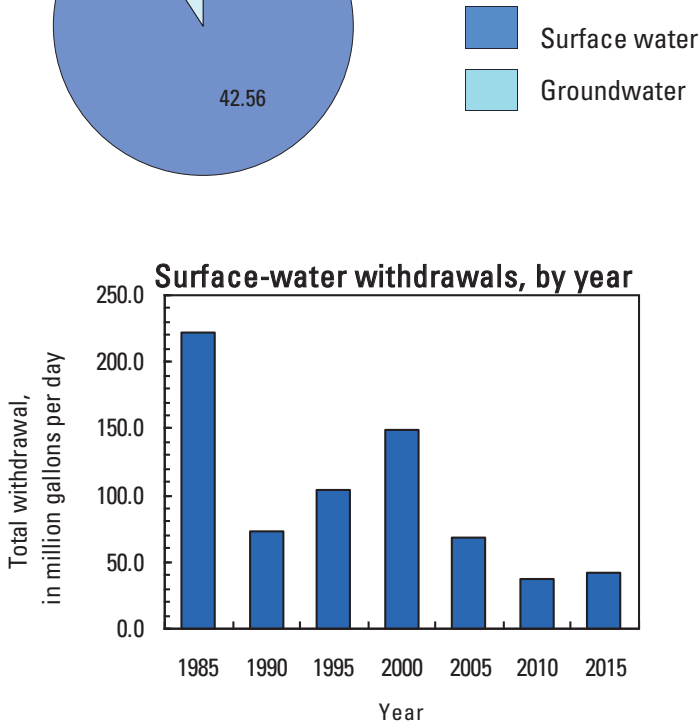

Groundwater withdrawals by aquifer

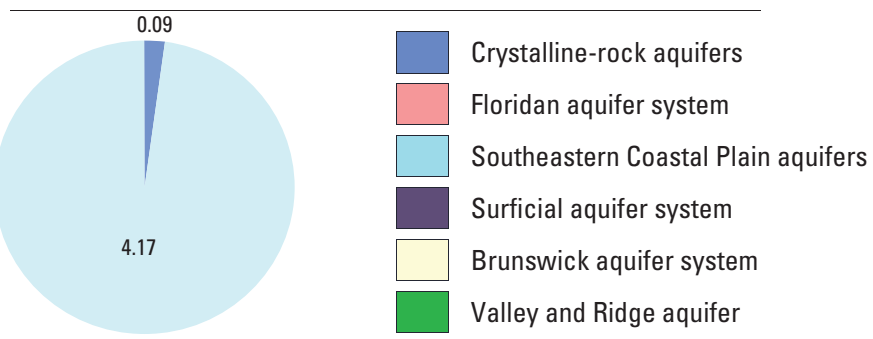

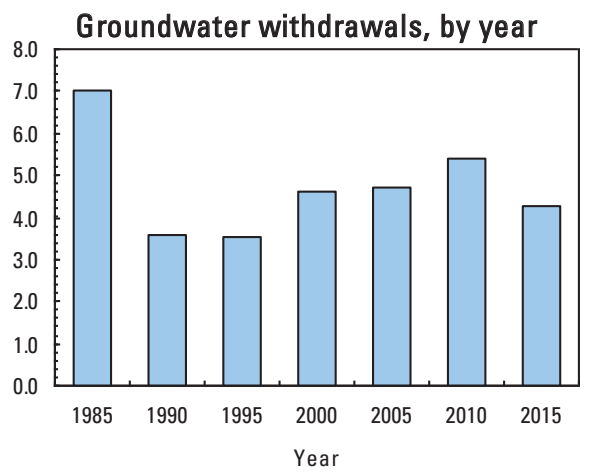




\section{BLECKLEY COUNTY}

Population

Population served by public supply-groundwater

5,184

Population served by public supply-surface water

0

Acres irrigated

14,700

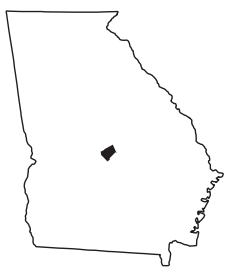

2015 WATER WITHDRAWALS AND ESTIMATED USE, IN MILLION GALLONS PER DAY

[--,not applicable; Mgal/d, million gallons per day]

\begin{tabular}{|c|c|c|c|c|c|c|c|c|}
\hline \multirow{3}{*}{ Category } & \multicolumn{3}{|c|}{ Withdrawals } & \multirow{3}{*}{$\begin{array}{c}\text { Total } \\
\text { use }\end{array}$} & \multirow{3}{*}{$\begin{array}{l}\text { Returns to } \\
\text { Surface }\end{array}$} & \multirow{2}{*}{\multicolumn{3}{|c|}{$\begin{array}{l}\text { Withdrawals by Major Industrial Groups } \\
\text { [NAICS, North American Industrial Classification System code] }\end{array}$}} \\
\hline & \multirow{2}{*}{$\begin{array}{c}\text { Ground- } \\
\text { water }\end{array}$} & \multirow{2}{*}{$\begin{array}{c}\text { Surface } \\
\text { water }\end{array}$} & \multirow[b]{2}{*}{ Total } & & & & & \\
\hline & & & & & & NAICS & Groundwater & Surface water \\
\hline Public supply & 0.20 & 0.00 & 0.20 & -- & -- & & & \\
\hline Domestic use & 0.53 & 0.00 & 0.53 & 0.62 & 0.00 & None & -- & -- \\
\hline Commercial use & 0.08 & 0.00 & 0.08 & 0.12 & 0.00 & & & \\
\hline Industrial use & 0.00 & 0.00 & 0.00 & 0 & 0.00 & & & \\
\hline Public-supply loss & -- & -- & -- & 0.07 & -- & & & \\
\hline Inter-county delivery & -- & -- & -- & 0 & - & & & \\
\hline Public wastewater treatment & t -- & -- & -- & -- & 1.19 & & & \\
\hline Mining use & 0.00 & 0.00 & 0.00 & 0.00 & 0.00 & Withdrawals & jor Public Sup & \\
\hline Irrigation (crop) & 3.59 & 2.13 & 5.72 & 5.72 & 0.00 & Name & Groundwater & Surface water \\
\hline Irrigation (golf) & 0.31 & 0.01 & 0.32 & 0.32 & 0.00 & City of Cochran & 0.20 & 0.00 \\
\hline Livestock-aquaculture & 0.04 & 0.02 & 0.06 & 0.06 & 0.00 & & & \\
\hline Thermoelectric power & 0.00 & 0.00 & 0.00 & 0.00 & 0.00 & & & \\
\hline TOTAL & 4.75 & 2.16 & 6.91 & 6.91 & 1.19 & & & \\
\hline
\end{tabular}

Total use is total withdrawal plus public supply deliveries and losses.

Withdrawals by water source

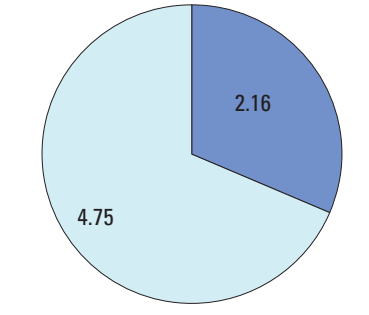

Withdrawals, in million gallons per day

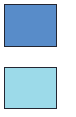

Surface water

Groundwater
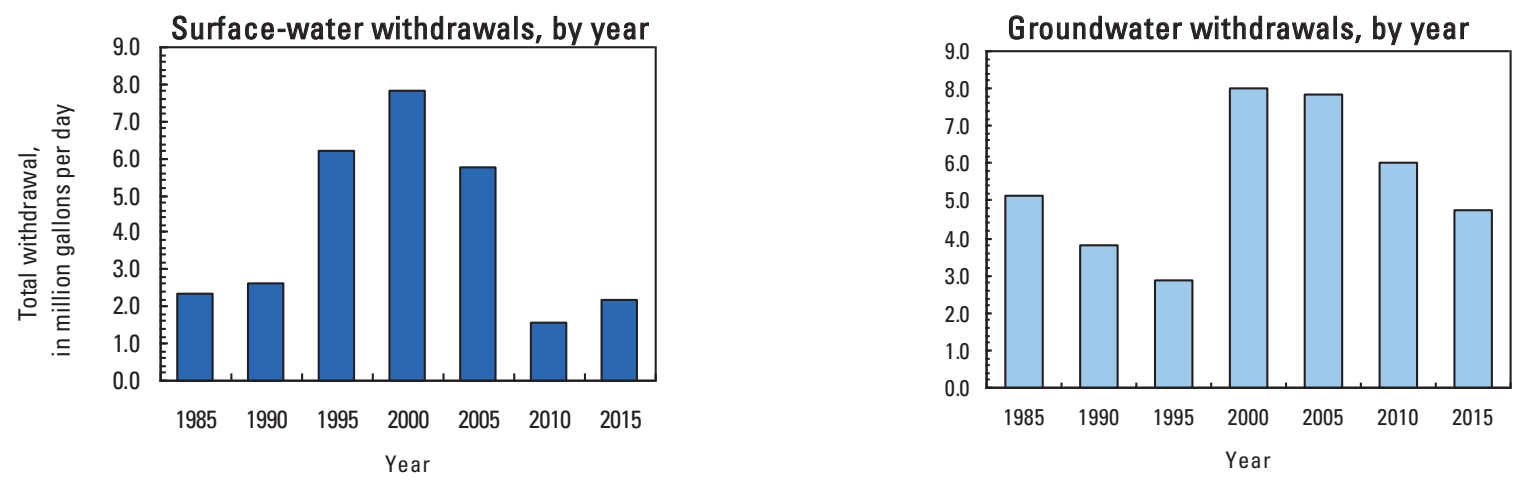


\section{BRANTLEY COUNTY}

Population

Population served by public supply-groundwater

Population served by public supply-surface water

Acres irrigated

2015 WATER WITHDRAWALS AND ESTIMATED USE, IN MILLION GALLONS PER DAY

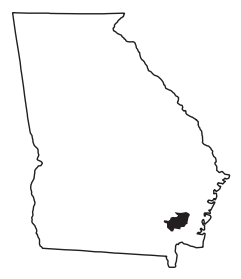

[--,not applicable; Mgal/d, million gallons per day]

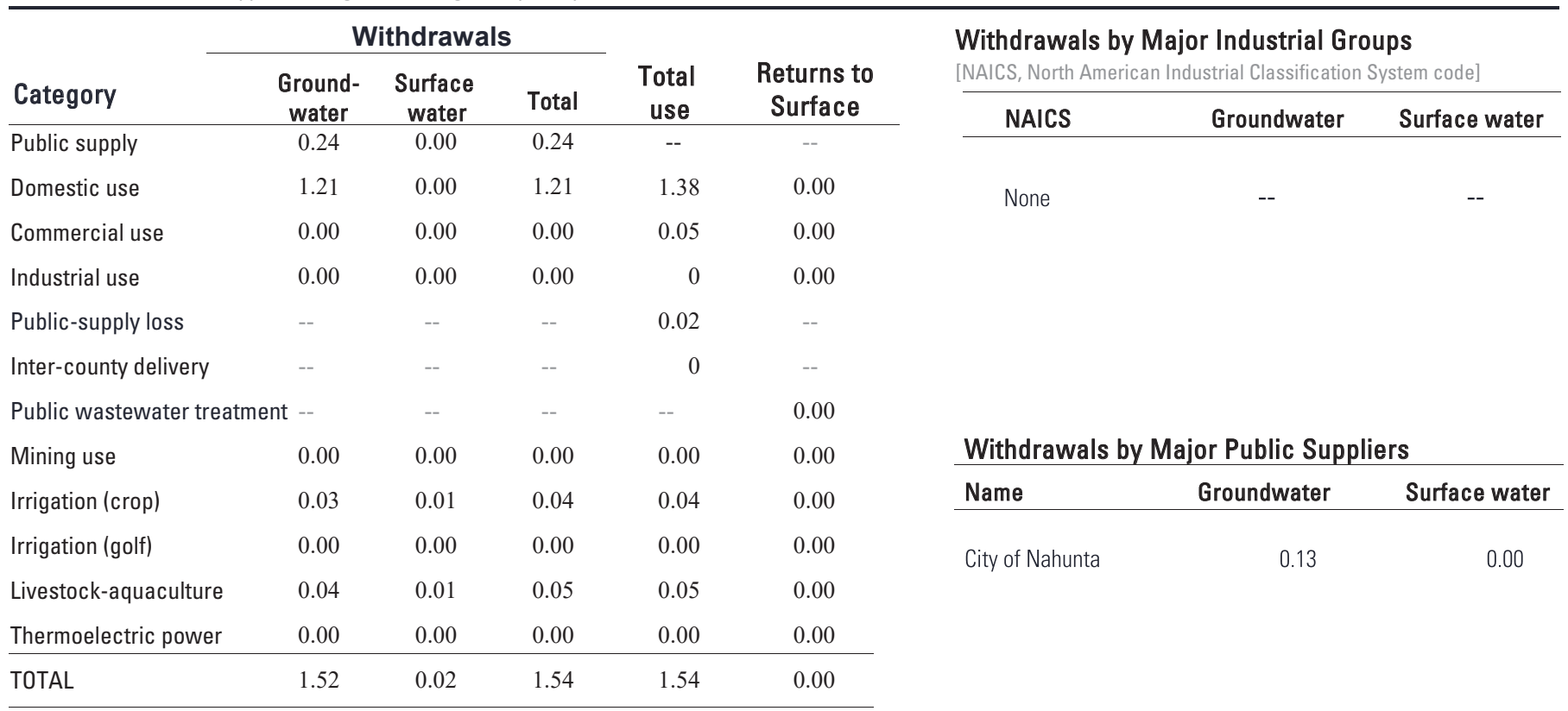

Total use is total withdrawal plus public supply deliveries and losses.

Withdrawals by water source

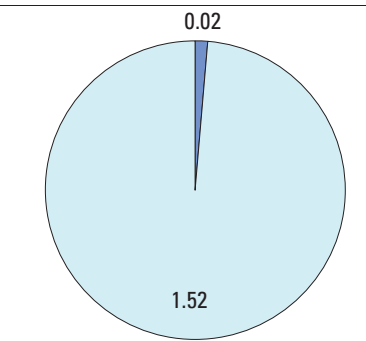

Withdrawals, in million gallons per day

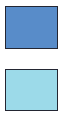

Surface water

Groundwater

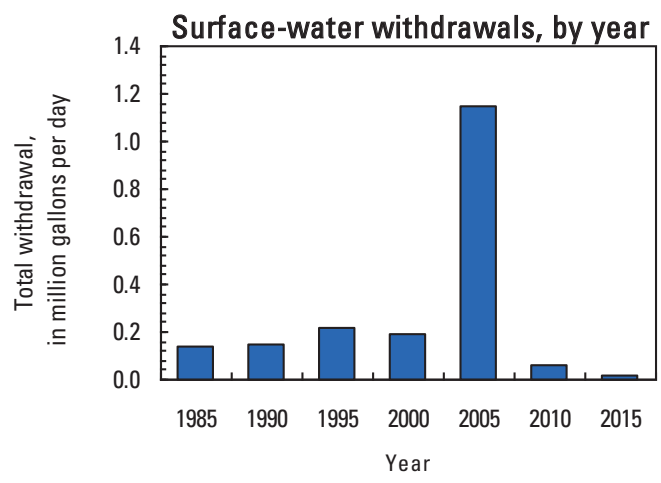

Groundwater withdrawals by aquifer
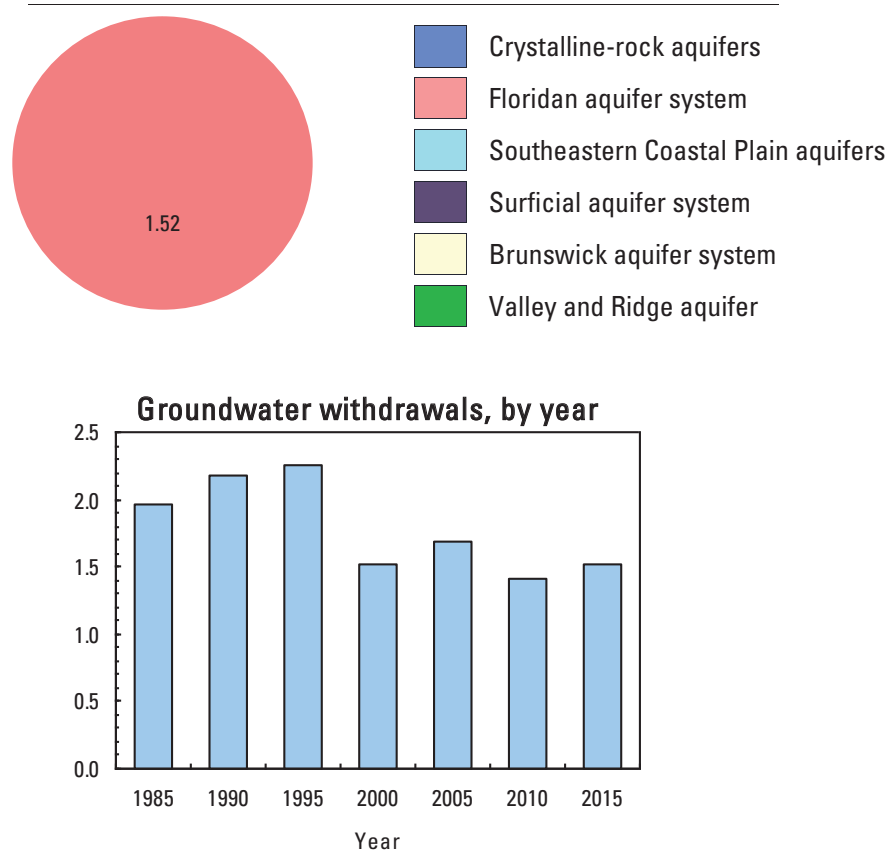


\section{BROOKS COUNTY}

Population

Population served by public supply-groundwater

7,696

Population served by public supply-surface water 0

Acres irrigated

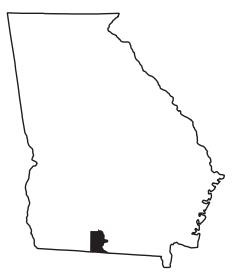

2015 WATER WITHDRAWALS AND ESTIMATED USE, IN MILLION GALLONS PER DAY

[--,not applicable; Mgal/d, million gallons per day]

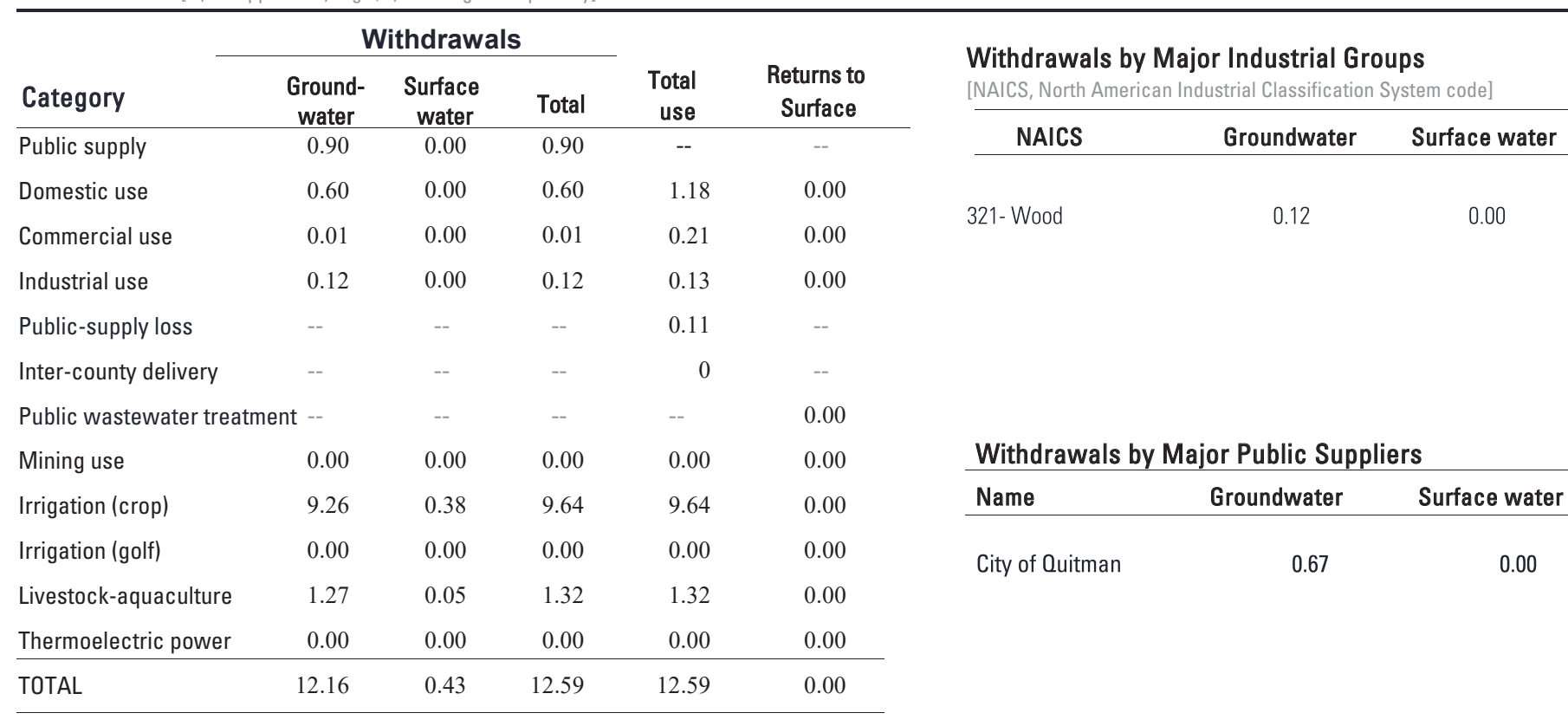

Total use is total withdrawal plus public supply deliveries and losses.

Withdrawals by water source

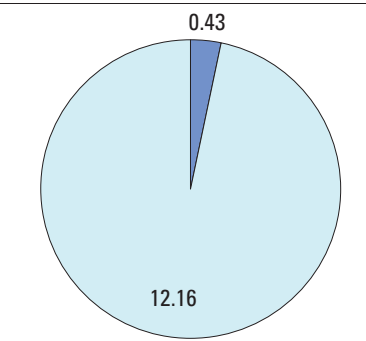

Withdrawals, in million

gallons per day

Surface water

Groundwater

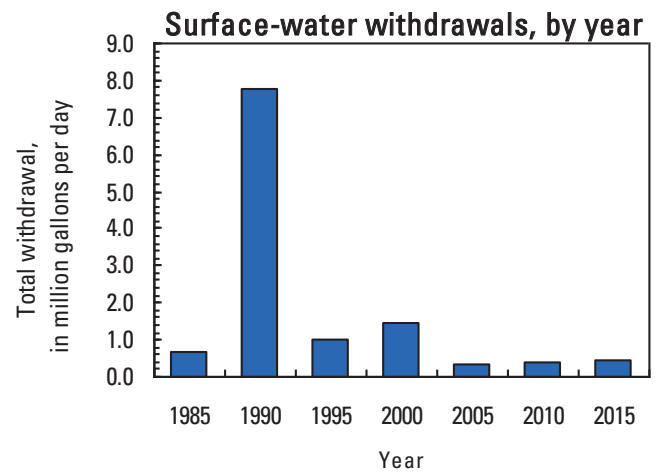

Groundwater withdrawals by aquifer

\begin{tabular}{ll}
\hline & Crystalline-rock aquifers \\
\hline & Floridan aquifer system \\
\hline $12.16 \quad$ Southeastern Coastal Plain aquifers \\
& Surficial aquifer system \\
& Brunswick aquifer system \\
& Valley and Ridge aquifer
\end{tabular}

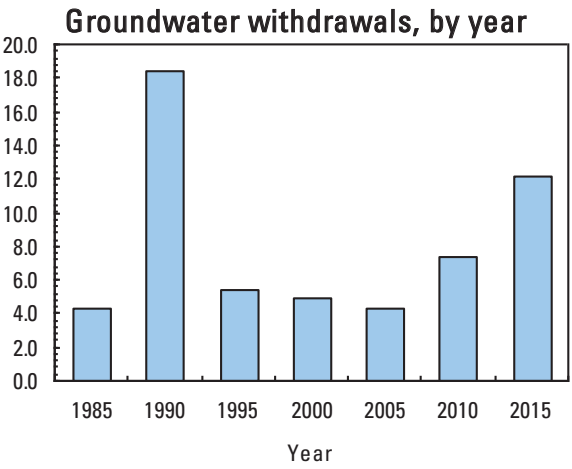




\section{BRYAN COUNTY}

Population

Population served by public supply-groundwater $\quad 26,217$

Population served by public supply-surface water $\quad 0$

Acres irrigated

2015 WATER WITHDRAWALS AND ESTIMATED USE, IN MILLION GALLONS PER DAY

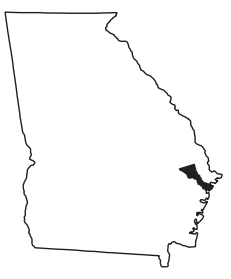

[--,not applicable; Mgal/d, million gallons per day]

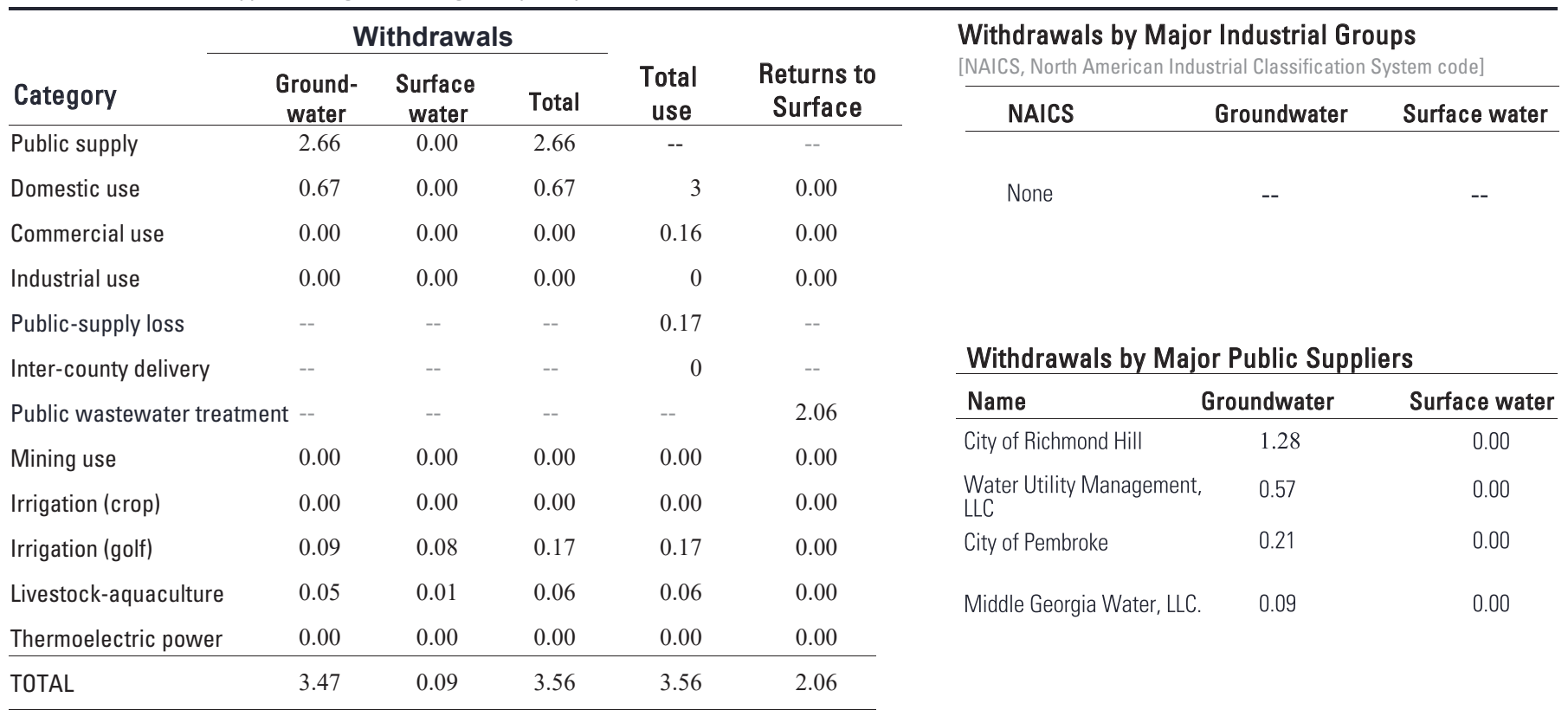

Total use is total withdrawal plus public supply deliveries and losses.

Withdrawals by water source

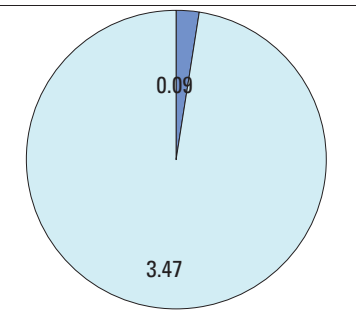

Withdrawals, in million gallons per day

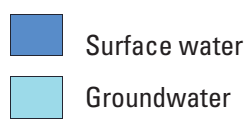

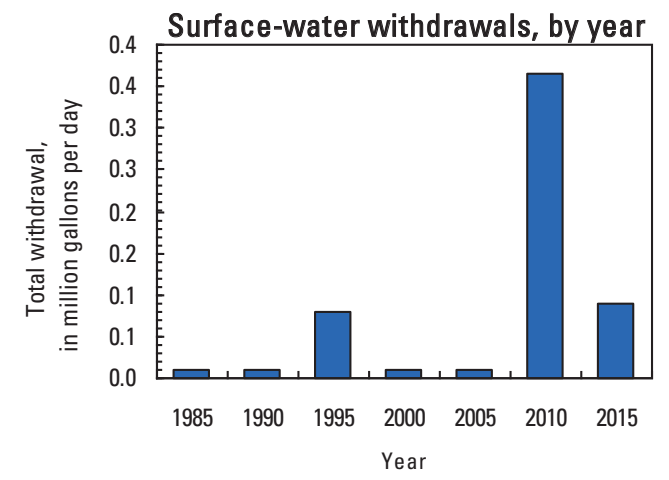

Groundwater withdrawals by aquifer

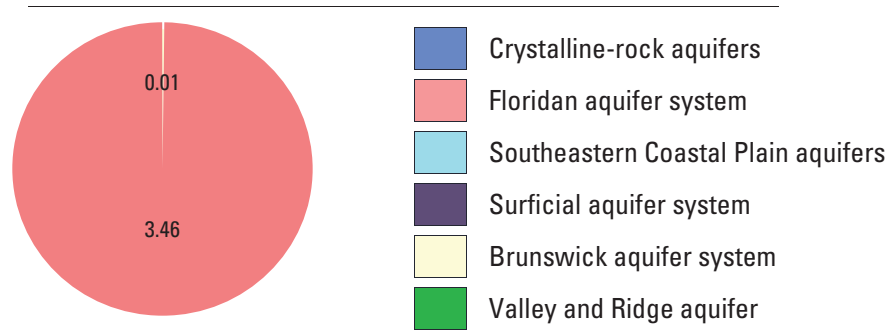

Groundwater withdrawals, by year

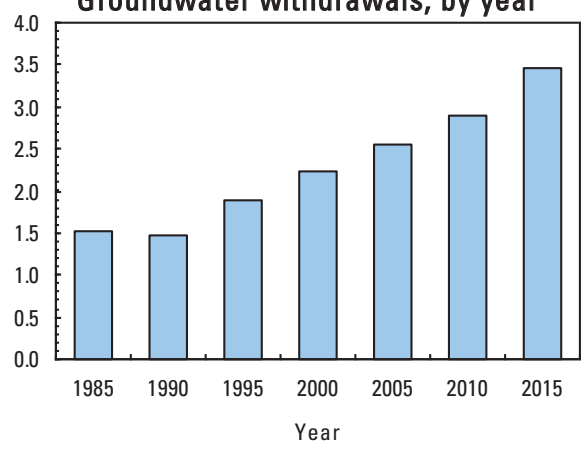




\section{BULLOCH COUNTY}

Population

Population served by public supply-groundwater $\quad 57,314$

Population served by public supply-surface water 0

Acres irrigated

17,850

2015 WATER WITHDRAWALS AND ESTIMATED USE, IN MILLION GALLONS PER DAY

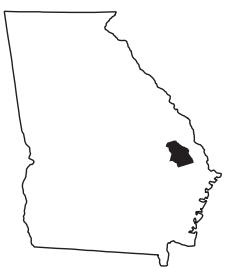

[--,not applicable; Mgal/d, million gallons per day]

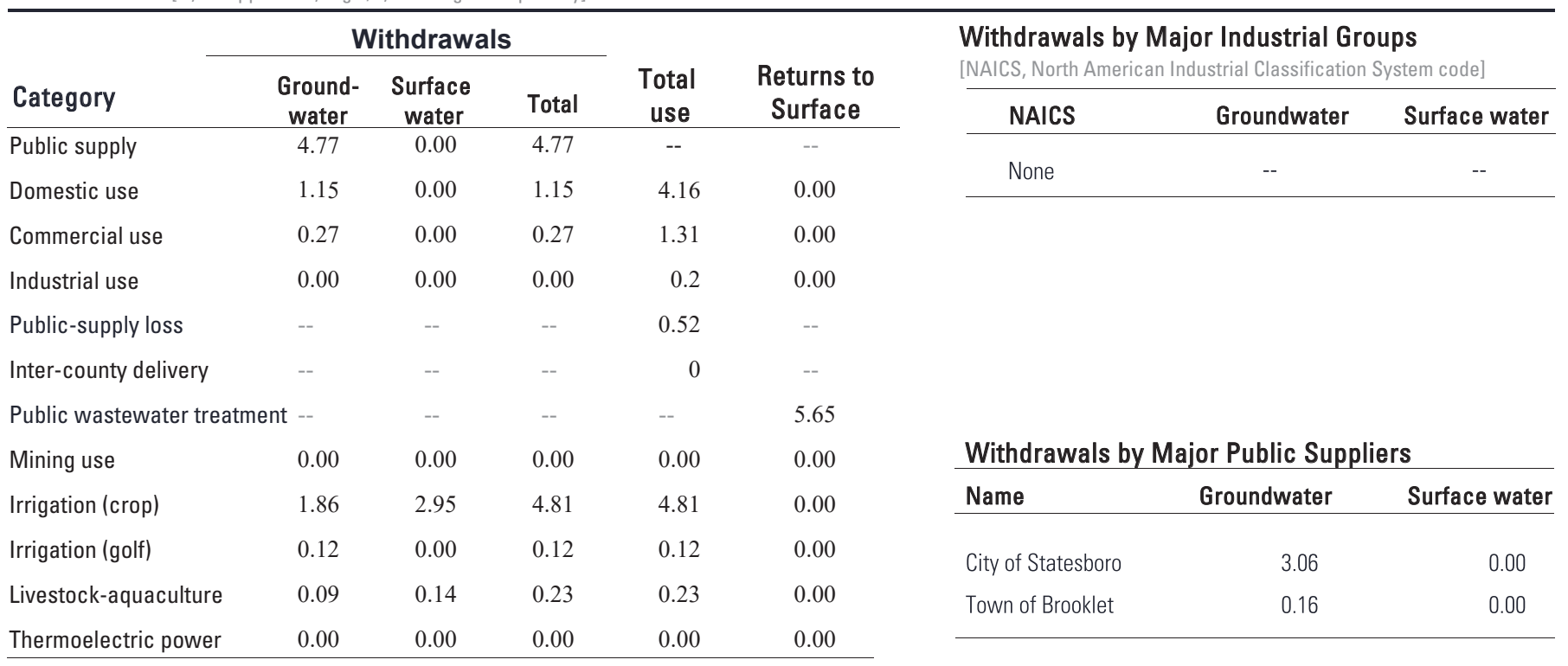

\begin{tabular}{llllll}
\hline TOTAL & 8.26 & 3.09 & 11.35 & 11.35 & 5.65
\end{tabular}

Total use is total withdrawal plus public supply deliveries and losses.

Withdrawals by water source

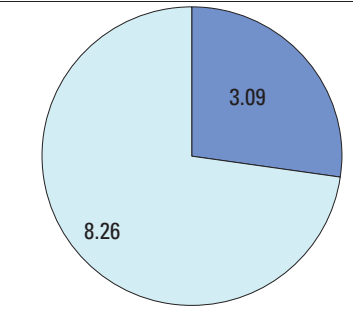

Withdrawals, in million

gallons per day

Surface water

Groundwater
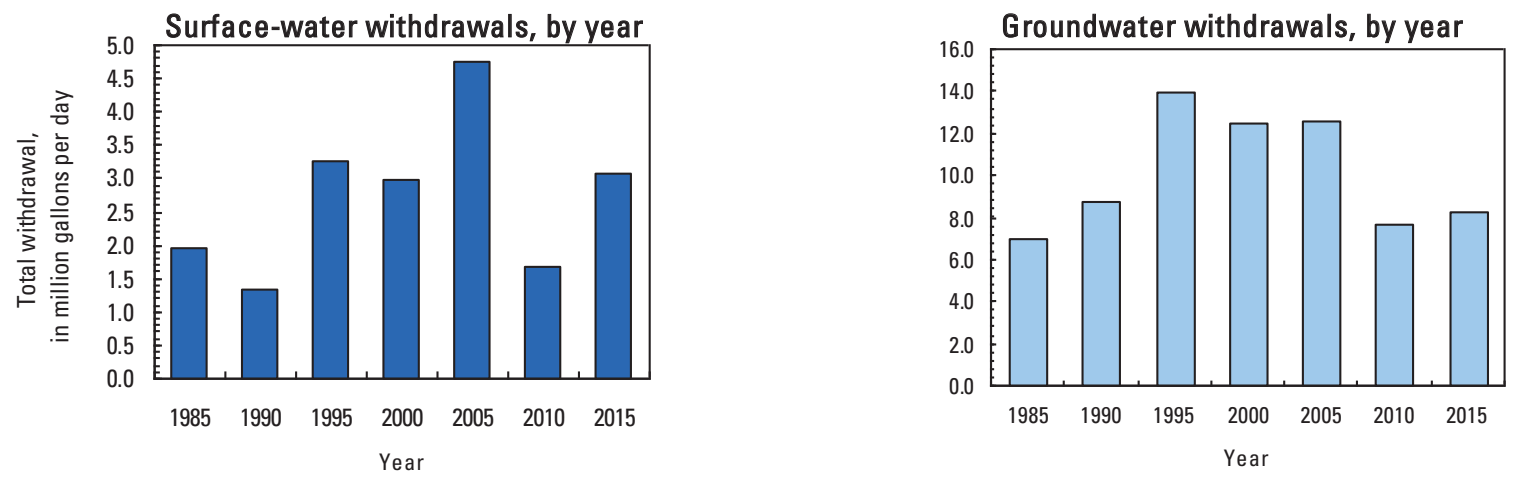


\section{BURKE COUNTY}

Population

Population served by public supply-groundwater

Population served by public supply-surface water

5,714

Acres irrigated

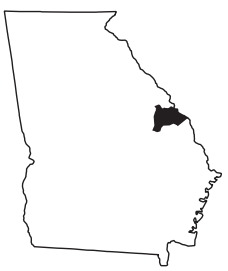

2015 WATER WITHDRAWALS AND ESTIMATED USE, IN MILLION GALLONS PER DAY

[--,not applicable; Mgal/d, million gallons per day]

\begin{tabular}{|c|c|c|c|c|c|c|c|c|}
\hline \multirow[b]{2}{*}{ Category } & \multicolumn{3}{|c|}{ Withdrawals } & \multirow{2}{*}{$\begin{array}{c}\text { Total } \\
\text { use }\end{array}$} & \multirow{2}{*}{$\begin{array}{l}\text { Returns to } \\
\text { Surface }\end{array}$} & \multirow{2}{*}{\multicolumn{3}{|c|}{$\begin{array}{l}\text { Withdrawals by Major Industrial Groups } \\
\text { [NAICS, North American Industrial Classification System code] }\end{array}$}} \\
\hline & Ground = & Surface & Total & & & & & \\
\hline Public supply & 0.85 & 0.00 & 0.85 & -- & -- & NAICS & Groundwater & Surface water \\
\hline Domestic use & 1.08 & 0.00 & 1.08 & 1.58 & 0.00 & \multirow{2}{*}{ 322- Paper, pulp } & \multirow{2}{*}{0.23} & \multirow{2}{*}{0.00} \\
\hline Commercial use & 0.01 & 0.00 & 0.01 & 0.09 & 0.00 & & & \\
\hline Industrial use & 0.23 & 0.00 & 0.23 & 0.34 & 0.00 & & & \\
\hline Public-supply loss & -- & -- & -- & 0.16 & -- & & & \\
\hline Inter-county delivery & -- & -- & -- & 0 & -- & & & \\
\hline Public wastewater treatmen & nt -- & -- & -- & -- & 1.24 & & & \\
\hline Mining use & 0.00 & 0.00 & 0.00 & 0.00 & 0.00 & \multicolumn{3}{|c|}{ Withdrawals by Major Public Suppliers } \\
\hline Irrigation (crop) & 15.15 & 2.64 & 17.79 & 17.79 & 0.00 & Name & Groundwater & Surface water \\
\hline Irrigation (golf) & 0.11 & 0.00 & 0.11 & 0.11 & 0.00 & City of Waynesboro & 0.64 & 0.00 \\
\hline Livestock-aquaculture & 0.76 & 0.13 & 0.89 & 0.89 & 0.00 & City of Sardis & 0.10 & 0.00 \\
\hline Thermoelectric power & 2.12 & 64.20 & 66.32 & 66.32 & 0.00 & & & \\
\hline TOTAL & 20.31 & 66.97 & 87.28 & 87.28 & 1.24 & & & \\
\hline
\end{tabular}

Total use is total withdrawal plus public supply deliveries and losses.

Withdrawals by water source

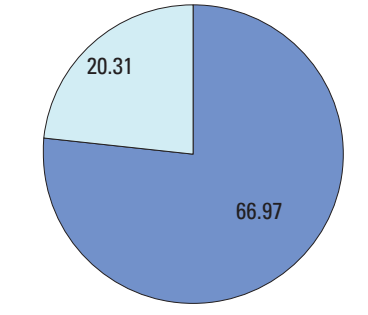

Withdrawals, in million gallons per day

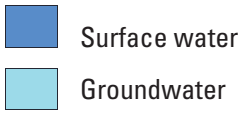

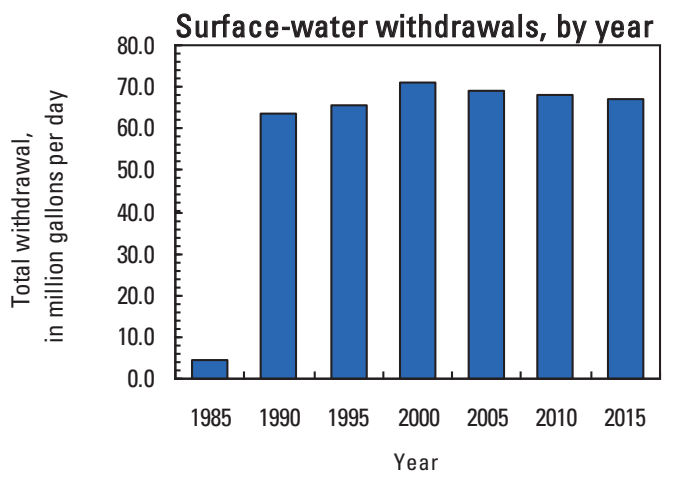

Groundwater withdrawals by aquifer

\begin{tabular}{|c|c|c|}
\hline \multirow{6}{*}{9.41} & \multirow{6}{*}{9.99} & Crystalline-rock aquifers \\
\hline & & Floridan aquifer system \\
\hline & & Southeastern Coastal Plain aquifers \\
\hline & & Surficial aquifer system \\
\hline & & Brunswick aquifer system \\
\hline & & Valley and Ridge aquifer \\
\hline
\end{tabular}

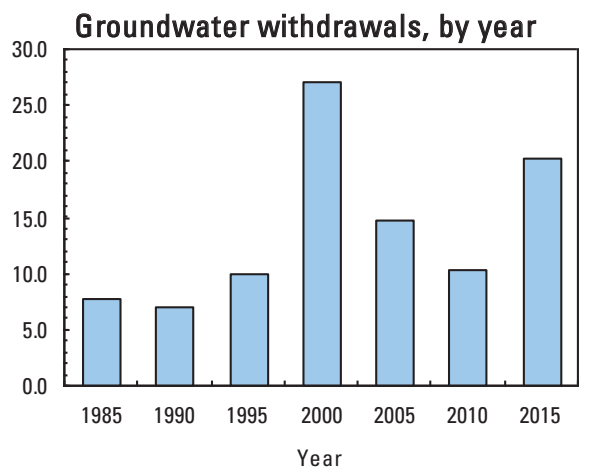




\section{BUTTS COUNTY}

Population

Population served by public supply-groundwater

Population served by public supply-surface water $\quad 21,600$

Acres irrigated

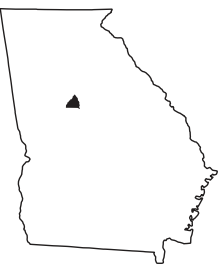

2015 WATER WITHDRAWALS AND ESTIMATED USE, IN MILLION GALLONS PER DAY

[--,not applicable; Mgal/d, million gallons per day]

\begin{tabular}{lccccc}
\hline & \multicolumn{3}{c}{ Withdrawals } & Total & Returns to \\
\cline { 2 - 4 } Category & $\begin{array}{c}\text { Ground } \\
\text { water }\end{array}$ & $\begin{array}{c}\text { Surface } \\
\text { water }\end{array}$ & Total & $\begin{array}{c}\text { Sse } \\
\text { Surface }\end{array}$ \\
\hline Public supply & 0.16 & 2.58 & 2.74 & -- & -- \\
Domestic use & 0.00 & 0.00 & 0.00 & 1.72 & 0.00 \\
Commercial use & 0.00 & 0.00 & 0.00 & 0.38 & 0.00 \\
Industrial use & 0.00 & 0.00 & 0.00 & 0.22 & 0.00 \\
Public-supply loss & -- & -- & -- & 0.42 & -- \\
Inter-county delivery & -- & -- & -- & -0.12 & -- \\
Public wastewater treatment & -- & -- & -- & -- & 0.31 \\
Mining use & 0.00 & 0.00 & 0.00 & 0.00 & 0.00 \\
Irrigation (crop) & 0.00 & 0.07 & 0.07 & 0.07 & 0.00 \\
Irrigation (golf) & 0.00 & 0.06 & 0.06 & 0.06 & 0.00 \\
Livestock-aquaculture & 0.00 & 0.00 & 0.00 & 0.00 & 0.00 \\
Thermoelectric power & 0.00 & 0.00 & 0.00 & 0.00 & 0.00 \\
\hline TOTAL & 0.16 & 2.71 & 2.87 & 2.75 & 0.31 \\
\hline
\end{tabular}

Withdrawals by Major Industrial Groups

[NAICS, North American Industrial Classification System code]

\begin{tabular}{lll}
\hline NAICS Groundwater & Surface water \\
\hline
\end{tabular}

Withdrawals by Major Public Suppliers

$\begin{array}{lcc}\text { Name } & \text { Groundwater } & \text { Surface water } \\ \text { Butts County Water \& } & 0.00 & 2.58 \\ \text { Sewer Authority/City of } & & \\ \text { Jackson/City of } & & \\ \text { Jenkinsburg } & & \end{array}$

Total use is total withdrawal plus public supply deliveries and losses. An estimate of $0.01 \mathrm{Mgal} / \mathrm{d}$ was delivered from neighboring counties in 2015. An estimate of $0.13 \mathrm{Mgal} / \mathrm{d}$ was delivered from Butts County to neighboring counties in 2015

\section{Withdrawals by water source}

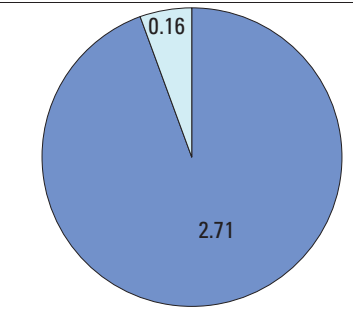

Withdrawals, in million gallons per day

Surface water

Groundwater

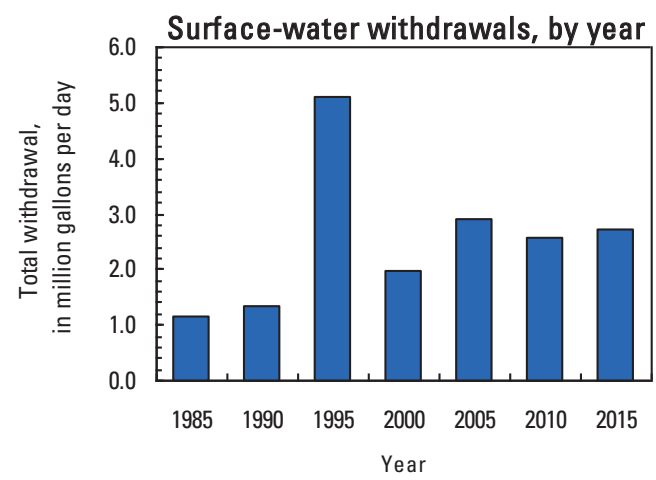

Groundwater withdrawals by aquifer

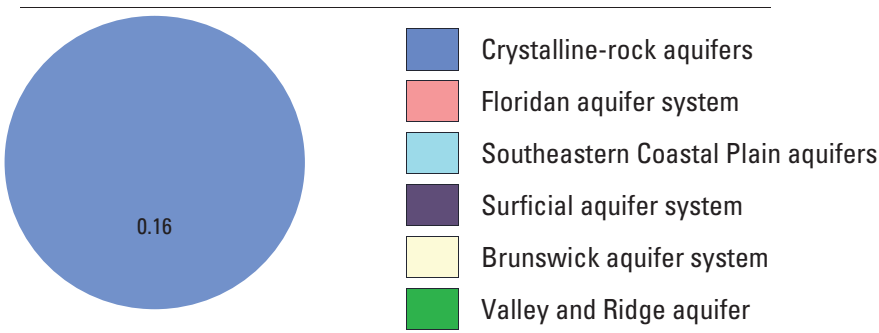




\section{CALHOUN COUNTY}

Population

Population served by public supply-groundwater

Population served by public supply-surface water

0

Acres irrigated

27,510

2015 WATER WITHDRAWALS AND ESTIMATED USE, IN MILLION GALLONS PER DAY

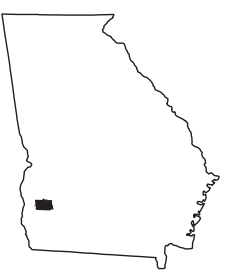

[--,not applicable; Mgal/d, million gallons per day]

\begin{tabular}{|c|c|c|c|c|c|}
\hline \multirow[b]{2}{*}{ Category } & \multicolumn{3}{|c|}{ Withdrawals } & \multirow[b]{2}{*}{$\begin{array}{c}\text { Total } \\
\text { use }\end{array}$} & \multirow[b]{2}{*}{$\begin{array}{c}\text { Returns to } \\
\text { Surface }\end{array}$} \\
\hline & $\begin{array}{c}\text { Ground- } \\
\text { water }\end{array}$ & $\begin{array}{c}\text { Surface } \\
\text { water }\end{array}$ & Total & & \\
\hline Public supply & 0.44 & 0.00 & 0.44 & -- & -- \\
\hline Domestic use & 0.22 & 0.00 & 0.22 & 0.44 & 0.00 \\
\hline Commercial use & 0.00 & 0.00 & 0.00 & 0.15 & 0.00 \\
\hline Industrial use & 0.00 & 0.00 & 0.00 & 0 & 0.00 \\
\hline Public-supply loss & -- & -- & -- & 0.07 & -- \\
\hline Inter-county delivery & -- & -- & -- & 0 & -- \\
\hline Public wastewater treatment & nt -- & -- & -- & -- & 0.56 \\
\hline Mining use & 0.00 & 0.00 & 0.00 & 0.00 & 0.00 \\
\hline Irrigation (crop) & 5.89 & 10.90 & 16.79 & 16.79 & 0.00 \\
\hline Irrigation (golf) & 0.00 & 0.00 & 0.00 & 0.00 & 0.00 \\
\hline Livestock-aquaculture & 0.06 & 0.12 & 0.18 & 0.18 & 0.00 \\
\hline Thermoelectric power & 0.00 & 0.00 & 0.00 & 0.00 & 0.00 \\
\hline TOTAL & 6.61 & 11.02 & 17.63 & 17.63 & 0.56 \\
\hline
\end{tabular}

Withdrawals by Major Industrial Groups

[NAICS, North American Industrial Classification System code]

NAICS Groundwater Surface water

None

Withdrawals by Major Public Suppliers

Name Groundwater Surface water

$\begin{array}{lll}\text { City of Edison } & 0.13 & 0.00 \\ \text { City of Leary } & 0.04 & 0.00 \\ \text { City of Morgan } & 0.23 & 0.00\end{array}$

Total use is total withdrawal plus public supply deliveries and losses.

Withdrawals by water source

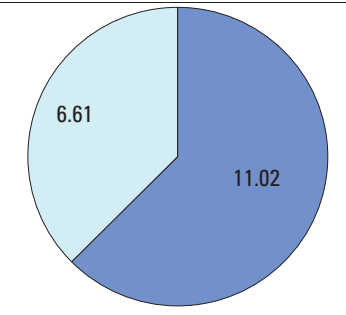

Withdrawals, in million

gallons per day

Surface water

Groundwater

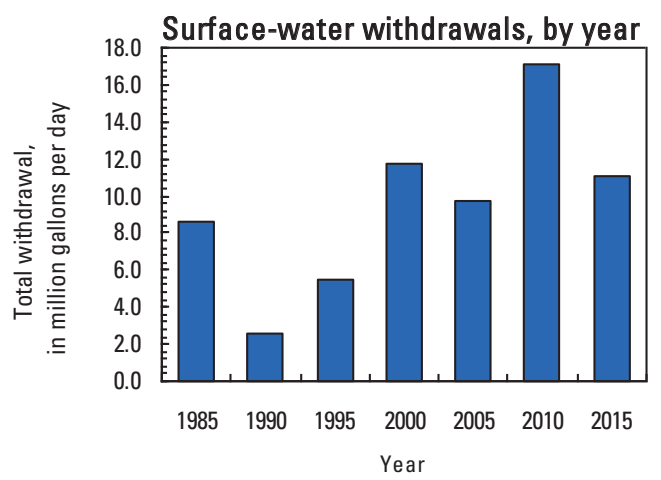

Groundwater withdrawals by aquifer
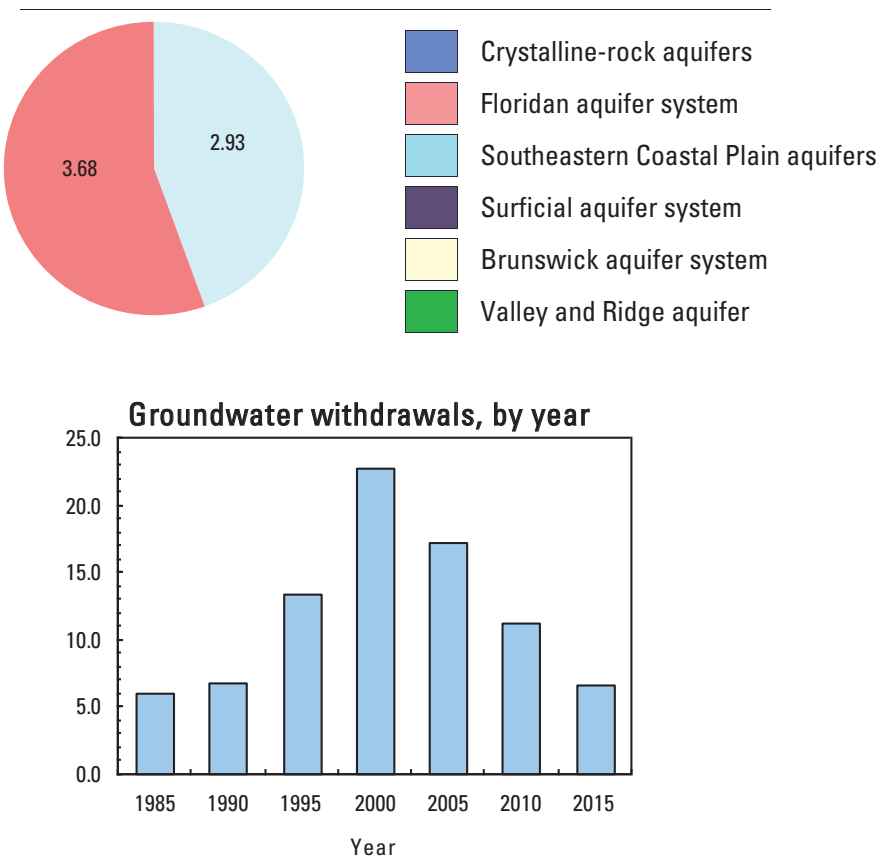


\section{CAMDEN COUNTY}

Population

52,102

Population served by public supply-groundwater

37,871

Population served by public supply-surface water

0

Acres irrigated

440

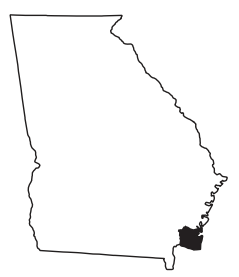

2015 WATER WITHDRAWALS AND ESTIMATED USE, IN MILLION GALLONS PER DAY

[--,not applicable; Mgal/d, million gallons per day]

\begin{tabular}{lcccccc}
\hline & \multicolumn{3}{c}{ Withdrawals } & & Total \\
\cline { 2 - 4 } Category & $\begin{array}{c}\text { Ground- } \\
\text { water }\end{array}$ & $\begin{array}{c}\text { Surface } \\
\text { water }\end{array}$ & $\begin{array}{c}\text { Total } \\
\text { use }\end{array}$ & $\begin{array}{c}\text { Returns to } \\
\text { Surface }\end{array}$ \\
\hline Public supply & 3.94 & 0.00 & 3.94 & -- & -- \\
Domestic use & 1.07 & 0.00 & 1.07 & 3.68 & 0.00 \\
Commercial use & 0.00 & 0.00 & 0.00 & 0.61 & 0.00 \\
Industrial use & 0.02 & 0.00 & 0.02 & 0.17 & 0.00 \\
Public-supply loss & -- & -- & -- & 0.57 & -- \\
Inter-county delivery & -- & -- & -- & 0 & -- \\
Public wastewater treatment & -- & -- & -- & -- & 4.14 \\
Mining use & 0.00 & 0.00 & 0.00 & 0.00 & 0.00 \\
Irrigation (crop) & 0.00 & 0.00 & 0.00 & 0.00 & 0.00 \\
Irrigation (golf) & 0.23 & 0.16 & 0.39 & 0.39 & 0.00 \\
Livestock-aquaculture & 0.00 & 0.01 & 0.01 & 0.01 & 0.00 \\
Thermoelectric power & 0.00 & 0.00 & 0.00 & 0.00 & 0.00 \\
\hline TOTAL & 5.26 & 0.17 & 5.43 & 5.43 & 4.14 \\
\hline
\end{tabular}

Withdrawals by Major Industrial Groups

[NAICS, North American Industrial Classification System code]

NAICS Groundwater Surface water

325- Chemicals

0.02

0.00

Withdrawals by Major Public Suppliers

\begin{tabular}{lcc} 
Name & Groundwater & Surface wate \\
\hline City of Kingsland & 1.55 & 0.00 \\
City of St. Marys & 1.42 & 0.00 \\
City of Woodbine & 0.10 & 0.00 \\
Naval Submarine Base & 0.82 & 0.00 \\
Kings Bay & & 0.00 \\
W \& D Utilities & 0.05 &
\end{tabular}

Total use is total withdrawal plus public supply deliveries and losses.

Withdrawals by water source

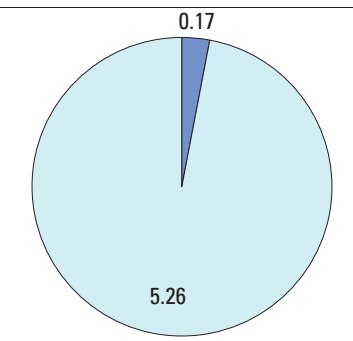

Withdrawals, in million

gallons per day

Surface water

Groundwater

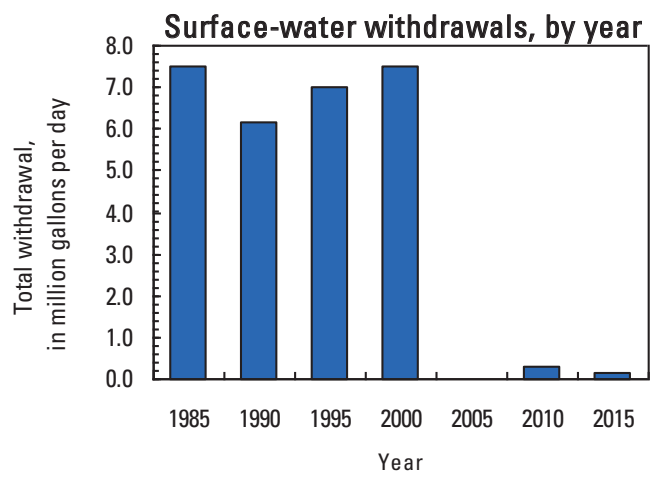

Groundwater withdrawals by aquifer
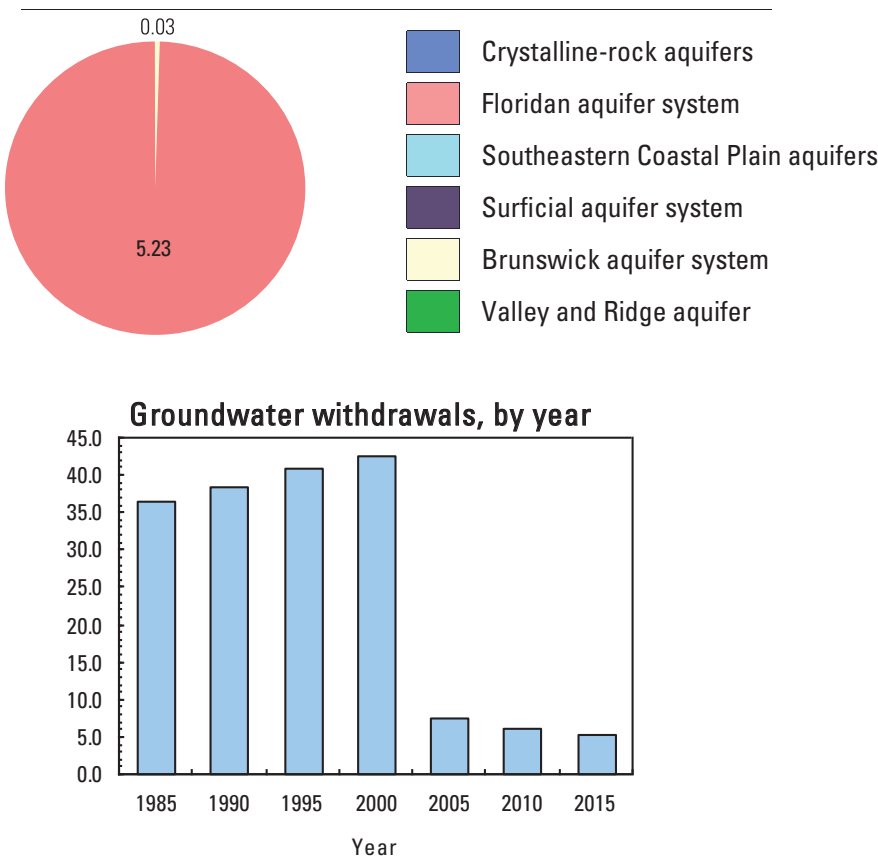


\section{CANDLER COUNTY}

Population

10,886

Population served by public supply-groundwater

6,655

Population served by public supply-surface water

0

Acres irrigated

2015 WATER WITHDRAWALS AND ESTIMATED USE, IN MILLION GALLONS PER DAY

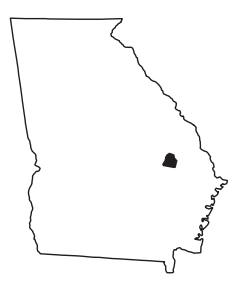

[--,not applicable; Mgal/d, million gallons per day]

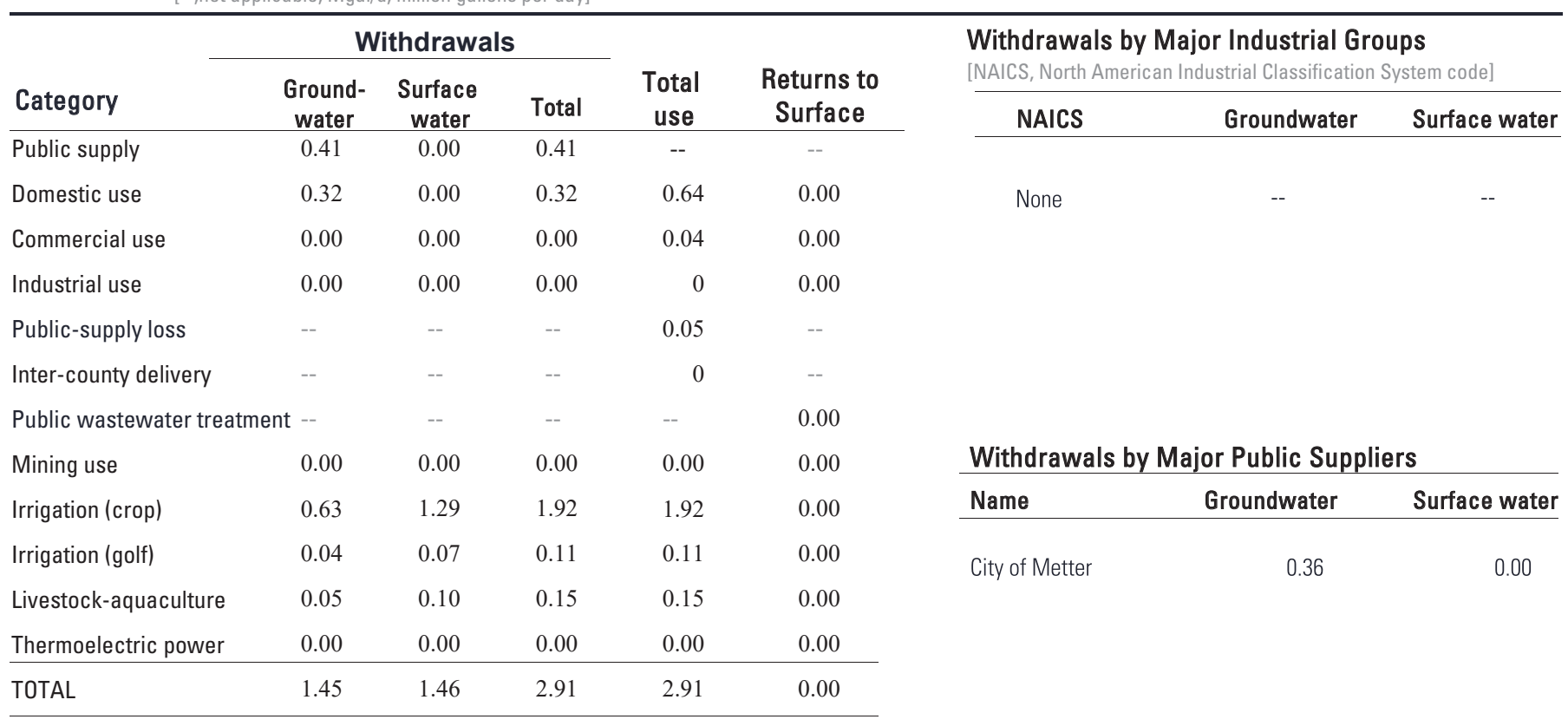

Total use is total withdrawal plus public supply deliveries and losses.

Withdrawals by water source

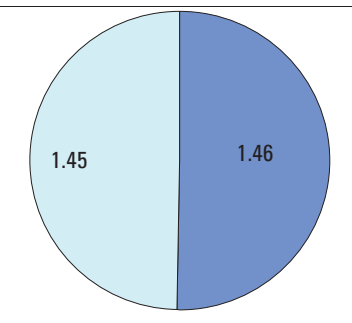

Withdrawals, in million

gallons per day

Surface water

Groundwater
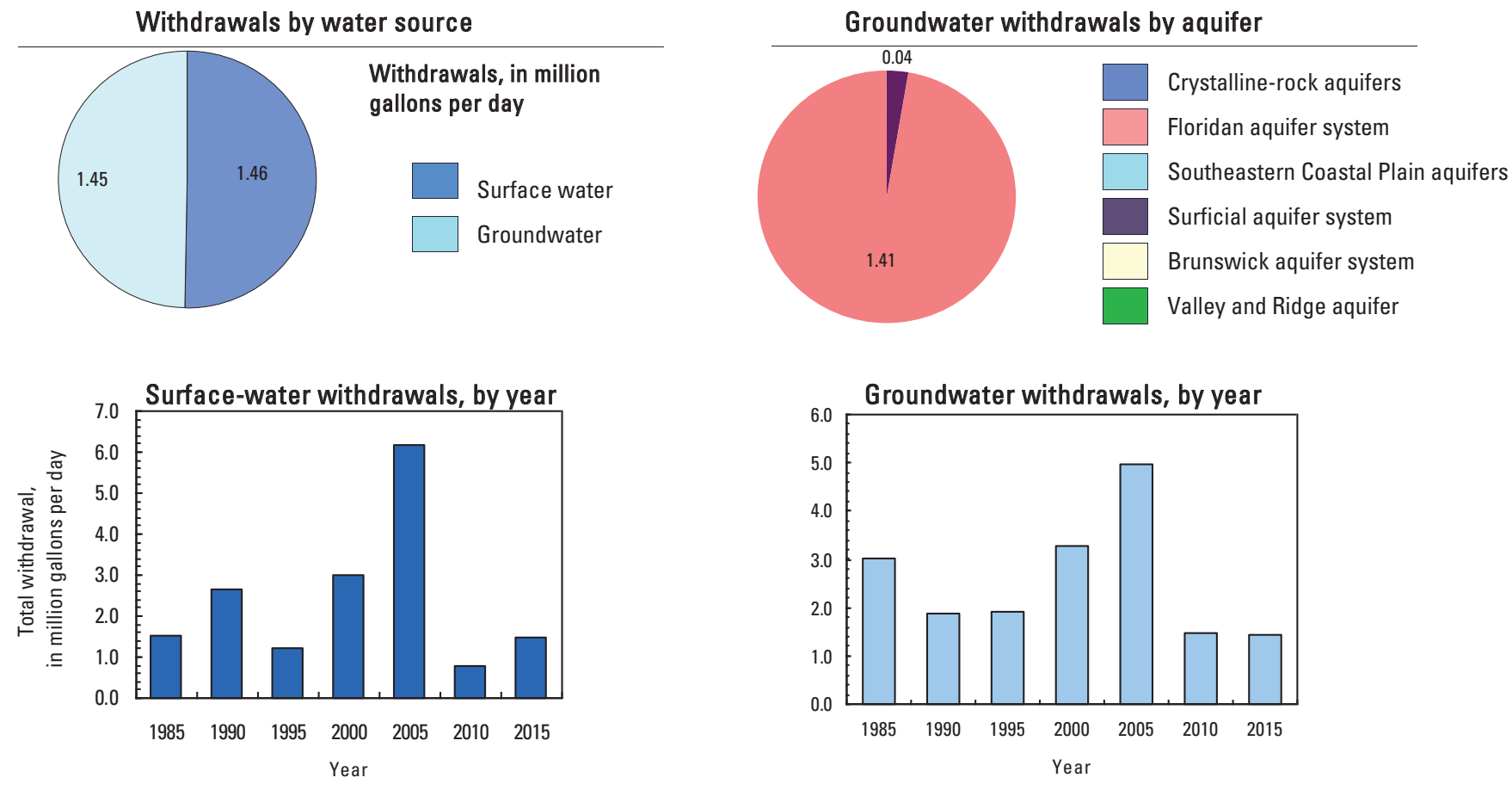


\section{CARROLL COUNTY}

Population

Population served by public supply-groundwater

Population served by public supply-surface water $\quad 94,939$

Acres irrigated

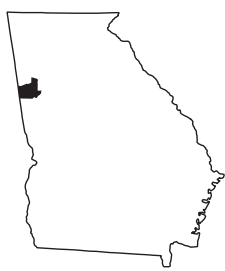

2015 WATER WITHDRAWALS AND ESTIMATED USE, IN MILLION GALLONS PER DAY

[--,not applicable; Mgal/d, million gallons per day]

\begin{tabular}{lcccccc}
\hline & \multicolumn{3}{c}{ Withdrawals } & & Total \\
\cline { 2 - 4 } Category & $\begin{array}{c}\text { Ground- } \\
\text { water }\end{array}$ & $\begin{array}{c}\text { Surface } \\
\text { water }\end{array}$ & $\begin{array}{c}\text { Total } \\
\text { use }\end{array}$ & $\begin{array}{c}\text { Returns to } \\
\text { Surface }\end{array}$ \\
\hline Public supply & 0.26 & 11.06 & 11.32 & -- & -- \\
Domestic use & 1.36 & 0.00 & 1.36 & 7.78 & 0.00 \\
Commercial use & 0.00 & 0.00 & 0.00 & 1.65 & 0.00 \\
Industrial use & 0.00 & 0.00 & 0.00 & 1.31 & 0.00 \\
Public-supply loss & -- & -- & -- & 1.94 & -- \\
Inter-county delivery & -- & -- & -- & 0 & -- \\
Public wastewater treatment & -- & -- & -- & -- & 1.76 \\
Mining use & 0.03 & 0.00 & 0.03 & 0.03 & 0.00 \\
Irrigation (crop) & 0.00 & 0.00 & 0.00 & 0.00 & 0.00 \\
Irrigation (golf) & 0.00 & 0.30 & 0.30 & 0.30 & 0.00 \\
Livestock-aquaculture & 0.46 & 0.69 & 1.15 & 1.15 & 0.00 \\
Thermoelectric power & 0.00 & 0.00 & 0.00 & 0.00 & 0.00 \\
\hline TOTAL & 2.11 & 12.05 & 14.16 & 14.16 & 1.76 \\
\hline
\end{tabular}

Withdrawals by Major Industrial Groups

[NAICS, North American Industrial Classification System code]

NAICS Groundwater Surface water

Withdrawals by Major Public Suppliers

\begin{tabular}{lcc} 
Name & Groundwater & Surface water \\
\hline Carroll County & 0.14 & 4.63 \\
City of Bowdon & 0.00 & 0.55 \\
City of Carrollton & 0.00 & 4.98 \\
City of Villa Rica & 0.00 & 0.90
\end{tabular}

Total use is total withdrawal plus public supply deliveries and losses.

Withdrawals by water source

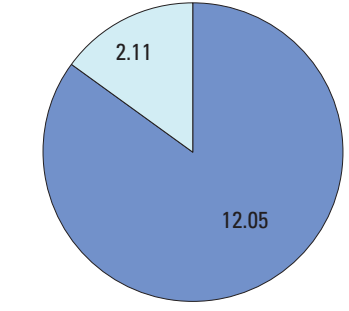

Withdrawals, in million

gallons per day

Surface water

Groundwater

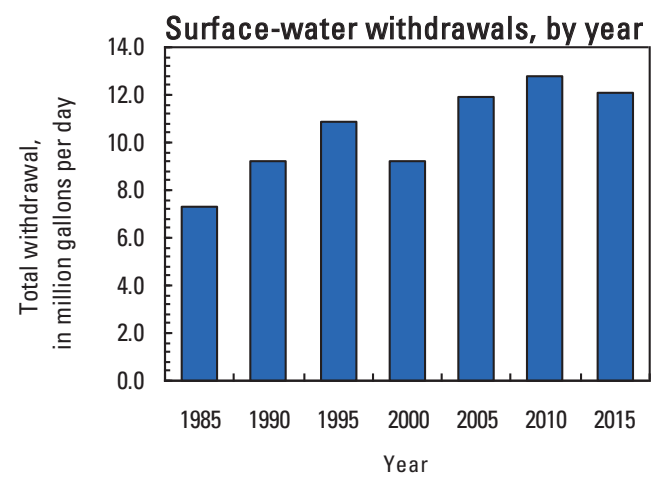

Groundwater withdrawals by aquifer
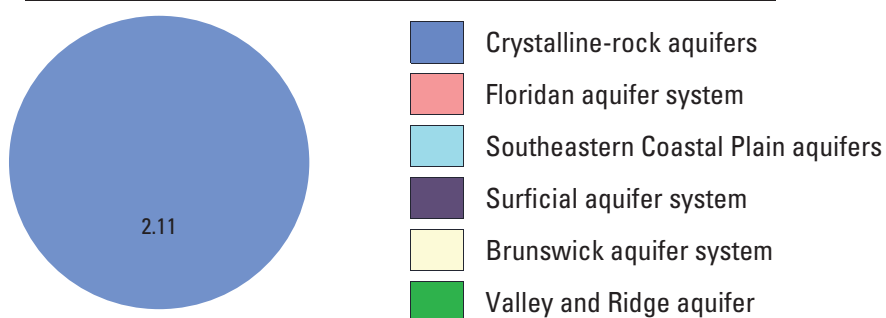

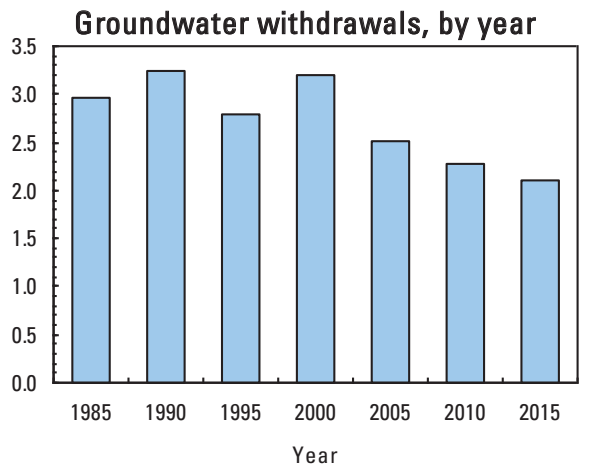




\section{CATOOSA COUNTY}

Population

Population served by public supply-groundwater

Population served by public supply-surface water

Acres irrigated
66,050

0

62,866

620

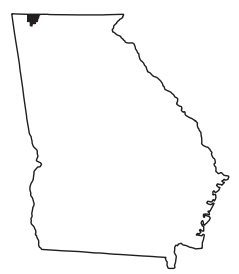

2015 WATER WITHDRAWALS AND ESTIMATED USE, IN MILLION GALLONS PER DAY

[--,not applicable; Mgal/d, million gallons per day]

\begin{tabular}{lccccc}
\hline & \multicolumn{3}{c}{ Withdrawals } & & Total \\
\cline { 2 - 4 } Category & $\begin{array}{c}\text { Ground- } \\
\text { water }\end{array}$ & $\begin{array}{c}\text { Surface } \\
\text { water }\end{array}$ & $\begin{array}{c}\text { Total } \\
\text { use }\end{array}$ & $\begin{array}{c}\text { Returns to } \\
\text { Surface }\end{array}$ \\
\hline Public supply & 0.00 & 4.68 & 4.68 & -- & -- \\
Domestic use & 0.24 & 0.00 & 0.24 & 5.11 & 0.00 \\
Commercial use & 0.00 & 0.00 & 0.00 & 0.45 & 0.00 \\
Industrial use & 0.00 & 0.00 & 0.00 & 0.06 & 0.00 \\
Public-supply loss & -- & -- & -- & -0.7 & -- \\
Inter-county delivery & -- & -- & -- & 1.91 & -- \\
Public wastewater treatment & -- & -- & -- & -- & 0.09 \\
Mining use & 0.04 & 0.00 & 0.04 & 0.04 & 0.00 \\
Irrigation (crop) & 0.08 & 0.15 & 0.23 & 0.23 & 0.00 \\
Irrigation (golf) & 0.21 & 0.26 & 0.47 & 0.47 & 0.00 \\
Livestock-aquaculture & 0.07 & 0.12 & 0.19 & 0.19 & 0.00 \\
Thermoelectric power & 0.00 & 0.00 & 0.00 & 0.00 & 0.00 \\
\hline TOTAL & 0.64 & 5.21 & 5.85 & 7.76 & 0.09 \\
\hline
\end{tabular}

Withdrawals by Major Industrial Groups

[NAICS, North American Industrial Classification System code]

NAICS Groundwater Surface water

None

Withdrawals by Major Public Suppliers

Name Groundwater Surface water

Catoosa Utility District

0.00

4.38

Authority

City of Ringgold

0.00

0.30

Total use is total withdrawal plus public supply deliveries and losses. An estimate of $1.99 \mathrm{Mgal} / \mathrm{d}$ was delivered from neighboring counties in 2015. An estimate of $0.08 \mathrm{Mgal} / \mathrm{d}$ was delivered from Catoosa County to neighboring counties in 2015.

\section{Withdrawals by water source}

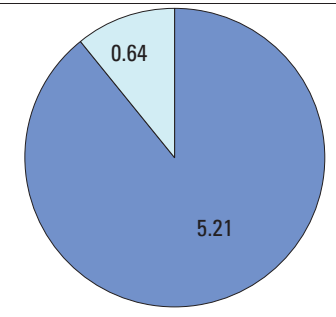

Withdrawals, in million gallons per day

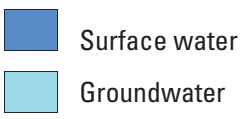

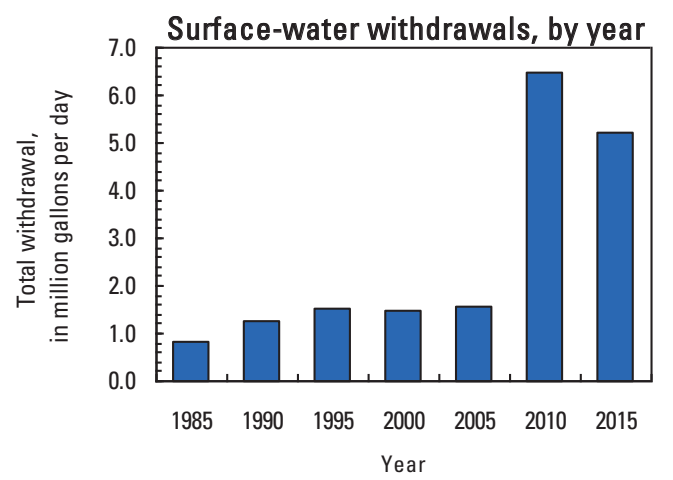

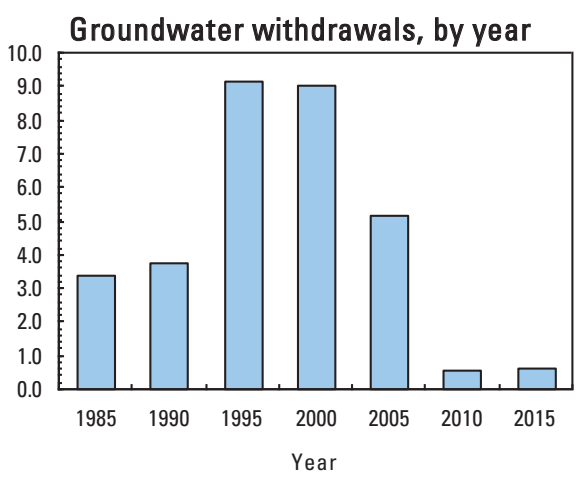




\section{CHARLTON COUNTY}

Population

12,965

Population served by public supply-groundwater

6,935

Population served by public supply-surface water

Acres irrigated

2015 WATER WITHDRAWALS AND ESTIMATED USE, IN MILLION GALLONS PER DAY

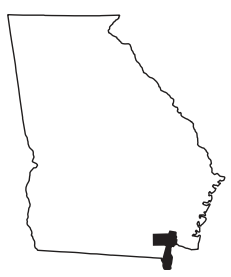

[--,not applicable; Mgal/d, million gallons per day]

\begin{tabular}{|c|c|c|c|c|c|c|c|c|}
\hline & \multicolumn{3}{|c|}{ Withdrawals } & \multirow{2}{*}{$\begin{array}{c}\text { Total } \\
\text { use }\end{array}$} & \multirow{2}{*}{$\begin{array}{c}\text { Returns to } \\
\text { Surface }\end{array}$} & \multirow{2}{*}{\multicolumn{3}{|c|}{$\begin{array}{l}\text { Withdrawals by Major Industrial Groups } \\
\text { [NAICS, North American Industrial Classification System code] }\end{array}$}} \\
\hline & $\begin{array}{c}\text { Ground- } \\
\text { water }\end{array}$ & $\begin{array}{c}\text { Surface } \\
\text { water }\end{array}$ & Total & & & & & \\
\hline Public supply & $\begin{array}{c}\text { Water } \\
0.76\end{array}$ & $\begin{array}{c}\text { Water } \\
0.00\end{array}$ & 0.76 & USE & -- & NAICS & Groundwater & Surface water \\
\hline Domestic use & 0.45 & 0.00 & 0.45 & 1.04 & 0.00 & 212 Mining & ד & 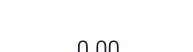 \\
\hline Commercial use & 0.00 & 0.00 & 0.00 & 0.08 & 0.00 & LIL- IVIIII) & 0.01 & 0.00 \\
\hline Industrial use & 0.07 & 0.00 & 0.07 & 0.07 & 0.00 & & & \\
\hline Public-supply loss & -- & -- & -- & 0.09 & -- & & & \\
\hline Inter-county delivery & -- & -- & -- & 0 & -- & & & \\
\hline Public wastewater treatment & t -- & -- & -- & -- & 1.29 & & & \\
\hline Mining use & 0.21 & 0.00 & 0.21 & 0.21 & 0.00 & Withdrawals & jor Public Sup & \\
\hline Irrigation (crop) & 0.00 & 0.00 & 0.00 & 0.00 & 0.00 & Name & Groundwater & Surface water \\
\hline Irrigation (golf) & 0.00 & 0.00 & 0.00 & 0.00 & 0.00 & City of Folkston & 0.70 & 0.00 \\
\hline Livestock-aquaculture & 0.02 & 0.01 & 0.03 & 0.03 & 0.00 & & & \\
\hline Thermoelectric power & 0.00 & 0.00 & 0.00 & 0.00 & 0.00 & & & \\
\hline TOTAL & 1.51 & 0.01 & 1.52 & 1.52 & 1.29 & & & \\
\hline
\end{tabular}

Total use is total withdrawal plus public supply deliveries and losses.

Withdrawals by water source

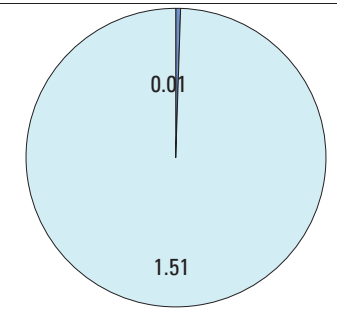

Withdrawals, in million

gallons per day

Surface water

Groundwater

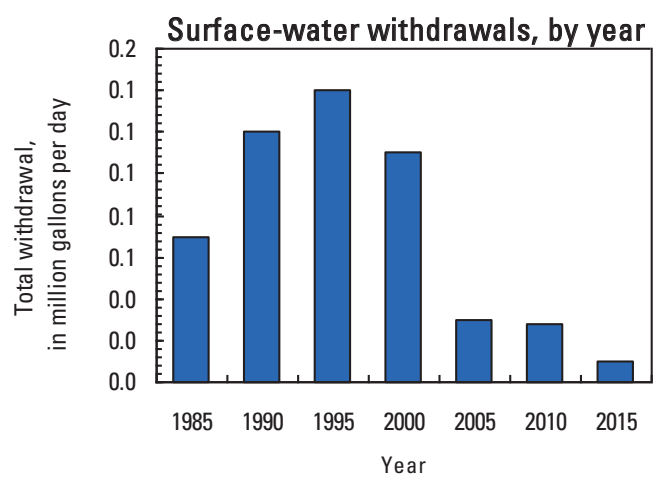

Groundwater withdrawals by aquifer

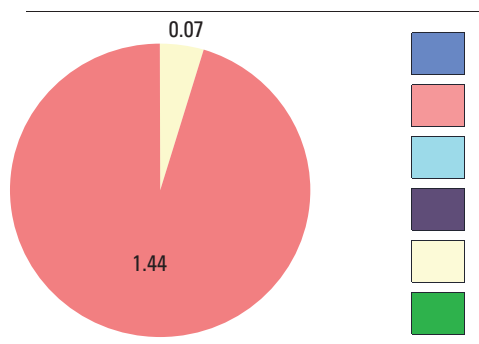

Crystalline-rock aquifers

Floridan aquifer system

Southeastern Coastal Plain aquifers

Surficial aquifer system

Brunswick aquifer system

Valley and Ridge aquifer

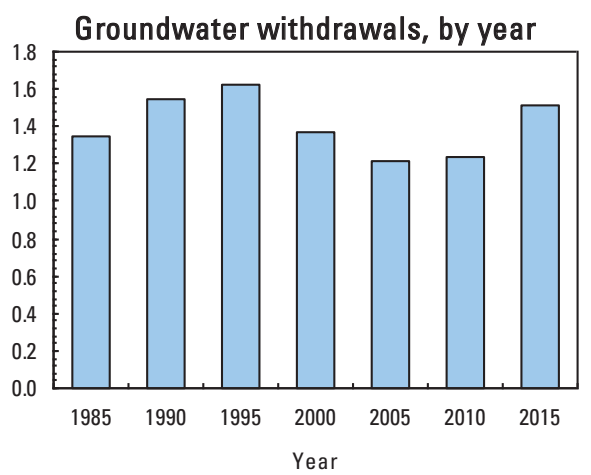




\section{CHATHAM COUNTY}

Population

286,956

Population served by public supply-groundwater

172,135

Population served by public supply-surface water 104,353

Acres irrigated

690

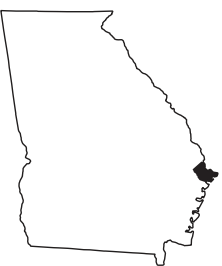

2015 WATER WITHDRAWALS AND ESTIMATED USE, IN MILLION GALLONS PER DAY

[--,not applicable; Mgal/d, million gallons per day]

\begin{tabular}{|c|c|c|c|c|c|}
\hline \multirow[b]{2}{*}{ Category } & \multicolumn{3}{|c|}{ Withdrawals } & \multirow[b]{2}{*}{$\begin{array}{c}\text { Total } \\
\text { use }\end{array}$} & \multirow[b]{2}{*}{$\begin{array}{c}\text { Returns to } \\
\text { Surface }\end{array}$} \\
\hline & $\begin{array}{c}\text { Ground- } \\
\text { water }\end{array}$ & $\begin{array}{c}\text { Surface } \\
\text { water }\end{array}$ & Total & & \\
\hline Public supply & 28.08 & 0.00 & 28.08 & -- & -- \\
\hline Domestic use & 0.29 & 0.00 & 0.29 & 16.12 & 0.00 \\
\hline Commercial use & 0.25 & 0.00 & 0.25 & 5.53 & 0.00 \\
\hline Industrial use & 17.00 & 23.76 & 40.76 & 60.37 & 0.00 \\
\hline Public-supply loss & -- & -- & -- & -12.64 & -- \\
\hline Inter-county delivery & -- & -- & -- & 21.7 & -- \\
\hline Public wastewater treatmen & ent -- & -- & -- & -- & 85.88 \\
\hline Mining use & 0.00 & 0.00 & 0.00 & 0.00 & 0.00 \\
\hline Irrigation (crop) & 0.03 & 0.00 & 0.03 & 0.03 & 0.00 \\
\hline Irrigation (golf) & 0.51 & 0.17 & 0.68 & 0.68 & 0.00 \\
\hline Livestock-aquaculture & 0.00 & 0.00 & 0.00 & 0.00 & 0.00 \\
\hline Thermoelectric power & 0.90 & 100.46 & 101.36 & 101.36 & 0.00 \\
\hline TOTAL & 47.06 & 124.4 & 171.5 & 193.2 & 85.9 \\
\hline
\end{tabular}

Total use is total withdrawal plus public supply deliveries and losses. An estimate of $23.8 \mathrm{Mgal} / \mathrm{d}$ was delivered from neighboring counties in 2015. An estimate of $2.1 \mathrm{Mgal} / \mathrm{d}$ was delivered from Chatham County to neighboring counties in 2015.

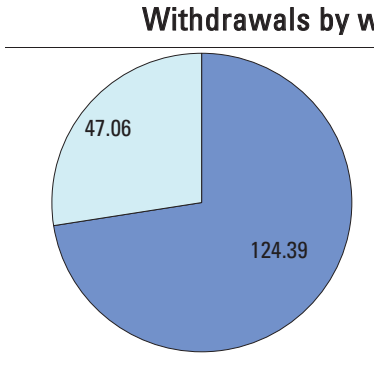

Withdrawals, in million gallons per day

Surface water

Groundwate

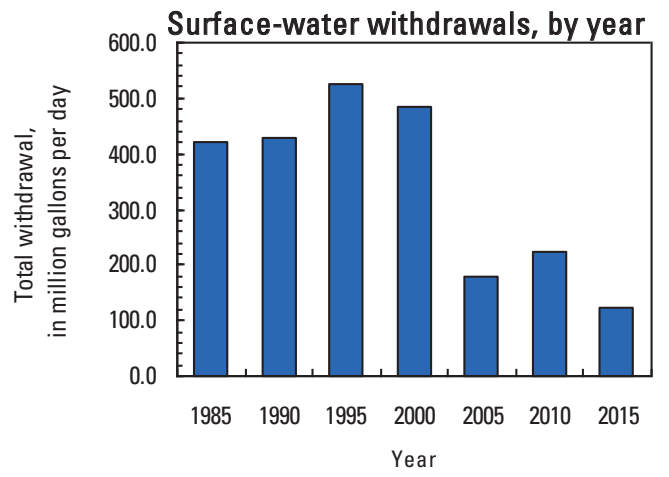

Withdrawals by Major Industrial Groups

[NAICS, North American Industrial Classification System code]

NAICS Groundwater Surface water

$\begin{array}{lrr}\text { 212- Mining } & 0.07 & 0.56 \\ \text { 311- Food } & 0.38 & 0.00 \\ \text { 321- Wood } & 0.05 & 0.00 \\ \text { 322- Paper, pulp } & 14.85 & 16.31\end{array}$

Withdrawals by Major Public Suppliers

\begin{tabular}{lcc} 
Name & Groundwater & Surface water \\
\hline City of Savannah & 20.41 & 0.00 \\
Skidaway Island Utilities, Inc. & 1.27 & 0.00 \\
Garden City & 0.90 & 0.00 \\
City of Tybee Island & 0.78 & 0.00 \\
Consolidated Utilties, Inc. & 0.64 & 0.00 \\
Hunter Army Airfield & 0.38 & 0.00 \\
City of Pooler & 0.31 & 0.00
\end{tabular}

City of Pooler

Groundwater withdrawals by aquifer
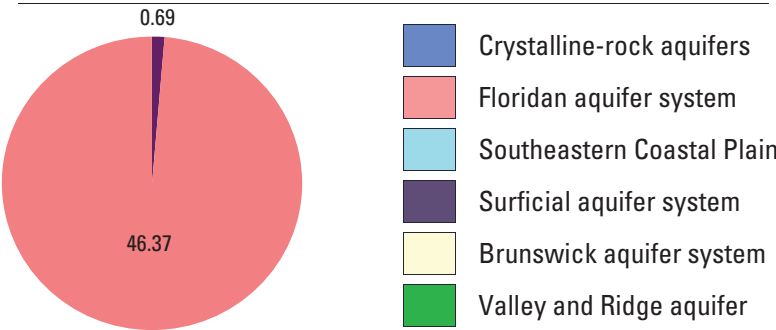


\section{CHATTAHOOCHEE COUNTY}

Population

Population served by public supply-groundwater $\quad 3,300$

Population served by public supply-surface water 0

Acres irrigated

0

2015 WATER WITHDRAWALS AND ESTIMATED USE, IN MILLION GALLONS PER DAY

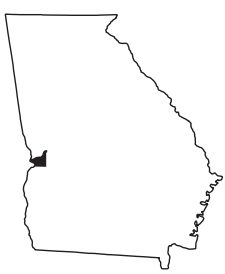

[--,not applicable; Mgal/d, million gallons per day]

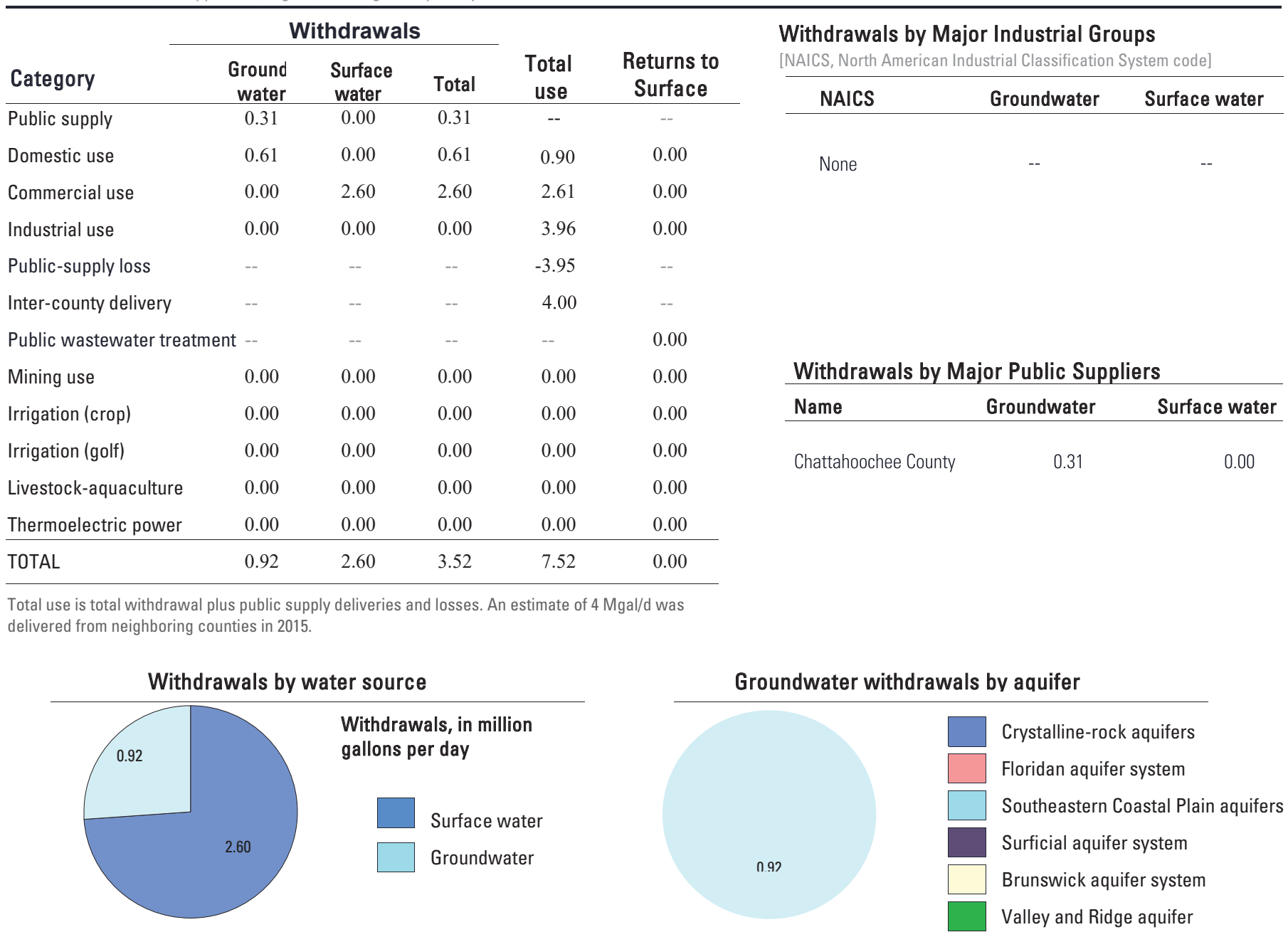
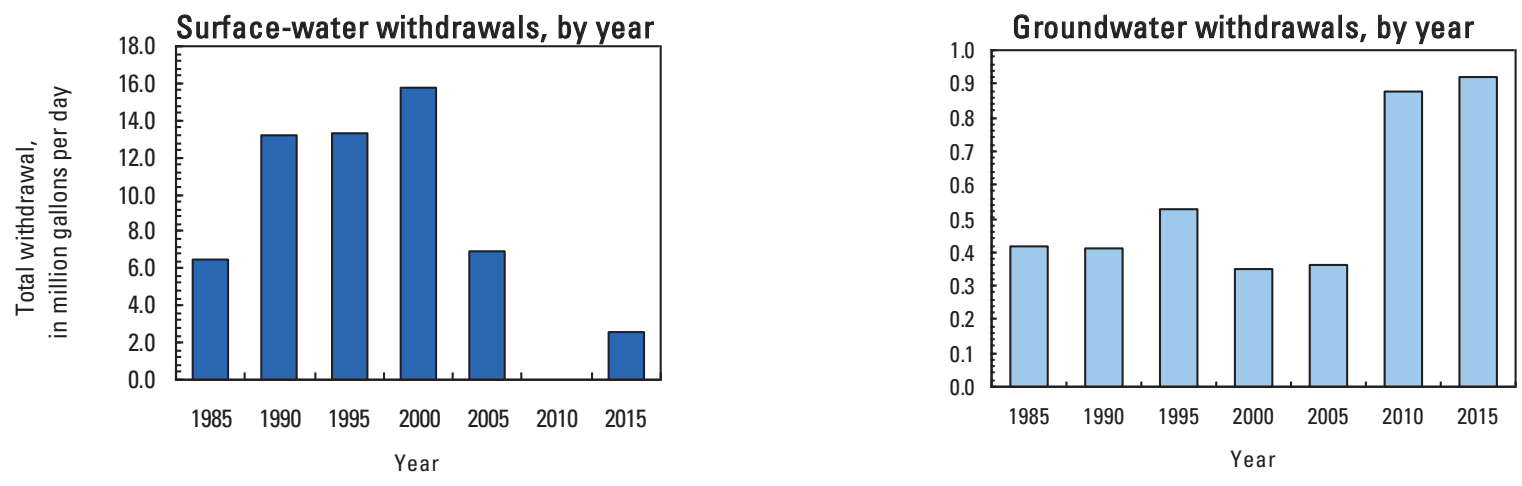


\section{CHATTOOGA COUNTY}

Population

Population served by public supply-groundwater

Population served by public supply-surface water 11,991

Acres irrigated

2015 WATER WITHDRAWALS AND ESTIMATED USE, IN MILLION GALLONS PER DAY

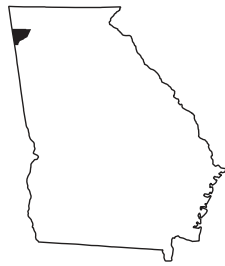

[--,not applicable; Mgal/d, million gallons per day]

\begin{tabular}{|c|c|c|c|c|c|}
\hline \multirow[b]{2}{*}{ Category } & \multicolumn{3}{|c|}{ Withdrawals } & \multirow[b]{2}{*}{$\begin{array}{c}\text { Total } \\
\text { use }\end{array}$} & \multirow[b]{2}{*}{$\begin{array}{c}\text { Returns to } \\
\text { Surface }\end{array}$} \\
\hline & $\begin{array}{l}\text { Ground } \\
\text { water }\end{array}$ & $\begin{array}{c}\text { Surface } \\
\text { water }\end{array}$ & Total & & \\
\hline Public supply & 0.99 & 1.81 & 2.80 & -- & -- \\
\hline Domestic use & 0.09 & 0.00 & 0.09 & 1.50 & 0.00 \\
\hline Commercial use & 0.00 & 0.00 & 0.00 & 0.21 & 0.00 \\
\hline Industrial use & 0.00 & 5.60 & 5.60 & 5.83 & 0.00 \\
\hline Public-supply loss & -- & -- & -- & 0.95 & -- \\
\hline Inter-county delivery & -- & -- & -- & -0.41 & -- \\
\hline Public wastewater treatmen & nt -- & -- & -- & -- & 8.52 \\
\hline Mining use & 0.01 & 0.00 & 0.01 & 0.01 & 0.00 \\
\hline Irrigation (crop) & 0.00 & 0.00 & 0.00 & 0.00 & 0.00 \\
\hline Irrigation (golf) & 0.00 & 0.00 & 0.00 & 0.00 & 0.00 \\
\hline Livestock-aquaculture & 0.43 & 0.88 & 1.31 & 1.31 & 0.00 \\
\hline Thermoelectric power & 0.00 & 0.00 & 0.00 & 0.00 & 0.00 \\
\hline TOTAL & 1.52 & 8.29 & 9.81 & 9.40 & 8.52 \\
\hline
\end{tabular}

Withdrawals by Major Industrial Groups

[NAICS, North American Industrial Classification System code]

NAICS Groundwater Surface water

$\begin{array}{lll}\text { 313- Textile mills } & 0.00 & 4.54 \\ \text { 314- Textiles } & 0.00 & 1.06\end{array}$

Withdrawals by Major Public Suppliers

\begin{tabular}{lcc} 
Name & Groundwater & Surface water \\
\hline Chattooga County & 0.08 & 0.00 \\
Town of Lyerly & 0.11 & 0.00 \\
City of Summerville & 0.00 & 1.81
\end{tabular}

Total use is total withdrawal plus public supply deliveries and losses. An estimate of $0.41 \mathrm{Mgal} / \mathrm{d}$ was delivered from Chattooga County to neighboring counties in 2015.

\section{Withdrawals by water source}

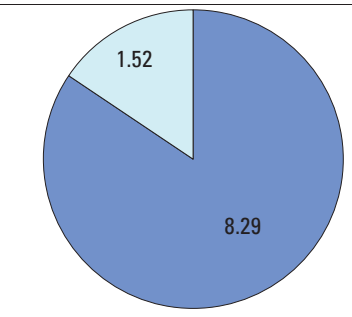

Withdrawals, in million gallons per day

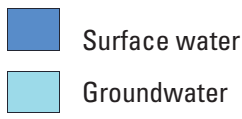

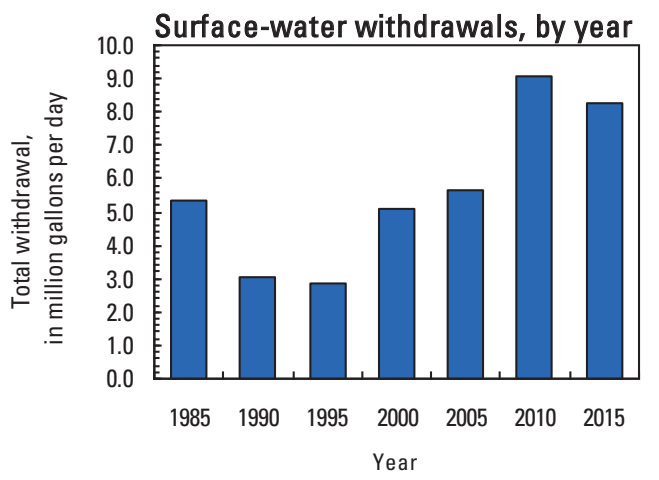

Groundwater withdrawals by aquifer

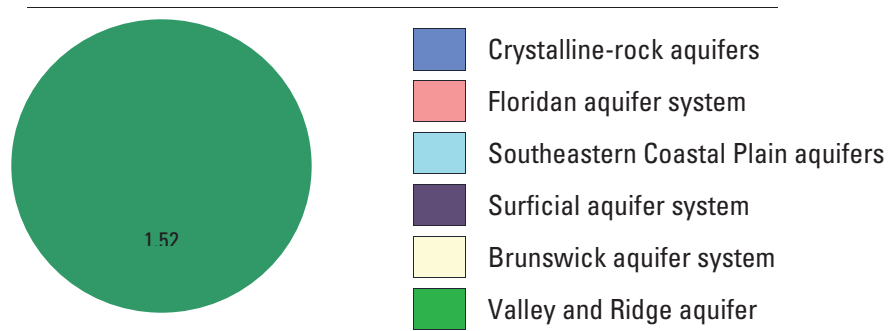




\section{CHEROKEE COUNTY}

Population

Population served by public supply-groundwater

4,834

Population served by public supply-surface water 213,689

Acres irrigated

1,410

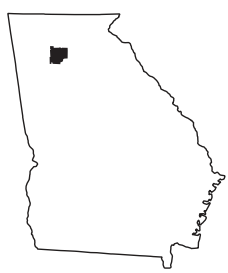

2015 WATER WITHDRAWALS AND ESTIMATED USE, IN MILLION GALLONS PER DAY

$[--$, not applicable; Mgal/d, million gallons per day $]$

\begin{tabular}{lccccc}
\hline & \multicolumn{3}{c}{ Withdrawals } & & Total \\
\cline { 2 - 4 } Category & $\begin{array}{c}\text { Ground- } \\
\text { water }\end{array}$ & $\begin{array}{c}\text { Surface } \\
\text { water }\end{array}$ & $\begin{array}{c}\text { Total } \\
\text { use }\end{array}$ & $\begin{array}{c}\text { Returns to } \\
\text { Surface }\end{array}$ \\
\hline Public supply & 0.40 & 18.89 & 19.29 & -- & -- \\
Domestic use & 0.48 & 0.00 & 0.48 & 15.41 & 0.00 \\
Commercial use & 0.00 & 0.00 & 0.00 & 1.32 & 0.00 \\
Industrial use & 0.01 & 0.00 & 0.01 & 0.59 & 0.00 \\
Public-supply loss & -- & -- & -- & 2.46 & -- \\
Inter-county delivery & -- & -- & -- & 0.78 & -- \\
Public wastewater treatment & -- & -- & -- & -- & 12.50 \\
Mining use & 0.14 & 0.00 & 0.14 & 0.14 & 0.00 \\
Irrigation (crop) & 0.09 & 0.15 & 0.24 & 0.24 & 0.00 \\
Irrigation (golf) & 0.58 & 0.95 & 1.53 & 1.53 & 0.00 \\
Livestock-aquaculture & 0.13 & 0.11 & 0.24 & 0.24 & 0.00 \\
Thermoelectric power & 0.00 & 0.00 & 0.00 & 0.00 & 0.00 \\
\hline TOTAL & 1.83 & 20.10 & 21.93 & 22.71 & 12.50 \\
\hline
\end{tabular}

Withdrawals by Major Industrial Groups

[NAICS, North American Industrial Classification System code]

NAICS Groundwater Surface water

311- Food

0.01

0.01

Withdrawals by Major Public Suppliers

\begin{tabular}{lcc} 
Name & Groundwater & Surface wate \\
\hline Cherokee County & 0.00 & 14.90 \\
City of Ball Ground & 0.21 & 0.00 \\
City of Canton & 0.00 & 3.99 \\
Lake Arrowhead Utility Co. & 0.17 & 0.00
\end{tabular}

Total use is total withdrawal plus public supply deliveries and losses. An estimate of $1.4 \mathrm{Mgal} / \mathrm{d}$ was delivered from neighboring counties in 2015. An estimate of $0.62 \mathrm{Mgal} / \mathrm{d}$ was delivered from Cherokee County to neighboring counties in 2015.

Withdrawals by water source

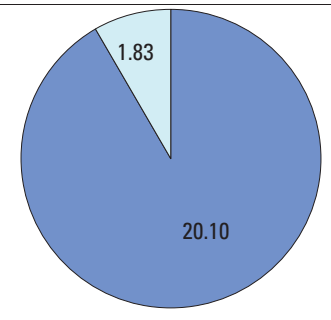

Withdrawals, in million gallons per day

Surface water

Groundwater
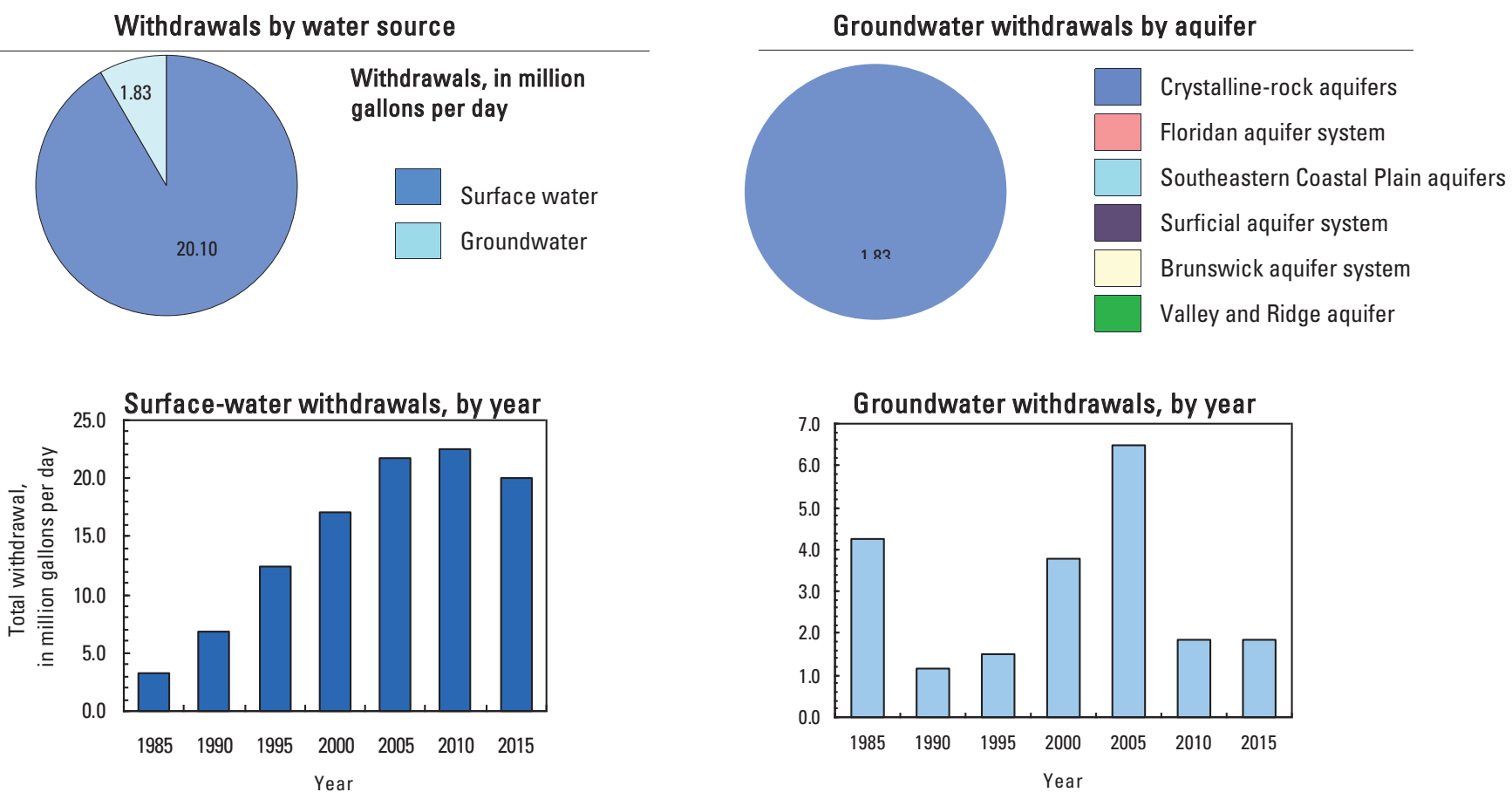


\section{CLARKE COUNTY}

Population

Population served by public supply-groundwater

Population served by public supply-surface water 120,789

Acres irrigated

2015 WATER WITHDRAWALS AND ESTIMATED USE, IN MILLION GALLONS PER DAY

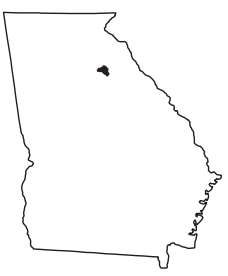

[--,not applicable; Mgal/d, million gallons per day

\begin{tabular}{lccccc}
\hline & \multicolumn{3}{c}{ Withdrawals } & & \\
\cline { 2 - 4 } Category & $\begin{array}{c}\text { Ground- } \\
\text { water }\end{array}$ & $\begin{array}{c}\text { Surface } \\
\text { water }\end{array}$ & $\begin{array}{c}\text { Total } \\
\text { Potal } \\
\text { use }\end{array}$ & $\begin{array}{c}\text { Returns to } \\
\text { Surface }\end{array}$ \\
\hline Public supply & 0.11 & 11.37 & 11.48 & -- & -- \\
Domestic use & 0.10 & 0.00 & 0.10 & 6.73 & 0.00 \\
Commercial use & 0.00 & 0.00 & 0.00 & 2.85 & 0.00 \\
Industrial use & 0.00 & 0.00 & 0.00 & 2.13 & 0.00 \\
Public-supply loss & -- & -- & -- & -0.13 & -- \\
Inter-county delivery & -- & -- & -- & 2.20 & -- \\
Public wastewater treatment & -- & -- & -- & -- & 14.99 \\
Mining use & 0.06 & 0.00 & 0.06 & 0.06 & 0.00 \\
Irrigation (crop) & 0.32 & 0.05 & 0.37 & 0.37 & 0.00 \\
Irrigation (golf) & 0.03 & 0.51 & 0.54 & 0.54 & 0.00 \\
Livestock-aquaculture & 0.05 & 0.01 & 0.06 & 0.06 & 0.00 \\
Thermoelectric power & 0.00 & 0.00 & 0.00 & 0.00 & 0.00 \\
\hline TOTAL & 0.67 & 11.94 & 12.61 & 14.81 & 14.99 \\
\hline
\end{tabular}

Withdrawals by Major Industrial Groups

[NAICS, North American Industrial Classification System code]

\begin{tabular}{ccc}
\hline NAICS Groundwater Surface water \\
\hline
\end{tabular}

None

Withdrawals by Major Public Suppliers

\begin{tabular}{lcc} 
Name & Groundwater & Surface water \\
\hline $\begin{array}{l}\text { Athens-Clarke County } \\
\begin{array}{l}\text { Public Utilities } \\
\text { Department }\end{array}\end{array}$ & 0.00 & 11.33 \\
& &
\end{tabular}

Total use is total withdrawal plus public supply deliveries and losses. An estimate of $2.2 \mathrm{Mgal} / \mathrm{d}$ was delivered from neighboring counties in 2015.

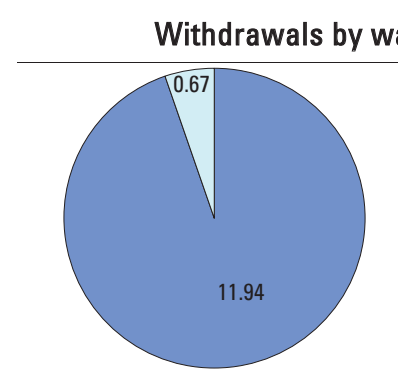

Withdrawals, in million

gallons per day
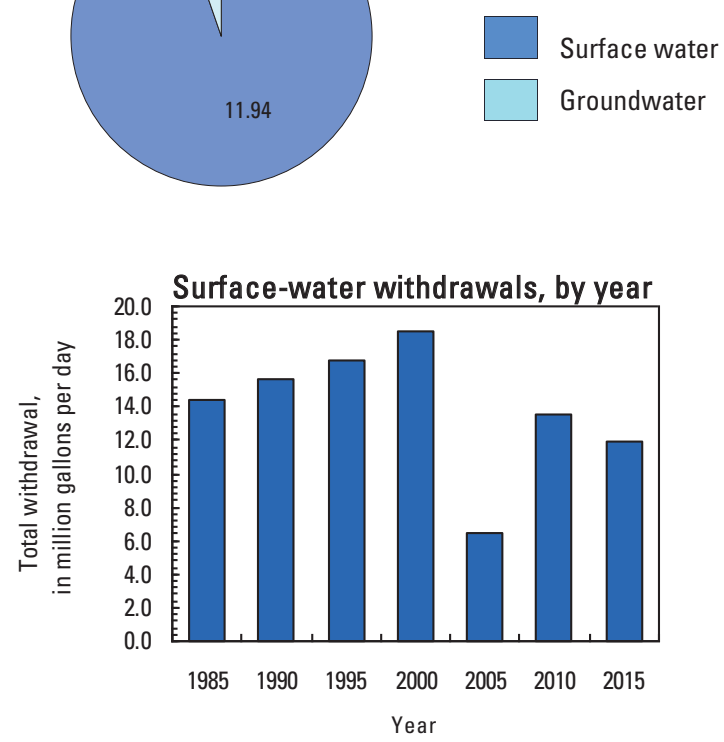

Groundwater withdrawals by aquifer

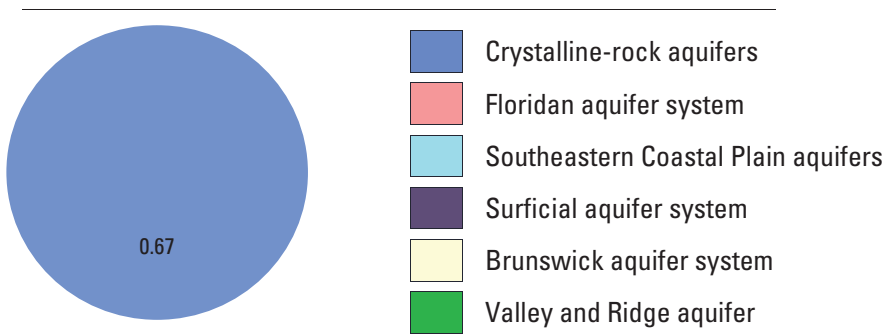




\section{CLAY COUNTY}

Population

Population served by public supply-groundwater

2,335

Population served by public supply-surface water

0

Acres irrigated

7,280

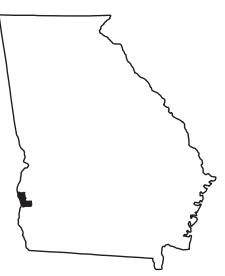

2015 WATER WITHDRAWALS AND ESTIMATED USE, IN MILLION GALLONS PER DAY

[--,not applicable; Mgal/d, million gallons per day]

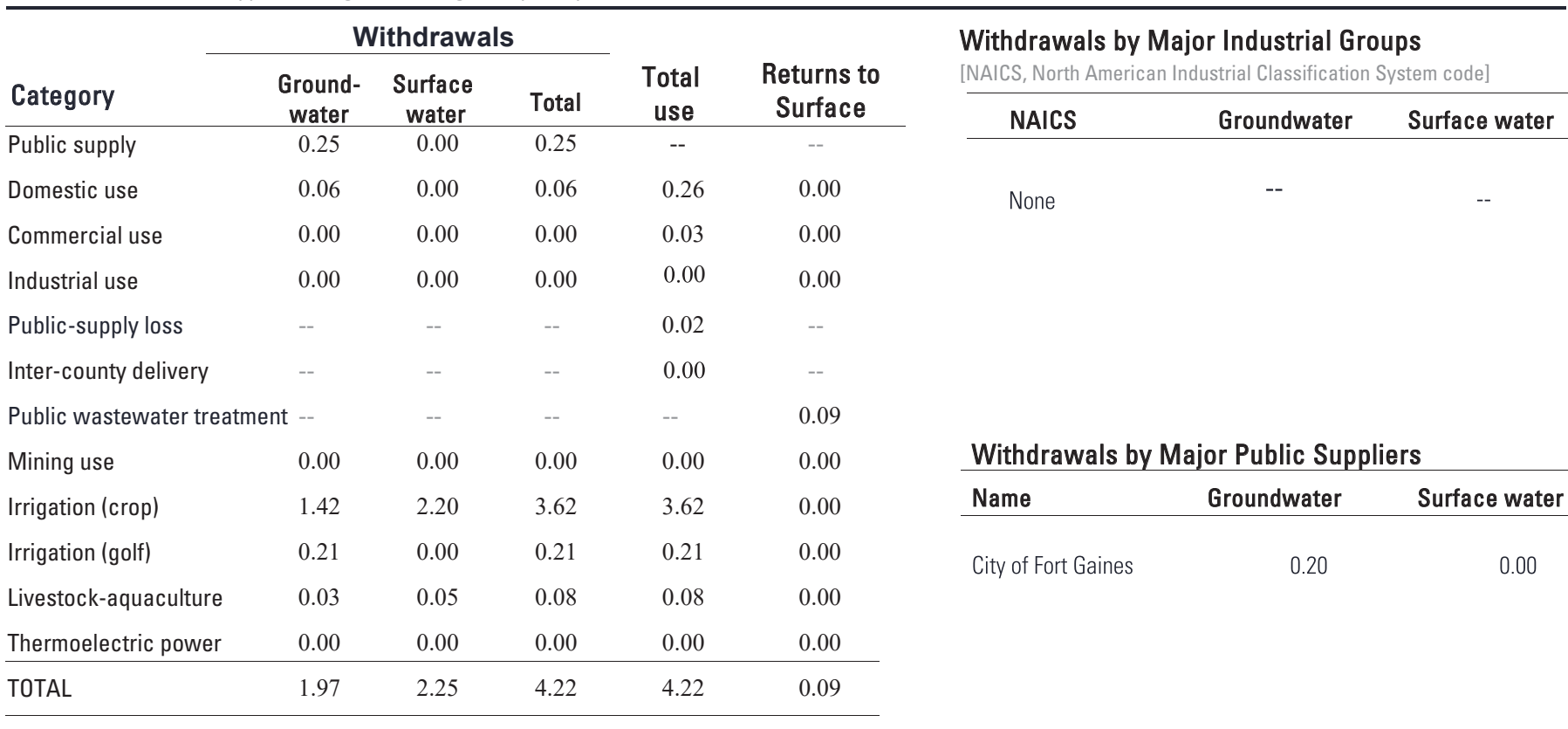

Total use is total withdrawal plus public supply deliveries and losses.

Withdrawals by water source

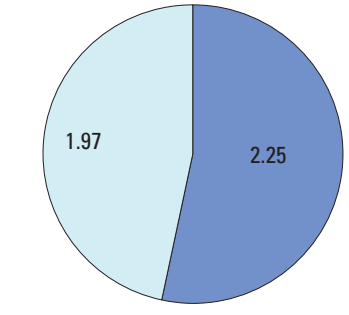

Withdrawals, in million gallons per day

Surface water

Groundwater

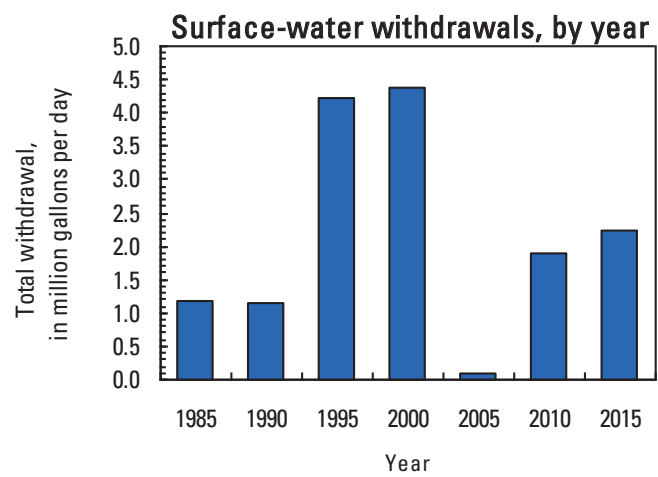

Groundwater withdrawals by aquifer
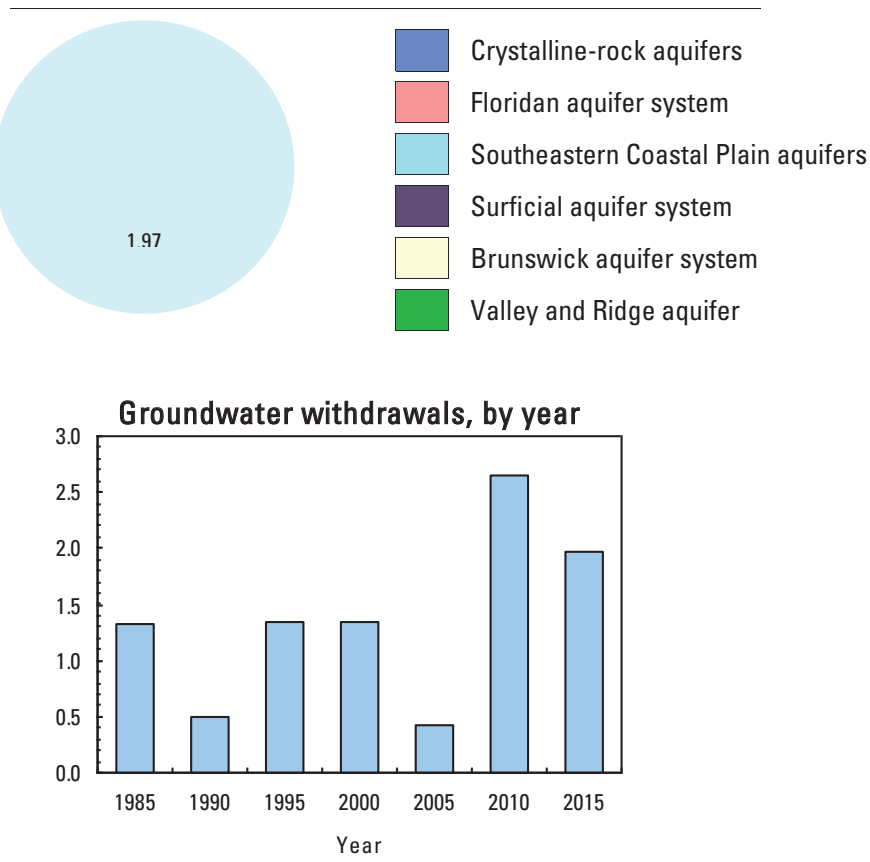


\section{CLAYTON COUNTY}

Population

Population served by public supply-groundwater

Population served by public supply-surface water

Acres irrigated
273,955

75

270,000

100

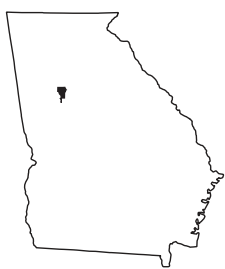

2015 WATER WITHDRAWALS AND ESTIMATED USE, IN MILLION GALLONS PER DAY

[--,not applicable; Mgal/d, million gallons per day]

\begin{tabular}{|c|c|c|c|c|c|c|c|c|}
\hline \multirow{3}{*}{ Category } & \multicolumn{3}{|c|}{ Withdrawals } & \multirow{3}{*}{$\begin{array}{c}\text { Total } \\
\text { use }\end{array}$} & \multirow{3}{*}{$\begin{array}{l}\text { Returns to } \\
\text { Surface }\end{array}$} & \multirow{2}{*}{\multicolumn{3}{|c|}{$\begin{array}{l}\text { Withdrawals by Major Industrial Groups } \\
\text { [NAICS, North American Industrial Classification System code] }\end{array}$}} \\
\hline & \multirow{2}{*}{$\begin{array}{l}\text { Ground- } \\
\text { water }\end{array}$} & \multirow{2}{*}{$\begin{array}{c}\text { Surface } \\
\text { water }\end{array}$} & \multirow[b]{2}{*}{ Total } & & & & & \\
\hline & & & & & & NAICS & Groundwater & Surface water \\
\hline Public supply & 0.04 & 10.72 & 10.76 & -- & -- & & & \\
\hline Domestic use & 0.29 & 0.00 & 0.29 & 20.88 & 0.00 & None & -- & -- \\
\hline Commercial use & 0.00 & 0.00 & 0.00 & 2.20 & 0.00 & & & \\
\hline Industrial use & 0.00 & 0.00 & 0.00 & 1.76 & 0.00 & & & \\
\hline Public-supply loss & -- & -- & -- & -13.79 & -- & & & \\
\hline Inter-county delivery & -- & -- & -- & 18.16 & -- & & & \\
\hline Public wastewater treatment & t -- & -- & -- & -- & 2.30 & & & \\
\hline Mining use & 0.20 & 0.02 & 0.22 & 0.22 & 0.00 & Withdrawals b & or Public Supp & \\
\hline Irrigation (crop) & 0.00 & 0.00 & 0.00 & 0.00 & 0.00 & Name & Groundwater & Surface water \\
\hline Irrigation (golf) & 0.00 & 0.10 & 0.10 & 0.10 & 0.00 & Clayton County & 0.03 & 15.14 \\
\hline Livestock-aquaculture & 0.00 & 0.02 & 0.02 & 0.02 & 0.00 & Water Authority & & \\
\hline Thermoelectric power & 0.00 & 0.00 & 0.00 & 0.00 & 0.00 & & & \\
\hline TOTAL & 0.53 & 10.86 & 11.39 & 29.55 & 2.30 & & & \\
\hline
\end{tabular}

Total use is total withdrawal plus public supply deliveries and losses. An estimate of $18.16 \mathrm{Mgal} / \mathrm{d}$ was delivered from neighboring counties in 2015.

\section{Withdrawals by water source}

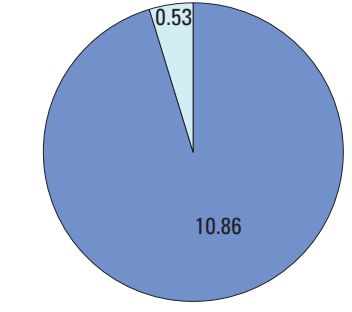

Withdrawals, in million

gallons per day

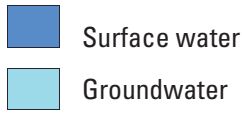

Surface-water withdrawals, by year

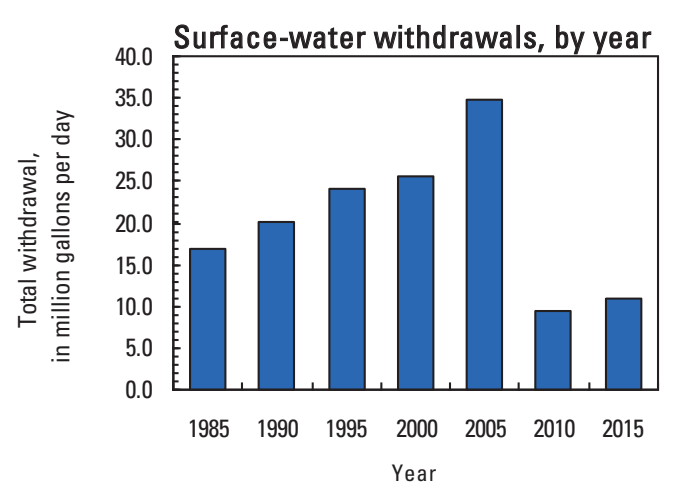

Groundwater withdrawals by aquifer

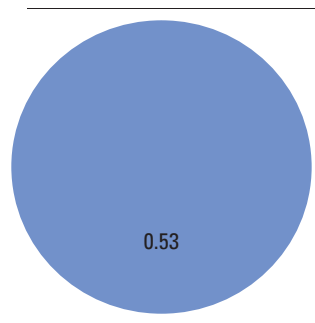

\begin{tabular}{l}
\hline Crystalline-rock aquifers \\
\hline$\quad$ Floridan aquifer system \\
\hline$\square$ Southeastern Coastal Plain aquifers \\
Surficial aquifer system \\
$\square \quad$ Brunswick aquifer system \\
\hline$\square \quad$ Valley and Ridge aquifer
\end{tabular}

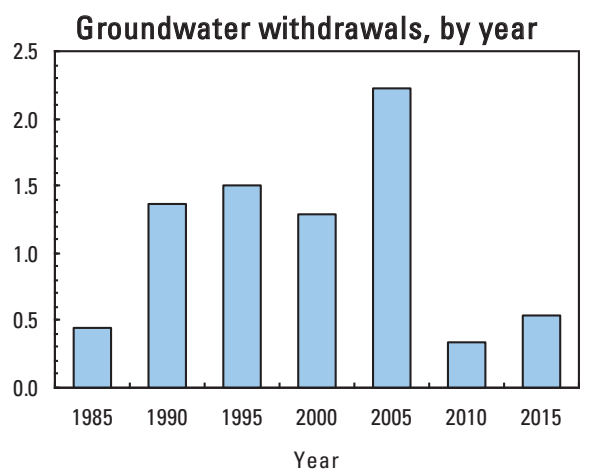




\section{CLINCH COUNTY}

Population

Population served by public supply-groundwater

3,945

Population served by public supply-surface water

Acres irrigated

2015 WATER WITHDRAWALS AND ESTIMATED USE, IN MILLION GALLONS PER DAY

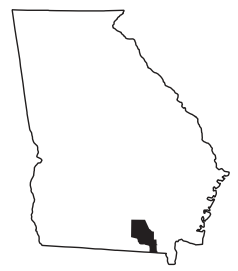

[--,not applicable; Mgal/d, million gallons per day]

\begin{tabular}{|c|c|c|c|c|c|c|c|c|}
\hline \multirow{3}{*}{ Category } & \multicolumn{3}{|c|}{ Withdrawals } & \multirow{3}{*}{$\begin{array}{c}\text { Total } \\
\text { use }\end{array}$} & \multirow{3}{*}{$\begin{array}{l}\text { Returns to } \\
\text { Surface }\end{array}$} & \multirow{2}{*}{\multicolumn{3}{|c|}{$\begin{array}{l}\text { Withdrawals by Major Industrial Groups } \\
\text { [NAICS, North American Industrial Classification System code] }\end{array}$}} \\
\hline & \multirow{2}{*}{$\begin{array}{c}\text { Ground- } \\
\text { water }\end{array}$} & \multirow{2}{*}{$\begin{array}{c}\text { Surface } \\
\text { water }\end{array}$} & \multirow[b]{2}{*}{ Total } & & & & & \\
\hline & & & & & & NAICS & Groundwater & Surface water \\
\hline Public supply & 0.45 & 0.00 & 0.45 & -- & -- & & & \\
\hline Domestic use & 0.22 & 0.00 & 0.22 & 0.48 & 0.00 & 332- Fabrication & 0.04 & -- \\
\hline Commercial use & 0.00 & 0.00 & 0.00 & 0.07 & 0.00 & & & \\
\hline Industrial use & 0.04 & 0.00 & 0.04 & 0.11 & 0.00 & & & \\
\hline Public-supply loss & -- & -- & -- & 0.05 & -- & & & \\
\hline Inter-county delivery & -- & -- & -- & 0.00 & -- & & & \\
\hline Public wastewater treatment & $\mathrm{t}--$ & -- & -- & -- & 0.41 & & & \\
\hline Mining use & 0.00 & 0.00 & 0.00 & 0.00 & 0.00 & Withdrawals by & jor Public Supp & \\
\hline Irrigation (crop) & 0.42 & 0.01 & 0.43 & 0.43 & 0.00 & Name & Groundwater & Surface water \\
\hline Irrigation (golf) & 0.00 & 0.00 & 0.00 & 0.00 & 0.00 & City of Homerville & 0.40 & 0.00 \\
\hline Livestock-aquaculture & 0.01 & 0.00 & 0.01 & 0.01 & 0.00 & & & \\
\hline Thermoelectric power & 0.00 & 0.00 & 0.00 & 0.00 & 0.00 & & & \\
\hline TOTAL & 1.14 & 0.01 & 1.15 & 1.15 & 0.41 & & & \\
\hline
\end{tabular}

Total use is total withdrawal plus public supply deliveries and losses.

Withdrawals by water source

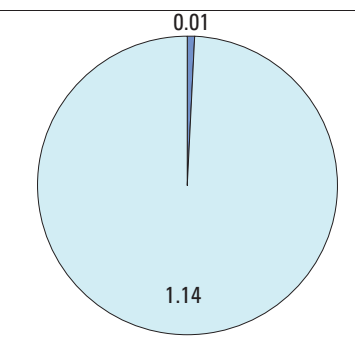

Withdrawals, in million gallons per day
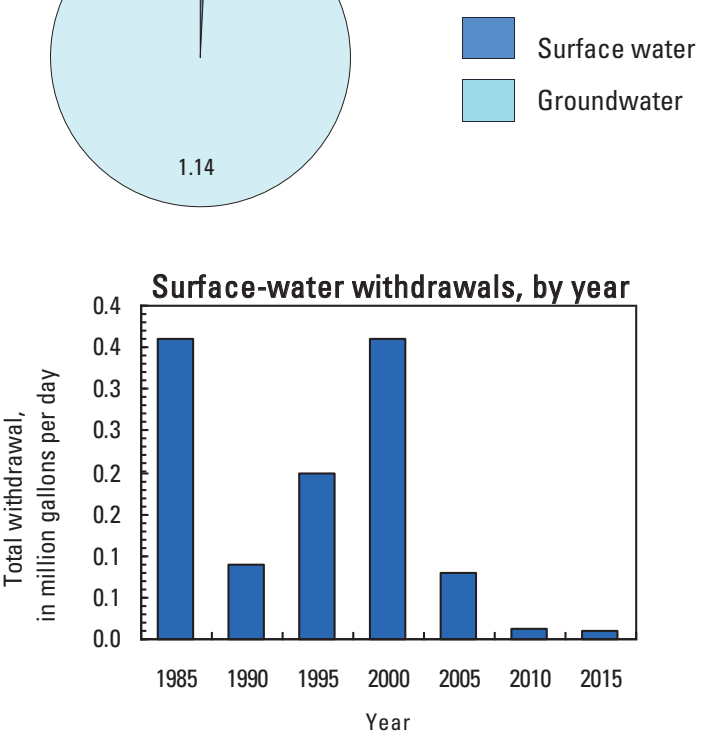

Groundwater withdrawals by aquifer

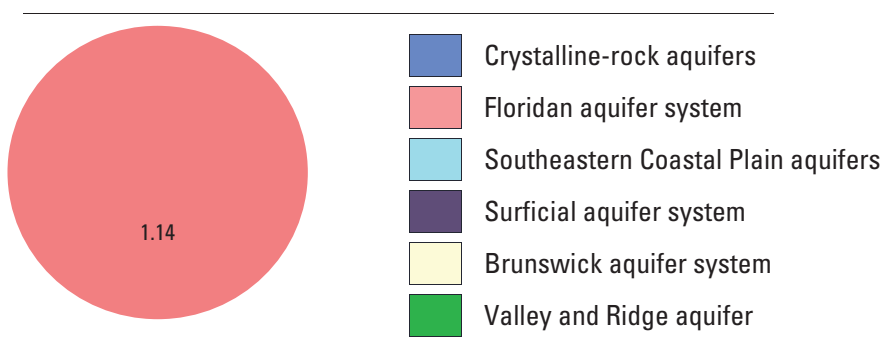

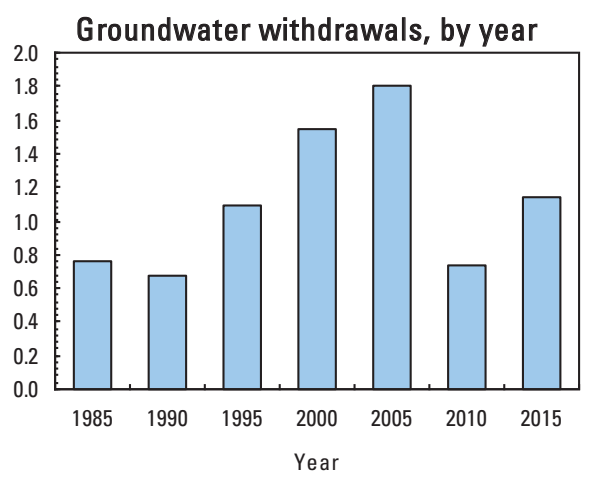




\section{COBB COUNTY}

Population

Population served by public supply-groundwater

Population served by public supply-surface water 650,254

Acres irrigated

2015 WATER WITHDRAWALS AND ESTIMATED USE, IN MILLION GALLONS PER DAY

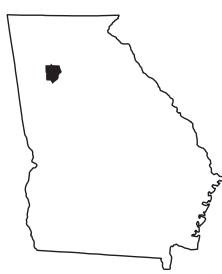

[--,not applicable; Mgal/d, million gallons per day]

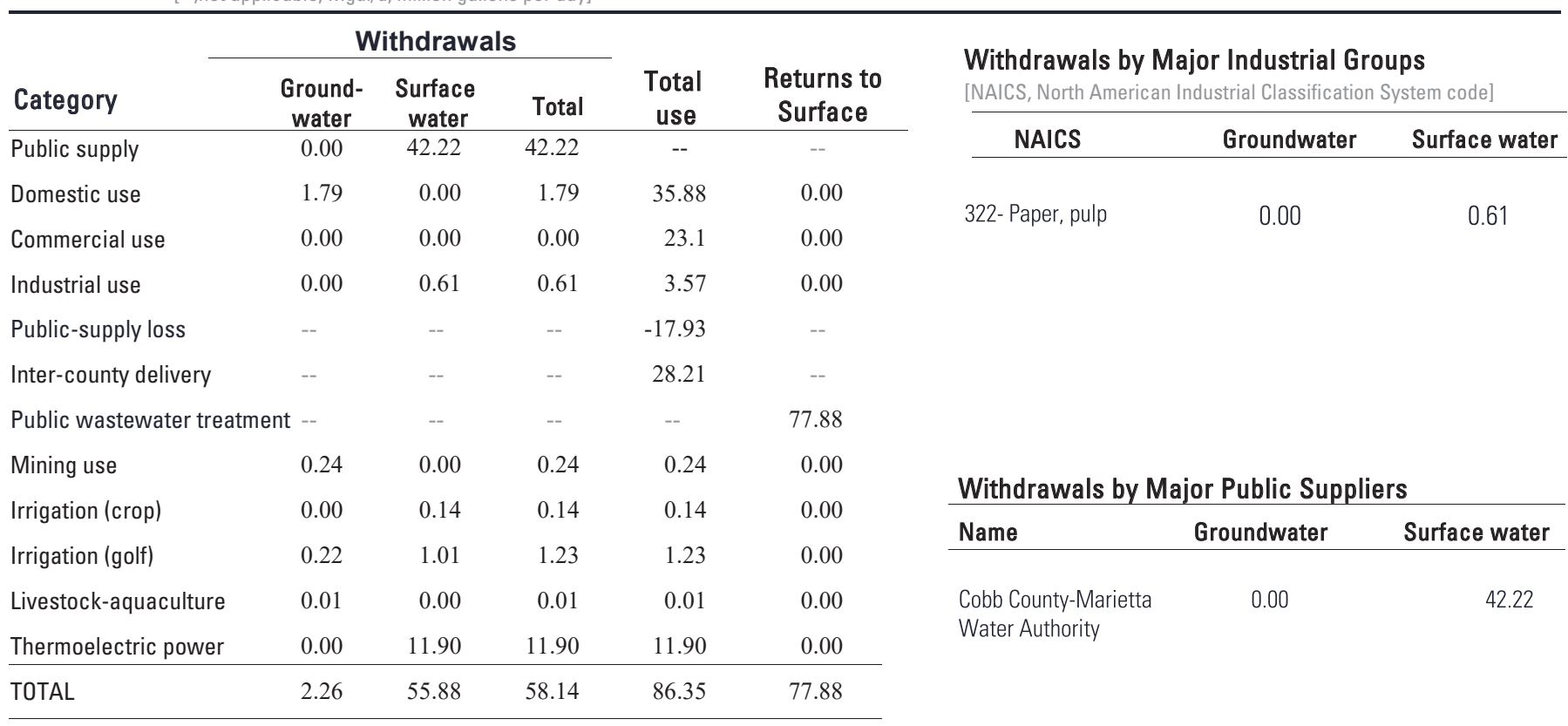

Total use is total withdrawal plus public supply deliveries and losses. An estimate of $40.1 \mathrm{Mgal} / \mathrm{d}$ was delivered from neighboring counties in 2015 . An estimate of $11.89 \mathrm{Mgal} / \mathrm{d}$ was delivered from Cobb County to neighboring counties in 2015.

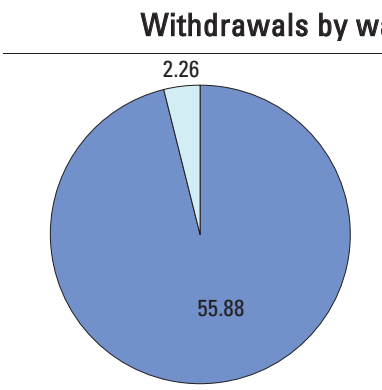

Withdrawals, in million gallons per day

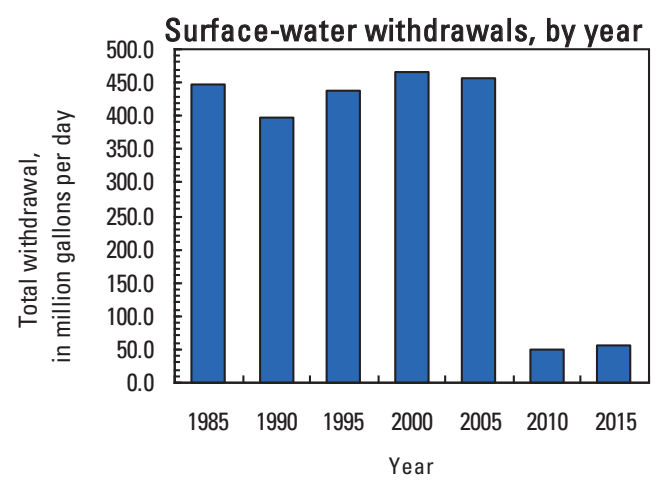

Surface water Groundwater
Groundwater withdrawals by aquifer

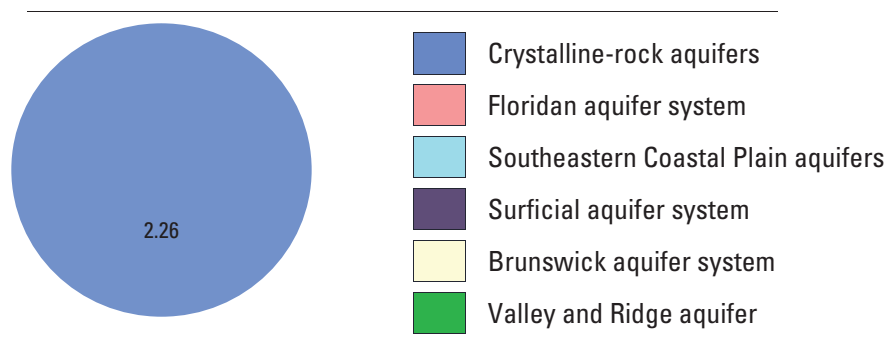

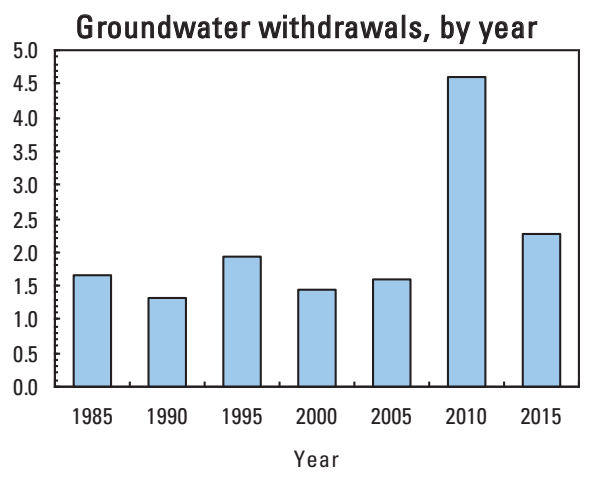




\section{COFFEE COUNTY}

Population

Population served by public supply-groundwater

Population served by public supply-surface water

0

Acres irrigated

2015 WATER WITHDRAWALS AND ESTIMATED USE, IN MILLION GALLONS PER DAY

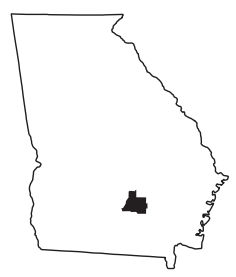

[--,not applicable; Mgal/d, million gallons per day]

\begin{tabular}{lccccc}
\hline & \multicolumn{3}{c}{ Withdrawals } & Total & Returns to \\
\cline { 2 - 4 } Category & $\begin{array}{c}\text { Ground } \\
\text { water }\end{array}$ & $\begin{array}{c}\text { Surface } \\
\text { water }\end{array}$ & Total & $\begin{array}{c}\text { Sure } \\
\text { Surface }\end{array}$ \\
\hline Public supply & 4.05 & 0.00 & 4.05 & -- & -- \\
Domestic use & 1.63 & 0.00 & 1.63 & 3.63 & 0.00 \\
Commercial use & 0.00 & 0.00 & 0.00 & 1.13 & 0.00 \\
Industrial use & 0.09 & 0.00 & 0.09 & 0.46 & 0.00 \\
Public-supply loss & -- & -- & -- & 0.55 & -- \\
Inter-county delivery & -- & -- & -- & 0.00 & -- \\
Public wastewater treatment & -- & -- & -- & -- & 4.32 \\
Mining use & 0.00 & 0.00 & 0.00 & 0.00 & 0.00 \\
Irrigation (crop) & 5.16 & 3.47 & 8.63 & 8.63 & 0.00 \\
Irrigation (golf) & 0.07 & 0.28 & 0.35 & 0.35 & 0.00 \\
Livestock-aquaculture & 0.37 & 0.25 & 0.62 & 0.62 & 0.00 \\
Thermoelectric power & 0.00 & 0.00 & 0.00 & 0.00 & 0.00 \\
\hline TOTAL & 11.37 & 4.00 & 15.37 & 15.37 & 4.32 \\
\hline
\end{tabular}

Withdrawals by Major Industrial Groups

[NAICS, North American Industrial Classification System code]

\begin{tabular}{lll}
\hline NAICS Groundwater & Surface water \\
\hline
\end{tabular}

326- Rubber, plastics

0.09

0.00

Withdrawals by Major Public Suppliers

\begin{tabular}{lcc}
\hline Name & Groundwater & Surface water \\
\hline City of Douglas & 3.34 & 0.00 \\
City of Nicholls & 0.34 & 0.00 \\
Coffee County- Oak Park & 0.12 & 0.00
\end{tabular}

Total use is total withdrawal plus public supply deliveries and losses.

Withdrawals by water source

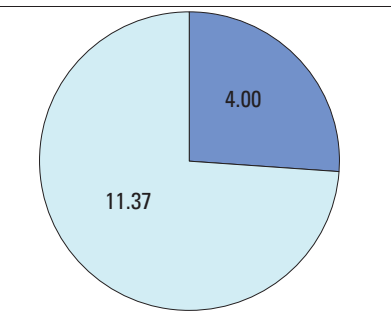

Withdrawals, in million gallons per day

Surface water

Groundwater

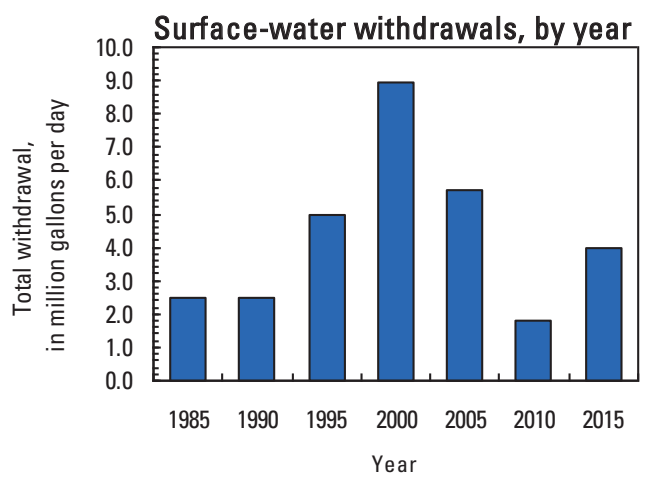

Groundwater withdrawals by aquifer

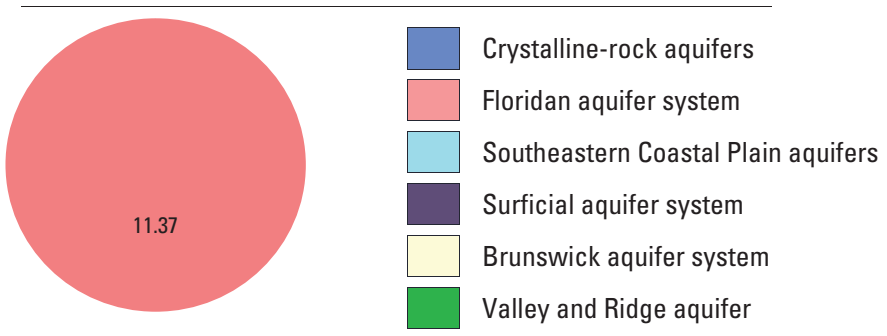

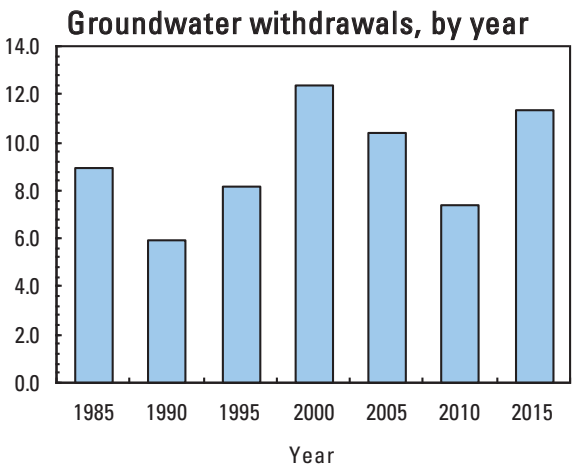




\section{COLQUITT COUNTY}

Population

45,844

Population served by public supply-groundwater

22,429

Population served by public supply-surface water

Acres irrigated

2015 WATER WITHDRAWALS AND ESTIMATED USE, IN MILLION GALLONS PER DAY

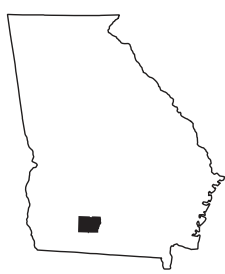

[--,not applicable; Mgal/d, million gallons per day]

\begin{tabular}{lcccccc}
\hline & \multicolumn{3}{c}{ Withdrawals } & & Total \\
\cline { 2 - 4 } Category & $\begin{array}{c}\text { Ground- } \\
\text { water }\end{array}$ & $\begin{array}{c}\text { Surface } \\
\text { water }\end{array}$ & $\begin{array}{c}\text { Total } \\
\text { Use }\end{array}$ & $\begin{array}{c}\text { Returns to } \\
\text { Surface }\end{array}$ \\
\hline Public supply & 3.11 & 0.00 & 3.11 & -- & -- \\
Domestic use & 1.76 & 0.00 & 1.76 & 3.27 & 0.00 \\
Commercial use & 0.00 & 0.00 & 0.00 & 0.64 & 0.00 \\
Industrial use & 1.14 & 0.00 & 1.14 & 1.21 & 0.00 \\
Public-supply loss & -- & -- & -- & 0.89 & -- \\
Inter-county delivery & -- & -- & -- & 0.00 & -- \\
Public wastewater treatment & -- & -- & -- & -- & 3.65 \\
Mining use & 0.00 & 0.00 & 0.00 & 0.00 & 0.00 \\
Irrigation (crop) & 7.64 & 12.58 & 20.22 & 20.22 & 0.00 \\
Irrigation (golf) & 0.00 & 0.00 & 0.00 & 0.00 & 0.00 \\
Livestock-aquaculture & 0.23 & 0.38 & 0.61 & 0.61 & 0.00 \\
Thermoelectric power & 0.00 & 0.00 & 0.00 & 0.00 & 0.00 \\
\hline TOTAL & 13.88 & 12.96 & 26.84 & 26.84 & 3.65 \\
\hline
\end{tabular}

Withdrawals by Major Industrial Groups

[NAICS, North American Industrial Classification System code]

NAICS Groundwater Surface water

311-Food

1.14

Withdrawals by Major Public Suppliers

Name Groundwater Surface water

$\begin{array}{lll}\text { City of Moultrie } & 2.49 & 0.00 \\ \text { City of Norman Park } & 0.17 & 0.00 \\ \text { Town of Riverside } & 0.02 & 0.00\end{array}$

Total use is total withdrawal plus public supply deliveries and losses.

Withdrawals by water source

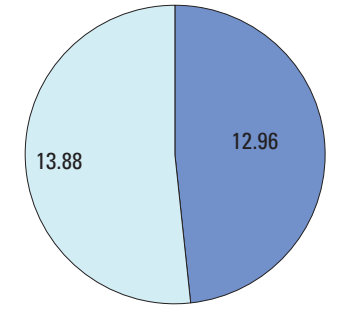

Withdrawals, in million gallons per day

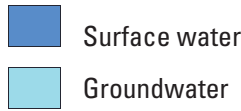

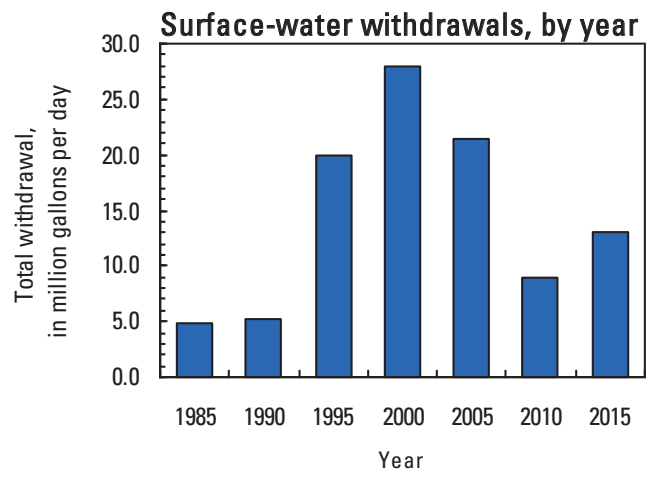

Groundwater withdrawals by aquifer
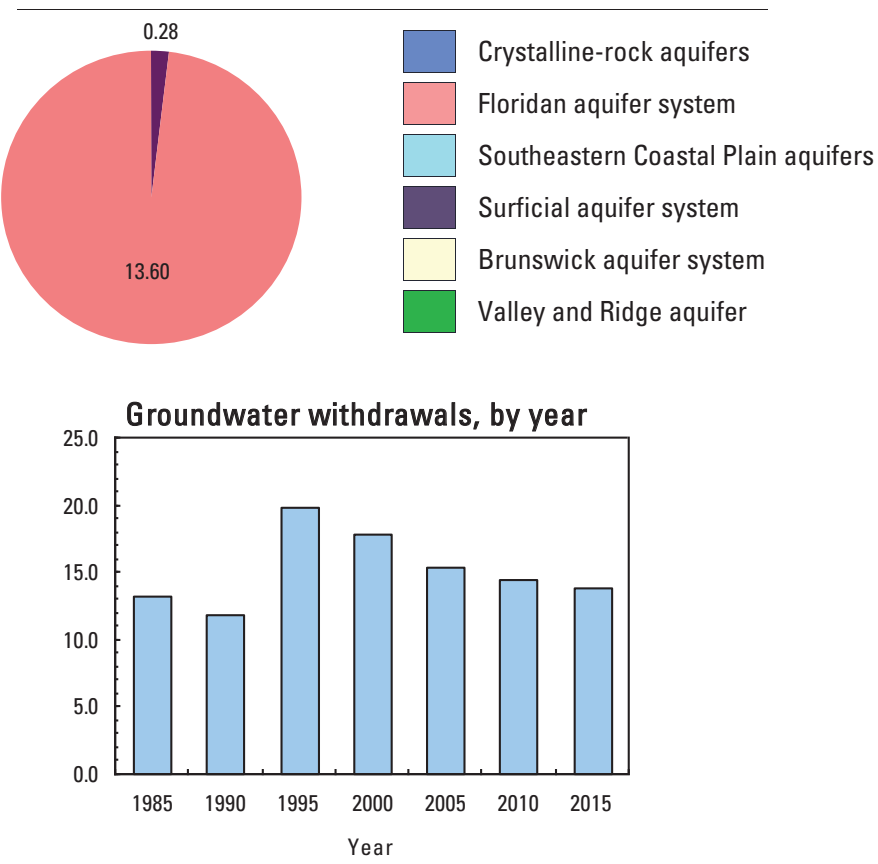


\section{COLUMBIA COUNTY}

Population

144,052

Population served by public supply-groundwater $\quad 12,062$

Population served by public supply-surface water 107,162

Acres irrigated

690

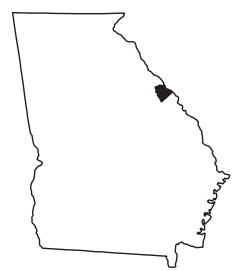

2015 WATER WITHDRAWALS AND ESTIMATED USE, IN MILLION GALLONS PER DAY

$[--$,not applicable; Mgal/d, million gallons per day]

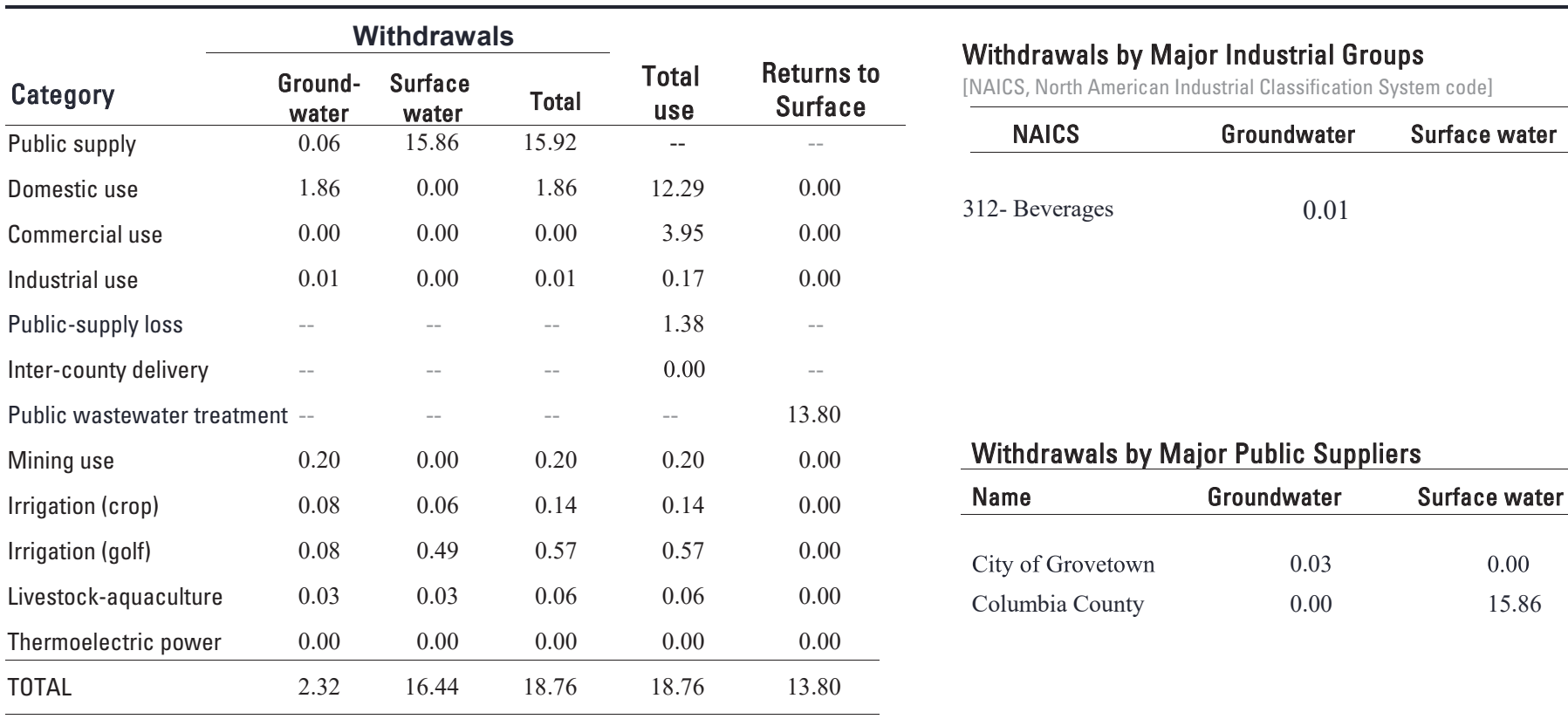

Total use is total withdrawal plus public supply deliveries and losses.

Withdrawals by water source

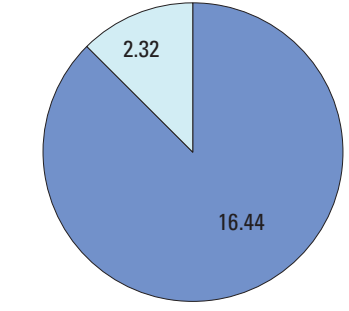

Withdrawals, in million

gallons per day

Surface water

Groundwater

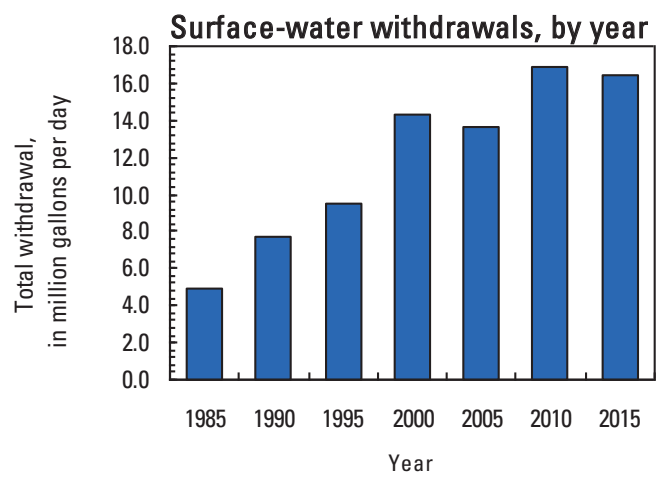

Groundwater withdrawals by aquifer

\begin{tabular}{lll}
\hline & Crystalline-rock aquifers \\
\hline & Floridan aquifer system \\
\hline & Southeastern Coastal Plain aquifers \\
\hline & Surficial aquifer system \\
$\square$ & Brunswick aquifer system \\
$\square$ & Valley and Ridge aquifer \\
\hline
\end{tabular}

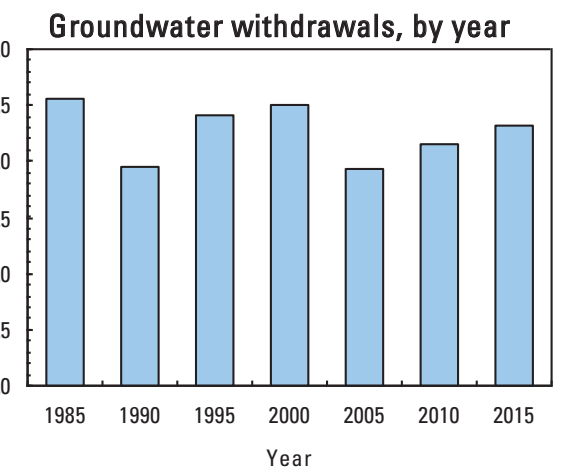




\section{COOK COUNTY}

Population

Population served by public supply-groundwater

Population served by public supply-surface water

Acres irrigated

2015 WATER WITHDRAWALS AND ESTIMATED

[--,not applicable; Mgal/d, million gallons per day]

\begin{tabular}{lccccc}
\hline & \multicolumn{3}{c}{ Withdrawals } & & Total \\
\cline { 2 - 4 } Category & $\begin{array}{c}\text { Ground- } \\
\text { water }\end{array}$ & $\begin{array}{c}\text { Surface } \\
\text { water }\end{array}$ & $\begin{array}{c}\text { Total } \\
\text { use }\end{array}$ & $\begin{array}{c}\text { Returns to } \\
\text { Surface }\end{array}$ \\
\hline Public supply & 1.40 & 0.00 & 1.40 & -- & -- \\
Domestic use & 0.62 & 0.00 & 0.62 & 1.5 & 0.00 \\
Commercial use & 0.00 & 0.00 & 0.00 & 0.28 & 0.00 \\
Industrial use & 0.00 & 0.00 & 0.00 & 0.02 & 0.00 \\
Public-supply loss & -- & -- & -- & 0.22 & -- \\
Inter-county delivery & -- & -- & -- & 0.00 & -- \\
Public wastewater treatment & -- & -- & -- & -- & 1.48 \\
Mining use & 0.11 & 0.00 & 0.11 & 0.11 & 0.00 \\
Irrigation (crop) & 6.53 & 1.24 & 7.77 & 7.77 & 0.00 \\
Irrigation (golf) & 0.00 & 0.13 & 0.13 & 0.13 & 0.00 \\
Livestock-aquaculture & 0.11 & 0.02 & 0.13 & 0.13 & 0.00 \\
Thermoelectric power & 0.00 & 0.00 & 0.00 & 0.00 & 0.00 \\
\hline TOTAL & 8.77 & 1.39 & 10.16 & 10.16 & 1.48 \\
\hline
\end{tabular}

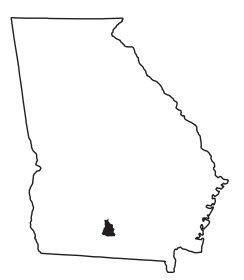

Withdrawals by Major Industrial Groups

[NAICS, North American Industrial Classification System code]

\begin{tabular}{lll}
\hline NAICS & Groundwater & Surface water \\
\hline
\end{tabular}

None

Withdrawals by Major Public Suppliers

Name Groundwater Surface water

$\begin{array}{lll}\text { City of Adel } & 0.08 & 0.00 \\ \text { City of Lenox } & 0.15 & 0.00 \\ \text { Town of Sparks } & 0.39 & 0.00\end{array}$

Total use is total withdrawal plus public supply deliveries and losses.

Withdrawals by water source

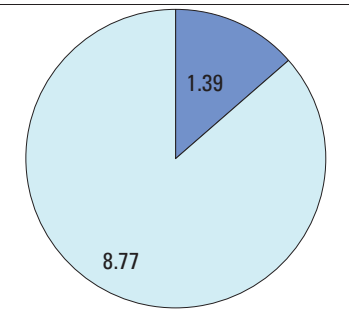

Withdrawals, in million

gallons per day

Surface water

Groundwater
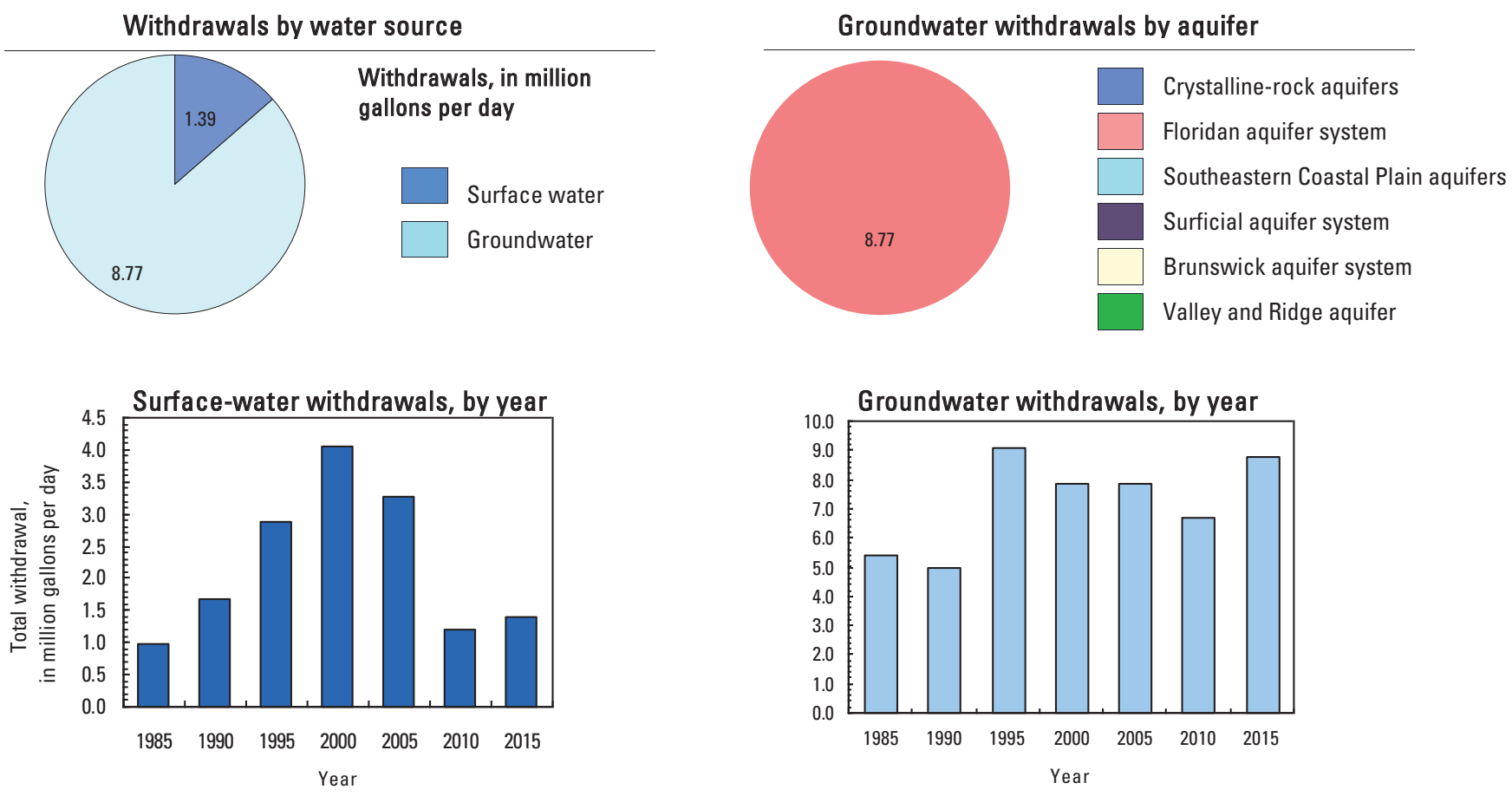
COWETA COUNTY

Population

138,427

Population served by public supply-groundwater $\quad 7,267$

Population served by public supply-surface water $\quad 95,966$

Acres irrigated

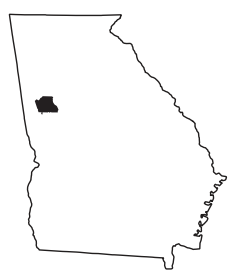

2015 WATER WITHDRAWALS AND ESTIMATED USE, IN MILLION GALLONS PER DAY

[--,not applicable; Mgal/d, million gallons per day]

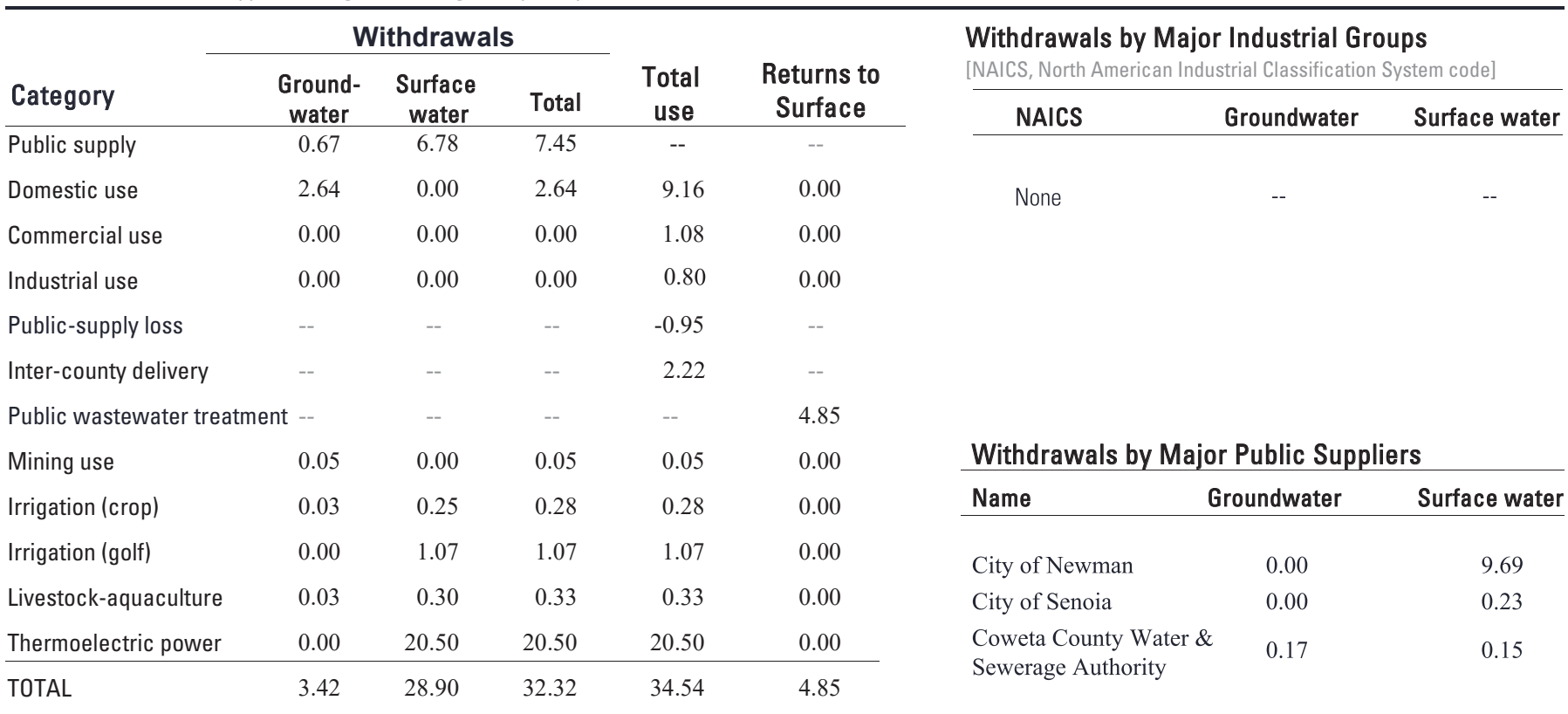

Total use is total withdrawal plus public supply deliveries and losses. An estimate of $2.466 \mathrm{Mgal} / \mathrm{d}$ was delivered from neighboring counties in 2015. An estimate of $0.25 \mathrm{Mgal} / \mathrm{d}$ was delivered from Coweta County to neighboring counties in 2015.

Withdrawals by water source

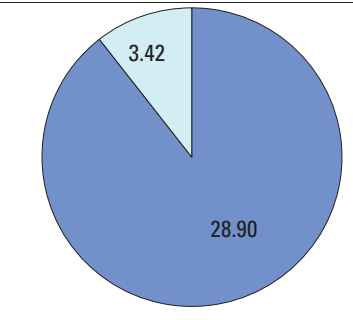

Withdrawals, in million

gallons per day

Surface water

Groundwater

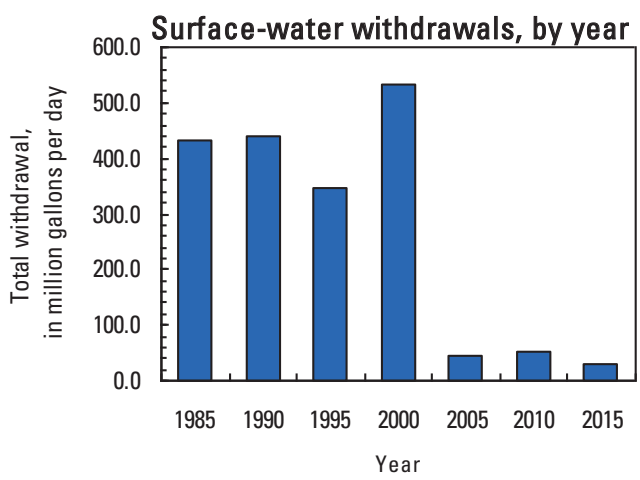

Groundwater withdrawals by aquifer

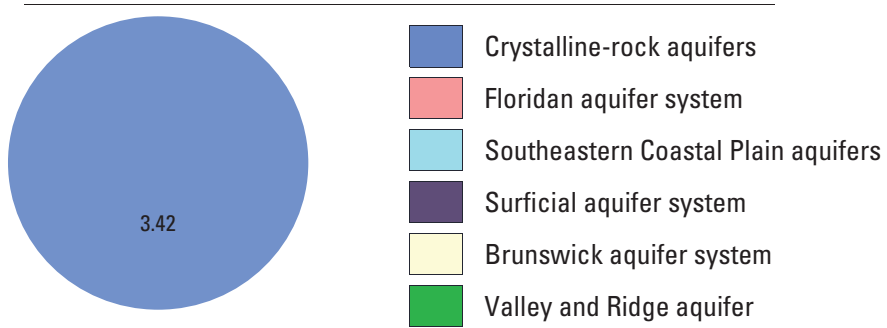

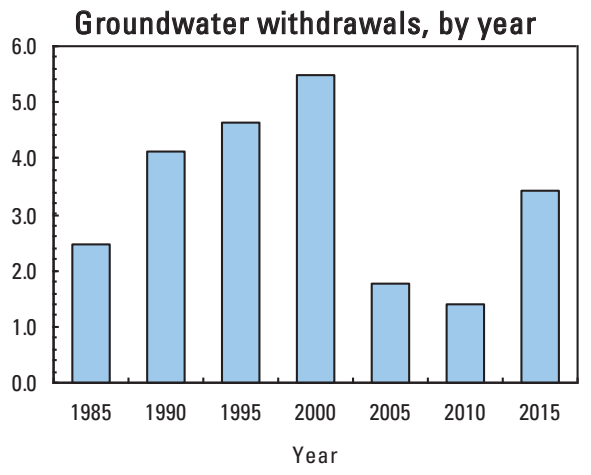




\section{CRAWFORD COUNTY}

Population

Population served by public supply-groundwater

3,565

Population served by public supply-surface water

0

Acres irrigated

2,910

2015 WATER WITHDRAWALS AND ESTIMATED USE, IN MILLION GALLONS PER DAY

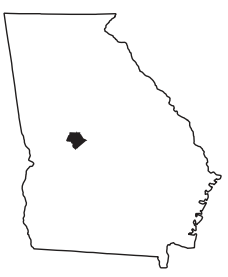

[--,not applicable; Mgal/d, million gallons per day]

\begin{tabular}{|c|c|c|c|c|c|c|c|c|}
\hline \multirow{3}{*}{ Category } & \multicolumn{3}{|c|}{ Withdrawals } & \multirow{3}{*}{$\begin{array}{c}\text { Total } \\
\text { use }\end{array}$} & \multirow{3}{*}{$\begin{array}{l}\text { Returns to } \\
\text { Surface }\end{array}$} & \multirow{2}{*}{\multicolumn{3}{|c|}{$\begin{array}{l}\text { Withdrawals by Major Industrial Groups } \\
\text { [NAICS, North American Industrial Classification System code] }\end{array}$}} \\
\hline & \multirow{2}{*}{$\begin{array}{c}\text { Ground- } \\
\text { water }\end{array}$} & \multirow{2}{*}{$\begin{array}{c}\text { Surface } \\
\text { water }\end{array}$} & \multirow{2}{*}{ Total } & & & & & \\
\hline & & & & & & \multirow[t]{3}{*}{ NAICS } & \multirow[t]{2}{*}{ Groundwater } & \multirow[t]{2}{*}{ Surface water } \\
\hline Public supply & 0.40 & 0.00 & 0.40 & -- & -- & & & \\
\hline Domestic use & 0.66 & 0.00 & 0.66 & 0.96 & 0.00 & & -- & -- \\
\hline Commercial use & 0.00 & 0.00 & 0.00 & 0.05 & 0.00 & & & \\
\hline Industrial use & 0.00 & 0.00 & 0.00 & 0.00 & 0.00 & & & \\
\hline Public-supply loss & -- & -- & -- & 0.05 & -- & & & \\
\hline Inter-county delivery & -- & -- & - & 0.00 & -- & & & \\
\hline Public wastewater treatment & t -- & -- & -- & -- & 0.16 & & & \\
\hline Mining use & 1.08 & 0.03 & 1.11 & 1.11 & 0.00 & \multicolumn{3}{|c|}{ Withdrawals by Major Public Suppliers } \\
\hline Irrigation (crop) & 1.26 & 0.00 & 1.26 & 1.26 & 0.00 & Name & Groundwater & Surface water \\
\hline Irrigation (golf) & 0.00 & 0.00 & 0.00 & 0.00 & 0.00 & \multirow{3}{*}{$\begin{array}{l}\text { Crawford County Water } \\
\text { System } \\
\text { City of Roberta }\end{array}$} & \multirow[t]{2}{*}{0.14} & \multirow[t]{2}{*}{0.00} \\
\hline Livestock-aquaculture & 0.09 & 0.11 & 0.20 & 0.20 & 0.00 & & & \\
\hline Thermoelectric power & 0.00 & 0.00 & 0.00 & 0.00 & 0.00 & & 0.23 & 0.00 \\
\hline TOTAL & 3.49 & 0.14 & 3.63 & 3.63 & 0.16 & & & \\
\hline
\end{tabular}

Total use is total withdrawal plus public supply deliveries and losses.

Withdrawals by water source

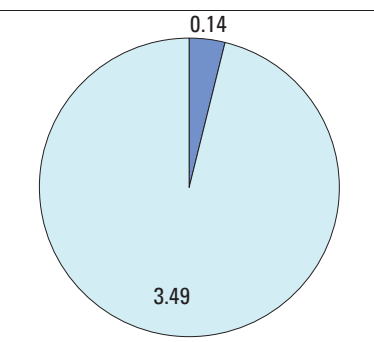

Withdrawals, in million

gallons per day

Surface water

Groundwater

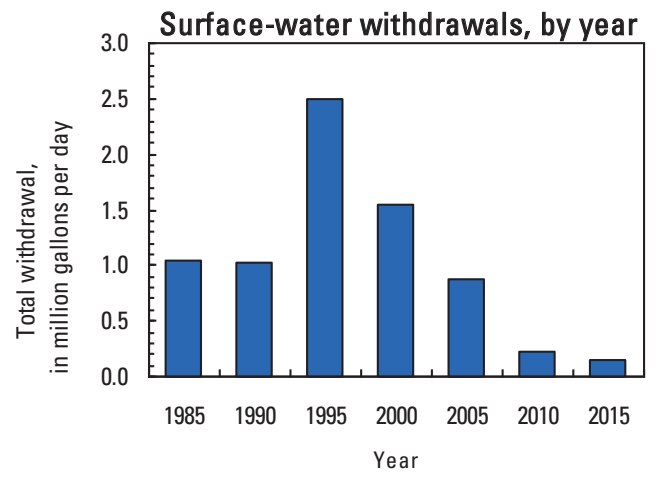

Groundwater withdrawals by aquifer
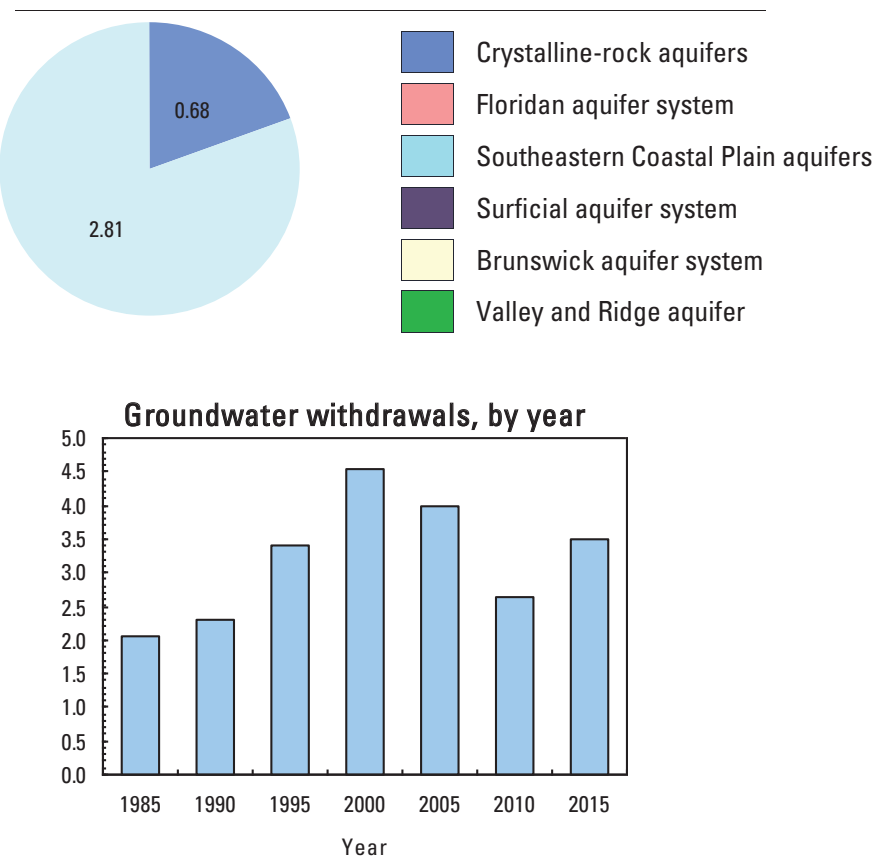


\section{CRISP COUNTY}

Population

Population served by public supply-groundwater

11,891

Population served by public supply-surface water

0

Acres irrigated

28,660

2015 WATER WITHDRAWALS AND ESTIMATED USE, IN MILLION GALLONS PER DA

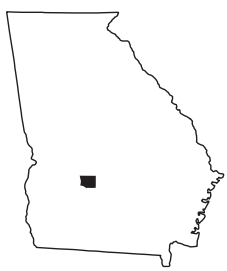

[--,not applicable; Mgal/d, million gallons per day]

\begin{tabular}{|c|c|c|c|c|c|c|c|c|}
\hline \multirow{2}{*}{ Category } & \multicolumn{3}{|c|}{ Withdrawals } & \multirow{2}{*}{$\begin{array}{c}\text { Total } \\
\text { use }\end{array}$} & \multirow{2}{*}{$\begin{array}{l}\text { Returns to } \\
\text { Surface }\end{array}$} & \multirow{2}{*}{\multicolumn{3}{|c|}{$\begin{array}{l}\text { Withdrawals by Major Industrial Groups } \\
\text { [NAICS, North American Industrial Classification System code] }\end{array}$}} \\
\hline & Ground- & Surface & Total & & & & & \\
\hline Public supply & $\begin{array}{l}\text { water } \\
2.01\end{array}$ & $\begin{array}{c}\text { water } \\
0.00\end{array}$ & 2.01 & $\begin{array}{c}\text { USe } \\
--\end{array}$ & surface & NAICS & Groundwater & Surface water \\
\hline Domestic use & 0.82 & 0.00 & 0.82 & 1.90 & 0.00 & \multirow{3}{*}{\multicolumn{2}{|c|}{ 321-Wood }} & \multirow{3}{*}{0.00} \\
\hline Commercial use & 0.00 & 0.00 & 0.00 & 0.56 & 0.00 & & & \\
\hline Industrial use & 0.15 & 0.00 & 0.15 & 0.16 & 0.00 & & & \\
\hline Public-supply loss & -- & -- & -- & 0.36 & -- & & & \\
\hline Inter-county delivery & -- & -- & -- & 0.00 & -- & & & \\
\hline Public wastewater treatment & $\mathrm{t}--$ & -- & -- & -- & 3.49 & & & \\
\hline Mining use & 0.00 & 0.00 & 0.00 & 0.00 & 0.00 & \multicolumn{3}{|c|}{ Withdrawals by Major Public Suppliers } \\
\hline Irrigation (crop) & 10.97 & 3.41 & 14.38 & 14.38 & 0.00 & Name Gro & oundwater & Surface water \\
\hline Irrigation (golf) & 0.21 & 0.00 & 0.21 & 0.21 & 0.00 & \multirow{4}{*}{ Crisp County Waterworks } & \multirow[t]{4}{*}{0.22} & \multirow[t]{4}{*}{0.00} \\
\hline Livestock-aquaculture & 0.11 & 0.03 & 0.14 & 0.14 & 0.00 & & & \\
\hline Thermoelectric power & 0.00 & 0.00 & 0.00 & 0.00 & 0.00 & & & \\
\hline TOTAL & 14.27 & 3.44 & 17.71 & 17.71 & 3.49 & & & \\
\hline
\end{tabular}

Total use is total withdrawal plus public supply deliveries and losses.

Withdrawals by water source

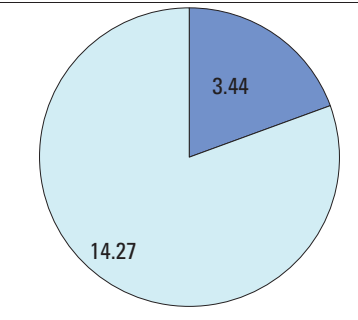

Withdrawals, in million

gallons per day

Surface water

Groundwater

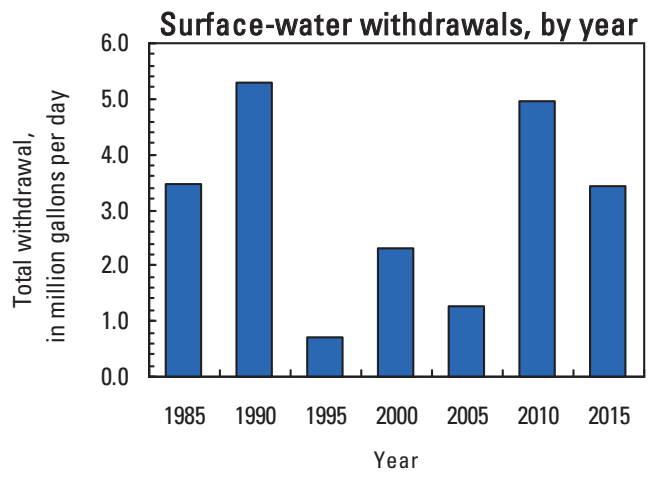

Groundwater withdrawals by aquifer

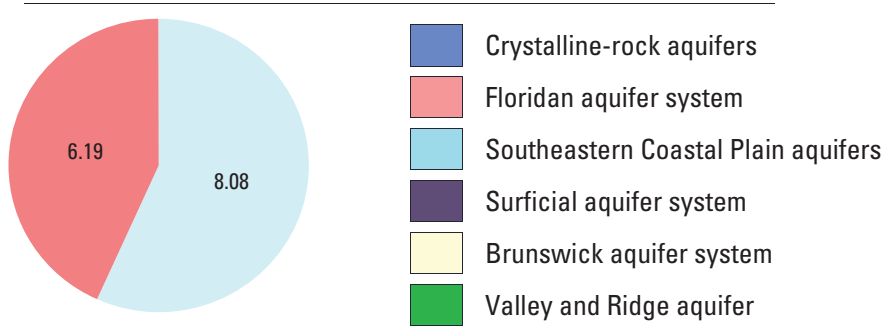




\section{DADE COUNTY}

Population

Population served by public supply-groundwater

Population served by public supply-surface water 15,147

Acres irrigated

2015 WATER WITHDRAWALS AND ESTIMATED USE, IN MILLION GALLONS PER DAY

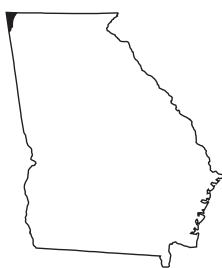

[--,not applicable; Mgal/d, million gallons per day]

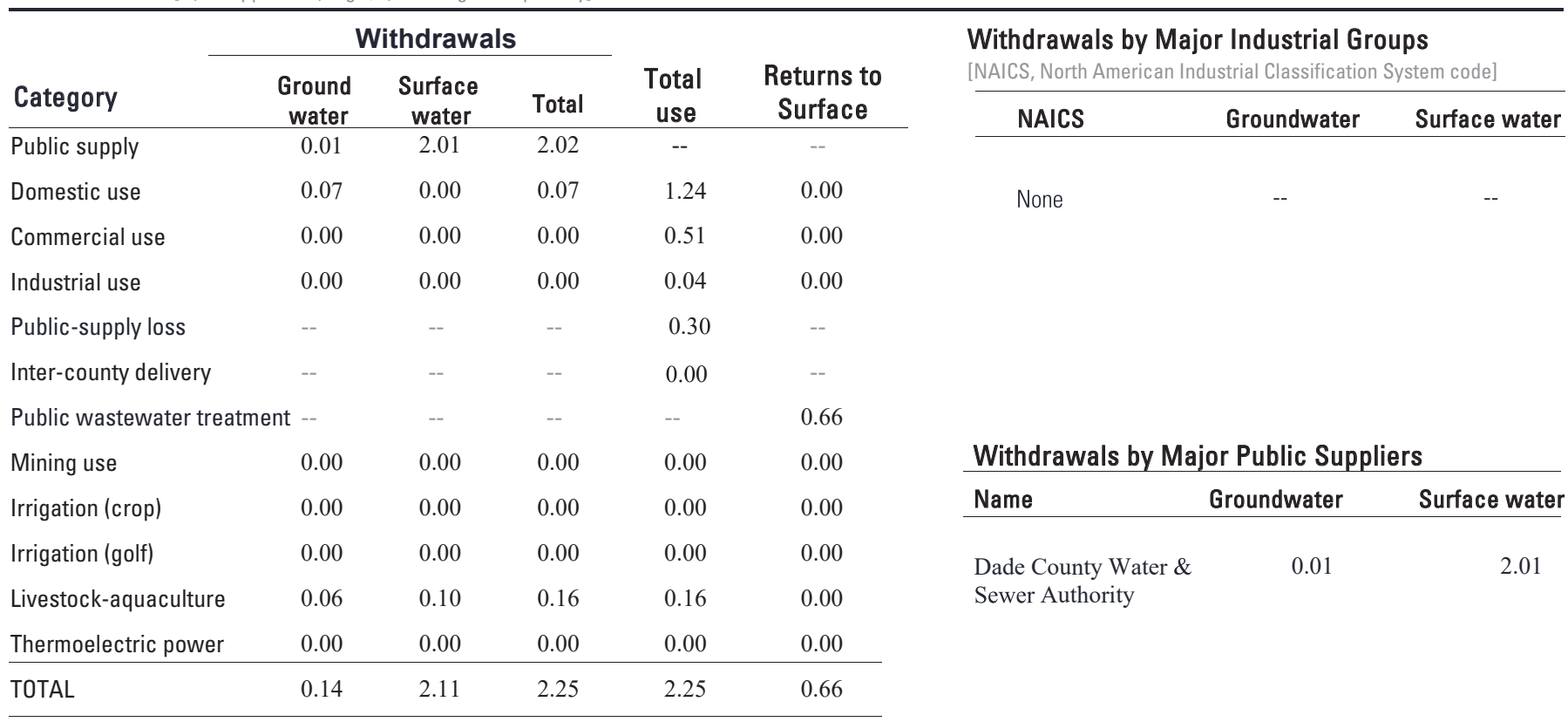

Total use is total withdrawal plus public supply deliveries and losses.

Withdrawals by water source

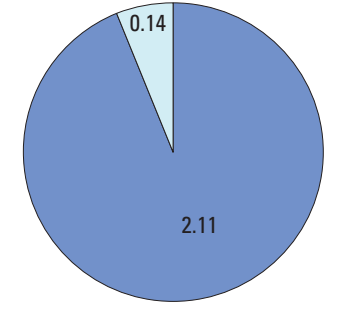

Withdrawals, in million gallons per day

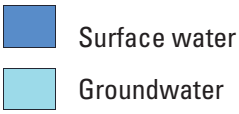

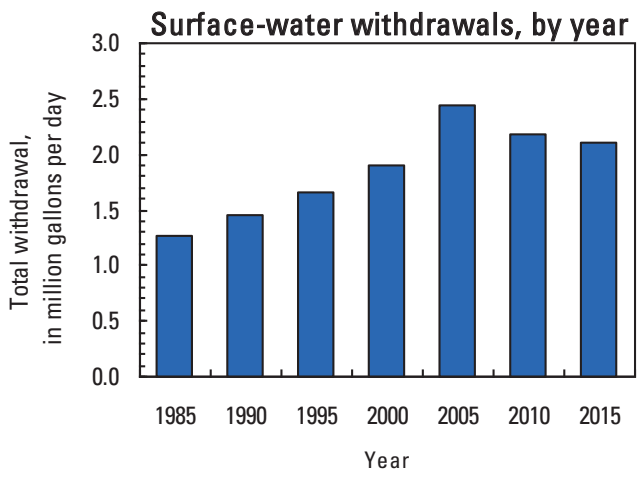

Groundwater withdrawals by aquifer

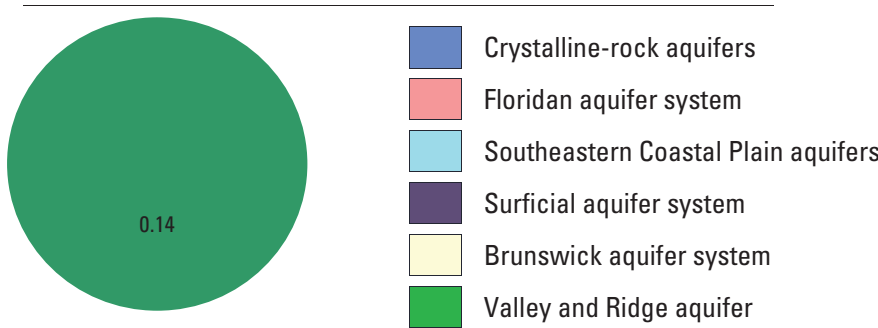




\section{DAWSON COUNTY}

Population

Population served by public supply-groundwater

Population served by public supply-surface water $\quad 14,571$

Acres irrigated

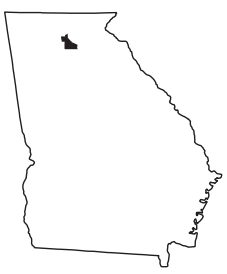

2015 WATER WITHDRAWALS AND ESTIMATED USE, IN MILLION GALLONS PER DAY

[--,not applicable; Mgal/d, million gallons per day]

\begin{tabular}{|c|c|c|c|c|c|}
\hline \multirow[b]{2}{*}{ Category } & \multicolumn{3}{|c|}{ Withdrawals } & \multirow[b]{2}{*}{$\begin{array}{c}\text { Total } \\
\text { use }\end{array}$} & \multirow[b]{2}{*}{$\begin{array}{c}\text { Returns to } \\
\text { Surface }\end{array}$} \\
\hline & $\begin{array}{l}\text { Ground- } \\
\text { water }\end{array}$ & $\begin{array}{c}\text { Surface } \\
\text { water }\end{array}$ & Total & & \\
\hline Public supply & 0.21 & 1.30 & 1.51 & -- & -- \\
\hline Domestic use & 0.50 & 0.00 & 0.50 & 1.52 & 0.00 \\
\hline Commercial use & 0.00 & 0.00 & 0.00 & 0.26 & 0.00 \\
\hline Industrial use & 0.00 & 0.00 & 0.00 & 0.02 & 0.00 \\
\hline Public-supply loss & -- & -- & -- & 0.21 & -- \\
\hline Inter-county delivery & -- & -- & -- & 0.11 & -- \\
\hline Public wastewater treatmen & nt -- & -- & -- & -- & 0.02 \\
\hline Mining use & 0.00 & 0.00 & 0.00 & 0.00 & 0.00 \\
\hline Irrigation (crop) & 0.00 & 0.00 & 0.00 & 0.00 & 0.00 \\
\hline Irrigation (golf) & 0.00 & 0.13 & 0.13 & 0.13 & 0.00 \\
\hline Livestock-aquaculture & 0.08 & 0.14 & 0.22 & 0.22 & 0.00 \\
\hline Thermoelectric power & 0.00 & 0.00 & 0.00 & 0.00 & 0.00 \\
\hline TOTAL & 0.79 & 1.57 & 2.36 & 2.47 & 0.02 \\
\hline
\end{tabular}

Withdrawals by Major Industrial Groups

[NAICS, North American Industrial Classification System code]

\begin{tabular}{lll}
\hline NAICS & Groundwater & Surface water \\
\hline None & -- & - \\
\\
Withdrawals by Major Public Suppliers \\
\hline Name & Groundwater Surface water
\end{tabular}

City of Dawsonville

0.18

0.00

Etowah Water \&

0.00

1.29

Total use is total withdrawal plus public supply deliveries and losses. An estimate of $0.11 \mathrm{Mgal} / \mathrm{d}$ was delivered from neighboring counties in 2015.

\section{Withdrawals by water source}

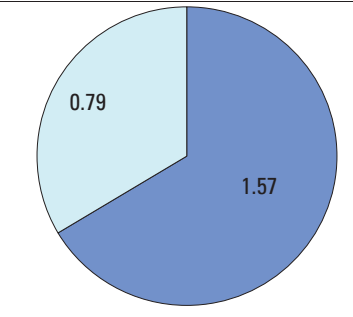

Withdrawals, in million gallons per day

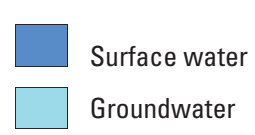

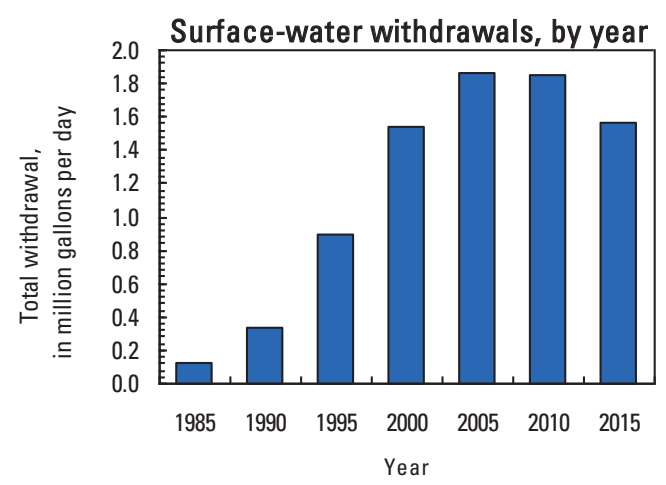

Groundwater withdrawals by aquifer

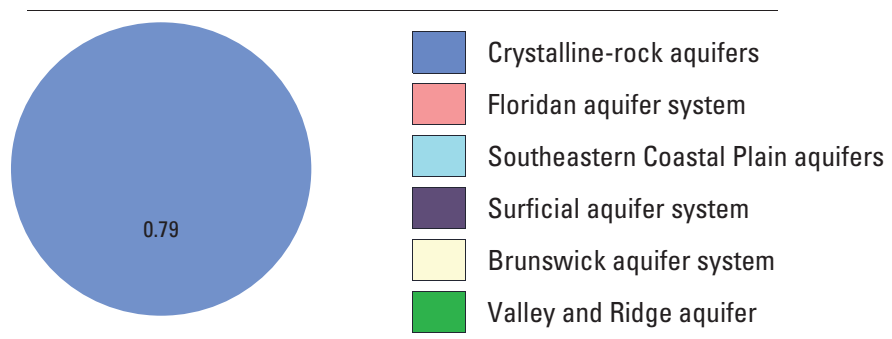




\section{DECATUR COUNTY}

Population

Population served by public supply-groundwater

Population served by public supply-surface water

Acres irrigated

2015 WATER WITHDRAWALS AND ESTIMATED USE, IN MILLION GALLONS PER DAY

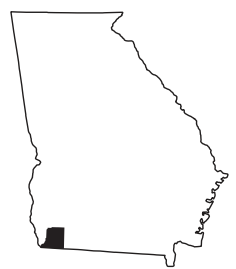

[--,not applicable; Mgal/d, million gallons per day]

\begin{tabular}{|c|c|c|c|c|c|c|c|c|}
\hline \multirow{2}{*}{ Category } & \multicolumn{3}{|c|}{ Withdrawals } & \multirow{2}{*}{$\begin{array}{c}\text { Total } \\
\text { use }\end{array}$} & \multirow{2}{*}{$\begin{array}{c}\text { Returns to } \\
\text { Surface }\end{array}$} & \multirow{2}{*}{\multicolumn{3}{|c|}{$\begin{array}{l}\text { Withdrawals by Major Industrial Groups } \\
\text { [NAICS, North American Industrial Classification System code] }\end{array}$}} \\
\hline & Ground - & Surface & Total & & & & & \\
\hline Public supply & $\begin{array}{c}\text { water } \\
2.43\end{array}$ & $\begin{array}{c}\text { water } \\
0.00\end{array}$ & 2.43 & $\begin{array}{c}\text { use } \\
--\end{array}$ & surtace & NAICS & Groundwater & Surface water \\
\hline Domestic use & 0.89 & 0.00 & 0.89 & 2.79 & 0.00 & \multirow{5}{*}{$\begin{array}{l}\text { 212- Mining } \\
\text { 313- Textile mills } \\
\text { 327- Stone, clay }\end{array}$} & \multirow{2}{*}{0.00} & \multirow{2}{*}{0.84} \\
\hline Commercial use & 0.00 & 0.00 & 0.00 & 0.30 & 0.00 & & & \\
\hline \multirow{2}{*}{ Industrial use } & \multirow{2}{*}{0.35} & \multirow{2}{*}{0.84} & \multirow{2}{*}{1.19} & \multirow{2}{*}{1.19} & \multirow{2}{*}{0.00} & & 0.15 & \multirow{3}{*}{0.00} \\
\hline & & & & & & & 0.20 & \\
\hline Public-supply loss & -- & -- & -- & 0.23 & -- & & & \\
\hline Inter-county delivery & -- & -- & -- & 0.00 & -- & & & \\
\hline Public wastewater treatmer & nt -- & -- & -- & -- & 3.35 & & & \\
\hline Mining use & 0.00 & 0.71 & 0.71 & 0.71 & 0.00 & \multicolumn{3}{|c|}{ Withdrawals by Major Public Suppliers } \\
\hline Irrigation (crop) & 55.18 & 0.95 & 56.13 & 56.13 & 0.00 & Name & Groundwater & Surface water \\
\hline Irrigation (golf) & 0.04 & 0.00 & 0.04 & 0.04 & 0.00 & City of Bainbridge & 2.31 & 0.00 \\
\hline Livestock-aquaculture & 0.42 & 0.01 & 0.43 & 0.43 & 0.00 & & & \\
\hline Thermoelectric power & 0.00 & 0.00 & 0.00 & 0.00 & 0.00 & & & \\
\hline TOTAL & 59.31 & 2.51 & 61.82 & 61.82 & 3.35 & & & \\
\hline
\end{tabular}

Total use is total withdrawal plus public supply deliveries and losses.

Withdrawals by water source

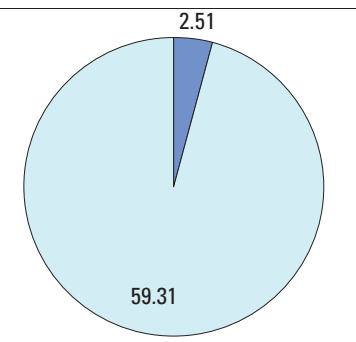

Withdrawals, in million gallons per day

Surface water

Groundwater

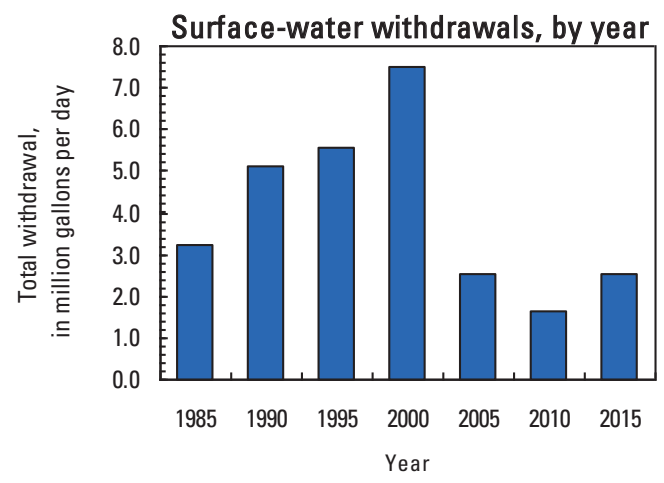

Groundwater withdrawals by aquifer

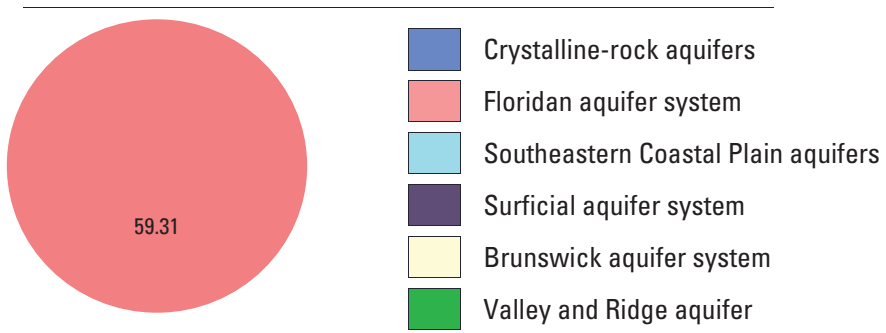

Groundwater withdrawals, by year

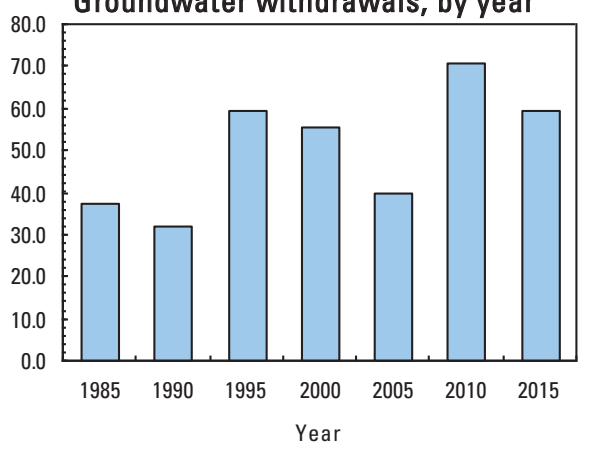




\section{DEKALB COUNTY}

Population

Population served by public supply-groundwater

Population served by public supply-surface water 734,871

Acres irrigated

2015 WATER WITHDRAWALS AND ESTIMATED USE, IN MILLION GALLONS PER DAY

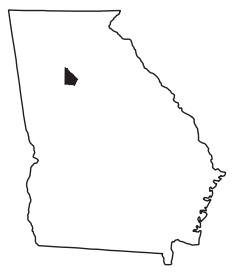

[--,not applicable; Mgal/d, million gallons per day]

\begin{tabular}{|c|c|c|c|c|c|}
\hline \multirow[b]{2}{*}{ Category } & \multicolumn{3}{|c|}{ Withdrawals } & \multirow[b]{2}{*}{$\begin{array}{c}\text { Total } \\
\text { use }\end{array}$} & \multirow[b]{2}{*}{$\begin{array}{c}\text { Returns to } \\
\text { Surface }\end{array}$} \\
\hline & $\begin{array}{c}\text { Ground- } \\
\text { water }\end{array}$ & $\begin{array}{c}\text { Surface } \\
\text { water }\end{array}$ & Total & & \\
\hline Public supply & 0.00 & 0.00 & 0.00 & -- & -- \\
\hline Domestic use & 0.00 & 0.00 & 0.00 & 43.11 & 0.00 \\
\hline Commercial use & 0.00 & 0.00 & 0.00 & 22.72 & 0.00 \\
\hline Industrial use & 0.00 & 0.00 & 0.00 & 5.83 & 0.00 \\
\hline Public-supply loss & -- & -- & -- & -71.66 & -- \\
\hline Inter-county delivery & -- & -- & -- & 83.22 & -- \\
\hline \multicolumn{2}{|c|}{ Public wastewater treatment -- } & -- & -- & -- & 40.16 \\
\hline Mining use & 0.20 & 0.00 & 0.20 & 0.20 & 0.00 \\
\hline Irrigation (crop) & 0.00 & 0.00 & 0.00 & 0.00 & 0.00 \\
\hline Irrigation (golf) & 0.45 & 1.03 & 1.48 & 1.48 & 0.00 \\
\hline Livestock-aquaculture & 0.00 & 0.00 & 0.00 & 0.00 & 0.00 \\
\hline Thermoelectric power & 0.00 & 0.00 & 0.00 & 0.00 & 0.00 \\
\hline TOTAL & 0.65 & 1.03 & 1.68 & 84.90 & 40.16 \\
\hline
\end{tabular}

Withdrawals by Major Industrial Groups

[NAICS, North American Industrial Classification System code]

NAICS Groundwater Surface water

Withdrawals by Major Public Suppliers

Name Groundwater Surface water

$\begin{array}{lll}\text { None }^{1} & 0.00 & 0.00\end{array}$

1DeKalb County withdraws water in Fulton County

Total use is total withdrawal plus public supply deliveries and losses. An estimate of $83.35 \mathrm{Mgal} / \mathrm{d}$ was delivered from neighboring counties in 2015. An estimate of $0.13 \mathrm{Mgal} / \mathrm{d}$ was delivered from DeKalb County to neighboring counties in 2015.

\section{Withdrawals by water source}

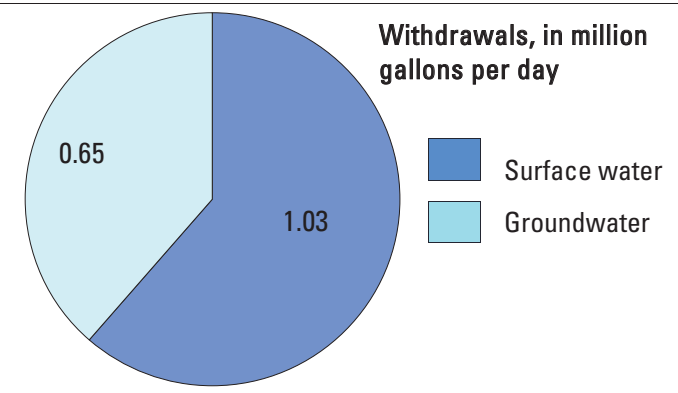

Surface-water withdrawals, by year

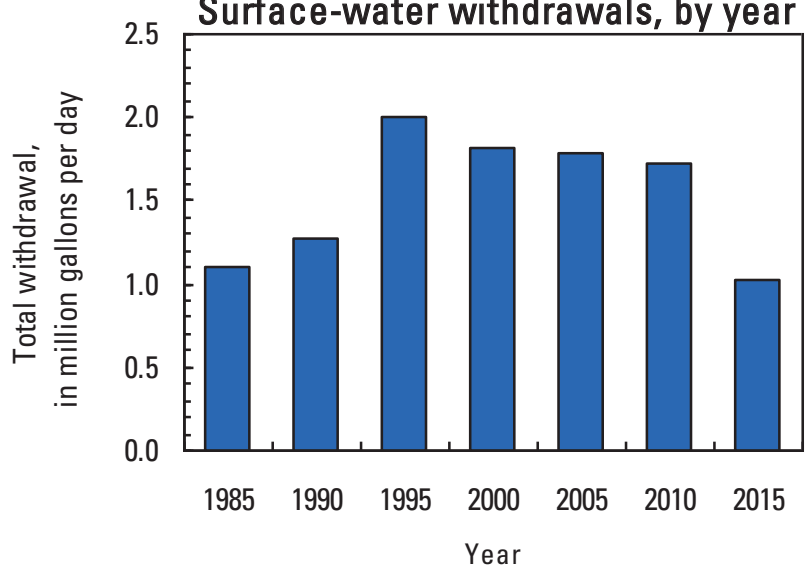

Groundwater withdrawals by aquifer
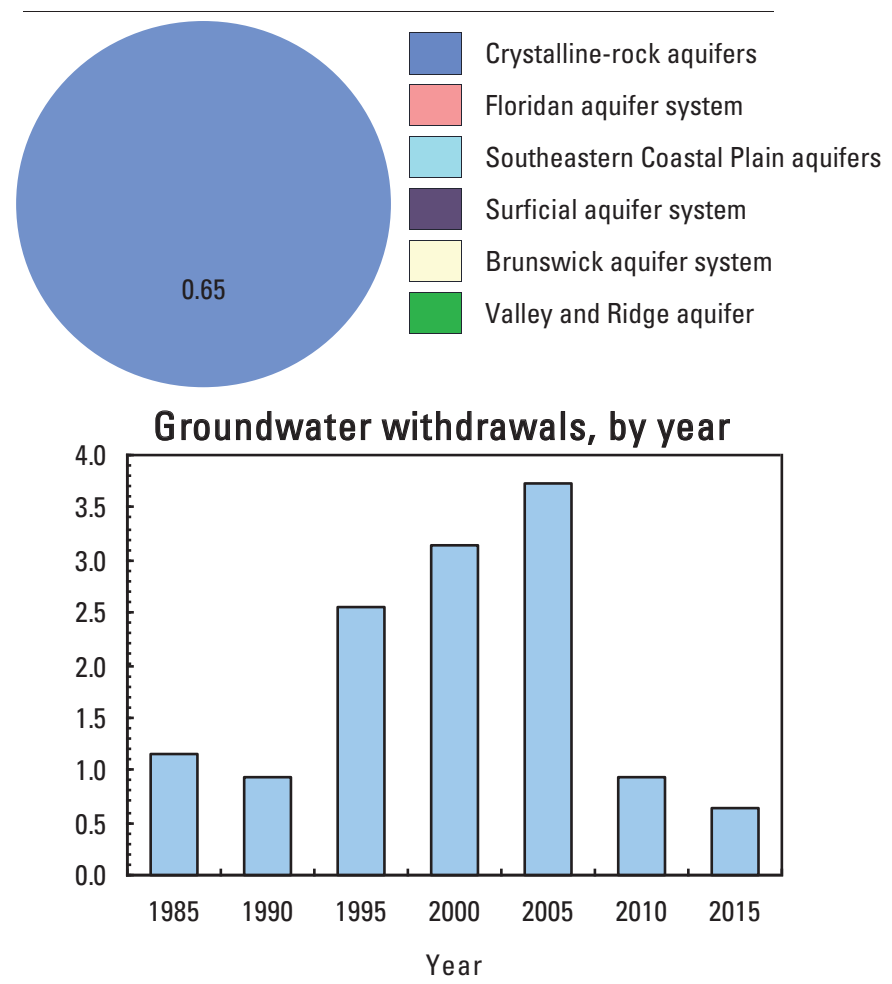


\section{DODGE COUNTY}

Population

Population served by public supply-groundwater

10,001

Population served by public supply-surface water

0

Acres irrigated

2015 WATER WITHDRAWALS AND ESTIMATED USE, IN MILLION GALLONS PER DAY

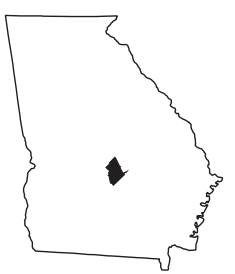

[--,not applicable; Mgal/d, million gallons per day]

\begin{tabular}{|c|c|c|c|c|c|c|c|c|}
\hline \multirow{2}{*}{ Category } & \multicolumn{3}{|c|}{ Withdrawals } & \multirow{2}{*}{$\begin{array}{c}\text { Total } \\
\text { use }\end{array}$} & \multirow{2}{*}{$\begin{array}{c}\text { Returns to } \\
\text { Surface }\end{array}$} & \multirow{2}{*}{\multicolumn{3}{|c|}{$\begin{array}{l}\text { Withdrawals by Major Industrial Groups } \\
\text { [NAICS, North American Industrial Classification System code] }\end{array}$}} \\
\hline & \multirow{2}{*}{$\begin{array}{c}\text { Ground- } \\
\text { water } \\
1.39\end{array}$} & \multirow{2}{*}{$\begin{array}{c}\text { Surface } \\
\text { water }\end{array}$} & \multirow{2}{*}{ Total } & & & & & \\
\hline Public supply & & & & & -- & NAICS & Groundwater & Surface water \\
\hline Domestic use & 0.82 & 0.00 & 0.82 & 1.57 & 0.00 & \multirow{3}{*}{ 322- Paper, pulp } & \multirow{3}{*}{0.02} & \multirow{3}{*}{0.00} \\
\hline Commercial use & 0.00 & 0.00 & 0.00 & 0.35 & 0.00 & & & \\
\hline Industrial use & 0.02 & 0.00 & 0.02 & 0.13 & 0.00 & & & \\
\hline Public-supply loss & -- & -- & -- & 0.18 & -- & & & \\
\hline Inter-county delivery & -- & -- & -- & 0.00 & -- & & & \\
\hline Public wastewater treatment & tt -- & -- & -- & -- & 0.90 & & & \\
\hline Mining use & 0.00 & 0.00 & 0.00 & 0.00 & 0.00 & \multicolumn{3}{|c|}{ Withdrawals by Major Public Suppliers } \\
\hline Irrigation (crop) & 3.54 & 3.16 & 6.70 & 6.70 & 0.00 & Name & Groundwater & Surface water \\
\hline Irrigation (golf) & 0.10 & 0.00 & 0.10 & 0.10 & 0.00 & \multirow[t]{4}{*}{ City of Eastman } & \multirow[t]{4}{*}{0.94} & \multirow[t]{4}{*}{0.00} \\
\hline Livestock-aquaculture & 0.16 & 0.14 & 0.30 & 0.30 & 0.00 & & & \\
\hline Thermoelectric power & 0.00 & 0.00 & 0.00 & 0.00 & 0.00 & & & \\
\hline TOTAL & 6.03 & 3.30 & 9.33 & 9.33 & 0.90 & & & \\
\hline
\end{tabular}

Total use is total withdrawal plus public supply deliveries and losses.

Withdrawals by water source

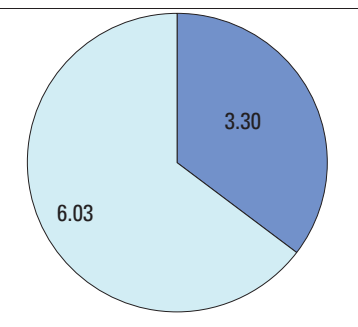

Withdrawals, in million

gallons per day

Surface water

Groundwater
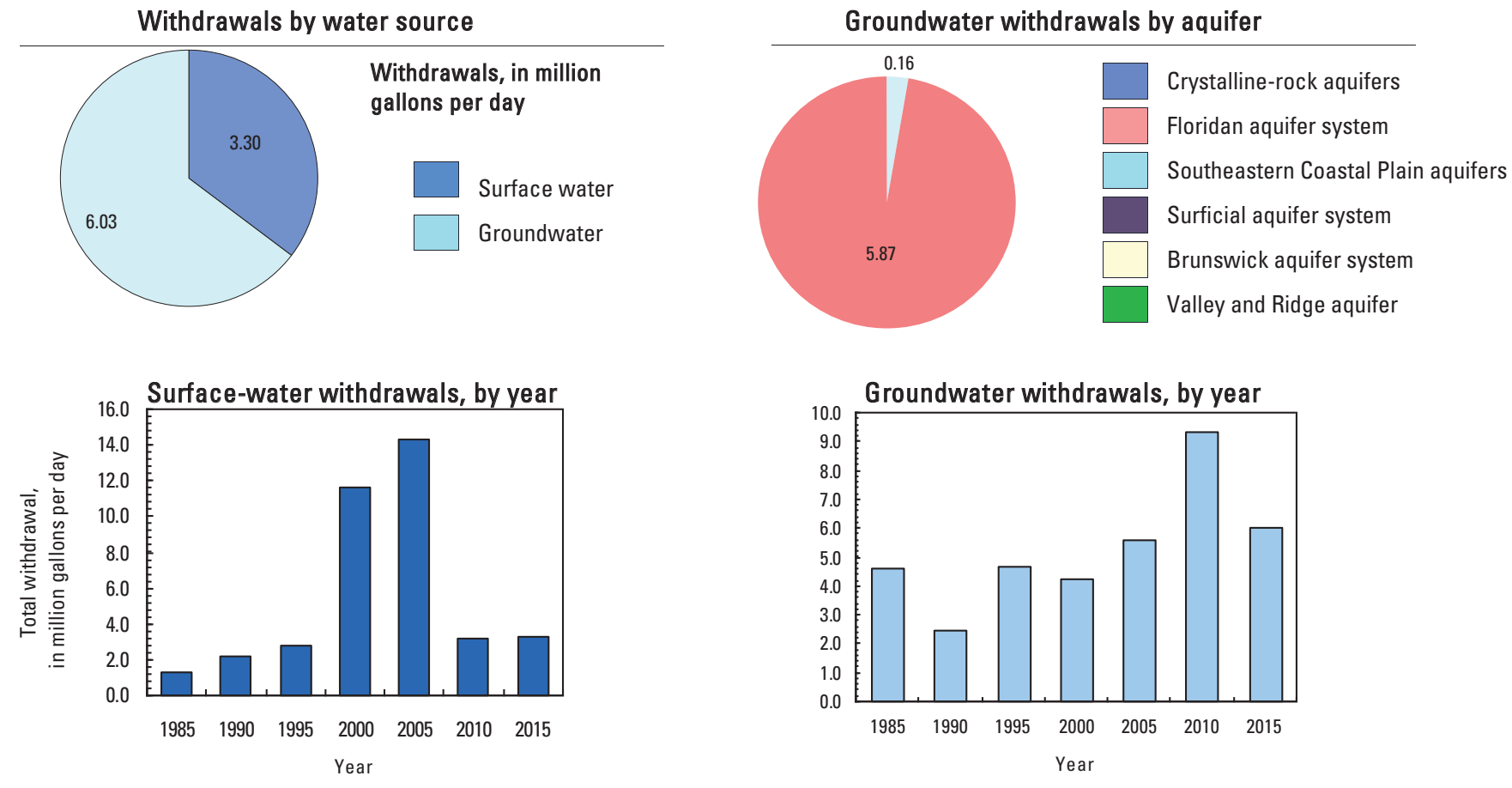


\section{DOOLY COUNTY}

Population

14,035

Population served by public supply-groundwater $\quad 8,863$

Population served by public supply-surface water 0

Acres irrigated

45,860

2015 WATER WITHDRAWALS AND ESTIMATED USE, IN MILLION GALLONS PER DAY

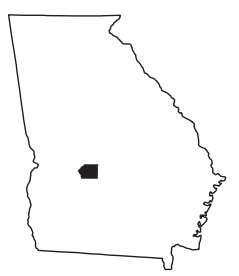

[--,not applicable; Mgal/d, million gallons per day]

\begin{tabular}{|c|c|c|c|c|c|}
\hline \multirow[b]{2}{*}{ Category } & \multicolumn{3}{|c|}{ Withdrawals } & \multirow[b]{2}{*}{$\begin{array}{c}\text { Total } \\
\text { use }\end{array}$} & \multirow[b]{2}{*}{$\begin{array}{c}\text { Returns to } \\
\text { Surface }\end{array}$} \\
\hline & $\begin{array}{l}\text { Ground- } \\
\text { water }\end{array}$ & $\begin{array}{c}\text { Surface } \\
\text { water }\end{array}$ & Total & & \\
\hline Public supply & 2.28 & 0.00 & 2.28 & -- & -- \\
\hline Domestic use & 0.39 & 0.00 & 0.39 & 1.13 & 0.00 \\
\hline Commercial use & 0.00 & 0.00 & 0.00 & 0.49 & 0.00 \\
\hline Industrial use & 0.00 & 0.00 & 0.00 & 0.50 & 0.00 \\
\hline Public-supply loss & -- & -- & -- & 0.55 & -- \\
\hline Inter-county delivery & -- & -- & -- & 0.00 & -- \\
\hline Public wastewater treatment & tt -- & -- & -- & -- & 0.24 \\
\hline Mining use & 0.00 & 0.00 & 0.00 & 0.00 & 0.00 \\
\hline Irrigation (crop) & 11.96 & 1.83 & 13.79 & 13.79 & 0.00 \\
\hline Irrigation (golf) & 0.00 & 0.00 & 0.00 & 0.00 & 0.00 \\
\hline Livestock-aquaculture & 0.16 & 0.02 & 0.18 & 0.18 & 0.00 \\
\hline Thermoelectric power & 0.00 & 0.00 & 0.00 & 0.00 & 0.00 \\
\hline TOTAL & 14.79 & 1.85 & 16.64 & 16.64 & 0.24 \\
\hline
\end{tabular}

Withdrawals by Major Industrial Groups

[NAICS, North American Industrial Classification System code]

NAICS Groundwater Surface water

None

Withdrawals by Major Public Suppliers

Name Groundwater Surface water

City of Vienna

1.57

0.00

City of Unadilla

0.28

0.00

City of Pinehurst

0.08

0.00

Total use is total withdrawal plus public supply deliveries and losses.

Withdrawals by water source

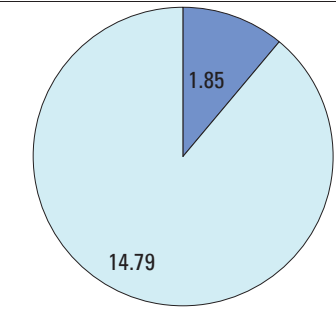

Withdrawals, in million

gallons per day

Surface water

Groundwater

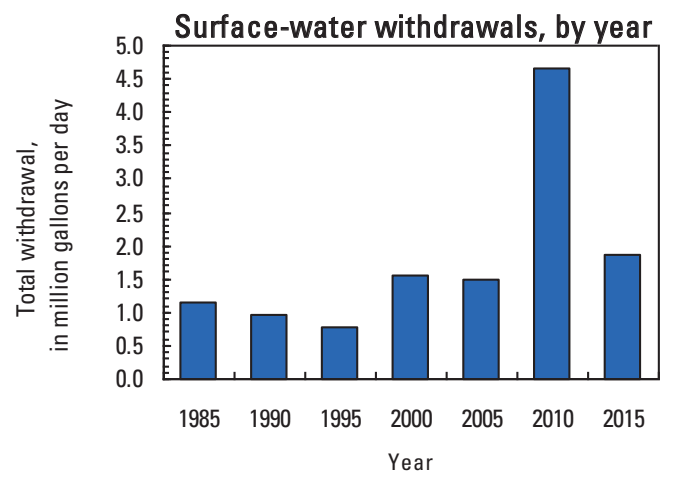

Groundwater withdrawals by aquifer

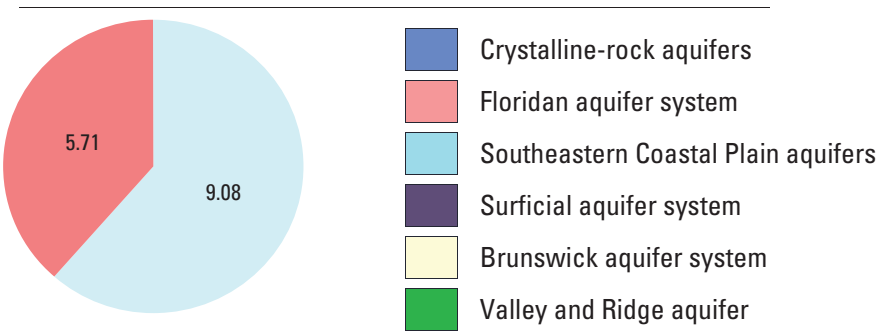

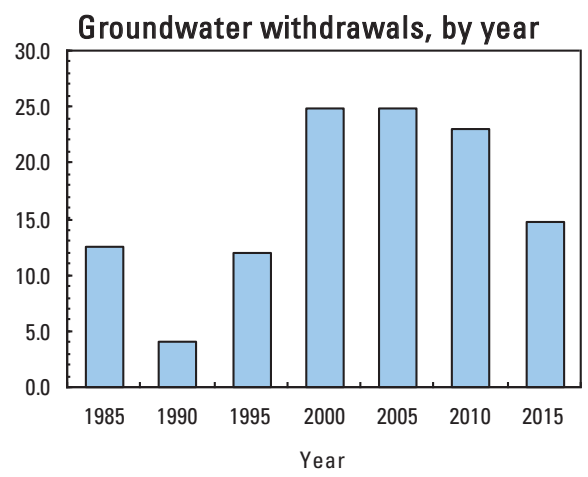




\section{DOUGHERTY COUNTY}

Population

Population served by public supply-groundwater

Population served by public supply-surface water

Acres irrigated

2015 WATER WITHDRAWALS AND ESTIMATED USE, IN MILLION GALLONS PER DAY

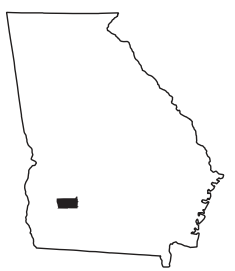

[--,not applicable; Mgal/d, million gallons per day]

\begin{tabular}{|c|c|c|c|c|c|c|c|c|}
\hline \multirow{2}{*}{ Category } & \multicolumn{3}{|c|}{ Withdrawals } & \multirow{2}{*}{$\begin{array}{c}\text { Total } \\
\text { use }\end{array}$} & \multirow{2}{*}{$\begin{array}{c}\text { Returns to } \\
\text { Surface }\end{array}$} & \multirow{2}{*}{\multicolumn{3}{|c|}{$\begin{array}{l}\text { Withdrawals by Major Industrial Groups } \\
\text { [NAICS, North American Industrial Classification System code] }\end{array}$}} \\
\hline & \multirow{2}{*}{$\begin{array}{c}\text { Ground- } \\
\text { water } \\
11.57\end{array}$} & \multirow{2}{*}{$\begin{array}{c}\begin{array}{c}\text { Surface } \\
\text { water }\end{array} \\
0.00\end{array}$} & \multirow{2}{*}{$\begin{array}{c}\text { Total } \\
11.57\end{array}$} & & & & & \\
\hline Public supply & & & & $\begin{array}{c}\text { Use } \\
--\end{array}$ & -- & NAICS & Groundwater & Surface water \\
\hline Domestic use & 0.29 & 0.00 & 0.29 & 6.50 & 0.00 & \multirow{4}{*}{$\begin{array}{l}\text { 312- Beverages } \\
\text { 322- Paper, pulp }\end{array}$} & \multirow{4}{*}{$\begin{array}{l}2.15 \\
5.40\end{array}$} & \multirow{4}{*}{$\begin{array}{l}0.00 \\
0.00\end{array}$} \\
\hline Commercial use & 0.00 & 0.00 & 0.00 & 309 & 000 & & & \\
\hline & & & & & & & & \\
\hline Industrial use & 7.55 & 0.00 & 7.55 & 8.08 & 0.00 & & & \\
\hline Public-supply loss & -- & -- & -- & 1.74 & -- & & & \\
\hline Inter-county delivery & -- & -- & -- & 0.00 & -- & & & \\
\hline \multicolumn{2}{|c|}{ Public wastewater treatment -- } & -- & - & -- & 19.69 & & & \\
\hline Mining use & 0.00 & 0.00 & 0.00 & 0.00 & 0.00 & \multicolumn{3}{|c|}{ Withdrawals by Major Public Suppliers } \\
\hline Irrigation (crop) & 13.64 & 0.01 & 13.65 & 13.65 & 0.00 & Name & Groundwater & Surface water \\
\hline Irrigation (golf) & 0.81 & 0.08 & 0.89 & 0.89 & 0.00 & City of Albany Utilities & 11.54 & 0.00 \\
\hline Livestock-aquaculture & 0.08 & 0.00 & 0.08 & 0.08 & 0.00 & & & \\
\hline Thermoelectric power & 0.00 & 18.83 & 18.83 & 18.83 & 0.00 & & & \\
\hline TOTAL & 33.94 & 18.92 & 52.86 & 52.86 & 19.69 & & & \\
\hline
\end{tabular}

Total use is total withdrawal plus public supply deliveries and losses.

Withdrawals by water source

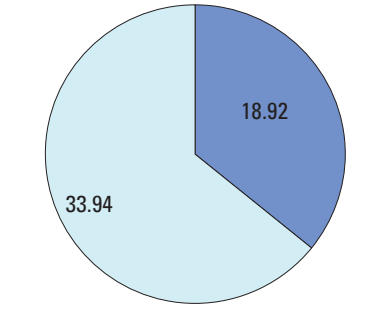

Withdrawals, in million gallons per day
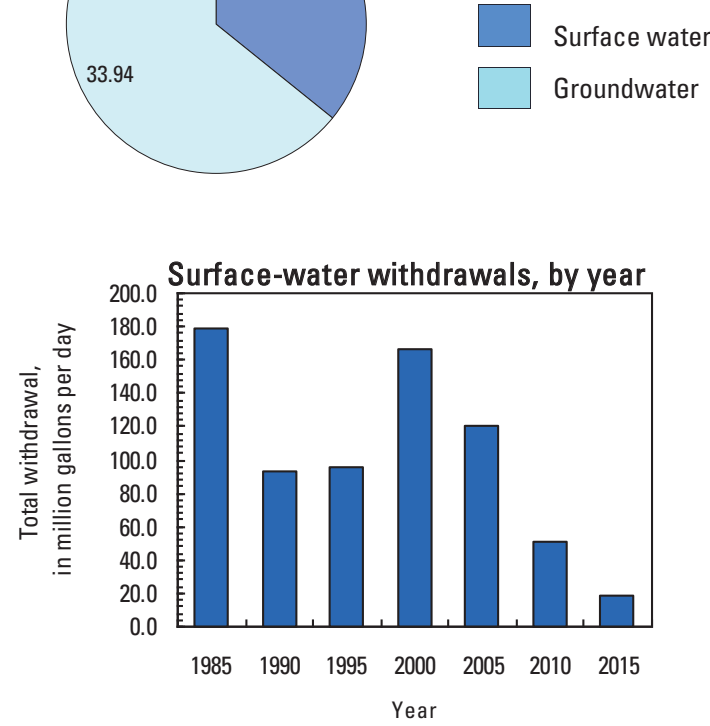

Groundwater withdrawals by aquifer

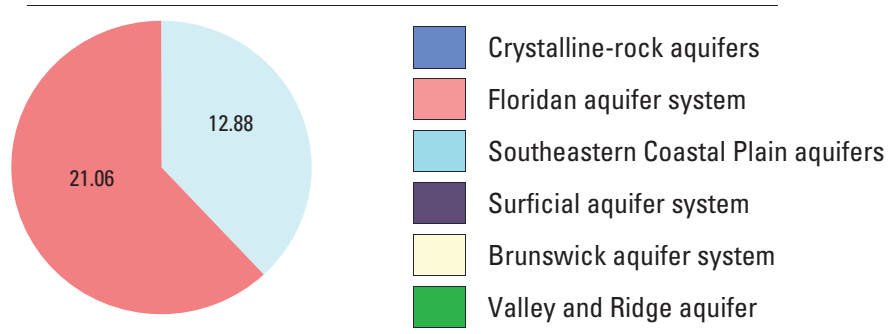

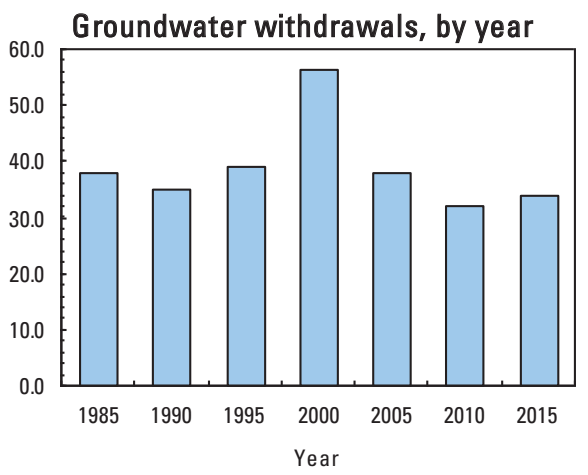




\section{DOUGLAS COUNTY}

Population

140,733

Population served by public supply-groundwater

Population served by public supply-surface water 134,793

Acres irrigated

370

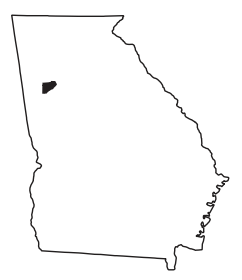

2015 WATER WITHDRAWALS AND ESTIMATED USE, IN MILLION GALLONS PER DAY

$[--$, not applicable; Mgal/d, million gallons per day]

\begin{tabular}{|c|c|c|c|c|c|}
\hline \multirow[b]{2}{*}{ Category } & \multicolumn{3}{|c|}{ Withdrawals } & \multirow[b]{2}{*}{$\begin{array}{c}\text { Total } \\
\text { use }\end{array}$} & \multirow[b]{2}{*}{$\begin{array}{c}\text { Returns to } \\
\text { Surface }\end{array}$} \\
\hline & $\begin{array}{c}\text { Ground- } \\
\text { water }\end{array}$ & $\begin{array}{c}\text { Surface } \\
\text { water }\end{array}$ & Total & & \\
\hline Public supply & 0.04 & 17.48 & 17.52 & -- & -- \\
\hline Domestic use & 0.15 & 0.00 & 0.15 & 6.67 & 0.00 \\
\hline Commercial use & 0.00 & 0.00 & 0.00 & 1.08 & 0.00 \\
\hline Industrial use & 0.00 & 0.00 & 0.00 & 0.32 & 0.00 \\
\hline Public-supply loss & -- & -- & -- & 9.60 & -- \\
\hline Inter-county delivery & -- & -- & -- & -7.97 & -- \\
\hline Public wastewater treatment & t -- & -- & -- & -- & 4.76 \\
\hline Mining use & 0.15 & 0.00 & 0.15 & 0.15 & 0.00 \\
\hline Irrigation (crop) & 0.00 & 0.00 & 0.00 & 0.00 & 0.00 \\
\hline Irrigation (golf) & 0.00 & 0.38 & 0.38 & 0.38 & 0.00 \\
\hline Livestock-aquaculture & 0.00 & 0.01 & 0.01 & 0.01 & 0.00 \\
\hline Thermoelectric power & 0.00 & 0.00 & 0.00 & 0.00 & 0.00 \\
\hline TOTAL & 0.34 & 17.87 & 18.21 & 10.24 & 4.76 \\
\hline
\end{tabular}

Withdrawals by Major Industrial Groups

[NAICS, North American Industrial Classification System code]

NAICS Groundwater Surface water

None

Withdrawals by Major Public Suppliers

Name Groundwater Surface water

Douglasville- Douglas

0.00

11.23

County Water and Sewer

Authority

City of East Point

0.00

6.25

Total use is total withdrawal plus public supply deliveries and losses. An estimate of $7.97 \mathrm{Mgal} / \mathrm{d}$ was

delivered from Douglas County to neighboring counties in 2015.
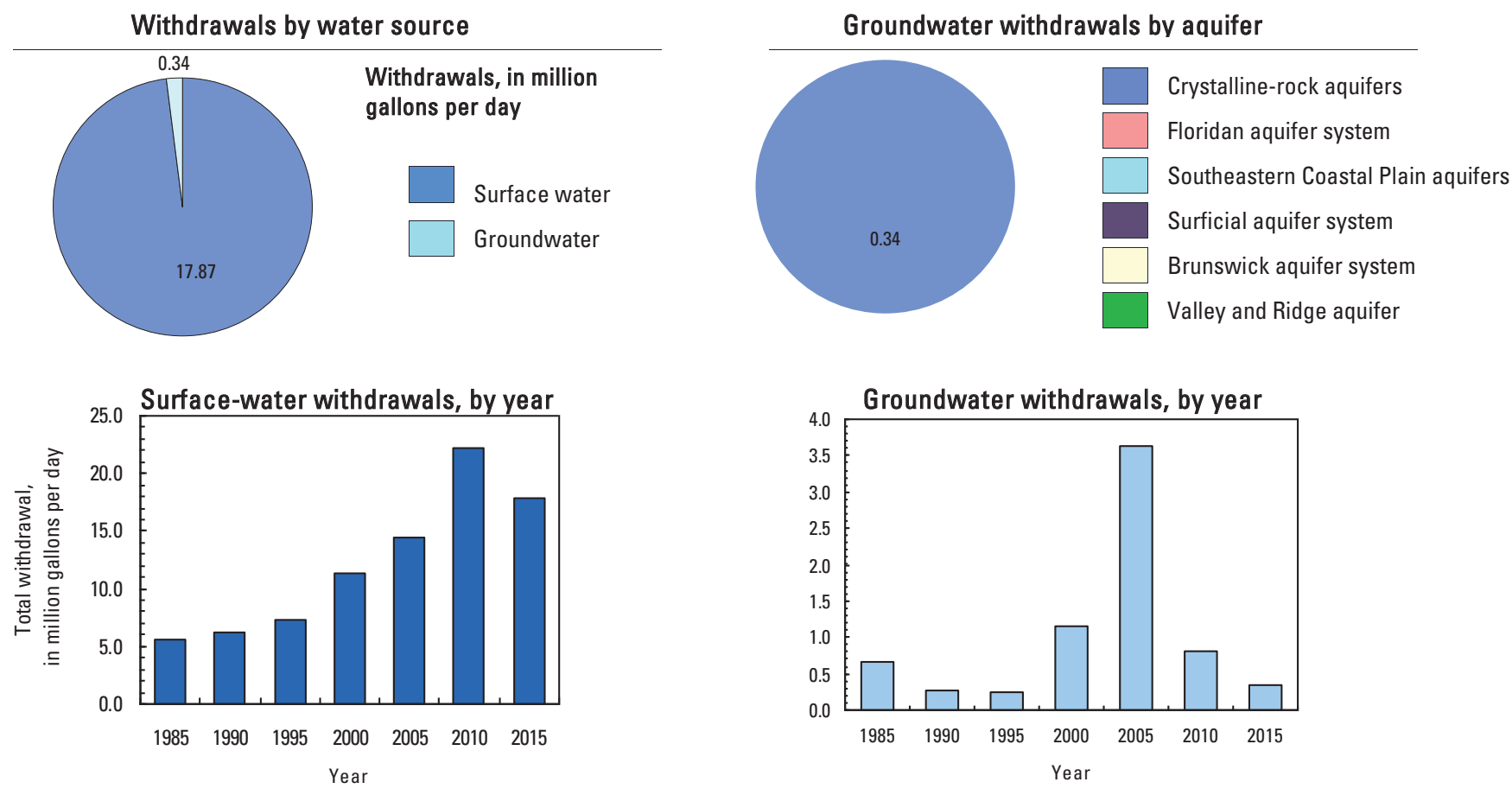


\section{EARLY COUNTY}

Population

Population served by public supply-groundwater $\quad 5,346$

Population served by public supply-surface water 0

Acres irrigated

2015 WATER WITHDRAWALS AND ESTIMATED USE, IN MILLION GALLONS PER DAY

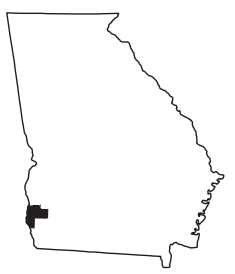

[--,not applicable; Mgal/d, million gallons per day]

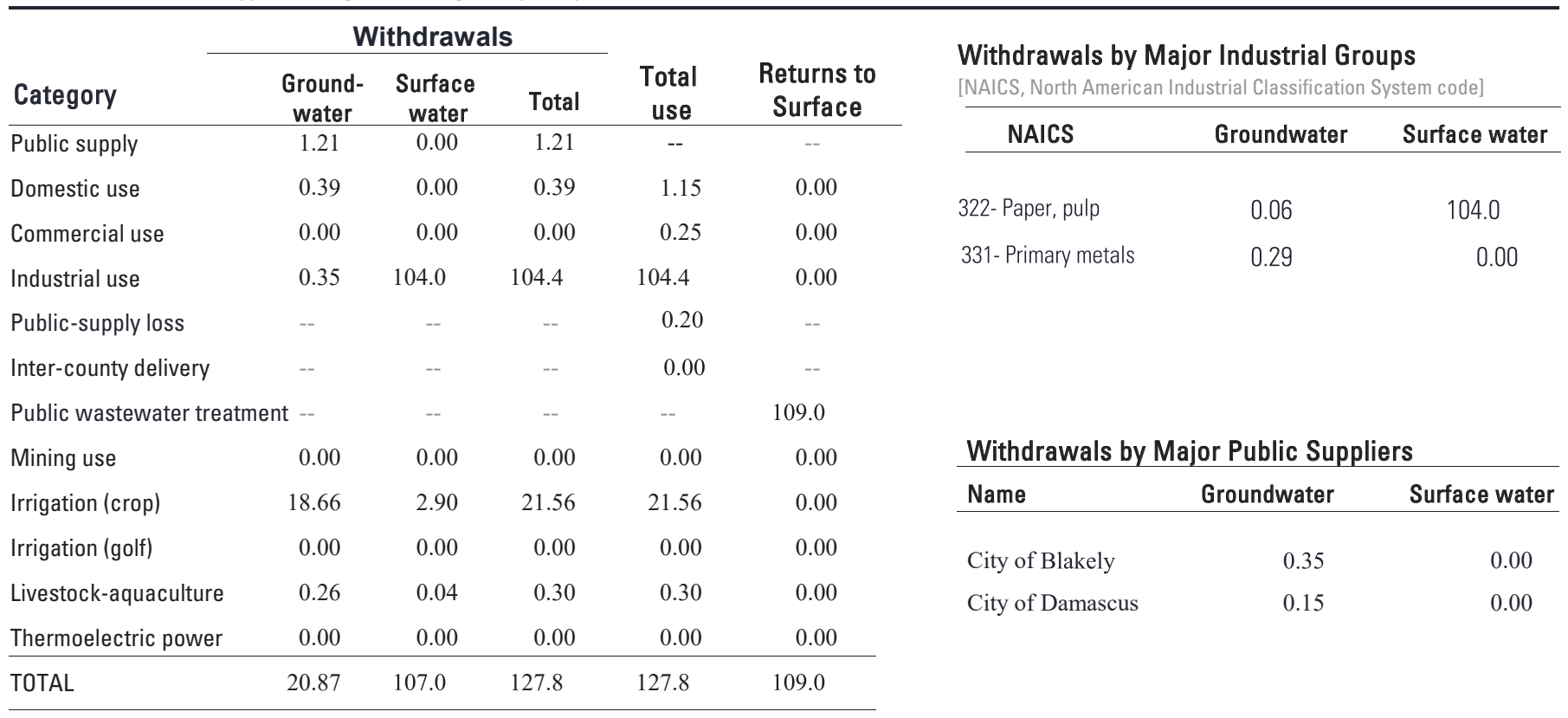

Total use is total withdrawal plus public supply deliveries and losses.

Withdrawals by water source

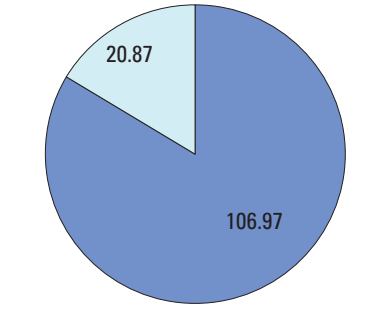

Withdrawals, in million gallons per day

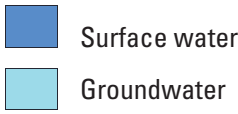

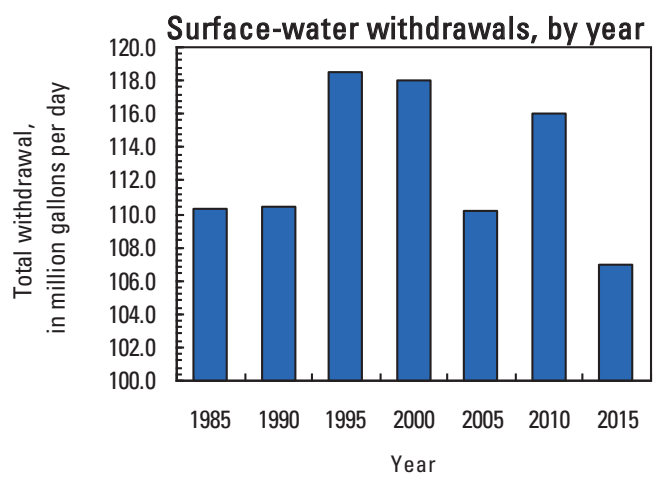

Groundwater withdrawals by aquifer

\begin{tabular}{lll}
\hline & Crystalline-rock aquifers \\
\hline & Floridan aquifer system \\
& & Southeastern Coastal Plain aquifers \\
& Surficial aquifer system \\
& Brunswick aquifer system \\
& Valley and Ridge aquifer
\end{tabular}

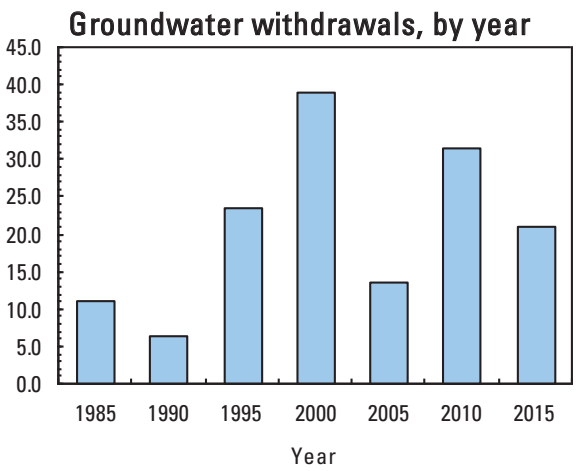




\section{ECHOLS COUNTY}

Population

Population served by public supply-groundwater

Population served by public supply-surface water

2015 WATER WITHDRAWALS AND ESTIMATED USE, IN MILLION GALLONS PER DAY

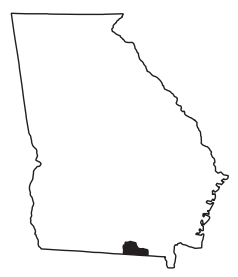

[--,not applicable; Mgal/d, million gallons per day]

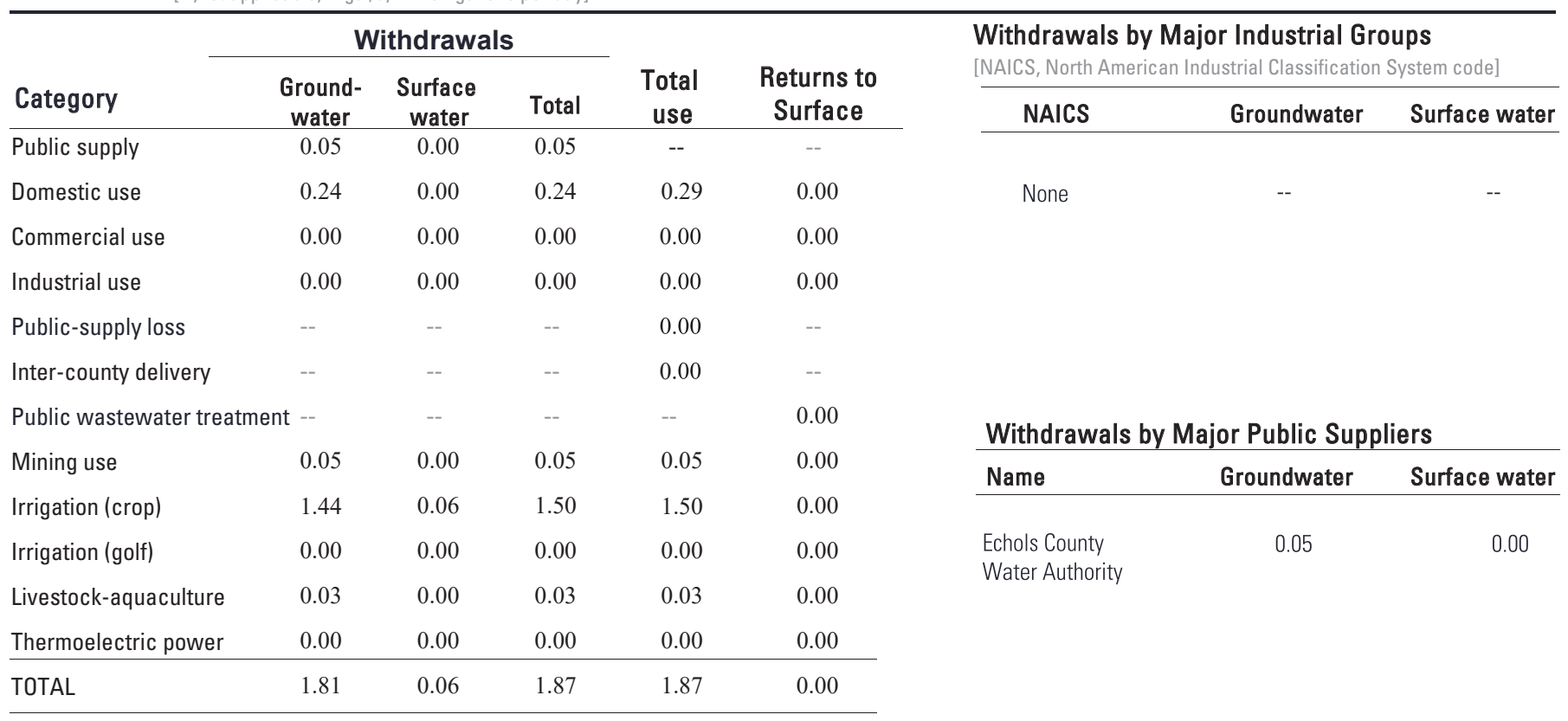

Total use is total withdrawal plus public supply deliveries and losses.

Withdrawals by water source

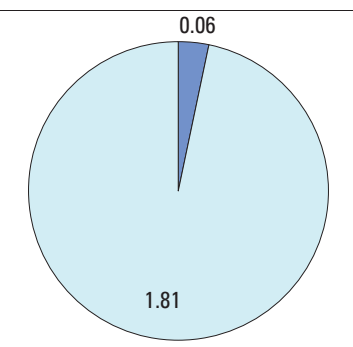

Withdrawals, in million

gallons per day

Surface water

Groundwater
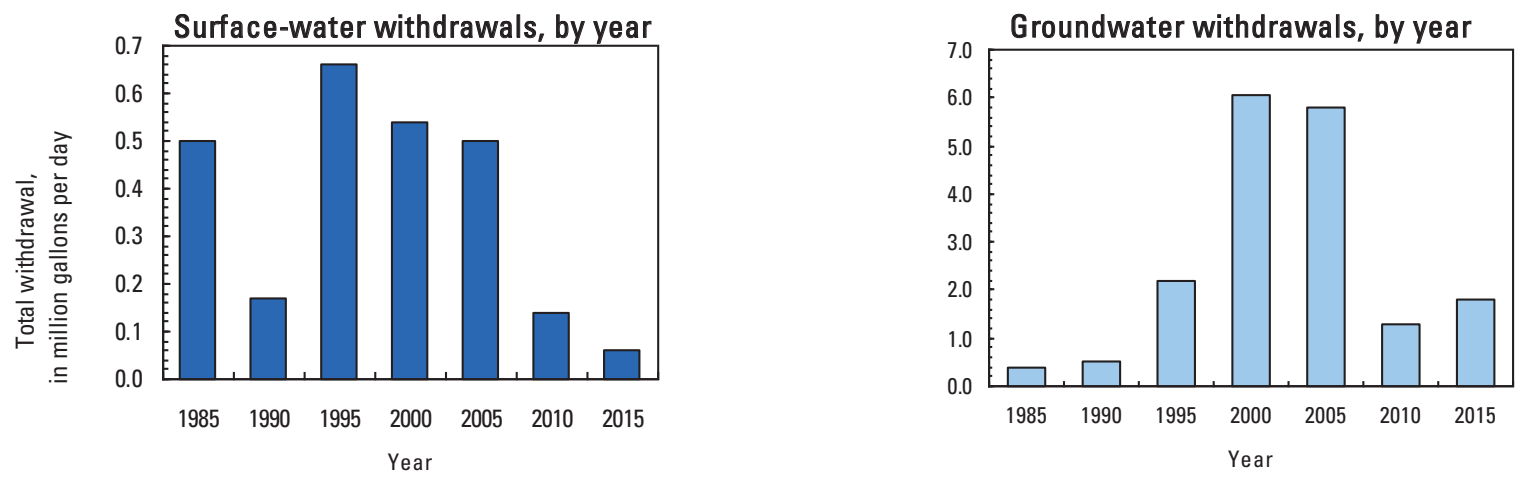


\section{EFFINGHAM COUNTY}

Population

Population served by public supply-groundwater

29,760

Population served by public supply-surface water $\quad 7,700$

Acres irrigated

2015 WATER WITHDRAWALS AND ESTIMATED USE, IN MILLION GALLONS PER DAY

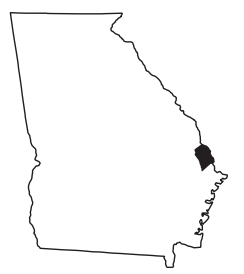

[--,not applicable; Mgal/d, million gallons per day]

\begin{tabular}{lccccc}
\hline & \multicolumn{3}{c}{ Withdrawals } & & Total \\
\cline { 2 - 4 } Category & $\begin{array}{c}\text { Ground- } \\
\text { water }\end{array}$ & $\begin{array}{c}\text { Surface } \\
\text { water }\end{array}$ & $\begin{array}{c}\text { Total } \\
\text { use }\end{array}$ & $\begin{array}{c}\text { Returns to } \\
\text { Surface }\end{array}$ \\
\hline Public supply & 2.77 & 33.03 & 35.80 & -- & -- \\
Domestic use & 1.47 & 0.00 & 1.47 & 5.16 & 0.00 \\
Commercial use & 0.00 & 0.00 & 0.00 & 0.39 & 0.00 \\
Industrial use & 0.73 & 13.17 & 13.90 & 14.38 & 0.00 \\
Public-supply loss & -- & -- & -- & 31.24 & -- \\
Inter-county delivery & -- & -- & -- & -30.54 & -- \\
Public wastewater treatment & -- & -- & -- & -- & 13.47 \\
Mining use & 0.25 & 0.00 & 0.25 & 0.25 & 0.00 \\
Irrigation (crop) & 0.99 & 0.09 & 1.08 & 1.08 & 0.00 \\
Irrigation (golf) & 0.00 & 0.00 & 0.00 & 0.00 & 0.00 \\
Livestock-aquaculture & 0.18 & 0.01 & 0.19 & 0.19 & 0.00 \\
Thermoelectric power & 0.06 & 70.77 & 70.83 & 70.83 & 0.00 \\
\hline TOTAL & 6.45 & 117.1 & 123.5 & 92.98 & 13.47 \\
\hline
\end{tabular}

Total use is total withdrawal plus public supply deliveries and losses. An estimate of $30.54 \mathrm{Mgal} / \mathrm{d}$ was delivered from Effingham County to neighboring counties in 2015.

Withdrawals by Major Industrial Groups

[NAICS, North American Industrial Classification System code]

\begin{tabular}{ccc}
\hline NAICS & Groundwater & Surface water \\
\hline
\end{tabular}

221- Electrical utility

0.06

0.06

322- Paper, pulp

0.73
Withdrawals by Major Public Suppliers

\begin{tabular}{lcc}
\hline Name & Groundwater & Surface wate \\
\hline City of Savannah & 0.00 & 33.03 \\
City of Rincon & 0.79 & 0.00 \\
City of Springfield & 0.51 & 0.00 \\
City of Guyton & 0.18 & 0.00 \\
Water Utility Management & 0.15 & 0.00
\end{tabular}

Company, LLC

\section{Withdrawals by water source}

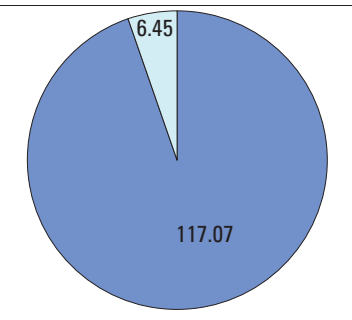

Withdrawals, in million gallons per day

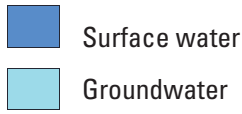

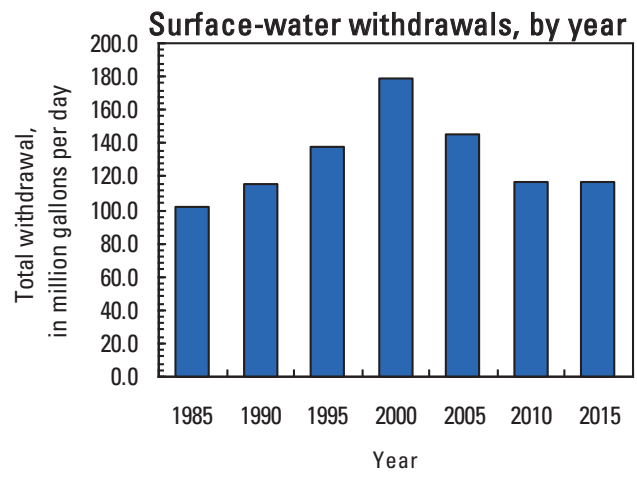

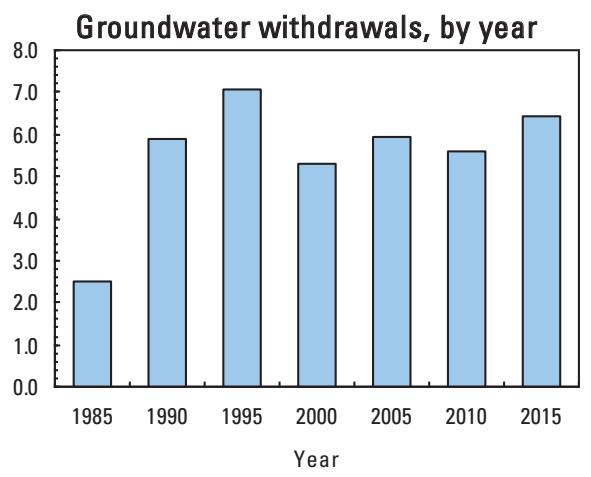

Groundwater withdrawals by aquifer

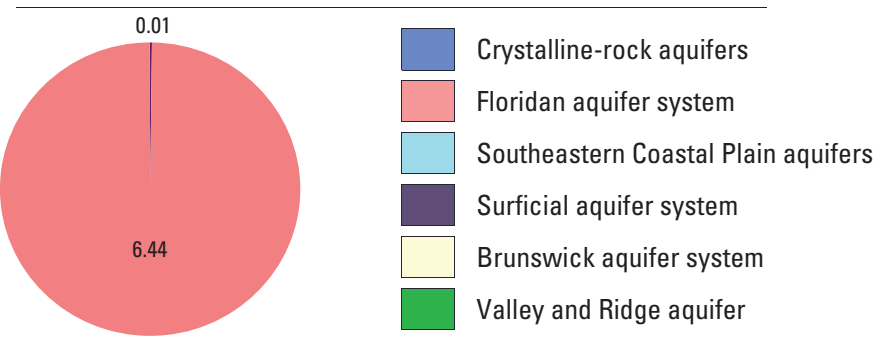




\section{ELBERT COUNTY}

Population

Population served by public supply-groundwater

Population served by public supply-surface water $\quad 7,800$

Acres irrigated

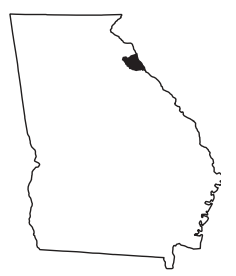

2015 WATER WITHDRAWALS AND ESTIMATED USE, IN MILLION GALLONS PER DAY

[--,not applicable; Mgal/d, million gallons per day]

\begin{tabular}{|c|c|c|c|c|c|c|c|c|}
\hline \multirow{3}{*}{ Category } & \multicolumn{3}{|c|}{ Withdrawals } & \multirow{3}{*}{$\begin{array}{c}\text { Total } \\
\text { use }\end{array}$} & \multirow{3}{*}{$\begin{array}{c}\text { Returns to } \\
\text { Surface }\end{array}$} & \multirow{2}{*}{\multicolumn{3}{|c|}{$\begin{array}{l}\text { Withdrawals by Major Industrial Groups } \\
\text { [NAICS, North American Industrial Classification System code] }\end{array}$}} \\
\hline & \multirow{2}{*}{$\begin{array}{l}\text { Ground } \\
\text { water }\end{array}$} & \multirow{2}{*}{$\begin{array}{l}\text { Surface } \\
\text { water }\end{array}$} & \multirow[b]{2}{*}{ Total } & & & & & \\
\hline & & & & & & NAICS & Groundwater & Surface water \\
\hline Public supply & 0.07 & 1.52 & 1.59 & -- & -- & & & \\
\hline Domestic use & 0.79 & 0.00 & 0.79 & 1.47 & 0.00 & None & -- & -- \\
\hline Commercial use & 0.02 & 0.00 & 0.02 & 0.19 & 0.00 & & & \\
\hline Industrial use & 0.00 & 0.00 & 0.00 & 0.52 & 0.00 & & & \\
\hline Public-supply loss & -- & -- & -- & 0.22 & -- & & & \\
\hline Inter-county delivery & -- & -- & -- & 0.00 & -- & & & \\
\hline Public wastewater treatment & t -- & -- & -- & -- & 1.52 & & & \\
\hline Mining use & 0.24 & 0.00 & 0.24 & 0.24 & 0.00 & Withdrawals & jor Public Supp & \\
\hline Irrigation (crop) & 0.29 & 0.07 & 0.36 & 0.36 & 0.00 & Name & Groundwater & Surface water \\
\hline Irrigation (golf) & 0.04 & 0.05 & 0.09 & 0.09 & 0.00 & City of Elberton & 0.00 & 1.52 \\
\hline Livestock-aquaculture & 0.32 & 0.10 & 0.42 & 0.42 & 0.00 & City of Bowman & 0.07 & 0.00 \\
\hline Thermoelectric power & 0.00 & 0.00 & 0.00 & 0.00 & 0.00 & & & \\
\hline TOTAL & 1.77 & 1.74 & 3.51 & 3.51 & 1.52 & & & \\
\hline
\end{tabular}

Total use is total withdrawal plus public supply deliveries and losses.

Withdrawals by water source

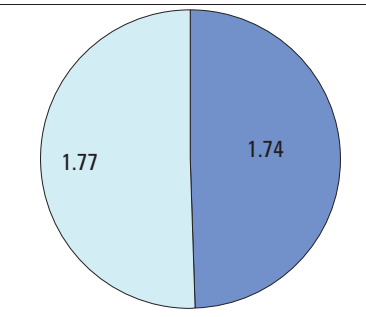

Withdrawals, in million gallons per day

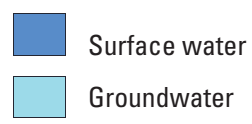

Groundwater withdrawals by aquifer

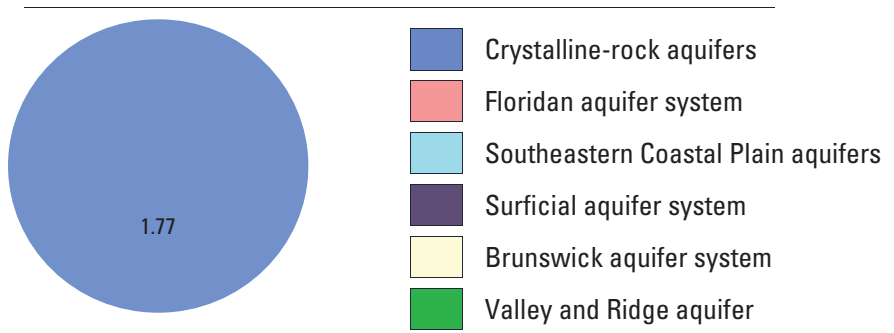

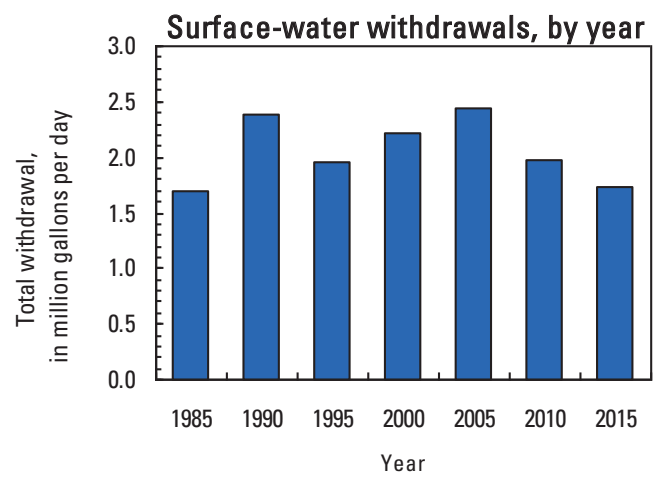

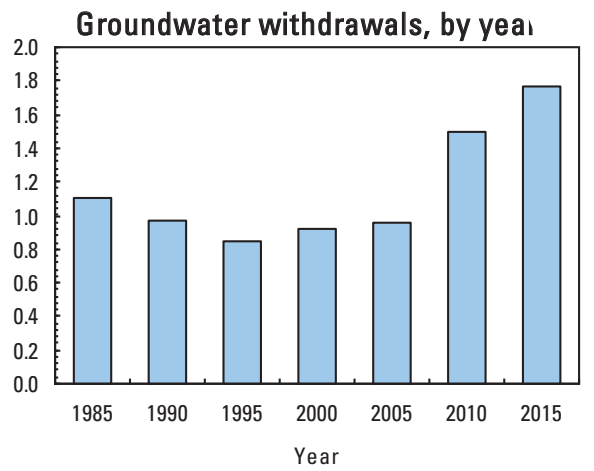




\section{EMANUEL COUNTY}

Population

Population served by public supply-groundwater

Population served by public supply-surface water

Acres irrigated

2015 WATER WITHDRAWALS AND ESTIMATED USE, IN MILLION GALLONS PER DAY

[--,not applicable; Mgal/d, million gallons per day]

\begin{tabular}{|c|c|c|c|c|c|c|c|c|}
\hline \multirow{2}{*}{ Category } & \multicolumn{3}{|c|}{ Withdrawals } & \multirow{2}{*}{$\begin{array}{c}\text { Total } \\
\text { use }\end{array}$} & \multirow{2}{*}{$\begin{array}{c}\text { Returns to } \\
\text { Surface }\end{array}$} & \multirow{2}{*}{\multicolumn{3}{|c|}{$\begin{array}{l}\text { Withdrawals by Major Industrial Groups } \\
\text { [NAICS, North American Industrial Classification System code] }\end{array}$}} \\
\hline & Ground- & Surface & Tatal & & & & & \\
\hline Public supply & Water & $\begin{array}{c}\text { Water } \\
0.00\end{array}$ & 1.59 & & -- & NAICS & Groundwater & Surface water \\
\hline Domestic use & 0.71 & 0.00 & 0.71 & 1.78 & 0.00 & \multirow{3}{*}{ 311- Food } & \multirow{3}{*}{0.74} & \multirow{3}{*}{0.00} \\
\hline Commercial use & 0.00 & 0.00 & 0.00 & 0.26 & 0.00 & & & \\
\hline Industrial use & 0.74 & 0.00 & 0.74 & 0.83 & 0.00 & & & \\
\hline Public-supply loss & -- & -- & -- & 0.17 & -- & & & \\
\hline Inter-county delivery & -- & -- & -- & 0.00 & -- & & & \\
\hline Public wastewater treatment & $t--$ & -- & -- & -- & 0.26 & & & \\
\hline Mining use & 0.00 & 0.00 & 0.00 & 0.00 & 0.00 & \multicolumn{3}{|c|}{ Withdrawals by Major Public Suppliers } \\
\hline Irrigation (crop) & 1.84 & 0.23 & 2.07 & 2.07 & 0.00 & Name & Groundwater & Surface water \\
\hline Irrigation (golf) & 0.00 & 0.00 & 0.00 & 0.00 & 0.00 & City of Swainsboro & 1.13 & 0.00 \\
\hline Livestock-aquaculture & 0.16 & 0.02 & 0.18 & 0.18 & 0.00 & Twin City & 0.22 & 0.00 \\
\hline Thermoelectric power & 0.00 & 0.00 & 0.00 & 0.00 & 0.00 & & & \\
\hline TOTAL & 5.04 & 0.25 & 5.29 & 5.29 & 0.26 & & & \\
\hline
\end{tabular}

Total use is total withdrawal plus public supply deliveries and losses.

Withdrawals by water source

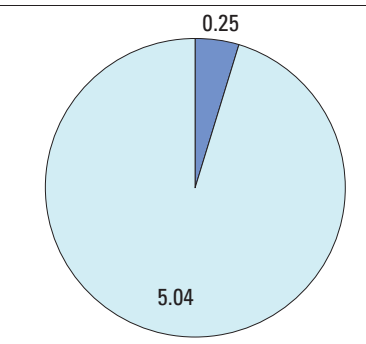

Withdrawals, in million

gallons per day

Surface water

Groundwater

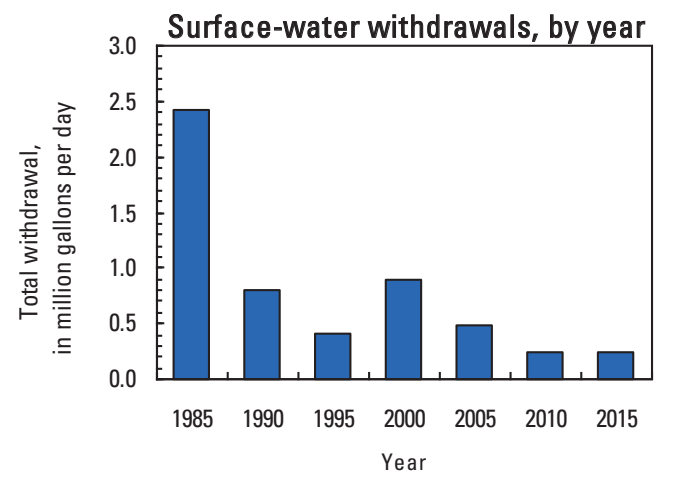

\section{Groundwater withdrawals by aquifer}
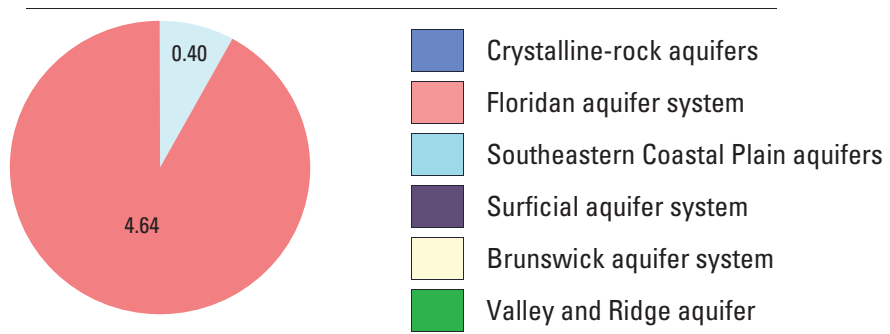

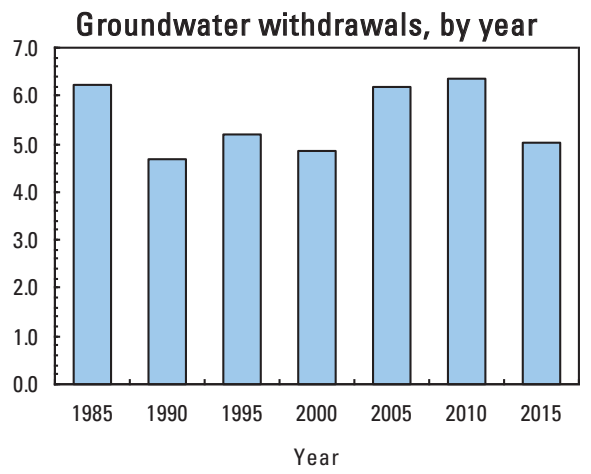




\section{EVANS COUNTY}

Population

Population served by public supply-groundwater

6,540

Population served by public supply-surface water

Acres irrigated
6,790

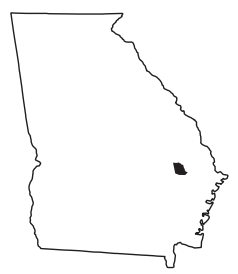

2015 WATER WITHDRAWALS AND ESTIMATED USE, IN MILLION GALLONS PER DAY

[--,not applicable; Mgal/d, million gallons per day]

\begin{tabular}{|c|c|c|c|c|c|c|c|c|}
\hline \multirow[b]{2}{*}{ Category } & \multicolumn{3}{|c|}{ Withdrawals } & \multirow{2}{*}{$\begin{array}{c}\text { Total } \\
\text { use }\end{array}$} & \multirow{2}{*}{$\begin{array}{c}\text { Returns to } \\
\text { Surface }\end{array}$} & \multirow{2}{*}{\multicolumn{3}{|c|}{$\begin{array}{l}\text { Withdrawals by Major Industrial Groups } \\
\text { [NAICS, North American Industrial Classification System code] }\end{array}$}} \\
\hline & Ground- & Surface & Total & & & & & \\
\hline Public supply & 0.54 & 0.00 & 0.54 & -- & -- & NAICS & Groundwater & Surface water \\
\hline Domestic use & 0.32 & 0.00 & 0.32 & 0.69 & 0.00 & \multirow{3}{*}{$\begin{array}{l}\text { 311- Food } \\
\text { 339- Misc. manufacturing }\end{array}$} & \multirow{2}{*}{1.72} & \multirow{2}{*}{0.00} \\
\hline Commercial use & 0.00 & 0.00 & 0.00 & 0.09 & 0.00 & & & \\
\hline Industrial use & 1.73 & 0.00 & 1.73 & 1.75 & 0.00 & & 0.01 & 0.00 \\
\hline Public-supply loss & -- & -- & -- & 0.06 & -- & & & \\
\hline Inter-county delivery & -- & -- & -- & 0.00 & -- & & & \\
\hline Public wastewater trea & ment -- & -- & -- & -- & 0.20 & & & \\
\hline Mining use & 0.00 & 0.00 & 0.00 & 0.00 & 0.00 & \multicolumn{3}{|c|}{ Withdrawals by Major Public Suppliers } \\
\hline Irrigation (crop) & 1.15 & 1.52 & 2.67 & 2.67 & 0.00 & Name & Groundwater & Surface wate। \\
\hline Irrigation (golf) & 0.06 & 0.00 & 0.06 & 0.06 & 0.00 & \multirow[t]{4}{*}{ City of Claxton } & \multirow[t]{2}{*}{0.42} & \multirow[t]{3}{*}{0.00} \\
\hline Livestock-aquaculture & 0.07 & 0.09 & 0.16 & 0.16 & 0.00 & & & \\
\hline Thermoelectric power & 0.00 & 0.00 & 0.00 & 0.00 & 0.00 & & & \\
\hline TOTAL & 3.87 & 1.61 & 5.48 & 5.48 & 0.20 & & & \\
\hline
\end{tabular}

Total use is total withdrawal plus public supply deliveries and losses.

Withdrawals by water source

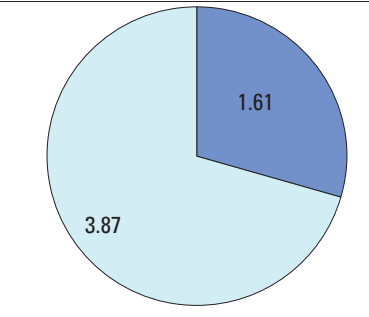

Withdrawals, in million gallons per day

Surface water

Groundwater
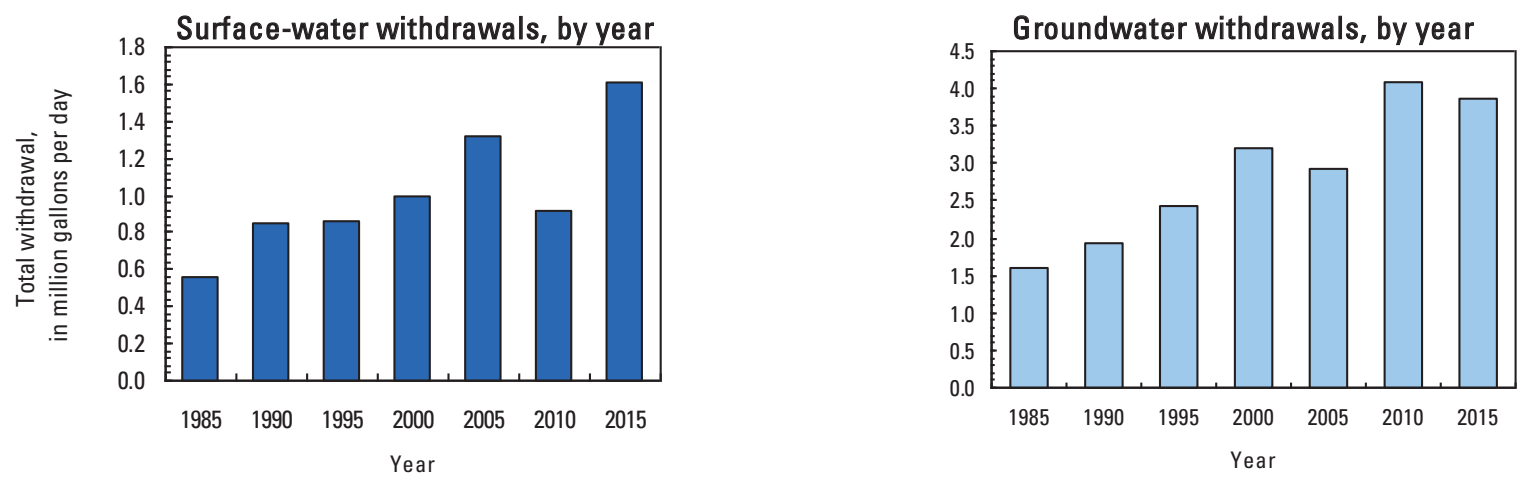


\section{FANNIN COUNTY}

Population

Population served by public supply-groundwater

Population served by public supply-surface water $\quad 12,526$

Acres irrigated

2015 WATER WITHDRAWALS AND ESTIMATED USE, IN MILLION GALLONS PER DAY

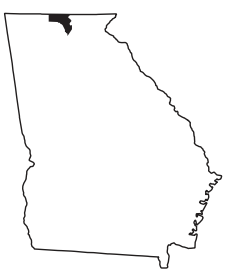

[--,not applicable; Mgal/d, million gallons per day]

\begin{tabular}{lccccc}
\hline & \multicolumn{3}{c}{ Withdrawals } & & \\
\cline { 2 - 4 } Category & $\begin{array}{c}\text { Ground } \\
\text { water }\end{array}$ & $\begin{array}{c}\text { Surface } \\
\text { water }\end{array}$ & Total & $\begin{array}{c}\text { Total } \\
\text { use }\end{array}$ & $\begin{array}{c}\text { Returns to } \\
\text { Surface }\end{array}$ \\
\hline Public supply & 0.09 & 1.66 & 1.75 & -- & -- \\
Domestic use & 0.71 & 0.00 & 0.71 & 1.7 & 0.00 \\
Commercial use & 0.00 & 0.00 & 0.00 & 0.36 & 0.00 \\
Industrial use & 0.00 & 0.00 & 0.00 & 0.04 & 0.00 \\
Public-supply loss & -- & -- & -- & 0.36 & -- \\
Inter-county delivery & -- & -- & -- & -0.12 & -- \\
Public wastewater treatment & -- & -- & -- & -- & 1.59 \\
Mining use & 0.00 & 0.00 & 0.00 & 0.00 & 0.00 \\
Irrigation (crop) & 0.01 & 0.01 & 0.02 & 0.02 & 0.00 \\
Irrigation (golf) & 0.00 & 0.00 & 0.00 & 0.00 & 0.00 \\
Livestock-aquaculture & 0.03 & 43.48 & 43.51 & 43.51 & 0.00 \\
Thermoelectric power & 0.00 & 0.00 & 0.00 & 0.00 & 0.00 \\
\hline TOTAL & 0.84 & 45.15 & 45.99 & 45.87 & 1.59 \\
\hline
\end{tabular}

Withdrawals by Major Industrial Groups

[NAICS, North American Industrial Classification System code]

\begin{tabular}{ccc}
\hline NAICS Groundwater & Surface water \\
\hline
\end{tabular}

None

Withdrawals by Major Public Suppliers

Name Groundwater Surface water

$\begin{array}{lll}\text { City of Blue Ridge } & 0.00 & 0.87 \\ \text { City of McCaysville } & 0.00 & 0.79 \\ \text { City of Morganton } & 0.08 & 0.00\end{array}$

Total use is total withdrawal plus public supply deliveries and losses. An estimate of $0.12 \mathrm{Mgal} / \mathrm{d}$ was delivered from Fannin County to neighboring counties in 2015.

Withdrawals by water source

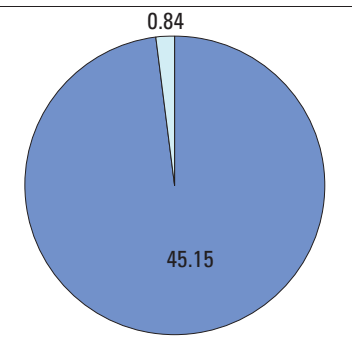

Withdrawals, in million

gallons per day

Surface water

Groundwater
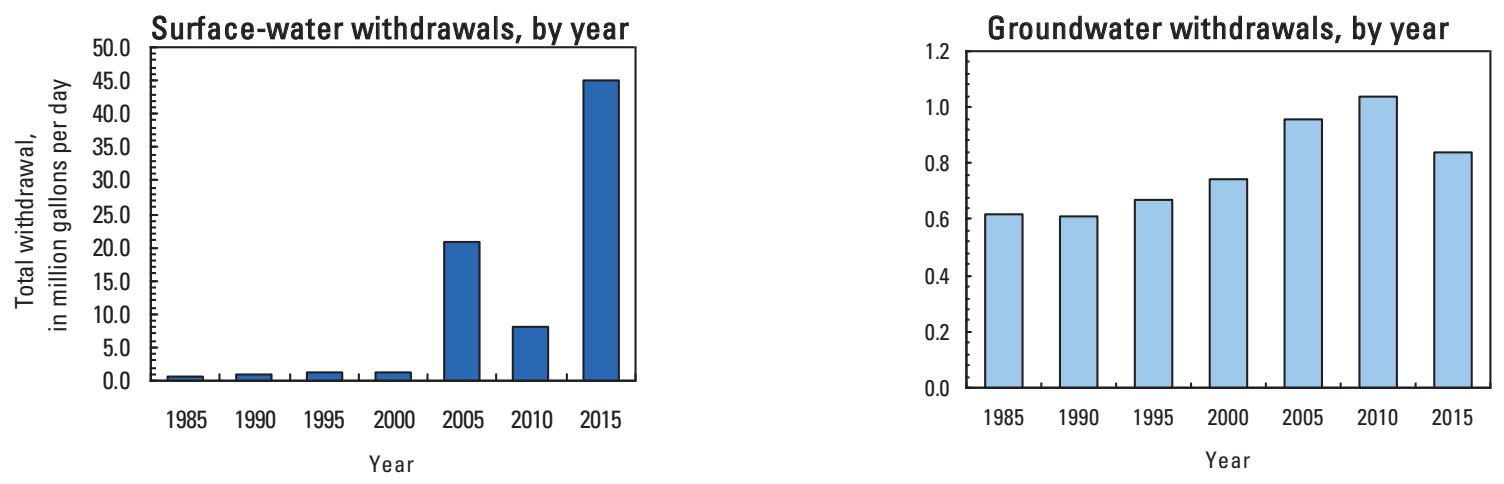


\section{FAYETTE COUNTY}

Population

110,714

Population served by public supply-groundwater $\quad 8,821$

Population served by public supply-surface water $\quad 83,097$

Acres irrigated

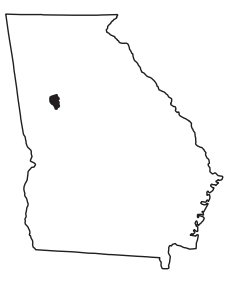

2015 WATER WITHDRAWALS AND ESTIMATED USE, IN MILLION GALLONS PER DAY

[--,not applicable; Mgal/d, million gallons per day]

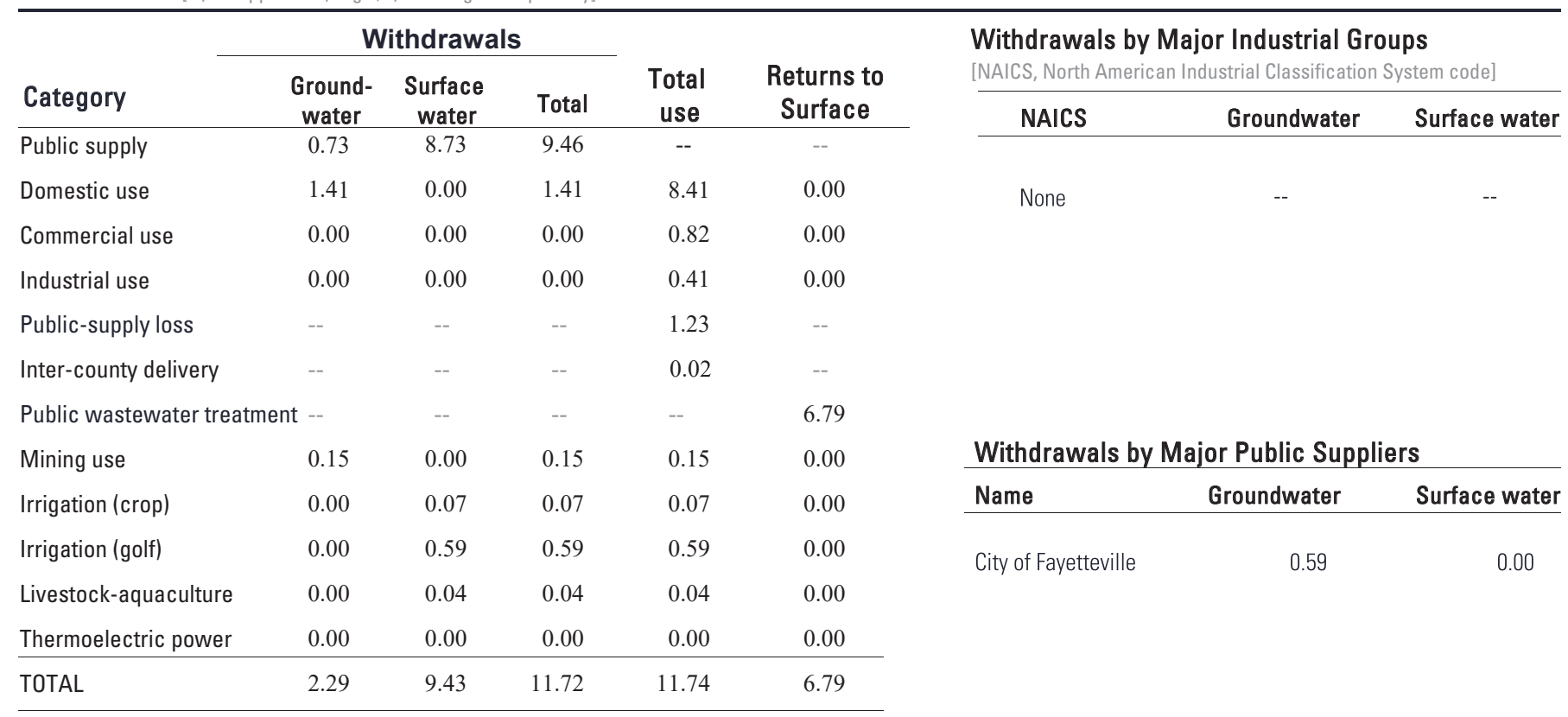

Total use is total withdrawal plus public supply deliveries and losses. An estimate of $0.02 \mathrm{Mgal} / \mathrm{d}$ was delivered from neighboring counties in 2015.

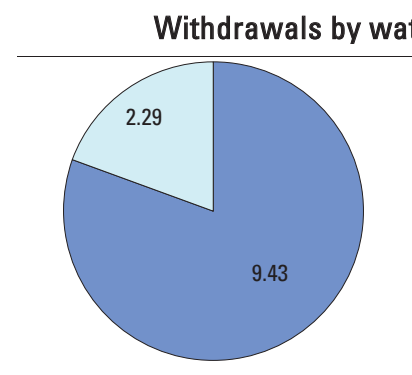

Withdrawals, in million gallons per day
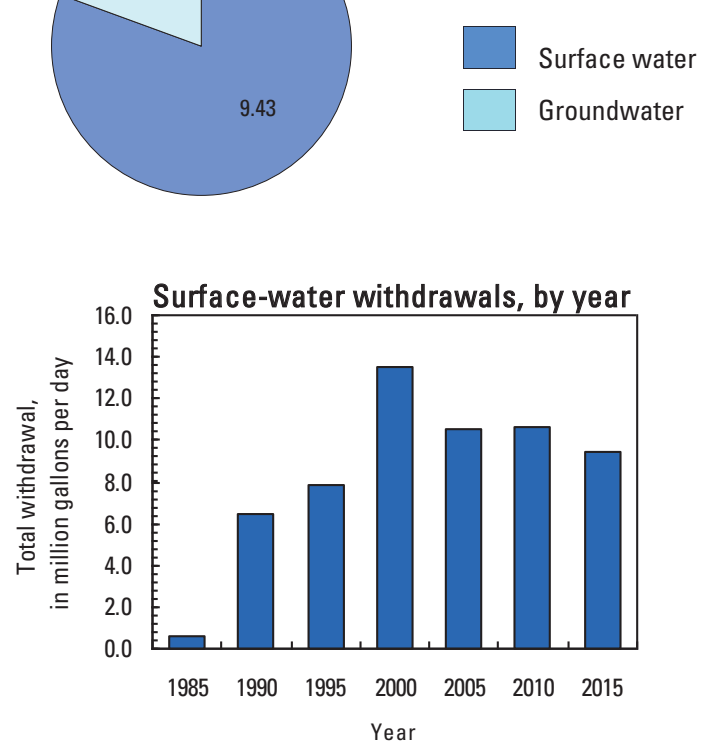

Groundwater withdrawals by aquifer

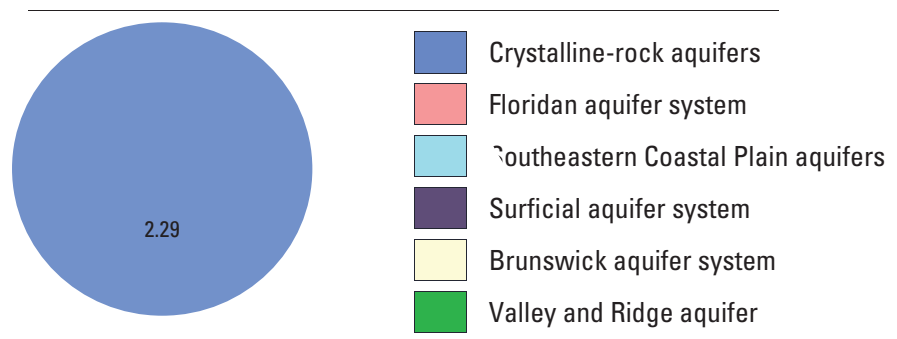




\section{FLOYD COUNTY}

Population

96,504

Population served by public supply-groundwater

6,684

Population served by public supply-surface water $\quad 84,820$

Acres irrigated

2015 WATER WITHDRAWALS AND ESTIMATED USE, IN MILLION GALLONS PER DAY

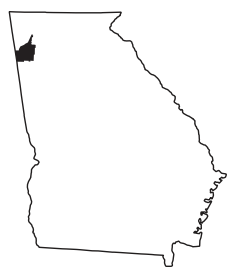

[--,not applicable; Mgal/d, million gallons per day]

\begin{tabular}{|c|c|c|c|c|c|}
\hline \multirow[b]{2}{*}{ Category } & \multicolumn{3}{|c|}{ Withdrawals } & \multirow[b]{2}{*}{$\begin{array}{c}\text { Total } \\
\text { use }\end{array}$} & \multirow[b]{2}{*}{$\begin{array}{c}\text { Returns to } \\
\text { Surface }\end{array}$} \\
\hline & $\begin{array}{c}\text { Ground- } \\
\text { water }\end{array}$ & $\begin{array}{c}\text { Surface } \\
\text { water }\end{array}$ & Total & & \\
\hline Public supply & 1.08 & 10.72 & 11.80 & -- & -- \\
\hline Domestic use & 0.38 & 0.00 & 0.38 & 6.61 & 0.00 \\
\hline Commercial use & 0.00 & 0.29 & 0.29 & 3.15 & 0.00 \\
\hline Industrial use & 0.10 & 22.90 & 23.00 & 24.07 & 0.00 \\
\hline Public-supply loss & -- & -- & -- & 1.64 & -- \\
\hline Inter-county delivery & -- & -- & -- & 0.47 & -- \\
\hline Public wastewater treatmen & nt -- & -- & -- & -- & 12.89 \\
\hline Mining use & 0.04 & 0.00 & 0.04 & 0.04 & 0.00 \\
\hline Irrigation (crop) & 0.33 & 0.60 & 0.93 & 0.93 & 0.00 \\
\hline Irrigation (golf) & 0.38 & 0.28 & 0.66 & 0.66 & 0.00 \\
\hline Livestock-aquaculture & 0.24 & 10.23 & 10.47 & 10.47 & 0.00 \\
\hline Thermoelectric power & 0.00 & 211.08 & 211.08 & 211.1 & 0.00 \\
\hline TOTAL & 2.55 & 256.1 & 258.7 & 259.1 & 12.89 \\
\hline
\end{tabular}

Withdrawals by Major Industrial Groups

[NAICS, North American Industrial Classification System code]

NAICS Groundwater Surface water

322- Paper, pulp

0.00

22.9

812- Laundry Services

0.10

0.00

Total use is total withdrawal plus public supply deliveries and losses. An estimate of $0.6 \mathrm{Mgal} / \mathrm{d}$ was delivered from neighboring counties in 2015. An estimate of $0.13 \mathrm{Mgal} / \mathrm{d}$ was delivered from Floyd

County to neighboring counties in 2015.

\section{Withdrawals by water source}

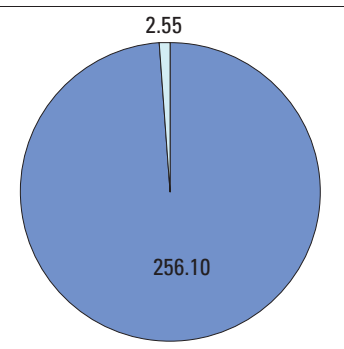

Withdrawals, in million gallons per day

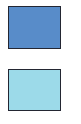

Surface water Groundwater

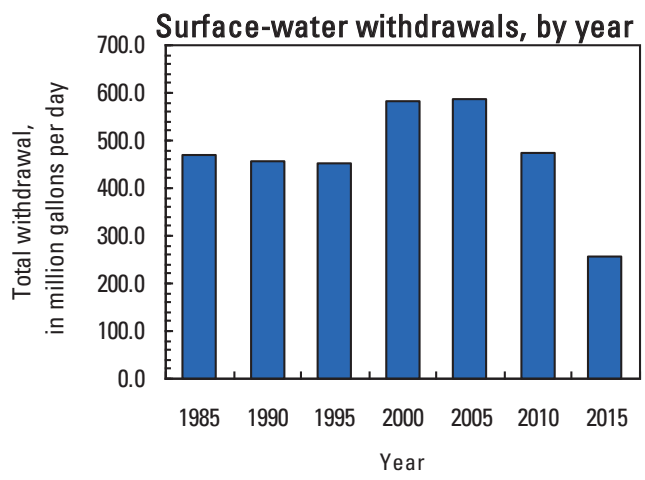

Withdrawals by Major Public Suppliers

Name Groundwater Surface water

$\begin{array}{lll}\text { City of Rome } & 0.00 & 7.13 \\ \text { Floyd County } & 0.72 & 2.72 \\ \text { City of Cave Spring } & 0.00 & 0.87\end{array}$




\section{FORSYTH COUNTY}

Population

Population served by public supply-groundwater

Population served by public supply-surface water 200,178

Acres irrigated

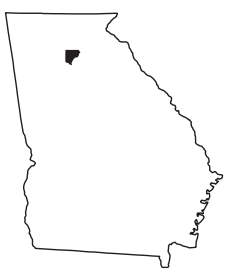

2015 WATER WITHDRAWALS AND ESTIMATED USE, IN MILLION GALLONS PER DAY

[--,not applicable; Mgal/d, million gallons per day]

\begin{tabular}{lccccc}
\hline & \multicolumn{3}{c}{ Withdrawals } & & \\
\cline { 2 - 4 } Category & $\begin{array}{c}\text { Ground } \\
\text { water }\end{array}$ & $\begin{array}{c}\text { Surface } \\
\text { water }\end{array}$ & $\begin{array}{c}\text { Total } \\
\text { Public supply }\end{array}$ & $\begin{array}{c}\text { Total } \\
\text { use }\end{array}$ & $\begin{array}{c}\text { Returns to } \\
\text { Surface }\end{array}$ \\
Domestic use & 0.33 & 20.60 & 20.61 & -- & -- \\
Commercial use & 0.00 & 0.00 & 0.33 & 12.67 & 0.00 \\
Industrial use & 0.00 & 0.00 & 0.00 & 3.33 & 0.00 \\
Public-supply loss & -- & -- & -- & 2.64 & 0.00 \\
Inter-county delivery & -- & -- & -- & -0.05 & -- \\
Public wastewater treatment & -- & -- & -- & -- & 4.44 \\
Mining use & 0.26 & 0.00 & 0.26 & 0.26 & 0.00 \\
Irrigation (crop) & 0.12 & 0.16 & 0.28 & 0.28 & 0.00 \\
Irrigation (golf) & 0.00 & 0.16 & 0.16 & 0.16 & 0.00 \\
Livestock-aquaculture & 0.01 & 6.31 & 6.32 & 6.32 & 0.00 \\
Thermoelectric power & 0.00 & 0.00 & 0.00 & 0.00 & 0.00 \\
\hline TOTAL & 0.73 & 27.23 & 27.96 & 27.91 & 4.44 \\
\hline
\end{tabular}

Withdrawals by Major Industrial Groups

[NAICS, North American Industrial Classification System code]

NAICS Groundwater Surface water

None

Withdrawals by Major Public Suppliers

\begin{tabular}{lcc} 
Name & Groundwater & Surface wate \\
\hline $\begin{array}{l}\text { Forsyth County Water } \\
\text { \& Sewer Authority }\end{array}$ & 0.00 & 12.73 \\
City of Cumming & 0.00 & 7.87
\end{tabular}

Total use is total withdrawal plus public supply deliveries and losses. An estimate of $0.05 \mathrm{Mgal} / \mathrm{d}$ was delivered from Forsyth County to neighboring counties in 2015.
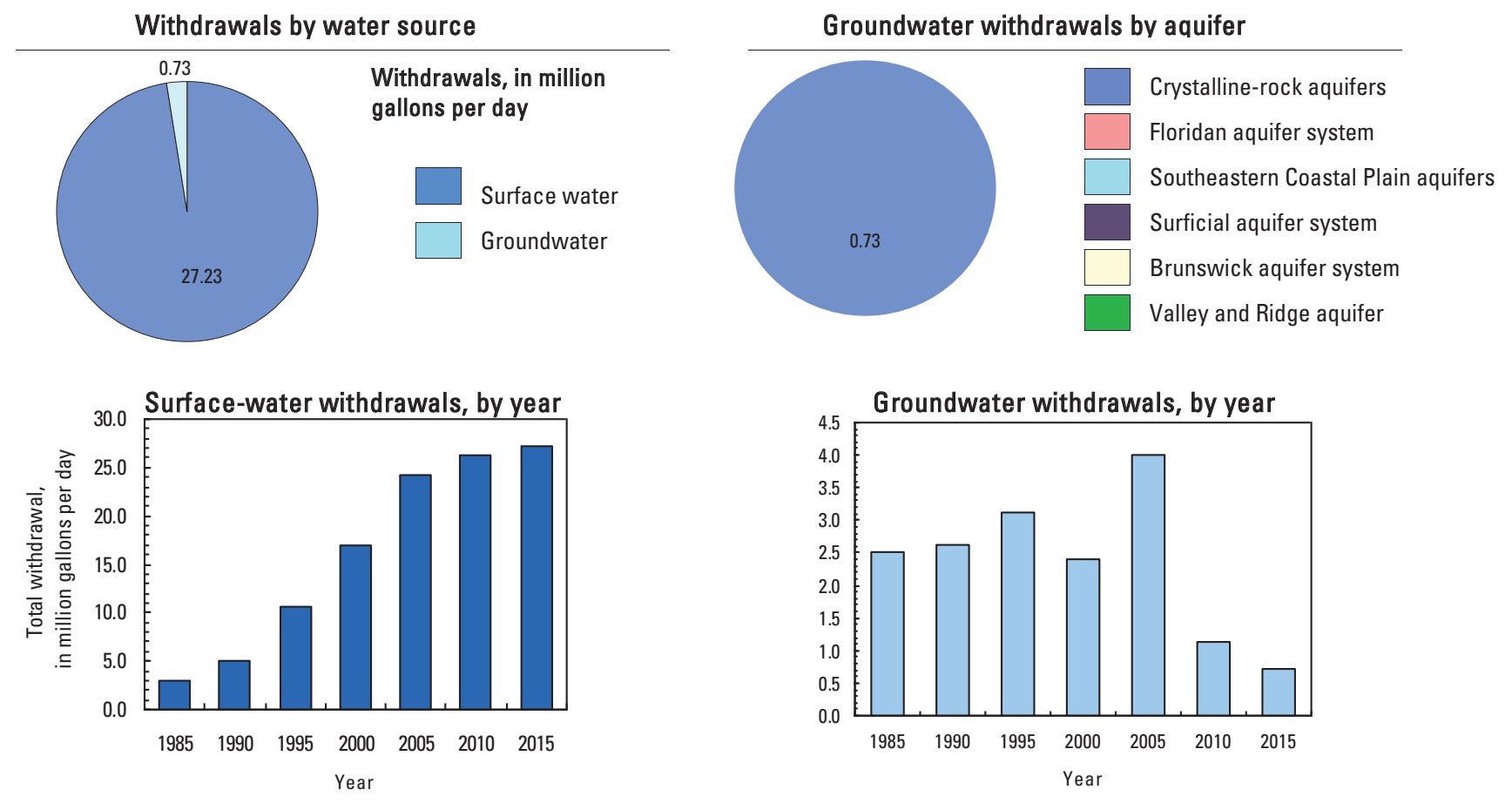


\section{FRANKLIN COUNTY}

Population

Population served by public supply-groundwater

Population served by public supply-surface water $\quad 12,452$

Acres irrigated

2015 WATER WITHDRAWALS AND ESTIMATED USE, IN MILLION GALLONS PER DAY

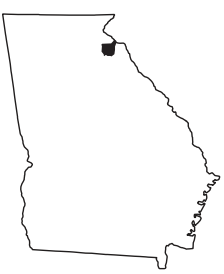

[--,not applicable; Mgal/d, million gallons per day]

\begin{tabular}{|c|c|c|c|c|c|c|c|c|}
\hline \multirow[b]{2}{*}{ Category } & \multicolumn{3}{|c|}{ Withdrawals } & \multirow[b]{2}{*}{$\begin{array}{c}\text { Total } \\
\text { use }\end{array}$} & \multirow[b]{2}{*}{$\begin{array}{l}\text { Returns to } \\
\text { Surface }\end{array}$} & \multicolumn{3}{|c|}{$\begin{array}{l}\text { Withdrawals by Major Industrial Groups } \\
\text { [NAICS, North American Industrial Classification System code] }\end{array}$} \\
\hline & $\begin{array}{c}\text { Ground- } \\
\text { water }\end{array}$ & $\begin{array}{c}\text { Surface } \\
\text { water }\end{array}$ & Total & & & NAICS & Groundwate & Surface wate \\
\hline Public supply & 0.59 & 1.36 & 1.95 & -- & -- & & & \\
\hline Domestic use & 0.46 & 0.00 & 0.46 & 1.36 & 0.00 & None & -- & -- \\
\hline Commercial use & 0.00 & 0.00 & 0.00 & 0.33 & 0.00 & & & \\
\hline Industrial use & 0.00 & 0.00 & 0.00 & 0.32 & 0.00 & & & \\
\hline Public-supply loss & -- & -- & -- & 0.40 & -- & & & \\
\hline Inter-county delivery & -- & -- & -- & -0.25 & -- & & & \\
\hline Public wastewater treatmen & nt -- & -- & -- & -- & 1.09 & & & \\
\hline Mining use & 0.00 & 0.00 & 0.00 & 0.00 & 0.00 & Withdrawals by $N$ & jor Public Sup & \\
\hline Irrigation (crop) & 0.07 & 0.00 & 0.07 & 0.07 & 0.00 & Name & Groundwater & Surface water \\
\hline Irrigation (golf) & 0.12 & 0.09 & 0.21 & 0.21 & 0.00 & City of Lavonia & 0.00 & 1.13 \\
\hline Livestock-aquaculture & 1.00 & 0.27 & 1.27 & 1.27 & 0.00 & The Franklin County & & \\
\hline Thermoelectric power & 0.00 & 0.00 & 0.00 & 0.00 & 0.00 & Public Utilities & 0.55 & 0.00 \\
\hline TOTAL & 2.24 & 1.72 & 3.96 & 3.71 & 1.09 & City of Royston & 0.00 & 0.23 \\
\hline
\end{tabular}

Total use is total withdrawal plus public supply deliveries and losses. An estimate of $0.12 \mathrm{Mgal} / \mathrm{d}$ was delivered from neighboring counties in 2015. An estimate of $0.37 \mathrm{Mgal} / \mathrm{d}$ was delivered from Franklin County to neighboring counties in 2015.

\section{Withdrawals by water source}

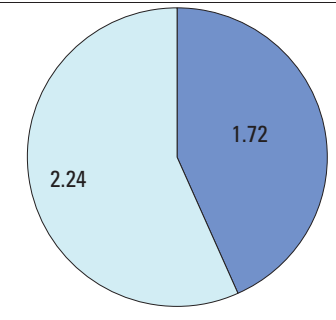

Withdrawals, in million gallons per day

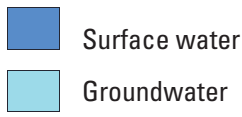

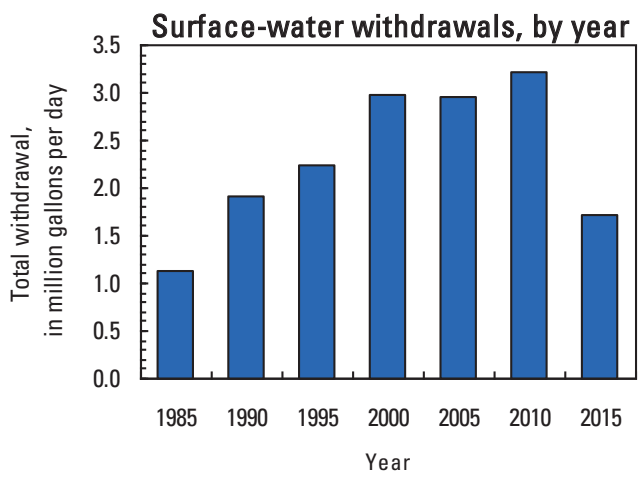

Groundwater withdrawals by aquifer

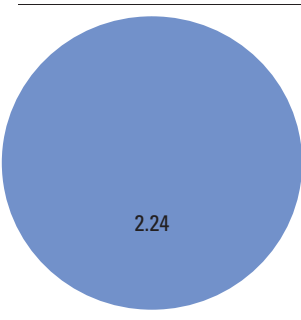

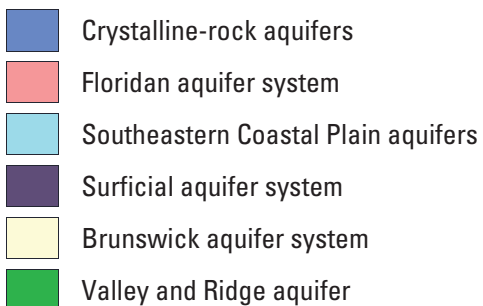

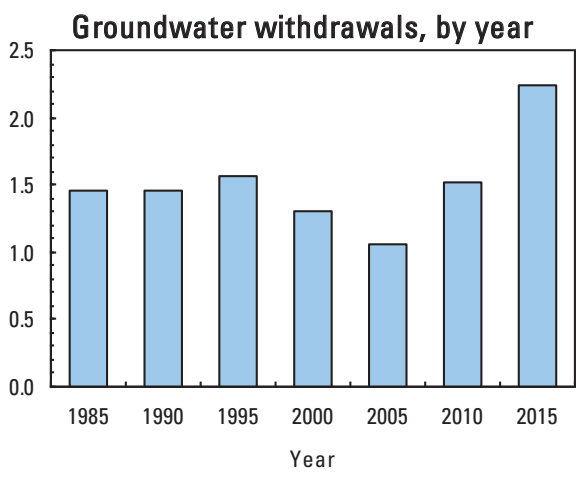




\section{FULTON COUNTY}

Population

$1,010,562$

Population served by public supply-groundwater

2,130

Population served by public supply-surface water $1,006,780$

Acres irrigated

2,180

2015 WATER WITHDRAWALS AND ESTIMATED USE. IN MILLION GALLONS PER DAY

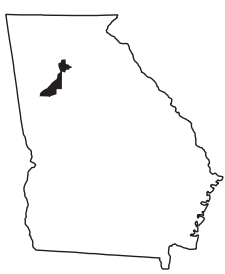

[--,not applicable; Mgal/d, million gallons per day]

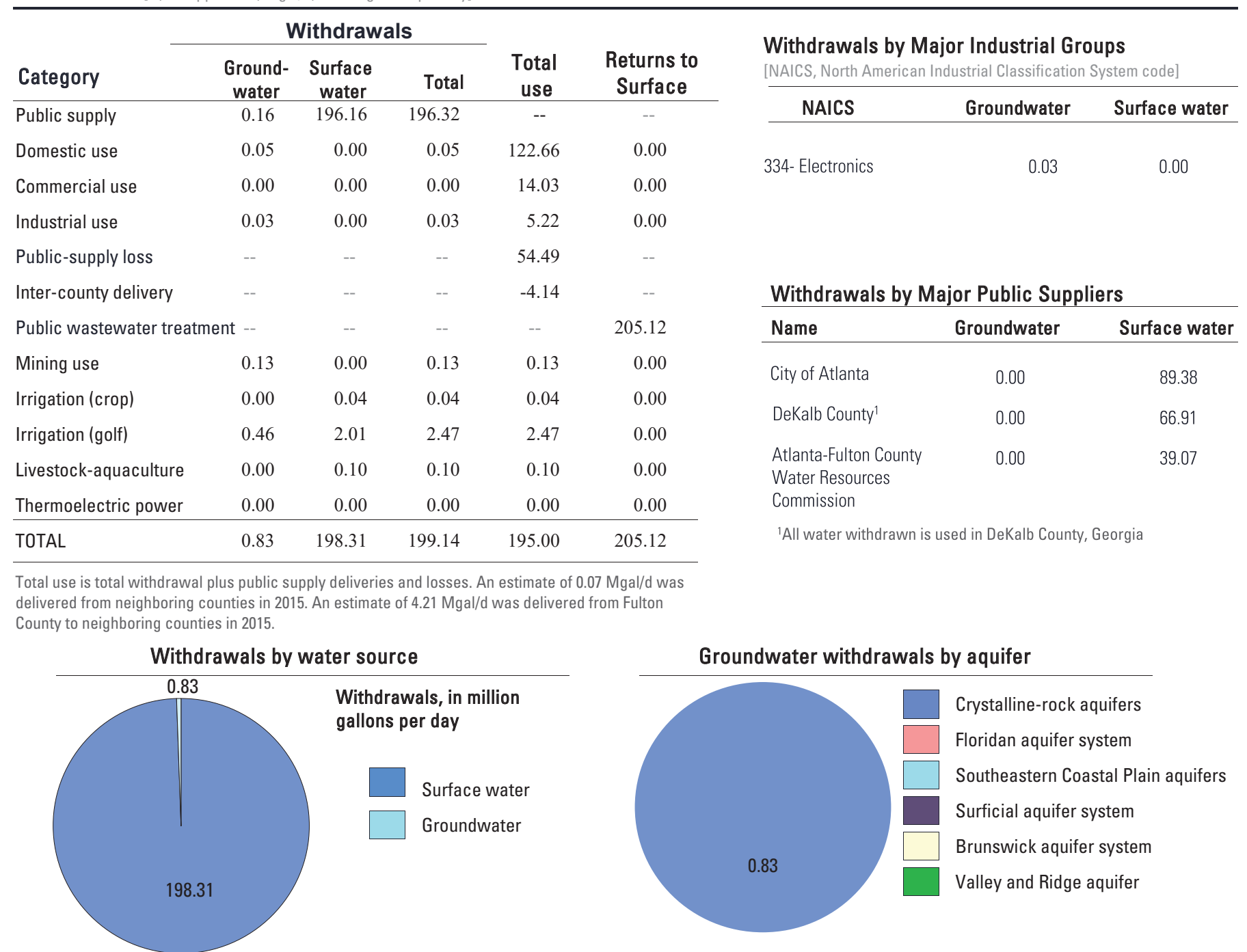
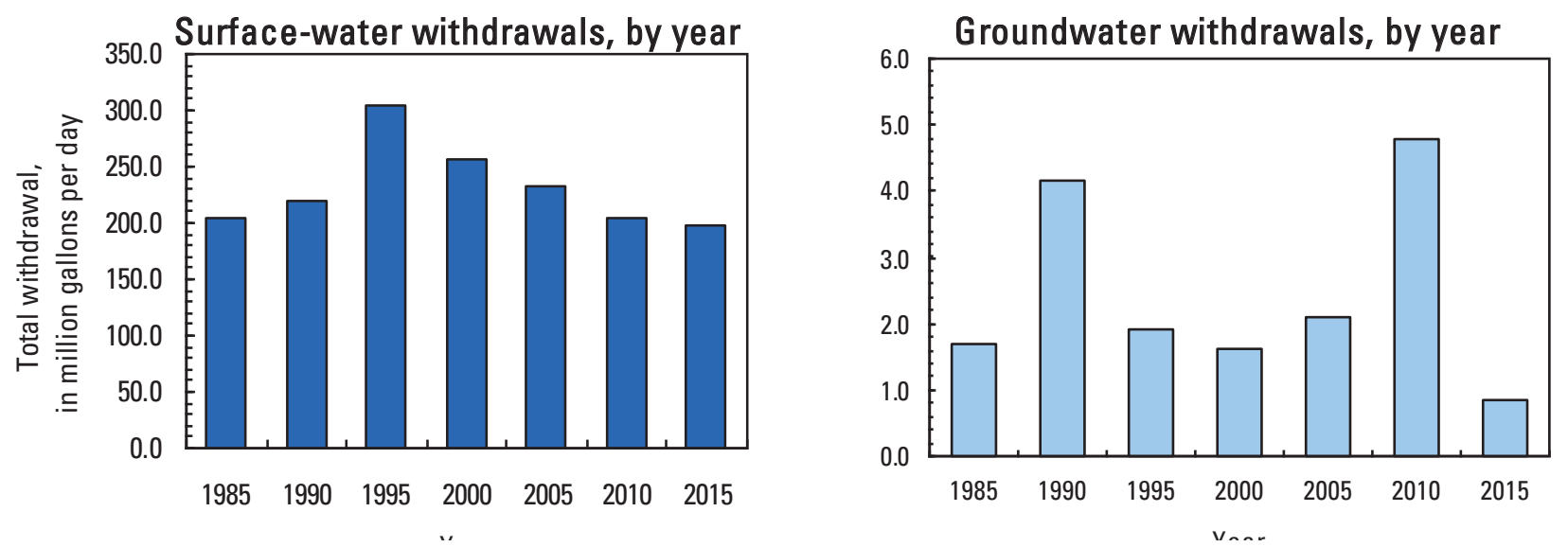


\section{GILMER COUNTY}

Population

Population served by public supply-groundwater

1,056

Population served by public supply-surface water $\quad 13,010$

Acres irrigated

200

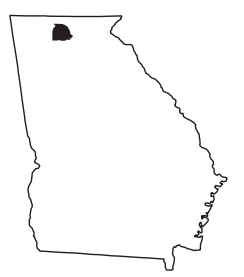

2015 WATER WITHDRAWALS AND ESTIMATED USE, IN MILLION GALLONS PER DAY

$[--$, not applicable; Mgal/d, million gallons per day]

\begin{tabular}{|c|c|c|c|c|c|}
\hline \multirow[b]{2}{*}{ Category } & \multicolumn{3}{|c|}{ Withdrawals } & \multirow[b]{2}{*}{$\begin{array}{c}\text { Total } \\
\text { use }\end{array}$} & \multirow[b]{2}{*}{$\begin{array}{c}\text { Returns to } \\
\text { Surface }\end{array}$} \\
\hline & $\begin{array}{l}\text { Ground- } \\
\text { water }\end{array}$ & $\begin{array}{c}\text { Surface } \\
\text { water }\end{array}$ & Total & & \\
\hline Public supply & 0.04 & 2.46 & 2.50 & -- & -- \\
\hline Domestic use & 1.15 & 0.00 & 1.15 & 1.71 & 0.00 \\
\hline Commercial use & 0.00 & 0.00 & 0.00 & 0.40 & 0.00 \\
\hline Industrial use & 2.48 & 0.00 & 2.48 & 3.79 & 0.00 \\
\hline Public-supply loss & -- & -- & -- & 0.23 & -- \\
\hline Inter-county delivery & -- & -- & -- & 0.19 & -- \\
\hline Public wastewater treatment & tt -- & -- & -- & -- & 4.62 \\
\hline Mining use & 0.08 & 0.00 & 0.08 & 0.08 & 0.00 \\
\hline Irrigation (crop) & 0.00 & 0.00 & 0.00 & 0.00 & 0.00 \\
\hline Irrigation (golf) & 0.00 & 0.21 & 0.21 & 0.21 & 0.00 \\
\hline Livestock-aquaculture & 0.28 & 0.53 & 0.81 & 0.81 & 0.00 \\
\hline Thermoelectric power & 0.00 & 0.00 & 0.00 & 0.00 & 0.00 \\
\hline TOTAL & 4.03 & 3.20 & 7.23 & 7.42 & 4.62 \\
\hline
\end{tabular}

Withdrawals by Major Industrial Groups

[NAICS, North American Industrial Classification System code]

NAICS Groundwater Surface water

212- Mining

1.14

0.00

327- Stone, clay

1.34

0.00

Withdrawals by Major Public Suppliers

Name Groundwater Surface water

Ellijay-Gilmer County

0.00

2.46

Water and Sewerage

Authority

Total use is total withdrawal plus public supply deliveries and losses. An estimate of $0.19 \mathrm{Mgal} / \mathrm{d}$ was delivered from neighboring counties in 2015.
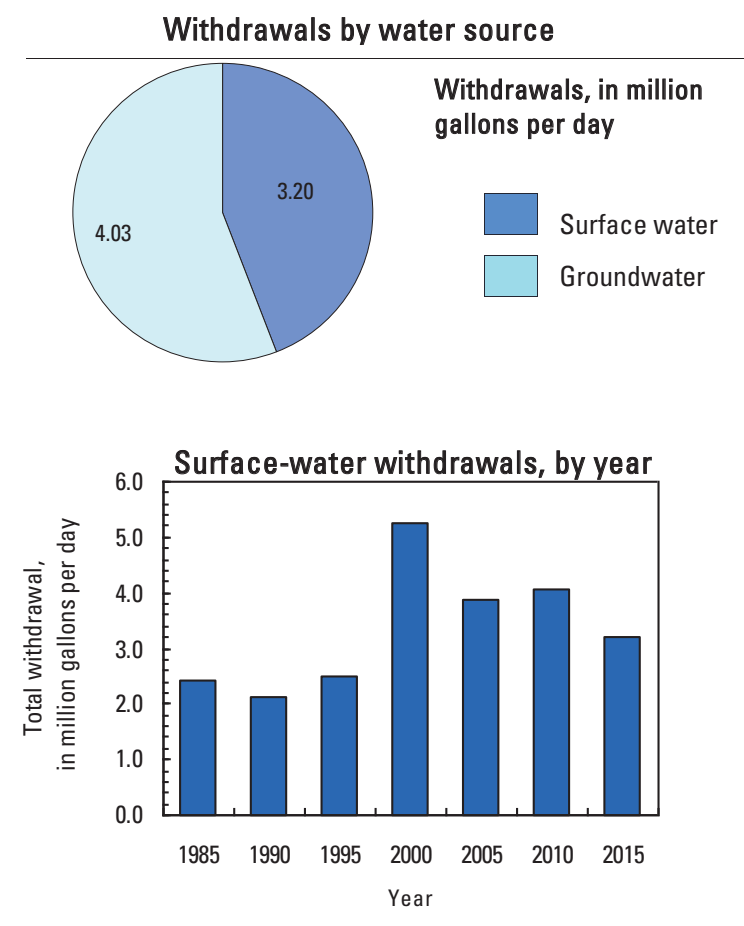

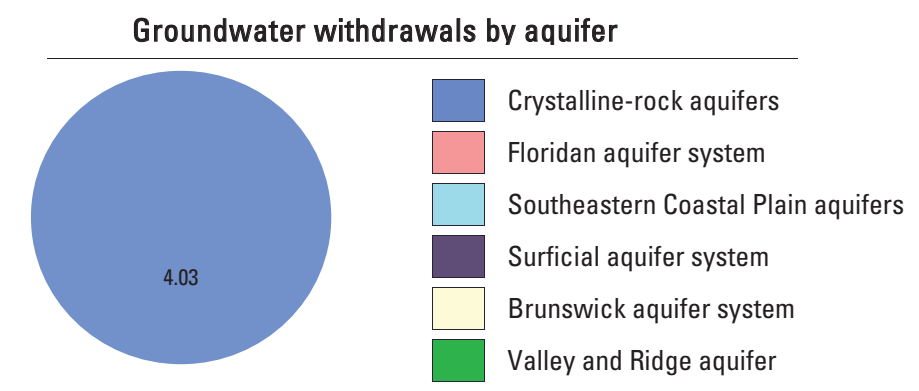




\section{GLASCOCK COUNTY}

Population

Population served by public supply-groundwater

Population served by public supply-surface water

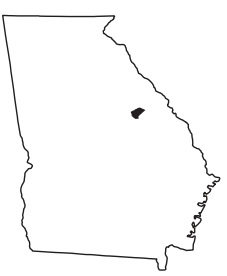

2015 WATER WITHDRAWALS AND ESTIMATED USE, IN MILLION GALLONS PER DAY

$[--$, not applicable; Mgal/d, million gallons per day]

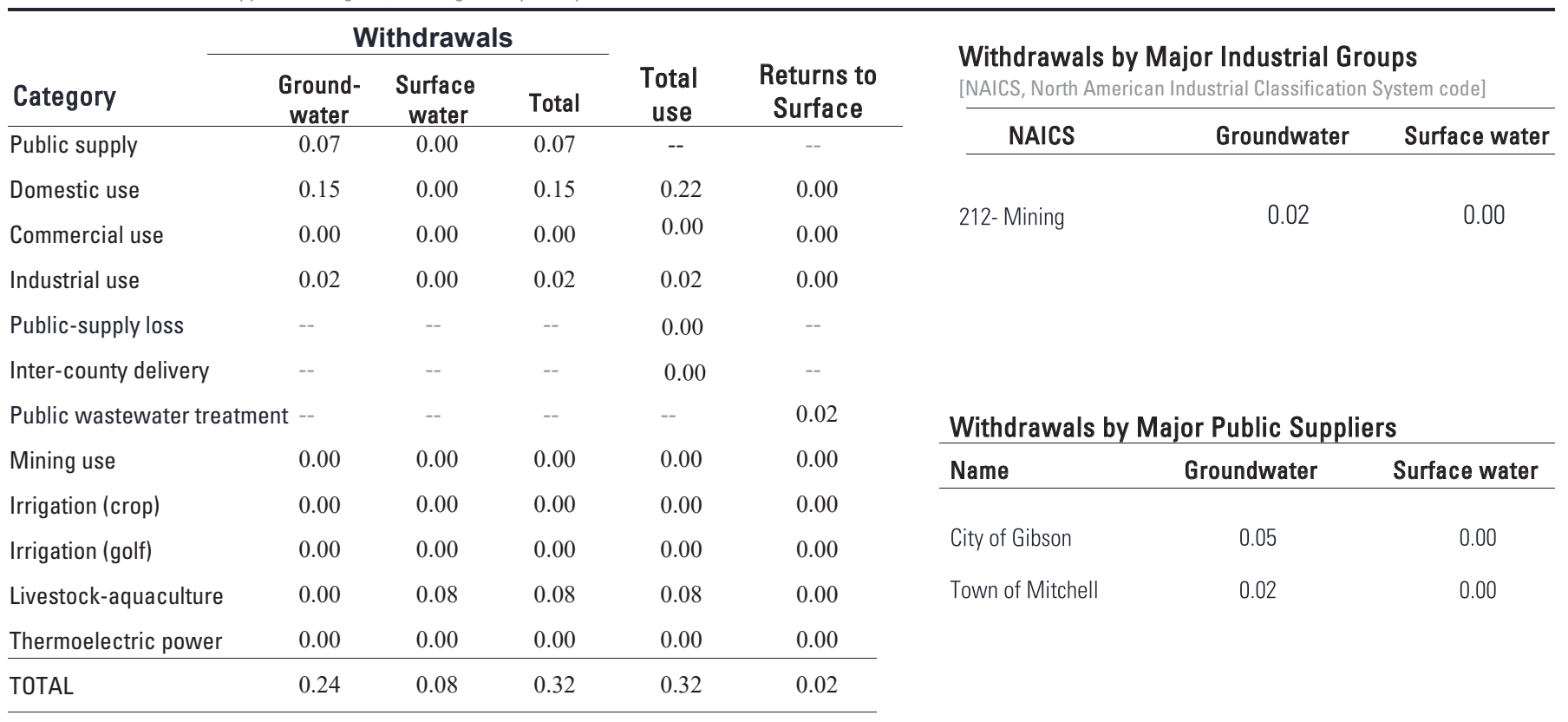

Total use is total withdrawal plus public supply deliveries and losses.

Withdrawals by water source

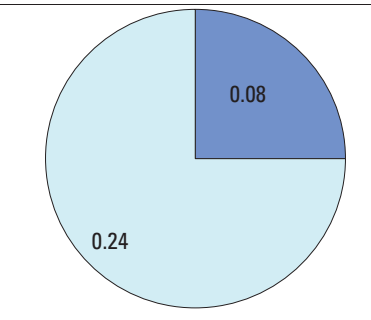

Withdrawals, in million gallons per day

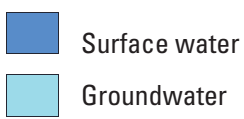

Groundwater
Groundwater withdrawals by aquifer
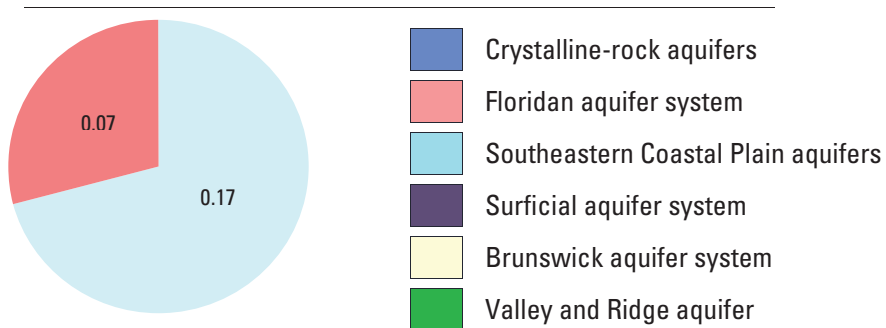
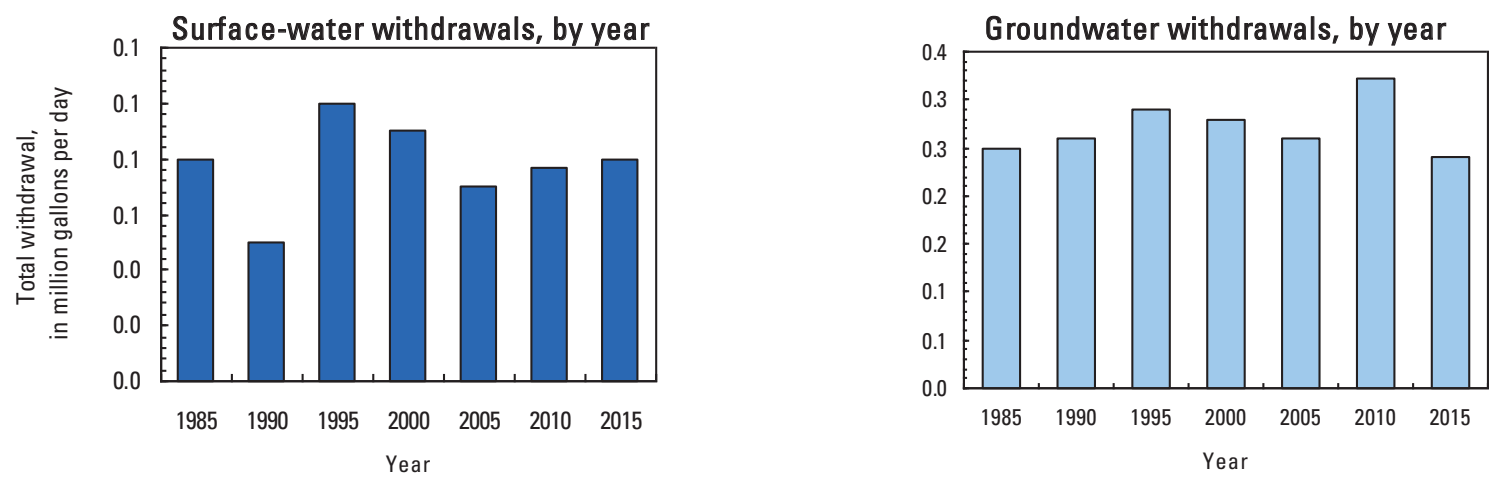


\section{GLYNN COUNTY}

Population

Population served by public supply-groundwater

Population served by public supply-surface water

0

Acres irrigated

2015 WATER WITHDRAWALS AND ESTIMATED USE, IN MILLION GALLONS PER DAY

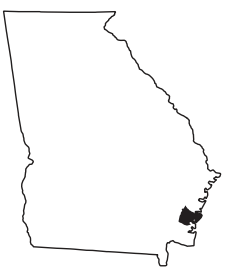

[--,not applicable; Mgal/d, million gallons per day]

\begin{tabular}{|c|c|c|c|c|c|}
\hline \multirow[b]{2}{*}{ Category } & \multicolumn{3}{|c|}{ Withdrawals } & \multirow[b]{2}{*}{$\begin{array}{c}\text { Total } \\
\text { use }\end{array}$} & \multirow[b]{2}{*}{$\begin{array}{c}\text { Returns to } \\
\text { Surface }\end{array}$} \\
\hline & $\begin{array}{c}\text { Ground- } \\
\text { water }\end{array}$ & $\begin{array}{c}\text { Surface } \\
\text { water }\end{array}$ & Total & & \\
\hline Public supply & 9.31 & 0.00 & 9.31 & -- & -- \\
\hline Domestic use & 0.71 & 0.00 & 0.71 & 6.00 & 0.00 \\
\hline Commercial use & 0.06 & 0.00 & 0.06 & 2.40 & 0.00 \\
\hline Industrial use & 29.53 & 0.00 & 29.53 & 29.75 & 0.00 \\
\hline Public-supply loss & -- & -- & -- & 1.46 & -- \\
\hline Inter-county delivery & -- & -- & -- & 0.00 & -- \\
\hline Public wastewater treatmen & nt -- & -- & -- & -- & 50.97 \\
\hline Mining use & 0.00 & 0.00 & 0.00 & 0.00 & 0.00 \\
\hline Irrigation (crop) & 0.00 & 0.00 & 0.00 & 0.00 & 0.00 \\
\hline Irrigation (golf) & 2.08 & 0.16 & 2.24 & 2.24 & 0.00 \\
\hline Livestock-aquaculture & 0.02 & 0.00 & 0.02 & 0.02 & 0.00 \\
\hline Thermoelectric power & 0.01 & 1.62 & 1.63 & 1.63 & 0.00 \\
\hline TOTAL & 41.72 & 1.78 & 43.50 & 43.50 & 50.97 \\
\hline
\end{tabular}

Withdrawals by Major Industrial Groups

[NAICS, North American Industrial Classification System code]

NAICS Groundwater Surface water

221- Electrical utility

0.00

311- Food

$\begin{array}{rr}0.01 & 0.00 \\ 0.27 & 0.00 \\ 0.01 & 0.00 \\ 24.66 & 0.00 \\ 4.59 & 0.00\end{array}$

Withdrawals by Major Public Suppliers

\begin{tabular}{lcc} 
Name & Groundwater & Surface water \\
\hline $\begin{array}{l}\text { Brunswick-Glynn County } \\
\text { Joint Water \& Sewer }\end{array}$ & 7.26 & 0.00 \\
Commission & & \\
Jekyll Island Authority & 0.58 & 0.00 \\
W \& D Utilities & 0.82 & 0.00
\end{tabular}

Total use is total withdrawal plus public supply deliveries and losses.

Withdrawals by water source

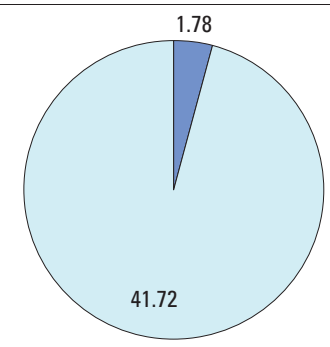

Withdrawals, in million

gallons per day

Surface water

Groundwater

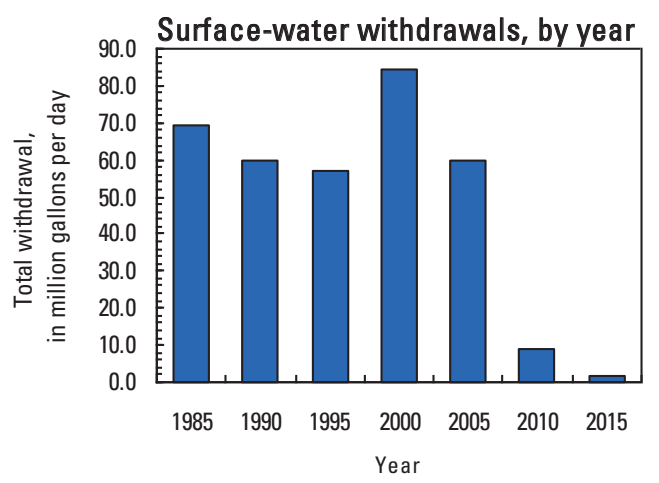

Groundwater withdrawals by aquifer
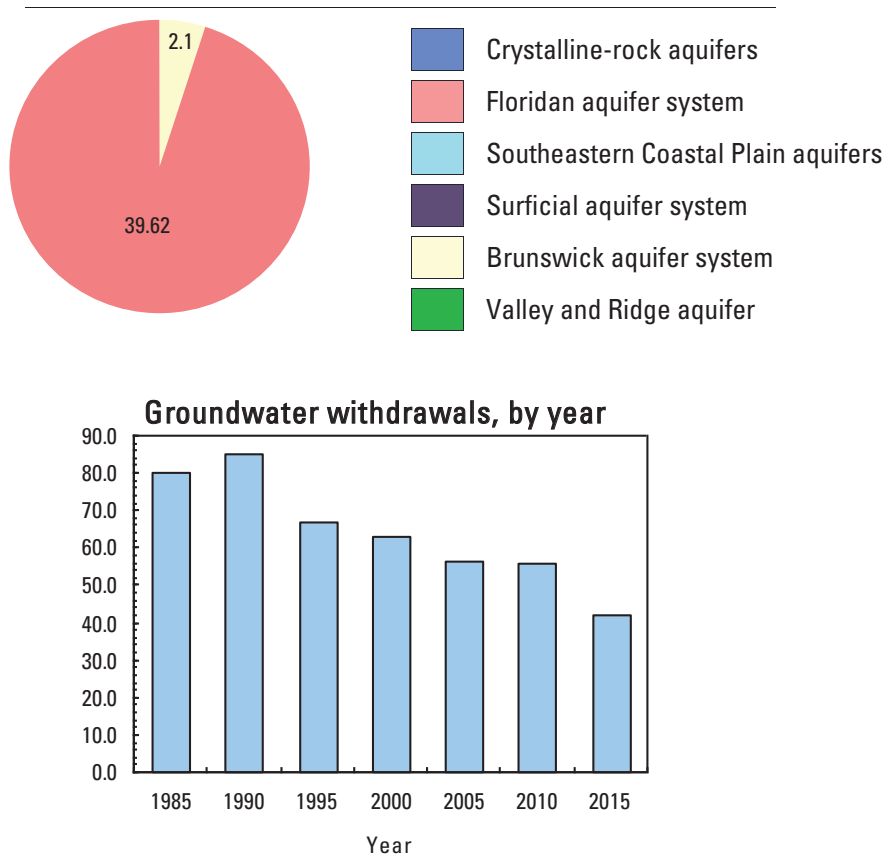


\section{GORDON COUNTY}

Population

Population served by public supply-groundwater

Population served by public supply-surface water $\quad 34,632$

Acres irrigated

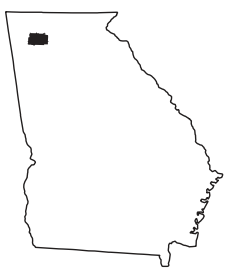

2015 WATER WITHDRAWALS AND ESTIMATED USE, IN MILLION GALLONS PER DAY

$[--$, not applicable; Mgal/d, million gallons per day]

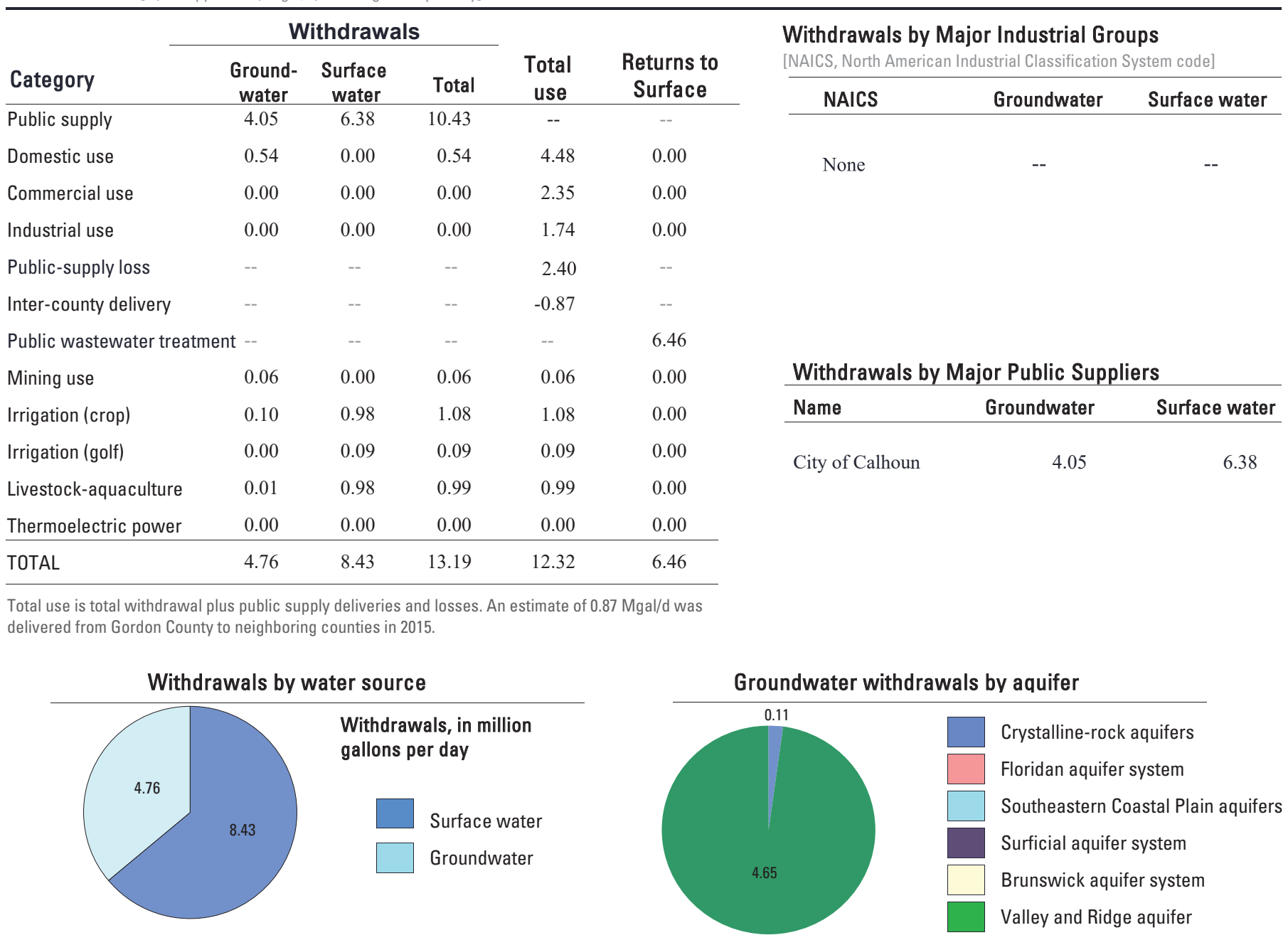
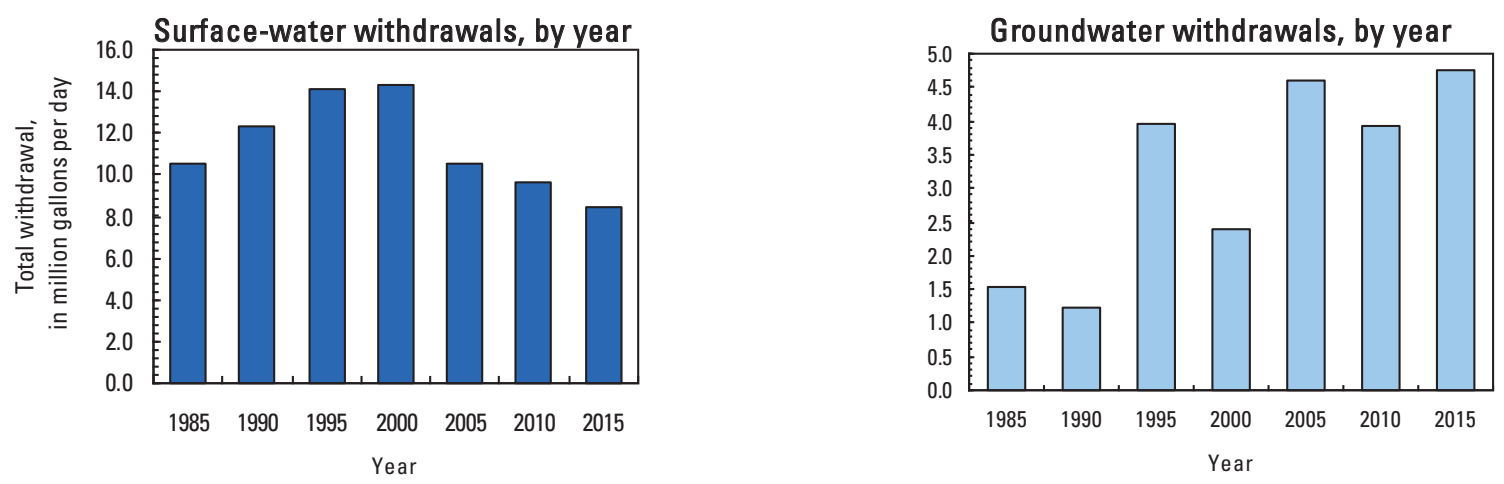


\section{GRADY COUNTY}

Population

Population served by public supply-groundwater

11,728

Population served by public supply-surface water

0

Acres irrigated

2015 WATER WITHDRAWALS AND ESTIMATED USE, IN MILLION GALLONS PER DAY

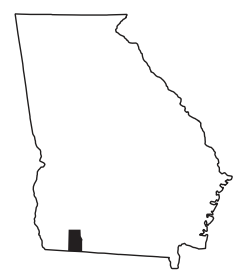

[--,not applicable; Mgal/d, million gallons per day]

\begin{tabular}{|c|c|c|c|c|c|c|c|c|}
\hline \multirow{3}{*}{ Category } & \multicolumn{3}{|c|}{ Withdrawals } & \multirow{3}{*}{$\begin{array}{c}\text { Total } \\
\text { use }\end{array}$} & \multirow{3}{*}{$\begin{array}{c}\text { Returns to } \\
\text { Surface }\end{array}$} & \multirow{2}{*}{\multicolumn{3}{|c|}{$\begin{array}{l}\text { Withdrawals by Major Industrial Groups } \\
\text { [NAICS, North American Industrial Classification System code] }\end{array}$}} \\
\hline & \multirow{2}{*}{$\begin{array}{l}\text { Ground- } \\
\text { water }\end{array}$} & \multirow{2}{*}{$\begin{array}{c}\text { Surface } \\
\text { water }\end{array}$} & \multirow[b]{2}{*}{ Total } & & & & & \\
\hline & & & & & & NAICS & Groundwater & Surface water \\
\hline Public supply & 1.73 & 0.00 & 1.73 & -- & -- & & & \\
\hline Domestic use & 1.01 & 0.00 & 1.01 & 2.22 & 0.00 & None & -- & -- \\
\hline Commercial use & 0.00 & 0.00 & 0.00 & 0.27 & 0.00 & & & \\
\hline Industrial use & 0.00 & 0.00 & 0.00 & 0.00 & 0.00 & & & \\
\hline Public-supply loss & -- & -- & -- & 0.25 & -- & & & \\
\hline Inter-county delivery & -- & -- & -- & 0.00 & -- & & & \\
\hline Public wastewater treatment & $\mathrm{t}--$ & -- & -- & -- & 1.95 & & & \\
\hline Mining use & 0.00 & 0.00 & 0.00 & 0.00 & 0.00 & Withdrawa & jor Public Sup & \\
\hline Irrigation (crop) & 3.59 & 1.84 & 5.43 & 5.43 & 0.00 & Name & Groundwater & Surface water \\
\hline Irrigation (golf) & 0.10 & 0.00 & 0.10 & 0.10 & 0.00 & City of Cairo & 1.58 & 0.00 \\
\hline Livestock-aquaculture & 0.31 & 0.16 & 0.47 & 0.47 & 0.00 & & & \\
\hline Thermoelectric power & 0.00 & 0.00 & 0.00 & 0.00 & 0.00 & & & \\
\hline TOTAL & 6.74 & 2.00 & 8.74 & 8.74 & 1.95 & & & \\
\hline
\end{tabular}

Total use is total withdrawal plus public supply deliveries and losses.

Withdrawals by water source

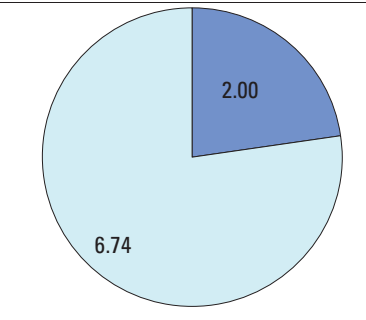

Withdrawals, in million gallons per day

Surface water

Groundwater
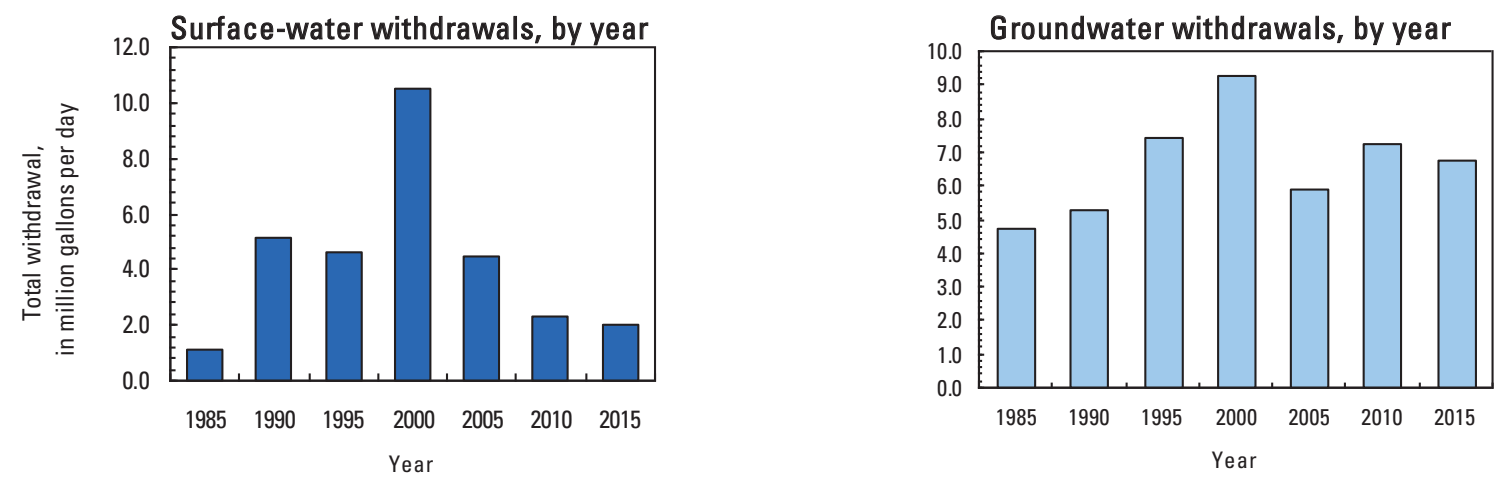


\section{GREENE COUNTY}

Population

Population served by public supply-groundwater

Population served by public supply-surface water $\quad 5,270$

Acres irrigated

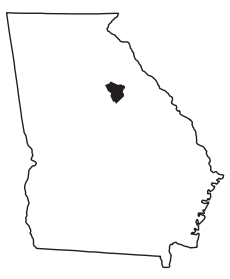

2015 WATER WITHDRAWALS AND ESTIMATED USE, IN MILLION GALLONS PER DAY

[--,not applicable; Mgal/d, million gallons per day]

\begin{tabular}{|c|c|c|c|c|c|}
\hline \multirow[b]{2}{*}{ Category } & \multicolumn{3}{|c|}{ Withdrawals } & \multirow[b]{2}{*}{$\begin{array}{c}\text { Total } \\
\text { use }\end{array}$} & \multirow[b]{2}{*}{$\begin{array}{c}\text { Returns to } \\
\text { Surface }\end{array}$} \\
\hline & $\begin{array}{l}\text { Ground- } \\
\text { water }\end{array}$ & $\begin{array}{c}\text { Surface } \\
\text { water }\end{array}$ & Total & & \\
\hline Public supply & 0.37 & 0.86 & 1.23 & -- & -- \\
\hline Domestic use & 0.49 & 0.00 & 0.49 & 1.46 & 0.00 \\
\hline Commercial use & 0.00 & 0.00 & 0.00 & 0.14 & 0.00 \\
\hline Industrial use & 0.00 & 0.00 & 0.00 & 0.00 & 0.00 \\
\hline Public-supply loss & -- & -- & -- & 0.12 & -- \\
\hline Inter-county delivery & -- & -- & -- & 0.00 & -- \\
\hline Public wastewater treatment & nt -- & -- & -- & -- & 0.09 \\
\hline Mining use & 0.10 & 0.00 & 0.10 & 0.10 & 0.00 \\
\hline Irrigation (crop) & 0.26 & 0.00 & 0.26 & 0.26 & 0.00 \\
\hline Irrigation (golf) & 0.00 & 0.88 & 0.88 & 0.88 & 0.00 \\
\hline Livestock-aquaculture & 0.48 & 0.00 & 0.48 & 0.48 & 0.00 \\
\hline Thermoelectric power & 0.00 & 0.00 & 0.00 & 0.00 & 0.00 \\
\hline TOTAL & 1.70 & 1.74 & 3.44 & 3.44 & 0.09 \\
\hline
\end{tabular}

Withdrawals by Major Industrial Groups

[NAICS, North American Industrial Classification System code]

\begin{tabular}{lll}
\hline NAICS & Groundwater & Surface water \\
\hline
\end{tabular}

None

Withdrawals by Major Public Suppliers

Name Groundwater Surface water

$\begin{array}{lll}\text { City of Greensboro } & 0.00 & 0.68\end{array}$

City of Union Point $\quad 0.00 \quad 0.18$

$\begin{array}{lll}\text { Piedmont Water Co } & 0.07 & 0.00\end{array}$

Total use is total withdrawal plus public supply deliveries and losses.

Withdrawals by water source

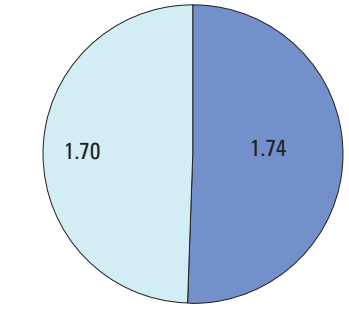

Withdrawals, in million gallons per day

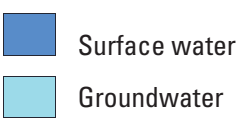

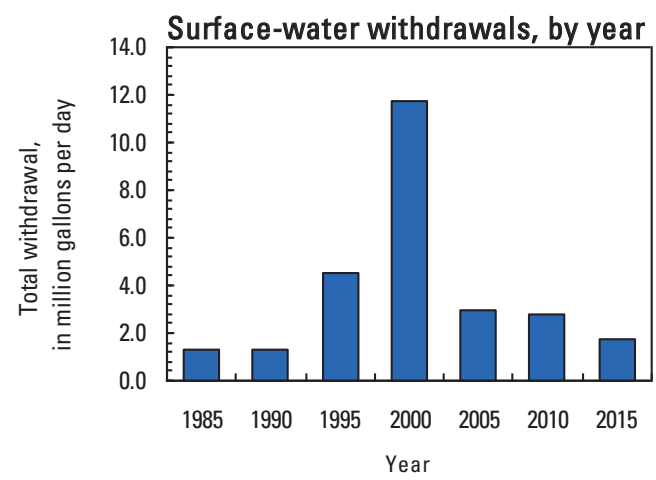

Groundwater withdrawals by aquifer

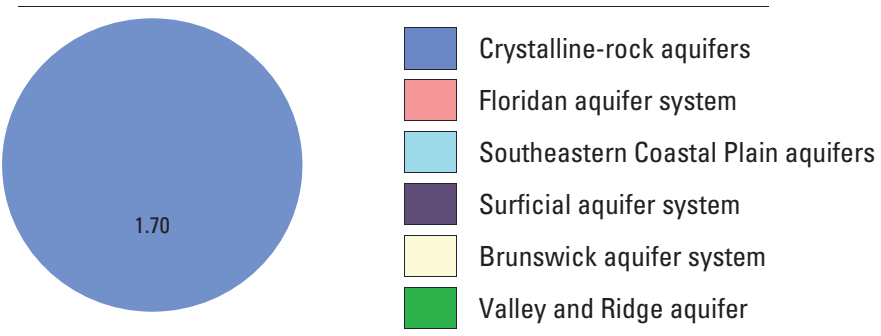

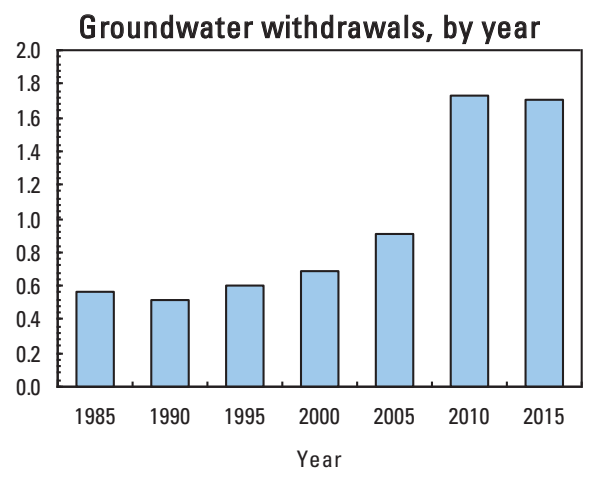




\section{GWINNETT COUNTY}

Population

895,823

Population served by public supply-groundwater

Population served by public supply-surface water 890,680

Acres irrigated

2015 WATER WITHDRAWALS AND ESTIMATED USE, IN MILLION GALLONS PER DAY

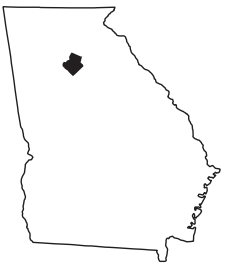

[--,not applicable; Mgal/d, million gallons per day]

\begin{tabular}{|c|c|c|c|c|c|}
\hline \multirow[b]{2}{*}{ Category } & \multicolumn{3}{|c|}{ Withdrawals } & \multirow[b]{2}{*}{$\begin{array}{c}\text { Total } \\
\text { use }\end{array}$} & \multirow[b]{2}{*}{$\begin{array}{c}\text { Returns to } \\
\text { Surface }\end{array}$} \\
\hline & $\begin{array}{l}\text { Ground- } \\
\text { water }\end{array}$ & $\begin{array}{c}\text { Surface } \\
\text { water }\end{array}$ & Total & & \\
\hline Public supply & 0.64 & 0.00 & 0.64 & -- & -- \\
\hline Domestic use & 0.00 & 0.00 & 0.00 & 51.2 & 0.00 \\
\hline Commercial use & 0.00 & 0.00 & 0.00 & 21.2 & 0.00 \\
\hline Industrial use & 0.00 & 0.00 & 0.00 & 0.46 & 0.00 \\
\hline Public-supply loss & -- & -- & -- & -72.22 & -- \\
\hline Inter-county delivery & -- & -- & -- & 70.07 & -- \\
\hline Public wastewater treatment & nt -- & -- & -- & -- & 51.52 \\
\hline Mining use & 0.24 & 0.01 & 0.25 & 0.25 & 0.00 \\
\hline Irrigation (crop) & 0.02 & 0.00 & 0.02 & 0.02 & 0.00 \\
\hline Irrigation (golf) & 0.04 & 1.28 & 1.32 & 1.32 & 0.00 \\
\hline Livestock-aquaculture & 0.00 & 0.00 & 0.00 & 0.00 & 0.00 \\
\hline Thermoelectric power & 0.00 & 0.00 & 0.00 & 0.00 & 0.00 \\
\hline TOTAL & 0.94 & 1.29 & 2.23 & 72.30 & 51.52 \\
\hline
\end{tabular}

Withdrawals by Major Industrial Groups

[NAICS, North American Industrial Classification System code]

NAICS Groundwater Surface water

None

Withdrawals by Major Public Suppliers'

Name Groundwater Surface water

City of Lawrenceville

0.48

0.00

${ }^{1}$ Gwinnett County Department of Water Resources withdrawls water from Lake Sidney Lanier at an intake location in Hall County

Total use is total withdrawal plus public supply deliveries and losses. An estimate of $70.07 \mathrm{Mgal} / \mathrm{d}$ was delivered from neighboring counties in 2015.
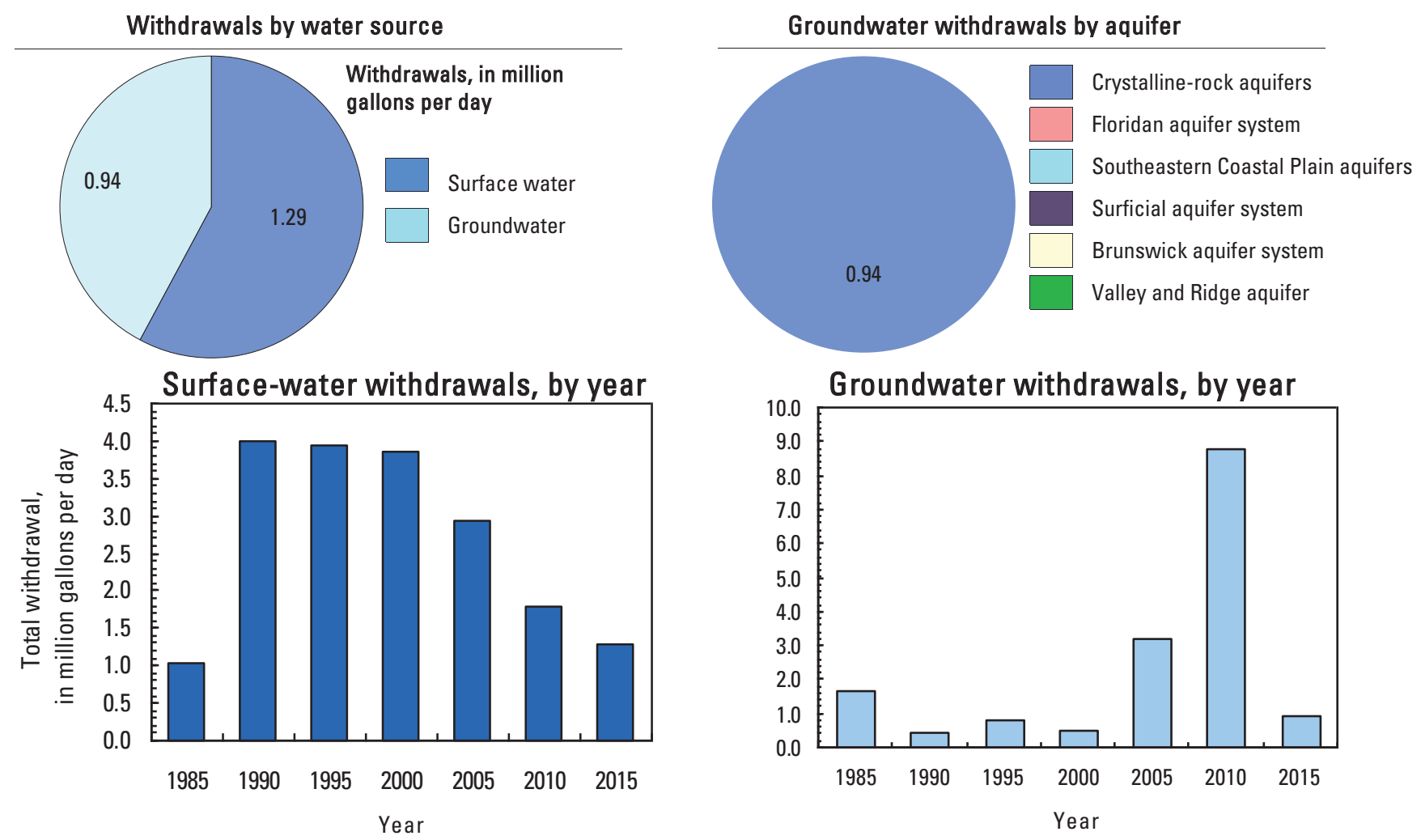


\section{HABERSHAM COUNTY}

Population

Population served by public supply-groundwater

Population served by public supply-surface water $\quad 20,128$

Acres irrigated

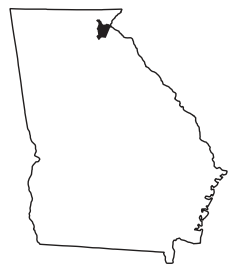

2015 WATER WITHDRAWALS AND ESTIMATED USE, IN MILLION GALLONS PER DAY

$[--$, not applicable; Mgal/d, million gallons per day]

\begin{tabular}{lccccc}
\hline & \multicolumn{3}{c}{ Withdrawals } & & Total \\
\cline { 2 - 4 } Category & $\begin{array}{c}\text { Ground- } \\
\text { water }\end{array}$ & $\begin{array}{c}\text { Surface } \\
\text { water }\end{array}$ & $\begin{array}{c}\text { Total } \\
\text { use }\end{array}$ & $\begin{array}{c}\text { Returns to } \\
\text { Surface }\end{array}$ \\
\hline Public supply & 0.72 & 4.94 & 5.66 & -- & -- \\
Domestic use & 0.60 & 0.00 & 0.60 & 3.46 & 0.00 \\
Commercial use & 0.00 & 0.00 & 0.00 & 0.58 & 0.00 \\
Industrial use & 0.00 & 0.00 & 0.00 & 1.51 & 0.00 \\
Public-supply loss & -- & -- & -- & 0.71 & -- \\
Inter-county delivery & -- & -- & -- & 0.12 & -- \\
Public wastewater treatment & -- & -- & -- & -- & 3.27 \\
Mining use & 0.02 & 0.00 & 0.02 & 0.02 & 0.00 \\
Irrigation (crop) & 0.02 & 0.20 & 0.22 & 0.22 & 0.00 \\
Irrigation (golf) & 0.00 & 0.00 & 0.00 & 0.00 & 0.00 \\
Livestock-aquaculture & 0.05 & 0.52 & 0.57 & 0.57 & 0.00 \\
Thermoelectric power & 0.00 & 0.00 & 0.00 & 0.00 & 0.00 \\
\hline TOTAL & 1.41 & 5.66 & 7.07 & 7.19 & 3.27 \\
\hline
\end{tabular}

Withdrawals by Major Industrial Groups

[NAICS, North American Industrial Classification System code]

\begin{tabular}{lll}
\hline NAICS & Groundwater & Surface water \\
\hline
\end{tabular}

Withdrawals by Major Public Suppliers

Name Groundwater Surface water

$\begin{array}{lll}\text { City of Alto } & 0.42 & 0.00 \\ \text { City of Baldwin } & 0.00 & 1.92 \\ \text { City of Clarkesville } & 0.00 & 0.55 \\ \text { City of Cornelia } & 0.14 & 2.47 \\ \text { City of Demorest } & 0.14 & 0.00\end{array}$

Total use is total withdrawal plus public supply deliveries and losses. An estimate of $0.12 \mathrm{Mgal} / \mathrm{d}$ was delivered from neighboring counties in 2015.

\section{Withdrawals by water source}

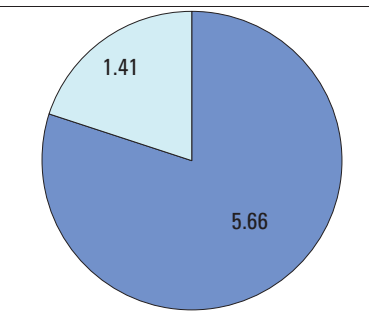

Withdrawals, in million

gallons per day

Surface water

Groundwater

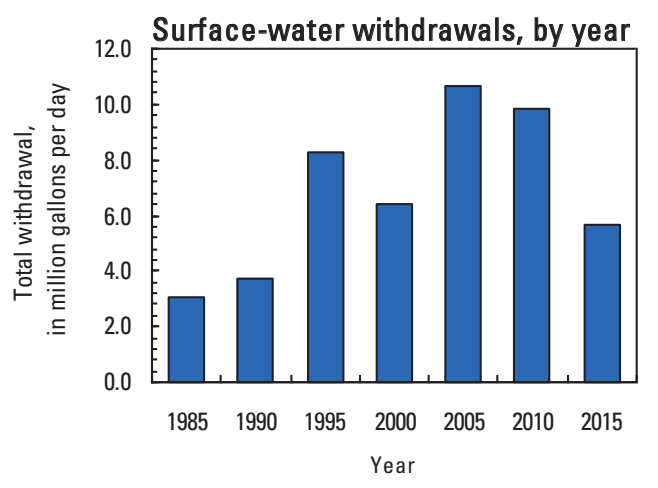

Groundwater withdrawals by aquifer
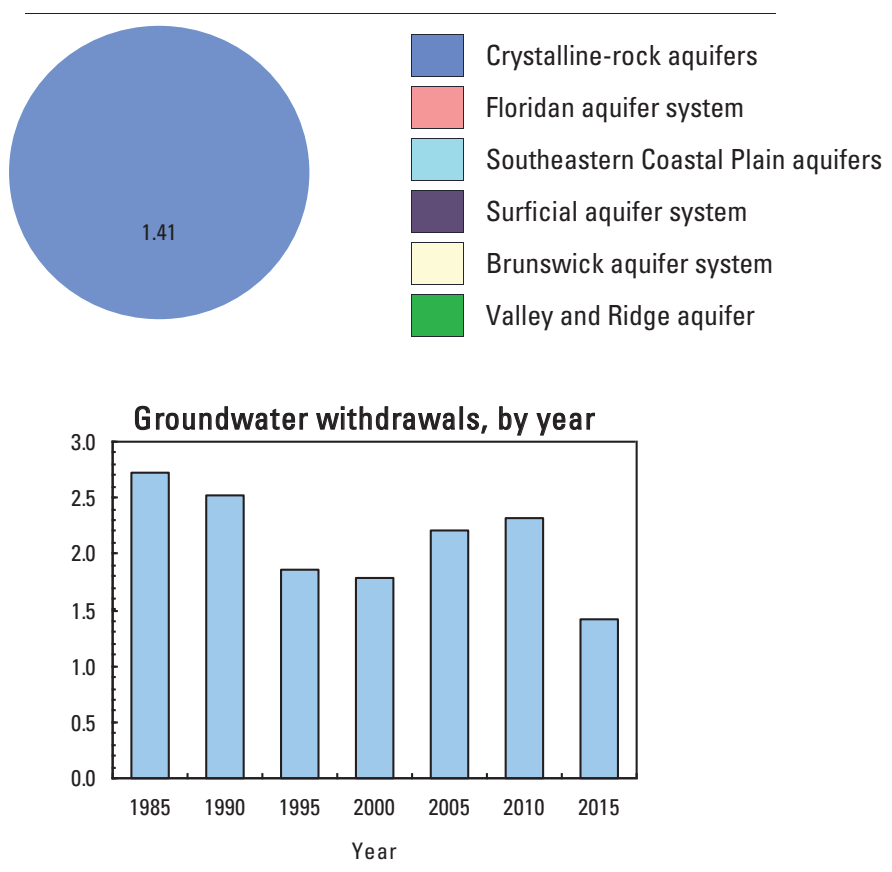


\section{HALL COUNTY}

Population

193,535

Population served by public supply-groundwater

7,593

Population served by public supply-surface water 147,263

Acres irrigated

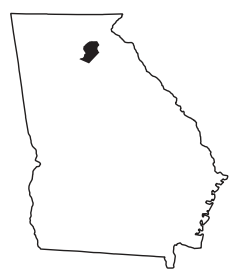

2015 WATER WITHDRAWALS AND ESTIMATED USE, IN MILLION GALLONS PER DAY

[--,not applicable; Mgal/d, million gallons per day]

\begin{tabular}{|c|c|c|c|c|c|}
\hline \multirow[b]{2}{*}{ Category } & \multicolumn{3}{|c|}{ Withdrawals } & \multirow[b]{2}{*}{$\begin{array}{c}\text { Total } \\
\text { use }\end{array}$} & \multirow[b]{2}{*}{$\begin{array}{c}\text { Returns to } \\
\text { Surface }\end{array}$} \\
\hline & $\begin{array}{c}\text { Ground- } \\
\text { water }\end{array}$ & $\begin{array}{c}\text { Surface } \\
\text { water }\end{array}$ & Total & & \\
\hline Public supply & 0.53 & 87.65 & 88.18 & -- & -- \\
\hline Domestic use & 1.06 & 0.00 & 1.06 & 6.32 & 0.00 \\
\hline Commercial use & 0.00 & 0.00 & 0.00 & 1.35 & 0.00 \\
\hline Industrial use & 0.39 & 0.05 & 0.44 & 3.46 & 0.00 \\
\hline Public-supply loss & -- & -- & -- & 78.55 & -- \\
\hline Inter-county delivery & -- & -- & -- & -70.07 & -- \\
\hline Public wastewater treat & ment -- & -- & -- & -- & 10.62 \\
\hline Mining use & 0.17 & 0.00 & 0.17 & 0.17 & 0.00 \\
\hline Irrigation (crop) & 0.06 & 0.03 & 0.09 & 0.09 & 0.00 \\
\hline Irrigation (golf) & 0.26 & 0.33 & 0.59 & 0.59 & 0.00 \\
\hline Livestock-aquaculture & 0.36 & 0.18 & 0.54 & 0.54 & 0.00 \\
\hline Thermoelectric power & 0.00 & 0.00 & 0.00 & 0.00 & 0.00 \\
\hline TOTAL & 2.83 & 88.24 & 91.07 & 18.00 & 10.62 \\
\hline
\end{tabular}

Total use is total withdrawal plus public supply deliveries and losses. An estimate of $70.07 \mathrm{Mgal} / \mathrm{d}$ was delivered from Hall County to neighboring counties in 2015.

Withdrawals by Major Industrial Groups

[NAICS, North American Industrial Classification System code]

\begin{tabular}{lcc}
\hline \multicolumn{1}{c}{ NAICS } & Groundwater & Surface water \\
\hline 311- Food & 0.39 & 0.00 \\
313- Textile mills & 0.00 & 0.05
\end{tabular}

Withdrawals by Major Public Suppliers

\begin{tabular}{lcc} 
Name & Groundwater & Surface water \\
\hline $\begin{array}{l}\text { Gwinnett Co. Department } \\
\text { of Water Resources }\end{array}$ & 0.00 & 68.69 \\
City of Gainesville & 0.00 & 17.58 \\
City of Buford & 0.00 & 1.38 \\
City of Flowery Branch & 0.22 & 0.00 \\
City of Lula & 0.17 & 0.00
\end{tabular}

City of Lula

0.17

0.00
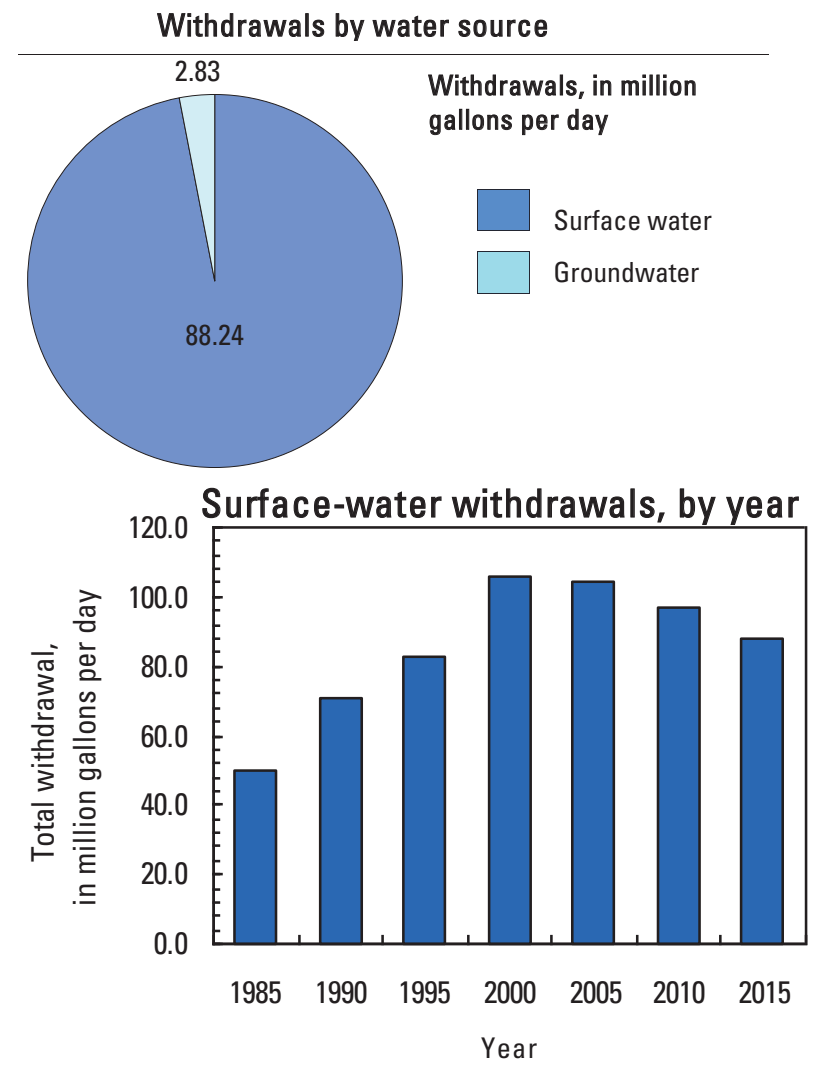
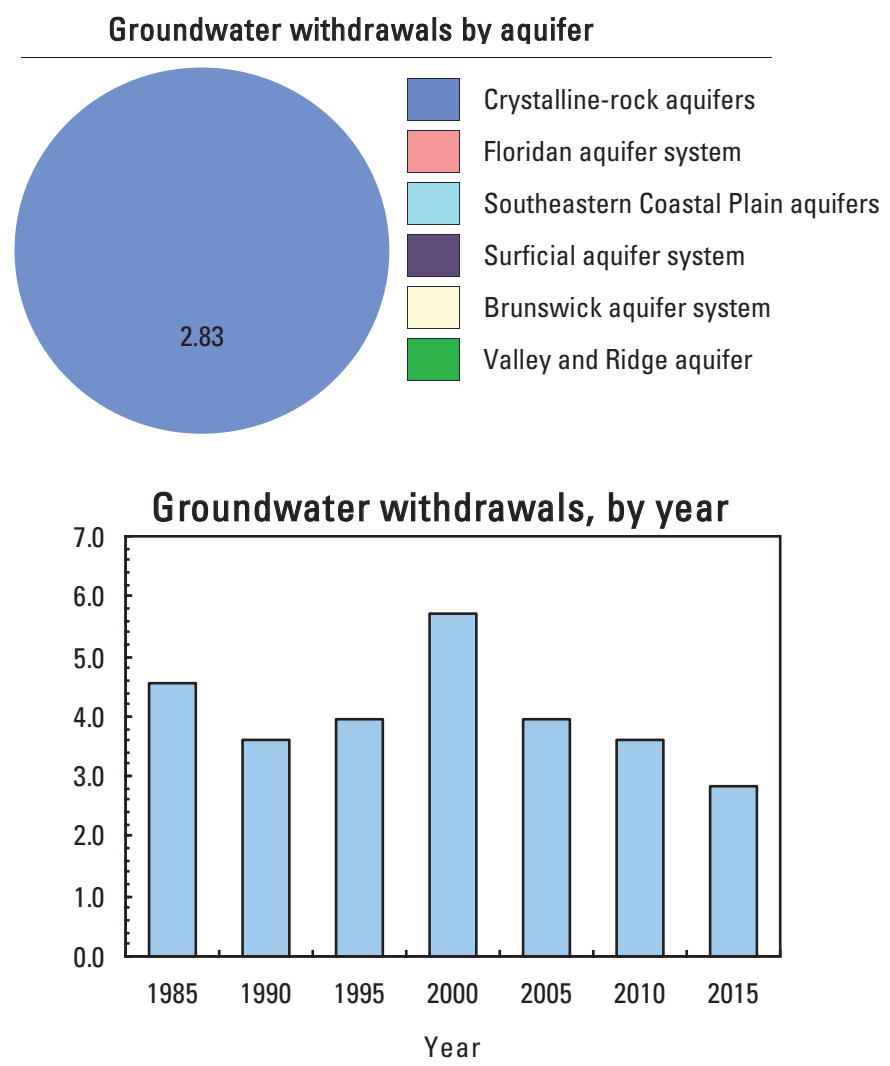


\section{HANCOCK COUNTY}

Population

Population served by public supply-groundwater

Population served by public supply-surface water $\quad 4,610$

Acres irrigated

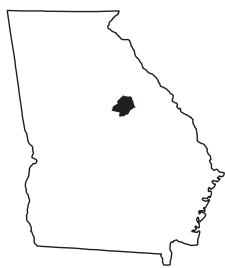

2015 WATER WITHDRAWALS AND ESTIMATED USE, IN MILLION GALLONS PER DAY

$[--$, not applicable; Mgal/d, million gallons per day]

\begin{tabular}{|c|c|c|c|c|c|}
\hline \multirow[b]{2}{*}{ Category } & \multicolumn{3}{|c|}{ Withdrawals } & \multirow[b]{2}{*}{$\begin{array}{c}\text { Total } \\
\text { use }\end{array}$} & \multirow[b]{2}{*}{$\begin{array}{c}\text { Returns to } \\
\text { Surface }\end{array}$} \\
\hline & $\begin{array}{l}\text { Ground- } \\
\text { water }\end{array}$ & $\begin{array}{c}\text { Surface } \\
\text { water }\end{array}$ & Total & & \\
\hline Public supply & 0.23 & 0.97 & 1.20 & -- & -- \\
\hline Domestic use & 0.06 & 0.00 & 0.06 & 0.51 & 0.00 \\
\hline Commercial use & 0.00 & 0.00 & 0.00 & 0.55 & 0.00 \\
\hline Industrial use & 0.00 & 0.00 & 0.00 & 0.00 & 0.00 \\
\hline Public-supply loss & -- & -- & -- & 0.20 & -- \\
\hline Inter-county delivery & -- & -- & -- & 0.00 & -- \\
\hline Public wastewater treatment & it -- & -- & -- & -- & 0.01 \\
\hline Mining use & 0.07 & 0.00 & 0.07 & 0.07 & 0.00 \\
\hline Irrigation (crop) & 0.45 & 0.11 & 0.56 & 0.56 & 0.00 \\
\hline Irrigation (golf) & 0.00 & 0.00 & 0.00 & 0.00 & 0.00 \\
\hline Livestock-aquaculture & 0.05 & 0.01 & 0.06 & 0.06 & 0.00 \\
\hline Thermoelectric power & 0.00 & 0.00 & 0.00 & 0.00 & 0.00 \\
\hline TOTAL & 0.86 & 1.09 & 1.95 & 1.95 & 0.01 \\
\hline
\end{tabular}

Withdrawals by Major Industrial Groups

[NAICS, North American Industrial Classification System code]

\begin{tabular}{lll}
\hline NAICS Groundwater & Surface water \\
\hline
\end{tabular}

None

Withdrawals by Major Public Suppliers

Name Groundwater Surface water

$\begin{array}{lll}\text { City of Sparta } & 0.00 & 0.97\end{array}$

Piedmont Water Company $\quad 0.17$

0.00

Total use is total withdrawal plus public supply deliveries and losses.

Withdrawals by water source

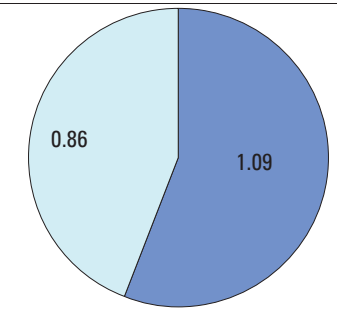

Withdrawals, in million gallons per day

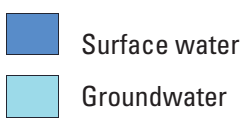

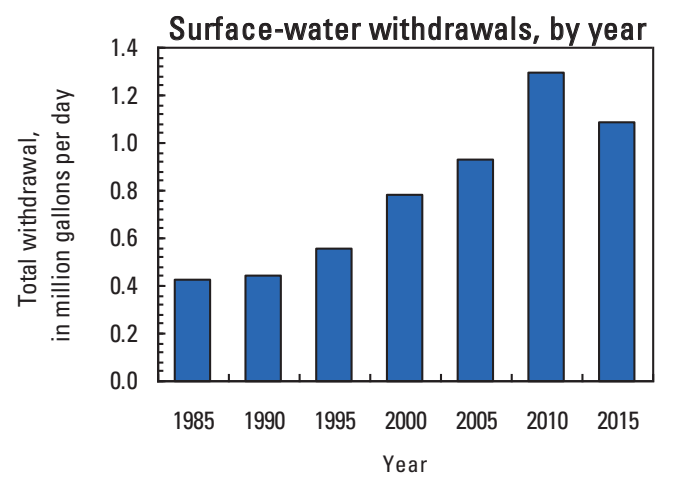

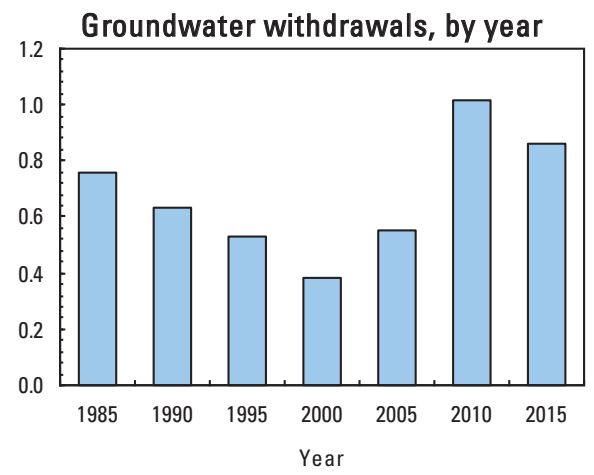




\section{HARALSON COUNTY}

Population

Population served by public supply-groundwater

Population served by public supply-surface water 23,234

Acres irrigated

2015 WATER WITHDRAWALS AND ESTIMATED USE, IN MILLION GALLONS PER DAY

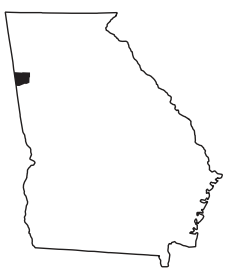

[--,not applicable; Mgal/d, million gallons per day]

\begin{tabular}{lccccc}
\hline & \multicolumn{3}{c}{ Withdrawals } & & \\
\cline { 2 - 4 } Category & $\begin{array}{c}\text { Ground } \\
\text { water }\end{array}$ & $\begin{array}{c}\text { Surface } \\
\text { water }\end{array}$ & Total & $\begin{array}{c}\text { Total } \\
\text { use }\end{array}$ & $\begin{array}{c}\text { Returns to } \\
\text { Surface }\end{array}$ \\
\hline Public supply & 0.11 & 2.42 & 2.53 & -- & -- \\
Domestic use & 0.38 & 0.00 & 0.38 & 1.98 & 0.00 \\
Commercial use & 0.00 & 0.00 & 0.00 & 0.46 & 0.00 \\
Industrial use & 0.00 & 0.00 & 0.00 & 0.05 & 0.00 \\
Public-supply loss & -- & -- & -- & 0.42 & -- \\
Inter-county delivery & -- & -- & -- & 0.20 & -- \\
Public wastewater treatment & -- & -- & -- & -- & 52.06 \\
Mining use & 0.00 & 0.00 & 0.00 & 0.00 & 0.00 \\
Irrigation (crop) & 0.00 & 0.00 & 0.00 & 0.00 & 0.00 \\
Irrigation (golf) & 0.00 & 0.14 & 0.14 & 0.14 & 0.00 \\
Livestock-aquaculture & 0.09 & 0.13 & 0.22 & 0.22 & 0.00 \\
Thermoelectric power & 0.00 & 0.00 & 0.00 & 0.00 & 0.00 \\
\hline TOTAL & 0.58 & 2.69 & 3.27 & 3.47 & 52.06 \\
\hline
\end{tabular}

Withdrawals by Major Industrial Groups

[NAICS, North American Industrial Classification System code]

NAICS Groundwater Surface water

None

Withdrawals by Major Public Suppliers

Name Groundwater Surface water

$\begin{array}{lll}\text { City of Bremen } & 0.00 & 0.27 \\ \text { Haralson County } & 0.11 & 2.15 \\ \text { Water Authority } & & \end{array}$

Total use is total withdrawal plus public supply deliveries and losses. An estimate of $0.2 \mathrm{Mgal} / \mathrm{d}$ was delivered from neighboring counties in 2015.

\section{Withdrawals by water source}

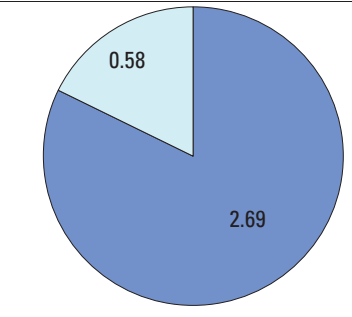

Withdrawals, in million gallons per day

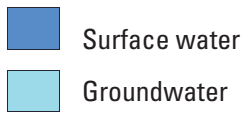

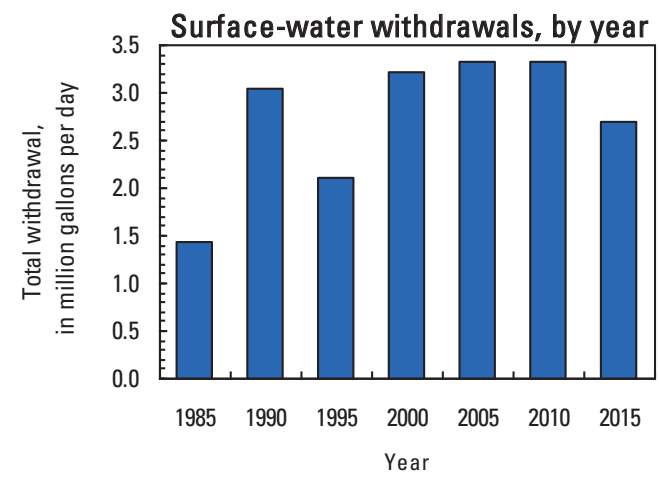

Groundwater withdrawals by aquifer

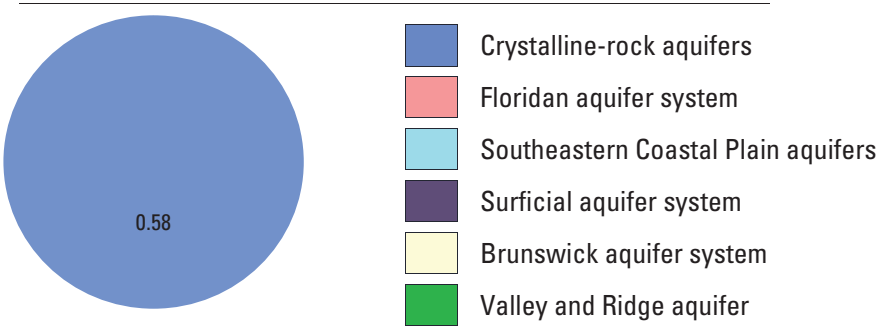




\section{HARRIS COUNTY}

Population

Population served by public supply-groundwater

Population served by public supply-surface water $\quad 20,132$

Acres irrigated

2015 WATER WITHDRAWALS AND ESTIMATED USE, IN MILLION GALLONS PER DAY

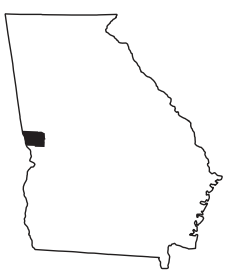

[--,not applicable; Mgal/d, million gallons per day]

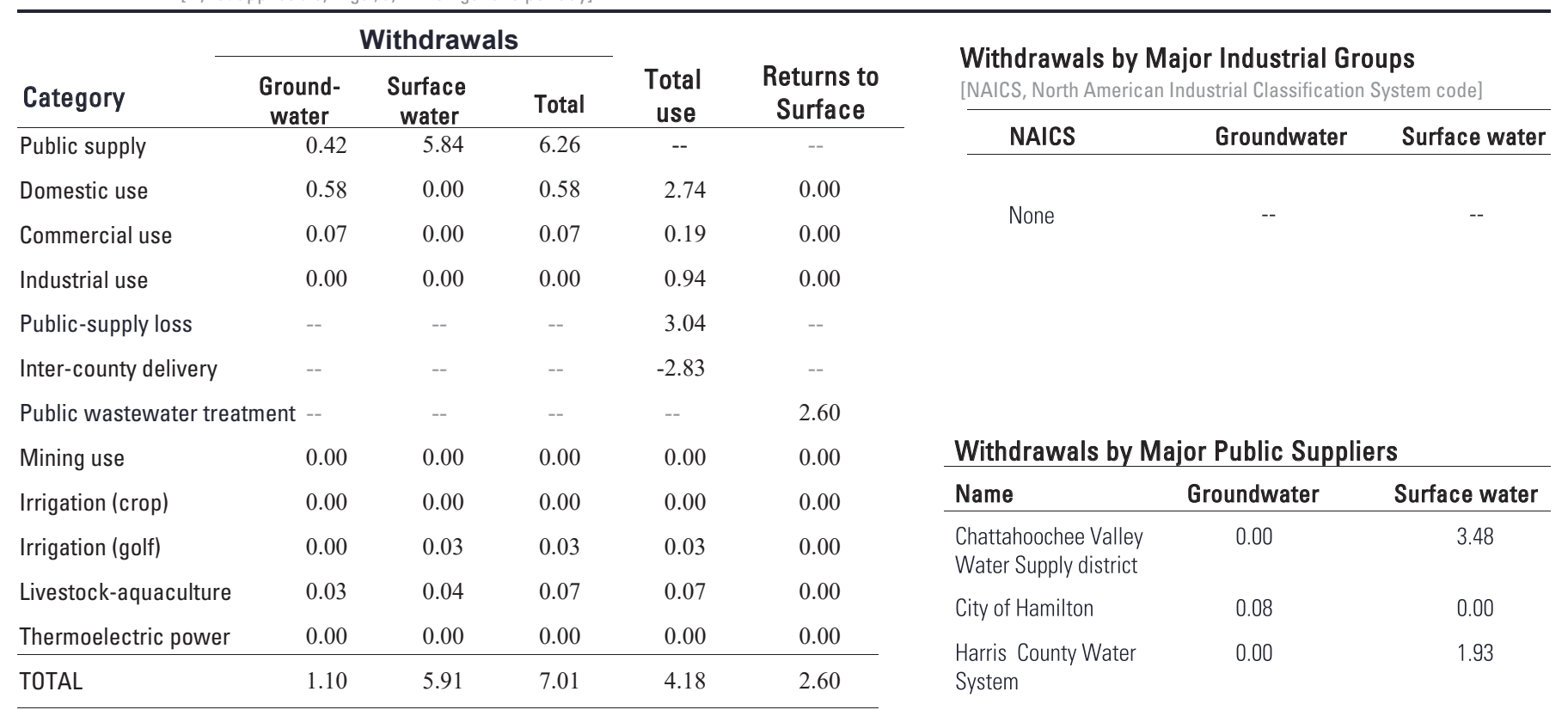

Total use is total withdrawal plus public supply deliveries and losses. An estimate of $0.9 \mathrm{Mgal} / \mathrm{d}$ was delivered from neighboring counties in 2015. An estimate of $3.73 \mathrm{Mgal} / \mathrm{d}$ was delivered from Harris County to neighboring counties in 2015.

\section{Withdrawals by water source}

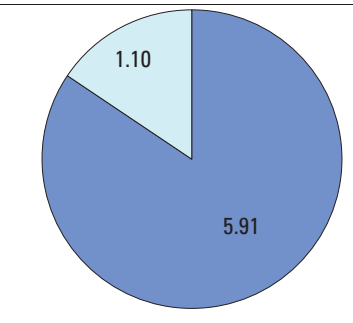

Withdrawals, in million gallons per day

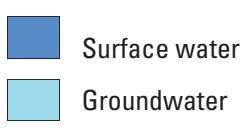

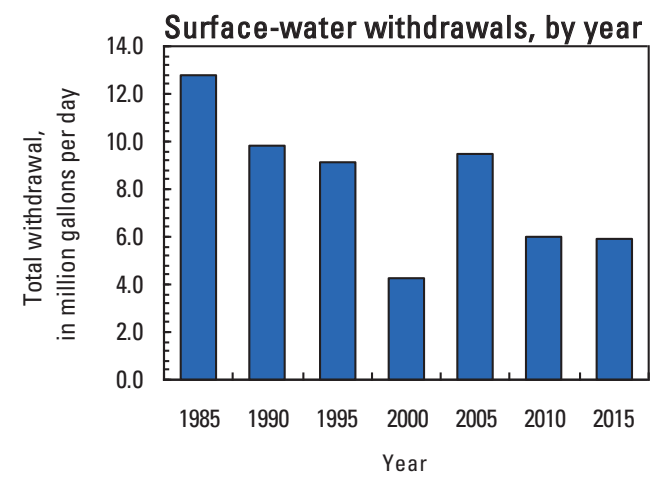

Groundwater withdrawals by aquifer

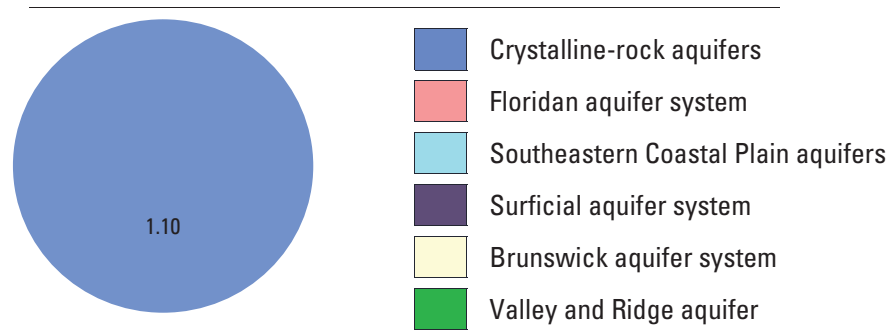




\section{HART COUNTY}

Population

Population served by public supply-groundwater

5,105

Population served by public supply-surface water

7,116

Acres irrigated

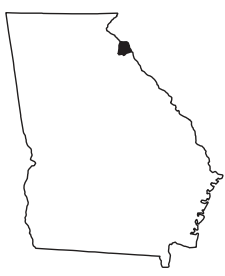

2015 WATER WITHDRAWALS AND ESTIMATED USE, IN MILLION GALLONS PER DAY

[--,not applicable; Mgal/d, million gallons per day]

\begin{tabular}{lccccc}
\hline & \multicolumn{3}{c}{ Withdrawals } & Total & Returns to \\
\cline { 2 - 5 } Category & $\begin{array}{c}\text { Ground- } \\
\text { water }\end{array}$ & $\begin{array}{c}\text { Surface } \\
\text { water }\end{array}$ & Total & $\begin{array}{c}\text { use } \\
\text { Surface }\end{array}$ \\
\hline Public supply & 0.25 & 1.21 & 1.46 & -- & -- \\
Domestic use & 1.00 & 0.00 & 1.00 & 2.07 & 0.00 \\
Commercial use & 0.00 & 0.00 & 0.00 & 0.38 & 0.00 \\
Industrial use & 0.00 & 0.00 & 0.00 & 0.04 & 0.00 \\
Public-supply loss & -- & -- & -- & -0.03 & -- \\
Inter-county delivery & -- & -- & -- & 0.29 & -- \\
Public wastewater treatment & -- & -- & -- & -- & 0.14 \\
Mining use & 0.02 & 0.00 & 0.02 & 0.02 & 0.00 \\
Irrigation (crop) & 0.21 & 0.54 & 0.75 & 0.75 & 0.00 \\
Irrigation (golf) & 0.00 & 0.17 & 0.17 & 0.17 & 0.00 \\
Livestock-aquaculture & 0.26 & 0.68 & 0.94 & 0.94 & 0.00 \\
Thermoelectric power & 0.00 & 0.00 & 0.00 & 0.00 & 0.00 \\
\hline TOTAL & 1.74 & 2.60 & 4.34 & 4.63 & 0.14 \\
\hline
\end{tabular}

Withdrawals by Major Industrial Groups

[NAICS, North American Industrial Classification System code]

NAICS Groundwater Surface water

None

Withdrawals by Major Public Suppliers

Name Groundwater Surface water

City of Hartwell $\quad 0.00 \quad 1.21$

City of Royston

0.15

0.00

Total use is total withdrawal plus public supply deliveries and losses. An estimate of $0.37 \mathrm{Mgal} / \mathrm{d}$ was delivered from neighboring counties in 2015. An estimate of $0.08 \mathrm{Mgal} / \mathrm{d}$ was delivered from Hart County to neighboring counties in 2015.

Withdrawals by water source

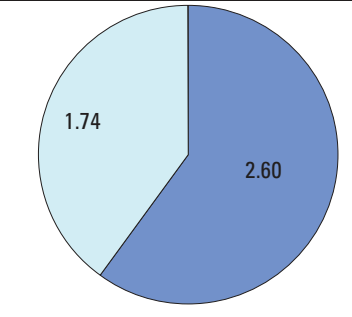

Withdrawals, in million gallons per day

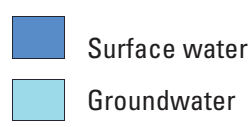

Groundwater withdrawals by aquifer

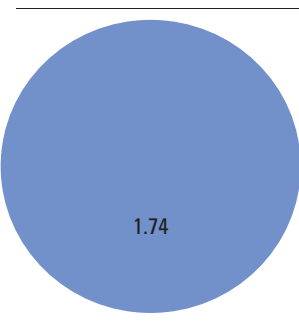

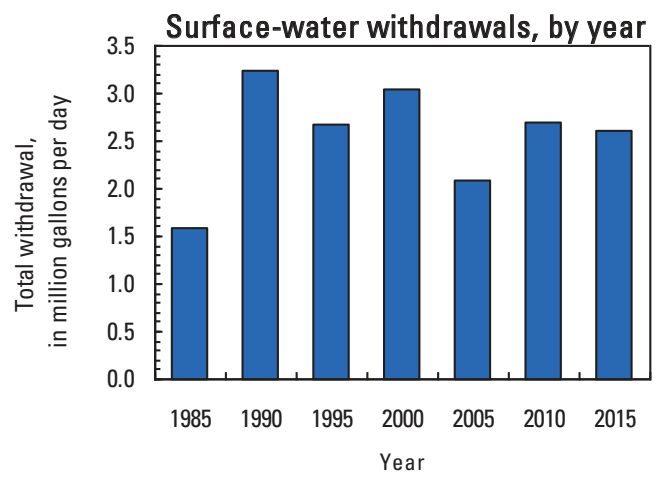

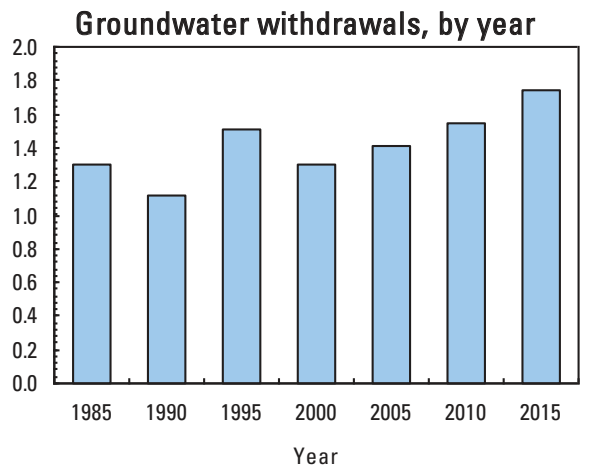




\section{HEARD COUNTY}

Population

Population served by public supply-groundwater

Population served by public supply-surface water $\quad 8,686$

Acres irrigated

0

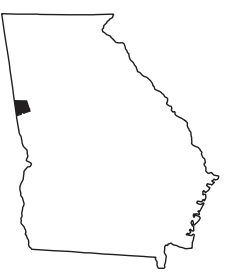

2015 WATER WITHDRAWALS AND ESTIMATED USE, IN MILLION GALLONS PER DAY

[--,not applicable; Mgal/d, million gallons per day]

\begin{tabular}{|c|c|c|c|c|c|c|c|c|}
\hline \multirow{3}{*}{ Category } & \multicolumn{3}{|c|}{ Withdrawals } & \multirow{3}{*}{$\begin{array}{c}\text { Total } \\
\text { use }\end{array}$} & \multirow{3}{*}{$\begin{array}{l}\text { Returns to } \\
\text { Surface }\end{array}$} & \multirow{2}{*}{\multicolumn{3}{|c|}{$\begin{array}{l}\text { Withdrawals by Major Industrial Groups } \\
\text { [NAICS, North American Industrial Classification System code] }\end{array}$}} \\
\hline & \multirow{2}{*}{$\begin{array}{l}\text { Ground- } \\
\text { water }\end{array}$} & \multirow{2}{*}{$\begin{array}{c}\text { Surface } \\
\text { water }\end{array}$} & \multirow{2}{*}{ Total } & & & & & \\
\hline & & & & & & \multirow[t]{4}{*}{ NAICS } & \multirow[t]{2}{*}{ Groundwater } & \multirow[t]{2}{*}{ Surface water } \\
\hline Public supply & 0.10 & 1.12 & 1.22 & -- & -- & & & \\
\hline Domestic use & 0.15 & 0.00 & 0.15 & 0.65 & 0.00 & & -- & -- \\
\hline Commercial use & 0.00 & 0.00 & 0.00 & 0.46 & 0.00 & & & \\
\hline Industrial use & 0.00 & 0.00 & 0.00 & 0.00 & 0.00 & & & \\
\hline Public-supply loss & -- & -- & -- & 0.26 & -- & & & \\
\hline Inter-county delivery & -- & -- & -- & 0.00 & -- & & & \\
\hline Public wastewater treatment & it -- & -- & -- & -- & 0.04 & & & \\
\hline Mining use & 0.00 & 0.00 & 0.00 & 0.00 & 0.00 & \multicolumn{3}{|c|}{ Withdrawals by Major Public Suppliers } \\
\hline Irrigation (crop) & 0.00 & 0.00 & 0.00 & 0.00 & 0.00 & Name & Groundwater & Surface water \\
\hline Irrigation (golf) & 0.00 & 0.00 & 0.00 & 0.00 & 0.00 & \multirow{4}{*}{$\begin{array}{l}\text { City of Ephesus } \\
\text { Heard County Water } \\
\text { Authority }\end{array}$} & 0.10 & 0.00 \\
\hline Livestock-aquaculture & 0.08 & 0.12 & 0.20 & 0.20 & 0.00 & & \multirow[t]{3}{*}{0.00} & \multirow[t]{3}{*}{1.12} \\
\hline Thermoelectric power & 0.00 & 14.55 & 14.55 & 14.55 & 0.00 & & & \\
\hline TOTAL & 0.33 & 15.79 & 16.12 & 16.12 & 0.04 & & & \\
\hline
\end{tabular}

Total use is total withdrawal plus public supply deliveries and losses.

Withdrawals by water source

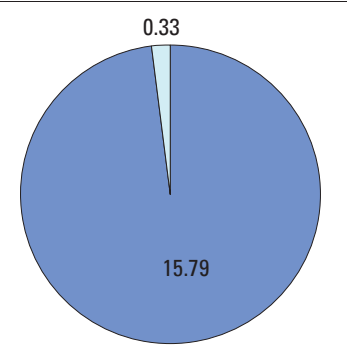

Withdrawals, in million

gallons per day

Surface water

Groundwater

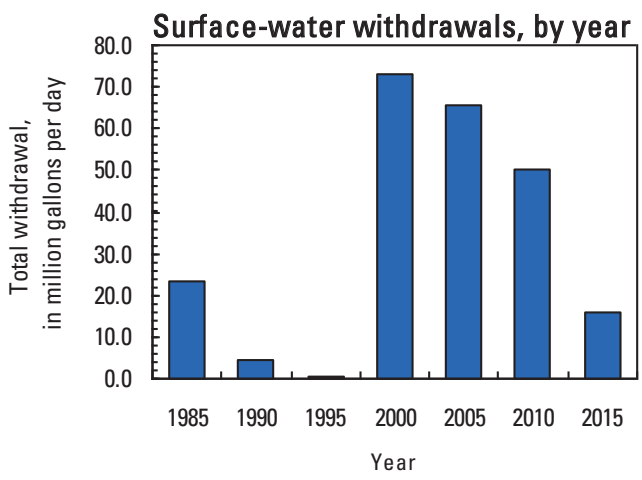

Groundwater withdrawals by aquifer

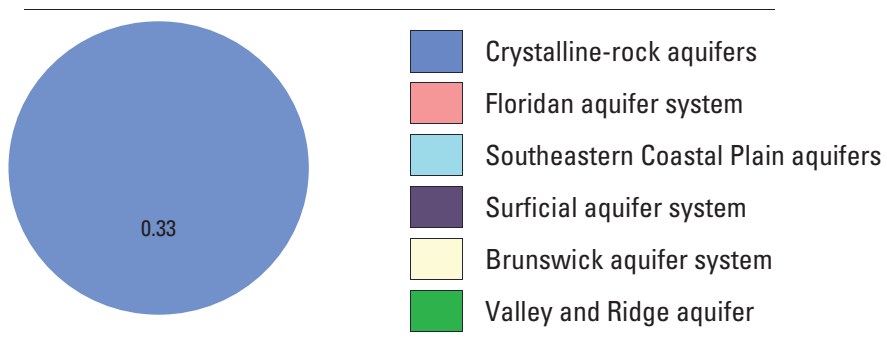

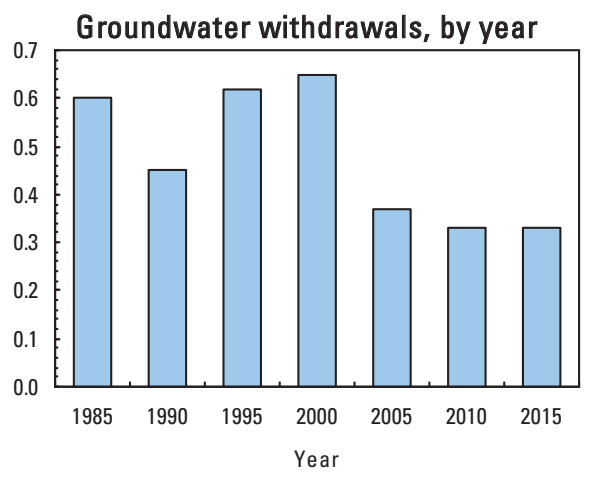




\section{HENRY COUNTY}

Population

217,739

Population served by public supply-groundwater $\quad 6,098$

Population served by public supply-surface water 183,108

Acres irrigated

2015 WATER WITHDRAWALS AND ESTIMATED USE, IN MILLION GALLONS PER DAY

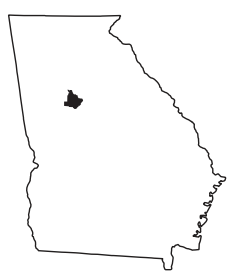

[--,not applicable; Mgal/d, million gallons per day]

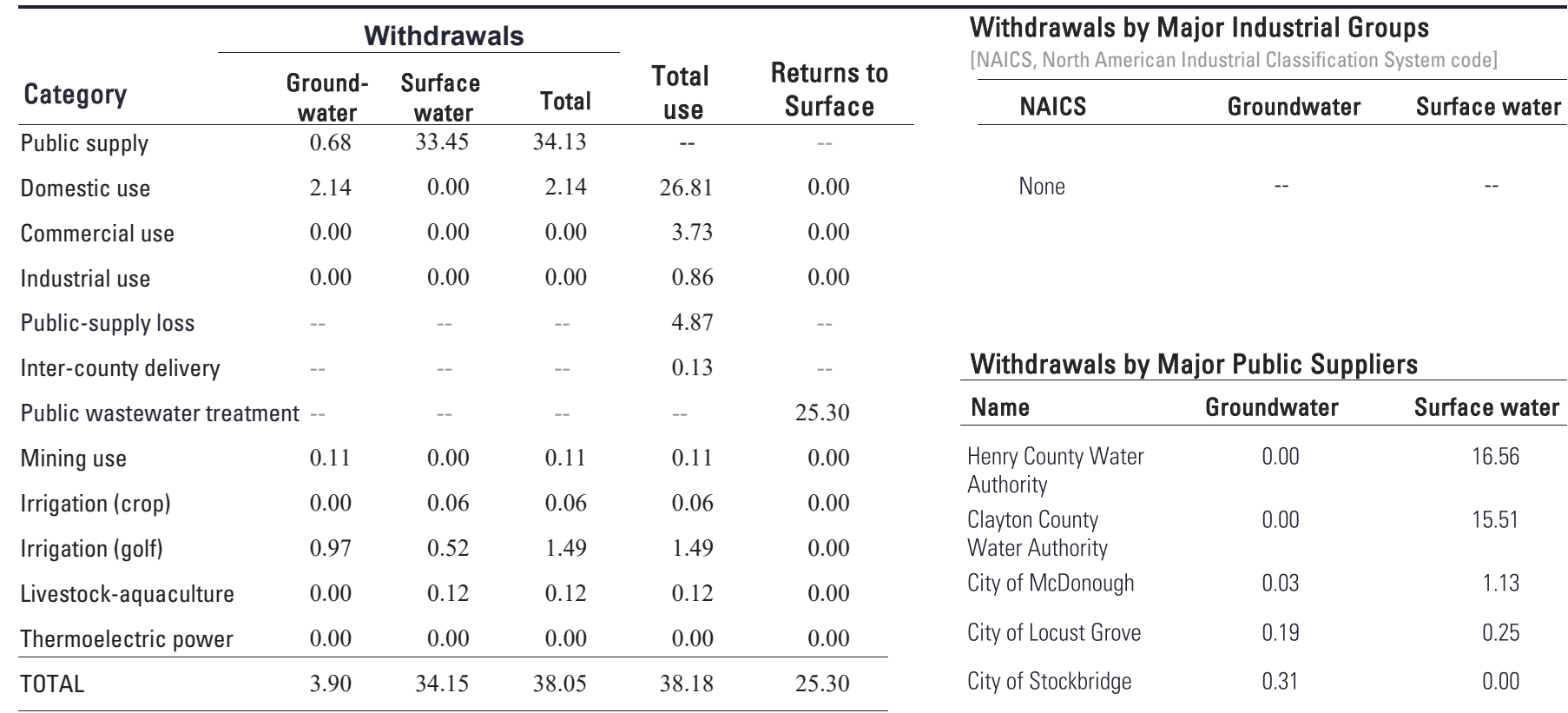

Total use is total withdrawal plus public supply deliveries and losses. An estimate of $0.13 \mathrm{Mgal} / \mathrm{d}$ was delivered from neighboring counties in 2015.
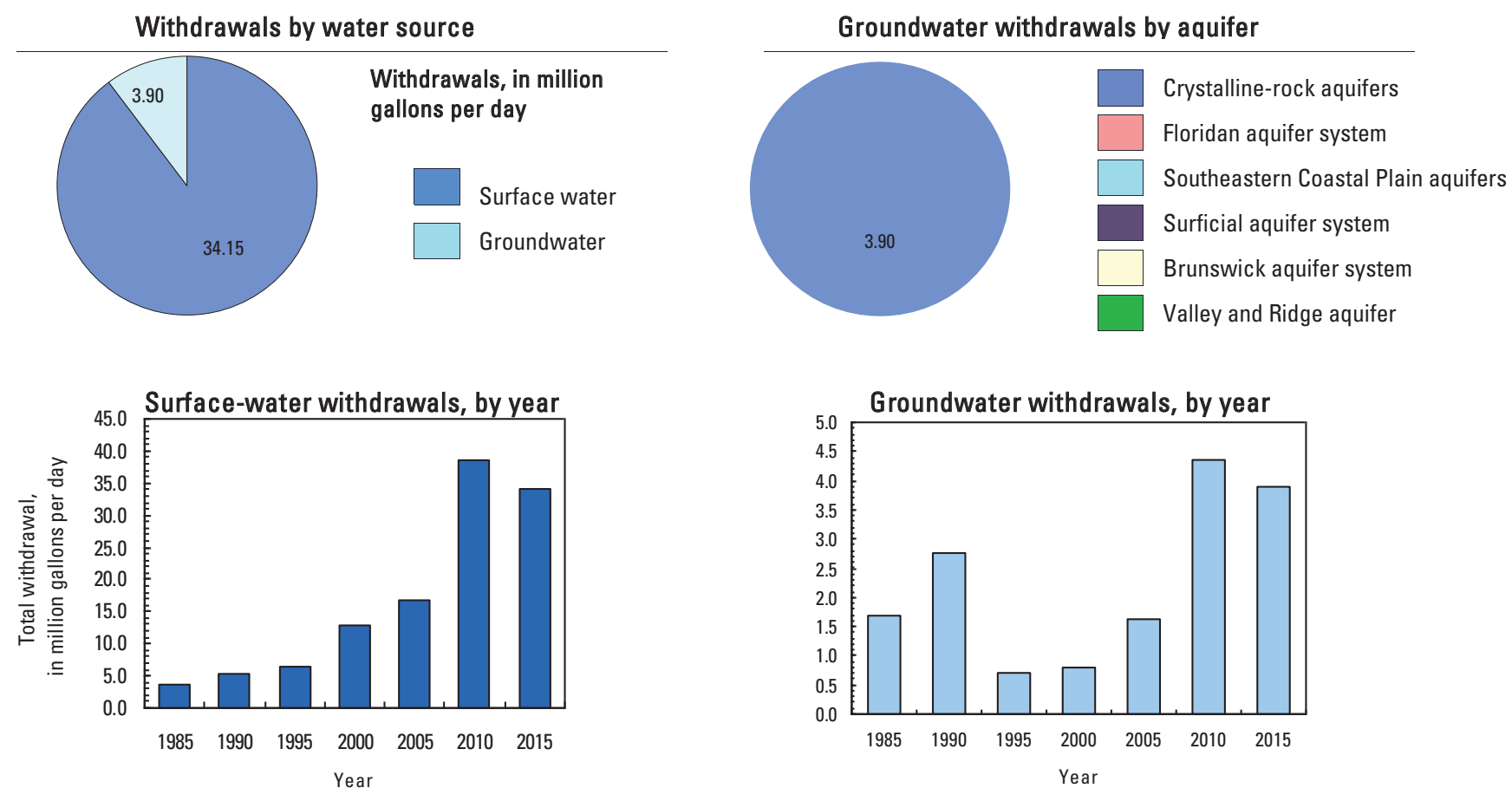


\section{HOUSTON COUNTY}

Population

Population served by public supply-groundwater $\quad 141,909$

Population served by public supply-surface water 0

Acres irrigated

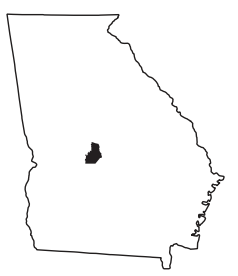

2015 WATER WITHDRAWALS AND ESTIMATED USE, IN MILLION GALLONS PER DAY

[--,not applicable; Mgal/d, million gallons per day]

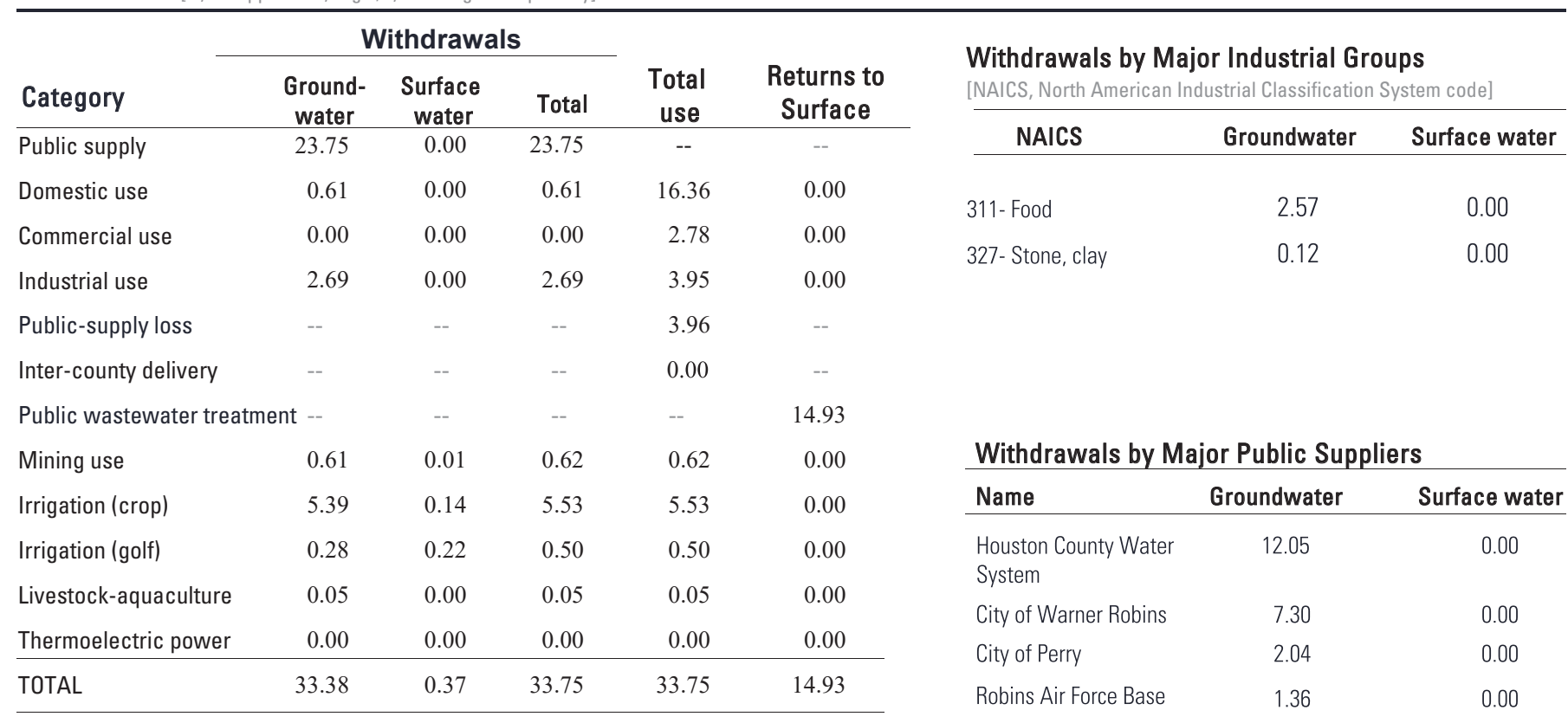

Total use is total withdrawal plus public supply deliveries and losses.

Withdrawals by water source
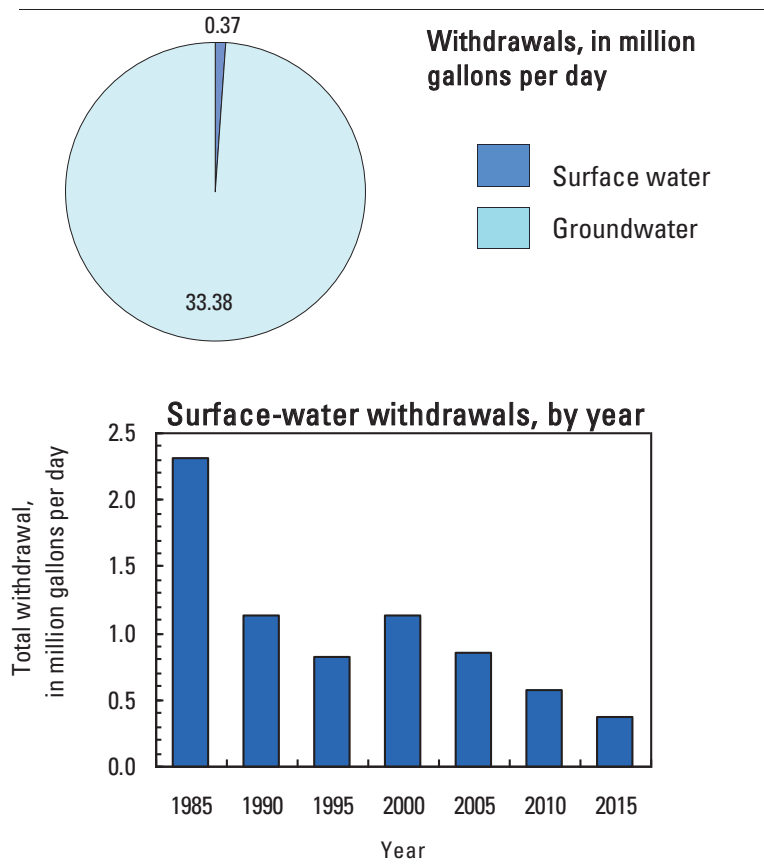

Groundwater withdrawals by aquifer
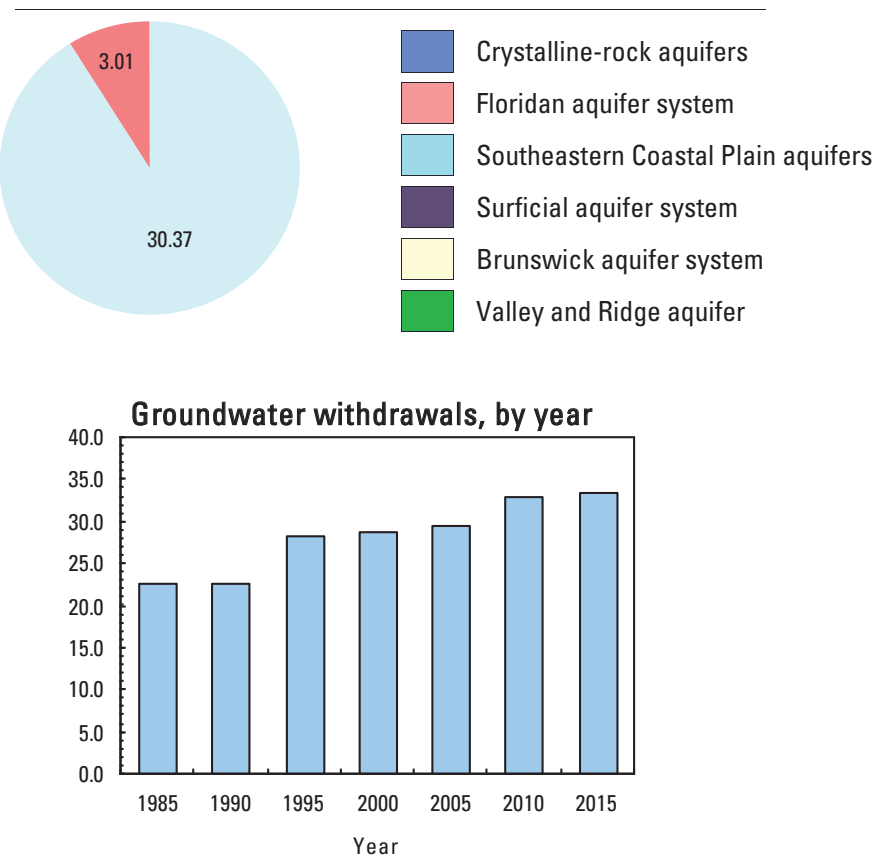


\section{IRWIN COUNTY}

Population

9,245

Population served by public supply-groundwater $\quad 3,107$

Population served by public supply-surface water $\quad 0$

Acres irrigated

2015 WATER WITHDRAWALS AND ESTIMATED USE, IN MILLION GALLONS PER DAY

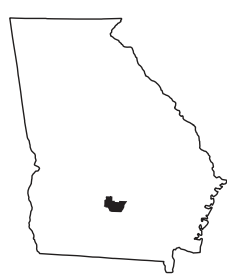

[--,not applicable; Mgal/d, million gallons per day]

\begin{tabular}{|c|c|c|c|c|c|c|c|c|}
\hline \multirow{3}{*}{ Category } & \multicolumn{3}{|c|}{ Withdrawals } & \multirow{3}{*}{$\begin{array}{c}\text { Total } \\
\text { use }\end{array}$} & \multirow{3}{*}{$\begin{array}{l}\text { Returns to } \\
\text { Surface }\end{array}$} & \multirow{2}{*}{\multicolumn{3}{|c|}{$\begin{array}{l}\text { Withdrawals by Major Industrial Groups } \\
\text { [NAICS, North American Industrial Classification System code] }\end{array}$}} \\
\hline & \multirow{2}{*}{$\begin{array}{c}\text { Ground- } \\
\text { water }\end{array}$} & \multirow{2}{*}{$\begin{array}{c}\text { Surface } \\
\text { water }\end{array}$} & \multirow[b]{2}{*}{ Total } & & & & & \\
\hline & & & & & & NAICS & Groundwater & Surface water \\
\hline Public supply & 0.44 & 0.00 & 0.44 & -- & -- & & & \\
\hline Domestic use & 0.46 & 0.00 & 0.46 & 0.68 & 0.00 & None & -- & -- \\
\hline Commercial use & 0.00 & 0.00 & 0.00 & 0.13 & 0.00 & & & \\
\hline Industrial use & 0.00 & 0.00 & 0.00 & 0.03 & 0.00 & & & \\
\hline Public-supply loss & -- & -- & - & 0.06 & -- & & & \\
\hline Inter-county delivery & -- & -- & - & 0.00 & -- & & & \\
\hline Public wastewater treatment & tt -- & -- & -- & -- & 0.00 & & & \\
\hline Mining use & 0.00 & 0.00 & 0.00 & 0.00 & 0.00 & Withdrawal & or Public Supp & \\
\hline Irrigation (crop) & 8.32 & 7.32 & 15.64 & 15.64 & 0.00 & Name & Groundwater & Surface water \\
\hline Irrigation (golf) & 0.14 & 0.00 & 0.14 & 0.14 & 0.00 & City of Ocilla & 0.40 & 0.00 \\
\hline Livestock-aquaculture & 0.09 & 0.08 & 0.17 & 0.17 & 0.00 & & & \\
\hline Thermoelectric power & 0.00 & 0.00 & 0.00 & 0.00 & 0.00 & & & \\
\hline TOTAL & 9.45 & 7.40 & 16.85 & 16.85 & 0.00 & & & \\
\hline
\end{tabular}

Total use is total withdrawal plus public supply deliveries and losses.

Withdrawals by water source

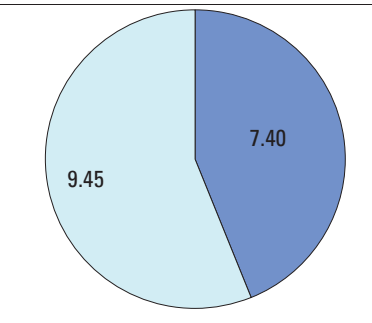

Withdrawals, in million gallons per day

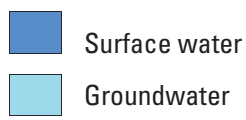

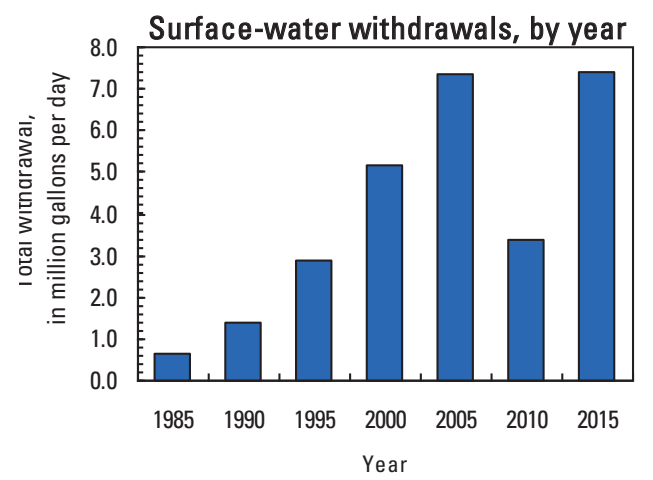

Groundwater withdrawals by aquifer

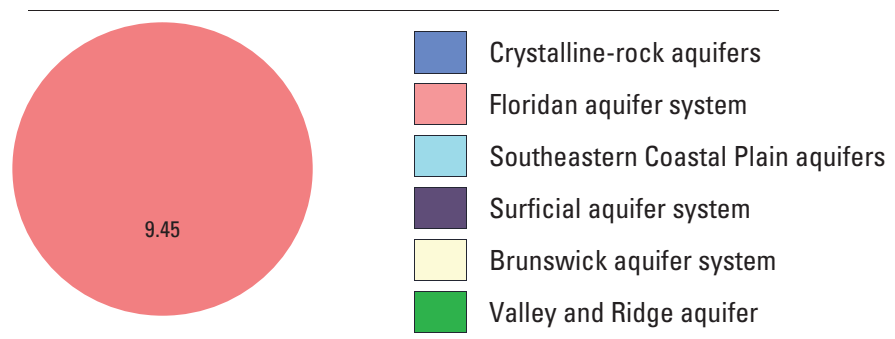




\section{JACKSON COUNTY}

Population

Population served by public supply-groundwater

Population served by public supply-surface water $\quad 46,638$

Acres irrigated

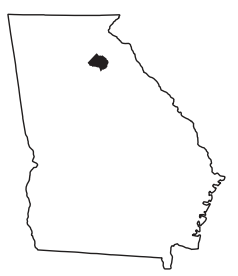

2015 WATER WITHDRAWALS AND ESTIMATED USE, IN MILLION GALLONS PER DAY

[--, not applicable; Mgal/d, million gallons per day]

\begin{tabular}{lccccc}
\hline & \multicolumn{3}{c}{ Withdrawals } & & Total \\
\cline { 2 - 4 } Category & $\begin{array}{c}\text { Ground- } \\
\text { water }\end{array}$ & $\begin{array}{c}\text { Surface } \\
\text { water }\end{array}$ & $\begin{array}{c}\text { Total } \\
\text { use }\end{array}$ & $\begin{array}{c}\text { Returns to } \\
\text { Surface }\end{array}$ \\
\hline Public supply & 0.34 & 9.18 & 9.52 & -- & -- \\
Domestic use & 0.88 & 0.00 & 0.88 & 4.86 & 0.00 \\
Commercial use & 0.00 & 0.00 & 0.00 & 1.34 & 0.00 \\
Industrial use & 0.52 & 0.00 & 0.52 & 1.02 & 0.00 \\
Public-supply loss & -- & -- & -- & 3.7 & -- \\
Inter-county delivery & -- & -- & -- & -2.59 & -- \\
Public wastewater treatment & -- & -- & -- & -- & 3.44 \\
Mining use & 0.09 & 0.00 & 0.09 & 0.09 & 0.00 \\
Irrigation (crop) & 0.07 & 0.02 & 0.09 & 0.09 & 0.00 \\
Irrigation (golf) & 0.00 & 0.20 & 0.20 & 0.20 & 0.00 \\
Livestock-aquaculture & 0.71 & 0.20 & 0.91 & 0.91 & 0.00 \\
Thermoelectric power & 0.00 & 0.00 & 0.00 & 0.00 & 0.00 \\
\hline TOTAL & 2.61 & 9.60 & 12.21 & 9.62 & 3.44 \\
\hline
\end{tabular}

Withdrawals by Major Industrial Groups

[NAICS, North American Industrial Classification System code]

NAICS Groundwater Surface water

None

Withdrawals by Major Public Suppliers

Name Groundwater Surface water

$\begin{array}{lll}\text { City of Braselton } & 0.29 & 0.00 \\ \text { City of Jefferson } & 0.00 & 1.23 \\ \text { Upper Oconee Basin } & 0.00 & 7.95\end{array}$

7.95

Total use is total withdrawal plus public supply deliveries and losses. An estimate of $0.02 \mathrm{Mgal} / \mathrm{d}$ was delivered from neighboring counties in 2015. An estimate of $2.61 \mathrm{Mgal} / \mathrm{d}$ was delivered from Jackson County to neighboring counties in 2015.

\section{Withdrawals by water source}

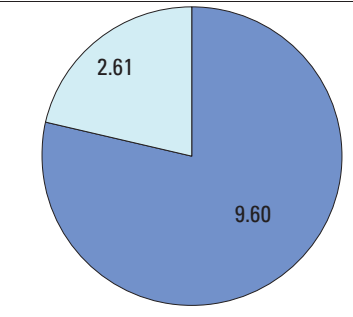

Withdrawals, in million

gallons per day

Surface water

Groundwater

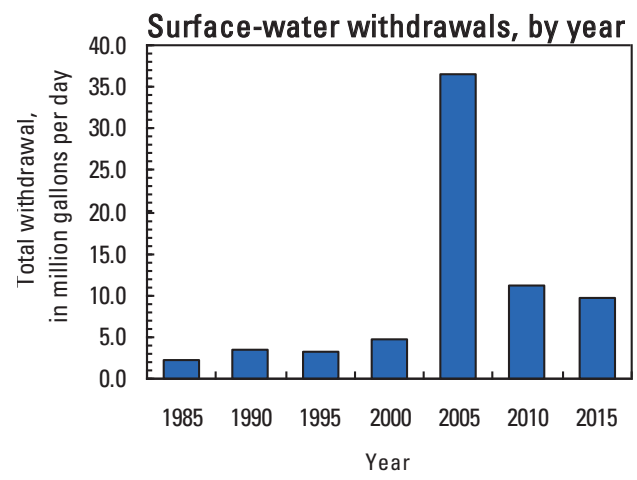

Groundwater withdrawals by aquifer

\begin{tabular}{ll}
\hline & Crystalline-rock aquifers \\
\hline & Floridan aquifer system \\
\hline & Southeastern Coastal Plain aquifers \\
& Surficial aquifer system \\
\hline$\quad$ Brunswick aquifer system \\
& Valley and Ridge aquifer
\end{tabular}

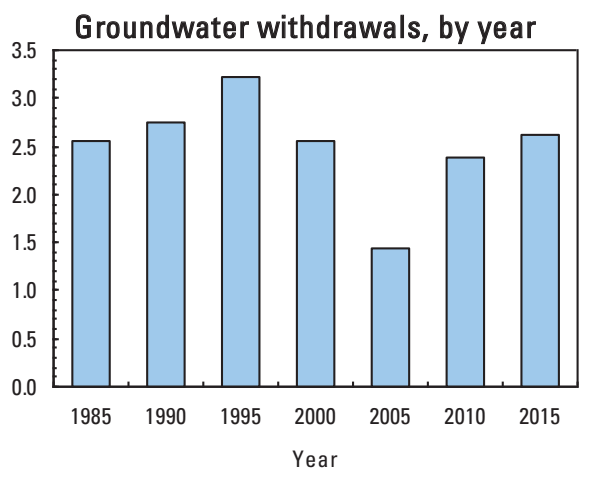




\section{JASPER COUNTY}

Population

Population served by public supply-groundwater

Population served by public supply-surface water $\quad 3,697$

Acres irrigated

2015 WATER WITHDRAWALS AND ESTIMATED USE, IN MILLION GALLONS PER DAY

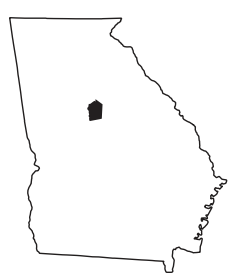

[--,not applicable; Mgal/d, million gallons per day]

\begin{tabular}{|c|c|c|c|c|c|c|c|c|}
\hline \multirow{3}{*}{ Category } & \multicolumn{3}{|c|}{ Withdrawals } & \multirow{3}{*}{$\begin{array}{c}\text { Total } \\
\text { use }\end{array}$} & \multirow{3}{*}{$\begin{array}{c}\text { Returns to } \\
\text { Surface }\end{array}$} & \multirow{2}{*}{\multicolumn{3}{|c|}{$\begin{array}{l}\text { Withdrawals by Major Industrial Groups } \\
\text { [NAICS, North American Industrial Classification System code] }\end{array}$}} \\
\hline & \multirow{2}{*}{$\begin{array}{l}\text { Ground - } \\
\text { water }\end{array}$} & \multirow{2}{*}{$\begin{array}{c}\text { Surface } \\
\text { water }\end{array}$} & \multirow{2}{*}{ Total } & & & & & \\
\hline & & & & & & \multirow{4}{*}{ None } & Groundwater & Surface water \\
\hline Public supply & 0.32 & 0.43 & 0.75 & -- & -- & & \multirow{4}{*}{--} & \multirow{4}{*}{-- } \\
\hline Domestic use & 0.45 & 0.00 & 0.45 & 0.97 & 0.00 & & & \\
\hline Commercial use & 0.00 & 0.00 & 0.00 & 0.17 & 0.00 & & & \\
\hline Industrial use & 0.00 & 0.00 & 0.00 & 0.03 & 0.00 & & & \\
\hline Public-supply loss & -- & -- & -- & 0.03 & -- & & & \\
\hline Inter-county delivery & -- & -- & - & 0.07 & -- & & & \\
\hline \multicolumn{2}{|l|}{ Public wastewater treatment -- } & -- & -- & -- & 0.02 & & & \\
\hline Mining use & 0.00 & 0.00 & 0.00 & 0.00 & 0.00 & \multicolumn{3}{|c|}{ Withdrawals by Major Public Suppliers } \\
\hline Irrigation (crop) & 0.33 & 0.04 & 0.37 & 0.37 & 0.00 & \multirow{4}{*}{$\begin{array}{l}\text { Name } \\
\text { City of Monticello } \\
\text { Turtle Cove Property } \\
\text { Owners Association }\end{array}$} & Groundwater & Surface water \\
\hline Irrigation (golf) & 0.00 & 0.00 & 0.00 & 0.00 & 0.00 & & \multirow{3}{*}{$\begin{array}{l}0.13 \\
0.16\end{array}$} & \multirow{3}{*}{$\begin{array}{l}0.43 \\
0.00\end{array}$} \\
\hline Livestock-aquaculture & 0.27 & 0.03 & 0.30 & 0.30 & 0.00 & & & \\
\hline Thermoelectric power & 0.00 & 0.00 & 0.00 & 0.00 & 0.00 & & & \\
\hline TOTAL & 1.37 & 0.50 & 1.87 & 1.94 & 0.02 & & & \\
\hline
\end{tabular}

Total use is total withdrawal plus public supply deliveries and losses. An estimate of $0.07 \mathrm{Mgal} / \mathrm{d}$ was delivered from neighboring counties in 2015.

Withdrawals by water source

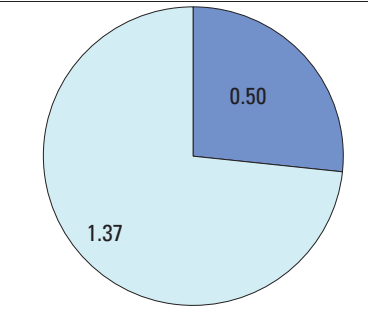

Withdrawals, in million gallons per day

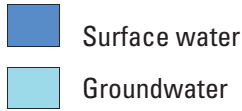

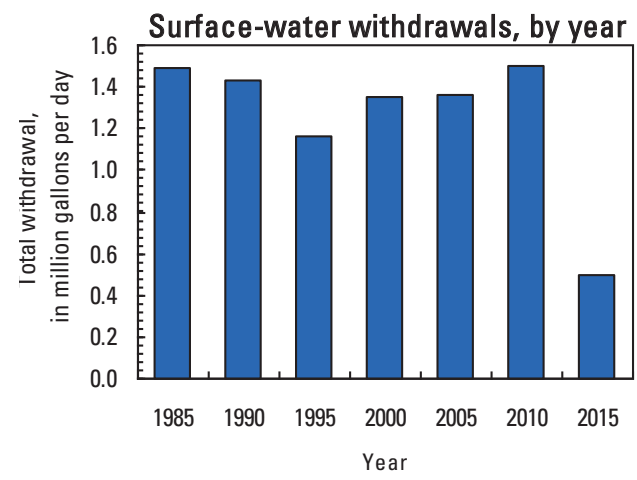

Groundwater withdrawals by aquifer

\begin{tabular}{|l|l}
\hline & Crystalline-rock aquifers \\
\hline & Floridan aquifer system \\
\hline & Southeastern Coastal Plain aquifers \\
& Surficial aquifer system \\
\hline$\quad$ Brunswick aquifer system \\
Valley and Ridge aquifer
\end{tabular}

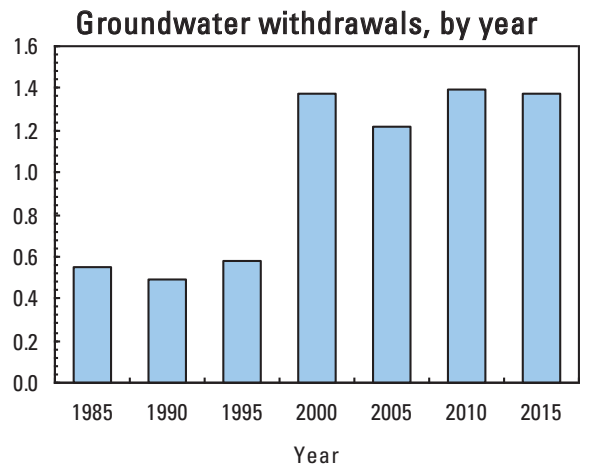




\section{JEFF DAVIS COUNTY}

Population

Population served by public supply-groundwater

6,331

Population served by public supply-surface water

0

Acres irrigated

11,030

2015 WATER WITHDRAWALS AND ESTIMATED USE, IN MILLION GALLONS PER DAY

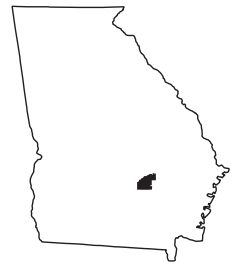

$[--$,not applicable; Mgal/d, million gallons per day]

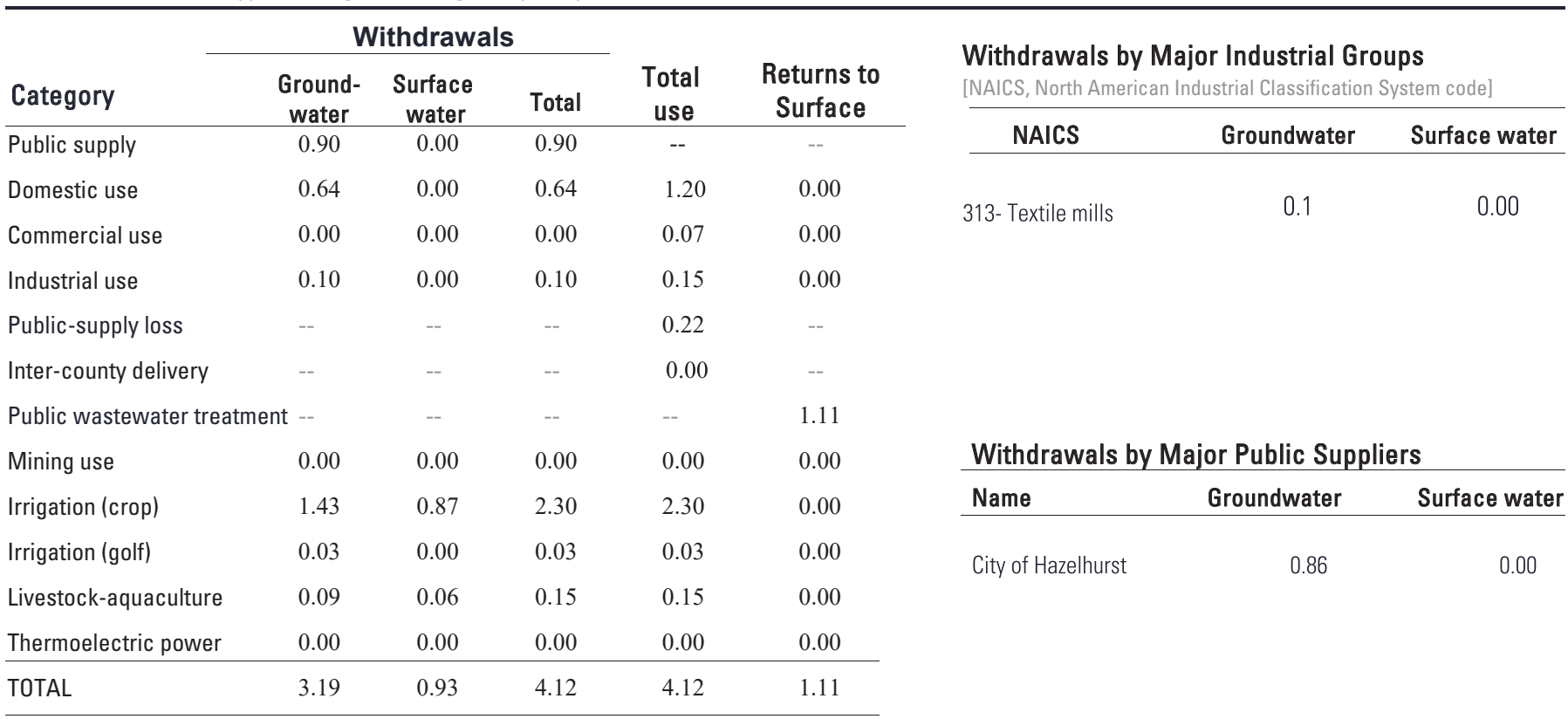

Total use is total withdrawal plus public supply deliveries and losses.

Withdrawals by water source

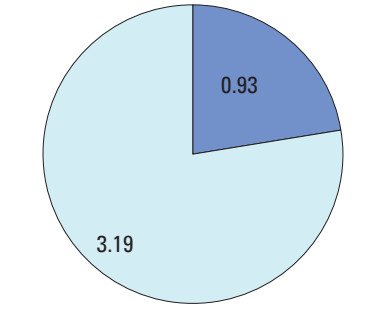

Withdrawals, in million

gallons per day

Surface water

Groundwater

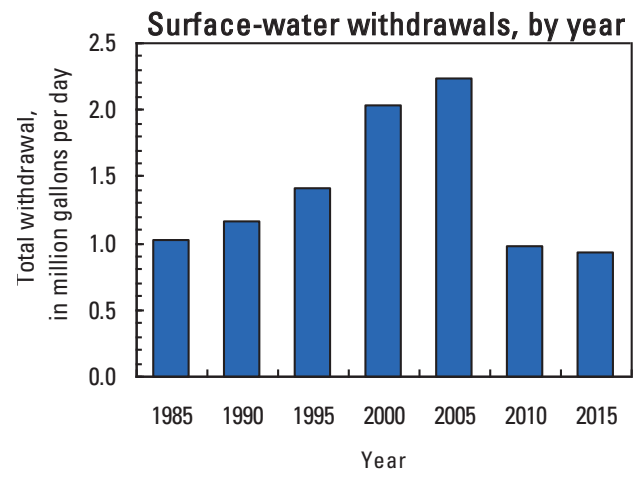

Groundwater withdrawals by aquifer

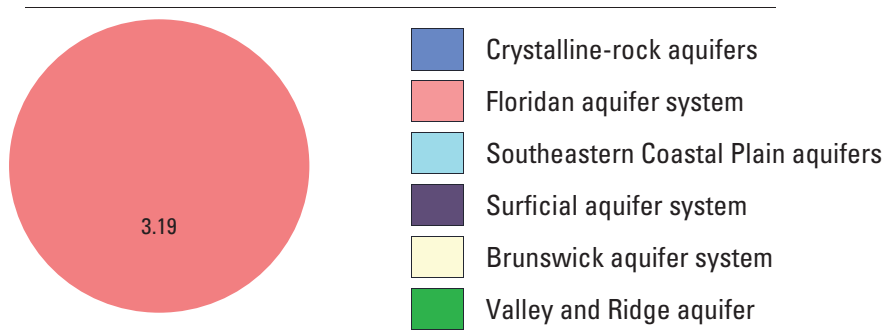




\section{JEFFERSON COUNTY}

Population

Population served by public supply-groundwater

9,590

Population served by public supply-surface water

0

Acres irrigated

2015 WATER WITHDRAWALS AND ESTIMATED USE, IN MILLION GALLONS PER DAY

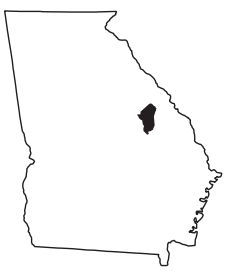

[--,not applicable; Mgal/d, million gallons per day]

\begin{tabular}{lccccc}
\hline & \multicolumn{3}{c}{ Withdrawals } & & \\
\cline { 2 - 4 } Category & $\begin{array}{c}\text { Ground } \\
\text { water }\end{array}$ & $\begin{array}{c}\text { Surface } \\
\text { water }\end{array}$ & Total & $\begin{array}{c}\text { Total } \\
\text { use }\end{array}$ & $\begin{array}{c}\text { Returns to } \\
\text { Surface }\end{array}$ \\
\hline Public supply & 1.32 & 0.00 & 1.32 & -- & -- \\
Domestic use & 0.49 & 0.00 & 0.49 & 1.38 & 0.00 \\
Commercial use & 0.00 & 0.00 & 0.00 & 0.26 & 0.00 \\
Industrial use & 4.11 & 2.97 & 7.08 & 7.11 & 0.00 \\
Public-supply loss & -- & -- & -- & 0.14 & -- \\
Inter-county delivery & -- & -- & -- & 0.00 & -- \\
Public wastewater treatment & -- & -- & -- & -- & 5.82 \\
Mining use & 0.00 & 0.00 & 0.00 & 0.00 & 0.00 \\
Irrigation (crop) & 14.24 & 1.60 & 15.84 & 15.84 & 0.00 \\
Irrigation (golf) & 0.03 & 0.00 & 0.03 & 0.03 & 0.00 \\
Livestock-aquaculture & 0.76 & 0.05 & 0.81 & 0.81 & 0.00 \\
Thermoelectric power & 0.00 & 0.00 & 0.00 & 0.00 & 0.00 \\
\hline TOTAL & 20.95 & 4.62 & 25.57 & 25.57 & 5.82 \\
\hline
\end{tabular}

Withdrawals by Major Industrial Groups

[NAICS, North American Industrial Classification System code]

\begin{tabular}{lll}
\hline NAICS Groundwater & Surface water \\
\hline
\end{tabular}

212- Mining

4.14

2.97

Withdrawals by Major Public Suppliers

Name Groundwater Surface water

City of Louisville $\quad 0.60 \quad 0.00$

City of Wadley $\quad 0.31 \quad 0.00$

City of Wrens

0.32

Total use is total withdrawal plus public supply deliveries and losses.

Withdrawals by water source

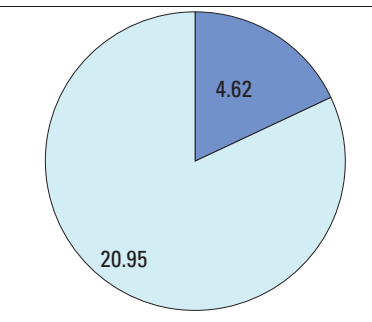

Withdrawals, in million gallons per day

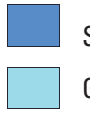

Surface water

Groundwater
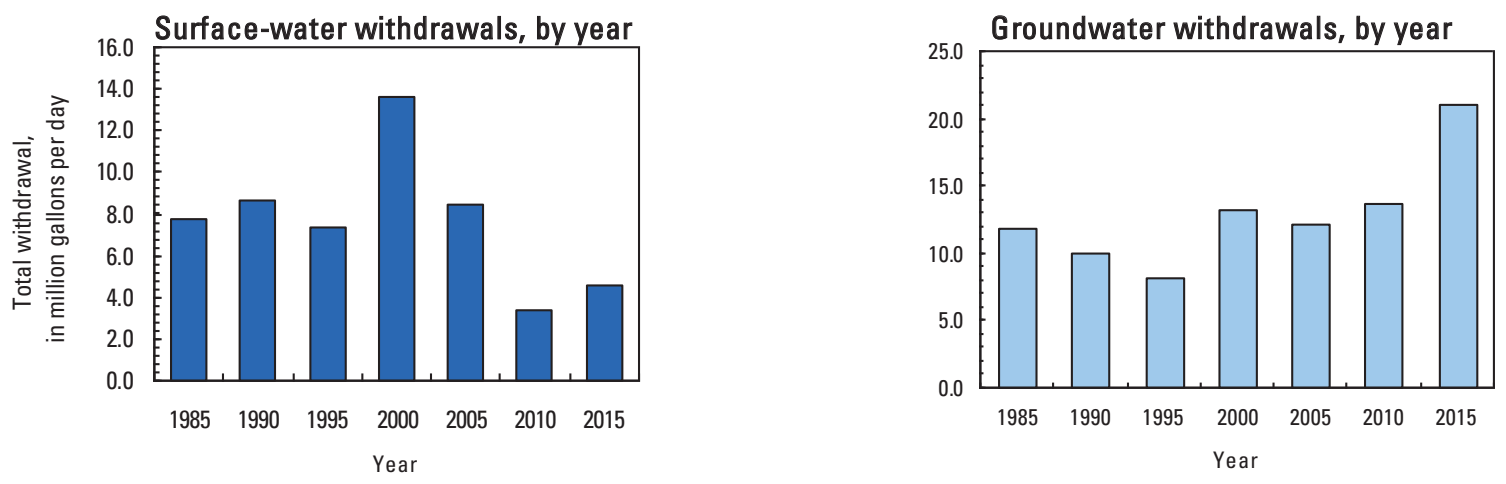


\section{JENKINS COUNTY}

Population

Population served by public supply-groundwater

Population served by public supply-surface water

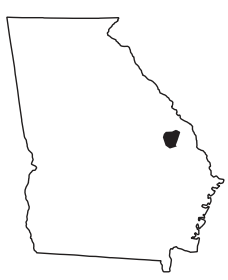

[--,not applicable; Mgal/d, million gallons per day]

\begin{tabular}{|c|c|c|c|c|c|c|c|c|}
\hline \multirow{2}{*}{ Category } & \multicolumn{3}{|c|}{ Withdrawals } & \multirow{3}{*}{$\begin{array}{c}\text { Total } \\
\text { use }\end{array}$} & \multirow{3}{*}{$\begin{array}{l}\text { Returns to } \\
\text { Surface }\end{array}$} & \multirow{2}{*}{\multicolumn{3}{|c|}{$\begin{array}{l}\text { Withdrawals by Major Industrial Groups } \\
\text { [NAICS, North American Industrial Classification System code] }\end{array}$}} \\
\hline & Ground & Surface & Total & & & & & \\
\hline Public supply & $\begin{array}{c}\text { water } \\
0.45\end{array}$ & $\begin{array}{c}\text { water } \\
0.00\end{array}$ & 0.45 & & & NAICS & Groundwater & Surface water \\
\hline Domestic use & 0.36 & 0.00 & 0.36 & 0.64 & 0.00 & \multirow{3}{*}{ 212- Mining } & \multirow{3}{*}{0.06} & \multirow{3}{*}{0.00} \\
\hline Commercial use & 0.00 & 0.00 & 0.00 & 0.08 & 0.00 & & & \\
\hline Industrial use & 0.07 & 0.00 & 0.07 & 0.07 & 0.00 & & & \\
\hline Public-supply loss & -- & -- & -- & 0.09 & -- & & & \\
\hline Inter-county delivery & -- & -- & -- & 0.00 & -- & & & \\
\hline Public wastewater treatment & tt -- & -- & -- & -- & 0.55 & & & \\
\hline Mining use & 0.00 & 0.00 & 0.00 & 0.00 & 0.00 & \multicolumn{3}{|c|}{ Withdrawals by Major Public Suppliers } \\
\hline Irrigation (crop) & 5.30 & 1.55 & 6.85 & 6.85 & 0.00 & Name & Groundwater & Surface water \\
\hline Irrigation (golf) & 0.00 & 0.00 & 0.00 & 0.00 & 0.00 & \multirow[t]{4}{*}{ City of Millen } & \multirow[t]{4}{*}{0.44} & \multirow[t]{4}{*}{0.00} \\
\hline Livestock-aquaculture & 0.23 & 0.04 & 0.27 & 0.27 & 0.00 & & & \\
\hline Thermoelectric power & 0.00 & 0.00 & 0.00 & 0.00 & 0.00 & & & \\
\hline TOTAL & 6.41 & 1.59 & 8.00 & 8.00 & 0.55 & & & \\
\hline
\end{tabular}

Total use is total withdrawal plus public supply deliveries and losses.

Withdrawals by water source

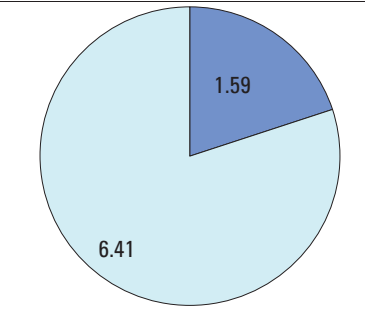

Withdrawals, in million gallons per day

Surface water

Groundwater

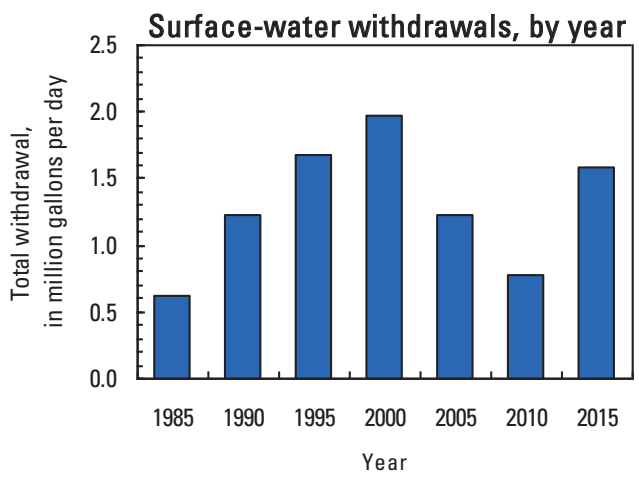

Groundwater withdrawals by aquifer

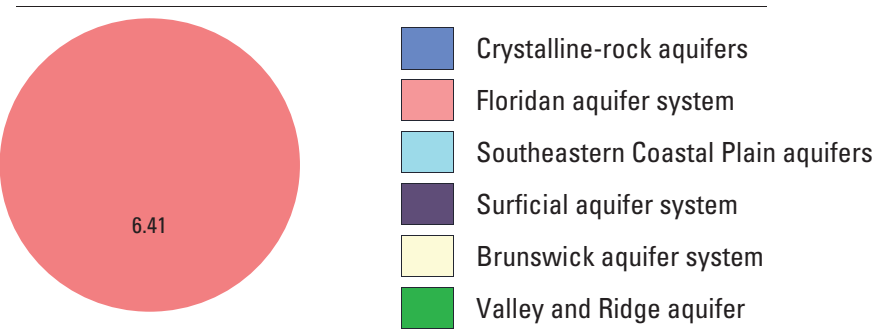




\section{JOHNSON COUNTY}

Population

9,656

Population served by public supply-groundwater $\quad 4,248$

Population served by public supply-surface water 0

Acres irrigated

2015 WATER WITHDRAWALS AND ESTIMATED USE, IN MILLION GALLONS PER DAY

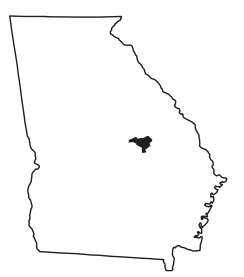

[--,not applicable; Mgal/d, million gallons per day]

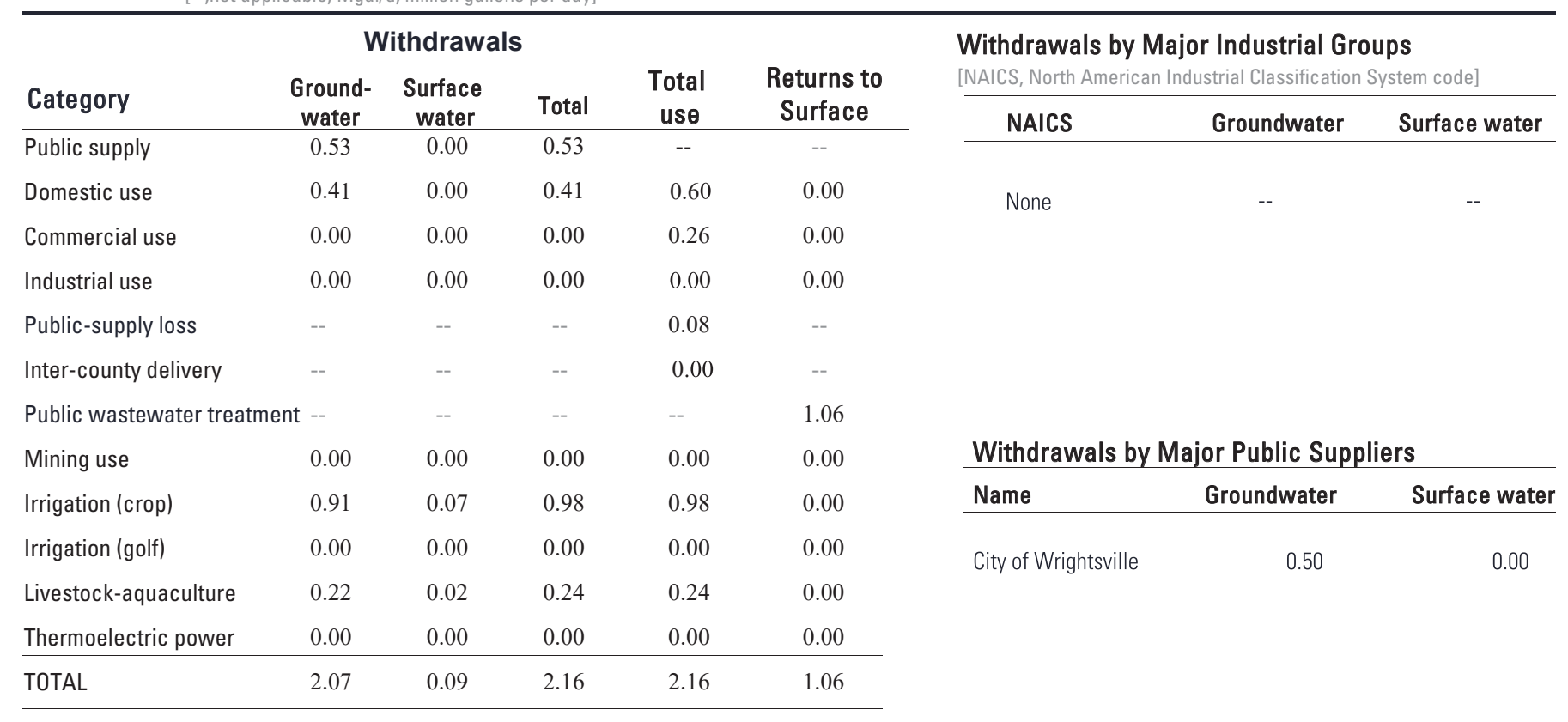

Total use is total withdrawal plus public supply deliveries and losses.

Withdrawals by water source

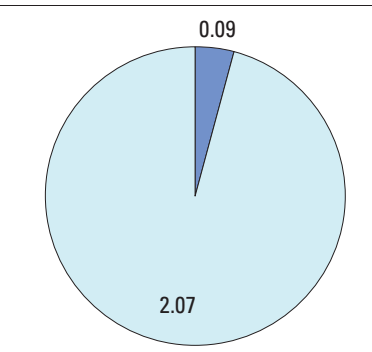

Withdrawals, in million gallons per day

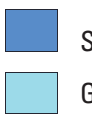

Surface water

Groundwater

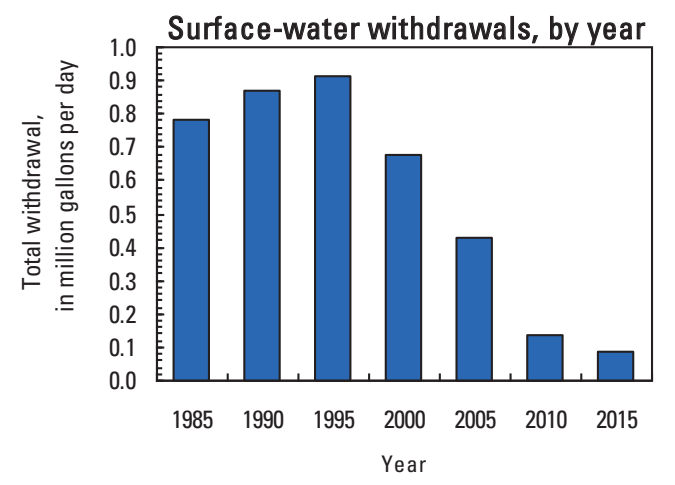

Groundwater withdrawals by aquifer
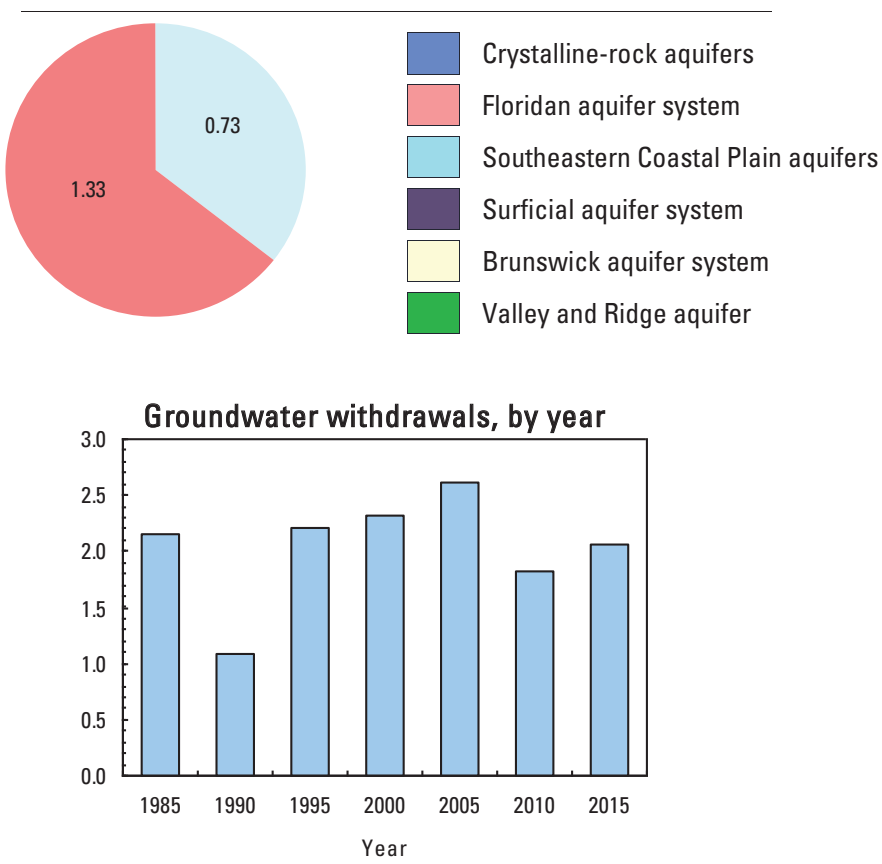


\section{JONES COUNTY}

Population

Population served by public supply-groundwater $\quad 15,349$

Population served by public supply-surface water 0

Acres irrigated

2015 WATER WITHDRAWALS AND ESTIMATED USE, IN MILLION GALLONS PER DAY

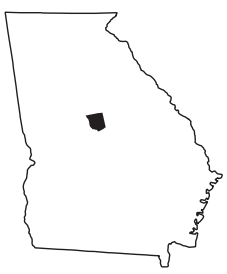

[--,not applicable; Mgal/d, million gallons per day]

\begin{tabular}{lcccccc}
\hline & \multicolumn{3}{c}{ Withdrawals } & & Total \\
\cline { 2 - 4 } Category & $\begin{array}{c}\text { Ground } \\
\text { water }\end{array}$ & $\begin{array}{c}\text { Surface } \\
\text { water }\end{array}$ & $\begin{array}{c}\text { Total } \\
\text { use }\end{array}$ & $\begin{array}{c}\text { Returns to } \\
\text { Surface }\end{array}$ \\
\hline Public supply & 1.69 & 1.04 & 2.73 & -- & -- \\
Domestic use & 0.99 & 0.00 & 0.99 & 2.28 & 0.00 \\
Commercial use & 0.00 & 0.00 & 0.00 & 0.14 & 0.00 \\
Industrial use & 0.00 & 0.00 & 0.00 & 0.00 & 0.00 \\
Public-supply loss & -- & -- & -- & 1.30 & -- \\
Inter-county delivery & -- & -- & -- & -1.04 & -- \\
Public wastewater treatment & -- & -- & -- & -- & 3.42 \\
Mining use & 0.48 & 0.01 & 0.49 & 0.49 & 0.00 \\
Irrigation (crop) & 0.00 & 0.00 & 0.00 & 0.00 & 0.00 \\
Irrigation (golf) & 0.00 & 0.16 & 0.16 & 0.16 & 0.00 \\
Livestock-aquaculture & 0.14 & 0.00 & 0.14 & 0.14 & 0.00 \\
Thermoelectric power & 0.00 & 0.00 & 0.00 & 0.00 & 0.00 \\
\hline TOTAL & 3.30 & 1.21 & 4.51 & 3.47 & 3.42 \\
\hline
\end{tabular}

Total use is total withdrawal plus public supply deliveries and losses. An estimate of $1.04 \mathrm{Mgal} / \mathrm{d}$ was delivered from Jones County to neighboring counties in 2015

Withdrawals by Major Industrial Groups

[NAICS, North American Industrial Classification System code]

\begin{tabular}{lll}
\hline NAICS Groundwater & Surface water \\
\hline
\end{tabular}

None

Withdrawals by Major Public Suppliers

\begin{tabular}{lcc} 
Name & Groundwater & Surface water \\
\hline Jones County & 1.23 & 0.00 \\
Macon Water Authority & 0.00 & 1.04 \\
City of Gray & 0.37 & 0.00
\end{tabular}

About $24 \mathrm{Mgal} / \mathrm{d}$ of water is withdrawn from the Ocmulgee River in Bibb County, Georgia delivered to Javors Lucas (Town Creek) Reservoir in Jones County. About $25 \mathrm{Mgal} / \mathrm{d}$ was withdrawn from the reservoir and delivered to Bibb County. Net withdrawal from Jones County was $1.04 \mathrm{Mgal} / \mathrm{d}$ in 2015.

\section{Withdrawals by water source}

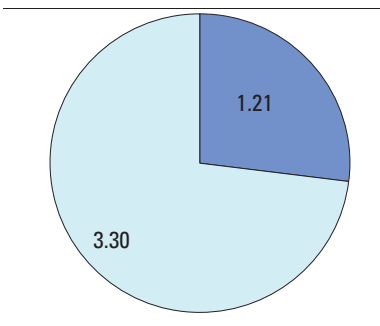

Withdrawals, in million

gallons per day

Surface water

Groundwater
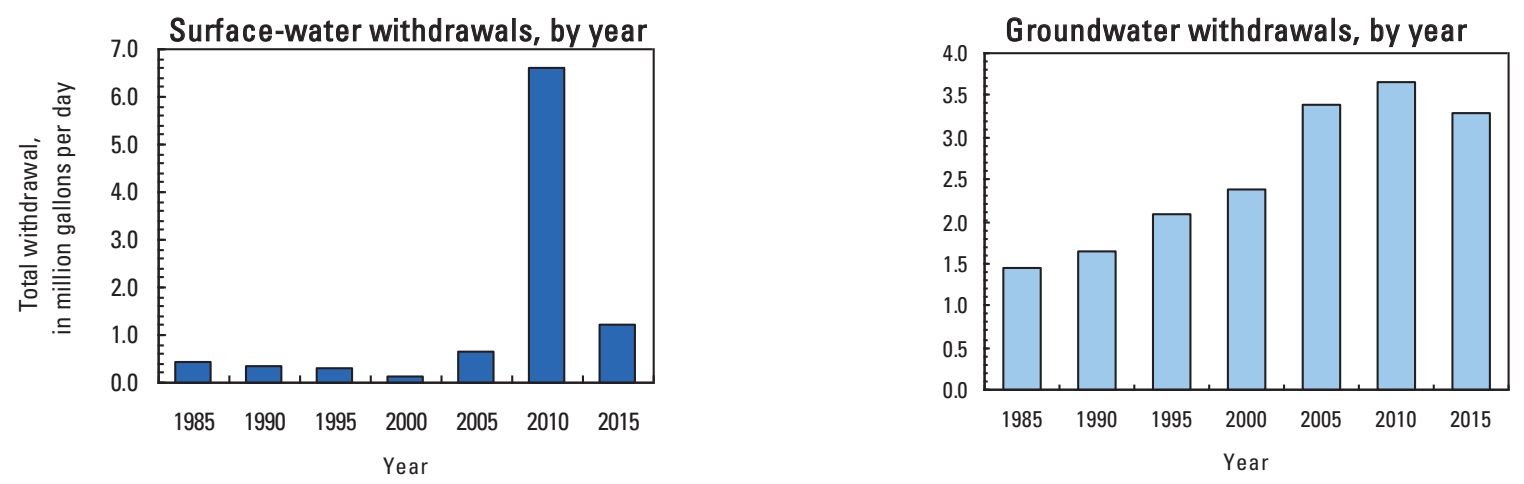


\section{LAMAR COUNTY}

Population

Population served by public supply-groundwater

Population served by public supply-surface water $\quad 7,657$

Acres irrigated

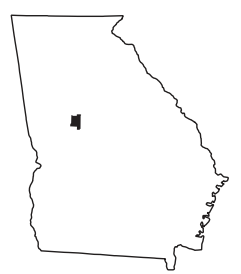

2015 WATER WITHDRAWALS AND ESTIMATED USE, IN MILLION GALLONS PER DAY

[--,not applicable; Mgal/d, million gallons per day]

\begin{tabular}{|c|c|c|c|c|c|c|c|c|}
\hline \multirow{3}{*}{ Category } & \multicolumn{3}{|c|}{ Withdrawals } & \multirow{3}{*}{$\begin{array}{c}\text { Total } \\
\text { use }\end{array}$} & \multirow{3}{*}{$\begin{array}{l}\text { Returns to } \\
\text { Surface }\end{array}$} & \multirow{2}{*}{\multicolumn{3}{|c|}{$\begin{array}{l}\text { Withdrawals by Major Industrial Groups } \\
\text { [NAICS, North American Industrial Classification System code] }\end{array}$}} \\
\hline & \multirow{2}{*}{$\begin{array}{c}\text { Ground- } \\
\text { water }\end{array}$} & \multirow{2}{*}{$\begin{array}{c}\text { Surface } \\
\text { water }\end{array}$} & \multirow{2}{*}{ Total } & & & & & \\
\hline & & & & & & NAICS & Groundwater & Surface water \\
\hline Public supply & 0.01 & 1.86 & 1.87 & -- & -- & & & \\
\hline Domestic use & 0.78 & 0.00 & 0.78 & 2.14 & 0.00 & None & -- & -- \\
\hline Commercial use & 0.00 & 0.00 & 0.00 & 0.22 & 0.00 & & & \\
\hline Industrial use & 0.00 & 0.00 & 0.00 & 0.01 & 0.00 & & & \\
\hline Public-supply loss & -- & -- & -- & 0.28 & -- & & & \\
\hline Inter-county delivery & -- & -- & -- & 0.00 & -- & & & \\
\hline Public wastewater treatment & tt -- & -- & -- & -- & 2.72 & & & \\
\hline Mining use & 0.00 & 0.00 & 0.00 & 0.00 & 0.00 & Withdrawals by & or Public Supp & \\
\hline Irrigation (crop) & 1.02 & 0.00 & 1.02 & 1.02 & 0.00 & Name & Groundwater & Surface water \\
\hline Irrigation (golf) & 0.00 & 0.08 & 0.08 & 0.08 & 0.00 & City of Barnesville & 0.00 & 1.86 \\
\hline Livestock-aquaculture & 0.26 & 0.00 & 0.26 & 0.26 & 0.00 & & & \\
\hline Thermoelectric power & 0.00 & 0.00 & 0.00 & 0.00 & 0.00 & & & \\
\hline TOTAL & 2.07 & 1.94 & 4.01 & 4.01 & 2.72 & & & \\
\hline
\end{tabular}

Total use is total withdrawal plus public supply deliveries and losses.

Withdrawals by water source

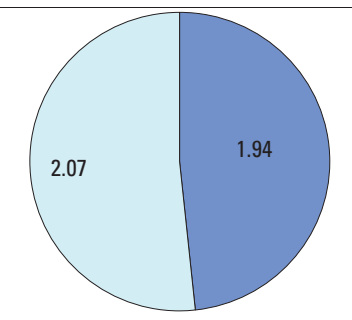

Withdrawals, in million

gallons per day

Surface water

Groundwater

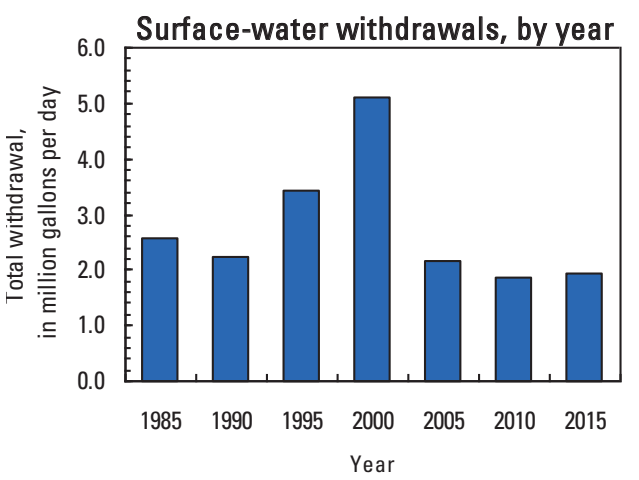

Groundwater withdrawals by aquifer
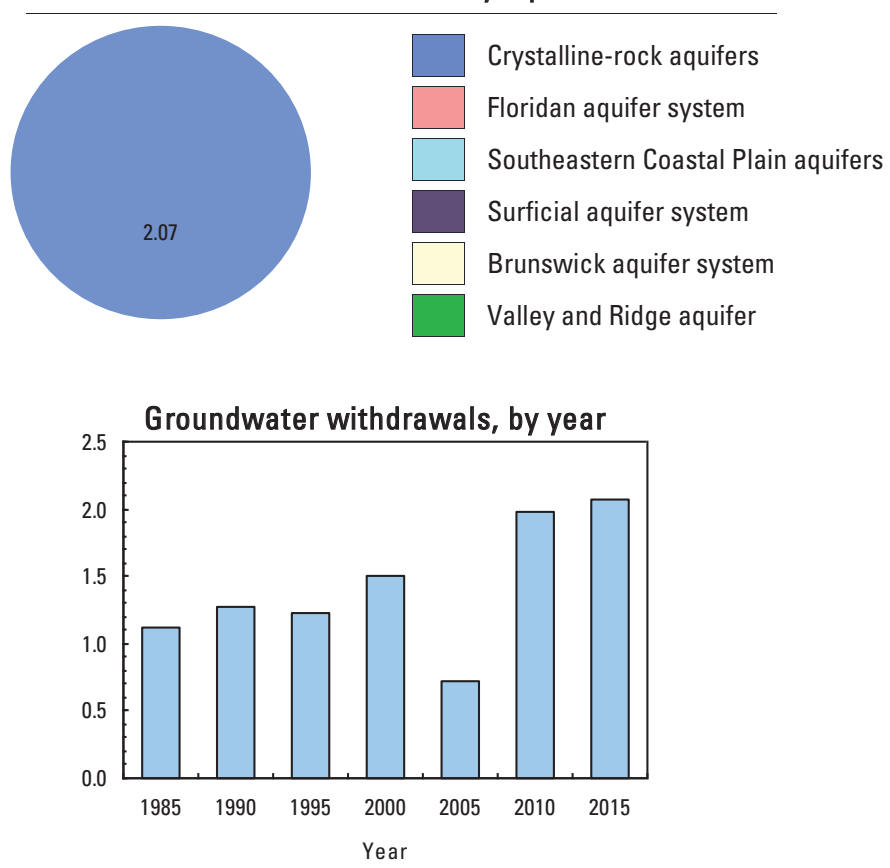


\section{LANIER COUNTY}

Population

10,312

Population served by public supply-groundwater

4,136

Population served by public supply-surface water

0

Acres irrigated

5,260

2015 WATER WITHDRAWALS AND ESTIMATED USE, IN MILLION GALLONS PER DAY

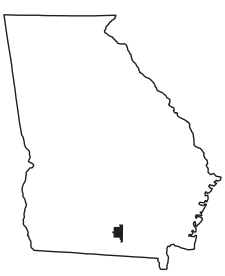

[--,not applicable; Mgal/d, million gallons per day]

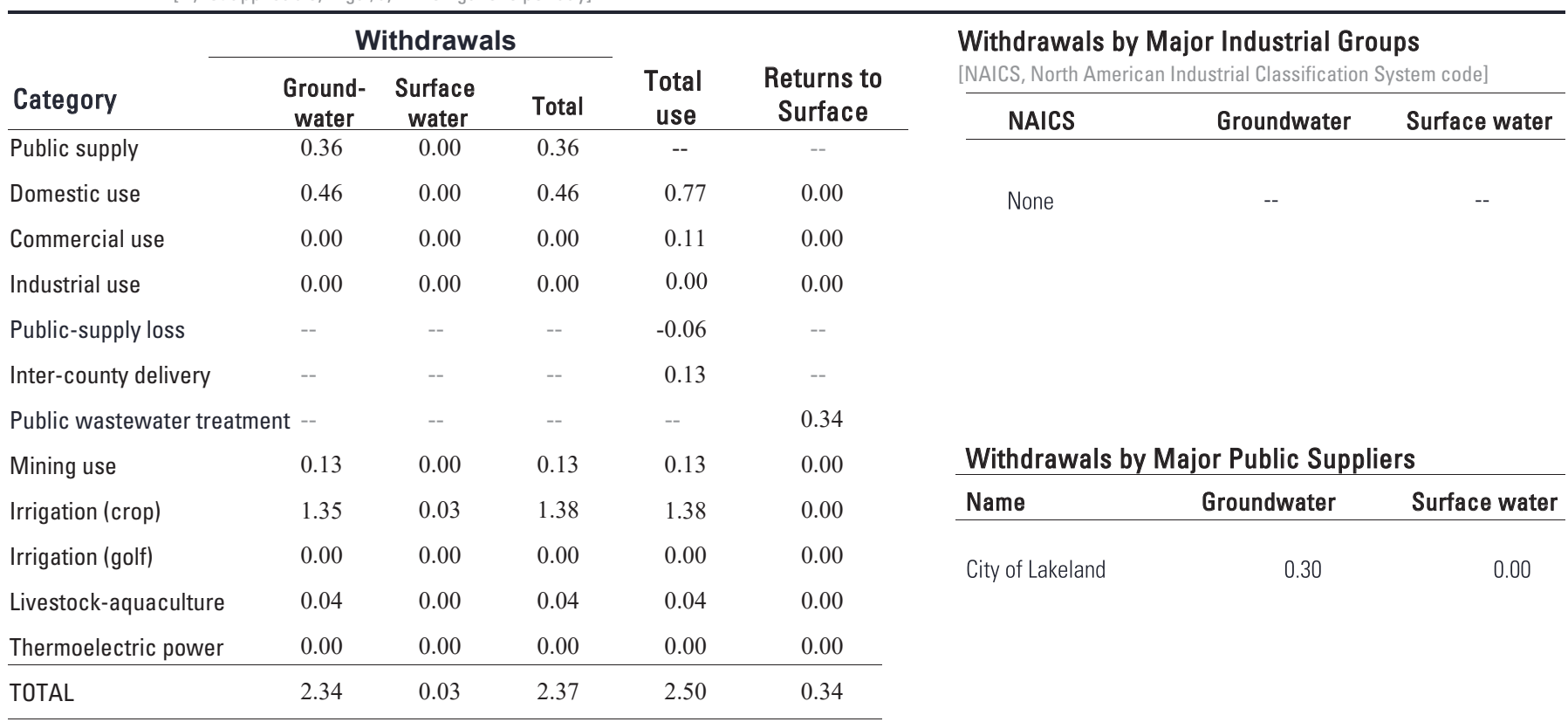

Total use is total withdrawal plus public supply deliveries and losses. An estimate of $0.13 \mathrm{Mgal} / \mathrm{d}$ was delivered from neighboring counties in 2015 .
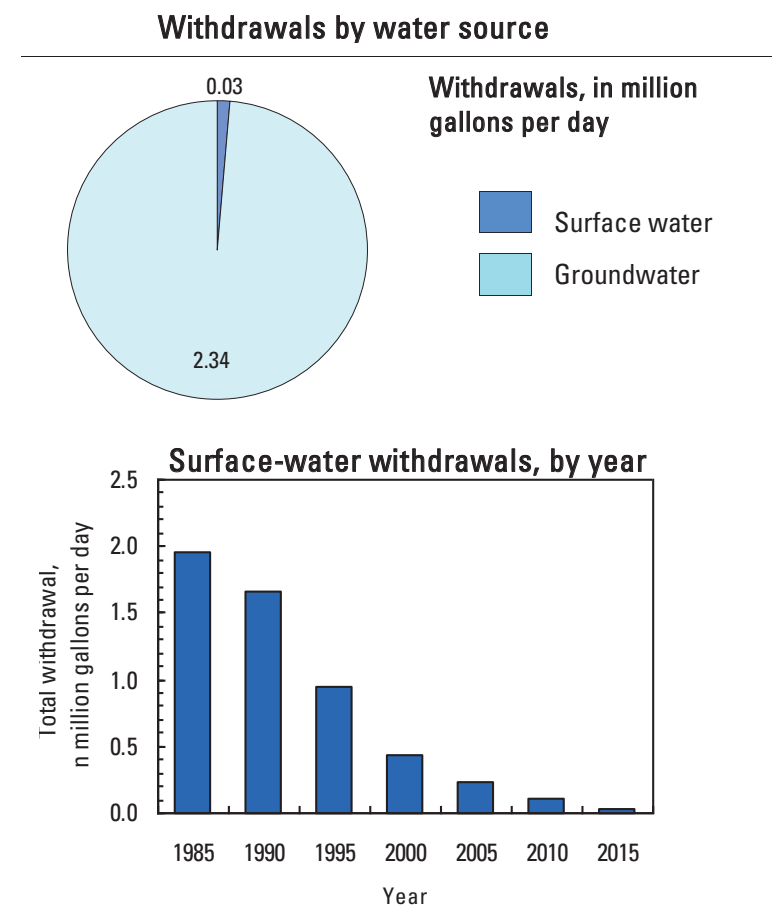

Groundwater withdrawals by aquifer

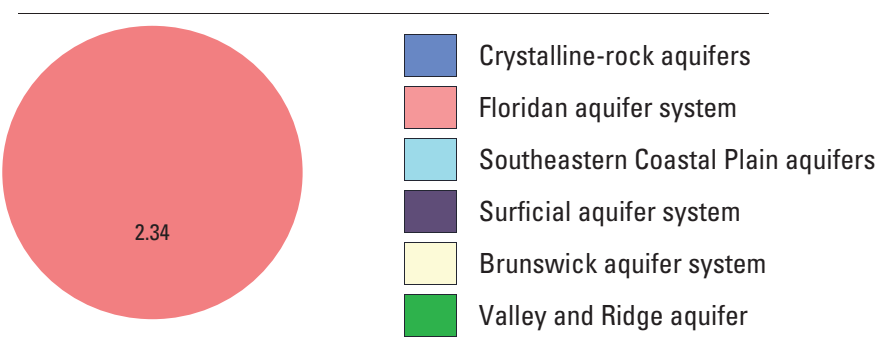

Groundwater withdrawals, by year

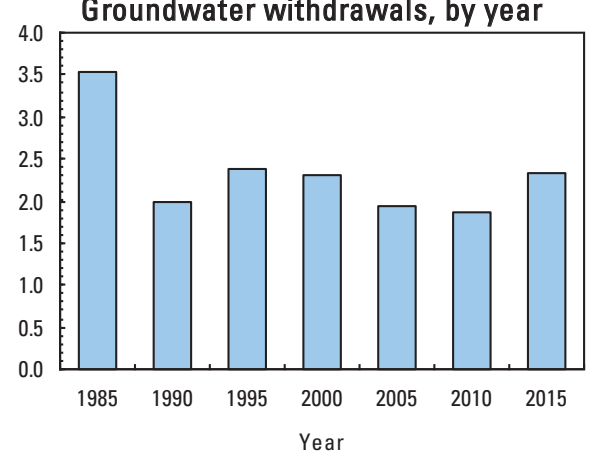




\section{LAURENS COUNTY}

Population

Population served by public supply-groundwater $\quad 14,533$

Population served by public supply-surface water $\quad 10,150$

Acres irrigated

2015 WATER WITHDRAWALS AND ESTIMATED USE, IN MILLION GALLONS PER DAY

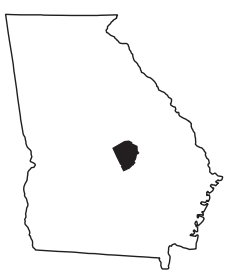

[--,not applicable; Mgal/d, million gallons per day]

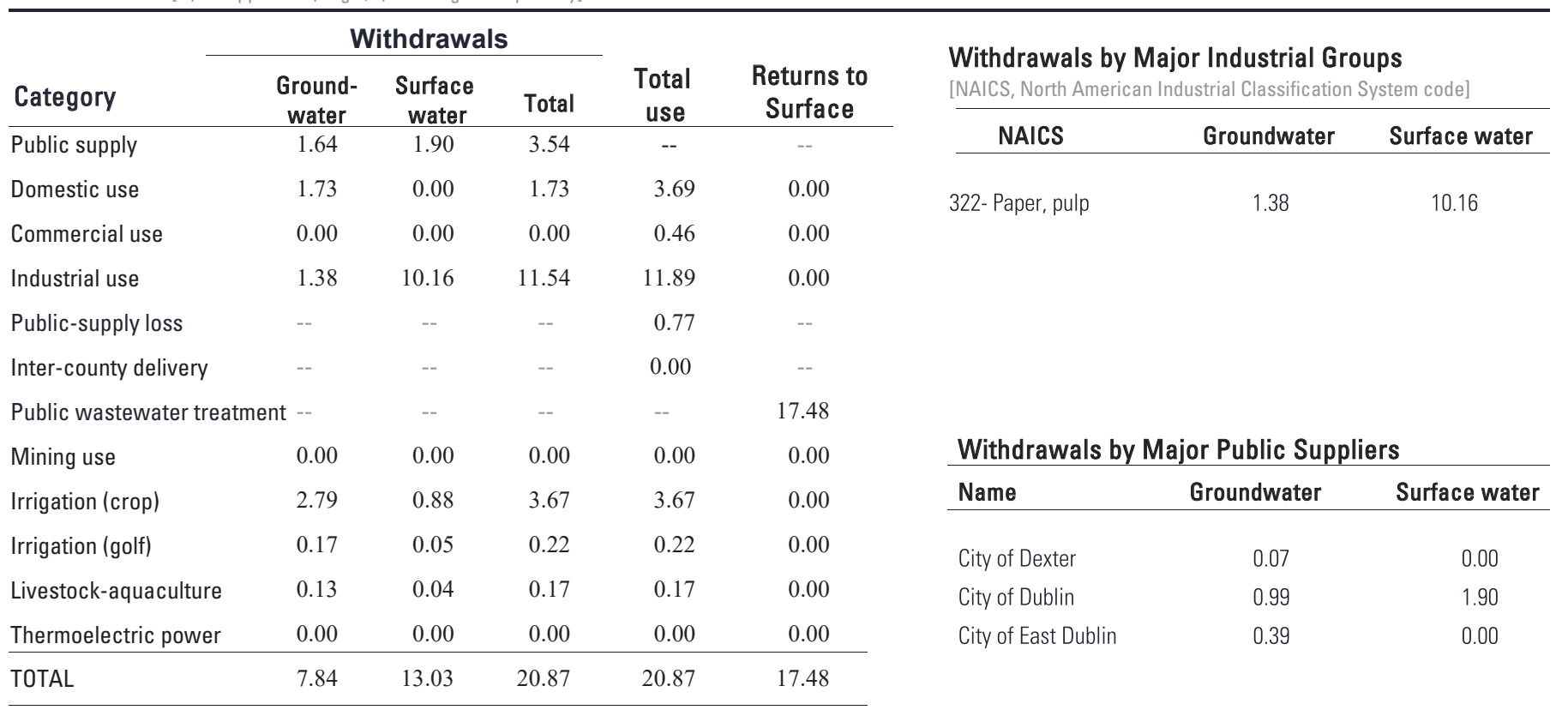

Total use is total withdrawal plus public supply deliveries and losses.

Withdrawals by water source

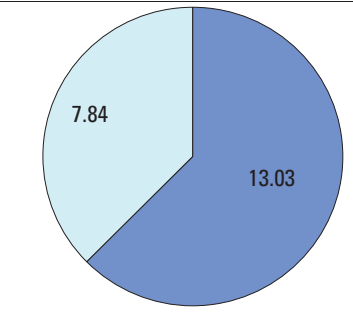

Withdrawals, in million

gallons per day

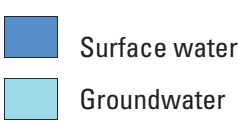

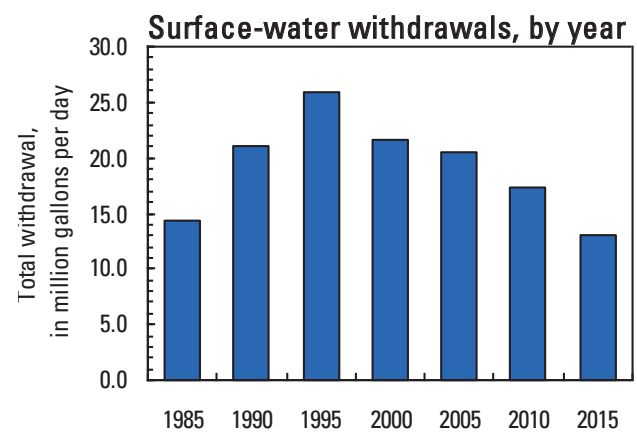

Groundwater withdrawals by aquifer
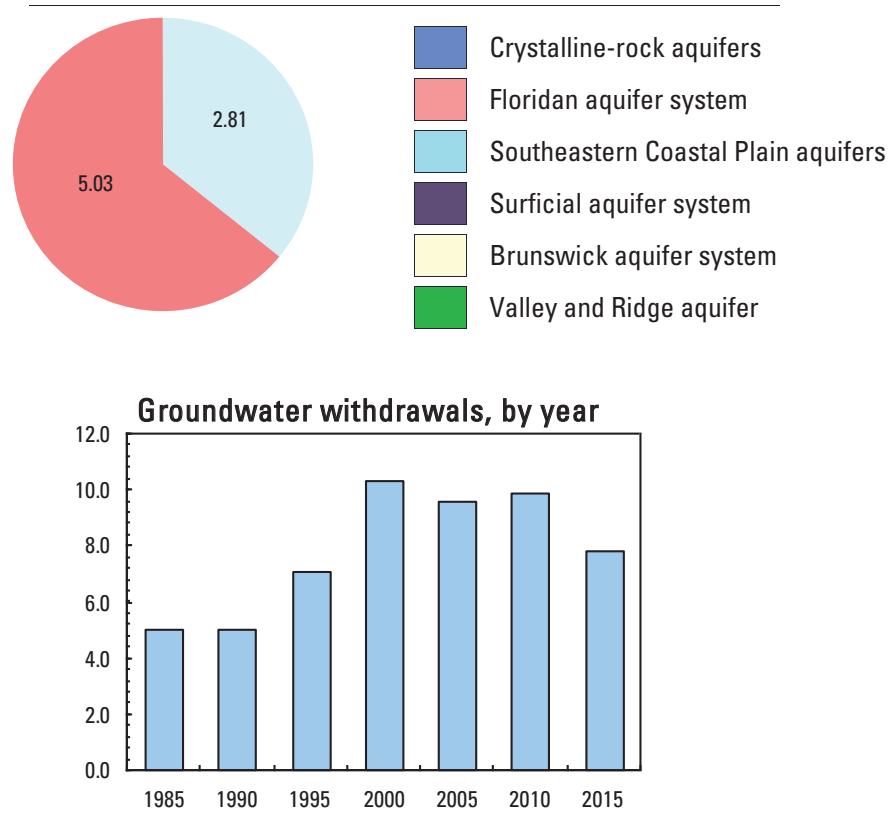


\section{LEE COUNTY}

Population

Population served by public supply-groundwater

Population served by public supply-surface water

Acres irrigated

29,202

18,727

0

42,830

[--,not applicable; Mgal/d, million gallons per day]

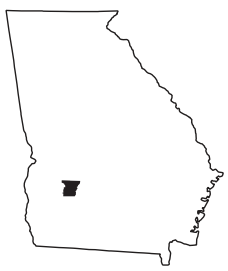

\begin{tabular}{|c|c|c|c|c|c|c|c|c|}
\hline \multirow{3}{*}{ Category } & \multicolumn{3}{|c|}{ Withdrawals } & \multirow{3}{*}{$\begin{array}{c}\text { Total } \\
\text { use }\end{array}$} & \multirow{3}{*}{$\begin{array}{l}\text { Returns to } \\
\text { Surface }\end{array}$} & \multirow{2}{*}{\multicolumn{3}{|c|}{$\begin{array}{l}\text { Withdrawals by Major Industrial Groups } \\
\text { [NAICS, North American Industrial Classification System code] }\end{array}$}} \\
\hline & \multirow{2}{*}{$\begin{array}{c}\text { Ground- } \\
\text { water } \\
1.87\end{array}$} & \multirow{3}{*}{$\begin{array}{c}\text { Surface } \\
\text { water }\end{array}$} & \multirow{2}{*}{ Total } & & & & & \\
\hline & & & & & & \multirow[t]{4}{*}{ NAICS } & \multirow[t]{2}{*}{ Groundwater } & \multirow{2}{*}{ Surface water } \\
\hline Public supply & & & 1.87 & -- & -- & & & \\
\hline Domestic use & 0.79 & 0.00 & 0.79 & 2.17 & 0.00 & & -- & -- \\
\hline Commercial use & 0.11 & 0.00 & 0.11 & 0.30 & 0.00 & & & \\
\hline Industrial use & 0.00 & 0.00 & 0.00 & 0.02 & 0.00 & & & \\
\hline Public-supply loss & & & & 0.28 & -- & & & \\
\hline Inter-county delivery & -- & -- & -- & 0.00 & -- & & & \\
\hline \multicolumn{2}{|c|}{ Public wastewater treatment -- } & -- & -- & -- & 1.15 & & & \\
\hline Mining use & 0.08 & 0.00 & 0.08 & 0.08 & 0.00 & \multicolumn{3}{|c|}{ Withdrawals by Major Public Suppliers } \\
\hline Irrigation (crop) & 23.45 & 3.81 & 27.26 & 27.26 & 0.00 & Name & Groundwater & Surface water \\
\hline Irrigation (golf) & 0.00 & 0.00 & 0.00 & 0.00 & 0.00 & City of Leesburg & 0.40 & 0.00 \\
\hline Livestock-aquaculture & 0.41 & 0.07 & 0.48 & 0.48 & 0.00 & \multirow{3}{*}{$\begin{array}{l}\text { Lee County Utilities } \\
\text { Authority }\end{array}$} & \multirow[t]{3}{*}{1.28} & \multirow[t]{3}{*}{0.00} \\
\hline Thermoelectric power & 0.00 & 0.00 & 0.00 & 0.00 & 0.00 & & & \\
\hline TOTAL & 26.71 & 3.88 & 30.59 & 30.59 & 1.15 & & & \\
\hline
\end{tabular}

Total use is total withdrawal plus public supply deliveries and losses.

Withdrawals by water source

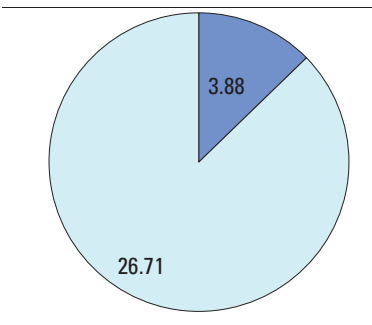

Withdrawals, in million

gallons per day

Surface water

Groundwater

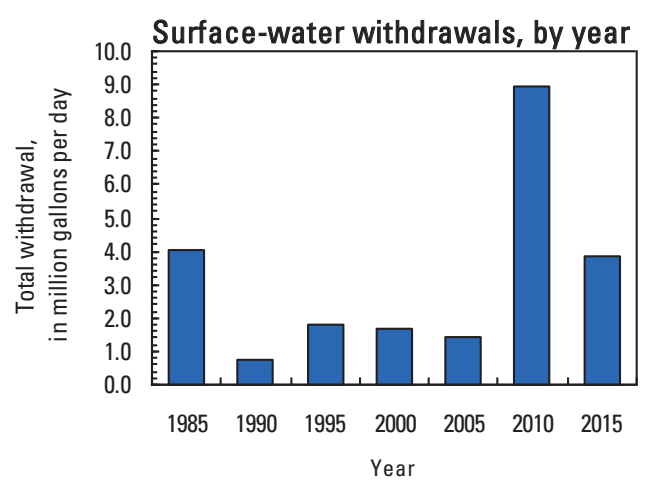

Groundwater withdrawals by aquifer

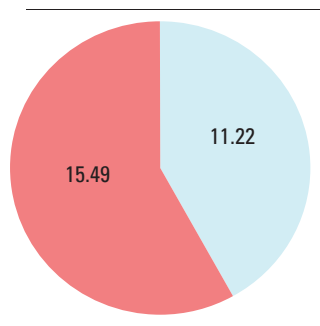

Crystalline-rock aquifers

Floridan aquifer system

Southeastern Coastal Plain aquifers

Surficial aquifer system

Brunswick aquifer system

Valley and Ridge aquifer
Groundwater withdrawals, by year

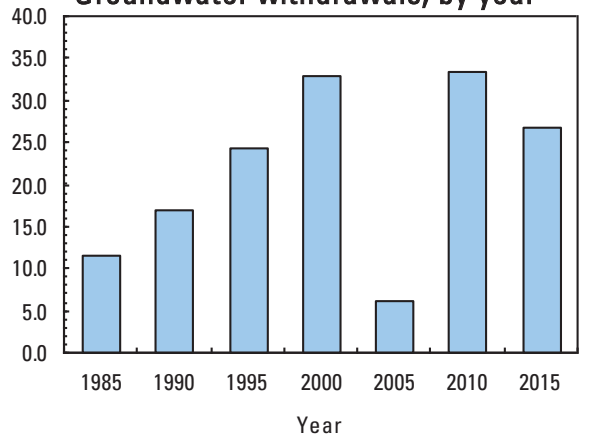




\section{LIBERTY COUNTY}

Population

Population served by public supply-groundwater

51,264

Population served by public supply-surface water 0

Acres irrigated

0

2015 WATER WITHDRAWALS AND ESTIMATED USE, IN MILLION GALLONS PER DAY

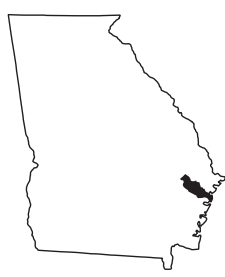

[--,not applicable; Mgal/d, million gallons per day]

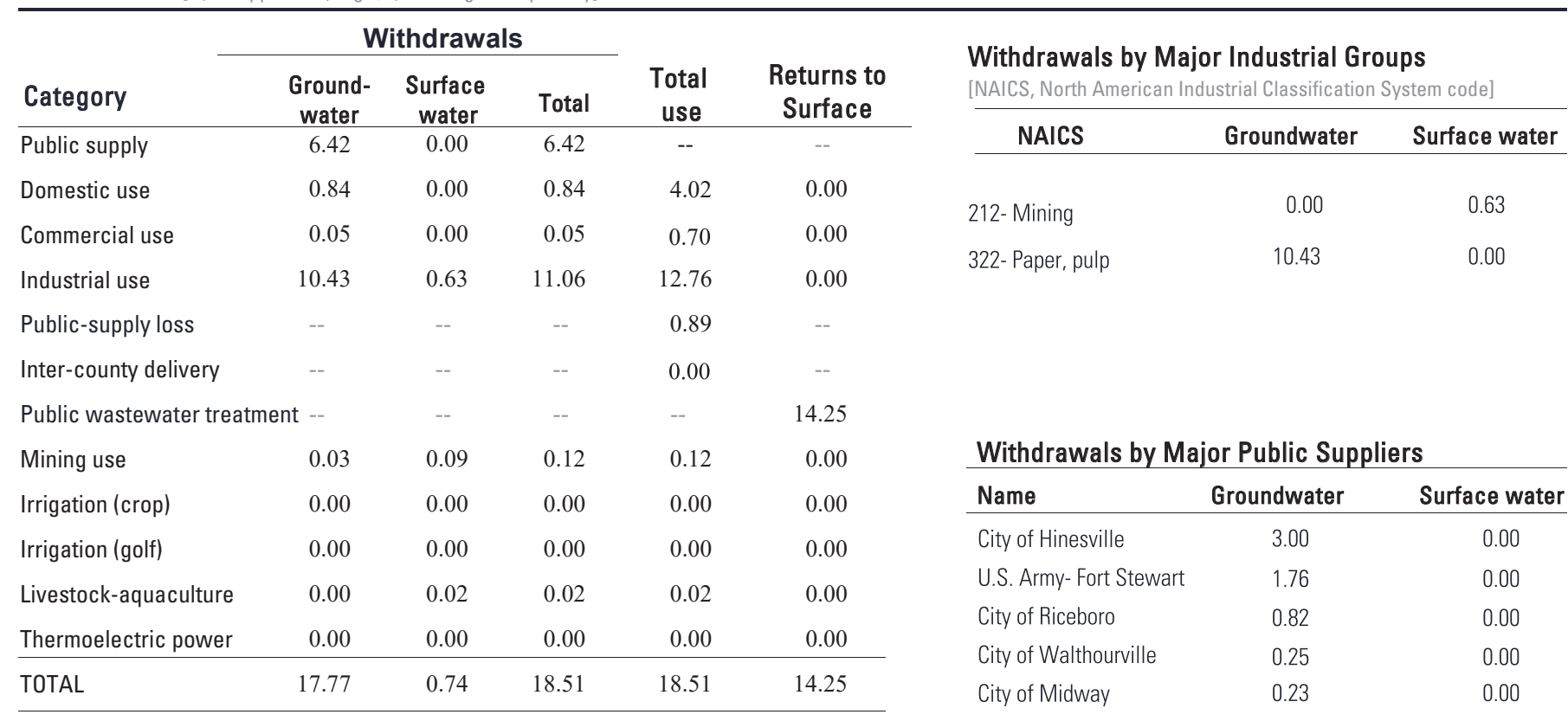

Total use is total withdrawal plus public supply deliveries and losses.

Withdrawals by water source

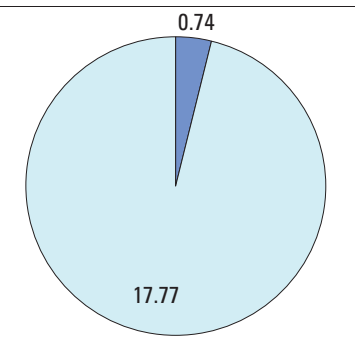

Withdrawals, in million

gallons per day

Surface water

Groundwater

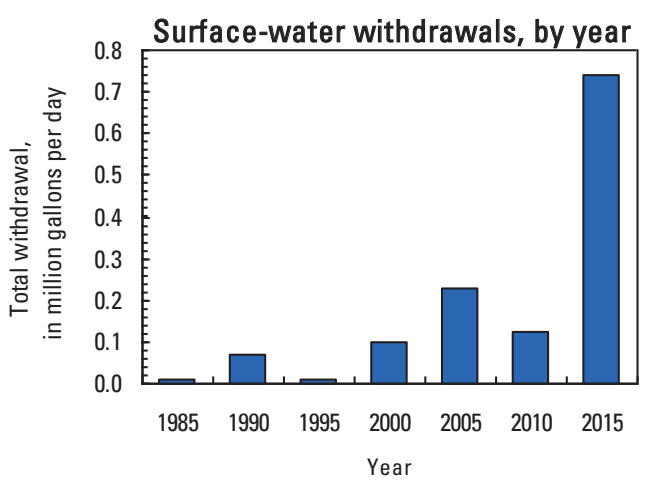

Groundwater withdrawals by aquifer
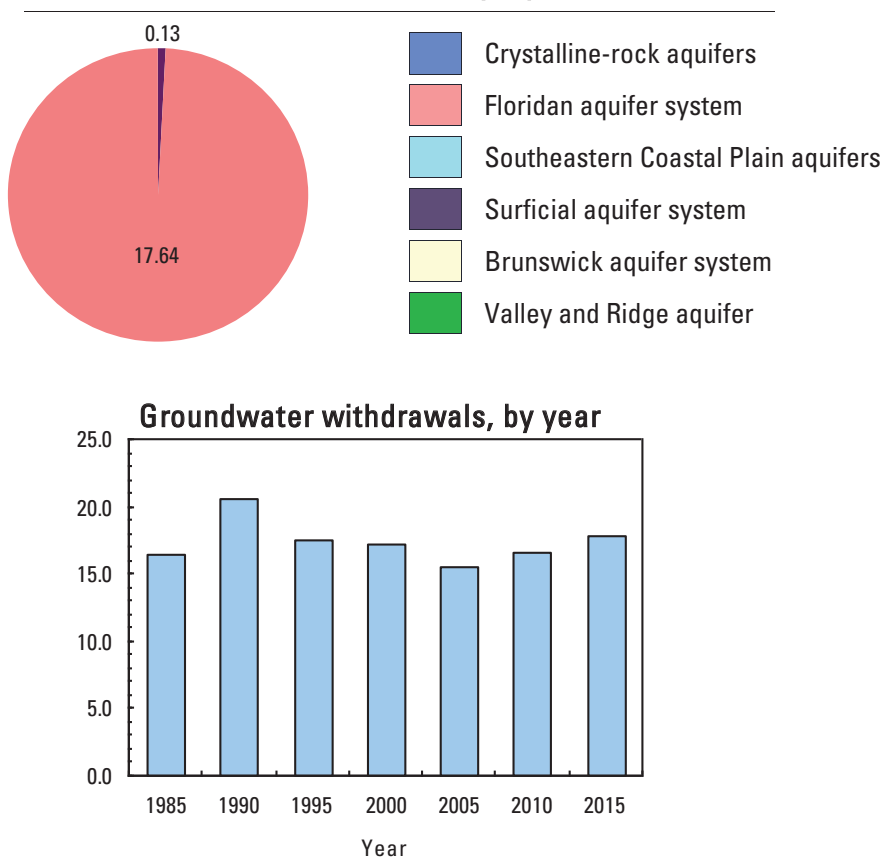


\section{LINCOLN COUNTY}

Population

Population served by public supply-groundwater

Population served by public supply-surface water $\quad 3,256$

Acres irrigated

\section{0}

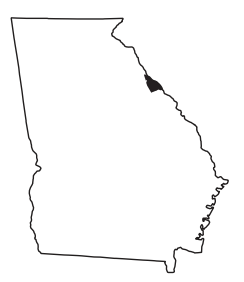

2015 WATER WITHDRAWALS AND ESTIMATED USE, IN MILLION GALLONS PER DAY

[--,not applicable; Mgal/d, million gallons per day]

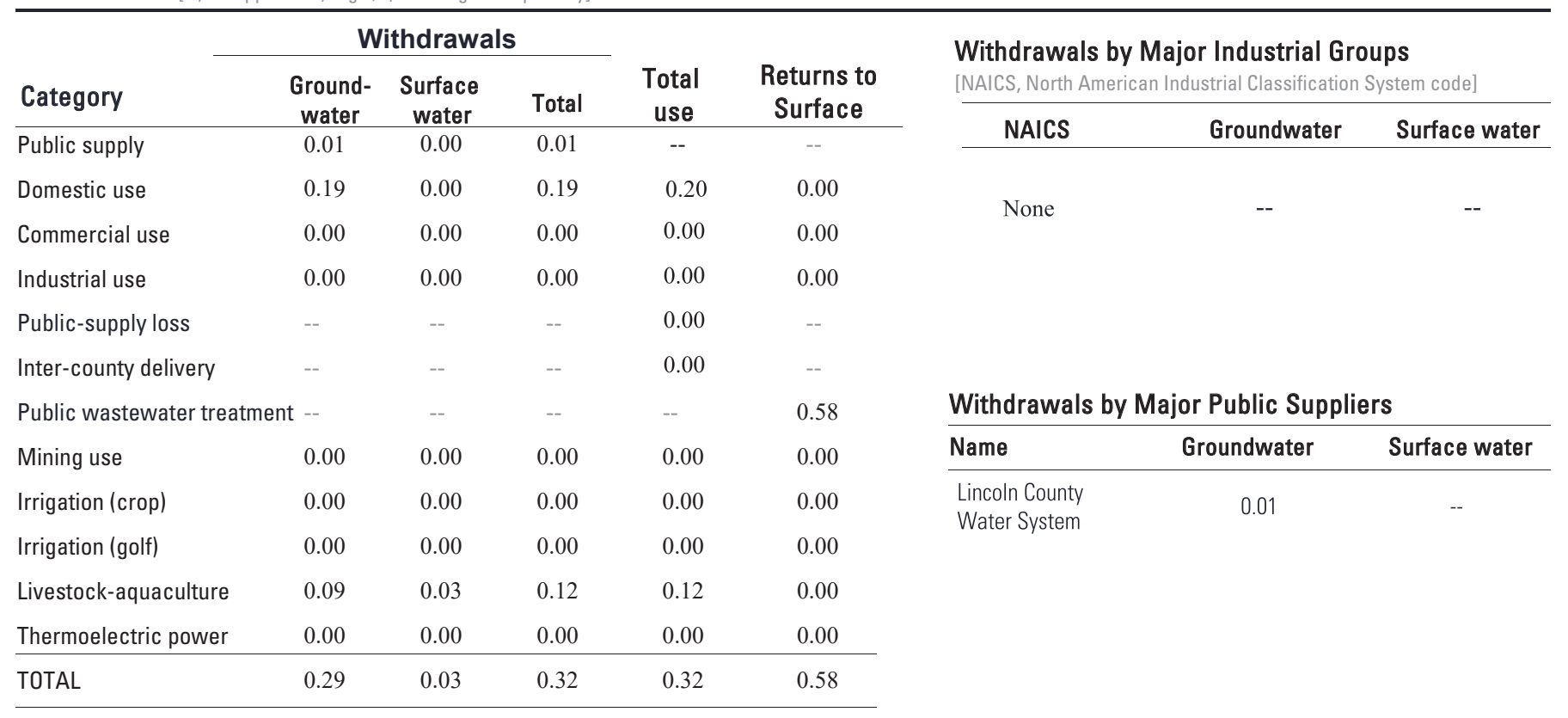

Total use is total withdrawal plus public supply deliveries and losses.

Withdrawals by water source

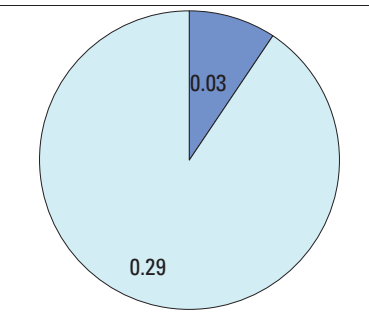

Withdrawals, in million gallons per day

Surface water

Groundwater

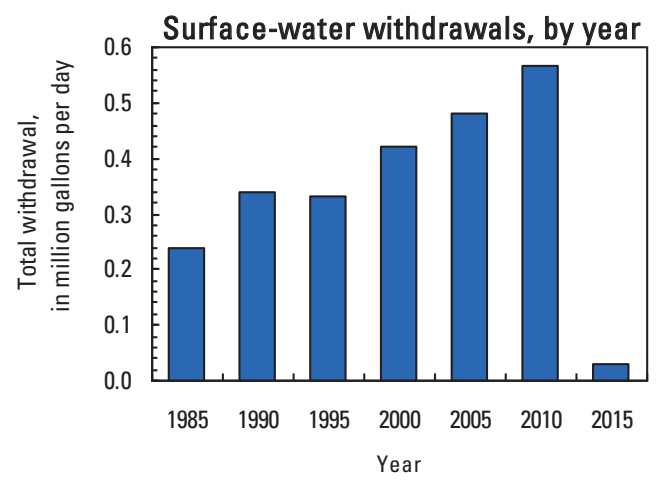

Groundwater withdrawals by aquifer

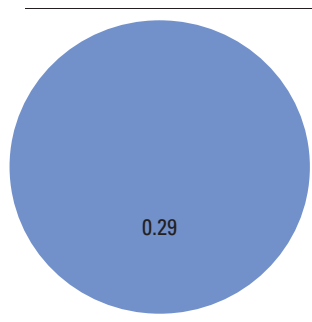

Crystalline-rock aquifers

Floridan aquifer system

Southeastern Coastal Plain aquifers

Surficial aquifer system

Brunswick aquifer system

Valley and Ridge aquifer

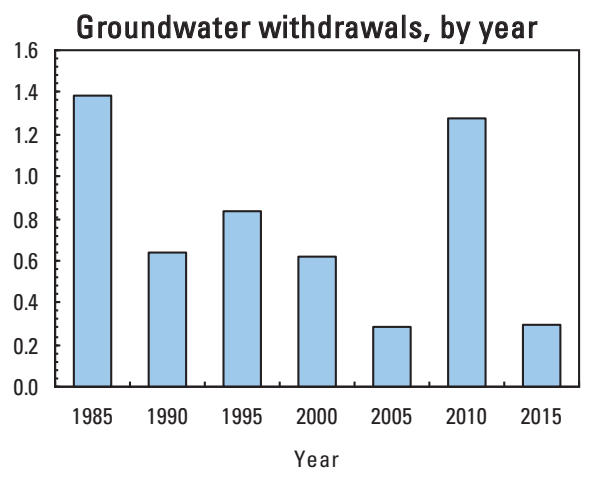




\section{LONG COUNTY}

Population

Population served by public supply-groundwater

Population served by public supply-surface water

Acres irrigated

2015 WATER WITHDRAWALS AND ESTIMATED USE, IN MILLION GALLONS PER DAY

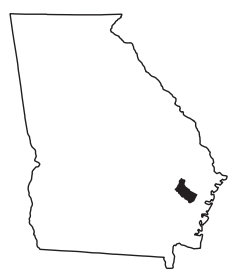

$[--$, not applicable; Mgal/d, million gallons per day]

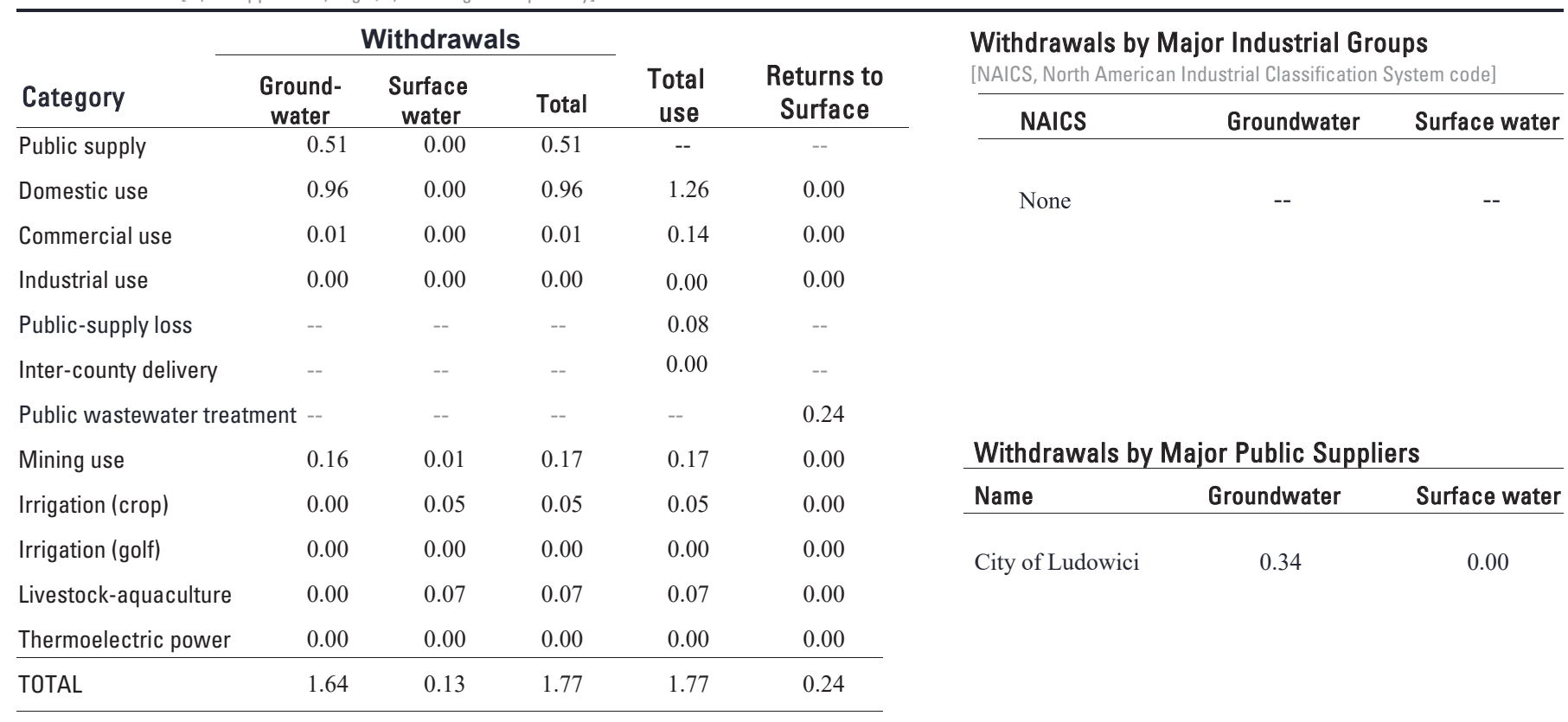

Total use is total withdrawal plus public supply deliveries and losses.

Withdrawals by water source

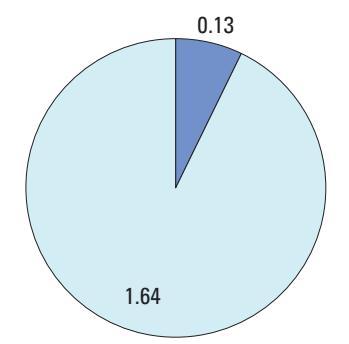

Withdrawals, in million

gallons per day

Surface water

Groundwater

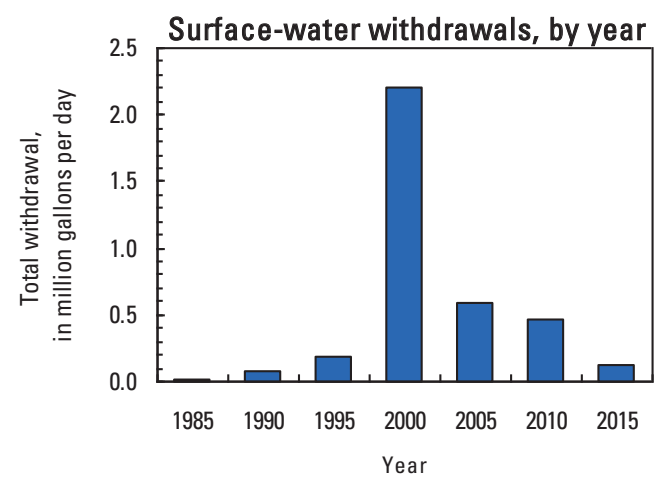

Groundwater withdrawals by aquifer
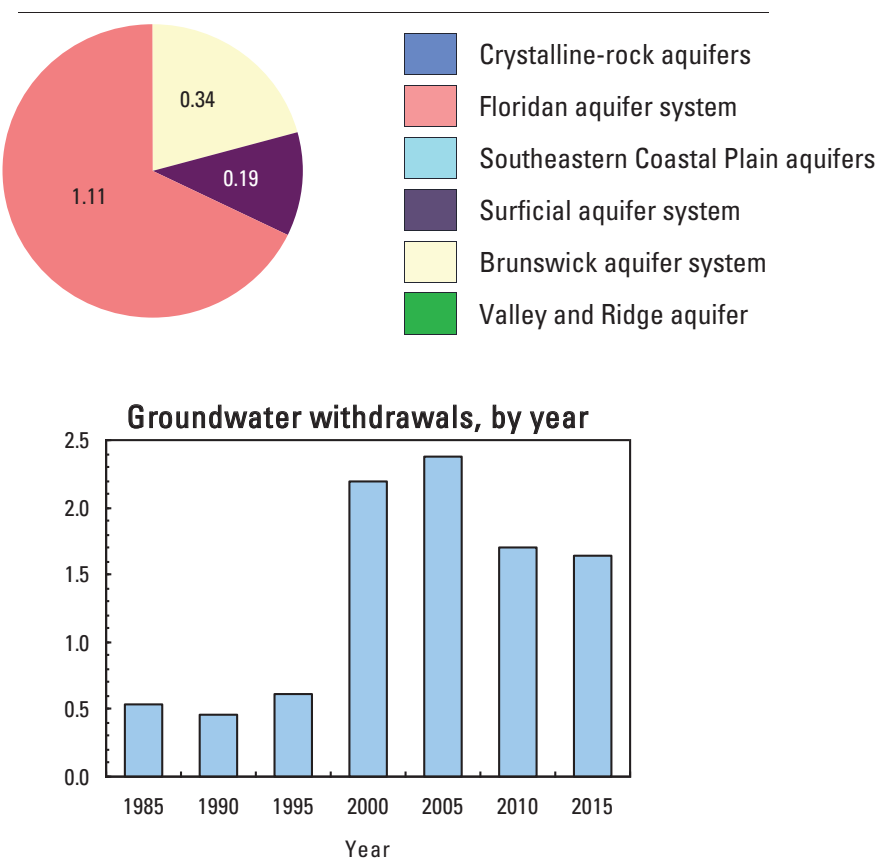


\section{LOWNDES COUNTY}

Population

Population served by public supply-groundwater

89,349

Population served by public supply-surface water

0

Acres irrigated

8,320

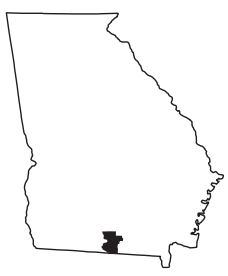

2015 WATER WITHDRAWALS AND ESTIMATED USE, IN MILLION GALLONS PER DAY

[--,not applicable; Mgal/d, million gallons per day]

\begin{tabular}{|c|c|c|c|c|c|}
\hline \multirow[b]{2}{*}{ Category } & \multicolumn{3}{|c|}{ Withdrawals } & \multirow[b]{2}{*}{$\begin{array}{c}\text { Total } \\
\text { use }\end{array}$} & \multirow[b]{2}{*}{$\begin{array}{c}\text { Returns to } \\
\text { Surface }\end{array}$} \\
\hline & $\begin{array}{c}\text { Ground- } \\
\text { water }\end{array}$ & $\begin{array}{c}\text { Surface } \\
\text { water }\end{array}$ & Total & & \\
\hline Public supply & 13.16 & 0.00 & 13.16 & -- & -- \\
\hline Domestic use & 1.76 & 0.00 & 1.76 & 8.96 & 0.00 \\
\hline Commercial use & 0.00 & 0.00 & 0.00 & 2.90 & 0.00 \\
\hline Industrial use & 11.26 & 0.00 & 11.26 & 12.33 & 0.00 \\
\hline Public-supply loss & -- & -- & -- & 1.99 & -- \\
\hline Inter-county delivery & -- & -- & -- & 0.00 & -- \\
\hline Public wastewater treatmer & ent -- & -- & -- & -- & 10.03 \\
\hline Mining use & 0.00 & 0.00 & 0.00 & 0.00 & 0.00 \\
\hline Irrigation (crop) & 1.38 & 0.22 & 1.60 & 1.60 & 0.00 \\
\hline Irrigation (golf) & 0.17 & 0.26 & 0.43 & 0.43 & 0.00 \\
\hline Livestock-aquaculture & 0.17 & 0.02 & 0.19 & 0.19 & 0.00 \\
\hline Thermoelectric power & 0.00 & 0.00 & 0.00 & 0.00 & 0.00 \\
\hline TOTAL & 27.90 & 0.50 & 28.40 & 28.40 & 10.03 \\
\hline
\end{tabular}

Withdrawals by Major Industrial Groups

[NAICS, North American Industrial Classification System code]

\begin{tabular}{lcc}
\hline \multicolumn{1}{c}{ NAICS } & Groundwater & Surface water \\
\hline 322- Paper, pulp & 10.86 & 0.00 \\
325- Chemicals & 0.36 & 0.00 \\
335- Instruments & 0.04 & 0.00
\end{tabular}

Total use is total withdrawal plus public supply deliveries and losses.

Withdrawals by water source

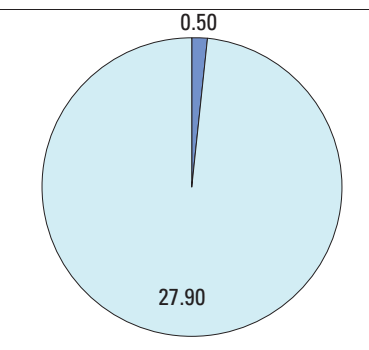

Withdrawals, in million gallons per day

Surface water

Groundwater

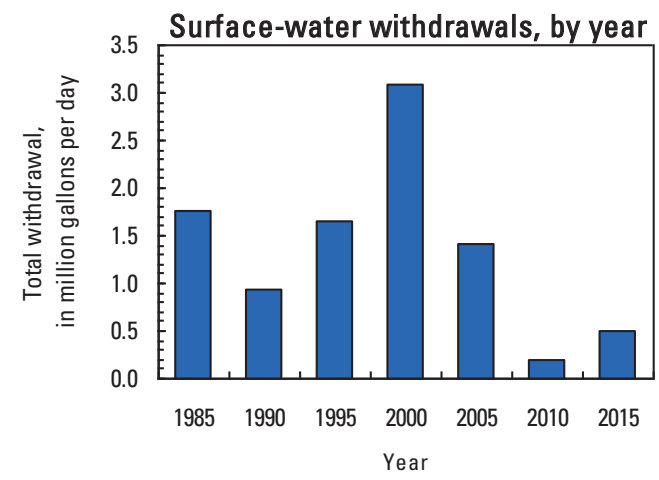

Withdrawals by Major Public Suppliers

\begin{tabular}{lcc} 
Name & Groundwater & Surface wate \\
\hline City of Valdosta & 10.11 & 0.00 \\
South Lowndes County & 0.98 & 0.00 \\
Water System & & \\
Lowndes County & 0.32 & 0.00 \\
Kinderlou Forest Water & 0.24 & 0.00 \\
System & & 0.00 \\
Moody Air Force Base & 0.28 &
\end{tabular}

Moody Air Force Base

0.28

0.00
Groundwater withdrawals by aquifer
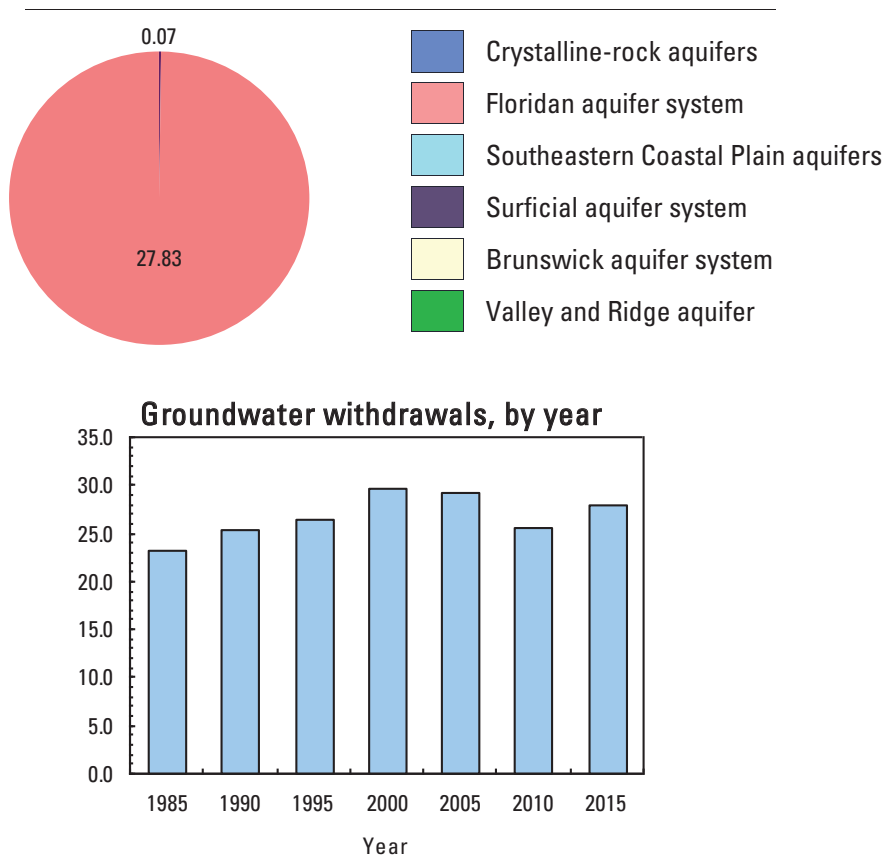


\section{LUMPKIN COUNTY}

Population

Population served by public supply-groundwater

3,581

Population served by public supply-surface water $\quad 4,954$

Acres irrigated

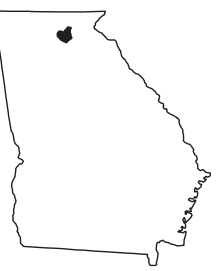

2015 WATER WITHDRAWALS AND ESTIMATED USE, IN MILLION GALLONS PER DAY

[--,not applicable; Mgal/d, million gallons per day]

\begin{tabular}{|c|c|c|c|c|c|c|c|c|}
\hline \multirow{3}{*}{ Category } & \multicolumn{3}{|c|}{ Withdrawals } & \multirow{3}{*}{$\begin{array}{c}\text { Total } \\
\text { use }\end{array}$} & \multirow{3}{*}{$\begin{array}{l}\text { Returns to } \\
\text { Surface }\end{array}$} & \multirow{2}{*}{\multicolumn{3}{|c|}{$\begin{array}{l}\text { Withdrawals by Major Industrial Groups } \\
\text { [NAICS, North American Industrial Classification System code] }\end{array}$}} \\
\hline & \multirow{2}{*}{$\begin{array}{l}\text { Ground - } \\
\text { water }\end{array}$} & \multirow{2}{*}{$\begin{array}{c}\text { Surface } \\
\text { water }\end{array}$} & \multirow{2}{*}{ Total } & & & & & \\
\hline & & & & & & \multirow{4}{*}{ None } & \multirow[t]{2}{*}{ Groundwater } & \multirow[t]{2}{*}{ Surface water } \\
\hline Public supply & 0.21 & 1.06 & 1.27 & -- & -- & & & \\
\hline Domestic use & 1.72 & 0.00 & 1.72 & 2.41 & 0.00 & & -- & -- \\
\hline Commercial use & 0.00 & 0.00 & 0.00 & 0.27 & 0.00 & & & \\
\hline Industrial use & 0.00 & 0.00 & 0.00 & 0.13 & 0.00 & & & \\
\hline Public-supply loss & -- & -- & -- & 0.18 & -- & & & \\
\hline Inter-county delivery & -- & -- & -- & 0.00 & -- & & & \\
\hline \multicolumn{2}{|c|}{ Public wastewater treatment -- } & -- & -- & -- & 1.03 & & & \\
\hline Mining use & 0.00 & 0.03 & 0.03 & 0.03 & 0.00 & \multicolumn{3}{|c|}{ Withdrawals by Major Public Suppliers } \\
\hline Irrigation (crop) & 0.09 & 0.02 & 0.11 & 0.11 & 0.00 & Name & Groundwater & Surface water \\
\hline Irrigation (golf) & 0.10 & 0.00 & 0.10 & 0.10 & 0.00 & \multirow[t]{4}{*}{ City of Dahlonega } & \multirow[t]{4}{*}{0.00} & \multirow[t]{4}{*}{1.06} \\
\hline Livestock-aquaculture & 0.13 & 0.03 & 0.16 & 0.16 & 0.00 & & & \\
\hline Thermoelectric power & 0.00 & 0.00 & 0.00 & 0.00 & 0.00 & & & \\
\hline TOTAL & 2.25 & 1.14 & 3.39 & 3.39 & 1.03 & & & \\
\hline
\end{tabular}

Total use is total withdrawal plus public supply deliveries and losses.

Withdrawals by water source

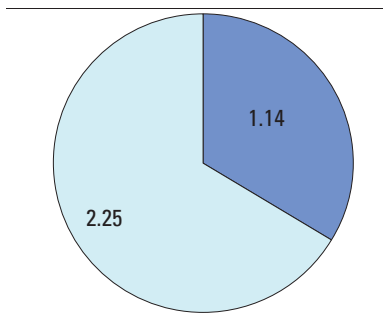

Withdrawals, in million gallons per day

Surface water Groundwater
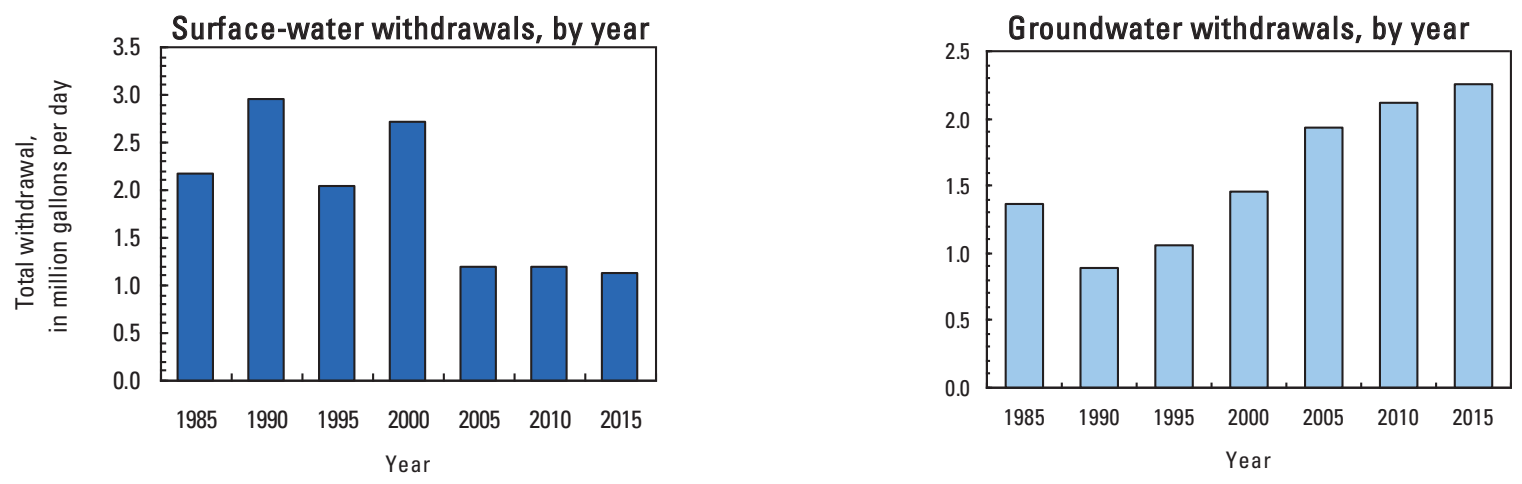


\section{MACON COUNTY}

Population

Population served by public supply-groundwater

8,486

Population served by public supply-surface water

0

Acres irrigated

2015 WATER WITHDRAWALS AND ESTIMATED USE, IN MILLION GALLONS PER DAY

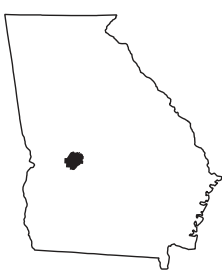

[--,not applicable; Mgal/d, million gallons per day]

\begin{tabular}{lccccc}
\hline & \multicolumn{3}{c}{ Withdrawals } & & Total \\
\cline { 2 - 4 } Category & $\begin{array}{c}\text { Ground- } \\
\text { water }\end{array}$ & $\begin{array}{c}\text { Surface } \\
\text { water }\end{array}$ & $\begin{array}{c}\text { Total } \\
\text { use }\end{array}$ & $\begin{array}{c}\text { Returns to } \\
\text { Surface }\end{array}$ \\
\hline Public supply & 1.03 & 0.00 & 1.03 & -- & -- \\
Domestic use & 0.39 & 0.00 & 0.39 & 0.95 & 0.00 \\
Commercial use & 0.00 & 0.00 & 0.00 & 0.09 & 0.00 \\
Industrial use & 0.53 & 9.78 & 10.31 & 10.49 & 0.00 \\
Public-supply loss & -- & -- & -- & 0.20 & -- \\
Inter-county delivery & -- & -- & -- & 0.00 & -- \\
Public wastewater treatment & -- & -- & -- & -- & 1.43 \\
Mining use & 0.00 & 0.00 & 0.00 & 0.00 & 0.00 \\
Irrigation (crop) & 9.33 & 2.65 & 11.98 & 11.98 & 0.00 \\
Irrigation (golf) & 0.00 & 0.00 & 0.00 & 0.00 & 0.00 \\
Livestock-aquaculture & 1.33 & 0.38 & 1.71 & 1.71 & 0.00 \\
Thermoelectric power & 0.00 & 0.00 & 0.00 & 0.00 & 0.00 \\
\hline TOTAL & 12.61 & 12.81 & 25.42 & 25.42 & 1.43 \\
\hline
\end{tabular}

Withdrawals by Major Industrial Groups

[NAICS, North American Industrial Classification System code]

\begin{tabular}{lcc}
\hline \multicolumn{1}{c}{ NAICS } & Groundwater & Surface water \\
\hline 212- Mining & 0.02 & 0.00 \\
322- Paper, pulp & 0.00 & 9.78 \\
325- Chemicals & 0.51 & 0.00
\end{tabular}

Withdrawals by Major Public Suppliers

\begin{tabular}{lcc} 
Name & Groundwater & Surface water \\
\hline City of Ideal & 0.04 & 0.00 \\
City of Marshallville & 0.14 & 0.00 \\
City of Montezuma & 0.39 & 0.00 \\
City of Oglethorpe & 0.46 & 0.00
\end{tabular}

Total use is total withdrawal plus public supply deliveries and losses.

Withdrawals by water source

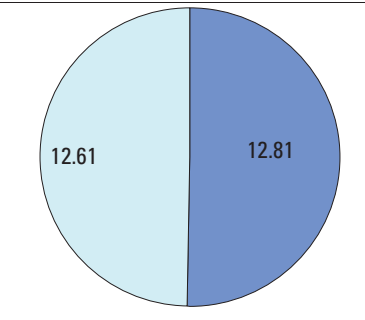

Withdrawals, in million gallons per day

Surface water

Groundwater
Groundwater withdrawals by aquifer

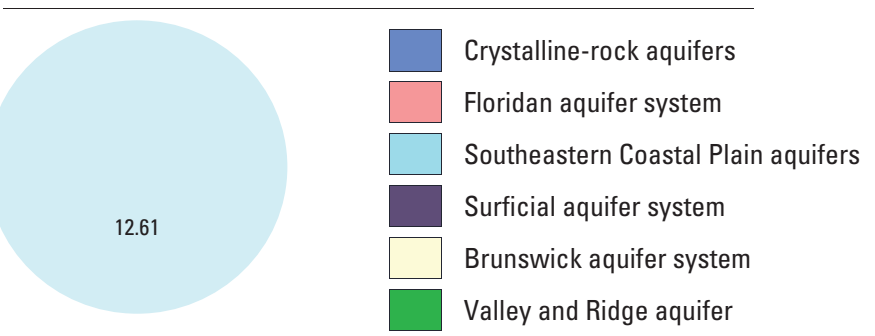

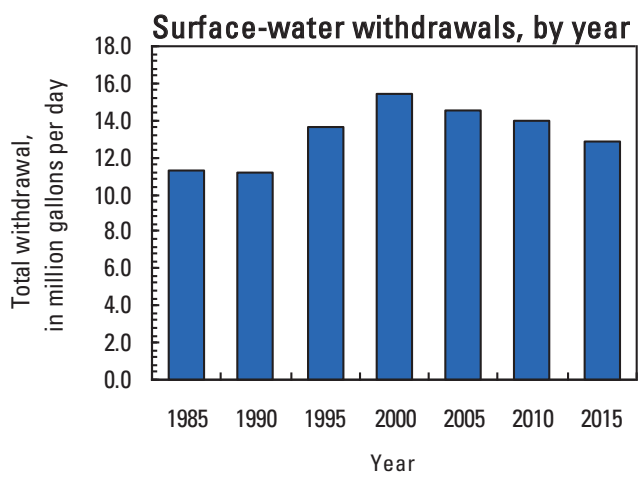

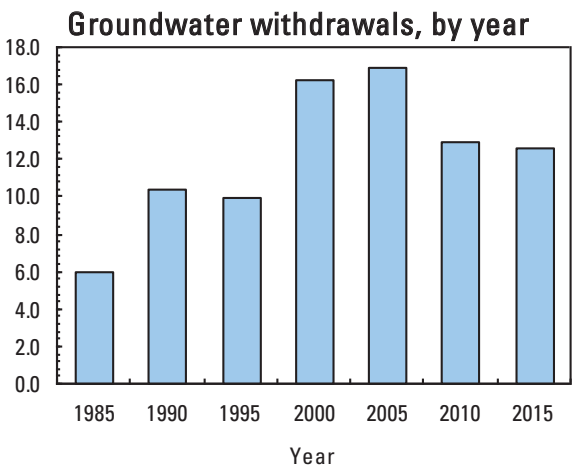




\section{MADISON COUNTY}

Population

Population served by public supply-groundwater

Population served by public supply-surface water

Acres irrigated

2015 WATER WITHDRAWALS AND ESTIMATED USE, IN MILLION GALLONS PER DAY

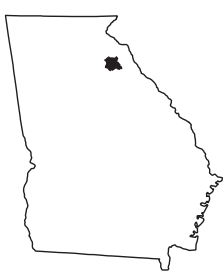

[--,not applicable; Mgal/d, million gallons per day]

\begin{tabular}{|c|c|c|c|c|c|c|c|c|}
\hline \multirow{2}{*}{ Category } & \multicolumn{3}{|c|}{ Withdrawals } & \multirow[b]{2}{*}{$\begin{array}{c}\text { Total } \\
\text { use }\end{array}$} & \multirow{2}{*}{$\begin{array}{c}\text { Returns to } \\
\text { Surface }\end{array}$} & \multirow{2}{*}{\multicolumn{3}{|c|}{$\begin{array}{l}\text { Withdrawals by Major Industrial Groups } \\
\text { [NAICS, North American Industrial Classification System code] }\end{array}$}} \\
\hline & Ground - & Surface & Total & & & & & \\
\hline Public supply & 0.53 & 0.00 & 0.53 & -- & -- & NAICS & Groundwater & Surface water \\
\hline Domestic use & 1.50 & 0.00 & 1.50 & 1.85 & 0.00 & \multirow{3}{*}{ 327- Stone, clay } & \multirow[b]{2}{*}{0.00} & \multirow{3}{*}{0.11} \\
\hline Commercial use & 0.00 & 0.00 & 0.00 & 0.04 & 0.00 & & & \\
\hline Industrial use & 0.00 & 0.11 & 0.11 & 0.12 & 0.00 & & & \\
\hline Public-supply loss & -- & -- & -- & 0.13 & -- & & & \\
\hline Inter-county delivery & -- & -- & -- & 0.00 & -- & & & \\
\hline Public wastewater treatment & $\mathrm{t}--$ & -- & - & -- & 0.01 & & & \\
\hline Mining use & 0.07 & 0.00 & 0.07 & 0.07 & 0.00 & \multicolumn{3}{|c|}{ Withdrawals by Major Public Suppliers } \\
\hline Irrigation (crop) & 0.00 & 0.00 & 0.00 & 0.00 & 0.00 & Name & Groundwater & Surface water \\
\hline Irrigation (golf) & 0.00 & 0.19 & 0.19 & 0.19 & 0.00 & City of Danielsville & 0.12 & 0.00 \\
\hline Livestock-aquaculture & 0.64 & 0.21 & 0.85 & 0.85 & 0.00 & City of Comer & 0.11 & 0.00 \\
\hline Thermoelectric power & 0.00 & 0.00 & 0.00 & 0.00 & 0.00 & & & \\
\hline TOTAL & 2.74 & 0.51 & 3.25 & 3.25 & 0.01 & & & \\
\hline
\end{tabular}

Total use is total withdrawal plus public supply deliveries and losses.

Withdrawals by water source

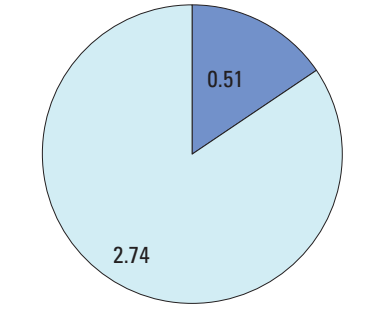

Withdrawals, in million gallons per day

Surface water

Groundwater
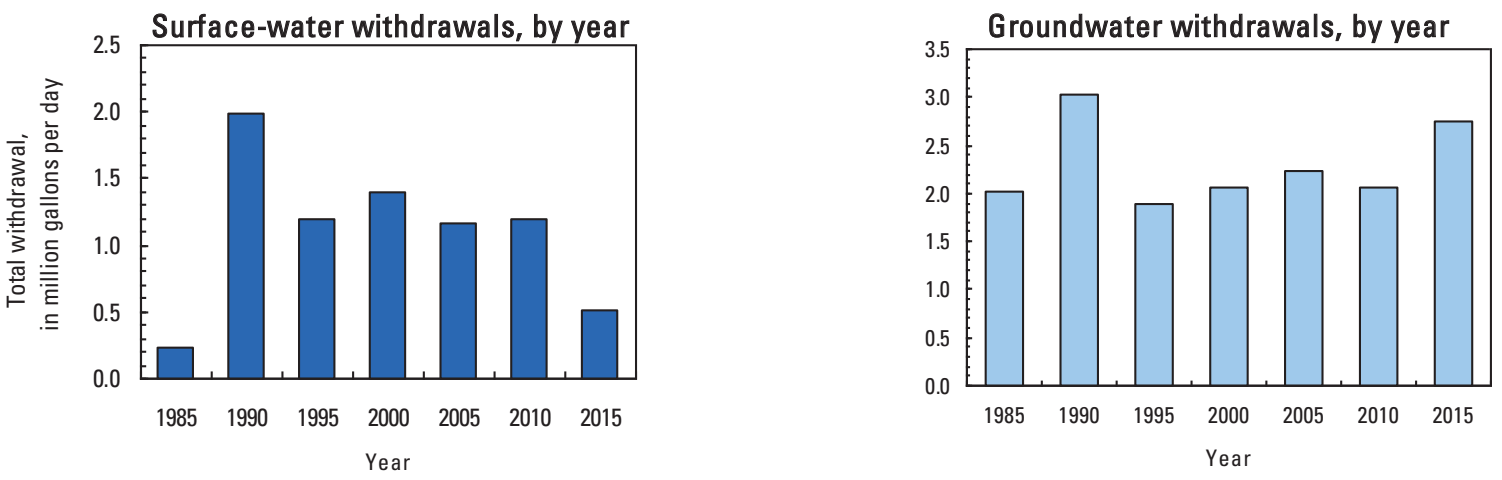


\section{MARION COUNTY}

Population

Population served by public supply-groundwater

5,554

Population served by public supply-surface water

0

Acres irrigated

2015 WATER WITHDRAWALS AND ESTIMATED USE, IN MILLION GALLONS PER DAY

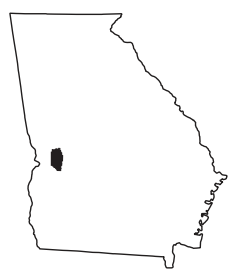

[--,not applicable; Mgal/d, million gallons per day]

\begin{tabular}{lccccc}
\hline & \multicolumn{3}{c}{ Withdrawals } & Total & Returns to \\
\cline { 2 - 5 } Category & $\begin{array}{c}\text { Ground } \\
\text { water }\end{array}$ & $\begin{array}{c}\text { Surface } \\
\text { water }\end{array}$ & Total & $\begin{array}{c}\text { Sse } \\
\text { Unrface }\end{array}$ \\
\hline Public supply & 1.41 & 0.00 & 1.41 & -- & -- \\
Domestic use & 0.24 & 0.00 & 0.24 & 1.16 & 0.00 \\
Commercial use & 0.00 & 0.00 & 0.00 & 0.12 & 0.00 \\
Industrial use & 0.00 & 0.44 & 0.44 & 0.54 & 0.00 \\
Public-supply loss & -- & -- & -- & 0.27 & -- \\
Inter-county delivery & -- & -- & -- & 0.00 & -- \\
Public wastewater treatment & -- & -- & -- & -- & 0.39 \\
Mining use & 0.95 & 1.07 & 2.02 & 2.02 & 0.00 \\
Irrigation (crop) & 0.21 & 0.64 & 0.85 & 0.85 & 0.00 \\
Irrigation (golf) & 0.00 & 0.00 & 0.00 & 0.00 & 0.00 \\
Livestock-aquaculture & 0.03 & 0.11 & 0.14 & 0.14 & 0.00 \\
Thermoelectric power & 0.00 & 0.00 & 0.00 & 0.00 & 0.00 \\
\hline TOTAL & 2.84 & 2.26 & 5.10 & 5.10 & 0.39 \\
\hline
\end{tabular}

Withdrawals by Major Industrial Groups

[NAICS, North American Industrial Classification System code]

\begin{tabular}{lll}
\hline NAICS & Groundwater Surface water \\
\hline
\end{tabular}

$\begin{array}{lll}\text { 212- Mining } & 0.00 & 0.44\end{array}$

Withdrawals by Major Public Suppliers

Name Groundwater Surface water

$\begin{array}{lll}\text { City of Buena Vista } & 0.67 & 0.00 \\ \text { Marion County } & 0.74 & 0.00 \\ \text { Water System } & & \end{array}$

Total use is total withdrawal plus public supply deliveries and losses.

Withdrawals by water source

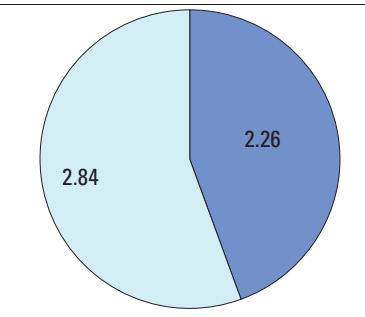

Withdrawals, in million gallons per day

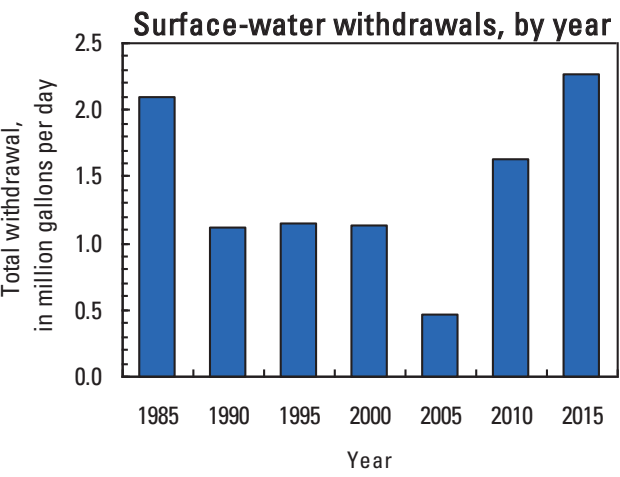

Groundwater withdrawals by aquifer

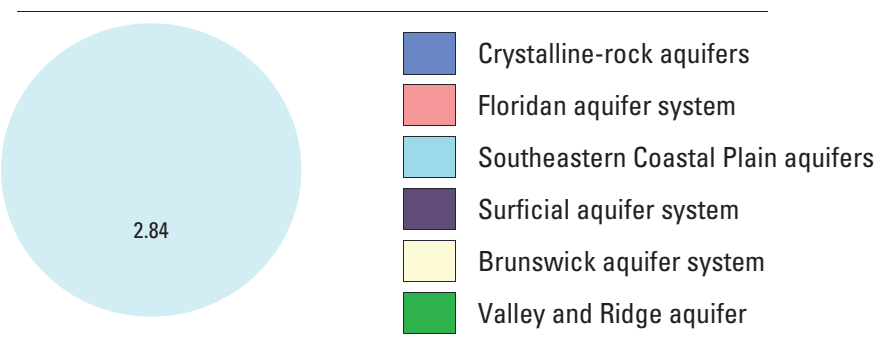

Groundwater withdrawals, by year

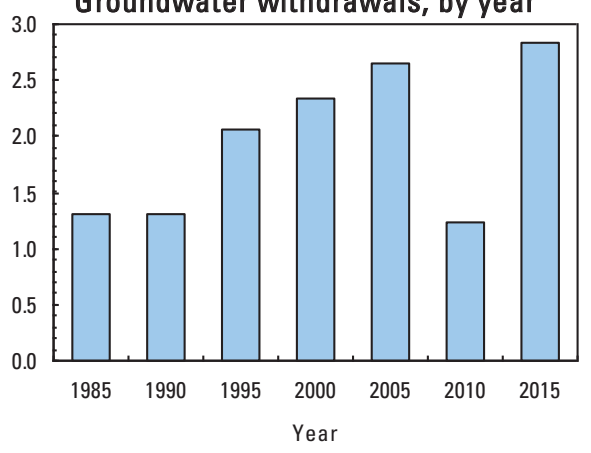




\section{MCDUFFIE COUNTY}

Population

Population served by public supply-groundwater

Population served by public supply-surface water

Acres irrigated

2015 WATER WITHDRAWALS AND ESTIMATED USE, IN MILLION GALLONS PER DAY

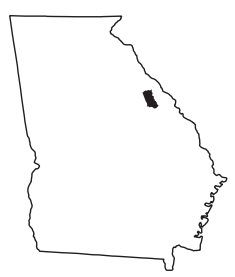

[--,not applicable; Mgal/d, million gallons per day]

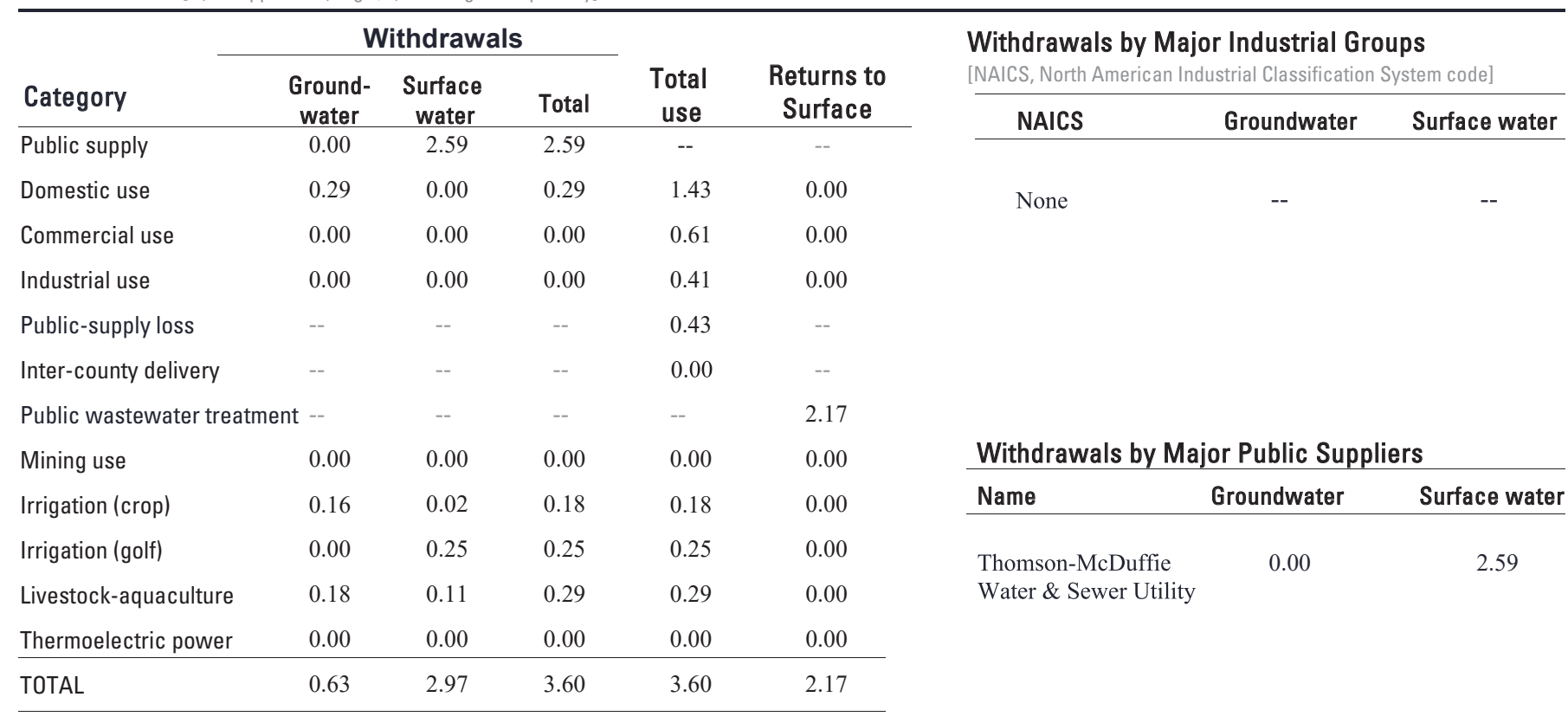

Total use is total withdrawal plus public supply deliveries and losses.

Withdrawals by water source

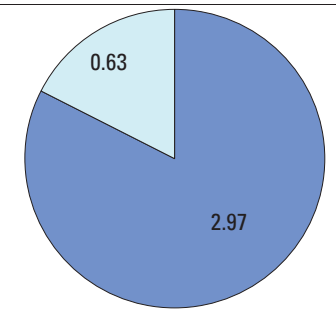

Withdrawals, in million

gallons per day

Surface water

Groundwater

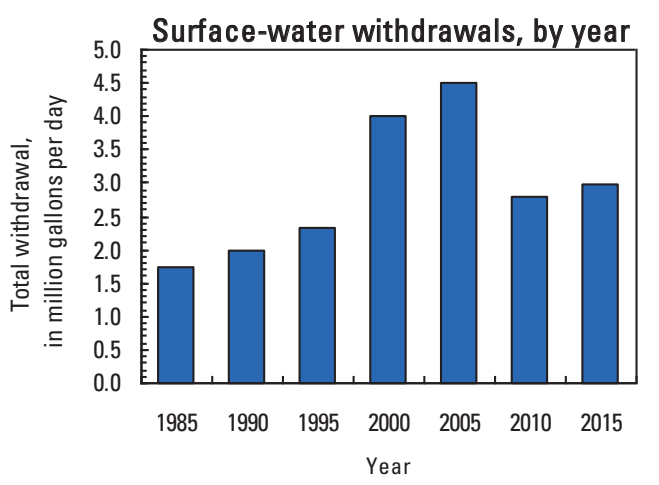

Groundwater withdrawals by aquifer
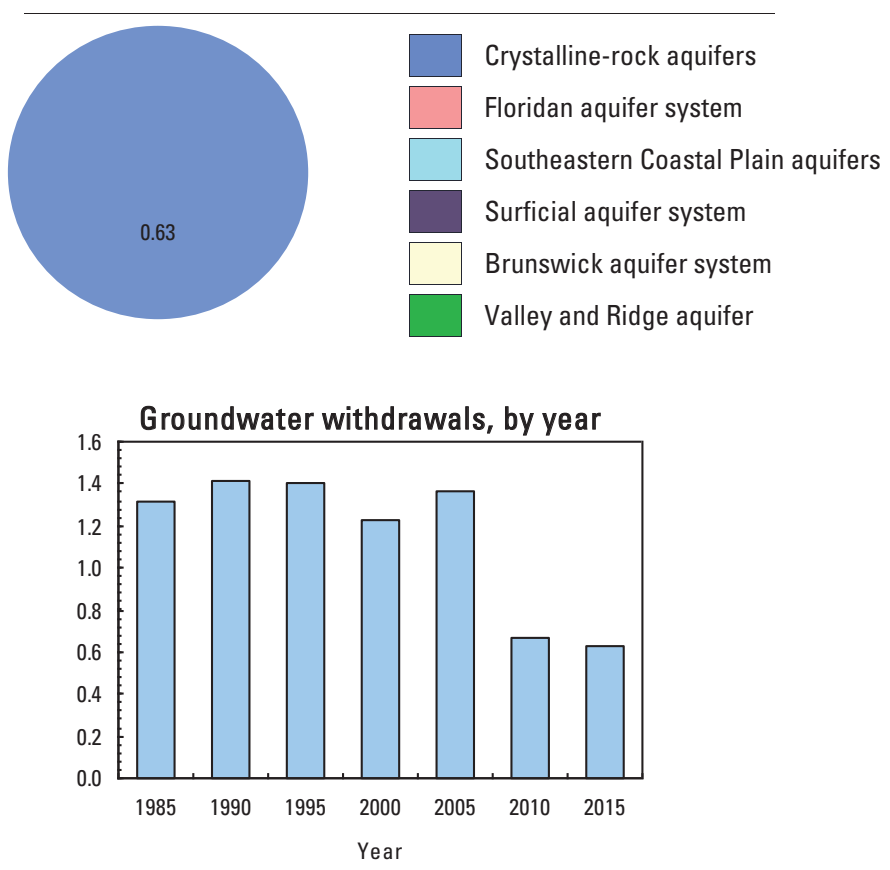


\section{MCINTOSH COUNTY}

Population

Population served by public supply-groundwater

Population served by public supply-surface water

Acres irrigated

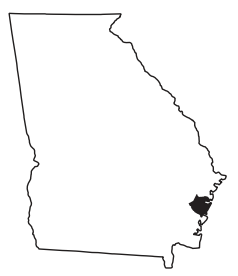

2015 WATER WITHDRAWALS AND ESTIMATED USE, IN MILLION GALLONS PER DAY

[--,not applicable; Mgal/d, million gallons per day]

\begin{tabular}{lccccc}
\hline & \multicolumn{3}{c}{ Withdrawals } & Total \\
\cline { 2 - 4 } Category & $\begin{array}{c}\text { Ground- } \\
\text { water }\end{array}$ & $\begin{array}{c}\text { Surface } \\
\text { water }\end{array}$ & $\begin{array}{c}\text { Total } \\
\text { use }\end{array}$ & $\begin{array}{c}\text { Returns to } \\
\text { Surface }\end{array}$ \\
\hline Public supply & 1.04 & 0.00 & 1.04 & -- & - \\
Domestic use & 0.26 & 0.00 & 0.26 & 1.2 & 0.00 \\
Commercial use & 0.00 & 0.00 & 0.00 & 0.04 & 0.00 \\
Industrial use & 0.02 & 0.00 & 0.02 & 0.02 & 0.00 \\
Public-supply loss & -- & -- & -- & 0.06 & -- \\
Inter-county delivery & -- & -- & -- & 0.00 & -- \\
Public wastewater treatment & -- & -- & -- & -- & 0.25 \\
Mining use & 0.00 & 0.00 & 0.00 & 0.00 & 0.00 \\
Irrigation (crop) & 0.00 & 0.00 & 0.00 & 0.00 & 0.00 \\
Irrigation (golf) & 0.16 & 0.00 & 0.16 & 0.16 & 0.00 \\
Livestock-aquaculture & 0.00 & 0.00 & 0.00 & 0.00 & 0.00 \\
Thermoelectric power & 0.00 & 0.00 & 0.00 & 0.00 & 0.00 \\
\hline TOTAL & 1.48 & 0.00 & 1.48 & 1.48 & 0.25 \\
\hline
\end{tabular}

Withdrawals by Major Industrial Groups

[NAICS, North American Industrial Classification System code]

\begin{tabular}{lll}
\hline NAICS & Groundwater & Surface water \\
\hline
\end{tabular}

339- Misc. manufacturing $\quad 0.02$

0.00

Total use is total withdrawal plus public supply deliveries and losses.

Withdrawals by Major Public Suppliers

\begin{tabular}{lcc} 
Name & Groundwater & Surface wat \\
\hline City of Darien & 0.39 & 0.00 \\
McIntosh County Water & 0.04 & 0.00 \\
System & & \\
Water Utility Management & 0.05 & 0.00 \\
W \& D Utilities & 0.20 & 0.00
\end{tabular}

Withdrawals by water source

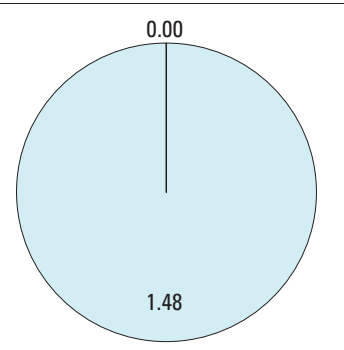

Withdrawals, in million gallons per day

Surface water

Groundwater

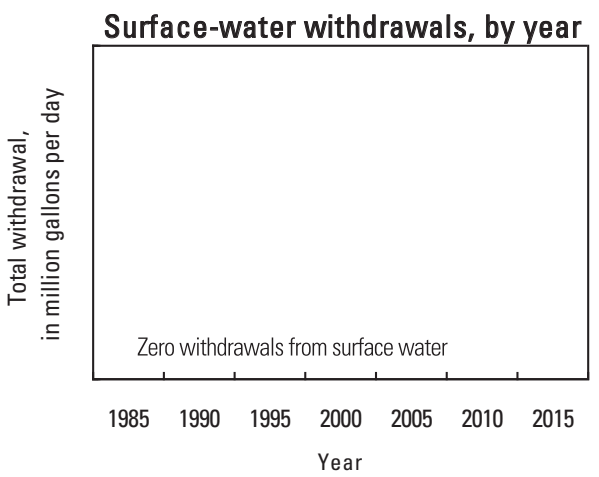

Groundwater withdrawals by aquifer

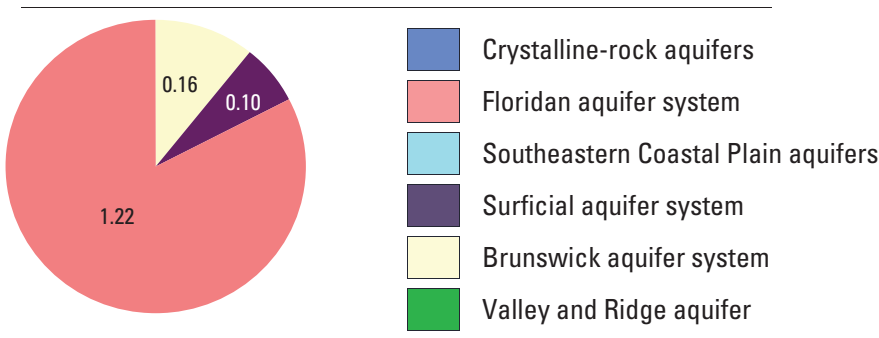

Groundwater withdrawals, by year

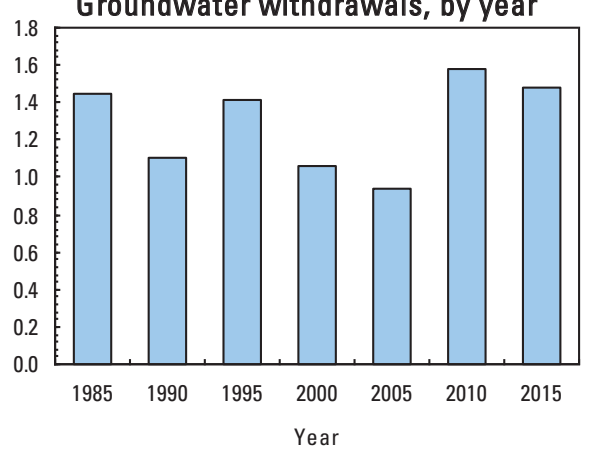




\section{MERIWETHER COUNTY}

Population

Population served by public supply-groundwater

Population served by public supply-surface water

Acres irrigated

300

2015 WATER WITHDRAWALS AND ESTIMATED USE, IN MILLION GALLONS PER DAY

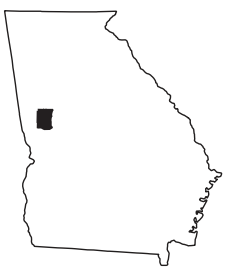

[--,not applicable; Mgal/d, million gallons per day]

\begin{tabular}{|c|c|c|c|c|c|c|c|c|}
\hline \multirow{2}{*}{ Category } & \multicolumn{3}{|c|}{ Withdrawals } & \multirow{2}{*}{$\begin{array}{c}\text { Total } \\
\text { use }\end{array}$} & \multirow{2}{*}{$\begin{array}{c}\text { Returns to } \\
\text { Surface }\end{array}$} & \multirow{2}{*}{\multicolumn{3}{|c|}{$\begin{array}{l}\text { Withdrawals by Major Industrial Groups } \\
\text { [NAICS, North American Industrial Classification System code] }\end{array}$}} \\
\hline & Ground- & Surface & Total & & & & & \\
\hline Public supply & $\begin{array}{c}\text { water } \\
0.18\end{array}$ & $\begin{array}{c}\text { water } \\
0.13\end{array}$ & 0.31 & & surtace & NAICS & Groundwater & Surface water \\
\hline Domestic use & 0.77 & 0.00 & 0.77 & 1.51 & 0.00 & \multirow{3}{*}{ 321- Wood } & \multirow{2}{*}{0.07} & \multirow{3}{*}{0.07} \\
\hline Commercial use & 0.06 & 0.14 & 0.20 & 0.45 & 0.00 & & & \\
\hline Industrial use & 0.07 & 0.00 & 0.07 & 0.08 & 0.00 & & & \\
\hline Public-supply loss & -- & -- & -- & -0.69 & -- & & & \\
\hline Inter-county delivery & -- & -- & -- & 0.93 & -- & & & \\
\hline Public wastewater treatmen & nt -- & -- & -- & -- & 0.57 & & & \\
\hline Mining use & 0.00 & 0.00 & 0.00 & 0.00 & 0.00 & \multicolumn{3}{|c|}{ Withdrawals by Major Public Suppliers } \\
\hline Irrigation (crop) & 0.09 & 0.04 & 0.13 & 0.13 & 0.00 & Name & Groundwater & Surface water \\
\hline Irrigation (golf) & 0.00 & 0.05 & 0.05 & 0.05 & 0.00 & City of Warm Springs & 0.00 & 0.13 \\
\hline Livestock-aquaculture & 0.14 & 0.17 & 0.31 & 0.31 & 0.00 & City of Woodbury & 0.00 & 0.13 \\
\hline Thermoelectric power & 0.00 & 0.00 & 0.00 & 0.00 & 0.00 & & & \\
\hline TOTAL & 1.31 & 0.53 & 1.84 & 2.77 & 0.57 & & & \\
\hline
\end{tabular}

Total use is total withdrawal plus public supply deliveries and losses. An estimate of $0.93 \mathrm{Mgal} / \mathrm{d}$ was delivered from neighboring counties in 2015.

Withdrawals by water source

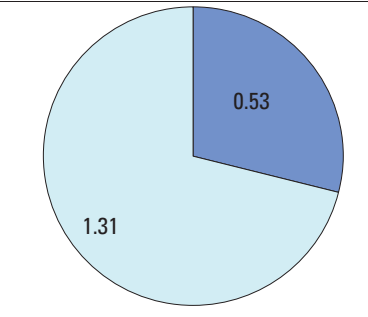

Withdrawals, in million gallons per day

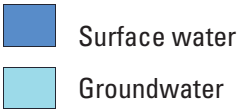

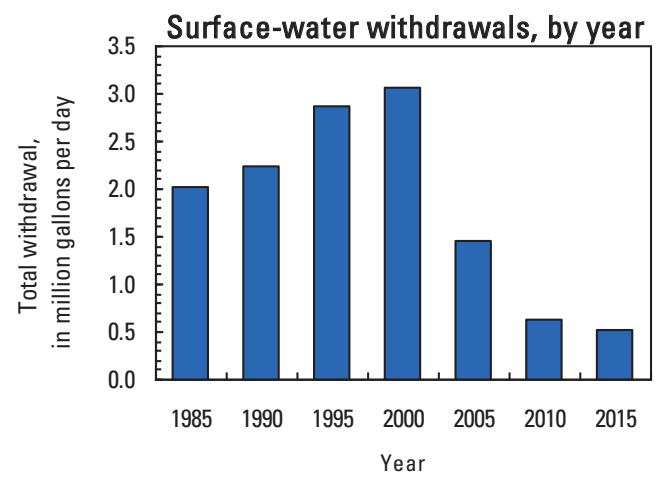

Groundwater withdrawals by aquifer
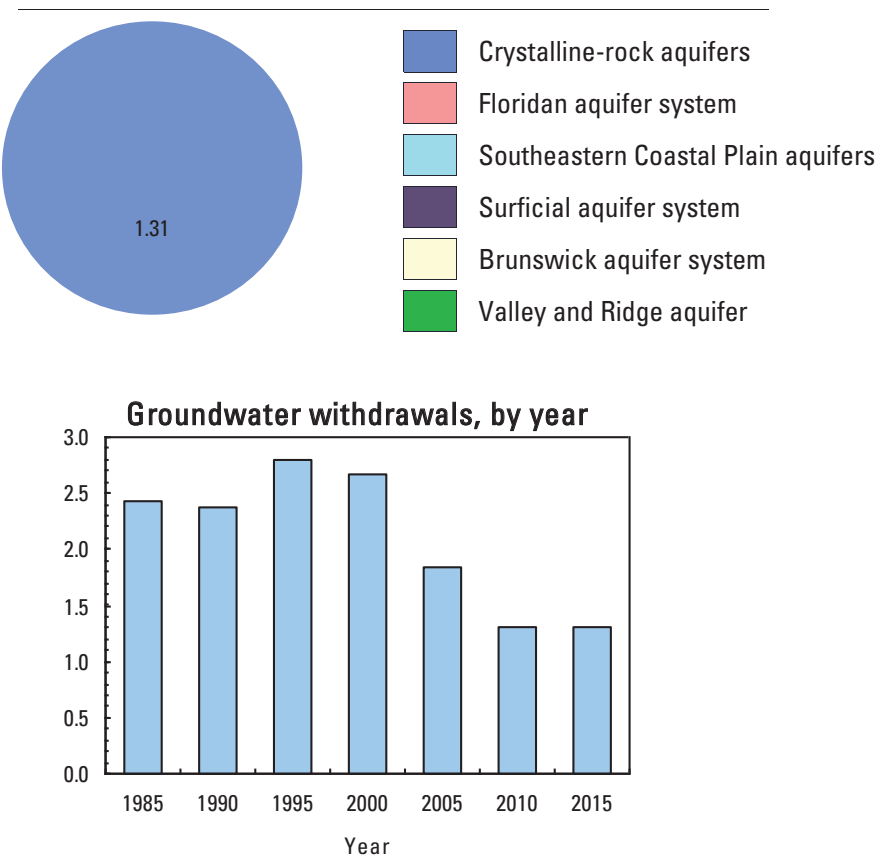


\section{MILLER COUNTY}

Population

Population served by public supply-groundwater

Population served by public supply-surface water

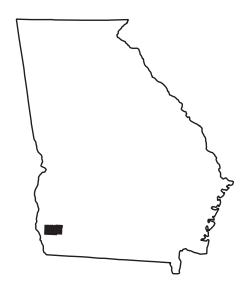

[--,not applicable; Mgal/d, million gallons per day]

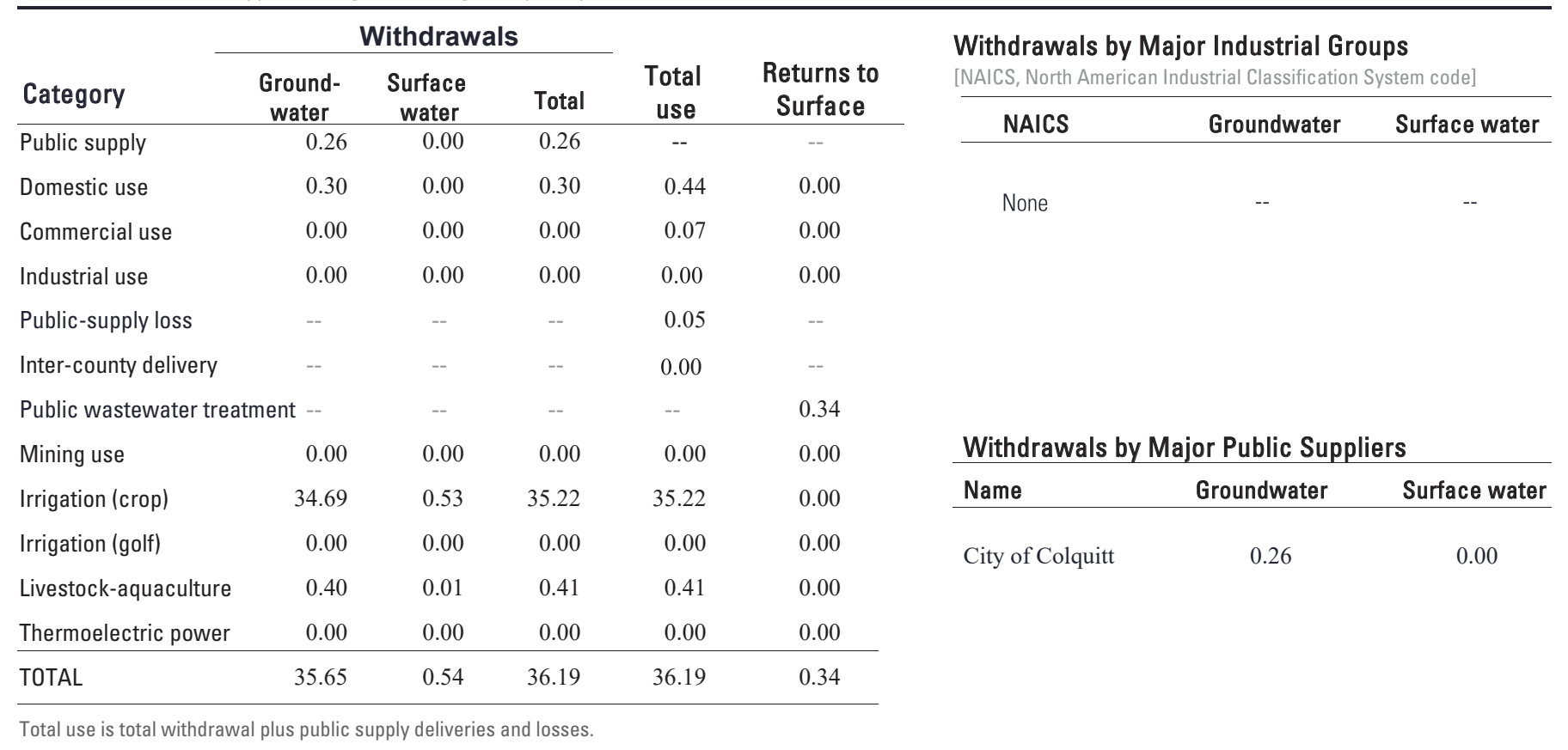

Withdrawals by water source
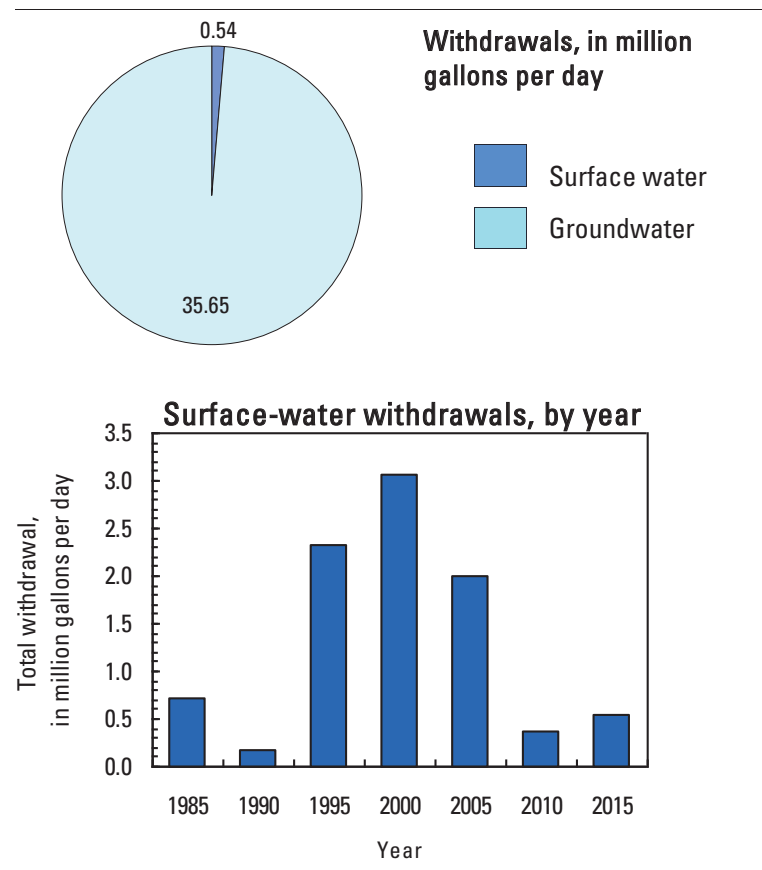

Groundwater withdrawals by aquifer

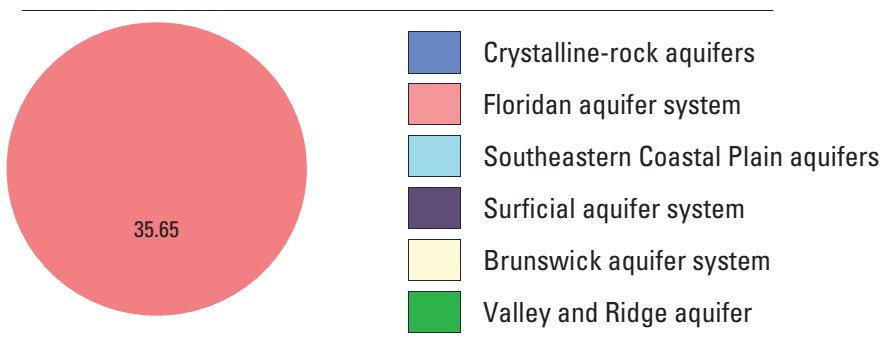

Groundwater withdrawals, by year

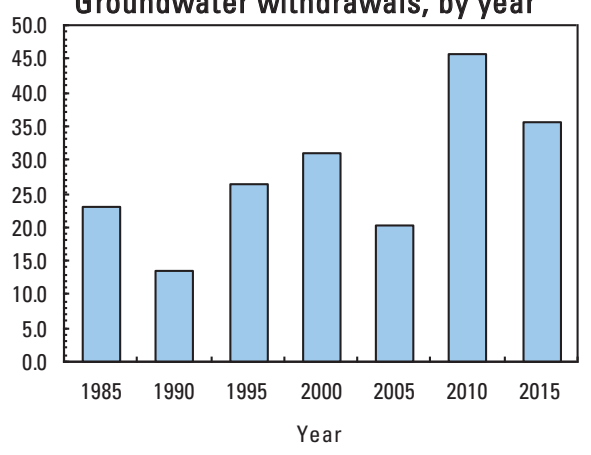




\section{MITCHELL COUNTY}

Population

Population served by public supply-groundwater

Population served by public supply-surface water

Acres irrigated

2015 WATER WITHDRAWALS AND ESTIMATED USE, IN MILLION GALLONS PER DAY

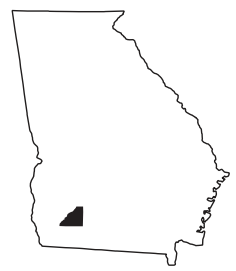

[--,not applicable; Mgal/d, million gallons per day]

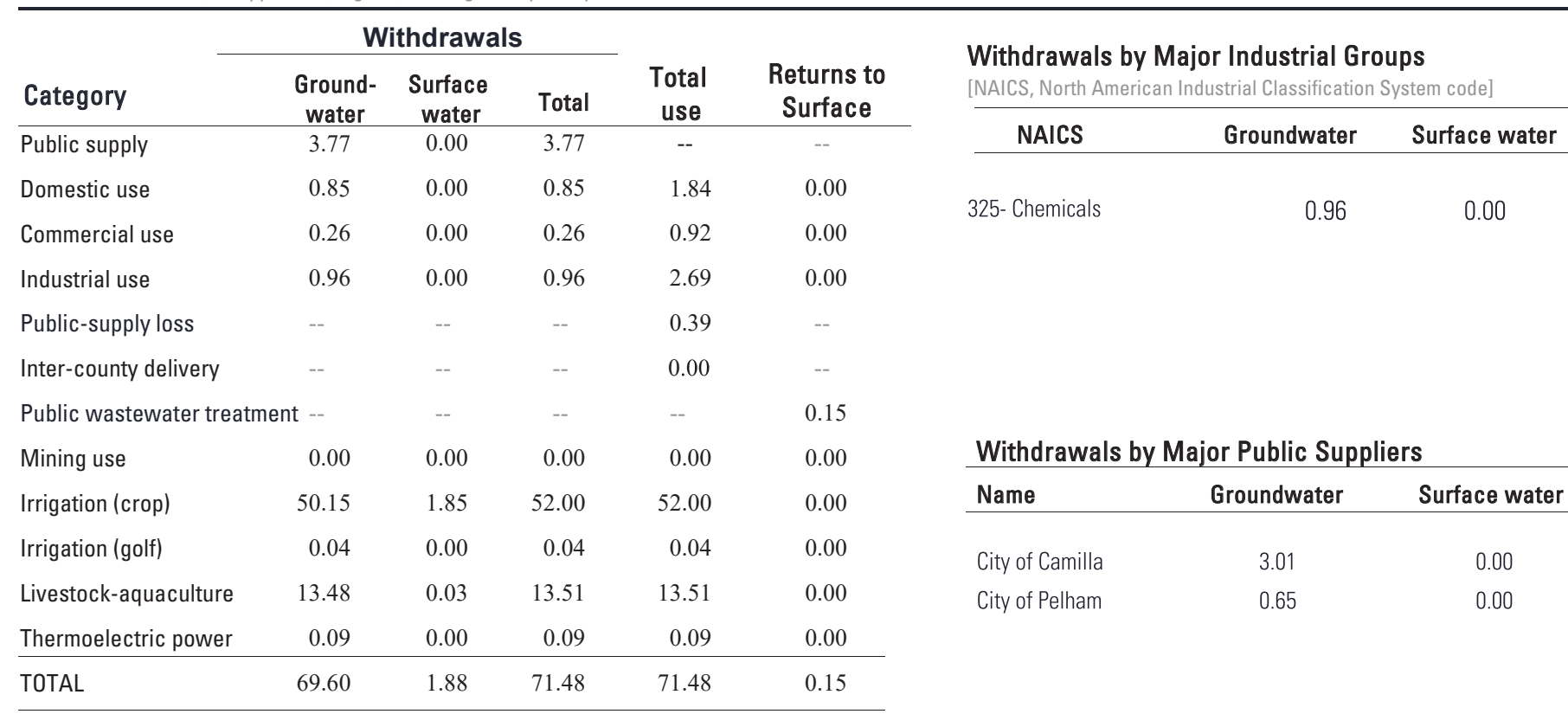

Total use is total withdrawal plus public supply deliveries and losses.

Withdrawals by water source

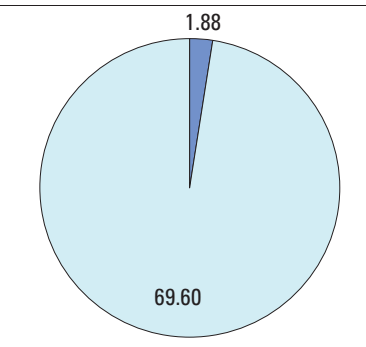

Withdrawals, in million

gallons per day

Surface water

Groundwater

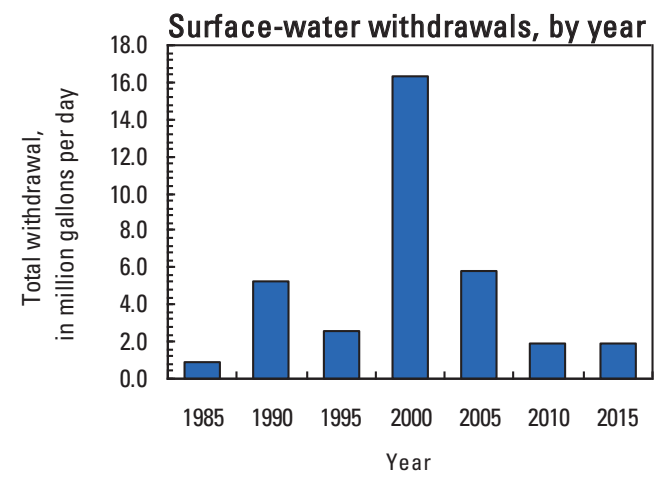

Groundwater withdrawals by aquifer

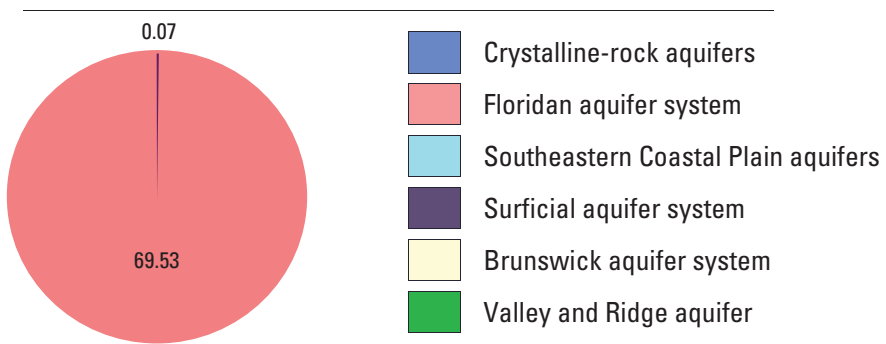

Groundwater withdrawals, by year

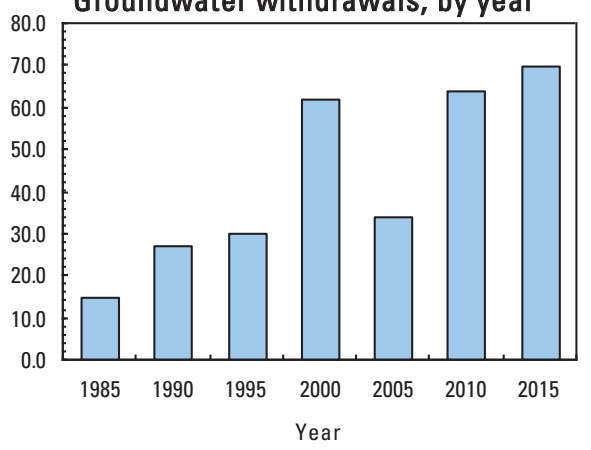




\section{MONROE COUNTY}

Population

Population served by public supply-groundwater

Population served by public supply-surface water $\quad 14,053$

Acres irrigated
430

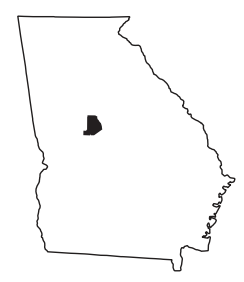

2015 WATER WITHDRAWALS AND ESTIMATED USE, IN MILLION GALLONS PER DAY

[--,not applicable; Mgal/d, million gallons per day]

\begin{tabular}{lcccccc}
\hline & \multicolumn{3}{c}{ Withdrawals } & & Total \\
\cline { 2 - 4 } Category & $\begin{array}{c}\text { Ground } \\
\text { water }\end{array}$ & $\begin{array}{c}\text { Surface } \\
\text { water }\end{array}$ & $\begin{array}{c}\text { Total } \\
\text { use }\end{array}$ & $\begin{array}{c}\text { Returns to } \\
\text { Surface }\end{array}$ \\
\hline Public supply & 0.01 & 1.57 & 1.58 & -- & -- \\
Domestic use & 0.96 & 0.00 & 0.96 & 2.35 & 0.00 \\
Commercial use & 0.00 & 0.00 & 0.00 & 0.23 & 0.00 \\
Industrial use & 0.00 & 0.00 & 0.00 & 0.02 & 0.00 \\
Public-supply loss & -- & -- & -- & -0.06 & -- \\
Inter-county delivery & -- & -- & -- & 0.36 & -- \\
Public wastewater treatment & -- & -- & -- & -- & 1.68 \\
Mining use & 0.16 & 0.00 & 0.16 & 0.16 & 0.00 \\
Irrigation (crop) & 0.09 & 0.01 & 0.10 & 0.10 & 0.00 \\
Irrigation (golf) & 0.16 & 0.17 & 0.33 & 0.33 & 0.00 \\
Livestock-aquaculture & 0.31 & 0.02 & 0.33 & 0.33 & 0.00 \\
Thermoelectric power & 0.06 & 44.33 & 44.39 & 44.39 & 0.00 \\
\hline TOTAL & 1.75 & 46.10 & 47.85 & 48.21 & 1.68 \\
\hline
\end{tabular}

Withdrawals by Major Industrial Groups

[NAICS, North American Industrial Classification System code]

NAICS Groundwater Surface water

None

Withdrawals by Major Public Suppliers

Name Groundwater Surface water

$\begin{array}{lll}\text { City of Forsyth } & 0.00 & 1.57\end{array}$

Total use is total withdrawal plus public supply deliveries and losses. An estimate of $0.36 \mathrm{Mgal} / \mathrm{d}$ was delivered from neighboring counties in 2015.

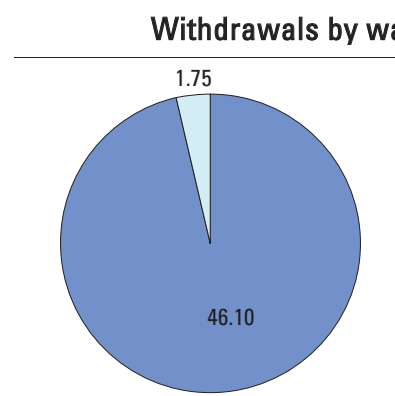

Withdrawals, in million gallons per day

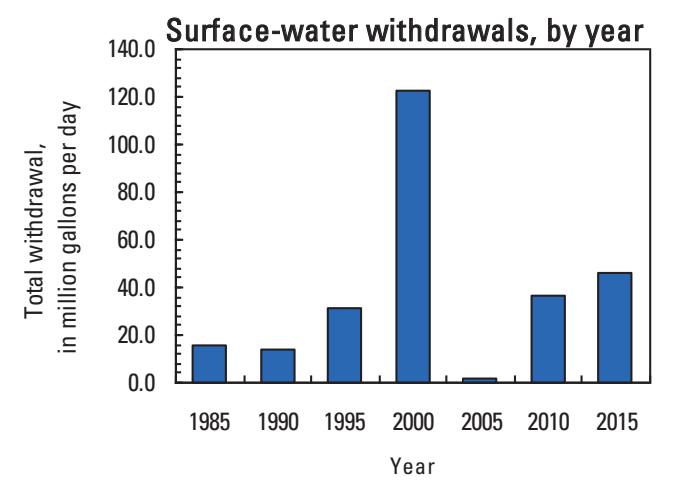

Groundwater withdrawals by aquifer
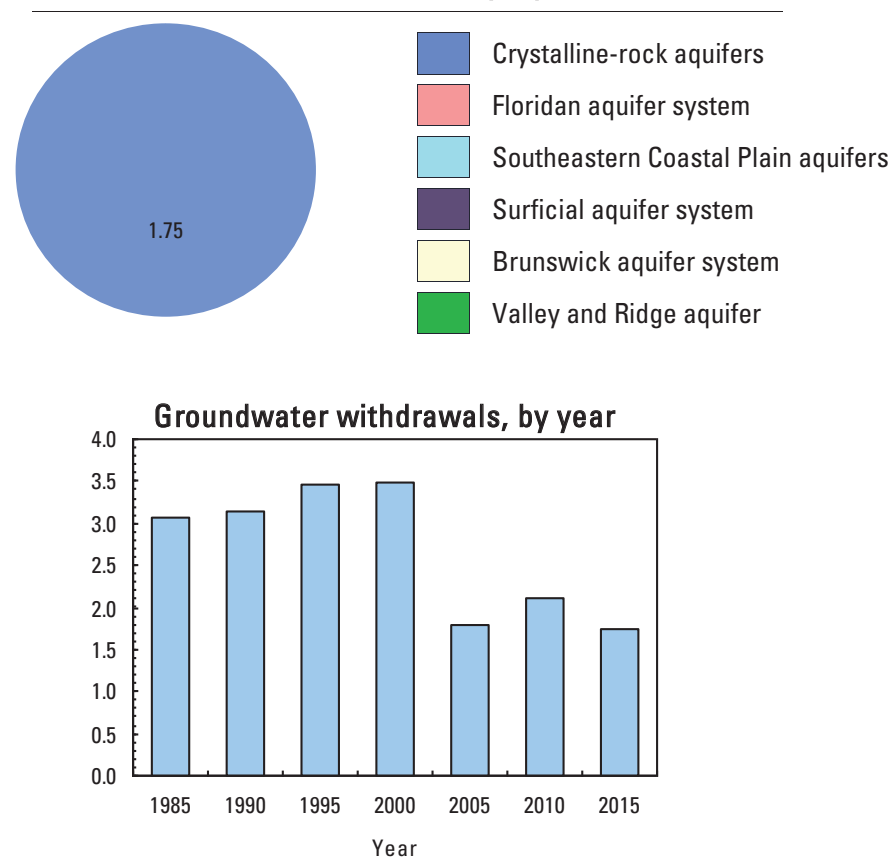


\section{MONTGOMERY COUNTY}

Population

Population served by public supply-groundwater

Population served by public supply-surface water

Acres irrigated

2015 WATER WITHDRAWALS AND ESTIMATED USE, IN MILLION GALLONS PER DAY

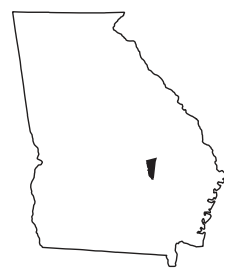

[--,not applicable; Mgal/d, million gallons per day]

\begin{tabular}{|c|c|c|c|c|c|c|c|c|}
\hline \multirow{3}{*}{ Category } & \multicolumn{3}{|c|}{ Withdrawals } & \multirow{3}{*}{$\begin{array}{c}\text { Total } \\
\text { use } \\
\end{array}$} & \multirow{3}{*}{$\begin{array}{l}\text { Returns to } \\
\text { Surface }\end{array}$} & \multirow{2}{*}{\multicolumn{3}{|c|}{$\begin{array}{l}\text { Withdrawals by Major Industrial Groups } \\
\text { [NAICS, North American Industrial Classification System code] }\end{array}$}} \\
\hline & \multirow{2}{*}{$\begin{array}{l}\text { Ground- } \\
\text { water }\end{array}$} & \multirow{2}{*}{$\begin{array}{c}\text { Surface } \\
\text { water }\end{array}$} & \multirow{2}{*}{ Total } & & & & & \\
\hline & & & & & & \multirow{4}{*}{ None } & \multirow[t]{2}{*}{ Groundwater } & \multirow[t]{2}{*}{ Surface water } \\
\hline Public supply & 0.18 & 0.00 & 0.18 & -- & -- & & & \\
\hline Domestic use & 0.38 & 0.00 & 0.38 & 0.56 & 0.00 & & -- & -- \\
\hline Commercial use & 0.00 & 0.00 & 0.00 & 0.03 & 0.00 & & & \\
\hline Industrial use & 0.00 & 0.00 & 0.00 & 0.00 & 0.00 & & & \\
\hline Public-supply loss & -- & -- & -- & -0.03 & -- & & & \\
\hline Inter-county delivery & -- & -- & -- & 0.04 & -- & & & \\
\hline Public wastewater treatment & t -- & -- & -- & -- & 0.46 & & & \\
\hline Mining use & 0.09 & 0.00 & 0.09 & 0.09 & 0.00 & \multicolumn{3}{|c|}{ Withdrawals by Major Public Suppliers } \\
\hline Irrigation (crop) & 0.61 & 0.24 & 0.85 & 0.85 & 0.00 & Name & Groundwater & Surface water \\
\hline Irrigation (golf) & 0.00 & 0.16 & 0.16 & 0.16 & 0.00 & \multirow[t]{4}{*}{ City of Mount Vernon } & \multirow[t]{4}{*}{0.06} & \multirow[t]{3}{*}{0.00} \\
\hline Livestock-aquaculture & 0.07 & 0.03 & 0.10 & 0.10 & 0.00 & & & \\
\hline Thermoelectric power & 0.00 & 0.00 & 0.00 & 0.00 & 0.00 & & & \\
\hline TOTAL & 1.33 & 0.43 & 1.76 & 1.80 & 0.46 & & & \\
\hline
\end{tabular}

Total use is total withdrawal plus public supply deliveries and losses. An estimate of $0.04 \mathrm{Mgal} / \mathrm{d}$ was delivered from neighboring counties in 2015.

Withdrawals by water source

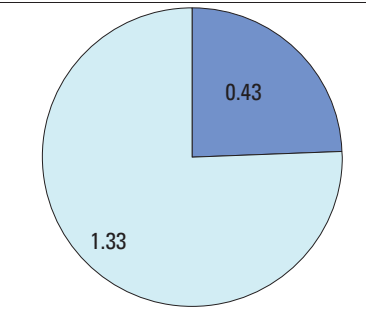

Withdrawals, in million gallons per day

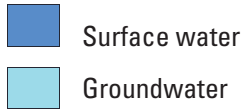

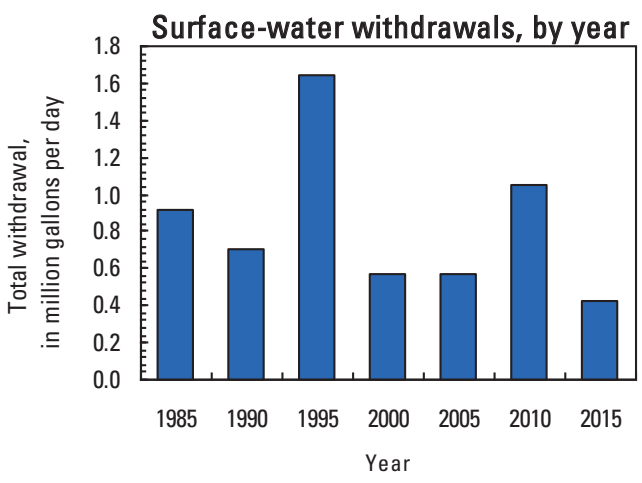

Groundwater withdrawals by aquifer

\begin{tabular}{ll}
\hline & Crystalline-rock aquifers \\
\hline & Floridan aquifer system \\
\hline & Southeastern Coastal Plain aquifers \\
& Surficial aquifer system \\
\hline$\quad$ Brunswick aquifer system \\
\hline$\quad$ Valley and Ridge aquifer
\end{tabular}

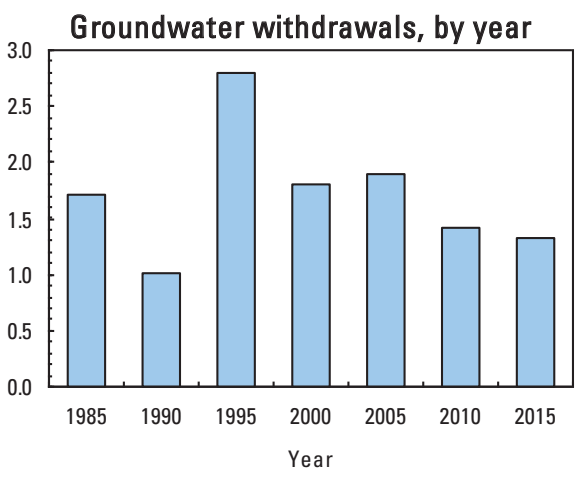




\section{MORGAN COUNTY}

Population

Population served by public supply-groundwater

Population served by public supply-surface water

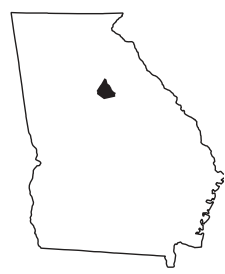

2015 WATER WITHDRAWALS AND ESTIMATED USE, IN MILLION GALLONS PER DAY

[--,not applicable; Mgal/d, million gallons per day $]$

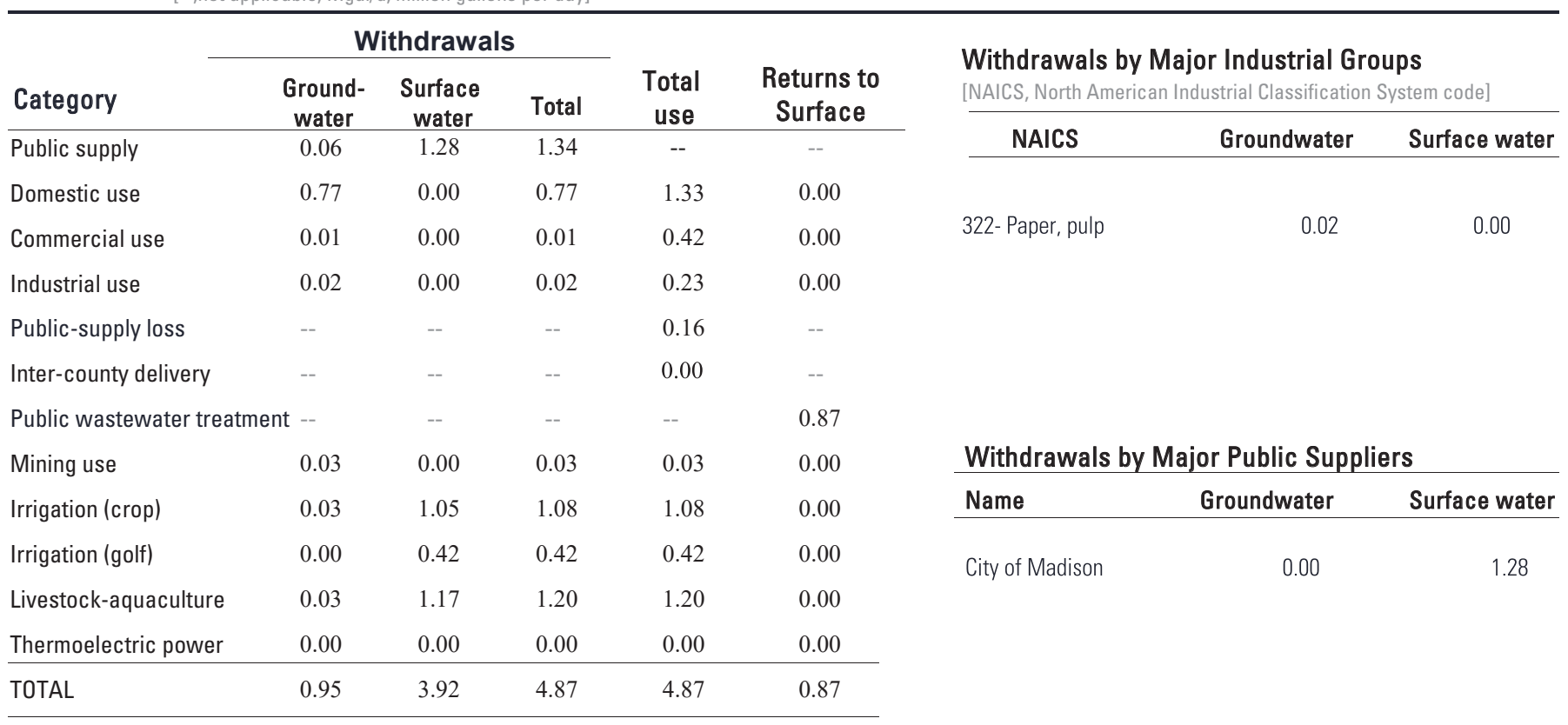

Total use is total withdrawal plus public supply deliveries and losses.

\section{Withdrawals by water source}

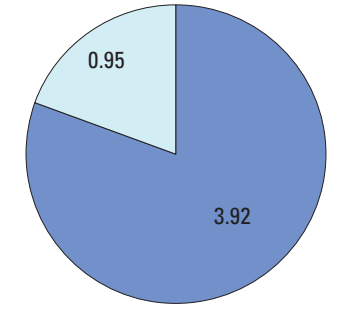

Withdrawals, in million

gallons per day

Surface water

Groundwater

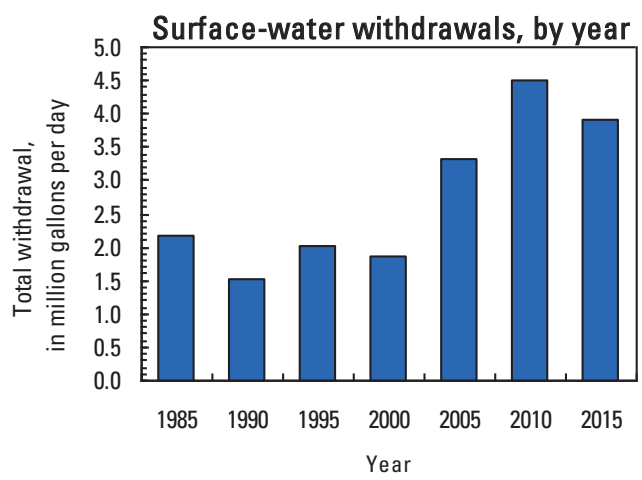

Groundwater withdrawals by aquifer

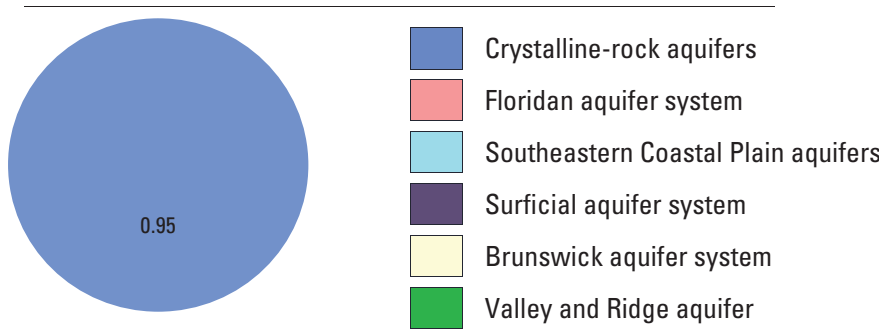




\section{MURRAY COUNTY}

Population

Population served by public supply-groundwater

Population served by public supply-surface water

17,257

Acres irrigated

300

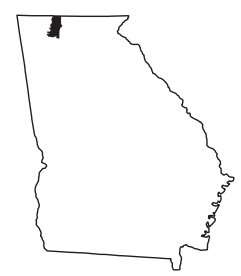

2015 WATER WITHDRAWALS AND ESTIMATED USE, IN MILLION GALLONS PER DAY

[--,not applicable; Mgal/d, million gallons per day]

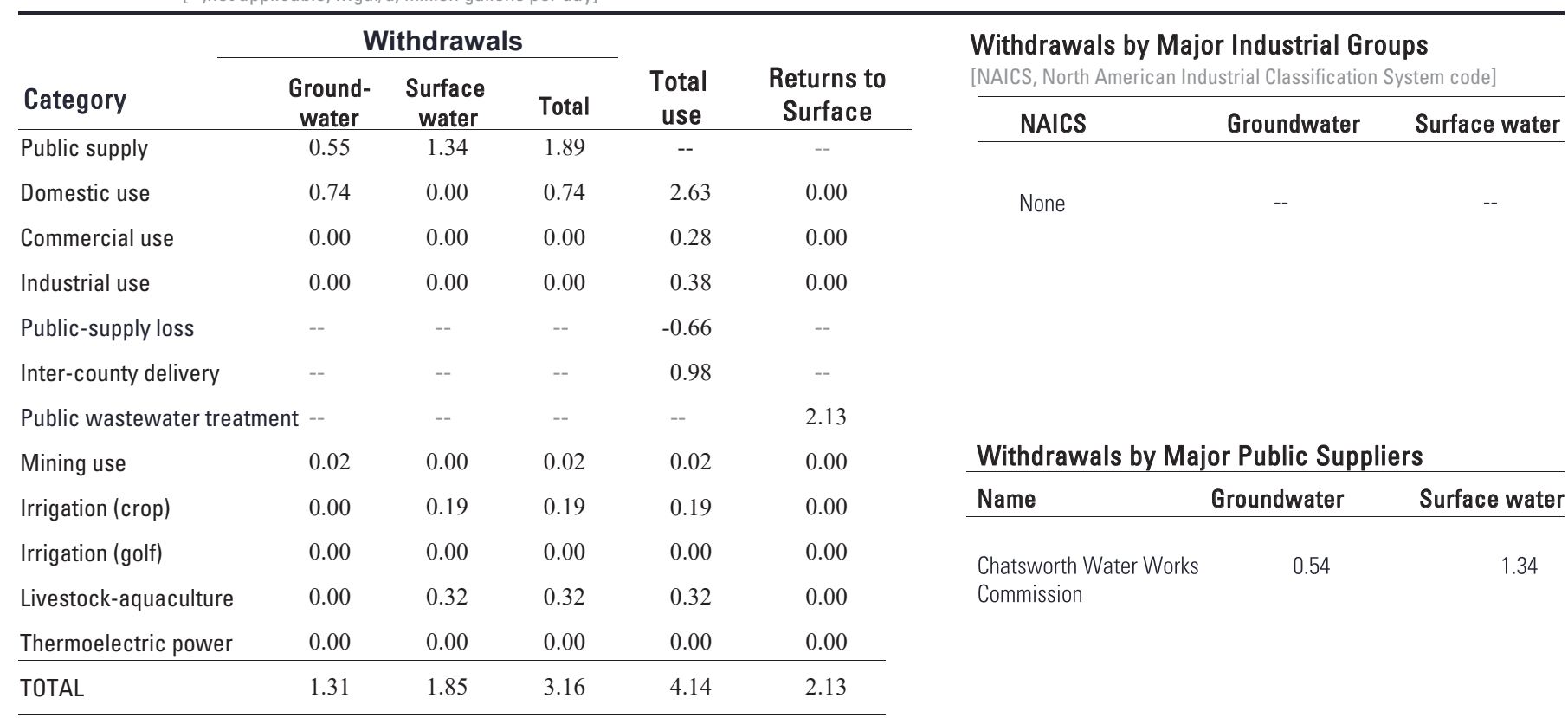

Total use is total withdrawal plus public supply deliveries and losses. An estimate of $0.98 \mathrm{Mgal} / \mathrm{d}$ was delivered from neighboring counties in 2015.

\section{Withdrawals by water source}

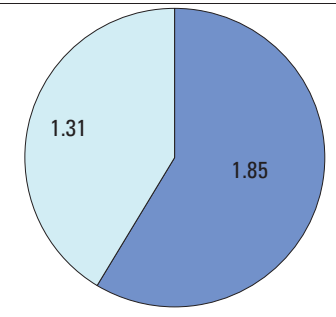

Withdrawals, in million gallons per day
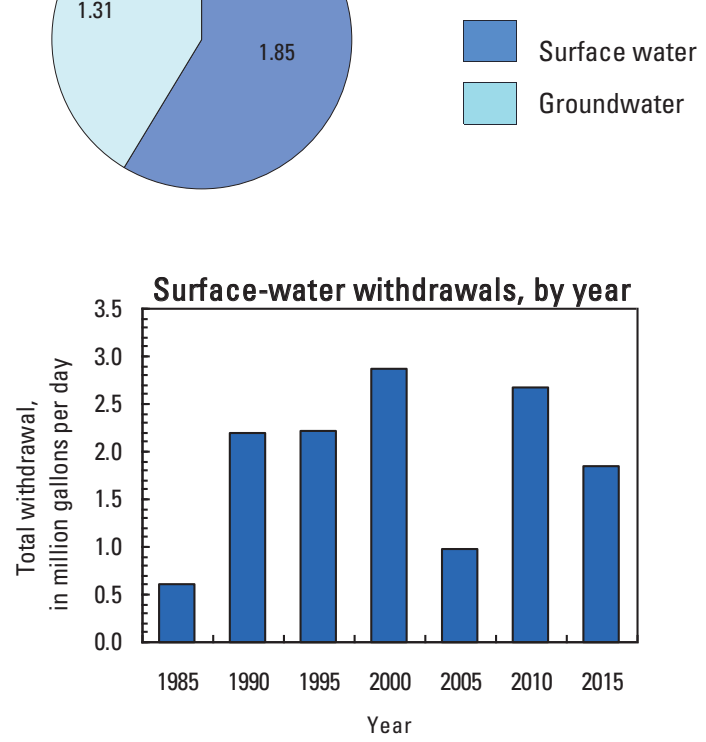

Groundwater withdrawals by aquifer

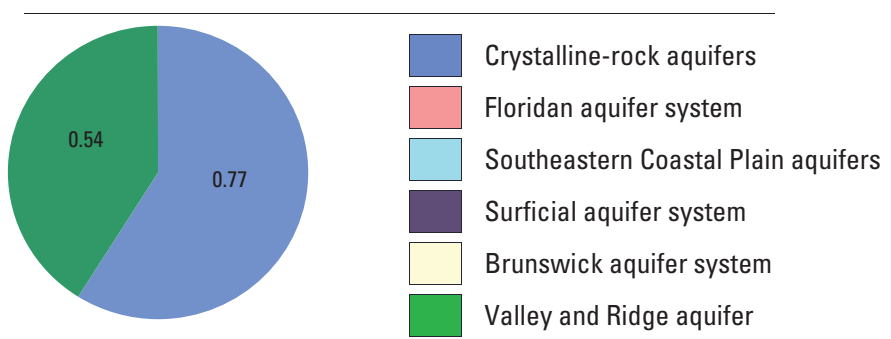

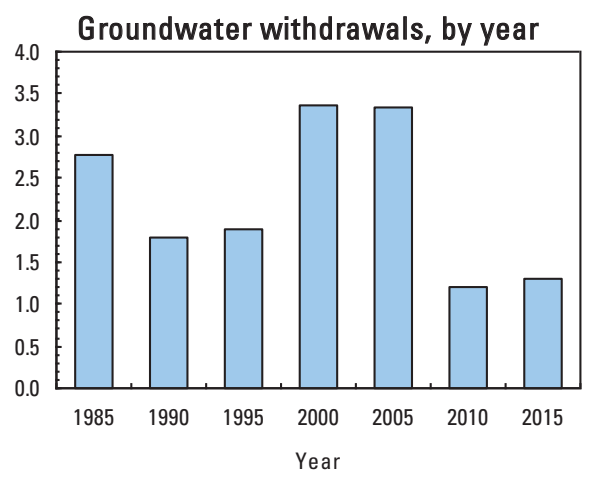




\section{MUSCOGEE COUNTY}

Population

Population served by public supply-groundwater

Population served by public supply-surface water 200,579

Acres irrigated

2015 WATER WITHDRAWALS AND ESTIMATED USE, IN MILLION GALLONS PER DAY

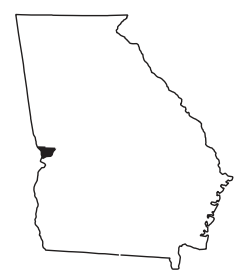

[--,not applicable; Mgal/d, million gallons per day]

\begin{tabular}{|c|c|c|c|c|c|c|c|c|}
\hline \multirow{3}{*}{ Category } & \multicolumn{3}{|c|}{ Withdrawals } & \multirow{3}{*}{$\begin{array}{c}\text { Total } \\
\text { use }\end{array}$} & \multirow{3}{*}{$\begin{array}{l}\text { Returns to } \\
\text { Surface }\end{array}$} & \multirow{2}{*}{\multicolumn{3}{|c|}{$\begin{array}{l}\text { Withdrawals by Major Industrial Groups } \\
\text { [NAICS, North American Industrial Classification System code] }\end{array}$}} \\
\hline & \multirow{2}{*}{$\begin{array}{c}\text { Ground- } \\
\text { water }\end{array}$} & \multirow{2}{*}{$\begin{array}{c}\text { Surface } \\
\text { water }\end{array}$} & \multirow[b]{2}{*}{ Total } & & & & & \\
\hline & & & & & & \multirow{4}{*}{ None } & \multirow[t]{2}{*}{ Groundwater } & \multirow[t]{2}{*}{ Surface water } \\
\hline Public supply & 0.00 & 36.77 & 36.77 & -- & -- & & & \\
\hline Domestic use & 0.00 & 0.00 & 0.00 & 17.03 & 0.00 & & -- & -- \\
\hline Commercial use & 0.00 & 0.00 & 0.00 & 6.35 & 0.00 & & & \\
\hline Industrial use & 0.00 & 0.00 & 0.00 & 1.72 & 0.00 & & & \\
\hline Public-supply loss & -- & -- & -- & 11.67 & -- & & & \\
\hline Inter-county delivery & -- & -- & -- & -6.52 & -- & & & \\
\hline Public wastewater treatment & t -- & -- & -- & -- & 107.38 & & & \\
\hline Mining use & 0.23 & 0.00 & 0.23 & 0.23 & 0.00 & \multicolumn{3}{|c|}{ Withdrawals by Major Public Suppliers } \\
\hline Irrigation (crop) & 0.00 & 0.00 & 0.00 & 0.00 & 0.00 & \multirow{4}{*}{$\begin{array}{l}\text { Name } \\
\text { City of Columbus } \\
\text { Smiths Water \& Sewer } \\
\text { Authority }\end{array}$} & Groundwater & Surface water \\
\hline Irrigation (golf) & 0.10 & 0.31 & 0.41 & 0.41 & 0.00 & & 0.00 & 34.95 \\
\hline Livestock-aquaculture & 0.00 & 0.00 & 0.00 & 0.00 & 0.00 & & \multirow[t]{2}{*}{0.00} & \multirow[t]{2}{*}{1.82} \\
\hline Thermoelectric power & 0.00 & 5.95 & 5.95 & 5.95 & 0.00 & & & \\
\hline TOTAL & 0.33 & 43.03 & 43.36 & 36.84 & 107.38 & & & \\
\hline
\end{tabular}

Total use is total withdrawal plus public supply deliveries and losses. An estimate of $6.52 \mathrm{Mgal} / \mathrm{d}$ was delivered from Muscogee County to neighboring counties in 2015.
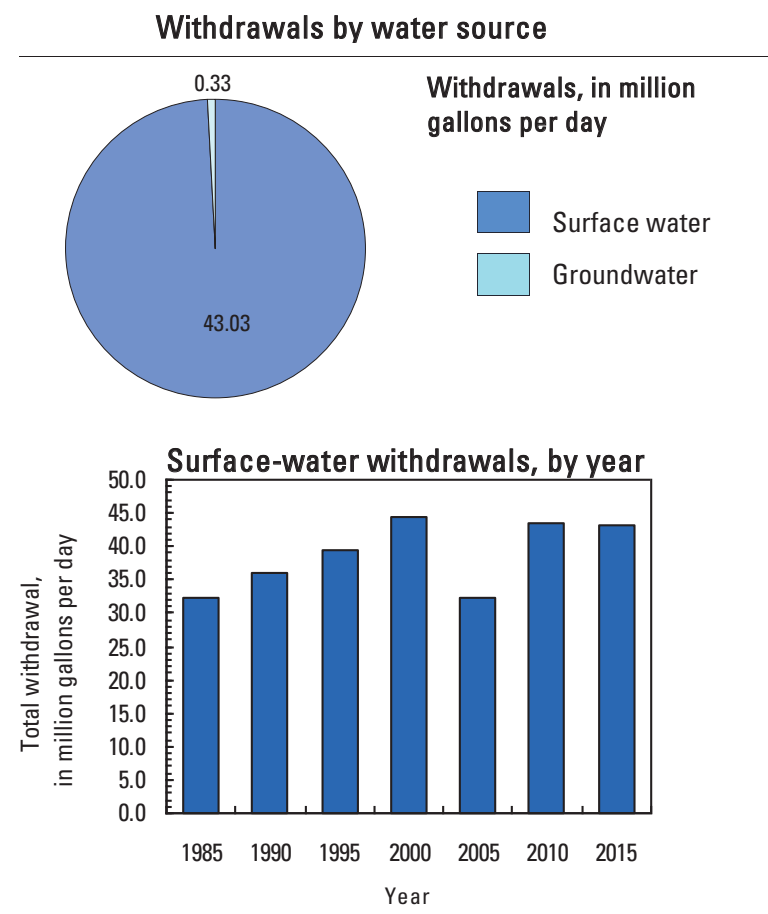
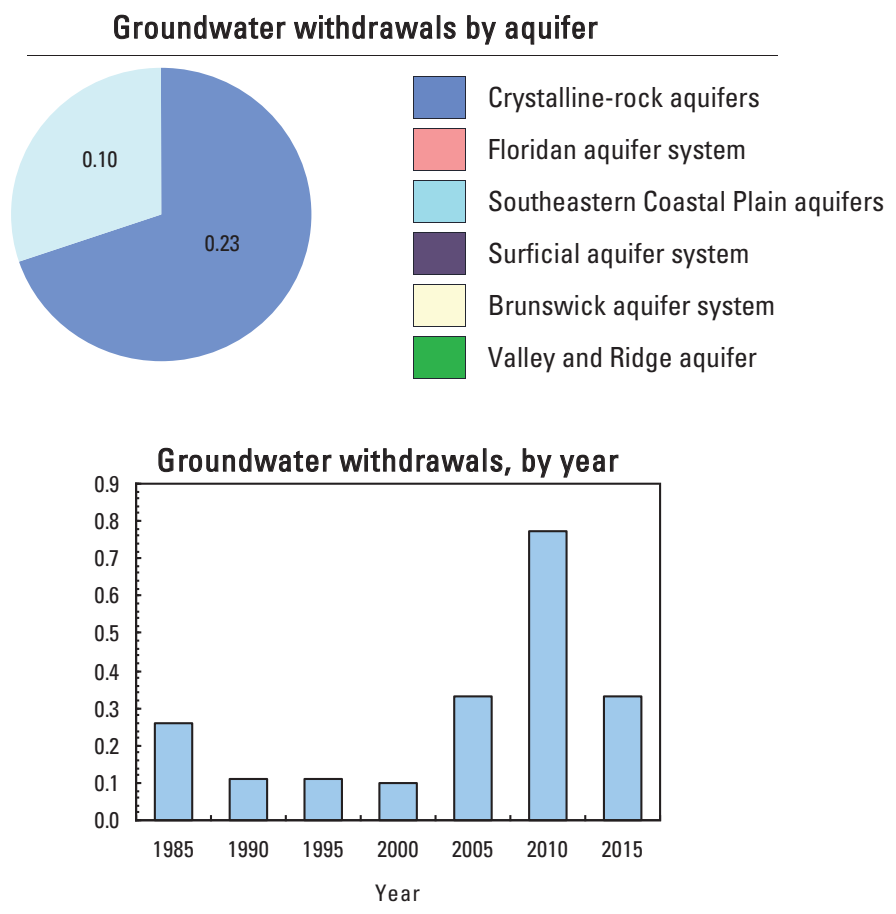


\section{NEWTON COUNTY}

Population

Population served by public supply-groundwater

Population served by public supply-surface water $\quad 86,600$

Acres irrigated

690

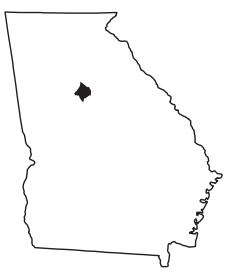

2015 WATER WITHDRAWALS AND ESTIMATED USE, IN MILLION GALLONS PER DAY

$[--$, not applicable; Mgal/d, million gallons per day]

\begin{tabular}{|c|c|c|c|c|c|}
\hline \multirow[b]{2}{*}{ Category } & \multicolumn{3}{|c|}{ Withdrawals } & \multirow[b]{2}{*}{$\begin{array}{c}\text { Total } \\
\text { use }\end{array}$} & \multirow[b]{2}{*}{$\begin{array}{c}\text { Returns to } \\
\text { Surface }\end{array}$} \\
\hline & $\begin{array}{l}\text { Ground- } \\
\text { water }\end{array}$ & $\begin{array}{c}\text { Surface } \\
\text { water }\end{array}$ & Total & & \\
\hline Public supply & 0.09 & 13.64 & 13.73 & -- & -- \\
\hline Domestic use & 1.30 & 0.00 & 1.30 & 7.65 & 0.00 \\
\hline Commercial use & 0.00 & 0.00 & 0.00 & 1.10 & 0.00 \\
\hline Industrial use & 0.00 & 0.00 & 0.00 & 0.48 & 0.00 \\
\hline Public-supply loss & -- & -- & -- & 5.80 & -- \\
\hline Inter-county delivery & -- & -- & -- & -4.38 & -- \\
\hline \multicolumn{2}{|c|}{ Public wastewater treatment -- } & -- & -- & -- & 0.01 \\
\hline Mining use & 0.05 & 0.00 & 0.05 & 0.05 & 0.00 \\
\hline Irrigation (crop) & 0.01 & 0.02 & 0.03 & 0.03 & 0.00 \\
\hline Irrigation (golf) & 0.21 & 0.29 & 0.50 & 0.50 & 0.00 \\
\hline Livestock-aquaculture & 0.05 & 0.10 & 0.15 & 0.15 & 0.00 \\
\hline Thermoelectric power & 0.00 & 0.00 & 0.00 & 0.00 & 0.00 \\
\hline TOTAL & 1.71 & 14.05 & 15.76 & 11.38 & 0.01 \\
\hline
\end{tabular}

Withdrawals by Major Industrial Groups

[NAICS, North American Industrial Classification System code]

NAICS Groundwater Surface water

None

Withdrawals by Major Public Suppliers

\begin{tabular}{lcc} 
Name & Groundwater & Surface water \\
\hline $\begin{array}{l}\text { Newton County Board } \\
\text { of Commissioners }\end{array}$ & 0.00 & 12.87 \\
$\begin{array}{l}\text { Newton County } \\
\begin{array}{l}\text { Water \& Sewerage } \\
\text { Authority }\end{array}\end{array}$ & 0.00 & 0.77 \\
\end{tabular}

Total use is total withdrawal plus public supply deliveries and losses. An estimate of $4.38 \mathrm{Mgal} / \mathrm{d}$ was telivered from Newton County to neighboring counties in 2015.

Withdrawals by water source

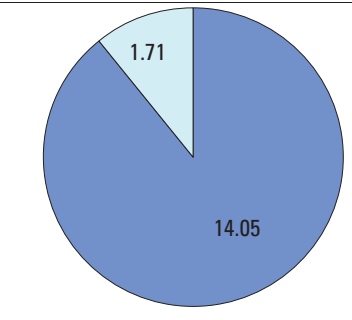

Withdrawals, in million gallons per day

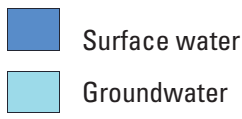

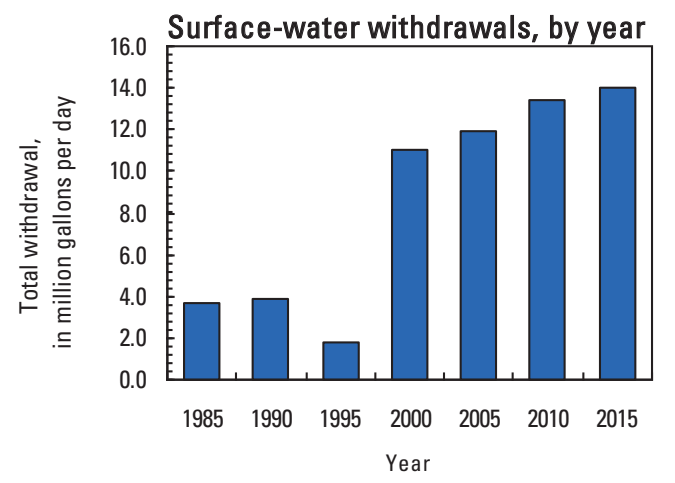

Groundwater withdrawals by aquifer

\begin{tabular}{|l|l}
\hline & Crystalline-rock aquifers \\
\hline & Floridan aquifer system \\
\hline & Southeastern Coastal Plain aquifers \\
& Surficial aquifer system \\
\hline$\quad$ Brunswick aquifer system \\
\hline$\quad$ Valley and Ridge aquifer
\end{tabular}

Groundwater withdrawals, by year

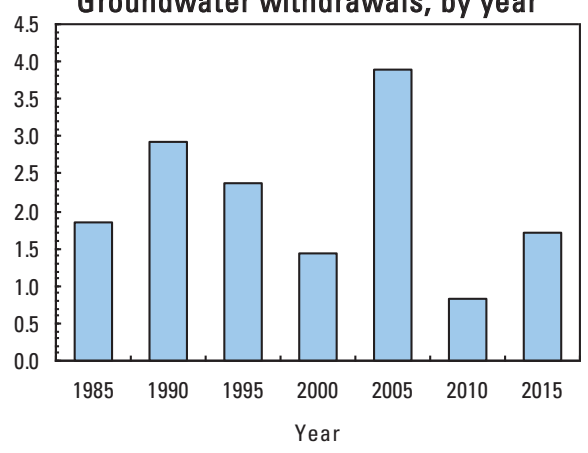




\section{OCONEE COUNTY}

Population

Population served by public supply-groundwater

Population served by public supply-surface water 19,831

Acres irrigated

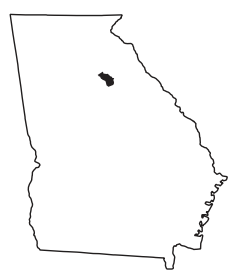

2015 WATER WITHDRAWALS AND ESTIMATED USE, IN MILLION GALLONS PER DAY

[--, not applicable; Mgal/d, million gallons per day]

\begin{tabular}{|c|c|c|c|c|c|c|c|c|}
\hline \multirow{3}{*}{ Category } & \multicolumn{3}{|c|}{ Withdrawals } & \multirow{3}{*}{$\begin{array}{c}\text { Total } \\
\text { use }\end{array}$} & \multirow{3}{*}{$\begin{array}{l}\text { Returns to } \\
\text { Surface }\end{array}$} & \multirow{2}{*}{\multicolumn{3}{|c|}{$\begin{array}{l}\text { Withdrawals by Major Industrial Groups } \\
\text { [NAICS, North American Industrial Classification System code] }\end{array}$}} \\
\hline & \multirow{2}{*}{$\begin{array}{l}\text { Ground- } \\
\text { water }\end{array}$} & \multirow{2}{*}{$\begin{array}{c}\text { Surface } \\
\text { water }\end{array}$} & \multirow{2}{*}{ Total } & & & & & \\
\hline & & & & & & NAICS & Groundwater & Surface water \\
\hline Public supply & 0.35 & 0.00 & 0.35 & -- & -- & & & \\
\hline Domestic use & 0.87 & 0.00 & 0.87 & 2.49 & 0.00 & None & -- & -- \\
\hline Commercial use & 0.00 & 0.00 & 0.00 & 0.86 & 0.00 & & & \\
\hline Industrial use & 0.00 & 0.00 & 0.00 & 0.04 & 0.00 & & & \\
\hline Public-supply loss & -- & -- & -- & -2.17 & -- & & & \\
\hline Inter-county delivery & -- & -- & -- & 2.47 & -- & & & \\
\hline Public wastewater treatment & t -- & -- & -- & -- & 0.09 & & & \\
\hline Mining use & 0.00 & 0.00 & 0.00 & 0.00 & 0.00 & Withdrawals by 1 & or Public Supp & \\
\hline Irrigation (crop) & 3.26 & 0.00 & 3.26 & 3.26 & 0.00 & Name & Groundwater & Surface water \\
\hline Irrigation (golf) & 0.00 & 0.18 & 0.18 & 0.18 & 0.00 & Oconee County Utility & 0.15 & 0.00 \\
\hline Livestock-aquaculture & 0.38 & 0.00 & 0.38 & 0.38 & 0.00 & Department & & \\
\hline Thermoelectric power & 0.00 & 0.00 & 0.00 & 0.00 & 0.00 & & & \\
\hline TOTAL & 4.86 & 0.18 & 5.04 & 7.51 & 0.09 & & & \\
\hline
\end{tabular}

Total use is total withdrawal plus public supply deliveries and losses. An estimate of $2.6 \mathrm{Mgal} / \mathrm{d}$ was delivered from neighboring counties in 2015. An estimate of $0.13 \mathrm{Mgal} / \mathrm{d}$ was delivered from 0 conee County to neighboring counties in 2015.

Withdrawals by water source

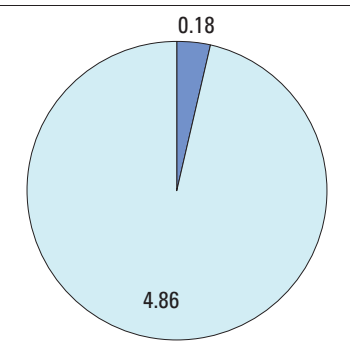

Withdrawals, in million gallons per day

Surface water

Groundwater

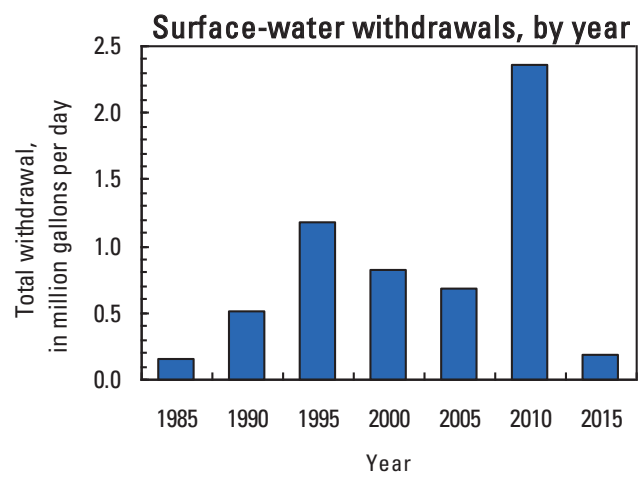

Groundwater withdrawals by aquifer
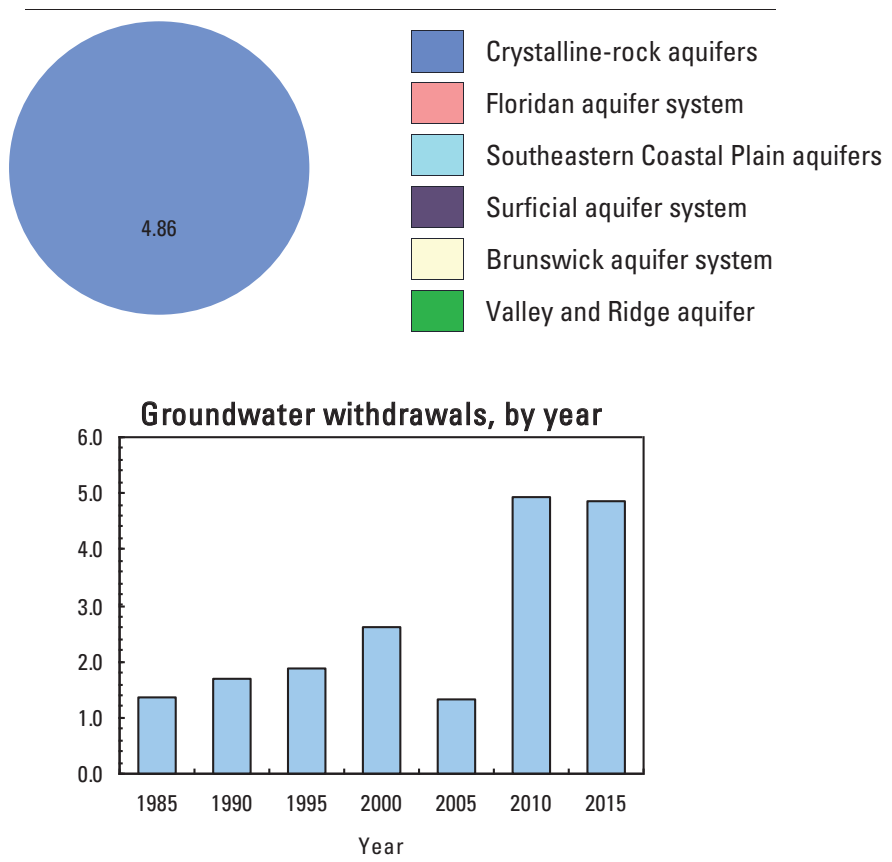


\section{OGLETHORPE COUNTY}

Population

Population served by public supply-groundwater

Population served by public supply-surface water

1,966

Acres irrigated

2015 WATER WITHDRAWALS AND ESTIMATED USE, IN MILLION GALLONS PER DAY

[--,not applicable; Mgal/d, million gallons per day]

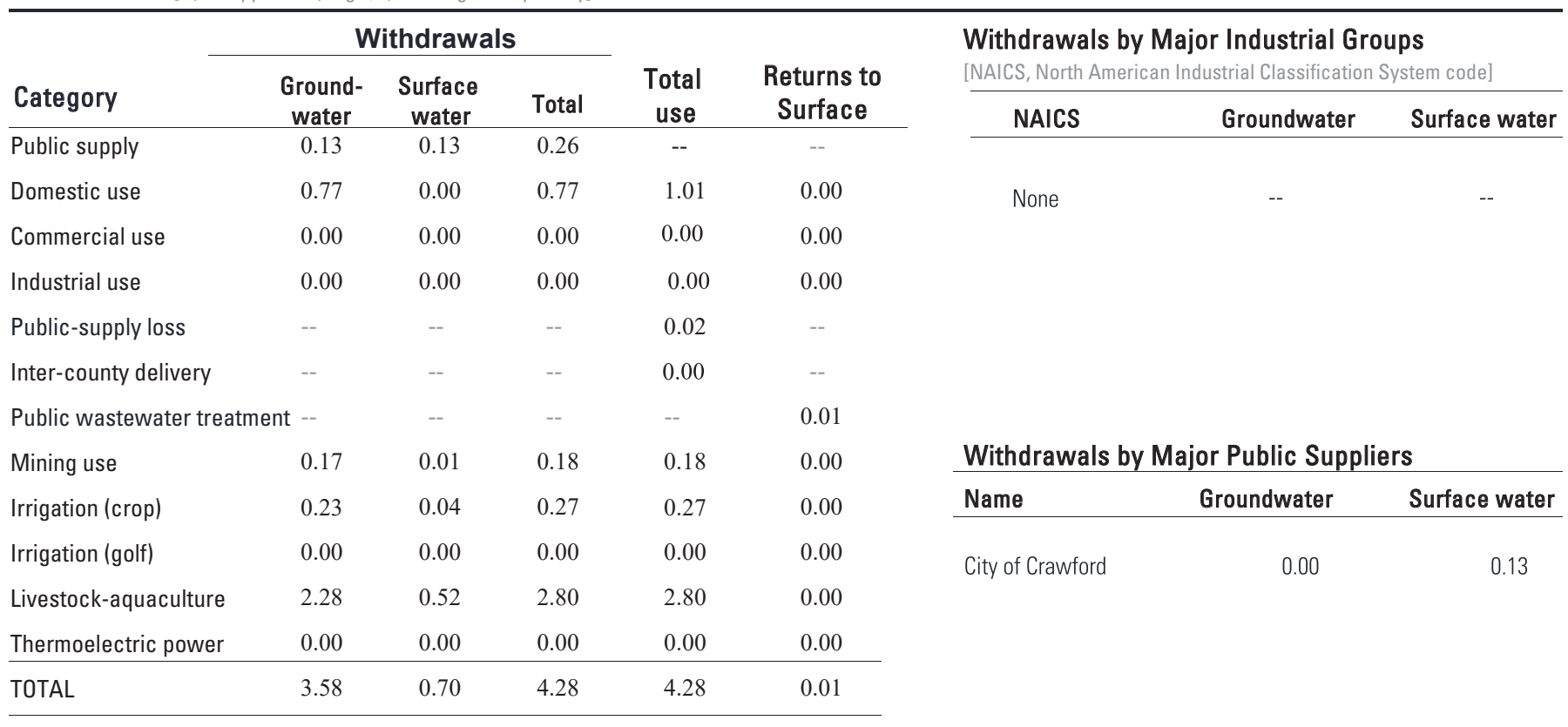

Total use is total withdrawal plus public supply deliveries and losses.

Withdrawals by water source

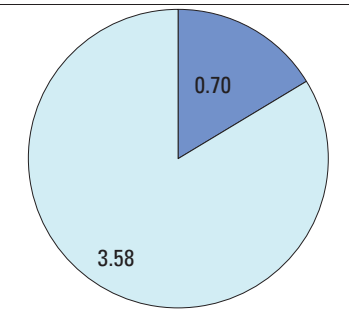

Withdrawals, in million

gallons per day

Surface water

Groundwater

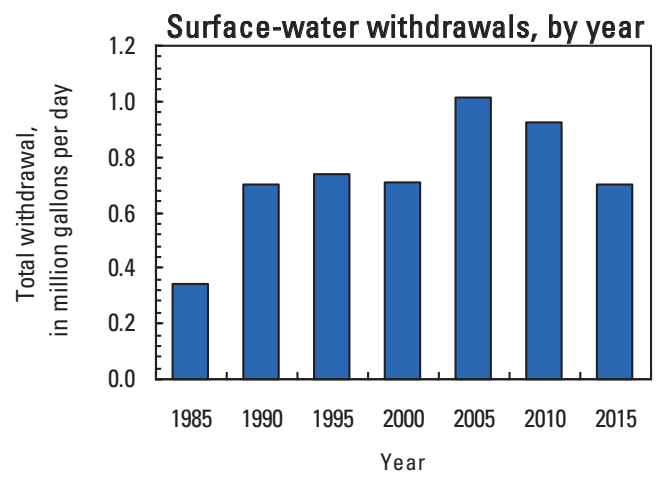

Groundwater withdrawals by aquifer

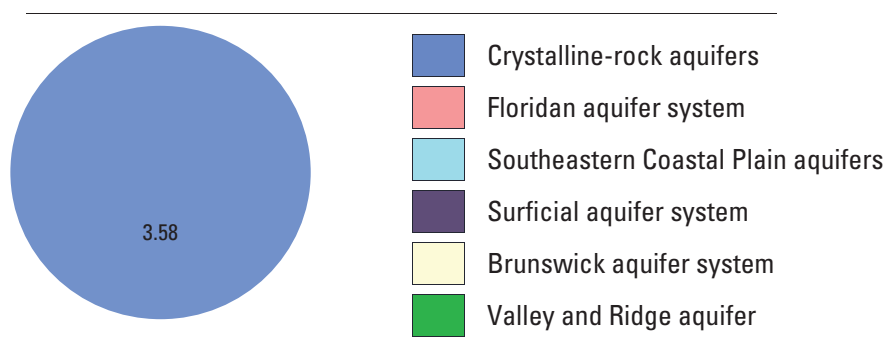

Groundwater withdrawals, by year

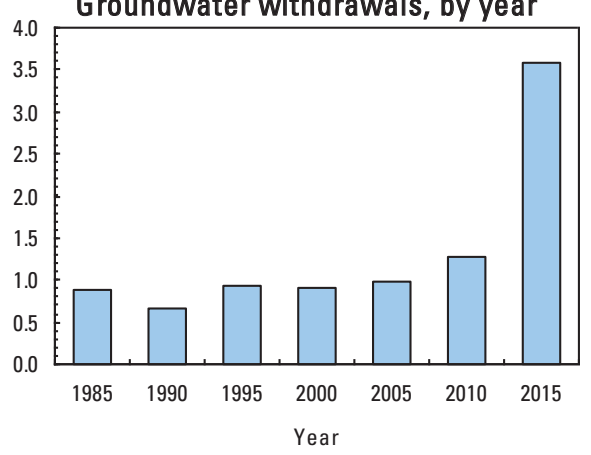




\section{PAULDING COUNTY}

Population

152,238

Population served by public supply-groundwater

1,614

Population served by public supply-surface water 120,760

Acres irrigated
460

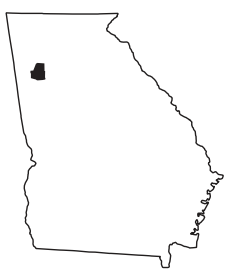

2015 WATER WITHDRAWALS AND ESTIMATED USE, IN MILLION GALLONS PER DAY

[--,not applicable; Mgal/d, million gallons per day]

\begin{tabular}{|c|c|c|c|c|c|c|c|c|}
\hline \multirow{3}{*}{ Category } & \multicolumn{3}{|c|}{ Withdrawals } & \multirow{3}{*}{$\begin{array}{c}\text { Total } \\
\text { use }\end{array}$} & \multirow{3}{*}{$\begin{array}{l}\text { Returns to } \\
\text { Surface }\end{array}$} & \multirow{2}{*}{\multicolumn{3}{|c|}{$\begin{array}{l}\text { Withdrawals by Major Industrial Groups } \\
\text { [NAICS, North American Industrial Classification System code] }\end{array}$}} \\
\hline & \multirow{2}{*}{$\begin{array}{c}\text { Ground- } \\
\text { water }\end{array}$} & \multirow{2}{*}{$\begin{array}{c}\text { Surface } \\
\text { water }\end{array}$} & \multirow{2}{*}{ Total } & & & & & \\
\hline & & & & & & \multirow{4}{*}{ None } & \multirow[t]{2}{*}{ Groundwater } & \multirow[t]{2}{*}{ Surface water } \\
\hline Public supply & 0.10 & 0.00 & 0.10 & -- & -- & & & \\
\hline Domestic use & 2.24 & 0.00 & 2.24 & 10.31 & 0.00 & & -- & -- \\
\hline Commercial use & 0.00 & 0.00 & 0.00 & 0.67 & 0.00 & & & \\
\hline Industrial use & 0.00 & 0.00 & 0.00 & 0.16 & 0.00 & & & \\
\hline Public-supply loss & -- & -- & -- & -8.80 & -- & & & \\
\hline Inter-county delivery & -- & -- & - & 10.53 & - & & & \\
\hline Public wastewater treatment & t -- & -- & -- & -- & 2.08 & & & \\
\hline Mining use & 0.08 & 0.00 & 0.08 & 0.08 & 0.00 & \multicolumn{3}{|c|}{ Withdrawals by Major Public Suppliers } \\
\hline Irrigation (crop) & 0.13 & 0.00 & 0.13 & 0.13 & 0.00 & \multirow{3}{*}{$\begin{array}{l}\text { Name } \\
\text { City of Dallas }\end{array}$} & Groundwater & Surface water \\
\hline Irrigation (golf) & 0.00 & 0.46 & 0.46 & 0.46 & 0.00 & & \multirow[t]{2}{*}{0.03} & \multirow[t]{2}{*}{0.00} \\
\hline Livestock-aquaculture & 0.09 & 0.00 & 0.09 & 0.09 & 0.00 & & & \\
\hline Thermoelectric power & 0.00 & 0.00 & 0.00 & 0.00 & 0.00 & & & \\
\hline TOTAL & 2.64 & 0.46 & 3.10 & 13.63 & 2.08 & & & \\
\hline
\end{tabular}

Total use is total withdrawal plus public supply deliveries and losses. An estimate of $10.53 \mathrm{Mgal} / \mathrm{d}$ was

delivered from neighboring counties in 2015.

\section{Withdrawals by water source}

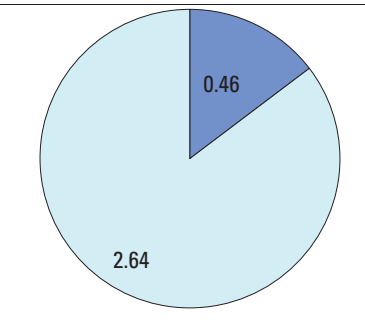

Withdrawals, in million

gallons per day

Surface water

Groundwater

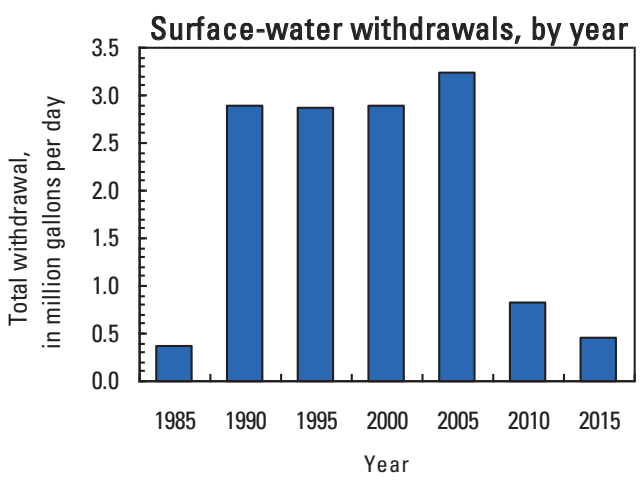

Groundwater withdrawals by aquifer
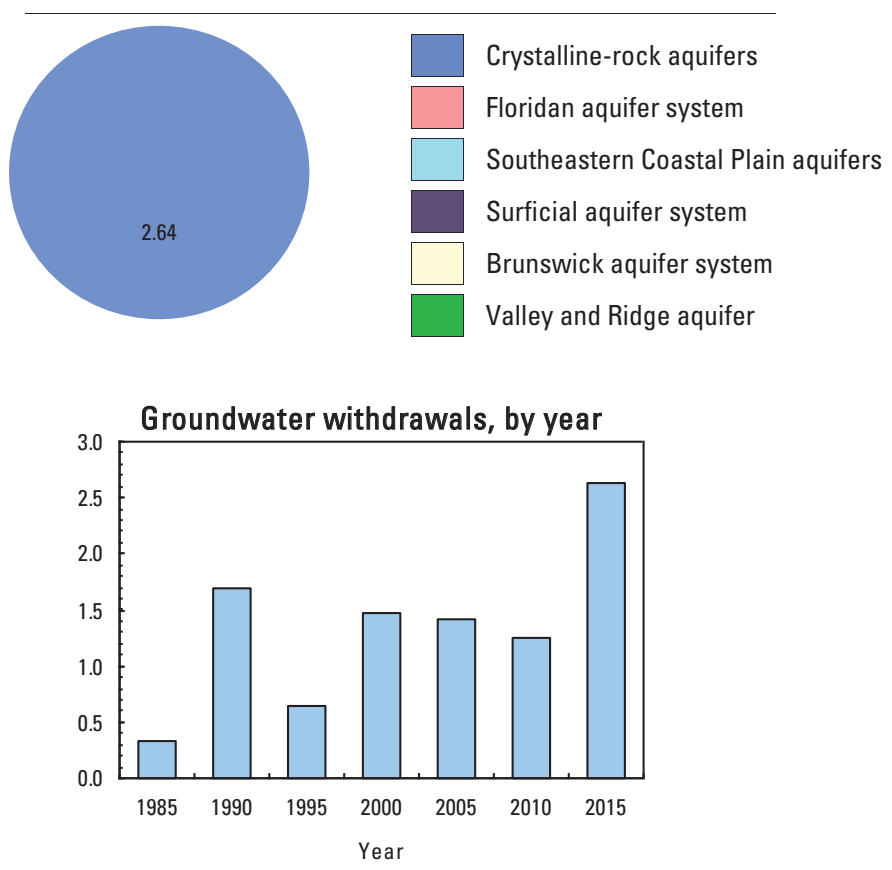


\section{PEACH COUNTY}

Population

Population served by public supply-groundwater

Population served by public supply-surface water

Acres irrigated

2015 WATER WITHDRAWALS AND ESTIMATED USE, IN MILLION GALLONS PER DAY

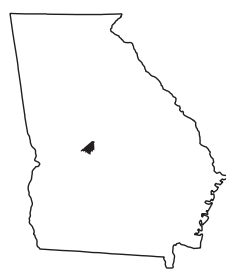

[--,not applicable; Mgal/d, million gallons per day]

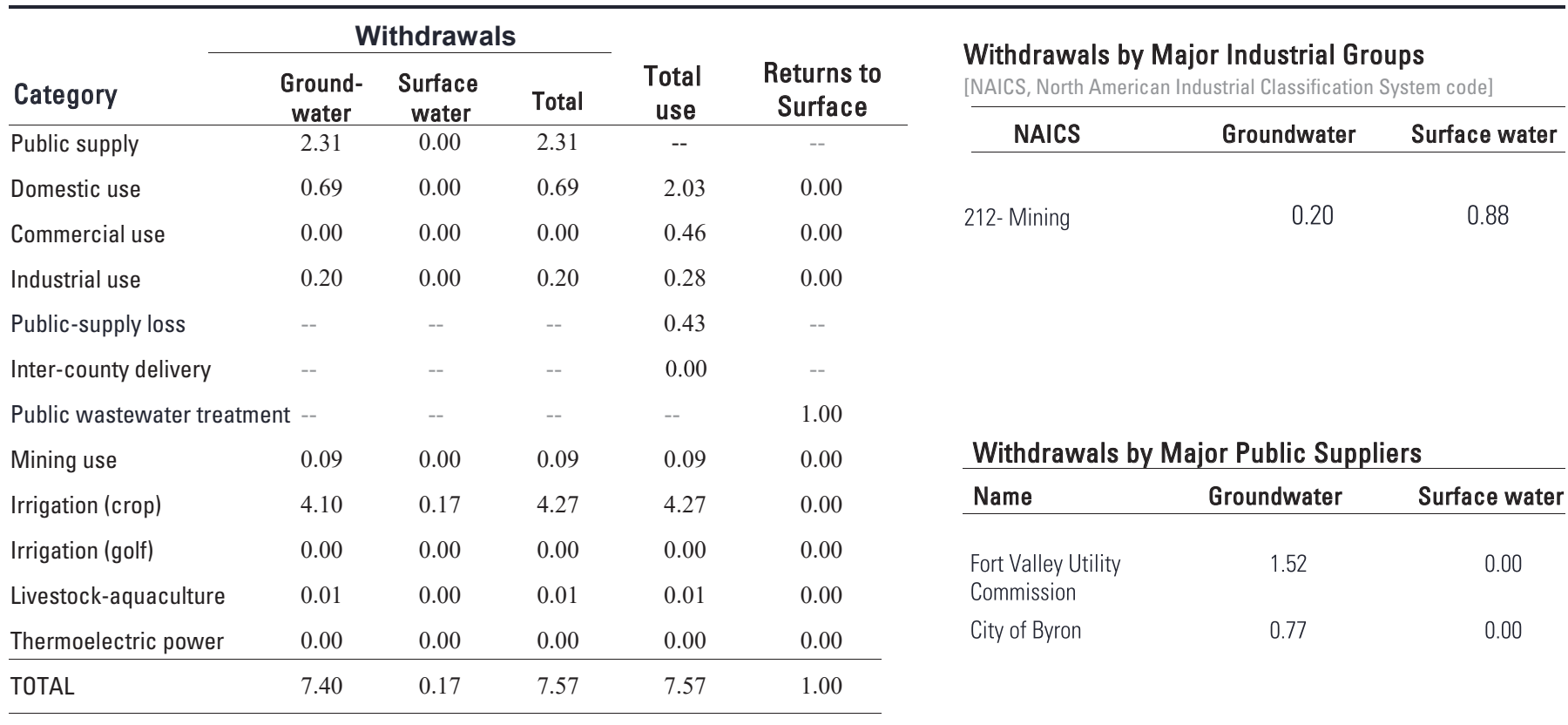

Total use is total withdrawal plus public supply deliveries and losses.

Withdrawals by water source

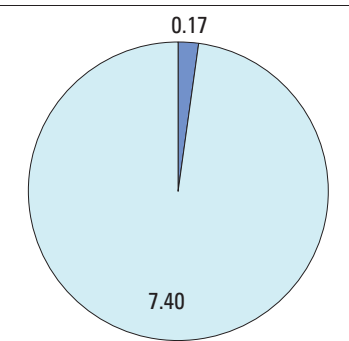

Withdrawals, in million gallons per day

Surface water

Groundwater

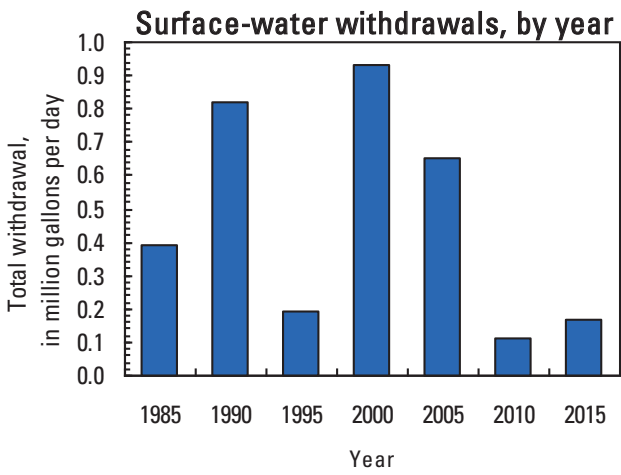

Groundwater withdrawals by aquifer

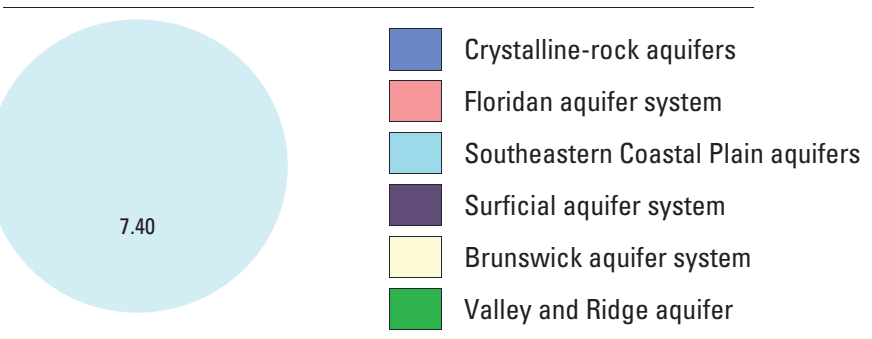

Groundwater withdrawals, by year

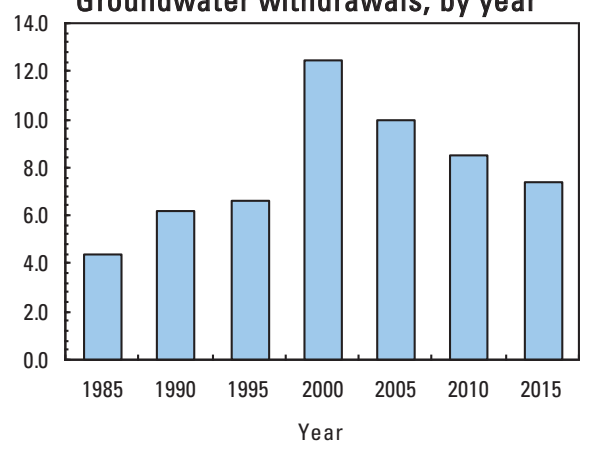




\section{PICKENS COUNTY}

Population

Population served by public supply-groundwater $\quad 14,654$

Population served by public supply-surface water $\quad 10,868$

Acres irrigated

2015 WATER WITHDRAWALS AND ESTIMATED USE, IN MILLION GALLONS PER DAY

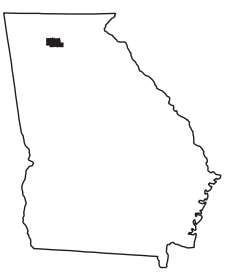

[--,not applicable; Mgal/d, million gallons per day]

\begin{tabular}{lccccc}
\hline & \multicolumn{3}{c}{ Withdrawals } & & Total \\
\cline { 2 - 4 } Category & $\begin{array}{c}\text { Ground- } \\
\text { water }\end{array}$ & $\begin{array}{c}\text { Surface } \\
\text { water }\end{array}$ & $\begin{array}{c}\text { Total } \\
\text { use }\end{array}$ & $\begin{array}{c}\text { Returns to } \\
\text { Surface }\end{array}$ \\
\hline Public supply & 0.89 & 1.88 & 2.77 & -- & -- \\
Domestic use & 0.36 & 0.00 & 0.36 & 2.73 & 0.00 \\
Commercial use & 0.00 & 0.00 & 0.00 & 0.34 & 0.00 \\
Industrial use & 1.12 & 0.00 & 1.12 & 1.21 & 0.00 \\
Public-supply loss & -- & -- & -- & -0.03 & -- \\
Inter-county delivery & -- & -- & -- & 0.55 & -- \\
Public wastewater treatment & -- & -- & -- & -- & 4.72 \\
Mining use & 0.30 & 0.00 & 0.30 & 0.30 & 0.00 \\
Irrigation (crop) & 0.00 & 0.00 & 0.00 & 0.00 & 0.00 \\
Irrigation (golf) & 0.00 & 0.09 & 0.09 & 0.09 & 0.00 \\
Livestock-aquaculture & 0.09 & 0.16 & 0.25 & 0.25 & 0.00 \\
Thermoelectric power & 0.00 & 0.00 & 0.00 & 0.00 & 0.00 \\
\hline TOTAL & 2.76 & 2.13 & 4.89 & 5.44 & 4.72 \\
\hline
\end{tabular}

Withdrawals by Major Industrial Groups

[NAICS, North American Industrial Classification System code]

\begin{tabular}{lll}
\hline NAICS Groundwater & Surface water \\
\hline
\end{tabular}

212- Mining

1.12

0.00

Total use is total withdrawal plus public supply deliveries and losses. An estimate of $0.55 \mathrm{Mgal} / \mathrm{d}$ was delivered from neighboring counties in 2015.

Withdrawals by Major Public Suppliers

\begin{tabular}{lcc} 
Name & Groundwater & Surface water \\
\hline City of Jasper & 0.89 & 1.00 \\
Big Canoe Utilities & 0.00 & 0.56 \\
Company & & \\
Bent Tree Water System & 0.00 & 0.32
\end{tabular}

\section{Withdrawals by water source}

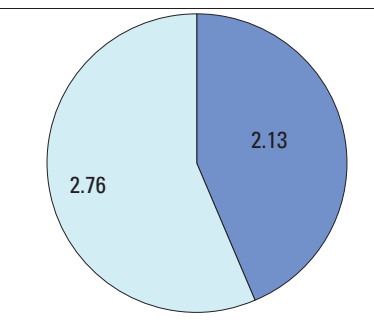

Withdrawals, in million gallons per day

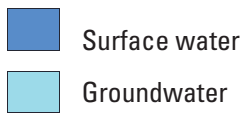

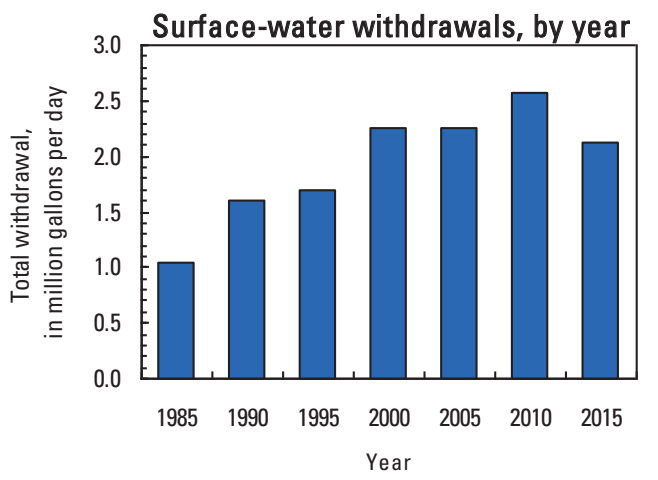

Groundwater withdrawals by aquifer

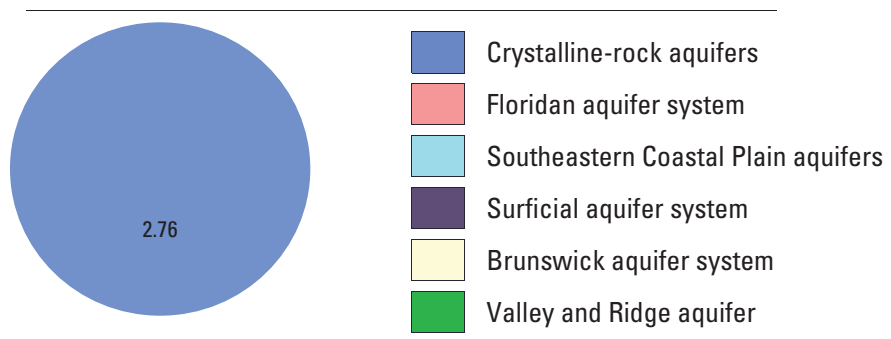

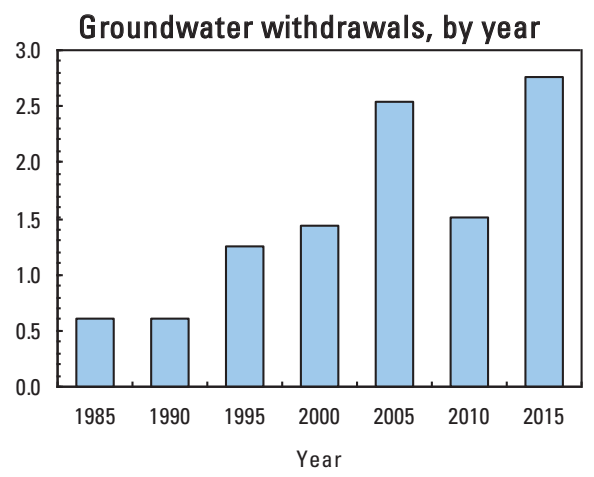




\section{PIERCE COUNTY}

Population

19,103

Population served by public supply-groundwater $\quad 7,997$

Population served by public supply-surface water $\quad 0$

Acres irrigated

2015 WATER WITHDRAWALS AND ESTIMATED USE, IN MILLION GALLONS PER DAY

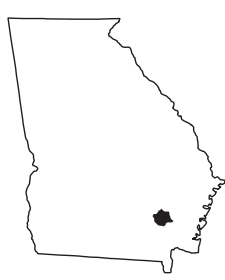

[--,not applicable; Mgal/d, million gallons per day]

\begin{tabular}{|c|c|c|c|c|c|c|c|c|}
\hline \multirow{3}{*}{$\begin{array}{l}\text { Category } \\
\text { Public supply }\end{array}$} & \multicolumn{3}{|c|}{ Withdrawals } & \multirow{2}{*}{$\begin{array}{c}\text { Total } \\
\text { use }\end{array}$} & \multirow{2}{*}{$\begin{array}{l}\text { Returns to } \\
\text { Surface }\end{array}$} & \multirow{2}{*}{\multicolumn{3}{|c|}{$\begin{array}{l}\text { Withdrawals by Major Industrial Groups } \\
\text { [NAICS, North American Industrial Classification System code] }\end{array}$}} \\
\hline & \multirow{2}{*}{$\begin{array}{c}\text { Ground- } \\
\text { water } \\
0.57\end{array}$} & \multirow{2}{*}{$\begin{array}{c}\begin{array}{c}\text { Surface } \\
\text { water }\end{array} \\
0.00\end{array}$} & \multirow{2}{*}{$\begin{array}{c}\text { Total } \\
0.57\end{array}$} & & & & & \\
\hline & & & & - & -- & NAICS & Groundwater & Surface water \\
\hline Domestic use & 0.83 & 0.00 & 0.83 & 1.25 & 0.00 & \multirow{3}{*}{$\begin{array}{l}\text { 212- Mining } \\
\text { 322- Paper, pulp }\end{array}$} & \multirow{3}{*}{$\begin{array}{l}0.07 \\
0.04\end{array}$} & \multirow{2}{*}{0.00} \\
\hline & 0.00 & 0.00 & 0.00 & 0.05 & 0.00 & & & \\
\hline Industrial use & 0.11 & 0.00 & 0.11 & 0.15 & 0.00 & & & 0.00 \\
\hline Public-supply loss & -- & -- & -- & 0.06 & -- & & & \\
\hline Inter-county delivery & -- & -- & -- & 0.00 & -- & & & \\
\hline \multicolumn{2}{|l|}{ Public wastewater treatment -- } & -- & -- & -- & 0.05 & & & \\
\hline Mining use & 0.00 & 0.00 & 0.00 & 0.00 & 0.00 & \multicolumn{3}{|c|}{ Withdrawals by Major Public Suppliers } \\
\hline Irrigation (crop) & 3.15 & 0.56 & 3.71 & 3.71 & 0.00 & Name & Groundwater & Surface water \\
\hline Irrigation (golf) & 0.07 & 0.00 & 0.07 & 0.07 & 0.00 & City of Blackshear & 0.43 & 0.00 \\
\hline Livestock-aquaculture & 0.14 & 0.03 & 0.17 & 0.17 & 0.00 & & & \\
\hline Thermoelectric power & 0.00 & 0.00 & 0.00 & 0.00 & 0.00 & & & \\
\hline TOTAL & 4.87 & 0.59 & 5.46 & 5.46 & 0.05 & & & \\
\hline
\end{tabular}

Withdrawals by water source

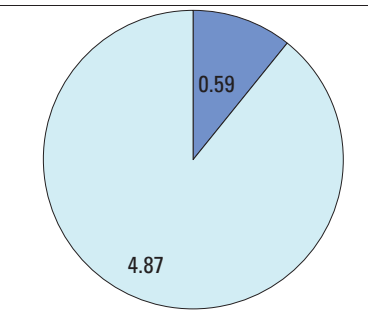

Withdrawals, in million gallons per day

Surface water

Groundwater

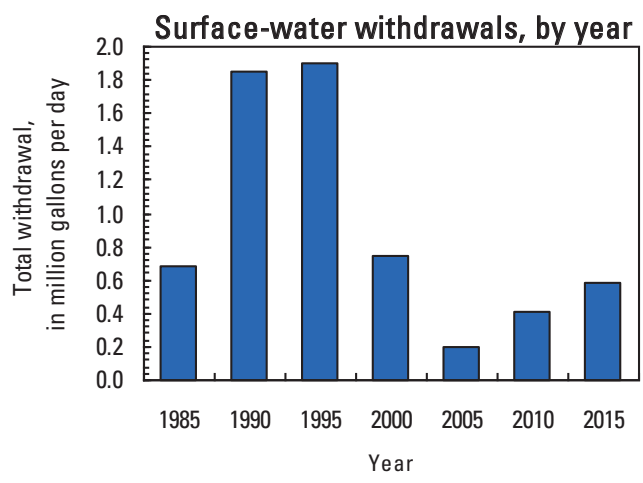

Groundwater withdrawals by aquifer

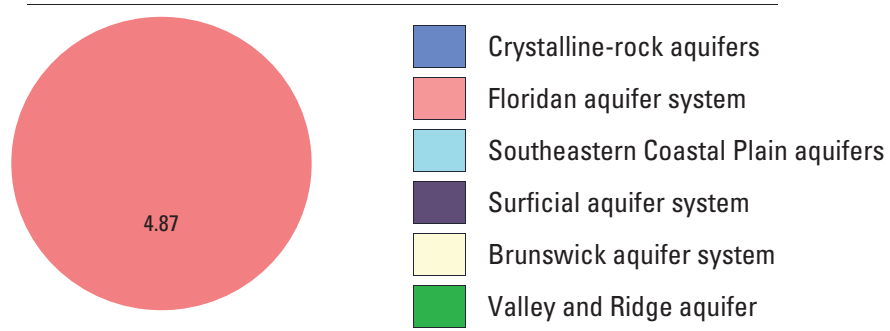

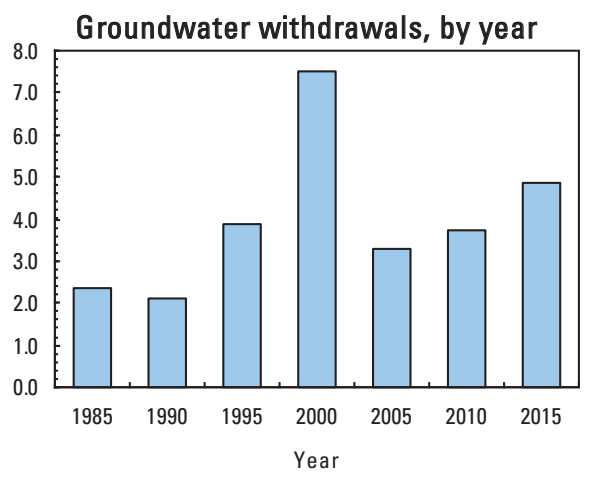




\section{PIKE COUNTY}

Population

Population served by public supply-groundwater $\quad 1,559$

Population served by public supply-surface water $\quad 2,016$

Acres irrigated

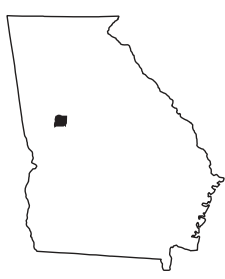

2015 WATER WITHDRAWALS AND ESTIMATED USE, IN MILLION GALLONS PER DAY

[--,not applicable; Mgal/d, million gallons per day]

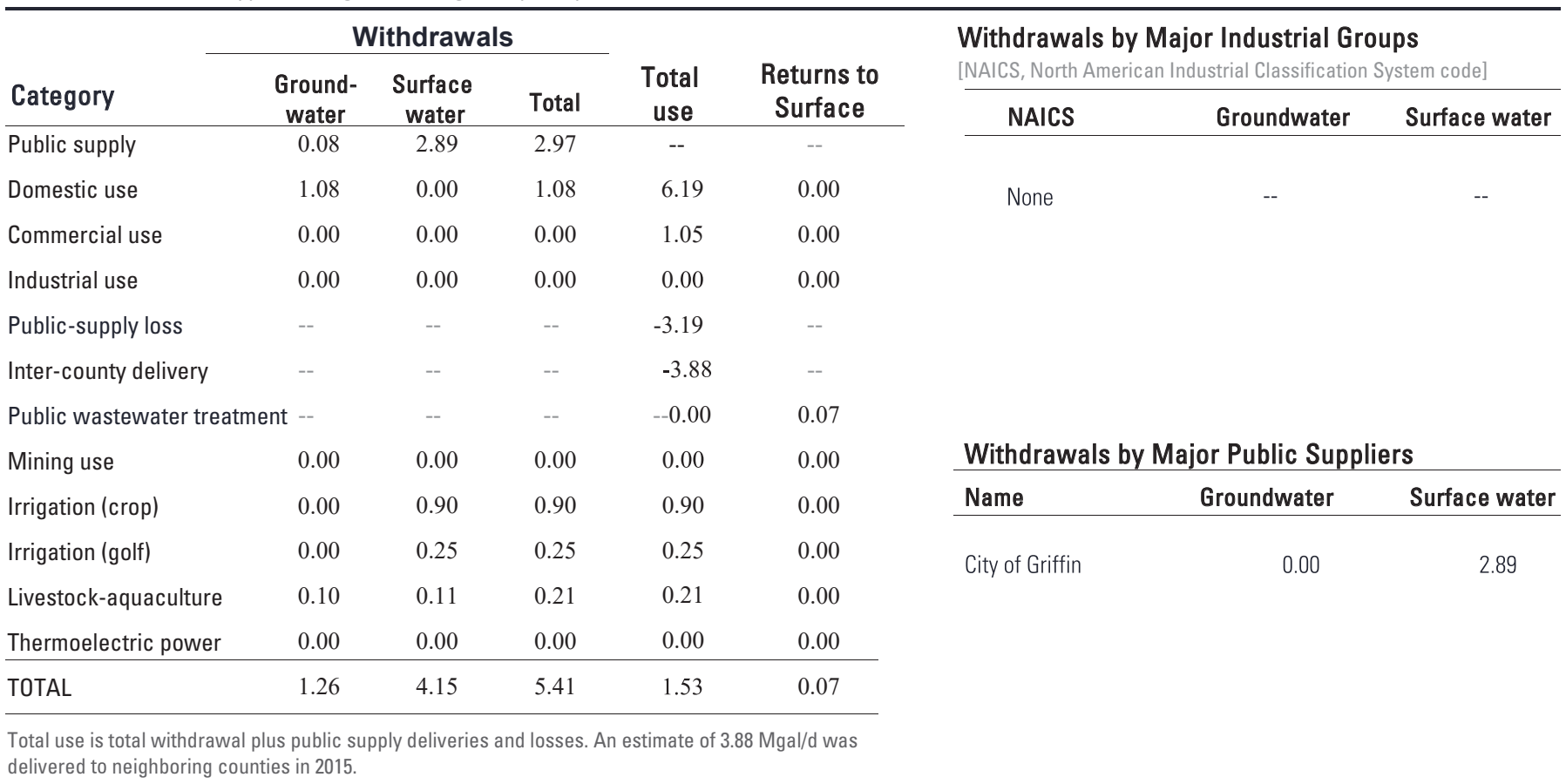

\section{Withdrawals by water source}

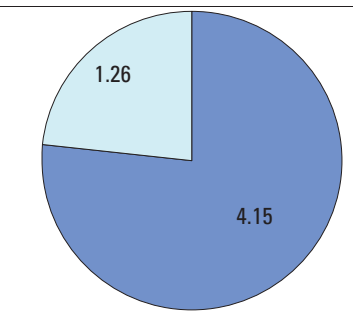

Withdrawals, in million

gallons per day

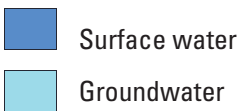

Groundwater withdrawals by aquifer

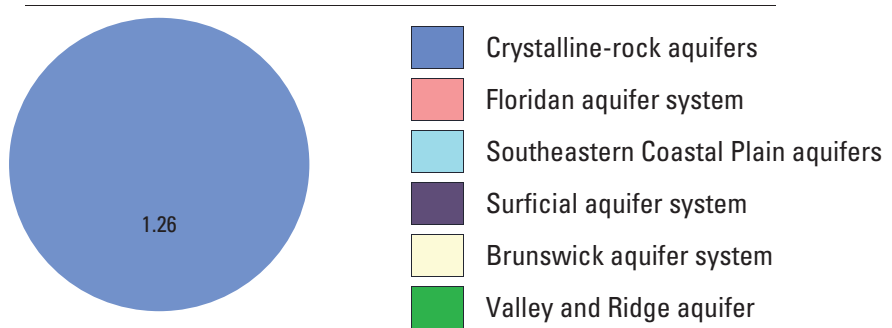

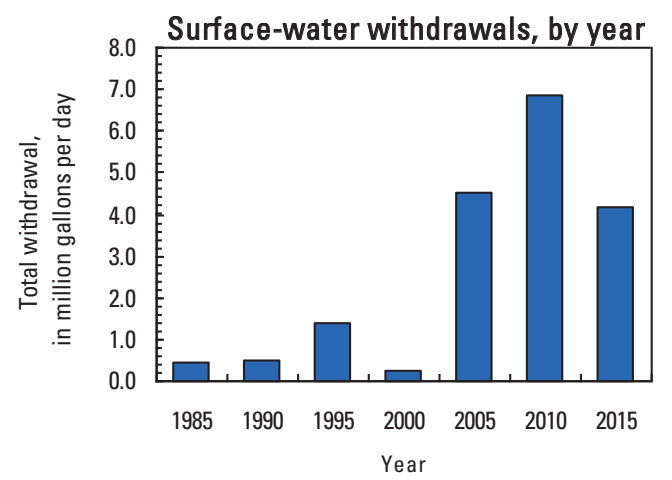

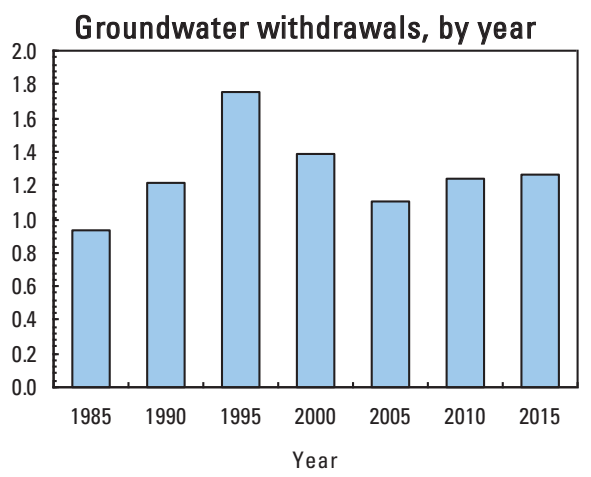




\section{POLK COUNTY}

Population

Population served by public supply-groundwater

Population served by public supply-surface water 33,811

Acres irrigated

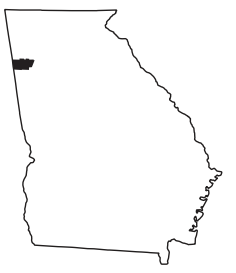

2015 WATER WITHDRAWALS AND ESTIMATED USE, IN MILLION GALLONS PER DAY

[--,not applicable; Mgal/d, million gallons per day]

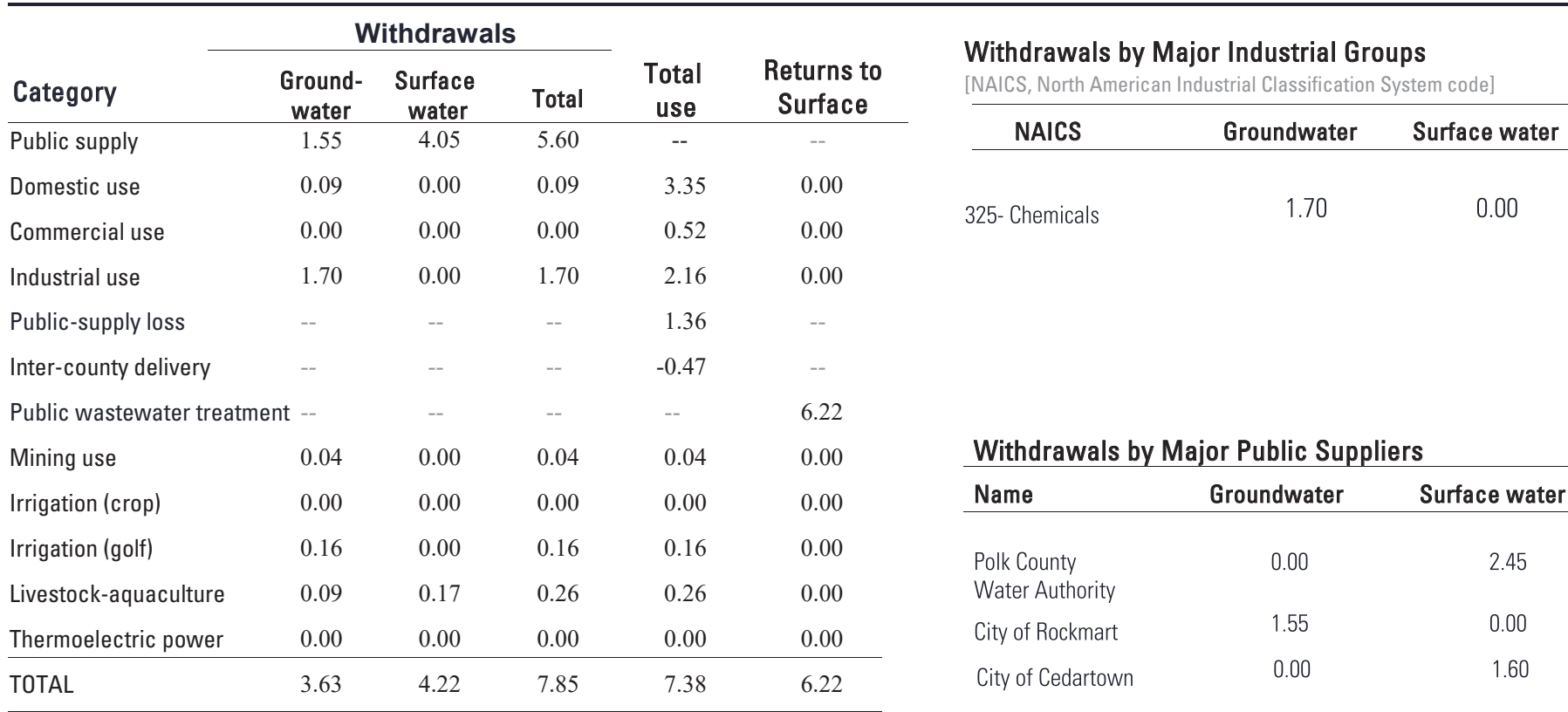

Total use is total withdrawal plus public supply deliveries and losses. An estimate of $0.47 \mathrm{Mgal} / \mathrm{d}$ was delivered from Polk County to neighboring counties in 2015.

Withdrawals by water source

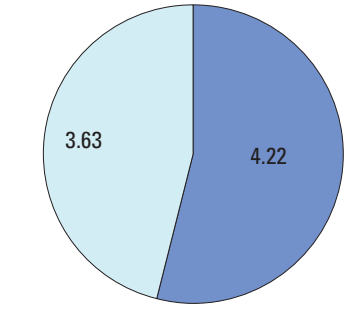

Withdrawals, in million gallons per day

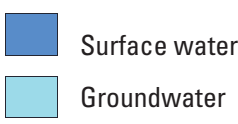

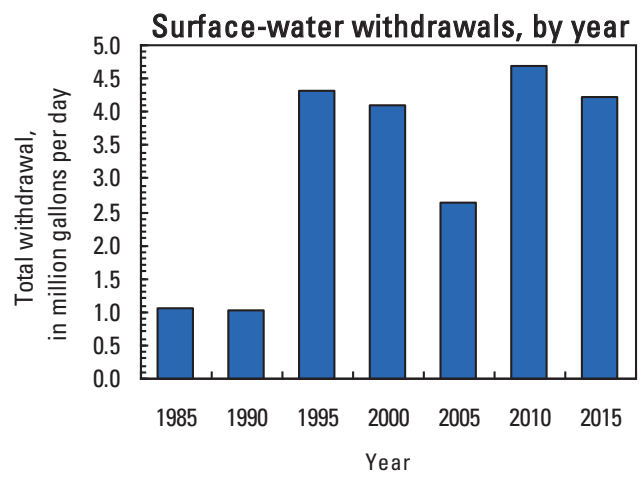

Groundwater withdrawals by aquifer

\begin{tabular}{|l|l}
\hline & Crystalline-rock aquifers \\
\hline & Floridan aquifer system \\
\hline & Southeastern Coastal Plain aquifers \\
& Surficial aquifer system \\
\hline & Brunswick aquifer system \\
& Valley and Ridge aquifer
\end{tabular}

Groundwater withdrawals, by year

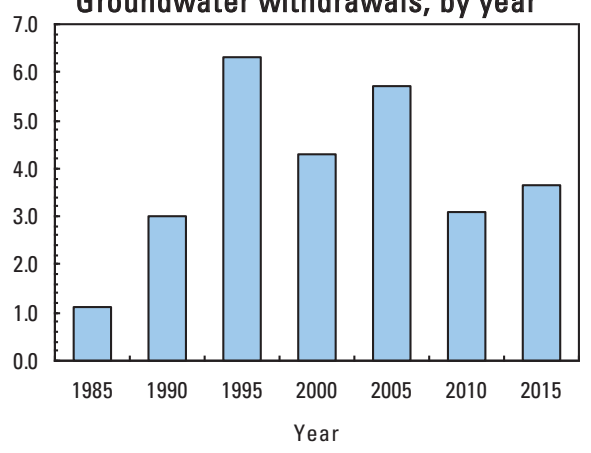




\section{PULASKI COUNTY}

Population

Population served by public supply-groundwater $\quad 5,343$

Population served by public supply-surface water 0

Acres irrigated

2015 WATER WITHDRAWALS AND ESTIMATED USE, IN MILLION GALLONS PER DAY

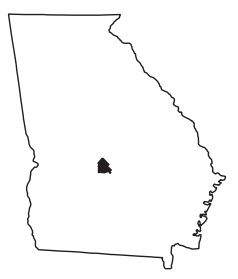

[--,not applicable; Mgal/d, million gallons per day]

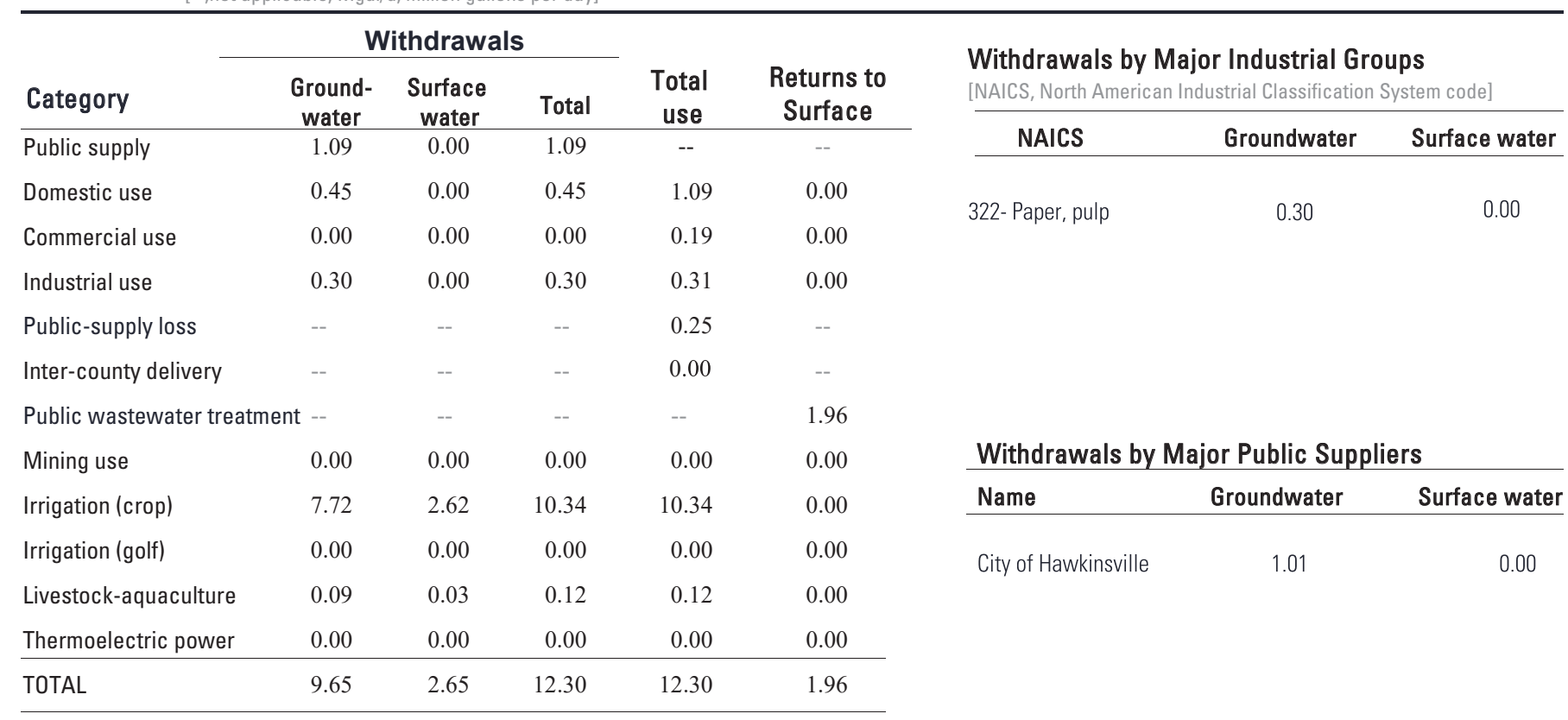

Total use is total withdrawal plus public supply deliveries and losses.

Withdrawals by water source

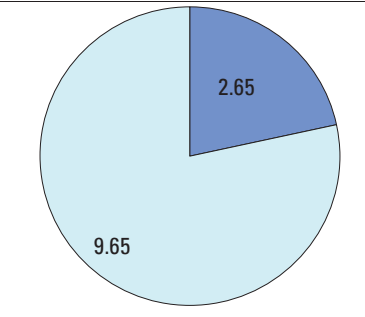

Withdrawals, in million

gallons per day

Surface water

Groundwater

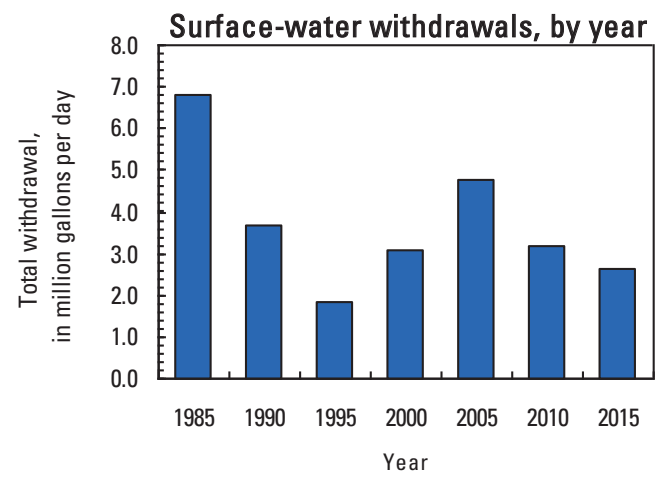

Groundwater withdrawals by aquifer

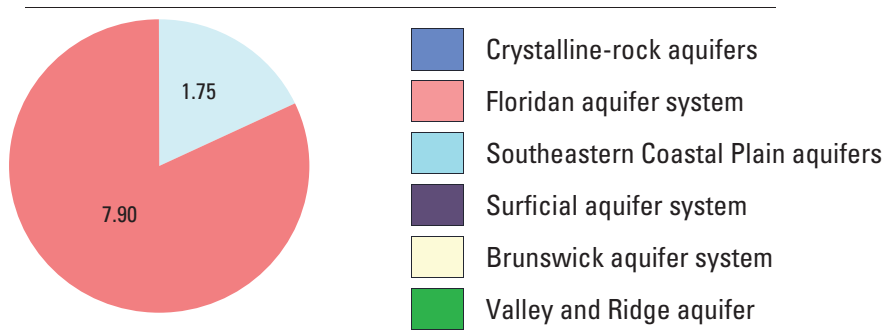

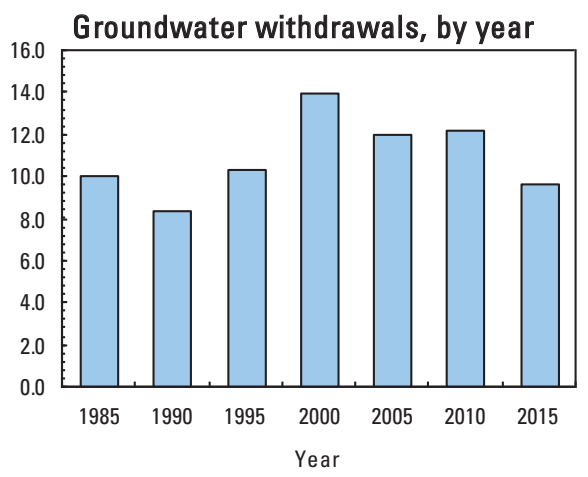




\section{PUTNAM COUNTY}

Population

Population served by public supply-groundwater

Population served by public supply-surface water $\quad 12,272$

Acres irrigated

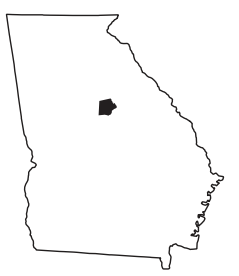

2015 WATER WITHDRAWALS AND ESTIMATED USE, IN MILLION GALLONS PER DAY

[--,not applicable; Mgal/d, million gallons per day]

\begin{tabular}{|c|c|c|c|c|c|c|c|c|}
\hline \multirow{2}{*}{ Category } & \multicolumn{3}{|c|}{ Withdrawals } & \multirow{2}{*}{$\begin{array}{c}\text { Total } \\
\text { use }\end{array}$} & \multirow{3}{*}{$\begin{array}{l}\text { Returns to } \\
\text { Surface }\end{array}$} & \multirow{2}{*}{\multicolumn{3}{|c|}{$\begin{array}{l}\text { Withdrawals by Major Industrial Groups } \\
\text { [NAICS, North American Industrial Classification System code] }\end{array}$}} \\
\hline & Ground- & Surface & Total & & & & & \\
\hline Public supply & $\begin{array}{c}\text { water } \\
0.50\end{array}$ & $\begin{array}{c}\text { water } \\
3.26\end{array}$ & 3.76 & & & NAICS & Groundwater & Surface water \\
\hline Domestic use & 0.18 & 0.00 & 0.18 & 0.92 & 0.00 & \multirow{3}{*}{ 321-Wood } & \multirow{3}{*}{0.07} & \multirow{3}{*}{0.00} \\
\hline Commercial use & 0.00 & 0.00 & 0.00 & 0.26 & 0.00 & & & \\
\hline Industrial use & 0.07 & 0.00 & 0.07 & 0.11 & 0.00 & & & \\
\hline Public-supply loss & -- & -- & -- & 2.72 & -- & & & \\
\hline Inter-county delivery & -- & -- & -- & -2.10 & -- & & & \\
\hline Public wastewater treatment & nt -- & -- & -- & -- & 107.82 & & & \\
\hline Mining use & 0.00 & 0.00 & 0.00 & 0.00 & 0.00 & \multicolumn{3}{|c|}{ Withdrawals by Major Public Suppliers } \\
\hline Irrigation (crop) & 0.20 & 0.05 & 0.25 & 0.25 & 0.00 & Name & Groundwater & Surface water \\
\hline Irrigation (golf) & 0.00 & 0.10 & 0.10 & 0.10 & 0.00 & \multirow[t]{4}{*}{ Sinclair Water Authority } & \multirow[t]{2}{*}{0.00} & \multirow[t]{3}{*}{3.26} \\
\hline Livestock-aquaculture & 0.59 & 0.15 & 0.74 & 0.74 & 0.00 & & & \\
\hline Thermoelectric power & 0.00 & 180.9 & 180.9 & 180.9 & 0.00 & & & \\
\hline TOTAL & 1.54 & 184.5 & 186.0 & 183.9 & 107.8 & & & \\
\hline
\end{tabular}

Total use is total withdrawal plus public supply deliveries and losses. An estimate of $2.1 \mathrm{Mgal} / \mathrm{d}$ was delivered from Putnam County to neighboring counties in 2015.

\section{Withdrawals by water source}

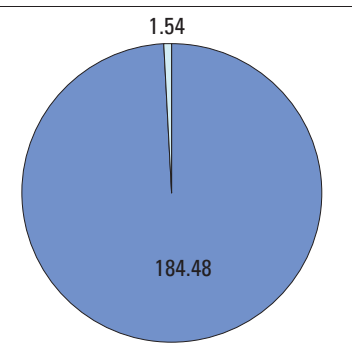

Withdrawals, in million

gallons per day

Surface water

Groundwater

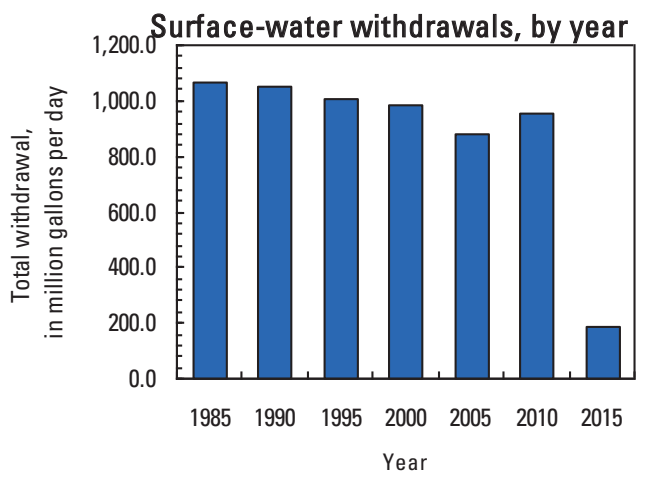

Groundwater withdrawals by aquifer
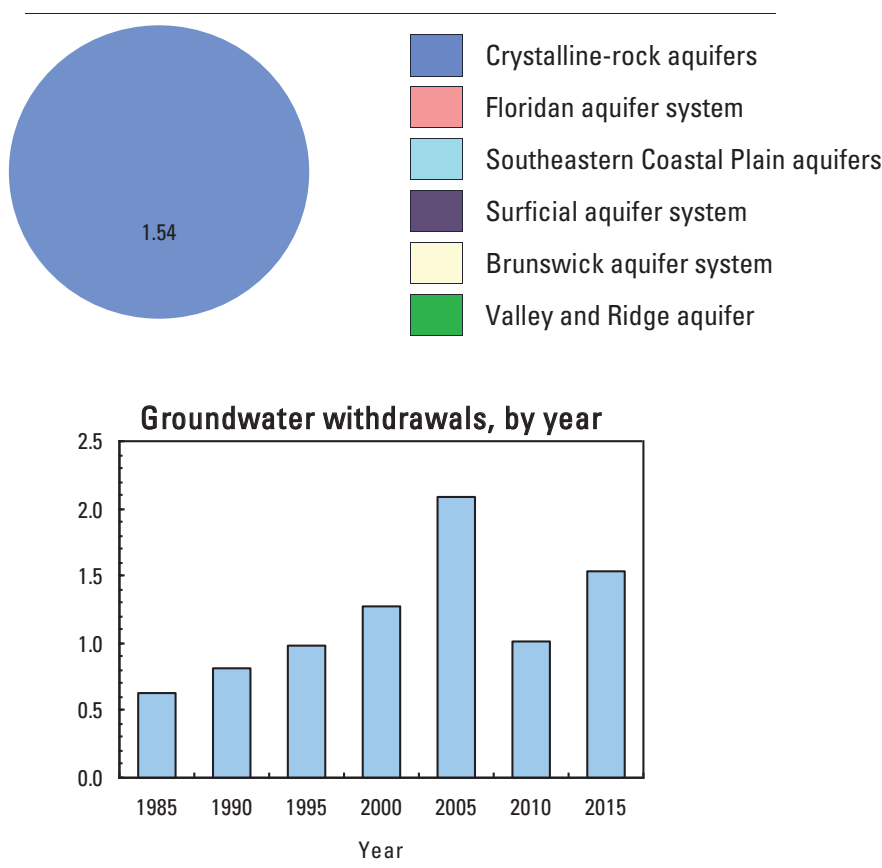


\section{QUITMAN COUNTY}

Population

Population served by public supply-groundwater

2,092

Population served by public supply-surface water 0

Acres irrigated

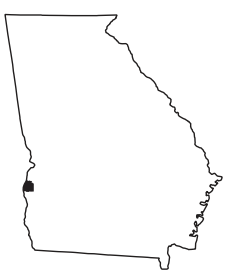

2015 WATER WITHDRAWALS AND ESTIMATED USE, IN MILLION GALLONS PER DAY

[--,not applicable; Mgal/d, million gallons per day]

\begin{tabular}{|c|c|c|c|c|c|c|c|c|}
\hline \multirow{3}{*}{ Category } & \multicolumn{3}{|c|}{ Withdrawals } & \multirow{3}{*}{$\begin{array}{c}\text { Total } \\
\text { use }\end{array}$} & \multirow{3}{*}{$\begin{array}{l}\text { Returns to } \\
\text { Surface }\end{array}$} & \multirow{2}{*}{\multicolumn{3}{|c|}{$\begin{array}{l}\text { Withdrawals by Major Industrial Groups } \\
\text { [NAICS, North American Industrial Classification System code] }\end{array}$}} \\
\hline & \multirow{2}{*}{$\begin{array}{c}\text { Ground - } \\
\text { water }\end{array}$} & \multirow{2}{*}{$\begin{array}{c}\text { Surface } \\
\text { water }\end{array}$} & \multirow{2}{*}{ Total } & & & & & \\
\hline & & & & & & \multirow[t]{2}{*}{ NAICS } & \multirow[t]{2}{*}{ Groundwater } & Surface water \\
\hline Public supply & 0.17 & 0.00 & 0.17 & -- & -- & & & \\
\hline Domestic use & 0.02 & 0.00 & 0.02 & 0.19 & 0.00 & \multirow[t]{3}{*}{ None } & \multirow[t]{3}{*}{--} & \multirow[t]{3}{*}{--} \\
\hline Commercial use & 0.00 & 0.00 & 0.00 & 0.00 & 0.00 & & & \\
\hline Industrial use & 0.00 & 0.00 & 0.00 & 0.00 & 0.00 & & & \\
\hline Public-supply loss & -- & -- & -- & 0.00 & -- & & & \\
\hline Inter-county delivery & -- & -- & -- & 0.00 & -- & & & \\
\hline \multicolumn{2}{|c|}{ Public wastewater treatment -- } & -- & -- & -- & 0.00 & \multicolumn{3}{|c|}{ Withdrawals by Major Public Suppliers } \\
\hline Mining use & 0.00 & 0.00 & 0.00 & 0.00 & 0.00 & \multirow[t]{2}{*}{ Name } & Groundwater & Surface water \\
\hline Irrigation (crop) & 0.00 & 0.05 & 0.05 & 0.05 & 0.00 & & \multirow{5}{*}{--} & \multirow{5}{*}{--} \\
\hline Irrigation (golf) & 0.00 & 0.00 & 0.00 & 0.00 & 0.00 & \multirow[t]{4}{*}{ None } & & \\
\hline Livestock-aquaculture & 0.00 & 0.00 & 0.00 & 0.00 & 0.00 & & & \\
\hline Thermoelectric power & 0.00 & 0.00 & 0.00 & 0.00 & 0.00 & & & \\
\hline TOTAL & 0.19 & 0.05 & 0.24 & 0.24 & 0.00 & & & \\
\hline
\end{tabular}

Total use is total withdrawal plus public supply deliveries and losses.

Withdrawals by water source

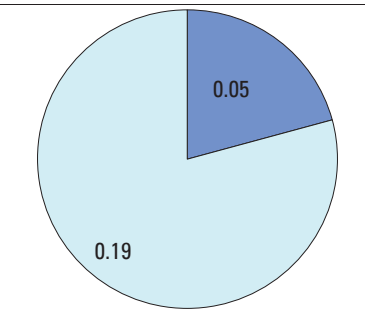

Withdrawals, in million

gallons per day

Surface water

Groundwater
Groundwater withdrawals by aquifer

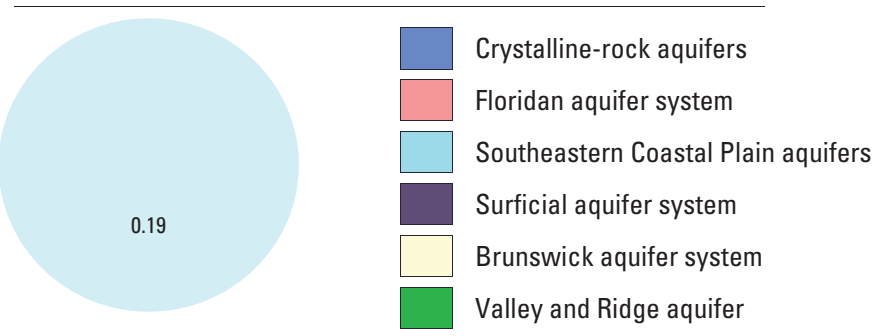

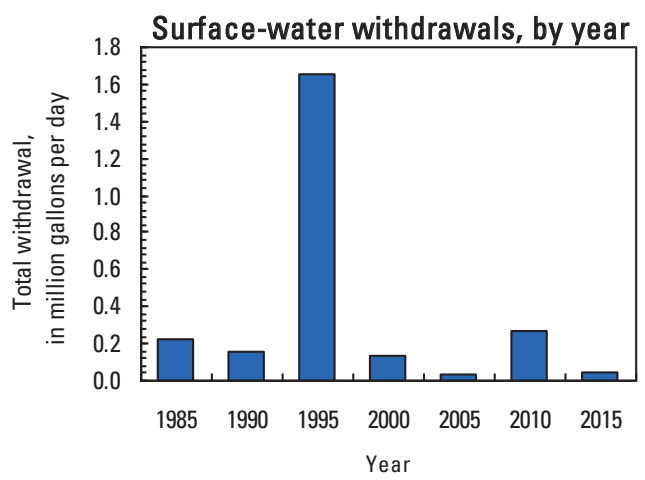

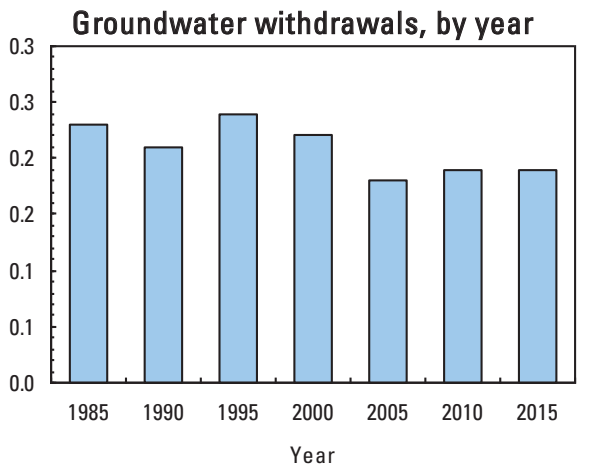




\section{RABUN COUNTY}

Population

Population served by public supply-groundwater

Population served by public supply-surface water $\quad 8,233$

Acres irrigated

2015 WATER WITHDRAWALS AND ESTIMATED USE, IN MILLION GALLONS PER DAY

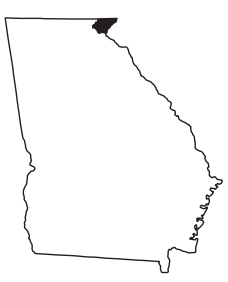

[--,not applicable; Mgal/d, million gallons per day]

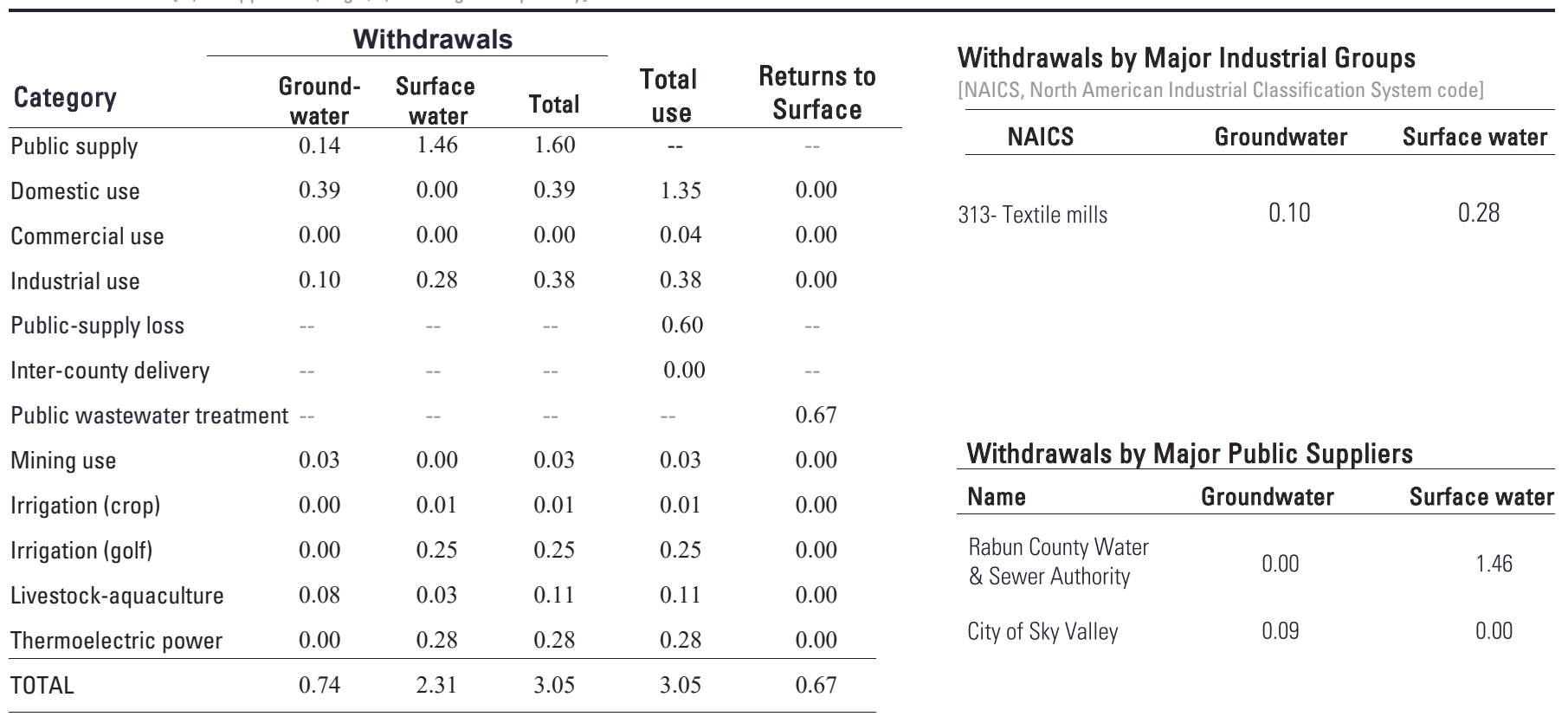

Total use is total withdrawal plus public supply deliveries and losses.

Withdrawals by water source

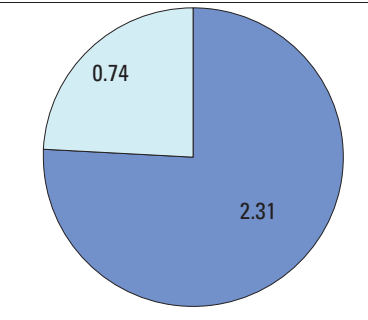

Withdrawals, in million gallons per day

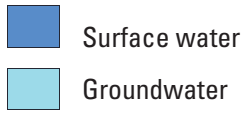

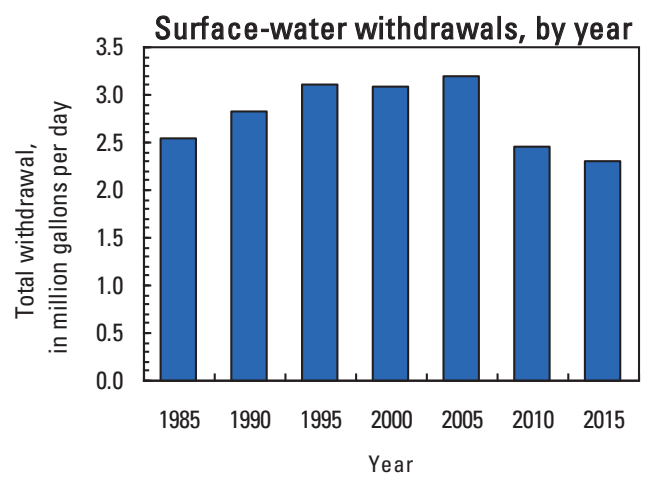

Groundwater withdrawals by aquifer

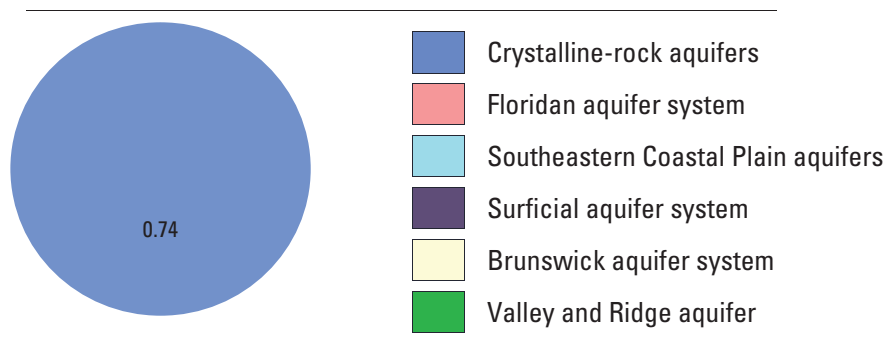

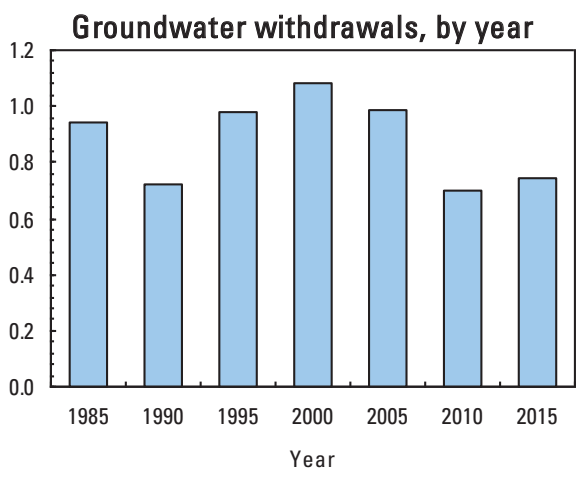




\section{RANDOLPH COUNTY}

Population

Population served by public supply-groundwater $\quad 5,016$

Population served by public supply-surface water 0

Acres irrigated

2015 WATER WITHDRAWALS AND ESTIMATED USE, IN MILLION GALLONS PER DAY

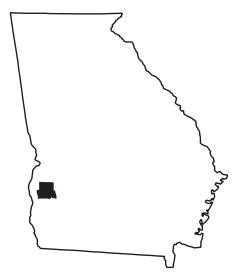

[--,not applicable; Mgal/d, million gallons per day]

\begin{tabular}{|c|c|c|c|c|c|c|c|c|}
\hline & \multicolumn{3}{|c|}{ Withdrawals } & \multirow{2}{*}{$\begin{array}{c}\text { Total } \\
\text { use }\end{array}$} & \multirow{2}{*}{$\begin{array}{c}\text { Returns to } \\
\text { Surface }\end{array}$} & \multirow{2}{*}{\multicolumn{3}{|c|}{$\begin{array}{l}\text { Withdrawals by Major Industrial Groups } \\
\text { [NAICS, North American Industrial Classification System code] }\end{array}$}} \\
\hline & $\begin{array}{l}\text { Ground- } \\
\text { water }\end{array}$ & $\begin{array}{c}\text { Surface } \\
\text { water }\end{array}$ & Total & & & & & \\
\hline Public supply & $\begin{array}{r}\text { water } \\
0.88\end{array}$ & $\begin{array}{r}\text { water } \\
0.00\end{array}$ & 0.88 & $\begin{array}{c}\text { Use } \\
--\end{array}$ & Sulace & NAICS & Groundwater & Surface water \\
\hline Domestic use & 0.16 & 0.00 & 0.16 & 0.76 & 0.00 & & & \\
\hline Commercial use & 0.00 & 0.00 & 0.00 & 0.12 & 0.00 & 311-Food & 0.17 & 0.00 \\
\hline Industrial use & 0.17 & 0.00 & 0.17 & 0.17 & 0.00 & & & \\
\hline Public-supply loss & -- & -- & -- & 0.16 & -- & & & \\
\hline Inter-county delivery & -- & -- & -- & 0.00 & -- & & & \\
\hline Public wastewater treatn & ment -- & -- & -- & -- & 0.47 & & & \\
\hline Mining use & 0.00 & 0.00 & 0.00 & 0.00 & 0.00 & Withdrawals & jor Public Sup & \\
\hline Irrigation (crop) & 8.53 & 9.24 & 17.77 & 17.77 & 0.00 & Name & Groundwater & Surface water \\
\hline Irrigation (golf) & 0.00 & 0.04 & 0.04 & 0.04 & 0.00 & City of Cuthbert & 0.49 & 0.00 \\
\hline Livestock-aquaculture & 0.03 & 0.03 & 0.06 & 0.06 & 0.00 & City of Shellman & 0.15 & 0.00 \\
\hline Thermoelectric power & 0.00 & 0.00 & 0.00 & 0.00 & 0.00 & & & \\
\hline TOTAL & 9.77 & 9.31 & 19.08 & 19.08 & 0.47 & & & \\
\hline
\end{tabular}

Total use is total withdrawal plus public supply deliveries and losses.

Withdrawals by water source

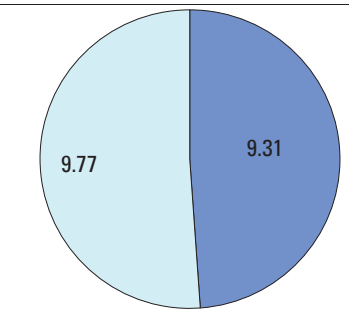

Withdrawals, in million

gallons per day

Surface water

Groundwater

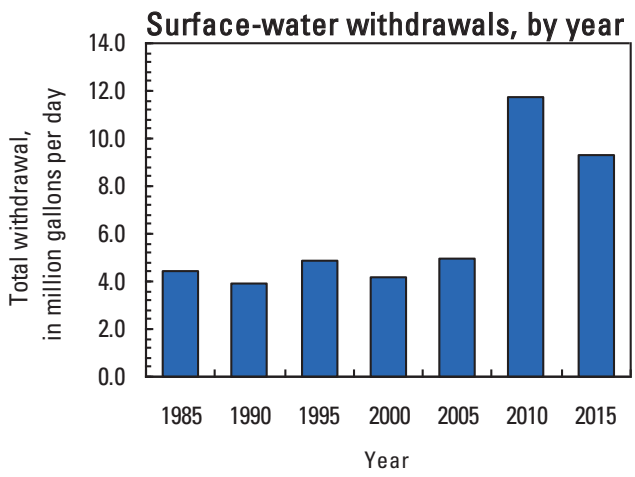

Groundwater withdrawals by aquifer

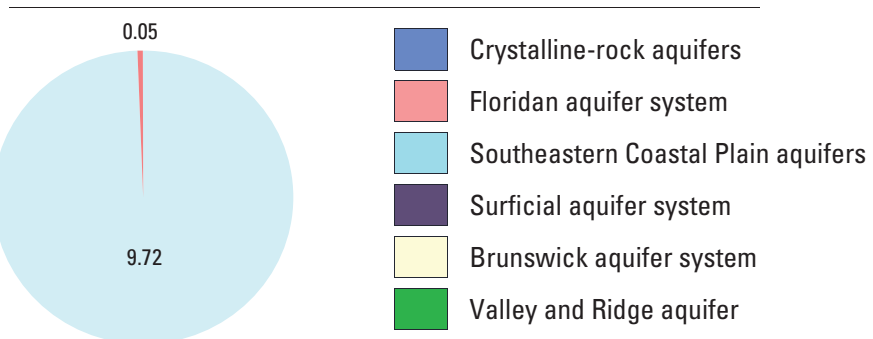

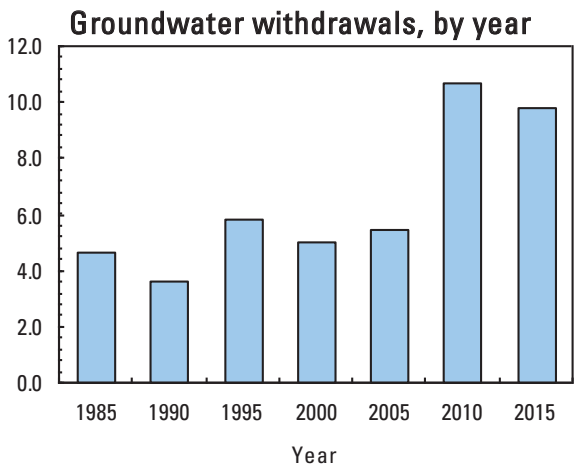




\section{RICHMOND COUNTY}

Population

Population served by public supply-groundwater $\quad 38,709$

Population served by public supply-surface water 157,125

Acres irrigated

2015 WATER WITHDRAWALS AND ESTIMATED USE, IN MILLION GALLONS PER DAY

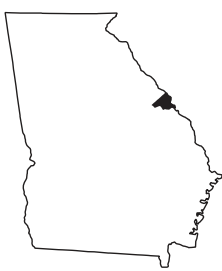

[--,not applicable; Mgal/d, million gallons per day]

\begin{tabular}{|c|c|c|c|c|c|}
\hline \multirow[b]{2}{*}{ Category } & \multicolumn{3}{|c|}{ Withdrawals } & \multirow[b]{2}{*}{$\begin{array}{c}\text { Total } \\
\text { use }\end{array}$} & \multirow[b]{2}{*}{$\begin{array}{c}\text { Returns to } \\
\text { Surface }\end{array}$} \\
\hline & $\begin{array}{c}\text { Ground- } \\
\text { water }\end{array}$ & $\begin{array}{c}\text { Surface } \\
\text { water }\end{array}$ & Total & & \\
\hline Public supply & 7.24 & 31.93 & 39.17 & -- & -- \\
\hline Domestic use & 0.45 & 0.00 & 0.45 & 13.69 & 0.00 \\
\hline Commercial use & 0.05 & 0.07 & 0.12 & 13.24 & 0.00 \\
\hline Industrial use & 1.34 & 64.81 & 66.15 & 71.16 & 0.00 \\
\hline Public-supply loss & -- & -- & -- & 7.80 & -- \\
\hline Inter-county delivery & -- & -- & -- & 0.00 & -- \\
\hline Public wastewater treatmen & t -- & -- & -- & -- & 96.41 \\
\hline Mining use & 2.16 & 0.00 & 2.16 & 2.16 & 0.00 \\
\hline Irrigation (crop) & 0.01 & 0.00 & 0.01 & 0.01 & 0.00 \\
\hline Irrigation (golf) & 0.15 & 0.43 & 0.58 & 0.58 & 0.00 \\
\hline Livestock-aquaculture & 0.04 & 0.00 & 0.04 & 0.04 & 0.00 \\
\hline Thermoelectric power & 0.00 & 0.00 & 0.00 & 0.00 & 0.00 \\
\hline TOTAL & 11.44 & 97.24 & 108.7 & 108.7 & 96.41 \\
\hline
\end{tabular}

Withdrawals by Major Industrial Groups

[NAICS, North American Industrial Classification System code]

NAICS Groundwater Surface water

321- Wood

$\begin{array}{lr}0.19 & 0.00 \\ 0.00 & 53.55 \\ 0.43 & 11.26 \\ 0.49 & 0.00\end{array}$

Withdrawals by Major Public Suppliers

Name Groundwater Surface water

Augusta Utilities $\quad 6.66 \quad 31.93$

City of Hephzibah $\quad 0.45 \quad 0.00$

City of Blyth $\quad 0.08 \quad 0.00$

Total use is total withdrawal plus public supply deliveries and losses.

Withdrawals by water source

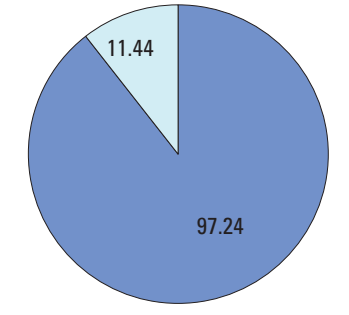

Withdrawals, in million gallons per day

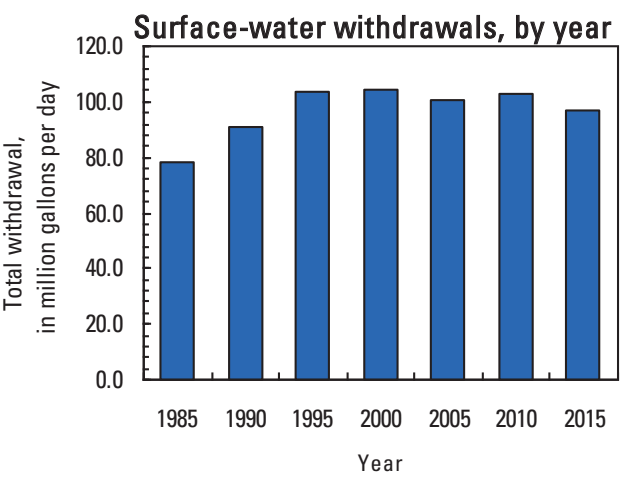

Groundwater withdrawals by aquifer

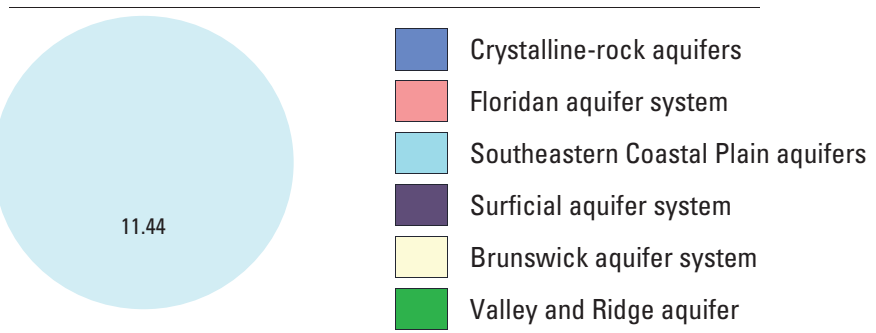

Groundwater withdrawals, by year

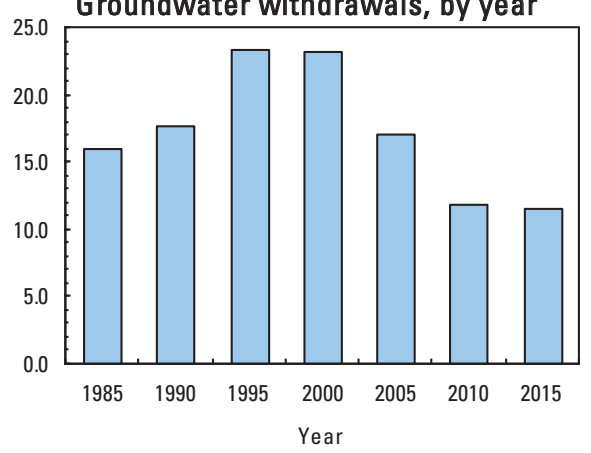




\section{ROCKDALE COUNTY}

Population

Population served by public supply-groundwater $\quad 2,716$

Population served by public supply-surface water $\quad 72,621$

Acres irrigated

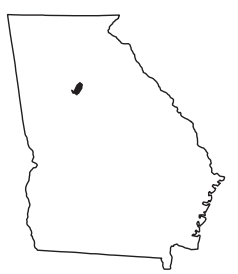

2015 WATER WITHDRAWALS AND ESTIMATED USE, IN MILLION GALLONS PER DAY

[--,not ap plicable; Mgal/d, million gallons per day]

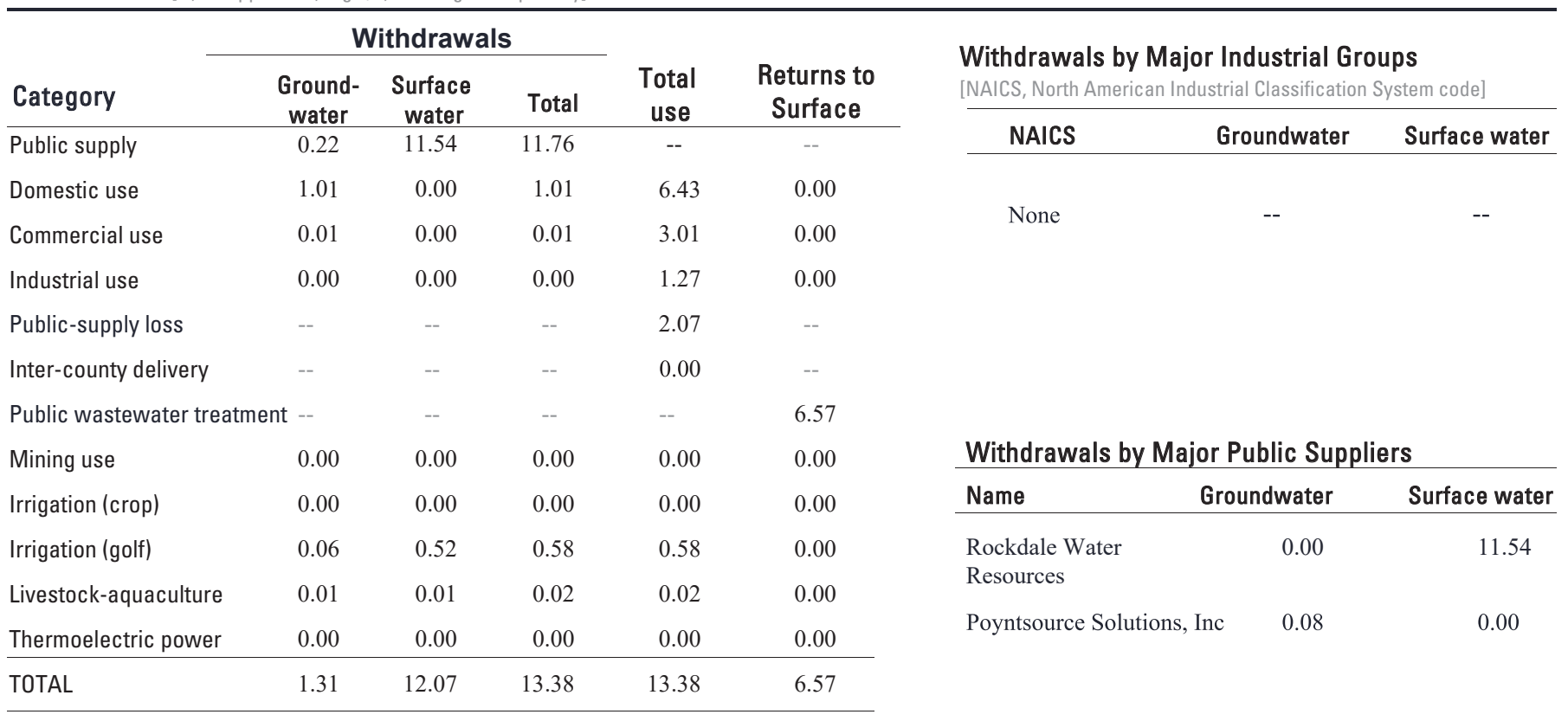

Total use is total withdrawal plus public supply deliveries and losses.

Withdrawals by water source

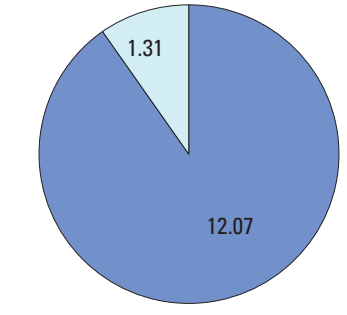

Withdrawals, in million

gallons per day

Surface water

Groundwater

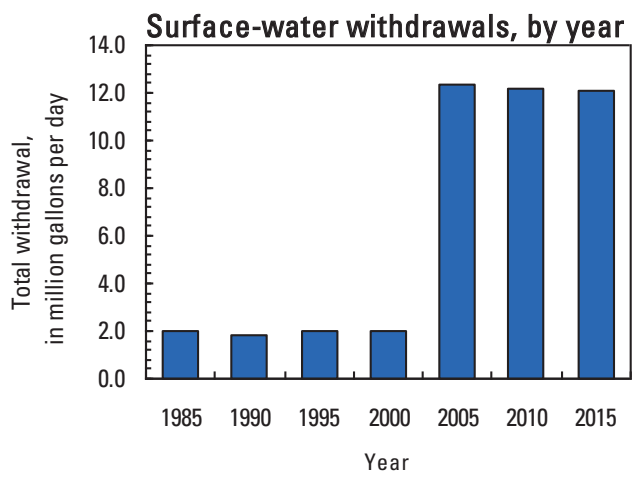

Groundwater withdrawals by aquifer

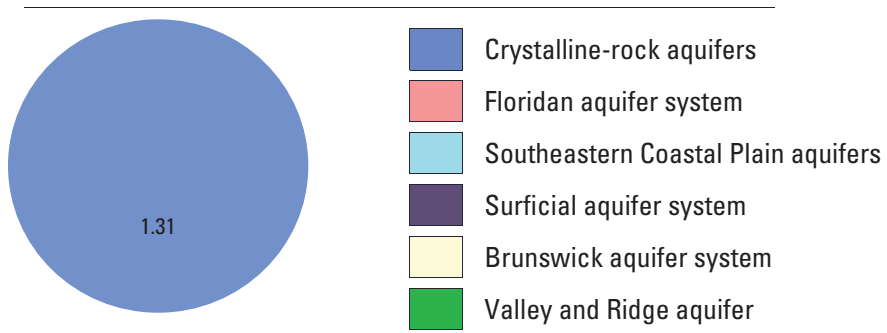

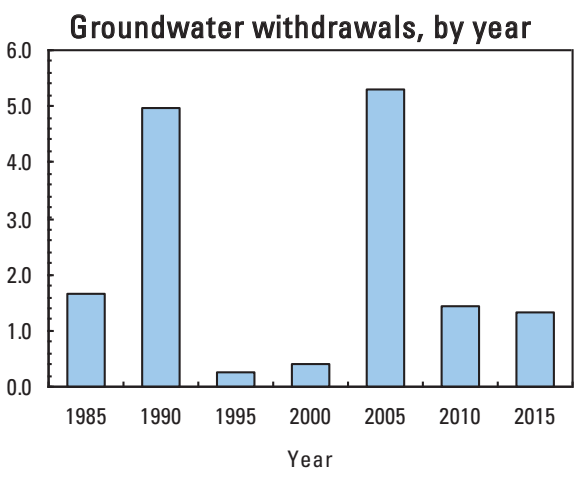




\section{SCHLEY COUNTY}

Population

Population served by public supply-groundwater

Population served by public supply-surface water

Acres irrigated

2015 WATER WITHDRAWALS AND ESTIMATED USE, IN MILLION GALLONS PER DAY

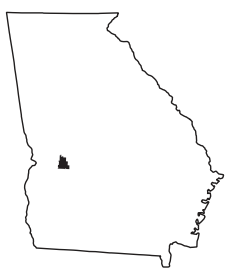

[--,not applicable; Mgal/d, million gallons per day]

\begin{tabular}{|c|c|c|c|c|c|c|c|c|}
\hline \multirow{2}{*}{ Category } & \multicolumn{3}{|c|}{ Withdrawals } & \multirow{3}{*}{$\begin{array}{c}\text { Total } \\
\text { use }\end{array}$} & \multirow{3}{*}{$\begin{array}{l}\text { Returns to } \\
\text { Surface }\end{array}$} & \multirow{2}{*}{\multicolumn{3}{|c|}{$\begin{array}{l}\text { Withdrawals by Major Industrial Groups } \\
\text { [NAICS, North American Industrial Classification System code] }\end{array}$}} \\
\hline & \multirow{2}{*}{$\begin{array}{l}\text { Ground- } \\
\text { water }\end{array}$} & \multirow{2}{*}{$\begin{array}{c}\text { Surface } \\
\text { water }\end{array}$} & Total & & & & & \\
\hline Public supply & & & 0.45 & & & \multirow[t]{3}{*}{ NAICS } & Groundwater & Surface water \\
\hline Domestic use & 0.08 & 0.00 & 0.08 & 0.41 & 0.00 & & & \\
\hline Commercial use & 0.00 & 0.00 & 0.00 & 0.03 & 0.00 & & -- & -- \\
\hline Industrial use & 0.00 & 0.00 & 0.00 & 0.01 & 0.00 & & & \\
\hline Public-supply loss & -- & -- & -- & 0.08 & -- & & & \\
\hline Inter-county delivery & -- & -- & -- & 0.00 & -- & & & \\
\hline Public wastewater treat & ment -- & -- & -- & -- & 0.30 & & & \\
\hline Mining use & 0.00 & 0.00 & 0.00 & 0.00 & 0.00 & \multicolumn{3}{|c|}{ Withdrawals by Major Public Suppliers } \\
\hline Irrigation (crop) & 0.07 & 0.04 & 0.11 & 0.11 & 0.00 & Name & Groundwater & Surface water \\
\hline Irrigation (golf) & 0.00 & 0.04 & 0.04 & 0.04 & 0.00 & City of Ellaville & 0.14 & 0.00 \\
\hline Livestock-aquaculture & 0.04 & 0.02 & 0.06 & 0.06 & 0.00 & Schley County Utilities & 0.31 & 0.00 \\
\hline Thermoelectric power & 0.00 & 0.00 & 0.00 & 0.00 & 0.00 & & & \\
\hline TOTAL & 0.64 & 0.10 & 0.74 & 0.74 & 0.30 & & & \\
\hline
\end{tabular}

Total use is total withdrawal plus public supply deliveries and losses.

Withdrawals by water source

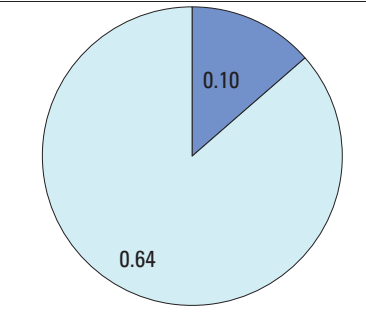

Withdrawals, in million gallons per day

Surface water

Groundwater

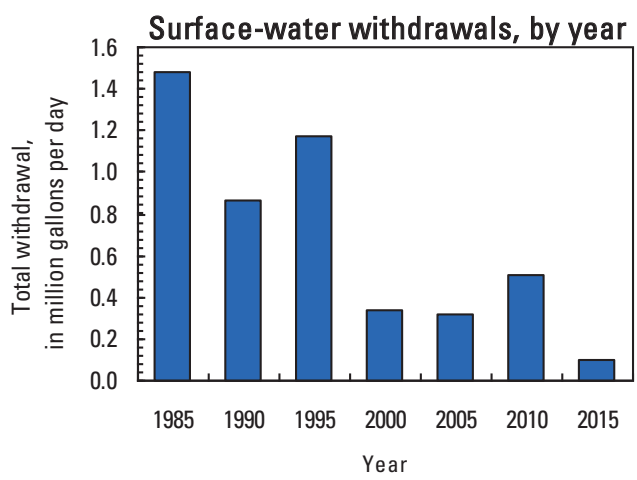

Groundwater withdrawals by aquifer
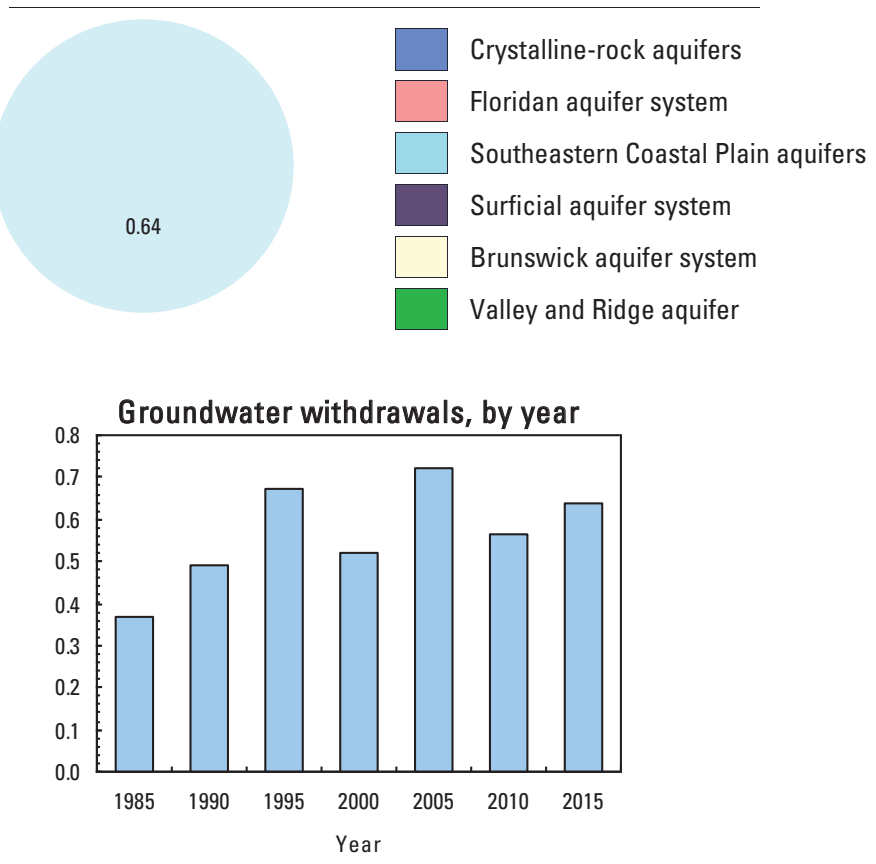


\section{SCREVEN COUNTY}

Population

Population served by public supply-groundwater

4,633

Population served by public supply-surface water

0

Acres irrigated

2015 WATER WITHDRAWALS AND ESTIMATED USE, IN MILLION GALLONS PER DAY

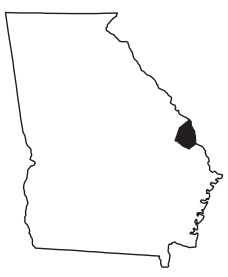

[--,not applicable; Mgal/d, million gallons per day]

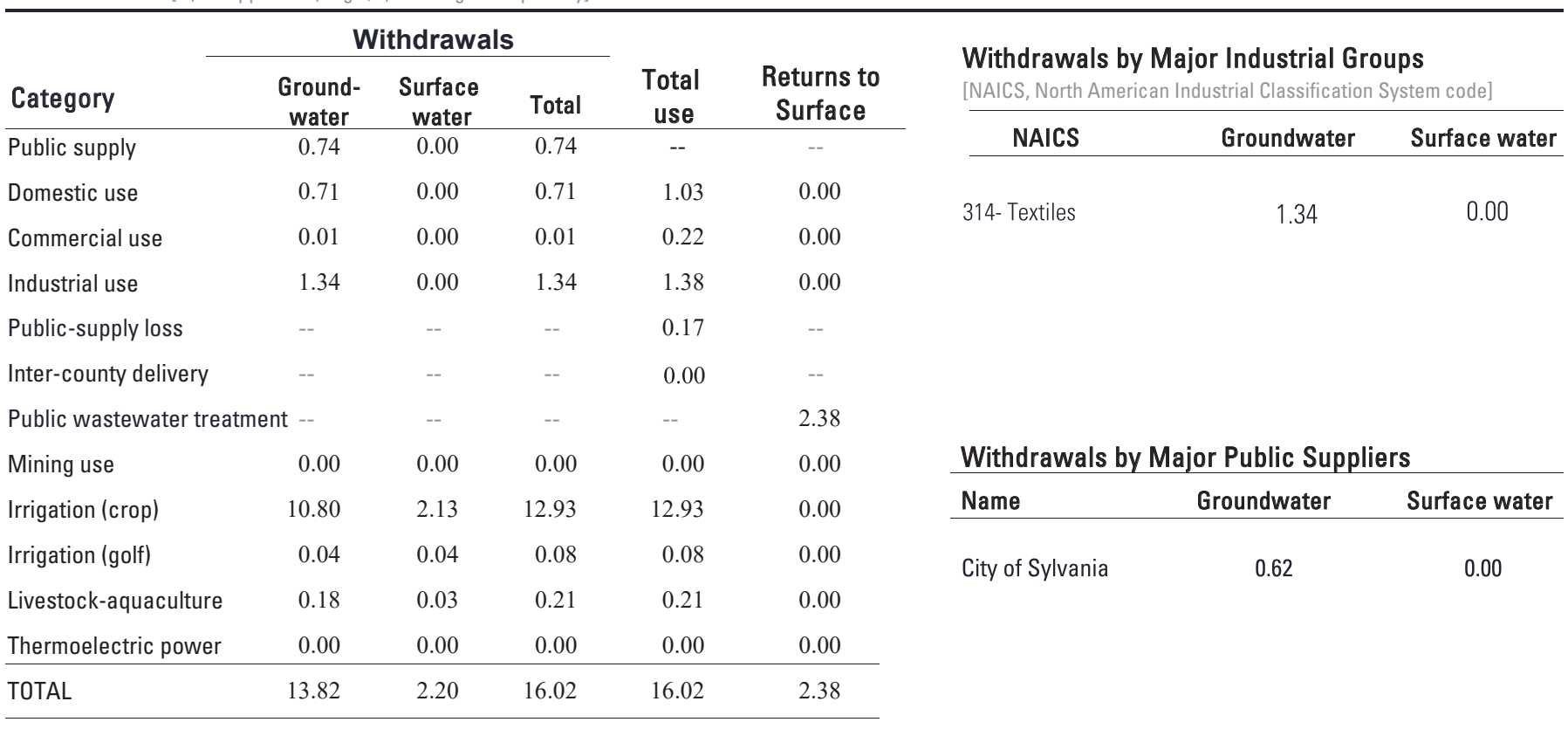

Total use is total withdrawal plus public supply deliveries and losses.

Withdrawals by water source

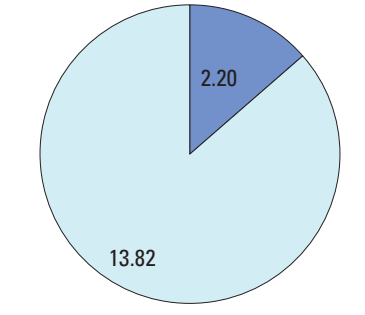

Withdrawals, in million

gallons per day

Surface water

Groundwater

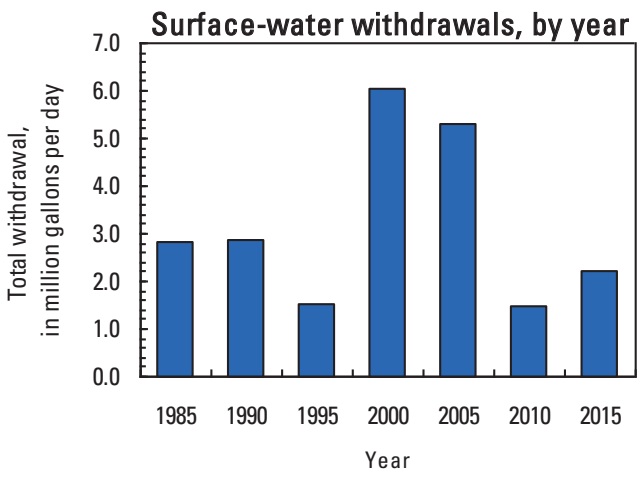

Groundwater withdrawals by aquifer

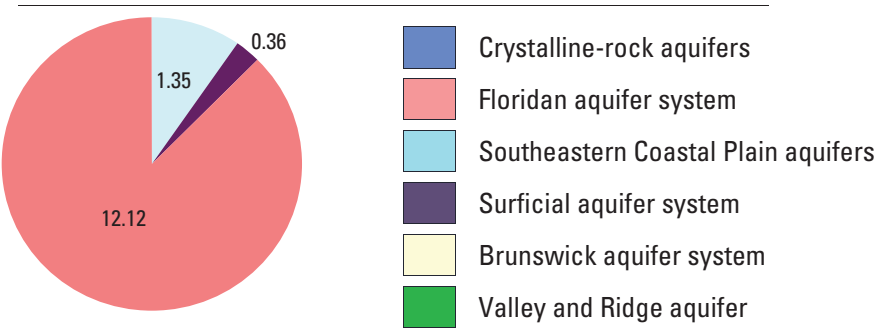

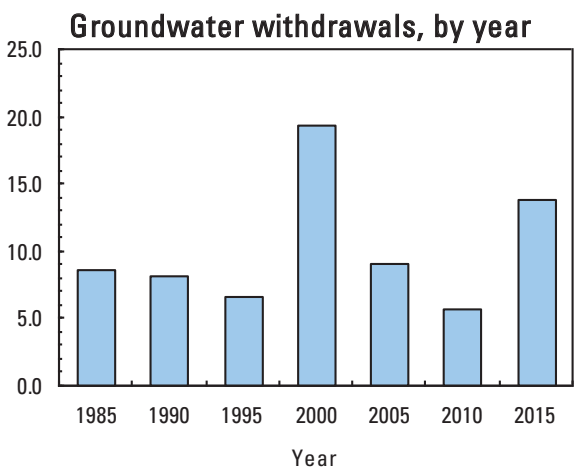




\section{SEMINOLE COUNTY}

Population

Population served by public supply-groundwater

Population served by public supply-surface water

Acres irrigated

2015 WATER WITHDRAWALS AND ESTIMATED USE, IN MILLION GALLONS PER DAY

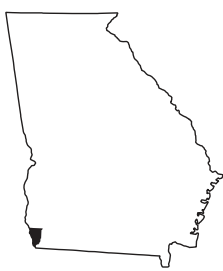

[--,not applicable; Mgal/d, million gallons per day]

\begin{tabular}{|c|c|c|c|c|c|c|c|c|}
\hline \multirow{3}{*}{ Category } & \multicolumn{3}{|c|}{ Withdrawals } & \multirow{3}{*}{$\begin{array}{c}\text { Total } \\
\text { use }\end{array}$} & \multirow{3}{*}{$\begin{array}{c}\text { Returns to } \\
\text { Surface }\end{array}$} & \multirow{2}{*}{\multicolumn{3}{|c|}{$\begin{array}{l}\text { Withdrawals by Major Industrial Groups } \\
\text { [NAICS, North American Industrial Classification System code] }\end{array}$}} \\
\hline & Ground- & Surface & & & & & & \\
\hline & water & water & Total & & & \multirow{2}{*}{ NAICS } & \multirow[t]{2}{*}{ Groundwater } & \multirow[t]{2}{*}{ Surface water } \\
\hline Public supply & 0.50 & 0.00 & 0.50 & -- & -- & & & \\
\hline Domestic use & 0.34 & 0.00 & 0.34 & 0.69 & 0.00 & \multirow{3}{*}{ None } & \multirow[t]{3}{*}{--} & \multirow[t]{3}{*}{--} \\
\hline Commercial use & 0.00 & 0.00 & 0.00 & 0.07 & 0.00 & & & \\
\hline Industrial use & 0.00 & 0.00 & 0.00 & 0.01 & 0.00 & & & \\
\hline Public-supply loss & -- & -- & -- & 0.07 & -- & & & \\
\hline Inter-county delivery & -- & -- & -- & 0.00 & -- & & & \\
\hline \multicolumn{2}{|l|}{ Public wastewater treatment -- } & -- & -- & -- & 0.42 & & & \\
\hline Mining use & 0.00 & 0.00 & 0.00 & 0.00 & 0.00 & \multicolumn{3}{|c|}{ Withdrawals by Major Public Suppliers } \\
\hline Irrigation (crop) & 43.76 & 0.33 & 44.09 & 44.09 & 0.00 & Name & Groundwater & Surface water \\
\hline Irrigation (golf) & 0.17 & 0.00 & 0.17 & 0.17 & 0.00 & \multirow{4}{*}{ City of Donalsonville } & \multirow[t]{4}{*}{0.44} & \multirow[t]{4}{*}{0.00} \\
\hline Livestock-aquaculture & 0.19 & 0.00 & 0.19 & 0.19 & 0.00 & & & \\
\hline Thermoelectric power & 0.00 & 0.00 & 0.00 & 0.00 & 0.00 & & & \\
\hline TOTAL & 44.96 & 0.33 & 45.29 & 45.29 & 0.42 & & & \\
\hline
\end{tabular}

Total use is total withdrawal plus public supply deliveries and losses.

Withdrawals by water source
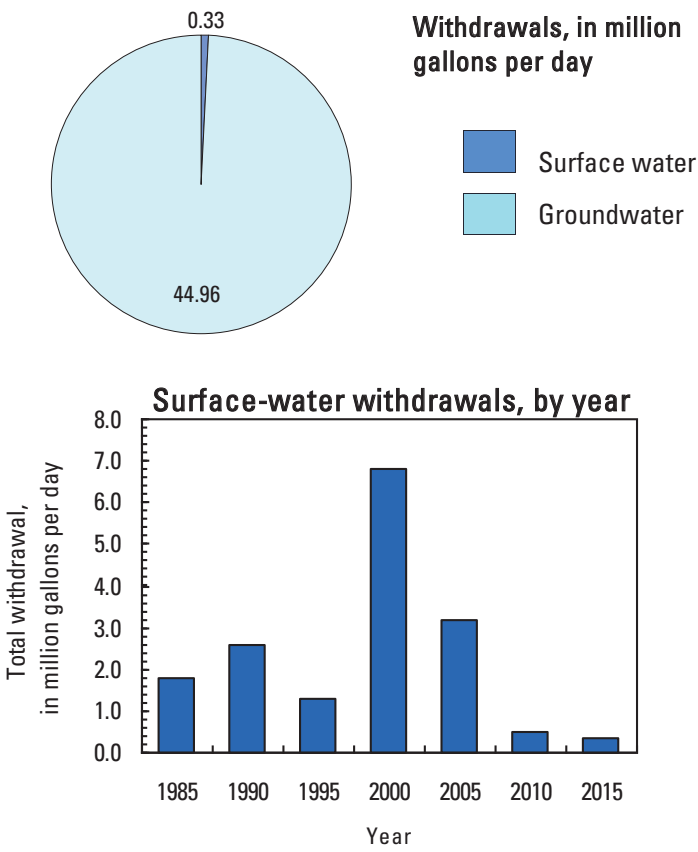

Groundwater withdrawals by aquifer

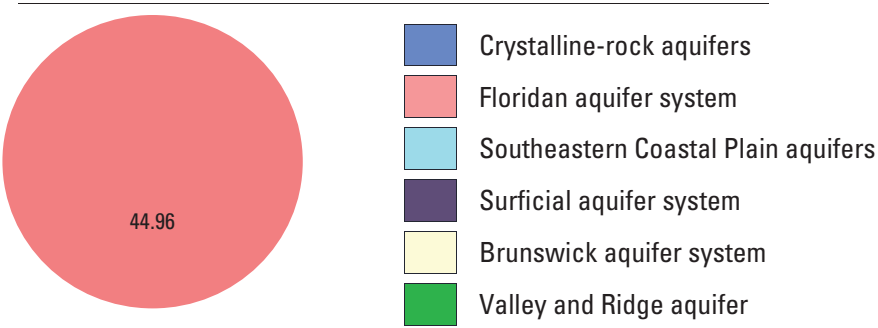

Groundwater withdrawals, by year

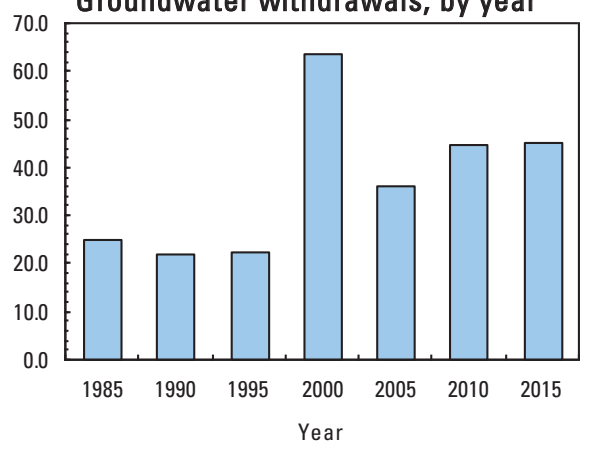




\section{SPALDING COUNTY}

Population

Population served by public supply-groundwater

Population served by public supply-surface water

Acres irrigated

450

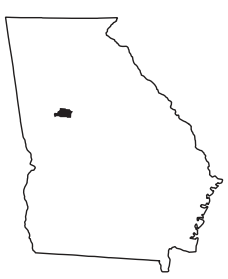

2015 WATER WITHDRAWALS AND ESTIMATED USE, IN MILLION GALLONS PER DAY

[--,not applicable; Mgal/d, million gallons per day]

\begin{tabular}{|c|c|c|c|c|c|c|c|c|}
\hline \multirow{3}{*}{ Category } & \multicolumn{3}{|c|}{ Withdrawals } & \multirow{3}{*}{$\begin{array}{c}\text { Total } \\
\text { use }\end{array}$} & \multirow{3}{*}{$\begin{array}{l}\text { Returns to } \\
\text { Surface }\end{array}$} & \multirow{2}{*}{\multicolumn{3}{|c|}{$\begin{array}{l}\text { Withdrawals by Major Industrial Groups } \\
\text { [NAICS, North American Industrial Classification System code] }\end{array}$}} \\
\hline & \multirow{2}{*}{$\begin{array}{l}\text { Ground- } \\
\text { water }\end{array}$} & \multirow{2}{*}{$\begin{array}{c}\text { Surface } \\
\text { water }\end{array}$} & \multirow[b]{2}{*}{ Total } & & & & & \\
\hline & & & & & & \multirow[t]{2}{*}{ NAICS } & \multirow[t]{2}{*}{ Groundwater } & \multirow{2}{*}{ Surface water } \\
\hline Public supply & 0.09 & 6.74 & 6.83 & -- & -- & & & \\
\hline Domestic use & 0.22 & 0.00 & 0.22 & 4.08 & 0.00 & \multirow[t]{5}{*}{ None } & \multirow[t]{5}{*}{--} & \multirow[t]{5}{*}{--} \\
\hline Commercial use & 0.00 & 0.00 & 0.00 & 0.91 & 0.00 & & & \\
\hline Industrial use & 0.00 & 0.00 & 0.00 & 0.28 & 0.00 & & & \\
\hline Public-supply loss & -- & -- & -- & 1.78 & -- & & & \\
\hline Inter-county delivery & -- & -- & -- & 0.46 & -- & & & \\
\hline \multicolumn{2}{|c|}{ Public wastewater treatment -- } & -- & -- & -- & 1.18 & \multicolumn{3}{|c|}{ Withdrawals by Major Public Suppliers } \\
\hline Mining use & 0.04 & 0.00 & 0.04 & 0.04 & 0.00 & Name & Groundwater & Surface water \\
\hline Irrigation (crop) & 0.02 & 0.01 & 0.03 & 0.03 & 0.00 & \multirow{5}{*}{ City of Griffin } & \multirow{5}{*}{0.00} & \multirow{5}{*}{6.74} \\
\hline Irrigation (golf) & 0.17 & 0.08 & 0.25 & 0.25 & 0.00 & & & \\
\hline Livestock-aquaculture & 0.03 & 0.02 & 0.05 & 0.05 & 0.00 & & & \\
\hline Thermoelectric power & 0.00 & 0.00 & 0.00 & 0.00 & 0.00 & & & \\
\hline TOTAL & 0.57 & 6.85 & 7.42 & 7.88 & 1.18 & & & \\
\hline
\end{tabular}

Total use is total withdrawal plus public supply deliveries and losses. An estimate of $2.89 \mathrm{Mgal} / \mathrm{d}$ was delivered from neighboring counties in 2015. An estimate of $2.43 \mathrm{Mgal} / \mathrm{d}$ was delivered from Spalding County to neighboring counties in 2015.

\section{Withdrawals by water source}

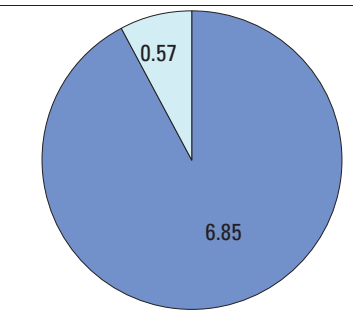

Withdrawals, in million gallons per day

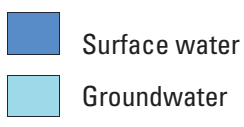

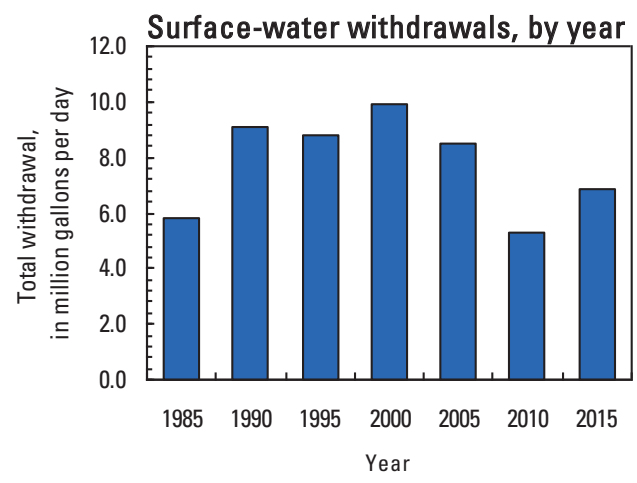

Groundwater withdrawals by aquifer
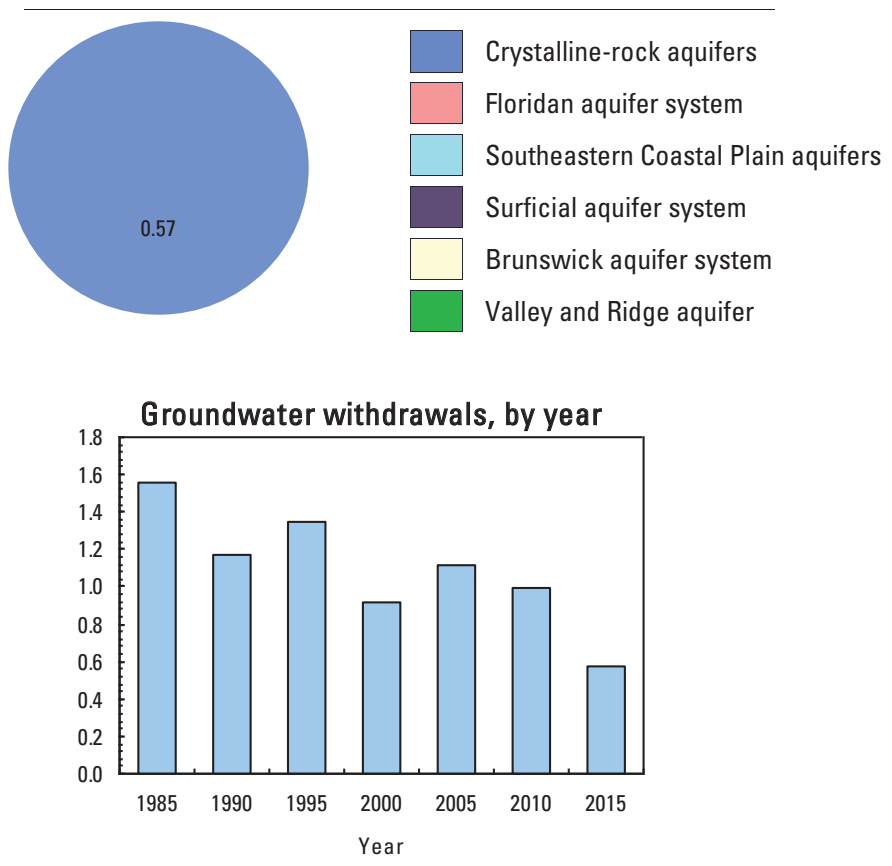


\section{STEPHENS COUNTY}

Population

Population served by public supply-groundwater

Population served by public supply-surface water $\quad 21,525$

Acres irrigated

2015 WATER WITHDRAWALS AND ESTIMATED USE, IN MILLION GALLONS PER DAY

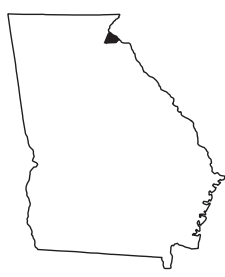

$[--$, not applicable; Mgal/d, million gallons per day

\begin{tabular}{lccccc}
\hline & \multicolumn{3}{c}{ Withdrawals } & & Total \\
\cline { 2 - 4 } Category & $\begin{array}{c}\text { Ground- } \\
\text { water }\end{array}$ & $\begin{array}{c}\text { Surface } \\
\text { water }\end{array}$ & $\begin{array}{c}\text { Total } \\
\text { use }\end{array}$ & $\begin{array}{c}\text { Returns to } \\
\text { Surface }\end{array}$ \\
\hline Public supply & 0.14 & 3.24 & 3.38 & -- & -- \\
Domestic use & 0.18 & 0.00 & 0.18 & 2.17 & 0.00 \\
Commercial use & 0.08 & 0.00 & 0.08 & 0.55 & 0.00 \\
Industrial use & 0.00 & 0.00 & 0.00 & 0.34 & 0.00 \\
Public-supply loss & -- & -- & -- & 0.58 & -- \\
Inter-county delivery & -- & -- & -- & -0.12 & -- \\
Public wastewater treatment & -- & -- & -- & -- & 1.51 \\
Mining use & 0.07 & 0.00 & 0.07 & 0.07 & 0.00 \\
Irrigation (crop) & 0.00 & 0.00 & 0.00 & 0.00 & 0.00 \\
Irrigation (golf) & 0.31 & 0.00 & 0.31 & 0.31 & 0.00 \\
Livestock-aquaculture & 0.25 & 0.08 & 0.33 & 0.33 & 0.00 \\
Thermoelectric power & 0.00 & 0.00 & 0.00 & 0.00 & 0.00 \\
\hline TOTAL & 1.03 & 3.32 & 4.35 & 4.23 & 1.51 \\
\hline
\end{tabular}

Withdrawals by Major Industrial Groups

[NAICS, North American Industrial Classification System code]

NAICS Groundwater Surface water

Withdrawals by Major Public Suppliers

Name Groundwater Surface water

City of Toccoa

0.00

Total use is total withdrawal plus public supply deliveries and losses. An estimate of $0.12 \mathrm{Mgal} / \mathrm{d}$ was delivered from Stephens County to neighboring counties in 2015.

Withdrawals by water source

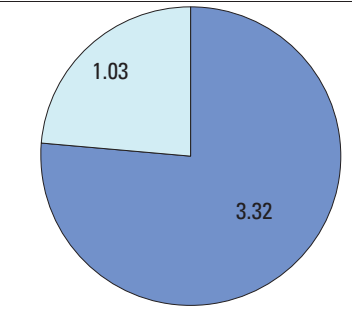

Withdrawals, in million gallons per day

Surface water

Groundwater

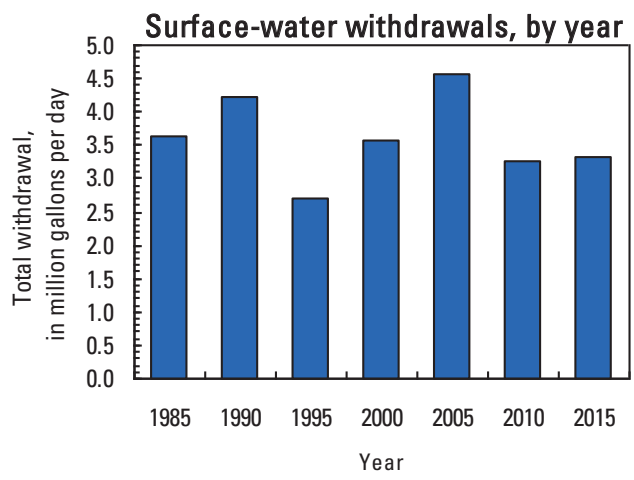

Groundwater withdrawals by aquifer
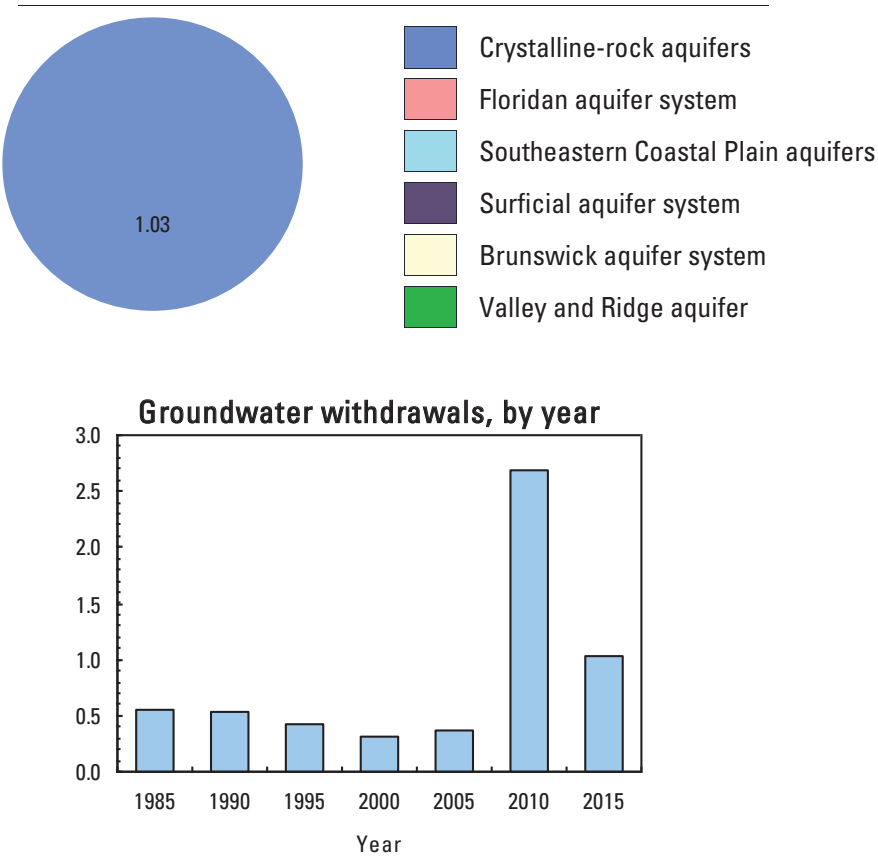


\section{STEWART COUNTY}

Population

Population served by public supply-groundwater

Population served by public supply-surface water

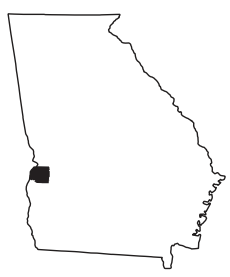

[--,not applicable; Mgal/d, million gallons per day]

\begin{tabular}{|c|c|c|c|c|c|c|c|c|}
\hline \multirow{3}{*}{ Category } & \multicolumn{3}{|c|}{ Withdrawals } & \multirow{3}{*}{$\begin{array}{c}\text { Total } \\
\text { use }\end{array}$} & \multirow{3}{*}{$\begin{array}{l}\text { Returns to } \\
\text { Surface }\end{array}$} & \multirow{2}{*}{\multicolumn{3}{|c|}{$\begin{array}{l}\text { Withdrawals by Major Industrial Groups } \\
\text { [NAICS, North American Industrial Classification System code] }\end{array}$}} \\
\hline & \multirow{2}{*}{$\begin{array}{l}\text { Ground } \\
\text { water }\end{array}$} & \multirow{2}{*}{$\begin{array}{c}\text { Surface } \\
\text { water }\end{array}$} & \multirow[b]{2}{*}{ Total } & & & & & \\
\hline & & & & & & \multirow[t]{4}{*}{ NAICS } & \multirow[t]{2}{*}{ Groundwater } & \multirow[t]{2}{*}{ Surface water } \\
\hline Public supply & 0.78 & 0.00 & 0.78 & -- & -- & & & \\
\hline Domestic use & 0.06 & 0.00 & 0.06 & 0.68 & 0.00 & & -- & -- \\
\hline Commercial use & 0.00 & 0.00 & 0.00 & 0.08 & 0.00 & & & \\
\hline Industrial use & 0.00 & 0.00 & 0.00 & 0.00 & 0.00 & & & \\
\hline Public-supply loss & -- & -- & -- & 0.08 & -- & & & \\
\hline Inter-county delivery & -- & -- & -- & 0.00 & -- & & & \\
\hline Public wastewater treatment & t -- & -- & -- & -- & 0.43 & & & \\
\hline Mining use & 0.00 & 0.00 & 0.00 & 0.00 & 0.00 & \multicolumn{3}{|c|}{ Withdrawals by Major Public Suppliers } \\
\hline Irrigation (crop) & 0.04 & 0.67 & 0.71 & 0.71 & 0.00 & Name & Groundwater & Surface water \\
\hline Irrigation (golf) & 0.00 & 0.00 & 0.00 & 0.00 & 0.00 & City of Richland & 0.37 & 0.00 \\
\hline Livestock-aquaculture & 0.00 & 0.01 & 0.01 & 0.01 & 0.00 & City of Lumpkin & 0.21 & 0.00 \\
\hline Thermoelectric power & 0.00 & 0.00 & 0.00 & 0.00 & 0.00 & & & \\
\hline TOTAL & 0.88 & 0.68 & 1.56 & 1.56 & 0.43 & & & \\
\hline
\end{tabular}

Total use is total withdrawal plus public supply deliveries and losses.

\section{Withdrawals by water source}

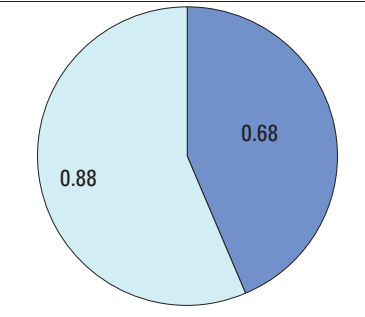

Withdrawals, in million gallons per day
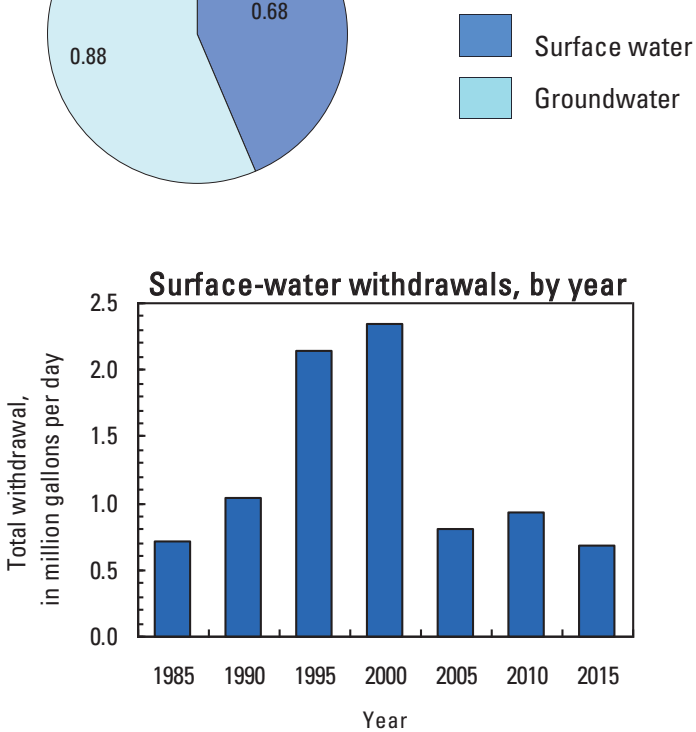

\section{Groundwater withdrawals by aquifer}
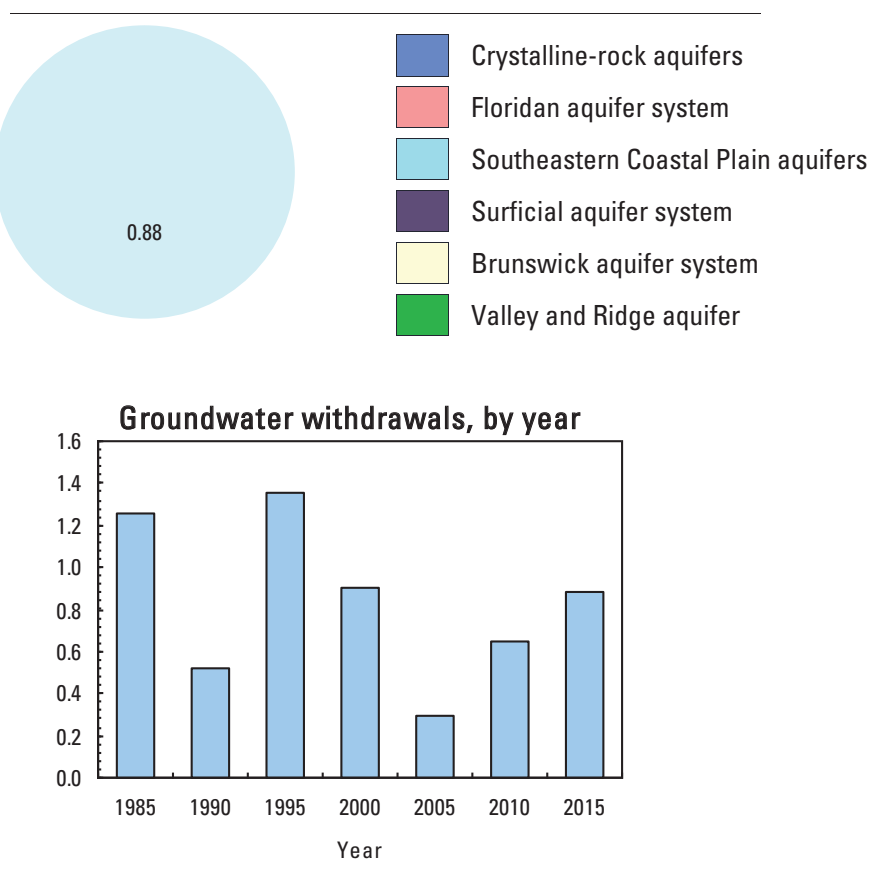


\section{SUMTER COUNTY}

Population

Population served by public supply-groundwater

Population served by public supply-surface water

Acres irrigated

2015 WATER WITHDRAWALS AND ESTIMATED USE, IN MILLION GALLONS PER DAY

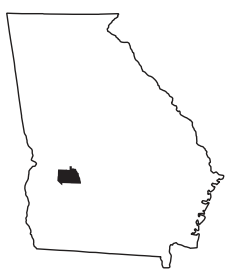

[--,not applicable; Mgal/d, million gallons per day]

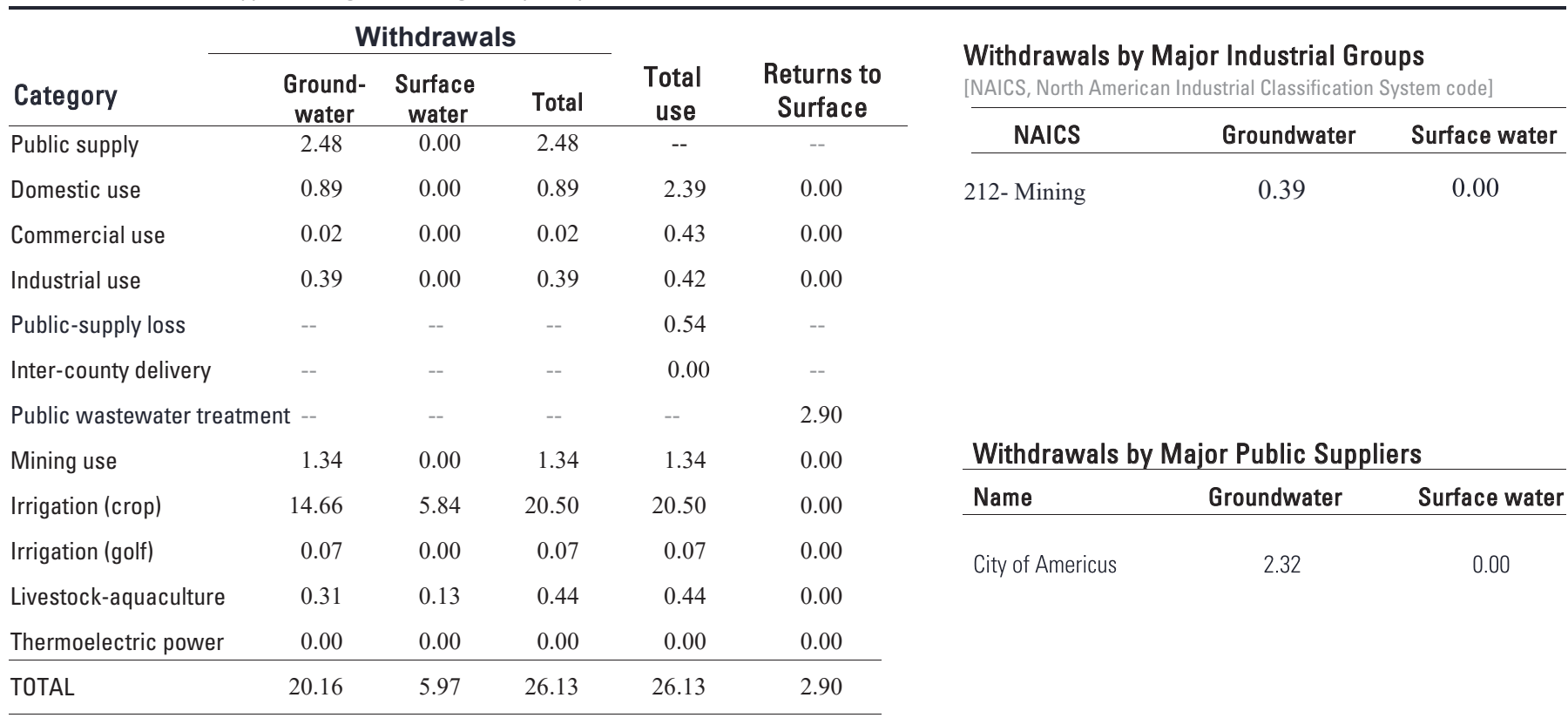

Total use is total withdrawal plus public supply deliveries and losses.

Withdrawals by water source

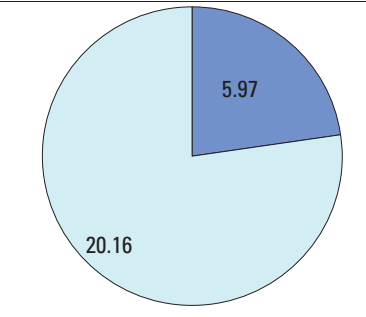

Withdrawals, in million gallons per day
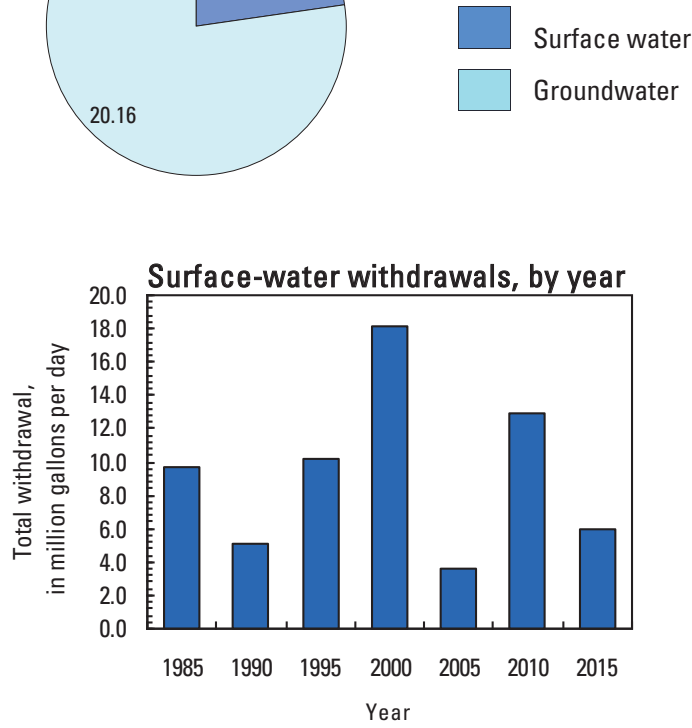

Groundwater withdrawals by aquifer

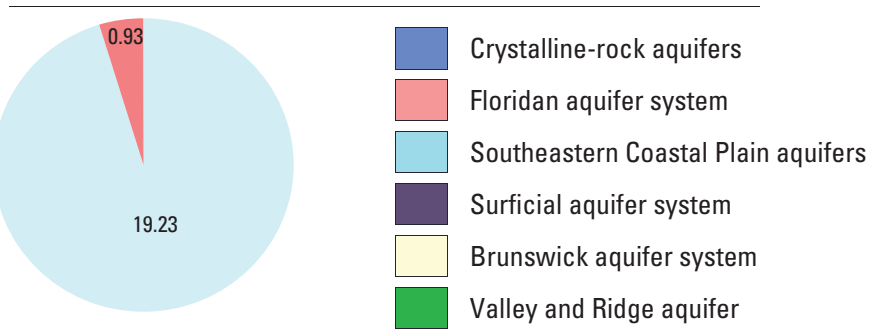

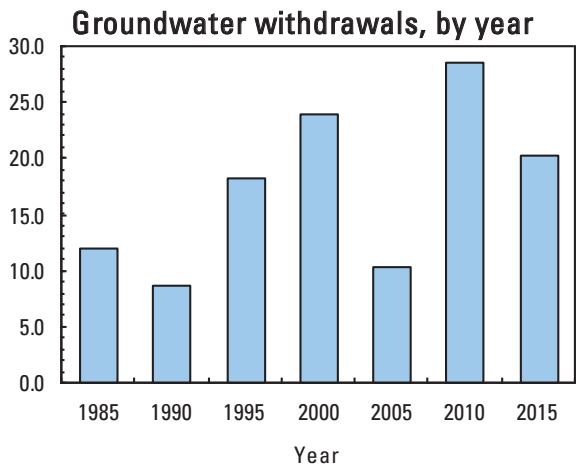




\section{TALBOT COUNTY}

Population

Population served by public supply-groundwater

Population served by public supply-surface water

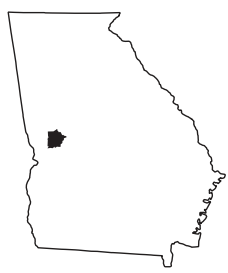

[--,not applicable; Mgal/d, million gallons per day]

\begin{tabular}{lccccc}
\hline & \multicolumn{3}{c}{ Withdrawals } & & Total \\
\cline { 2 - 4 } Category & $\begin{array}{c}\text { Ground } \\
\text { water }\end{array}$ & $\begin{array}{c}\text { Surface } \\
\text { water }\end{array}$ & $\begin{array}{c}\text { Total } \\
\text { use }\end{array}$ & $\begin{array}{c}\text { Returns to } \\
\text { Surface }\end{array}$ \\
\hline Public supply & 0.10 & 1.23 & 1.33 & -- & -- \\
Domestic use & 0.09 & 0.00 & 0.09 & 1.75 & 0.00 \\
Commercial use & 0.00 & 0.00 & 0.00 & 0.39 & 0.00 \\
Industrial use & 0.00 & 0.00 & 0.00 & 0.00 & 0.00 \\
Public-supply loss & -- & -- & -- & -0.72 & -- \\
Inter-county delivery & -- & -- & -- & -1.04 & -- \\
Public wastewater treatment & -- & -- & -- & -- & 0.24 \\
Mining use & 0.31 & 0.00 & 0.31 & 0.31 & 0.00 \\
Irrigation (crop) & 0.00 & 0.00 & 0.00 & 0.00 & 0.00 \\
Irrigation (golf) & 0.00 & 0.00 & 0.00 & 0.00 & 0.00 \\
Livestock-aquaculture & 0.06 & 0.04 & 0.10 & 0.10 & 0.00 \\
Thermoelectric power & 0.00 & 0.00 & 0.00 & 0.00 & 0.00 \\
\hline TOTAL & 0.56 & 1.27 & 1.83 & 0.79 & 0.24 \\
\hline
\end{tabular}

Withdrawals by Major Industrial Groups

[NAICS, North American Industrial Classification System code]

NAICS Groundwater Surface water

$$
\text { None }
$$

Withdrawals by Major Public Suppliers

\begin{tabular}{lcc} 
Name & Groundwater & Surface wate \\
\hline City of Manchester & 0.00 & 1.23 \\
City of Talbotton & 0.09 & 0.00
\end{tabular}

Total use is total withdrawal plus public supply deliveries and losses. An estimate of $0.07 \mathrm{Mgla} / \mathrm{d}$ was delivered from neighboring counties and $1.11 \mathrm{Mgal} / \mathrm{d}$ was delivered to neighboring counties in 2015 .

\section{Withdrawals by water source}

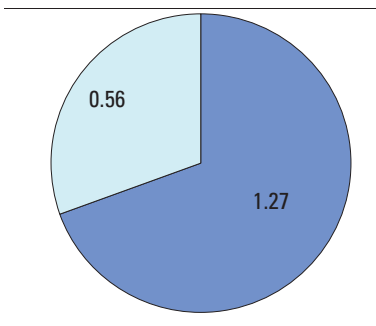

Withdrawals, in million gallons per day

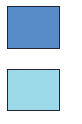

Surface water

Groundwater
Groundwater withdrawals by aquifer

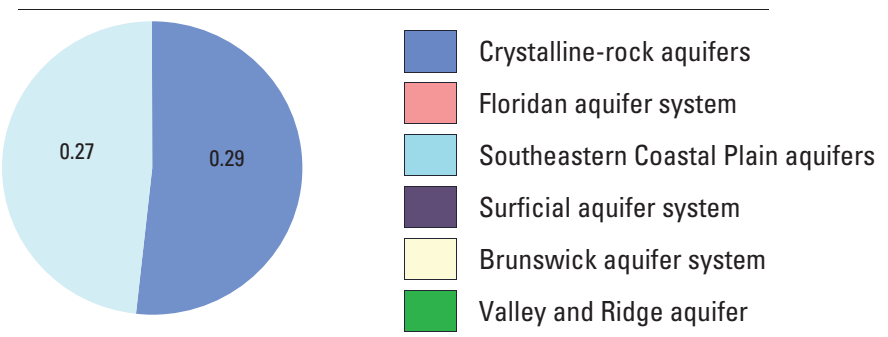

Groundwater withdrawals, by year
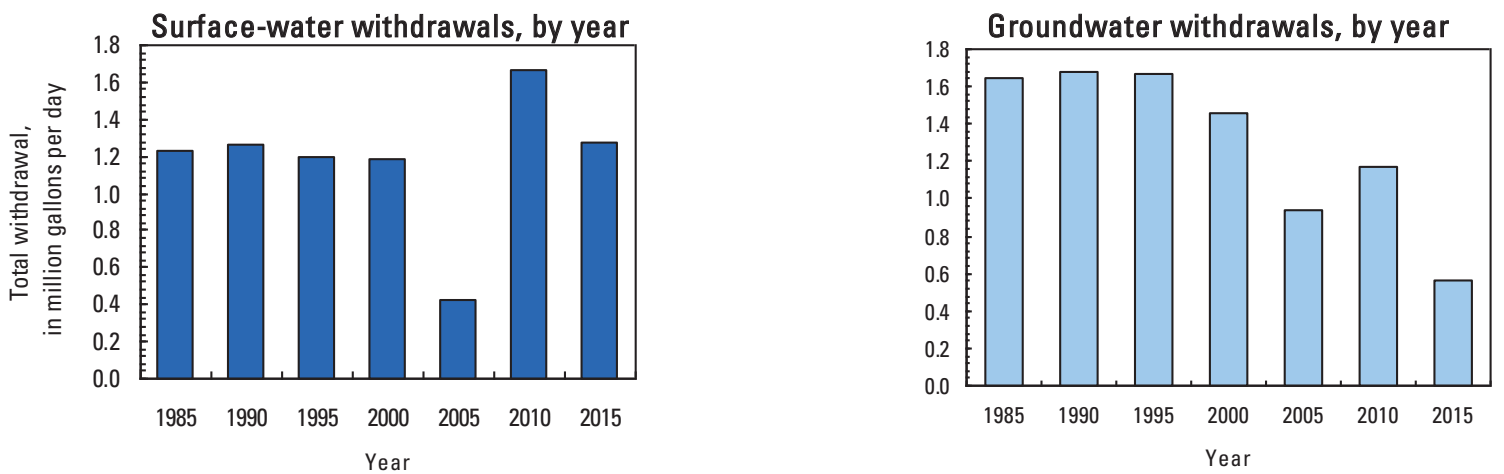


\section{TALIAFERRO COUNTY}

Population

Population served by public supply-groundwater

Population served by public supply-surface water

Acres irrigated

\section{WATER WITHDRAWALS AND ESTIMATED USE, IN MILLION GALLONS PER DAY}

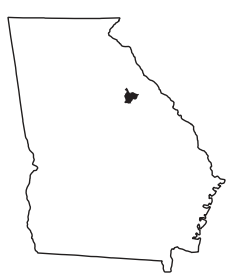

[--,not applicable; Mgal/d, million gallons per day]

\begin{tabular}{|c|c|c|c|c|c|c|c|c|}
\hline \multirow{3}{*}{ Category } & \multicolumn{3}{|c|}{ Withdrawals } & \multirow{3}{*}{$\begin{array}{c}\text { Total } \\
\text { use }\end{array}$} & \multirow{3}{*}{$\begin{array}{l}\text { Returns to } \\
\text { Surface }\end{array}$} & \multirow{2}{*}{\multicolumn{3}{|c|}{$\begin{array}{l}\text { Withdrawals by Major Industrial Groups } \\
\text { [NAICS, North American Industrial Classification System code] } \\
\end{array}$}} \\
\hline & \multirow{2}{*}{$\begin{array}{l}\text { Ground } \\
\text { water }\end{array}$} & \multirow{2}{*}{$\begin{array}{c}\text { Surface } \\
\text { water }\end{array}$} & \multirow{2}{*}{ Total } & & & & & \\
\hline & & & & & & \multirow{2}{*}{ NAICS } & \multirow[t]{2}{*}{ Groundwater } & \multirow{2}{*}{ Surface water } \\
\hline Public supply & 0.05 & 0.00 & 0.05 & -- & -- & & & \\
\hline Domestic use & 0.08 & 0.00 & 0.08 & 0.13 & 0.00 & \multirow{3}{*}{ None } & \multirow[t]{3}{*}{-- } & \multirow[t]{3}{*}{--} \\
\hline Commercial use & 0.00 & 0.00 & 0.00 & 0.00 & 0.00 & & & \\
\hline Industrial use & 0.00 & 0.00 & 0.00 & 0.00 & 0.00 & & & \\
\hline Public-supply loss & -- & -- & -- & 0.00 & -- & & & \\
\hline Inter-county delivery & -- & -- & - & 0.00 & -- & & & \\
\hline Public wastewater treatment & $t--$ & -- & -- & -- & 0.01 & & & \\
\hline Mining use & 0.00 & 0.00 & 0.00 & 0.00 & 0.00 & \multicolumn{3}{|c|}{ Withdrawals by Major Public Suppliers } \\
\hline Irrigation (crop) & 0.00 & 0.00 & 0.00 & 0.00 & 0.00 & Name & Groundwater & Surface water \\
\hline Irrigation (golf) & 0.00 & 0.00 & 0.00 & 0.00 & 0.00 & \multirow{4}{*}{ City of Crawfordville } & \multirow{4}{*}{0.05} & \multirow{4}{*}{--} \\
\hline Livestock-aquaculture & 0.00 & 0.00 & 0.00 & 0.00 & 0.00 & & & \\
\hline Thermoelectric power & 0.00 & 0.00 & 0.00 & 0.00 & 0.00 & & & \\
\hline TOTAL & 0.13 & 0.00 & 0.13 & 0.13 & 0.01 & & & \\
\hline
\end{tabular}

Total use is total withdrawal plus public supply deliveries and losses.

Withdrawals by water source

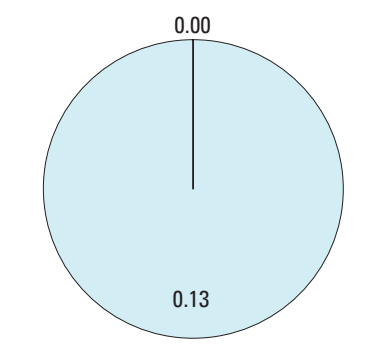

Withdrawals, in million gallons per day

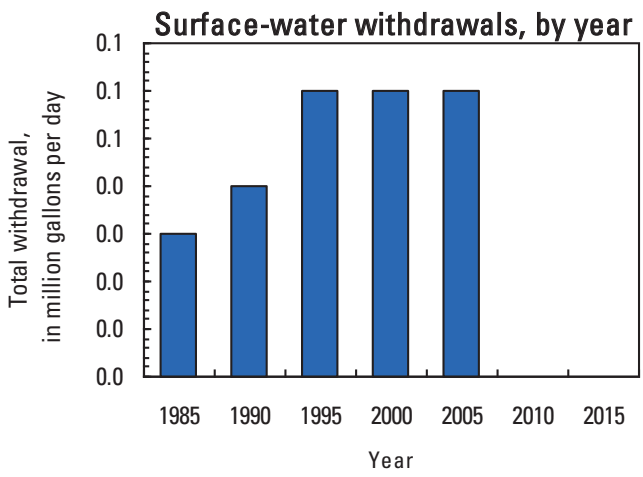

Groundwater withdrawals by aquifer
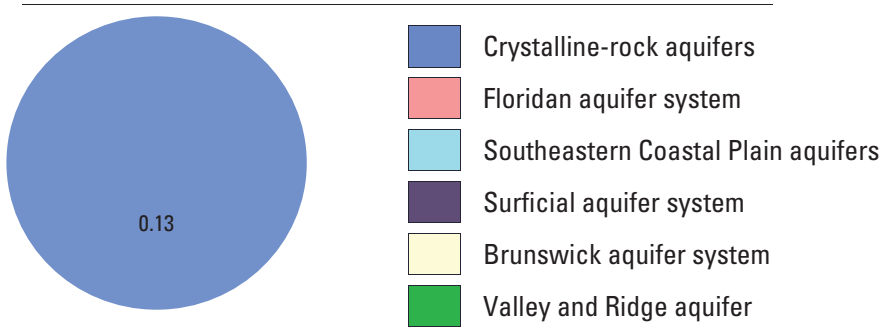

Groundwater withdrawals, by year

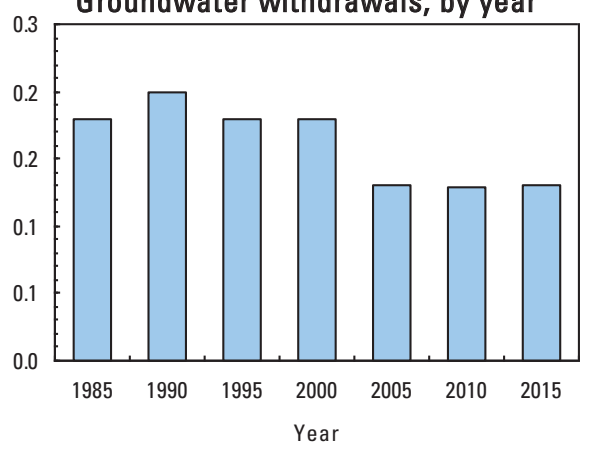


TATTNALL COUNTY

Population

Population served by public supply-groundwater

9,605

Population served by public supply-surface water

0

Acres irrigated

18,190

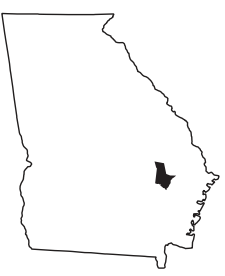

2015 WATER WITHDRAWALS AND ESTIMATED USE, IN MILLION GALLONS PER DAY

[--,not applicable; Mgal/d, million gallons per day

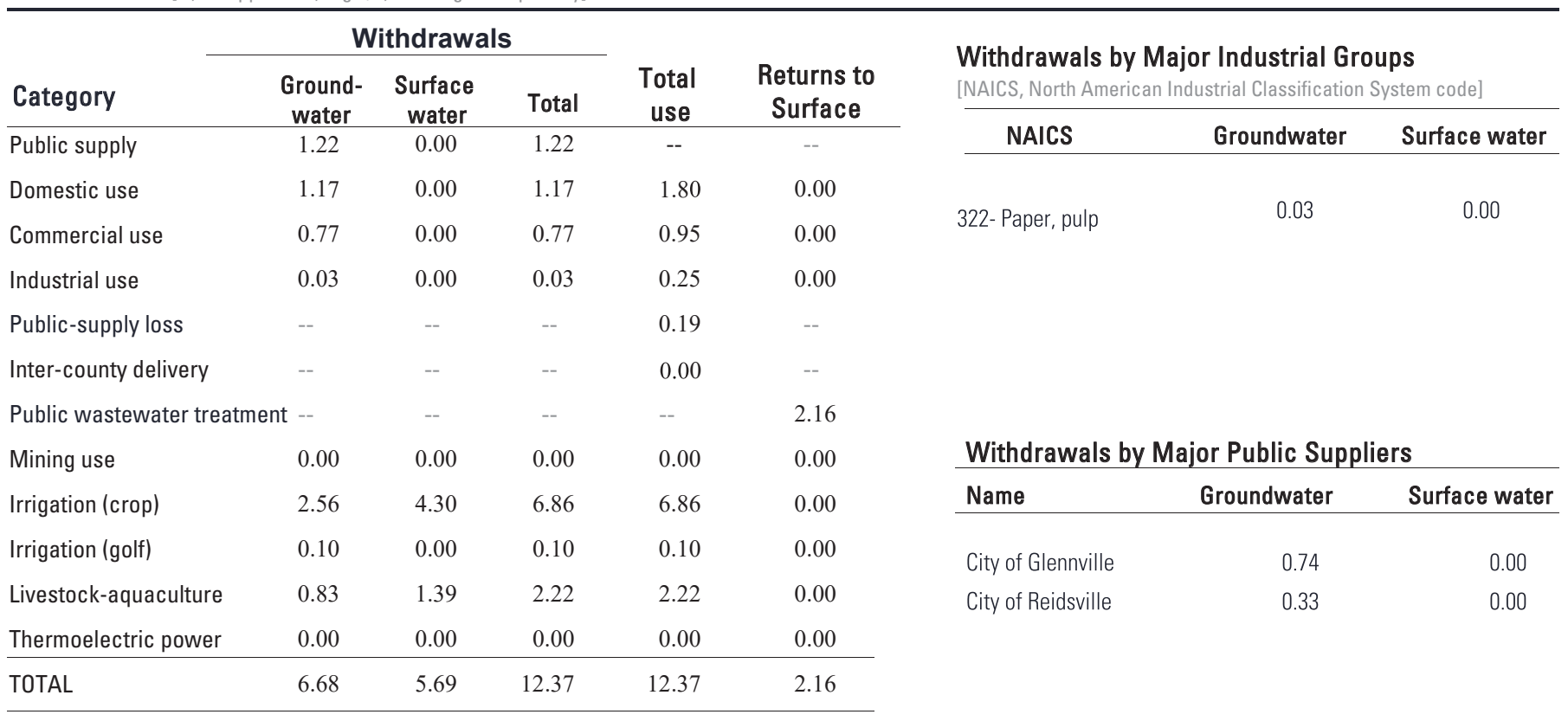

Total use is total withdrawal plus public supply deliveries and losses.

Withdrawals by water source

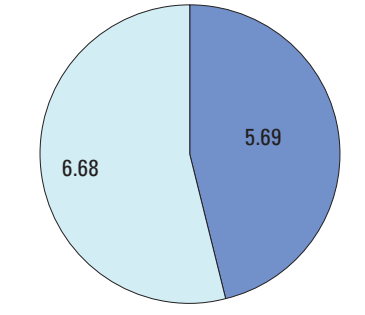

Withdrawals, in million

gallons per day

Surface water

Groundwater

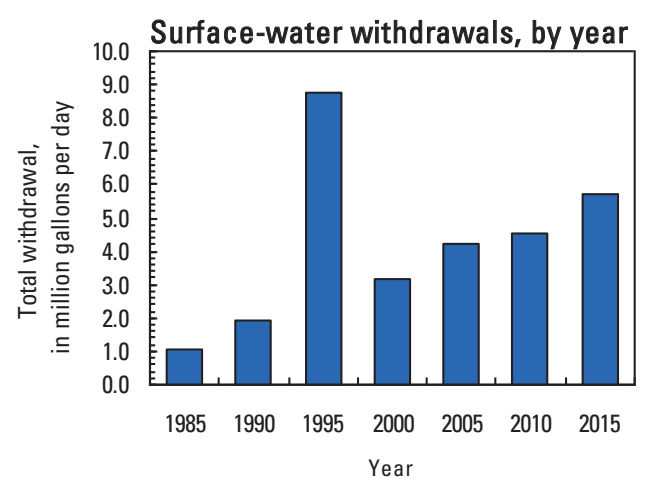

Groundwater withdrawals by aquifer

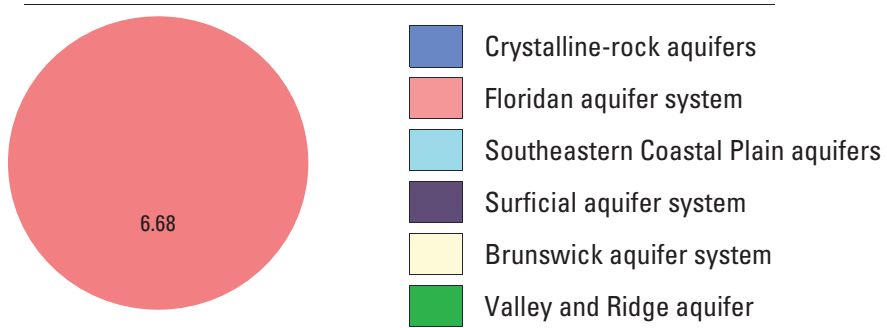

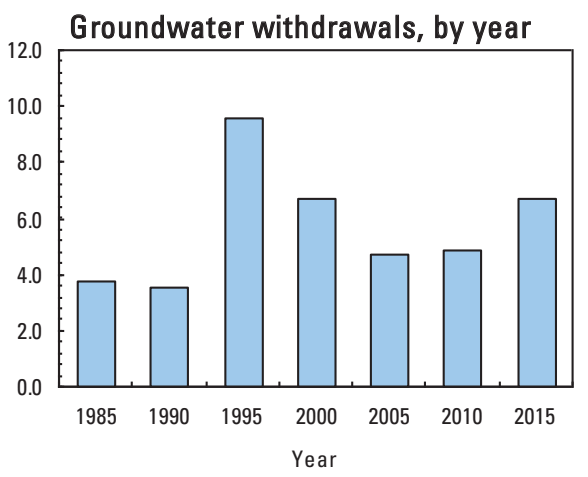




\section{TAYLOR COUNTY}

Population

Population served by public supply-groundwater

Population served by public supply-surface water

Acres irrigated

2015 WATER WITHDRAWALS AND ESTIMATED USE, IN MILLION GALLONS PER DAY

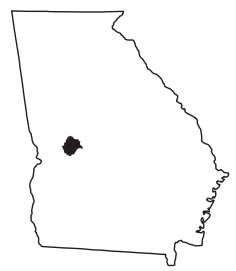

[--,not applicable; Mgal/d, million gallons per day]

\begin{tabular}{|c|c|c|c|c|c|c|c|c|}
\hline \multirow{2}{*}{ Category } & \multicolumn{3}{|c|}{ Withdrawals } & \multirow{2}{*}{$\begin{array}{c}\text { Total } \\
\text { use }\end{array}$} & \multirow{2}{*}{$\begin{array}{c}\text { Returns to } \\
\text { Surface }\end{array}$} & \multirow{2}{*}{\multicolumn{3}{|c|}{$\begin{array}{l}\text { Withdrawals by Major Industrial Groups } \\
\text { [NAICS, North American Industrial Classification System code] }\end{array}$}} \\
\hline & Ground- & Surface & Tatal & & & & & \\
\hline Public supply & $\begin{array}{c}\text { water } \\
0.62\end{array}$ & $\begin{array}{c}\text { water } \\
0.00\end{array}$ & 0.62 & $\begin{array}{c}\text { use } \\
--\end{array}$ & $\begin{array}{c}\text { Surtace } \\
--\end{array}$ & NAICS & Groundwater & Surface water \\
\hline Domestic use & 0.29 & 0.00 & 0.29 & 0.75 & 0.00 & \multirow{3}{*}{ 212- Mining } & \multirow{3}{*}{0.00} & \multirow{3}{*}{1.74} \\
\hline Commercial use & 0.01 & 0.00 & 0.01 & 0.08 & 0.00 & & & \\
\hline Industrial use & 0.00 & 1.74 & 1.74 & 1.74 & 0.00 & & & \\
\hline Public-supply loss & -- & -- & -- & 0.09 & -- & & & \\
\hline Inter-county delivery & -- & -- & -- & 0.00 & -- & & & \\
\hline Public wastewater treatment & t -- & -- & -- & -- & 1.64 & & & \\
\hline Mining use & 0.03 & 0.58 & 0.61 & 0.61 & 0.00 & \multicolumn{3}{|c|}{ Withdrawals by Major Public Suppliers } \\
\hline Irrigation (crop) & 0.01 & 0.28 & 0.29 & 0.29 & 0.00 & Name & Groundwater & Surface water \\
\hline Irrigation (golf) & 0.00 & 0.00 & 0.00 & 0.00 & 0.00 & City of Butler & 0.45 & 0.00 \\
\hline Livestock-aquaculture & 0.00 & 0.07 & 0.07 & 0.07 & 0.00 & City of Reynolds & 0.16 & 0.00 \\
\hline Thermoelectric power & 0.00 & 0.00 & 0.00 & 0.00 & 0.00 & & & \\
\hline TOTAL & 0.96 & 2.67 & 3.63 & 3.63 & 1.64 & & & \\
\hline
\end{tabular}

Total use is total withdrawal plus public supply deliveries and losses.

Withdrawals by water source

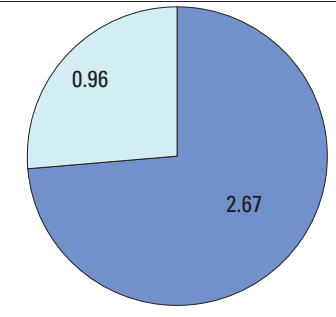

Withdrawals, in million

gallons per day

Surface water

Groundwater
0.96

\section{Groundwater withdrawals by aquifer}
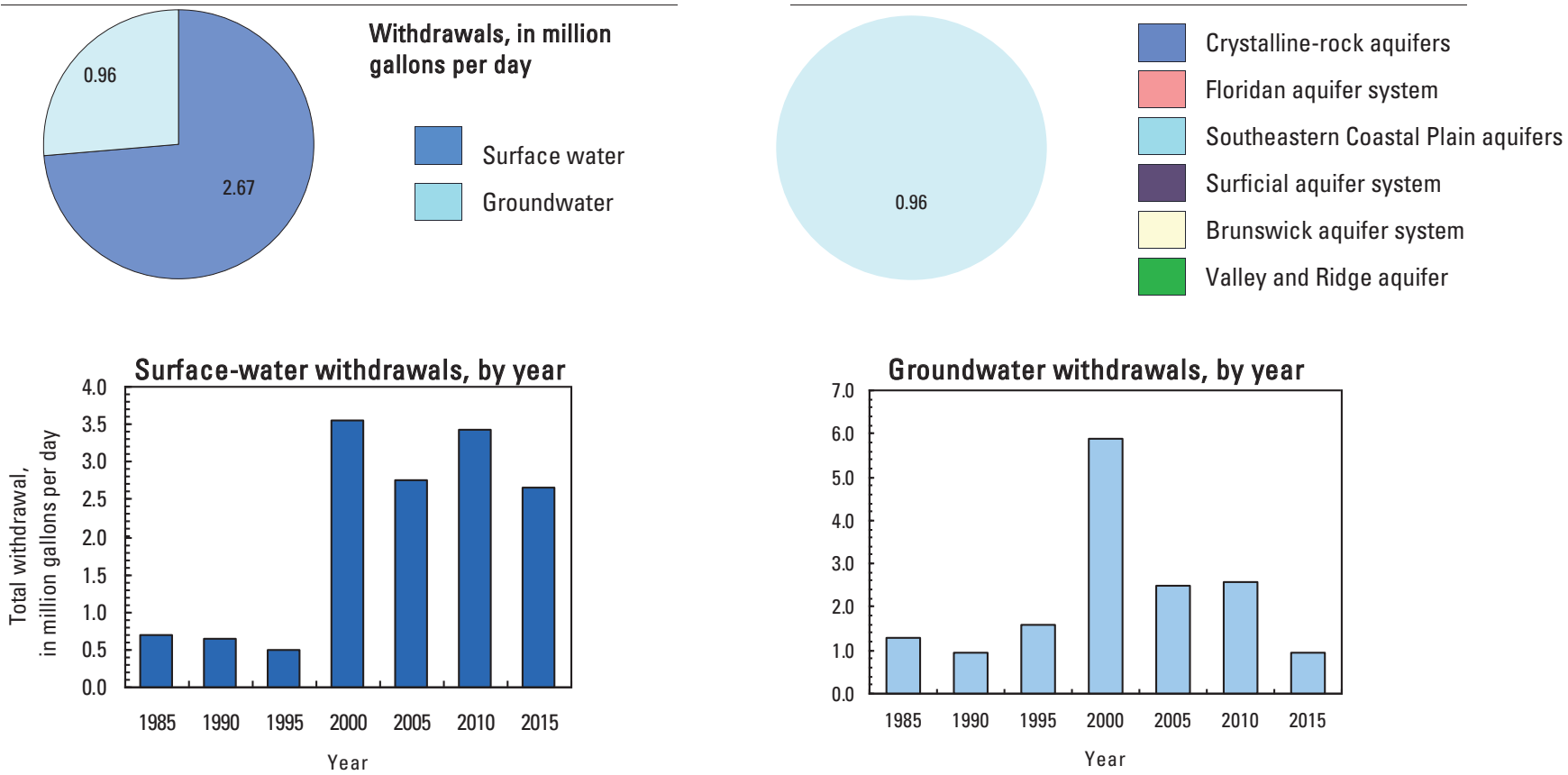

Groundwater withdrawals, by year

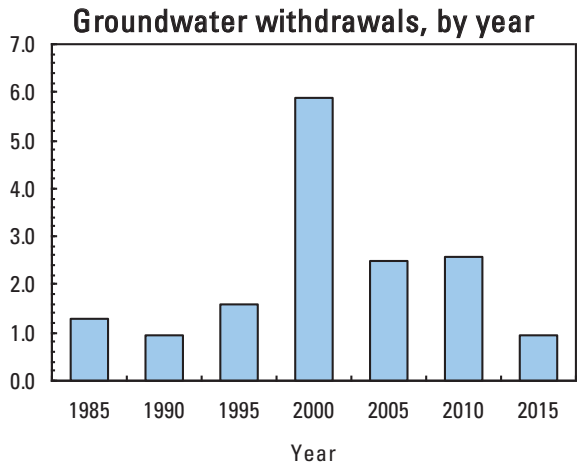




\section{TELFAIR COUNTY}

Population

Population served by public supply-groundwater

9,267

Population served by public supply-surface water

0

Acres irrigated

10,240

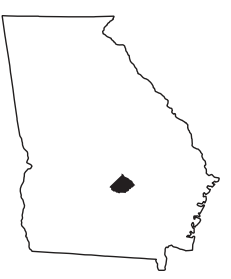

2015 WATER WITHDRAWALS AND ESTIMATED USE, IN MILLION GALLONS PER DAY

[--,not applicable; Mgal/d, million gallons per day]

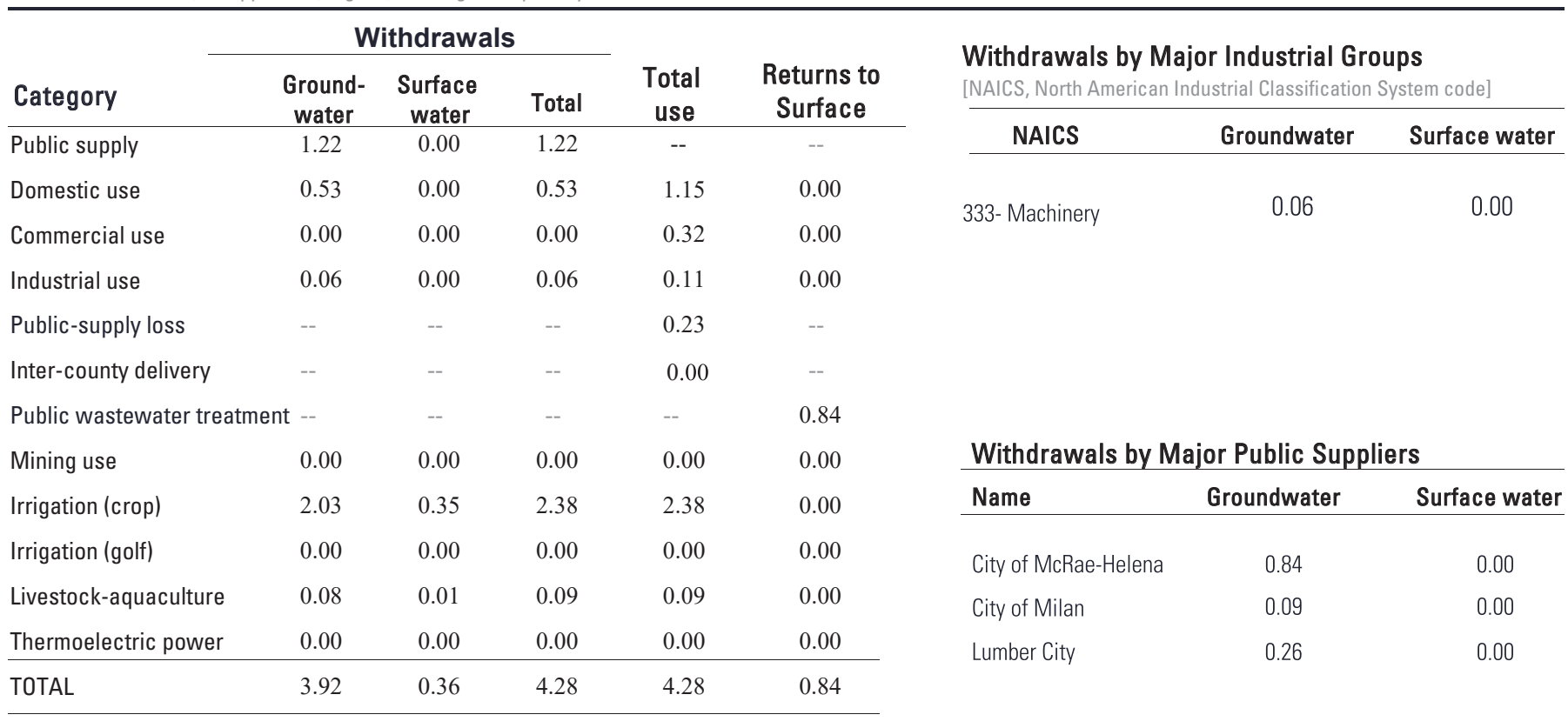

Total use is total withdrawal plus public supply deliveries and losses.

Withdrawals by water source

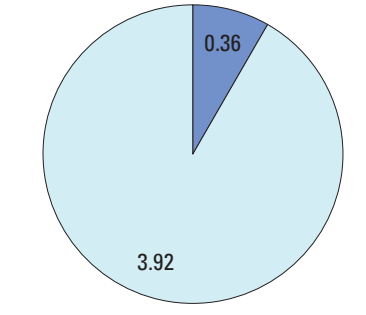

Withdrawals, in million gallons per day

Surface water

Groundwater

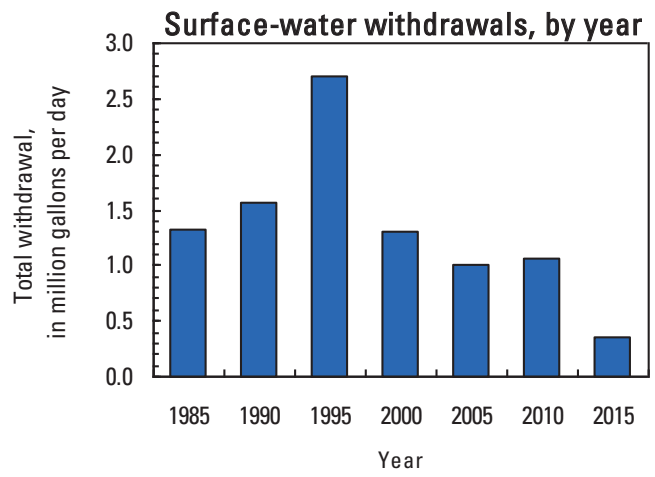

Groundwater withdrawals by aquifer

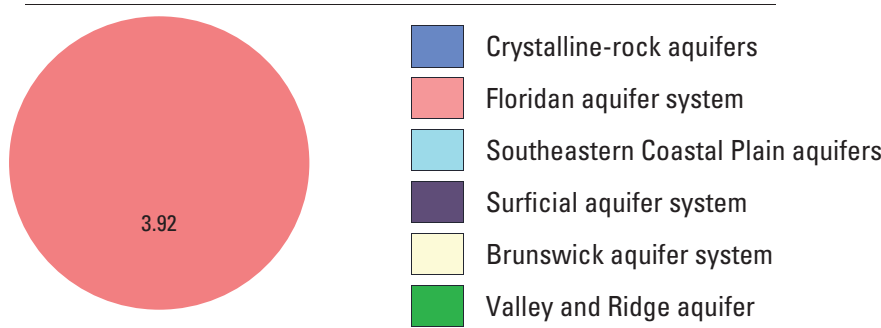




\section{TERRELL COUNTY}

Population

Population served by public supply-groundwater

Population served by public supply-surface water

Acres irrigated

30,060

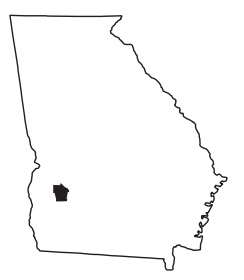

2015 WATER WITHDRAWALS AND ESTIMATED USE, IN MILLION GALLONS PER DAY

[--,not applicable; Mgal/d, million gallons per day]

\begin{tabular}{|c|c|c|c|c|c|c|c|c|}
\hline \multirow{2}{*}{ Category } & \multicolumn{3}{|c|}{ Withdrawals } & \multirow{3}{*}{$\begin{array}{c}\text { Total } \\
\text { use }\end{array}$} & \multirow{2}{*}{$\begin{array}{c}\text { Returns to } \\
\text { Surface }\end{array}$} & \multirow{2}{*}{\multicolumn{3}{|c|}{$\begin{array}{l}\text { Withdrawals by Major Industrial Groups } \\
\text { [NAICS, North American Industrial Classification System code] }\end{array}$}} \\
\hline & \multirow{2}{*}{$\begin{array}{c}\text { Ground- } \\
\text { water } \\
1.44\end{array}$} & \multirow{2}{*}{$\begin{array}{c}\begin{array}{c}\text { Surface } \\
\text { water }\end{array} \\
0.00\end{array}$} & \multirow{2}{*}{ Total } & & & & & \\
\hline & & & & & $\begin{array}{c}\text { Surtace } \\
--\end{array}$ & \multirow[t]{3}{*}{ NAICS } & Groundwater & Surface water \\
\hline Domestic use & 0.22 & 0.00 & 0.22 & 0.71 & 0.00 & & & \\
\hline Commercial use & 0.02 & 0.00 & 0.02 & 0.17 & 0.00 & & -- & -- \\
\hline Industrial use & 0.00 & 0.00 & 0.00 & 0.25 & 0.00 & & & \\
\hline Public-supply loss & -- & -- & -- & 0.55 & -- & & & \\
\hline Inter-county delivery & -- & -- & -- & 0.00 & -- & & & \\
\hline \multicolumn{2}{|l|}{ Public wastewater treatment -- } & -- & -- & -- & 1.99 & & & \\
\hline Mining use & 0.00 & 0.00 & 0.00 & 0.00 & 0.00 & \multicolumn{3}{|c|}{ Withdrawals by Major Public Suppliers } \\
\hline Irrigation (crop) & 9.75 & 5.11 & 14.86 & 14.86 & 0.00 & Name & Groundwater & Surface water \\
\hline Irrigation (golf) & 0.00 & 0.03 & 0.03 & 0.03 & 0.00 & City of Dawson & 1.33 & 0.00 \\
\hline Livestock-aquaculture & 0.02 & 0.01 & 0.03 & 0.03 & 0.00 & & & \\
\hline Thermoelectric power & 0.00 & 0.00 & 0.00 & 0.00 & 0.00 & & & \\
\hline TOTAL & 11.45 & 5.15 & 16.60 & 16.60 & 1.99 & & & \\
\hline
\end{tabular}

Total use is total withdrawal plus public supply deliveries and losses.

Withdrawals by water source

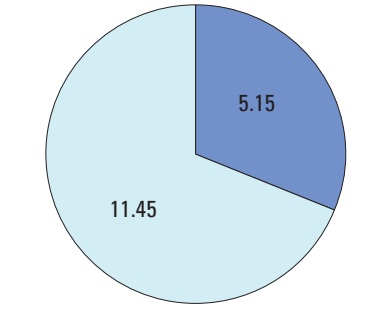

Withdrawals, in million gallons per day

Surface water

Groundwater

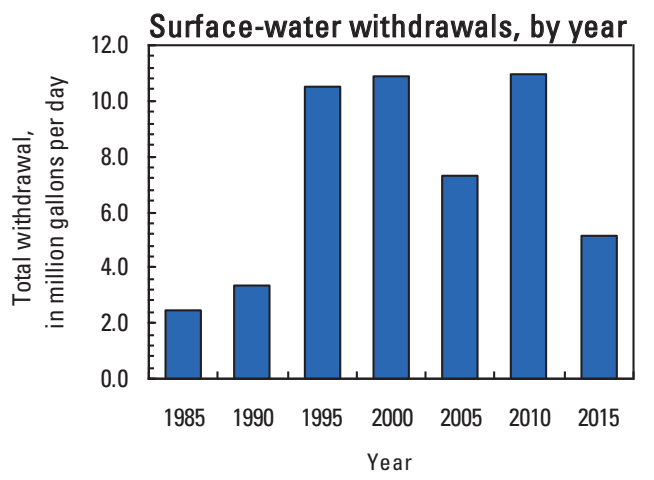

\section{Groundwater withdrawals by aquifer}

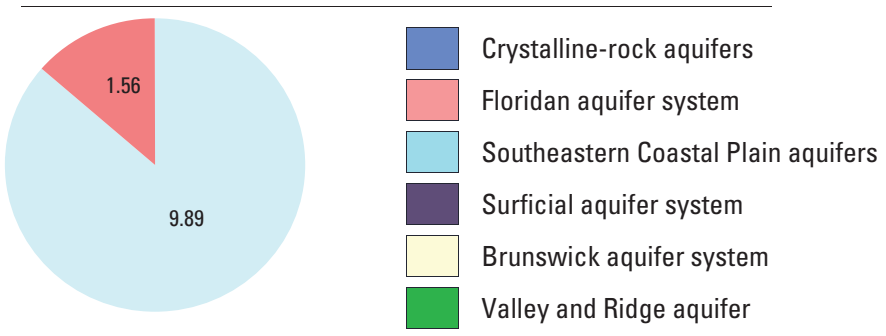

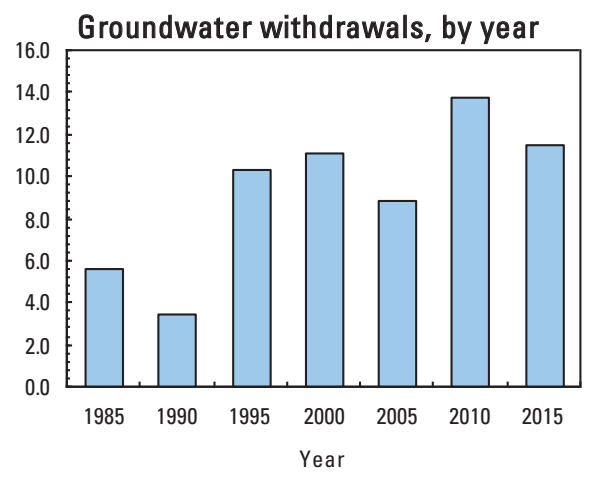




\section{THOMAS COUNTY}

Population

Population served by public supply-groundwater $\quad 31,354$

Population served by public supply-surface water 0

Acres irrigated

2015 WATER WITHDRAWALS AND ESTIMATED USE, IN MILLION GALLONS PER DAY

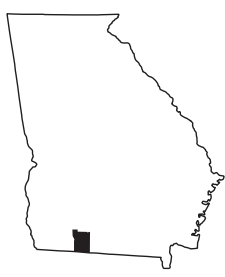

[--,not applicable; Mgal/d, million gallons per day]

\begin{tabular}{lccccc}
\hline & \multicolumn{3}{c}{ Withdrawals } & & Total \\
\cline { 2 - 4 } Category & $\begin{array}{c}\text { Ground- } \\
\text { water }\end{array}$ & $\begin{array}{c}\text { Surface } \\
\text { water }\end{array}$ & $\begin{array}{c}\text { Total } \\
\text { use }\end{array}$ & $\begin{array}{c}\text { Returns to } \\
\text { Surface }\end{array}$ \\
\hline Public supply & 5.64 & 0.00 & 5.64 & -- & -- \\
Domestic use & 1.03 & 0.00 & 1.03 & 3.56 & 0.00 \\
Commercial use & 0.01 & 0.00 & 0.01 & 1.25 & 0.00 \\
Industrial use & 0.17 & 0.00 & 0.17 & 0.54 & 0.00 \\
Public-supply loss & -- & -- & -- & 1.50 & -- \\
Inter-county delivery & -- & -- & -- & 0.00 & -- \\
Public wastewater treatment & -- & -- & -- & -- & 5.81 \\
Mining use & 0.85 & 0.01 & 0.86 & 0.86 & 0.00 \\
Irrigation (crop) & 3.09 & 0.44 & 3.53 & 3.53 & 0.00 \\
Irrigation (golf) & 0.16 & 0.00 & 0.16 & 0.16 & 0.00 \\
Livestock-aquaculture & 0.26 & 0.04 & 0.30 & 0.30 & 0.00 \\
Thermoelectric power & 0.00 & 0.00 & 0.00 & 0.00 & 0.00 \\
\hline TOTAL & 11.21 & 0.49 & 11.70 & 11.70 & 5.81 \\
\hline
\end{tabular}

Withdrawals by Major Industrial Groups

[NAICS, North American Industrial Classification System code]

\begin{tabular}{lcc}
\hline \multicolumn{1}{c}{ NAICS } & Groundwater & Surface water \\
\hline 212- Mining & 0.10 & 0.00 \\
$\begin{array}{l}\text { 561- Administrative } \\
\text { services }\end{array}$ & 0.07 & 0.00
\end{tabular}

Withdrawals by Major Public Suppliers

\begin{tabular}{lcc}
\hline Name & Groundwater & Surface wate \\
\hline City of Boston & 0.38 & 0.00 \\
City of Coolidge & 0.05 & 0.00 \\
City of Meigs & 0.18 & 0.00 \\
City of Thomasville & 4.69 & 0.00
\end{tabular}

Total use is total withdrawal plus public supply deliveries and losses.

Withdrawals by water source

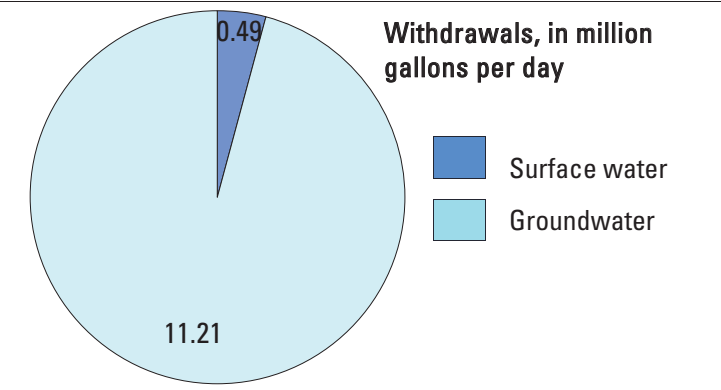

Surface-water withdrawals, by year

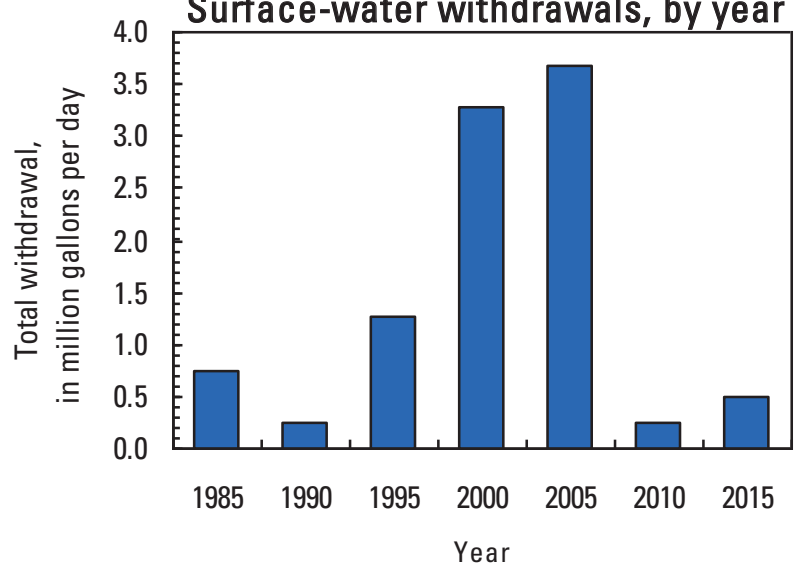

Groundwater withdrawals by aquifer
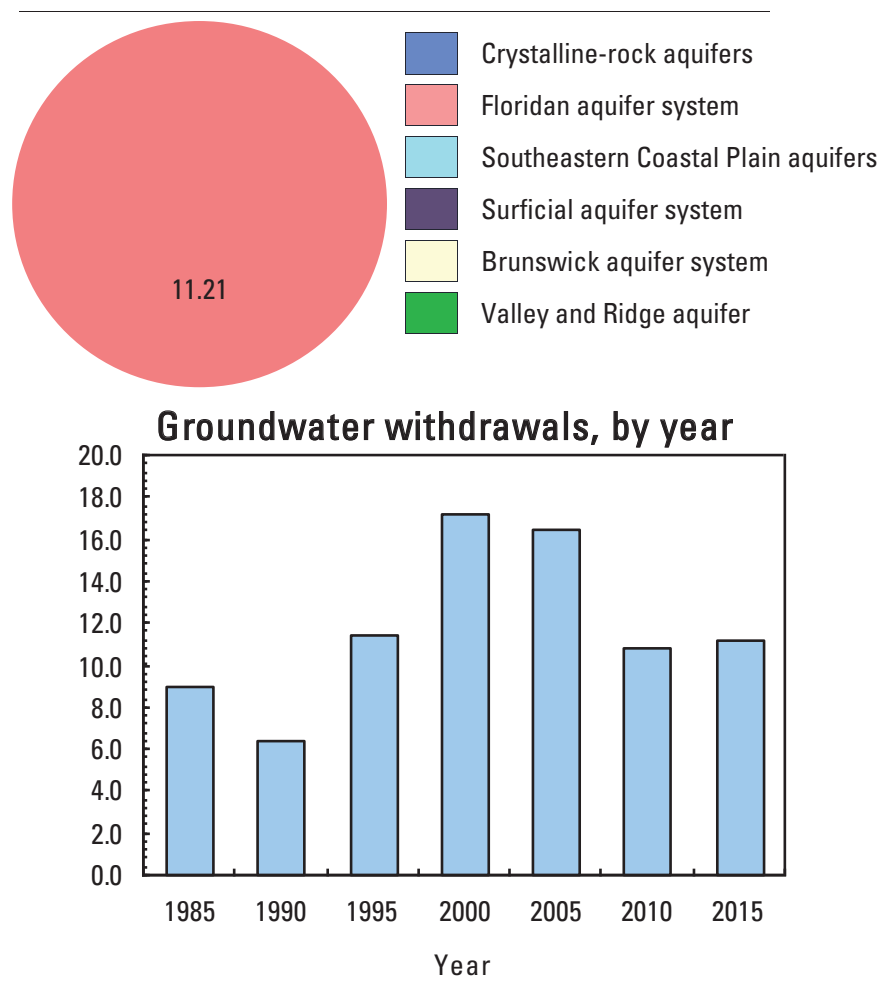


\section{TIFT COUNTY}

Population

Population served by public supply-groundwater

Population served by public supply-surface water

Acres irrigated

2015 WATER WITHDRAWALS AND ESTIMATED USE, IN MILLION GALLONS PER DAY

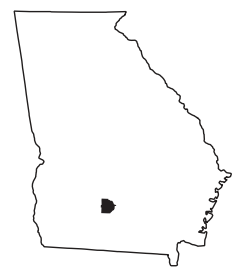

[--,not applicable; Mgal/d, million gallons per day]

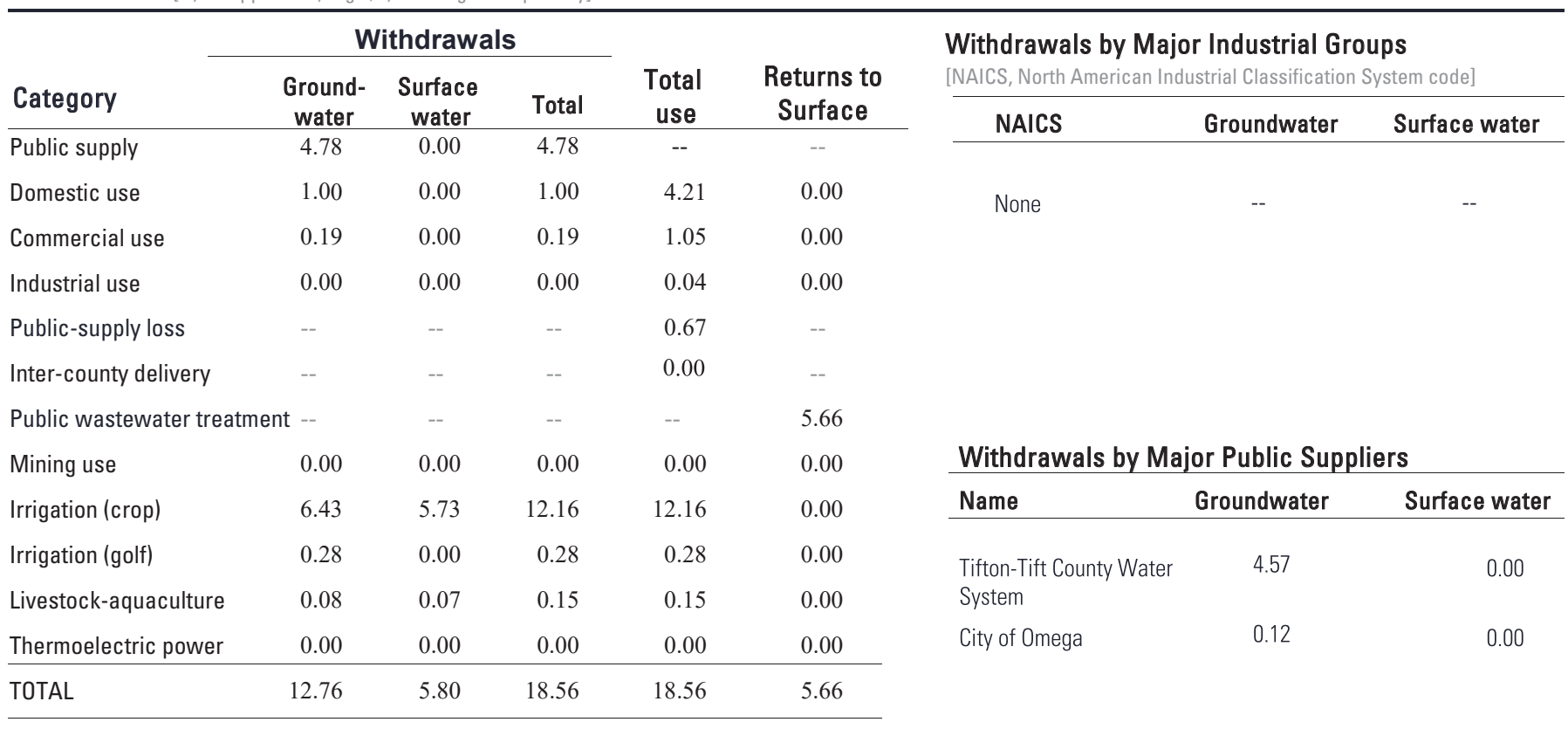

Total use is total withdrawal plus public supply deliveries and losses.

Withdrawals by water source

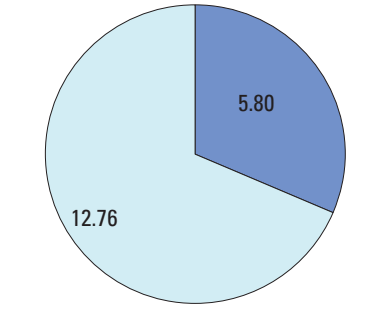

Withdrawals, in million gallons per day

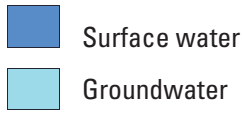

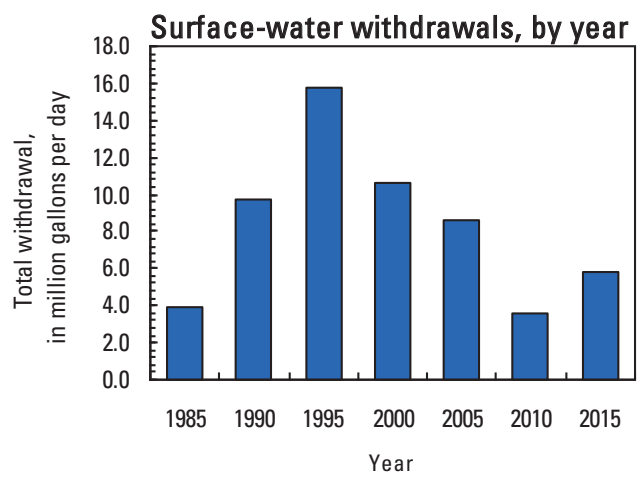

Groundwater withdrawals by aquifer
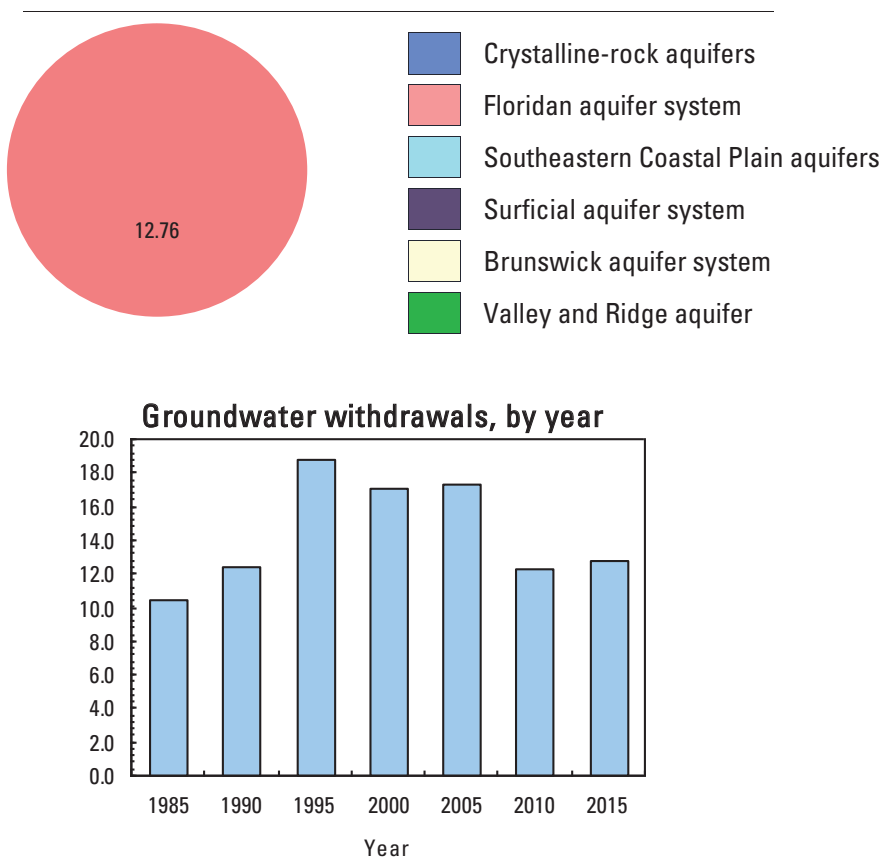


\section{TOOMBS COUNTY}

Population

Population served by public supply-groundwater

Population served by public supply-surface water Acres irrigated
15,020

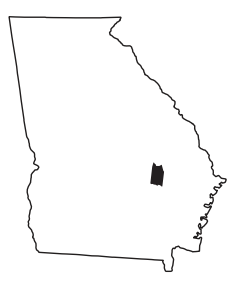

2015 WATER WITHDRAWALS AND ESTIMATED USE, IN MILLION GALLONS PER DAY

[--,not applicable; Mgal/d, million gallons per day]

\begin{tabular}{|c|c|c|c|c|c|c|c|c|}
\hline \multirow{3}{*}{ Category } & \multicolumn{3}{|c|}{ Withdrawals } & \multirow{3}{*}{$\begin{array}{c}\text { Total } \\
\text { use }\end{array}$} & \multirow{3}{*}{$\begin{array}{l}\text { Returns to } \\
\text { Surface }\end{array}$} & \multirow{2}{*}{\multicolumn{3}{|c|}{$\begin{array}{l}\text { Withdrawals by Major Industrial Groups } \\
\text { [NAICS, North American Industrial Classification System code] }\end{array}$}} \\
\hline & \multirow{2}{*}{$\begin{array}{c}\text { Ground - } \\
\text { water }\end{array}$} & \multirow{2}{*}{$\begin{array}{c}\text { Surface } \\
\text { water }\end{array}$} & \multirow[b]{2}{*}{ Total } & & & & & \\
\hline & & & & & & NAICS & Groundwater & Surface water \\
\hline Public supply & 2.84 & 0.00 & 2.84 & -- & -- & & & \\
\hline Domestic use & 0.66 & 0.00 & 0.66 & 2.07 & 0.00 & None & -- & -- \\
\hline Commercial use & 0.00 & 0.00 & 0.00 & 0.51 & 0.00 & & & \\
\hline Industrial use & 0.00 & 0.00 & 0.00 & 0.43 & 0.00 & & & \\
\hline Public-supply loss & -- & -- & -- & 0.49 & -- & & & \\
\hline Inter-county delivery & -- & -- & -- & 0.00 & -- & & & \\
\hline Public wastewater treatment & t -- & -- & -- & -- & 1.58 & & & \\
\hline Mining use & 0.06 & 0.00 & 0.06 & 0.06 & 0.00 & Withdrawal & jor Public Sup & \\
\hline Irrigation (crop) & 4.38 & 3.96 & 8.34 & 8.34 & 0.00 & Name & Groundwater & Surface water \\
\hline Irrigation (golf) & 0.03 & 0.06 & 0.09 & 0.09 & 0.00 & City of Vidalia & 1.48 & 0.00 \\
\hline Livestock-aquaculture & 0.06 & 0.26 & 0.32 & 0.32 & 0.00 & City of Lyons & 1.27 & 0.00 \\
\hline Thermoelectric power & 0.00 & 0.00 & 0.00 & 0.00 & 0.00 & & & \\
\hline TOTAL & 8.03 & 4.28 & 12.31 & 12.31 & 1.58 & & & \\
\hline
\end{tabular}

Total use is total withdrawal plus public supply deliveries and losses.

Withdrawals by water source

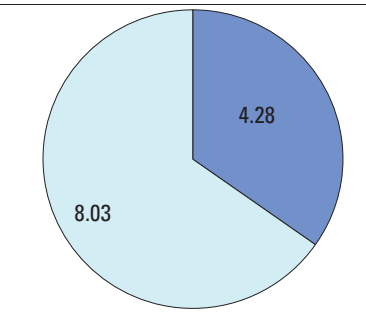

Withdrawals, in million gallons per day

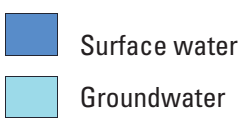

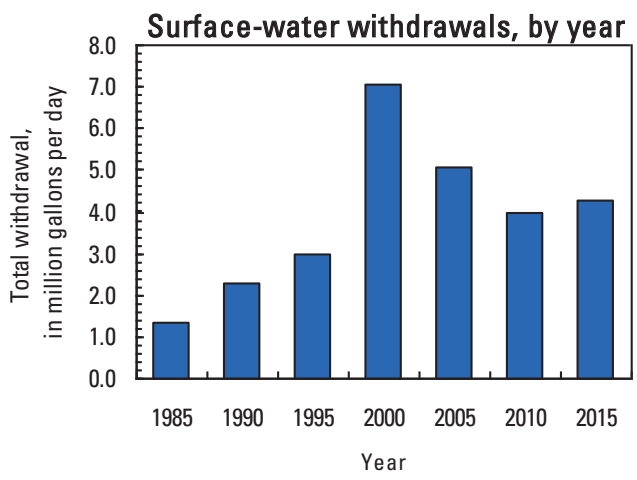

Groundwater withdrawals by aquifer

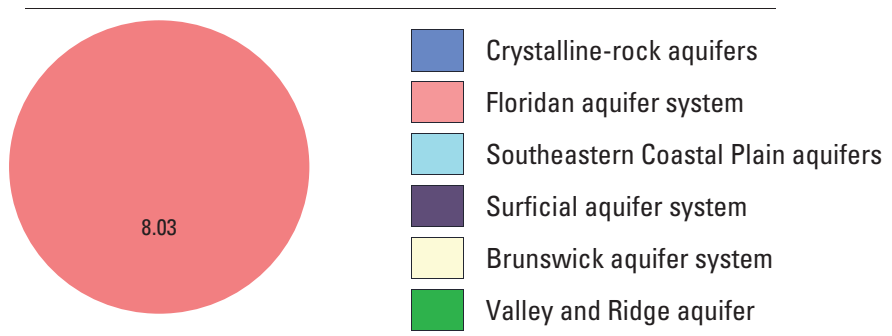




\section{TOWNS COUNTY}

Population

11,182

Population served by public supply-groundwater

Population served by public supply-surface water

Acres irrigated

90

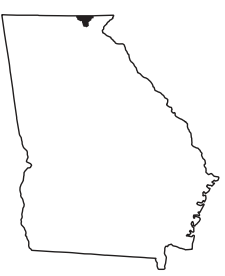

2015 WATER WITHDRAWALS AND ESTIMATED USE, IN MILLION GALLONS PER DAY

[--,not applicable; Mgal/d, million gallons per day]

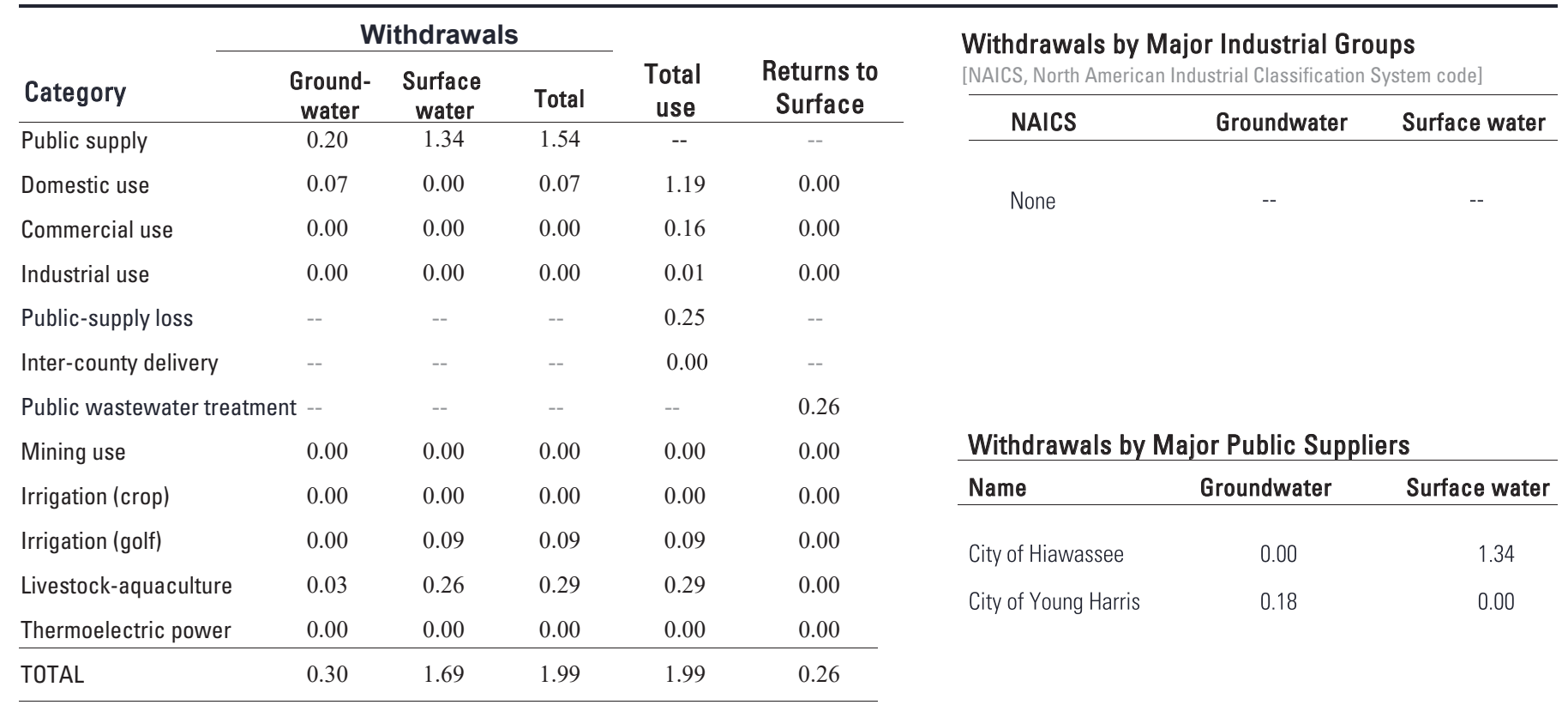

Total use is total withdrawal plus public supply deliveries and losses.

Withdrawals by water source

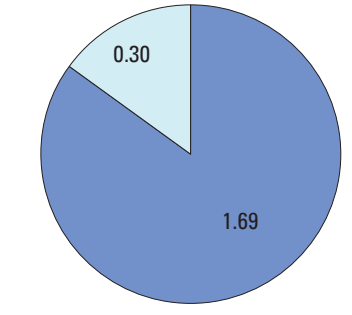

Withdrawals, in million

gallons per day

Surface water

Groundwater

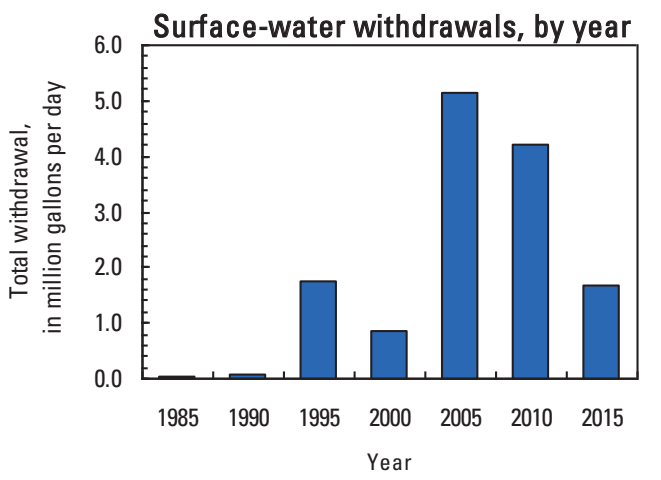

Groundwater withdrawals by aquifer

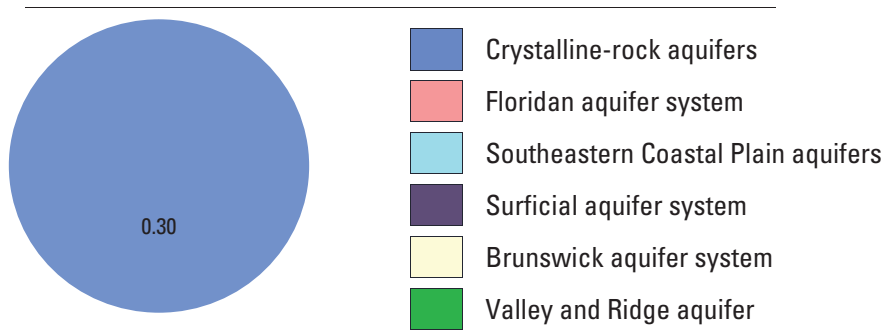

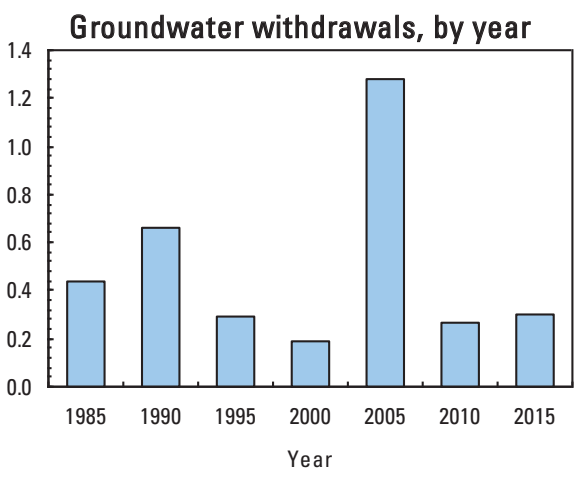




\section{TREUTLEN COUNTY}

Population

Population served by public supply-groundwater

Population served by public supply-surface water

0

Acres irrigated

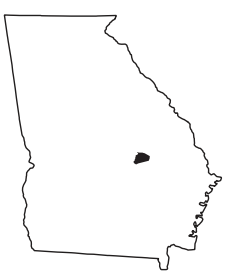

2015 WATER WITHDRAWALS AND ESTIMATED USE, IN MILLION GALLONS PER DAY

[--,not applicable; Mgal/d, million gallons per day]

\begin{tabular}{|c|c|c|c|c|c|c|c|c|}
\hline \multirow{2}{*}{ Category } & \multicolumn{3}{|c|}{ Withdrawals } & \multirow{3}{*}{$\begin{array}{c}\text { Total } \\
\text { use }\end{array}$} & \multirow{3}{*}{$\begin{array}{l}\text { Returns to } \\
\text { Surface }\end{array}$} & \multirow{2}{*}{\multicolumn{3}{|c|}{$\begin{array}{l}\text { Withdrawals by Major Industrial Groups } \\
\text { [NAICS, North American Industrial Classification System code] }\end{array}$}} \\
\hline & \multirow{2}{*}{$\begin{array}{c}\text { Ground- } \\
\text { water }\end{array}$} & \multirow{2}{*}{$\begin{array}{c}\begin{array}{c}\text { Surface } \\
\text { water }\end{array} \\
0.00\end{array}$} & Total & & & & & \\
\hline Public supply & & & $\begin{array}{c}\text { Total } \\
0.30\end{array}$ & & & NAICS & Groundwater & Surface water \\
\hline Domestic use & 0.32 & 0.00 & 0.32 & 0.49 & 0.00 & \multirow{2}{*}{ None } & \multirow{2}{*}{--} & \multirow{2}{*}{--} \\
\hline Commercial use & 0.00 & 0.00 & 0.00 & 0.05 & 0.00 & & & \\
\hline Inter-county delivery & -- & -- & -- & -0.04 & -- & & & \\
\hline \multicolumn{2}{|l|}{ Public wastewater treatment -- } & -- & -- & -- & 0.49 & & & \\
\hline Mining use & 0.00 & 0.00 & 0.00 & 0.00 & 0.00 & \multicolumn{3}{|c|}{ Withdrawals by Major Public Suppliers } \\
\hline Irrigation (crop) & 0.67 & 1.06 & 1.73 & 1.73 & 0.00 & Name & Groundwater & Surface water \\
\hline Thermoelectric power & 0.00 & 0.00 & 0.00 & 0.00 & 0.00 & & & \\
\hline TOTAL & 1.30 & 1.08 & 2.38 & 2.34 & 0.49 & & & \\
\hline
\end{tabular}

Total use is total withdrawal plus public supply deliveries and losses. An estimate of $0.04 \mathrm{Mgal} / \mathrm{d}$ was

delivered from Treutlen County to neighboring counties in 2015.

\section{Withdrawals by water source}

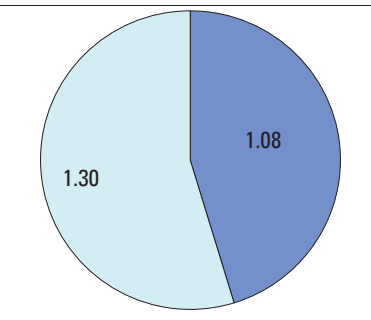

Withdrawals, in million

gallons per day

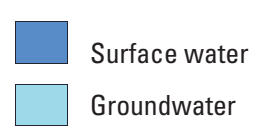

Groundwater withdrawals by aquifer

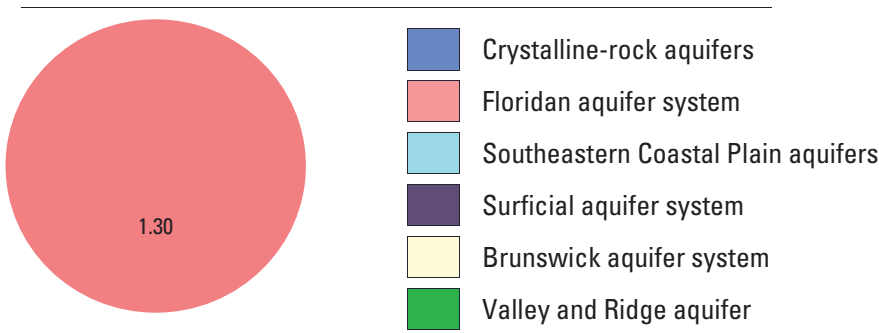

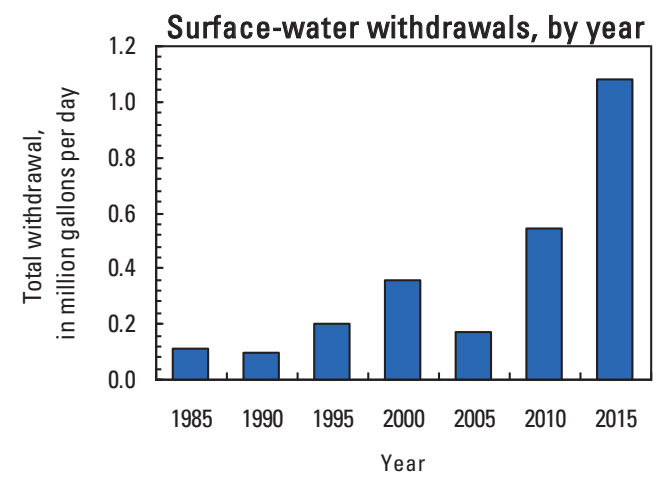

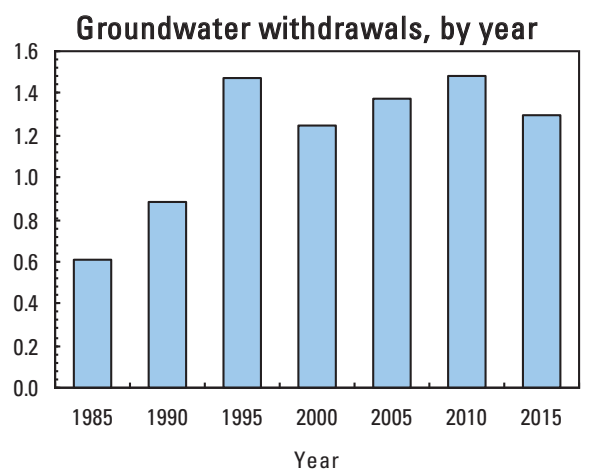




\section{TROUP COUNTY}

Population

69,763

Population served by public supply-groundwater

Population served by public supply-surface water $\quad 49,509$

Acres irrigated

2015 WATER WITHDRAWALS AND ESTIMATED USE, IN MILLION GALLONS PER DAY

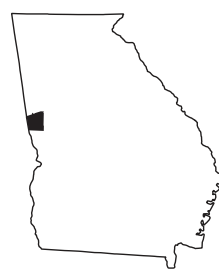

[--,not applicable; Mgal/d, million gallons per day]

\begin{tabular}{|c|c|c|c|c|c|c|c|c|}
\hline \multirow{3}{*}{ Category } & \multicolumn{3}{|c|}{ Withdrawals } & \multirow{3}{*}{$\begin{array}{c}\text { Total } \\
\text { use }\end{array}$} & \multirow{3}{*}{$\begin{array}{c}\text { Returns to } \\
\text { Surface }\end{array}$} & \multirow{2}{*}{\multicolumn{3}{|c|}{$\begin{array}{l}\text { Withdrawals by Major Industrial Groups } \\
\text { [NAICS, North American Industrial Classification System code] }\end{array}$}} \\
\hline & \multirow{2}{*}{$\begin{array}{c}\text { Ground- } \\
\text { water } \\
0.00\end{array}$} & \multirow{2}{*}{$\begin{array}{c}\text { Surface } \\
\text { water }\end{array}$} & \multirow{2}{*}{ Total } & & & & & \\
\hline & & & & & & NAICS & Groundwater & Surface water \\
\hline rudic supply & & 0.54 & 0.34 & -- & -- & & & \\
\hline Domestic use & 1.52 & 0.00 & 1.52 & 5.04 & 0.00 & \multirow{3}{*}{ None } & \multirow{2}{*}{--} & \multirow{3}{*}{--} \\
\hline Commercial use & 0.01 & 0.00 & 0.01 & 2.02 & 0.00 & & & \\
\hline Industrial use & 0.00 & 0.00 & 0.00 & 1.74 & 0.00 & & & \\
\hline Public-supply loss & -- & -- & -- & 1.27 & -- & & & \\
\hline Inter-county delivery & -- & -- & -- & 0.00 & -- & & & \\
\hline Public wastewater treatment & it -- & -- & -- & -- & 6.36 & & & \\
\hline Mining use & 0.04 & 0.00 & 0.04 & 0.04 & 0.00 & \multicolumn{3}{|c|}{ Withdrawals by Major Public Suppliers } \\
\hline Irrigation (crop) & 0.00 & 0.00 & 0.00 & 0.00 & 0.00 & Name & Groundwater & Surface water \\
\hline Irrigation (golf) & 0.00 & 0.08 & 0.08 & 0.08 & 0.00 & City of Lagrange & 0.00 & 7.35 \\
\hline Livestock-aquaculture & 0.05 & 0.08 & 0.13 & 0.13 & 0.00 & City of West Point & 0.00 & 1.19 \\
\hline Thermoelectric power & 0.00 & 0.00 & 0.00 & 0.00 & 0.00 & & & \\
\hline TOTAL & 1.62 & 8.70 & 10.32 & 10.32 & 6.36 & & & \\
\hline
\end{tabular}

Total use is total withdrawal plus public supply deliveries and losses. An estimate of $0.25 \mathrm{Mgal} / \mathrm{d}$ was delivered from neighboring counties in 2015. An estimate of $0.25 \mathrm{Mgal} / \mathrm{d}$ was delivered from Troup County to neighboring counties in 2015.

\section{Withdrawals by water source}

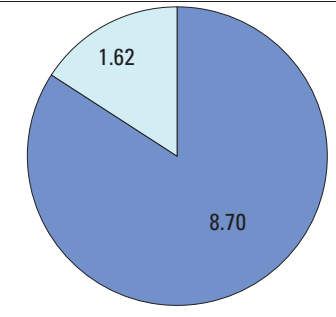

Withdrawals, in million gallons per day

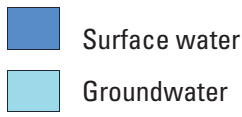

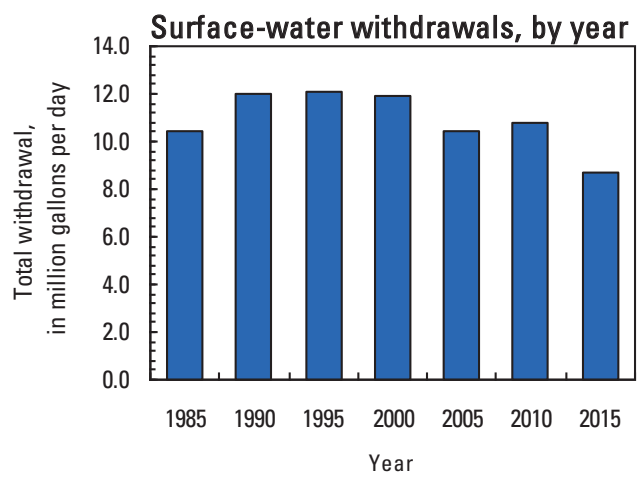

Groundwater withdrawals by aquifer

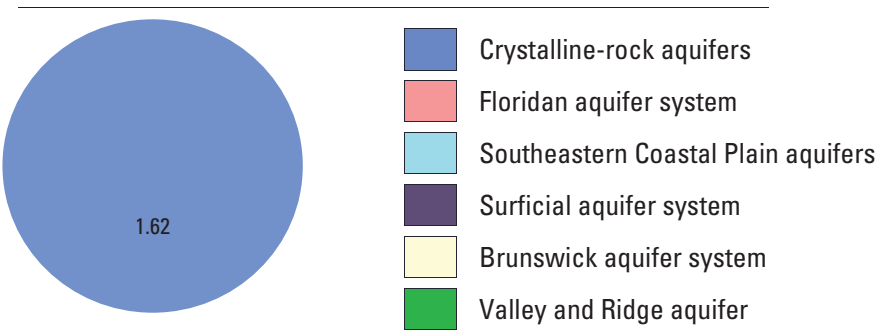

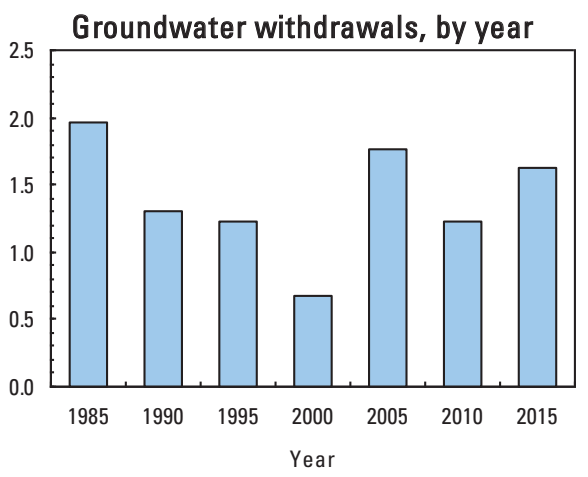




\section{TURNER COUNTY}

Population

Population served by public supply-groundwater

5,387

Population served by public supply-surface water

0

Acres irrigated

2015 WATER WITHDRAWALS AND ESTIMATED USE, IN MILLION GALLONS PER DAY

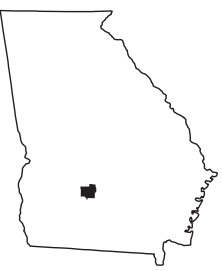

$[--$,not applicable; Mgal/d, million gallons per day]

\begin{tabular}{|c|c|c|c|c|c|c|c|c|}
\hline \multirow{3}{*}{ Category } & \multicolumn{3}{|c|}{ Withdrawals } & \multirow{3}{*}{$\begin{array}{c}\text { Total } \\
\text { use }\end{array}$} & \multirow{3}{*}{$\begin{array}{l}\text { Returns to } \\
\text { Surface }\end{array}$} & \multirow{2}{*}{\multicolumn{3}{|c|}{$\begin{array}{l}\text { Withdrawals by Major Industrial Groups } \\
\text { [NAICS, North American Industrial Classification System code] }\end{array}$}} \\
\hline & \multirow{2}{*}{$\begin{array}{l}\text { Ground- } \\
\text { water }\end{array}$} & \multirow{2}{*}{$\begin{array}{c}\text { Surface } \\
\text { water }\end{array}$} & \multirow{2}{*}{ Total } & & & & & \\
\hline & & & & & & \multirow[t]{3}{*}{ NAICS } & Groundwater & Surface water \\
\hline Domestic use & 0.21 & 0.00 & 0.21 & 0.71 & 0.00 & & & \\
\hline Commercial use & 0.02 & 0.00 & 0.02 & 0.16 & 0.00 & & -- & - \\
\hline Inter-county delivery & -- & -- & -- & 0.00 & -- & & & \\
\hline \multicolumn{2}{|l|}{ Public wastewater treatment -- } & -- & -- & -- & 0.99 & & & \\
\hline Mining use & 0.00 & 0.00 & 0.00 & 0.00 & 0.00 & \multicolumn{3}{|c|}{ Withdrawals by Major Public Suppliers } \\
\hline Irrigation (crop) & 7.28 & 4.07 & 11.35 & 11.35 & 0.00 & Name & Groundwater & Surface water \\
\hline TOTAL & 8.50 & 4.15 & 12.65 & 12.65 & 0.99 & & & \\
\hline
\end{tabular}

Total use is total withdrawal plus public supply deliveries and losses.

Withdrawals by water source

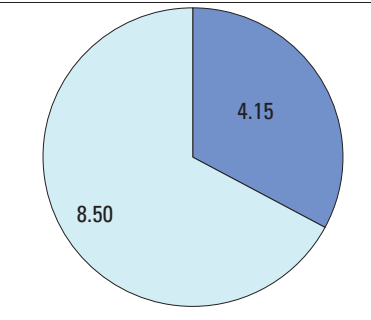

Withdrawals, in million gallons per day

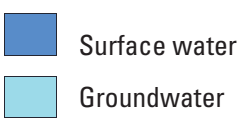

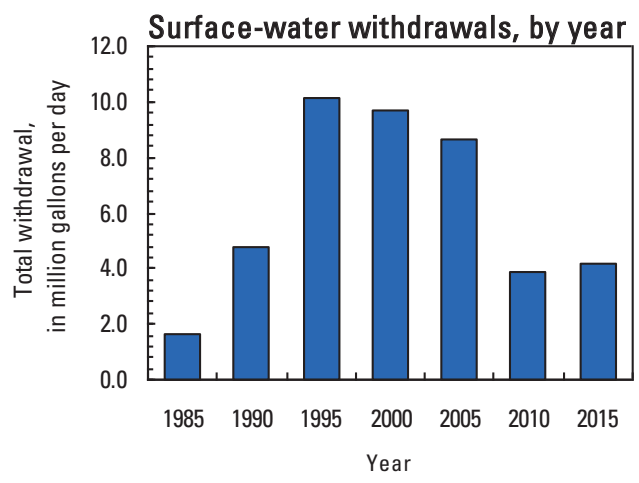

Groundwater withdrawals by aquifer

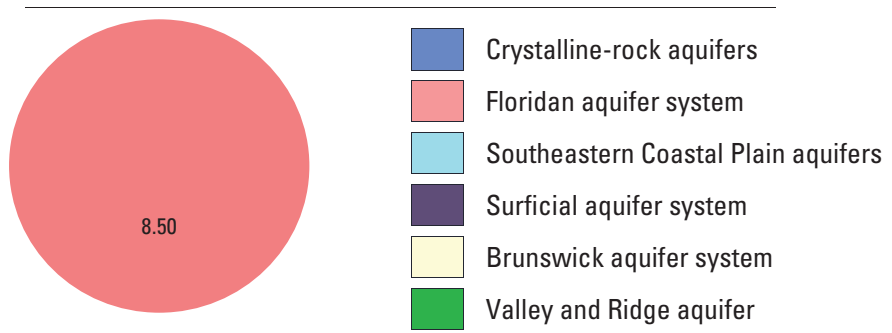




\section{TWIGGS COUNTY}

Population

8,390

Population served by public supply-groundwater $\quad 2,371$

Population served by public supply-surface water $\quad 0$

Acres irrigated

2015 WATER WITHDRAWALS AND ESTIMATED USE, IN MILLION GALLONS PER DAY

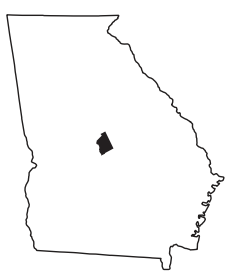

[--,not applicable; Mgal/d, million gallons per day]

\begin{tabular}{|c|c|c|c|c|c|c|c|c|}
\hline \multirow[b]{2}{*}{ Category } & \multicolumn{3}{|c|}{ Withdrawals } & \multirow{2}{*}{$\begin{array}{c}\text { Total } \\
\text { use }\end{array}$} & \multirow{2}{*}{$\begin{array}{c}\text { Returns to } \\
\text { Surface }\end{array}$} & \multirow{2}{*}{\multicolumn{3}{|c|}{$\begin{array}{l}\text { Withdrawals by Major Industrial Groups } \\
\text { [NAICS, North American Industrial Classification System code] }\end{array}$}} \\
\hline & Ground- & Surface & Total & & & & & \\
\hline Public supply & $\begin{array}{c}\text { Water } \\
0.45\end{array}$ & $\begin{array}{c}\text { water } \\
0.00\end{array}$ & 0.45 & & -- & NAICS & Groundwater & Surface water \\
\hline Domestic use & 0.45 & 0.00 & 0.45 & 0.70 & 0.00 & \multirow{3}{*}{ 212-Mining } & \multirow[b]{2}{*}{3.35} & \multirow{3}{*}{0.00} \\
\hline Commercial use & 0.00 & 0.00 & 0.00 & 0.14 & 0.00 & & & \\
\hline Industrial use & 3.35 & 0.00 & 3.35 & 3.35 & 0.00 & & & \\
\hline Public-supply loss & -- & -- & -- & 0.06 & -- & & & \\
\hline Inter-county delivery & -- & -- & -- & 0.00 & -- & & & \\
\hline Public wastewater treatment & t -- & -- & -- & -- & 3.89 & & & \\
\hline Mining use & 0.00 & 0.00 & 0.00 & 0.00 & 0.00 & \multicolumn{3}{|c|}{ Withdrawals by Major Public Suppliers } \\
\hline Irrigation (crop) & 1.22 & 0.04 & 1.26 & 1.26 & 0.00 & Name & Groundwater & Surface water \\
\hline Irrigation (golf) & 0.00 & 0.00 & 0.00 & 0.00 & 0.00 & \multirow{4}{*}{$\begin{array}{l}\text { City of Jeffersonville } \\
\text { Twiggs County Water } \\
\text { Department }\end{array}$} & \multirow{3}{*}{0.05} & \multirow{3}{*}{0.00} \\
\hline Livestock-aquaculture & 0.03 & 0.00 & 0.03 & 0.03 & 0.00 & & & \\
\hline Thermoelectric power & 0.00 & 0.00 & 0.00 & 0.00 & 0.00 & & & \\
\hline TOTAL & 5.50 & 0.04 & 5.54 & 5.54 & 3.89 & & & \\
\hline
\end{tabular}

Total use is total withdrawal plus public supply deliveries and losses.

Withdrawals by water source

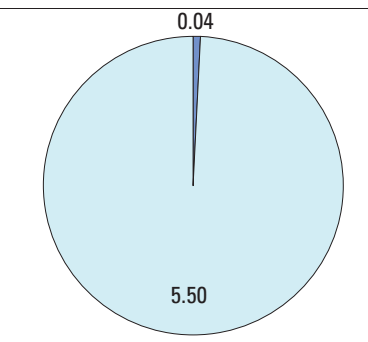

Withdrawals, in million gallons per day

Surface water

Groundwater

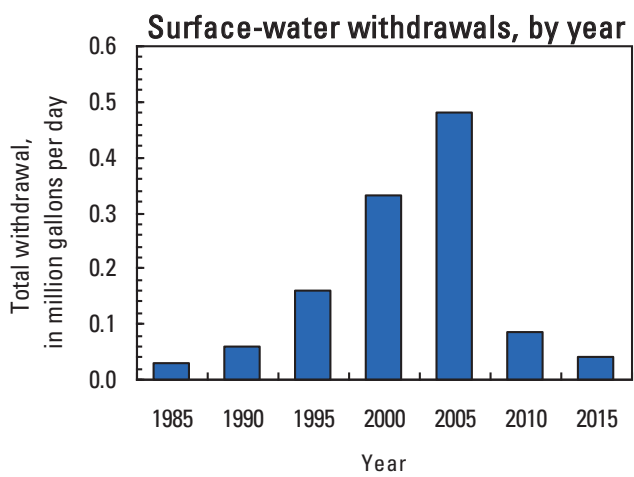

Groundwater withdrawals by aquifer

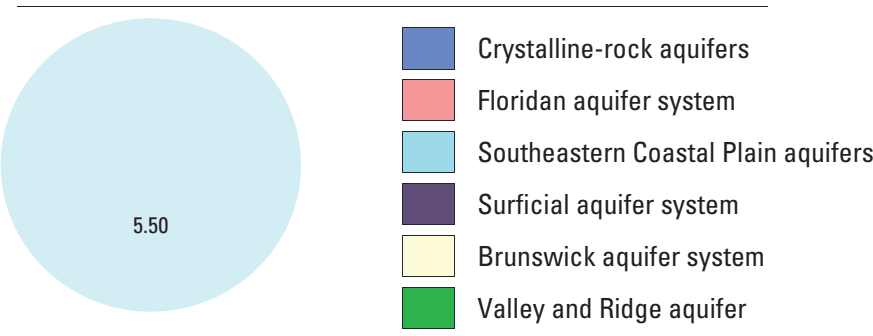

Groundwater withdrawals, by year

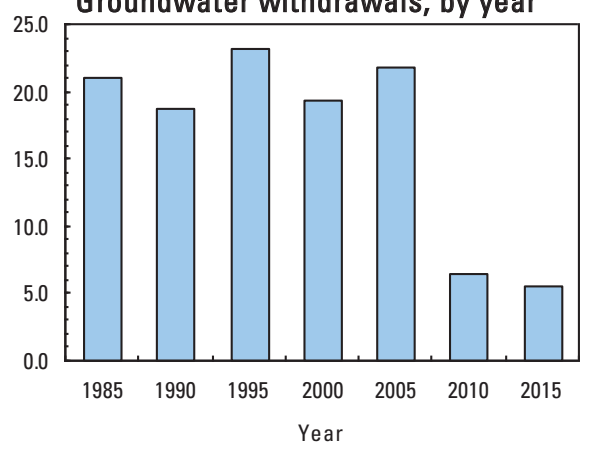




\section{UNION COUNTY}

Population

Population served by public supply-groundwater

Population served by public supply-surface water $\quad 10,797$

Acres irrigated

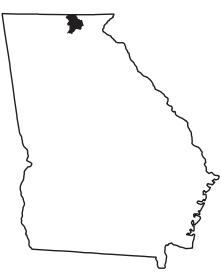

2015 WATER WITHDRAWALS AND ESTIMATED USE, IN MILLION GALLONS PER DAY

[--,not applicable; Mgal/d, million gallons per day]

\begin{tabular}{|c|c|c|c|c|c|c|c|c|}
\hline \multirow{2}{*}{ Category } & \multicolumn{3}{|c|}{ Withdrawals } & \multirow{2}{*}{$\begin{array}{c}\text { Total } \\
\text { use }\end{array}$} & \multirow{2}{*}{$\begin{array}{l}\text { Returns to } \\
\text { Surface }\end{array}$} & \multirow{2}{*}{\multicolumn{3}{|c|}{$\begin{array}{l}\text { Withdrawals by Major Industrial Groups } \\
\text { [NAICS, North American Industrial Classification System code] }\end{array}$}} \\
\hline & Ground- & Surface & Total & & & & & \\
\hline Public supply & $\begin{array}{l}\text { water } \\
0.86\end{array}$ & $\begin{array}{c}\text { water } \\
0.96\end{array}$ & 1.82 & & surtace & NAICS & Groundwater & Surface water \\
\hline Domestic use & 0.02 & 0.00 & 0.02 & 1.09 & 0.00 & \multirow{3}{*}{ None } & \multirow[b]{2}{*}{--} & \multirow{3}{*}{--} \\
\hline Commercial use & 0.00 & 0.00 & 0.00 & 0.22 & 0.00 & & & \\
\hline Industrial use & 0.00 & 0.00 & 0.00 & 0.18 & 0.00 & & & \\
\hline Public-supply loss & -- & -- & -- & 0.35 & -- & & & \\
\hline Inter-county delivery & -- & -- & -- & 0.00 & -- & & & \\
\hline Public wastewater treatment & t -- & -- & -- & -- & 0.36 & & & \\
\hline Mining use & 0.03 & 0.00 & 0.03 & 0.03 & 0.00 & \multicolumn{3}{|c|}{ Withdrawals by Major Public Suppliers } \\
\hline Irrigation (crop) & 0.00 & 0.00 & 0.00 & 0.00 & 0.00 & Name & Groundwater & Surface water \\
\hline Irrigation (golf) & 0.00 & 0.19 & 0.19 & 0.19 & 0.00 & \multirow{4}{*}{$\begin{array}{l}\text { City of Blairsville } \\
\text { Notla Water Authority }\end{array}$} & 0.24 & 0.24 \\
\hline Livestock-aquaculture & 0.02 & 0.04 & 0.06 & 0.06 & 0.00 & & 0.19 & 0.72 \\
\hline Thermoelectric power & 0.00 & 0.00 & 0.00 & 0.00 & 0.00 & & & \\
\hline TOTAL & 0.93 & 1.19 & 2.12 & 2.12 & 0.36 & & & \\
\hline
\end{tabular}

Total use is total withdrawal plus public supply deliveries and losses.

Withdrawals by water source

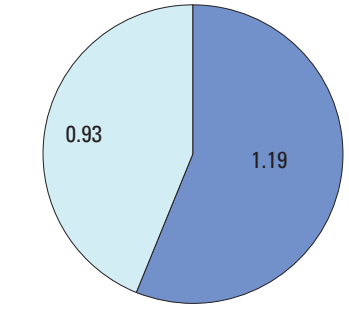

Withdrawals, in million gallons per day

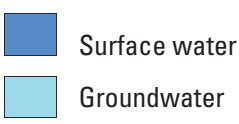

Groundwater withdrawals by aquifer

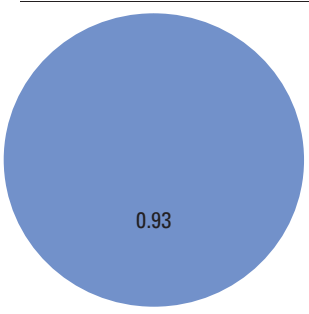

Crystalline-rock aquifers

Floridan aquifer system

Southeastern Coastal Plain aquifers

Surficial aquifer system

Brunswick aquifer system

Valley and Ridge aquifer
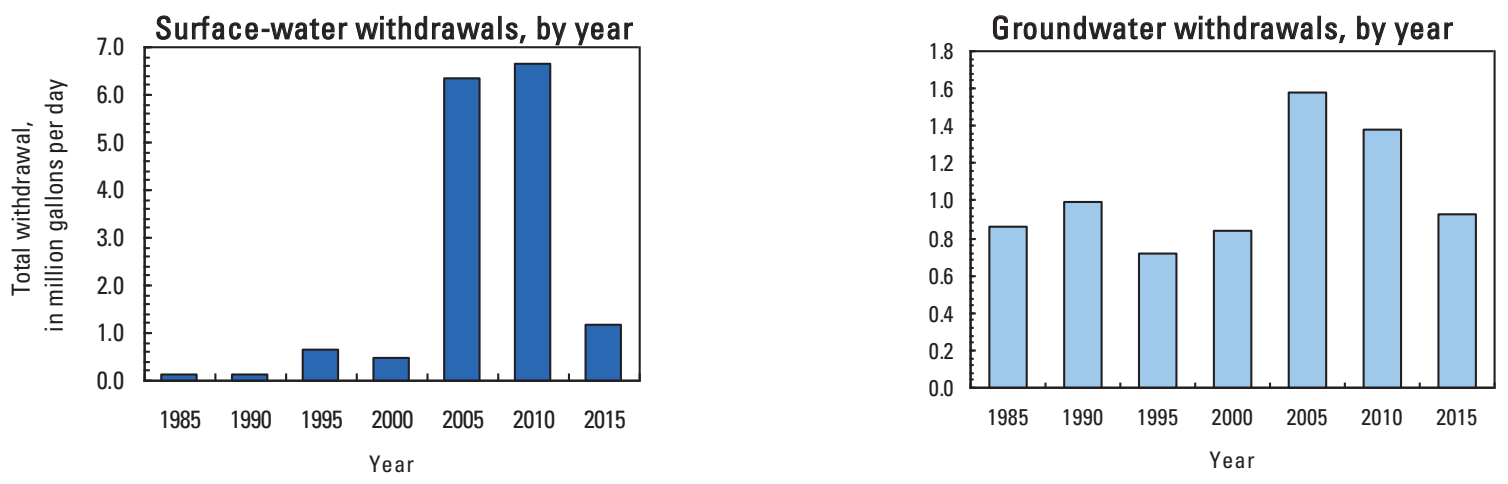


\section{UPSON COUNTY}

Population

Population served by public supply-groundwater

Population served by public supply-surface water 11,410

Acres irrigated

950

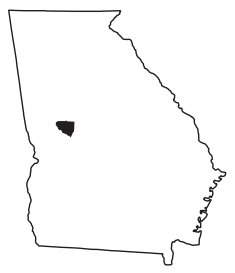

2015 WATER WITHDRAWALS AND ESTIMATED USE, IN MILLION GALLONS PER DAY

[--,not applicable; Mgal/d, million gallons per day]

\begin{tabular}{lcccccc}
\hline & \multicolumn{3}{c}{ Withdrawals } & & Total \\
\cline { 2 - 4 } Category & $\begin{array}{c}\text { Ground- } \\
\text { water }\end{array}$ & $\begin{array}{c}\text { Surface } \\
\text { water }\end{array}$ & $\begin{array}{c}\text { Total } \\
\text { use }\end{array}$ & $\begin{array}{c}\text { Returns to } \\
\text { Surface }\end{array}$ \\
\hline Public supply & 0.54 & 2.48 & 3.02 & -- & -- \\
Domestic use & 0.87 & 0.00 & 0.87 & 2.67 & 0.00 \\
Commercial use & 0.00 & 0.00 & 0.00 & 0.44 & 0.00 \\
Industrial use & 0.00 & 0.28 & 0.28 & 0.35 & 0.00 \\
Public-supply loss & -- & -- & -- & 0.71 & -- \\
Inter-county delivery & -- & -- & -- & 0.00 & -- \\
Public wastewater treatment & -- & -- & -- & -- & 2.83 \\
Mining use & 0.00 & 0.00 & 0.00 & 0.00 & 0.00 \\
Irrigation (crop) & 0.00 & 0.40 & 0.40 & 0.40 & 0.00 \\
Irrigation (golf) & 0.00 & 0.00 & 0.00 & 0.00 & 0.00 \\
Livestock-aquaculture & 0.02 & 0.24 & 0.26 & 0.26 & 0.00 \\
Thermoelectric power & 0.00 & 0.00 & 0.00 & 0.00 & 0.00 \\
\hline TOTAL & 1.43 & 3.40 & 4.83 & 4.83 & 2.83 \\
\hline
\end{tabular}

Withdrawals by Major Industrial Groups

[NAICS, North American Industrial Classification System code]

NAICS Groundwater Surface water

313- Textile mills

0.00

0.28

Withdrawals by Major Public Suppliers

\begin{tabular}{lcc} 
Name & Groundwater & Surface water \\
\hline City of Thomaston & 0.00 & 2.48 \\
Upson County & 0.36 & 0.00 \\
Water Department & &
\end{tabular}

Total use is total withdrawal plus public supply deliveries and losses.

Withdrawals by water source

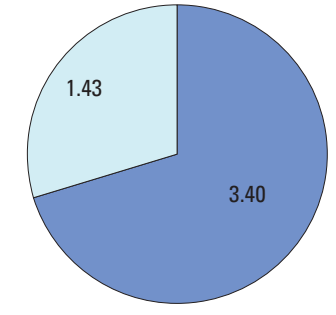

Withdrawals, in million gallons per day

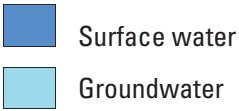

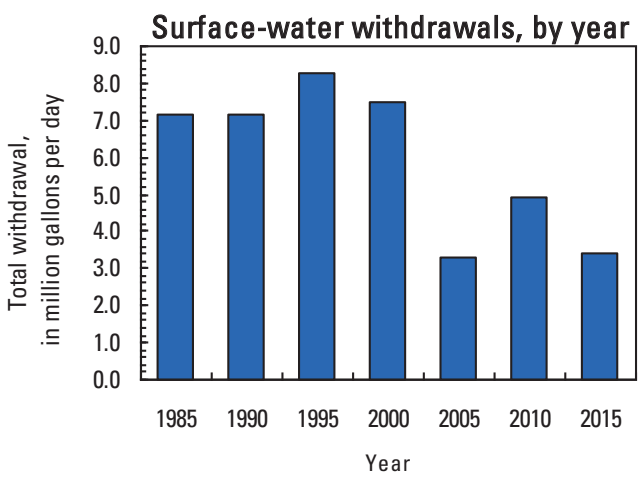

Groundwater withdrawals by aquifer

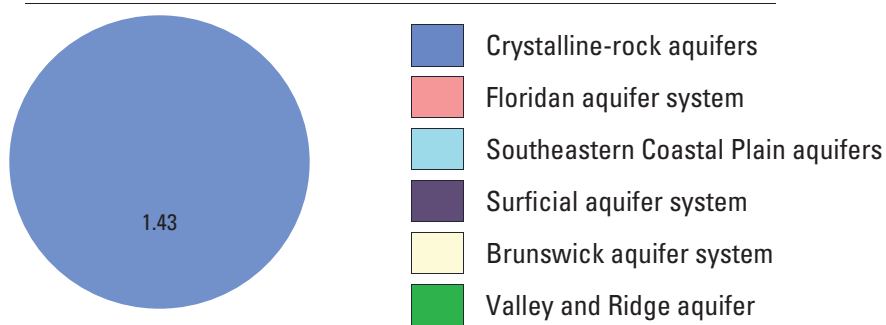

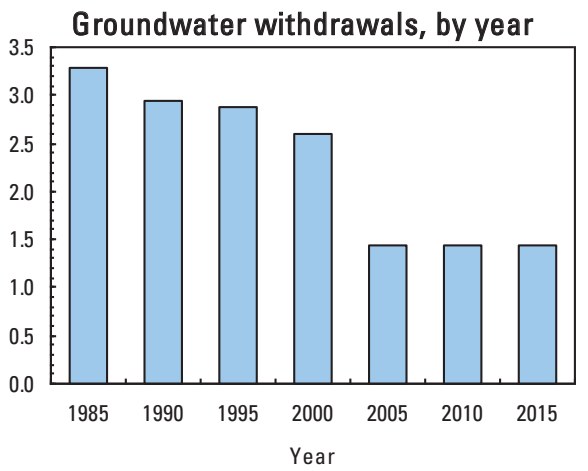




\section{WALKER COUNTY}

Population

Population served by public supply-groundwater $\quad 48,338$

Population served by public supply-surface water $\quad 13,259$

Acres irrigated

2015 WATER WITHDRAWALS AND ESTIMATED USE, IN MILLION GALLONS PER DAY

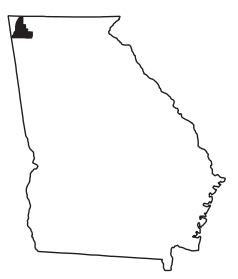

[--,not applicable; Mgal/d, million gallons per day]

\begin{tabular}{|c|c|c|c|c|c|}
\hline \multirow[b]{2}{*}{ Category } & \multicolumn{3}{|c|}{ Withdrawals } & \multirow[b]{2}{*}{$\begin{array}{c}\text { Total } \\
\text { use }\end{array}$} & \multirow[b]{2}{*}{$\begin{array}{c}\text { Returns to } \\
\text { Surface }\end{array}$} \\
\hline & $\begin{array}{c}\text { Ground- } \\
\text { water }\end{array}$ & $\begin{array}{c}\text { Surface } \\
\text { water }\end{array}$ & Total & & \\
\hline Public supply & 5.58 & 1.04 & 6.62 & -- & -- \\
\hline Domestic use & 0.49 & 0.00 & 0.49 & 5.32 & 0.00 \\
\hline Commercial use & 0.00 & 0.00 & 0.00 & 1.65 & 0.00 \\
\hline Industrial use & 0.03 & 0.60 & 0.63 & 1.04 & 0.00 \\
\hline Public-supply loss & -- & -- & -- & -0.27 & - \\
\hline Inter-county delivery & -- & -- & -- & 2.32 & - \\
\hline Public wastewater treatmen & t -- & -- & -- & -- & 4.48 \\
\hline Mining use & 0.00 & 0.00 & 0.00 & 0.00 & 0.00 \\
\hline Irrigation (crop) & 0.00 & 0.00 & 0.00 & 0.00 & 0.00 \\
\hline Irrigation (golf) & 0.00 & 0.00 & 0.00 & 0.00 & 0.00 \\
\hline Livestock-aquaculture & 0.18 & 0.34 & 0.52 & 0.52 & 0.00 \\
\hline Thermoelectric power & 0.00 & 0.00 & 0.00 & 0.00 & 0.00 \\
\hline TOTAL & 6.28 & 1.98 & 8.26 & 10.58 & 4.48 \\
\hline
\end{tabular}

Withdrawals by Major Industrial Groups

[NAICS, North American Industrial Classification System code]

\begin{tabular}{lll}
\hline NAICS & Groundwater Surface water \\
\hline
\end{tabular}

313- Textile mills

0.03

0.60

Withdrawals by Major Public Suppliers

\begin{tabular}{lcc} 
Name & Groundwater & Surface wat \\
\hline $\begin{array}{l}\text { Water County Water \& } \\
\text { Sewerage Authority }\end{array}$ & 3.60 & 0.00 \\
$\begin{array}{l}\text { Walker County Rural Water } \\
\text { \& Sewer Authority }\end{array}$ & 0.33 & 0.00 \\
City of Lafayette & 0.88 & 1.04 \\
City of Chickamauga & 0.77 & 0.00
\end{tabular}

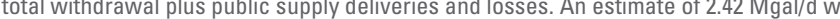

delivered from neighboring counties in 2015. An estimate of $0.1 \mathrm{Mgal} / \mathrm{d}$ was delivered from Walker

County to neighboring counties in 2015.

\section{Withdrawals by water source}

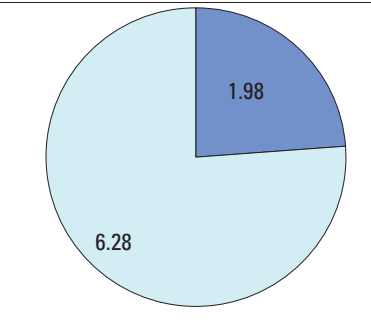

Withdrawals, in million

gallons per day

Surface water

Groundwater

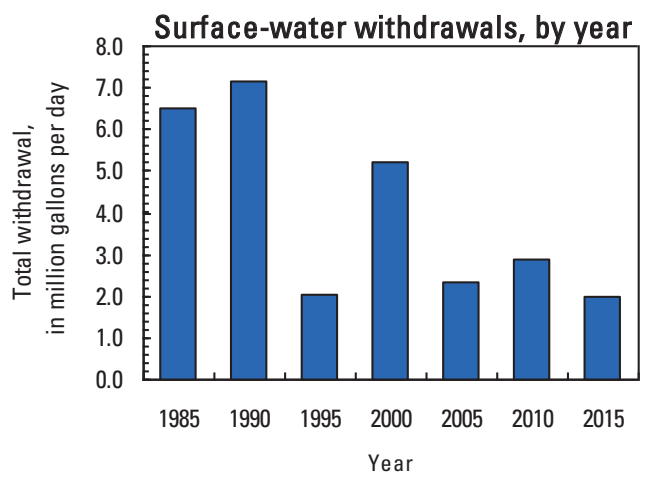

Groundwater withdrawals by aquifer
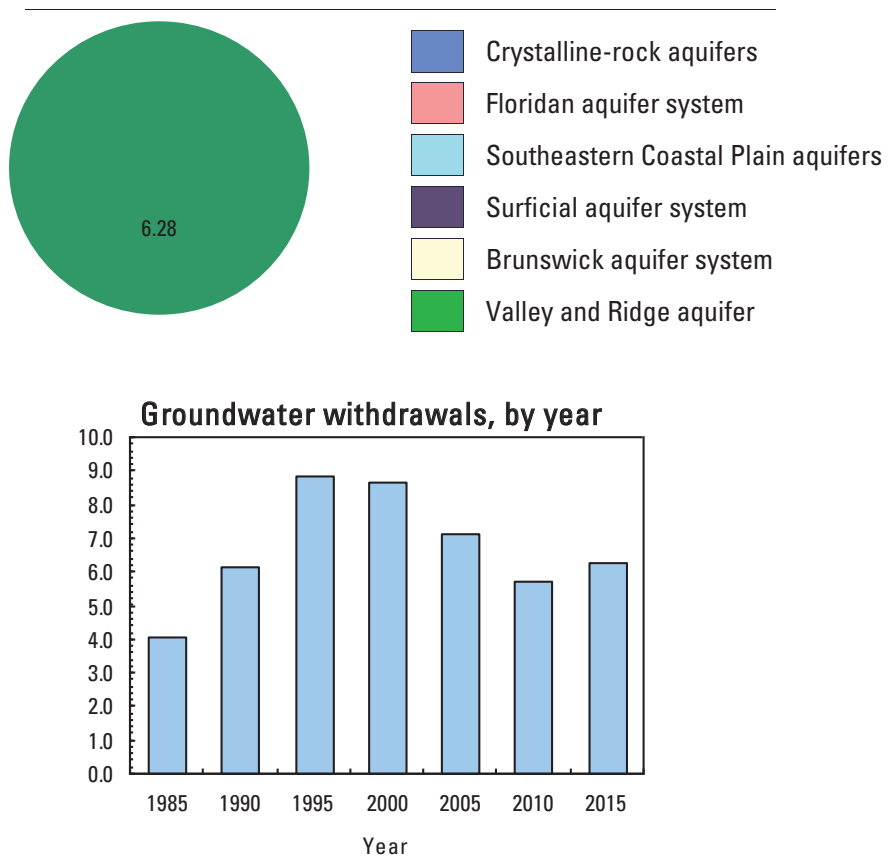


\section{WALTON COUNTY}

Population

88,399

Population served by public supply-groundwater

Population served by public supply-surface water $\quad 62,361$

Acres irrigated

2015 WATER WITHDRAWALS AND ESTIMATED USE, IN MILLION GALLONS PER DAY

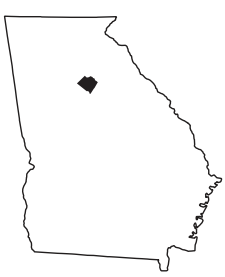

[--,not applicable; Mgal/d, million gallons per day]

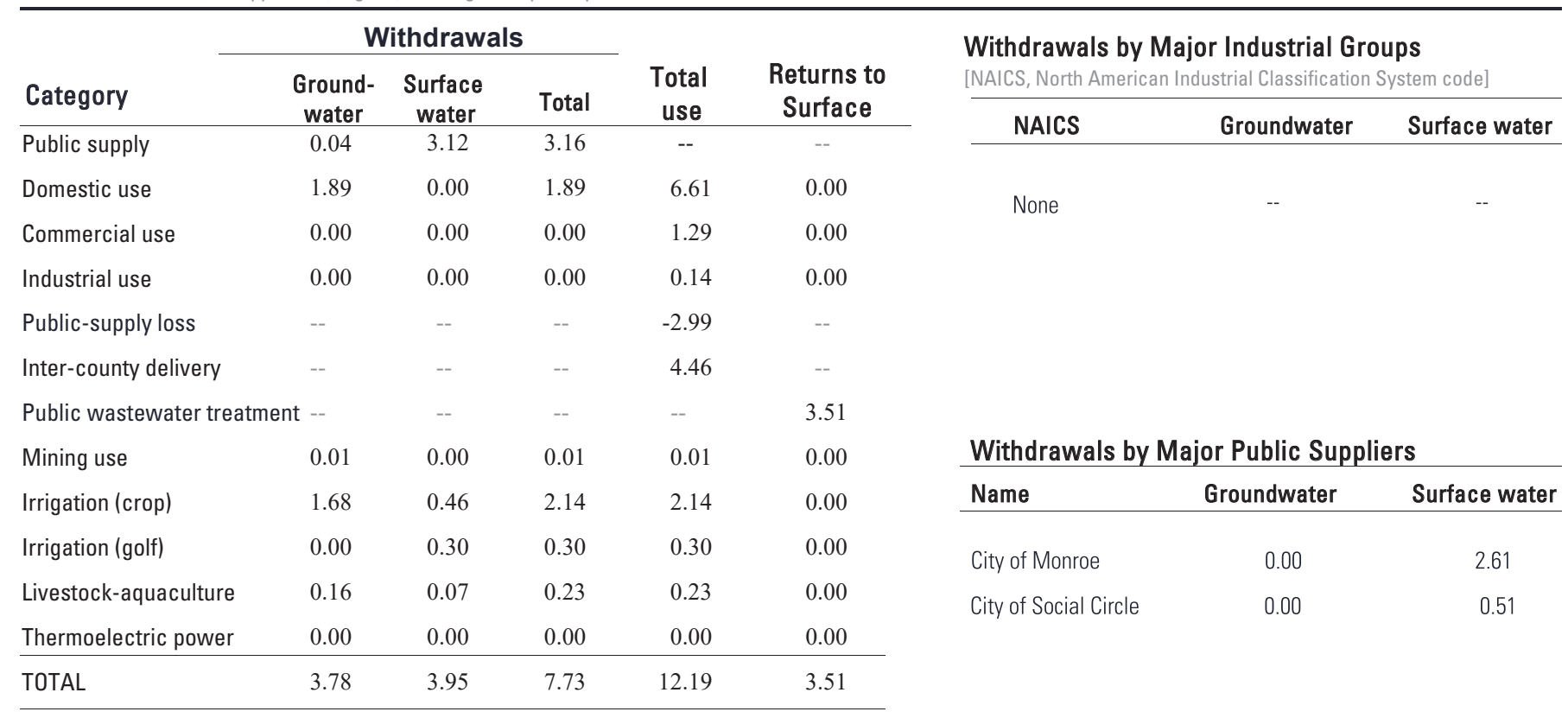

Total use is total withdrawal plus public supply deliveries and losses. An estimate of $4.46 \mathrm{Mgal} / \mathrm{d}$ was delivered from neighboring counties in 2015.

\section{Withdrawals by water source}

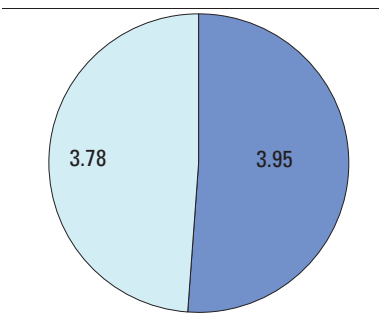

Withdrawals, in million gallons per day

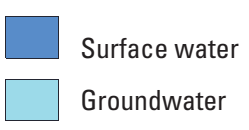

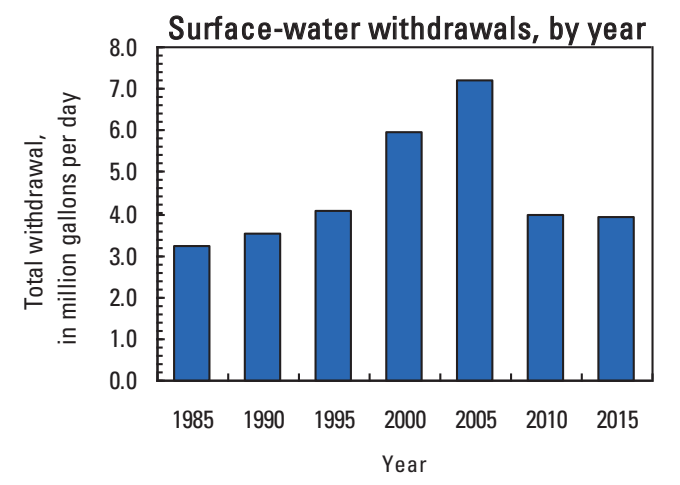

Groundwater withdrawals by aquifer

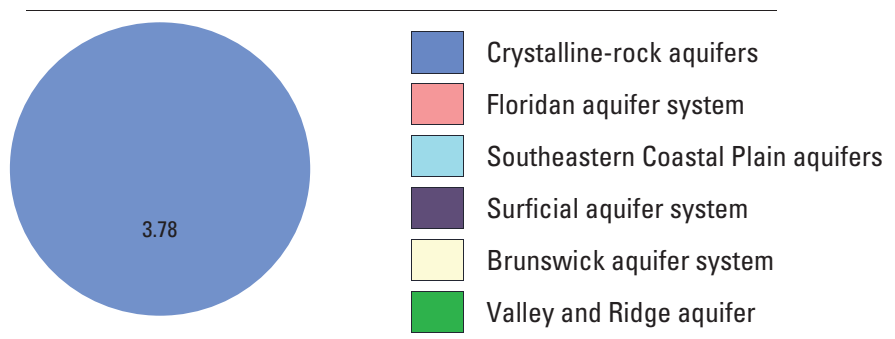




\section{WARE COUNTY}

Population

Population served by public supply-groundwater $\quad 31,485$

Population served by public supply-surface water $\quad 0$

Acres irrigated

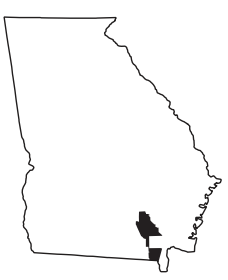

2015 WATER WITHDRAWALS AND ESTIMATED USE, IN MILLION GALLONS PER DAY

[--,not applicable; Mgal/d, million gallons per day]

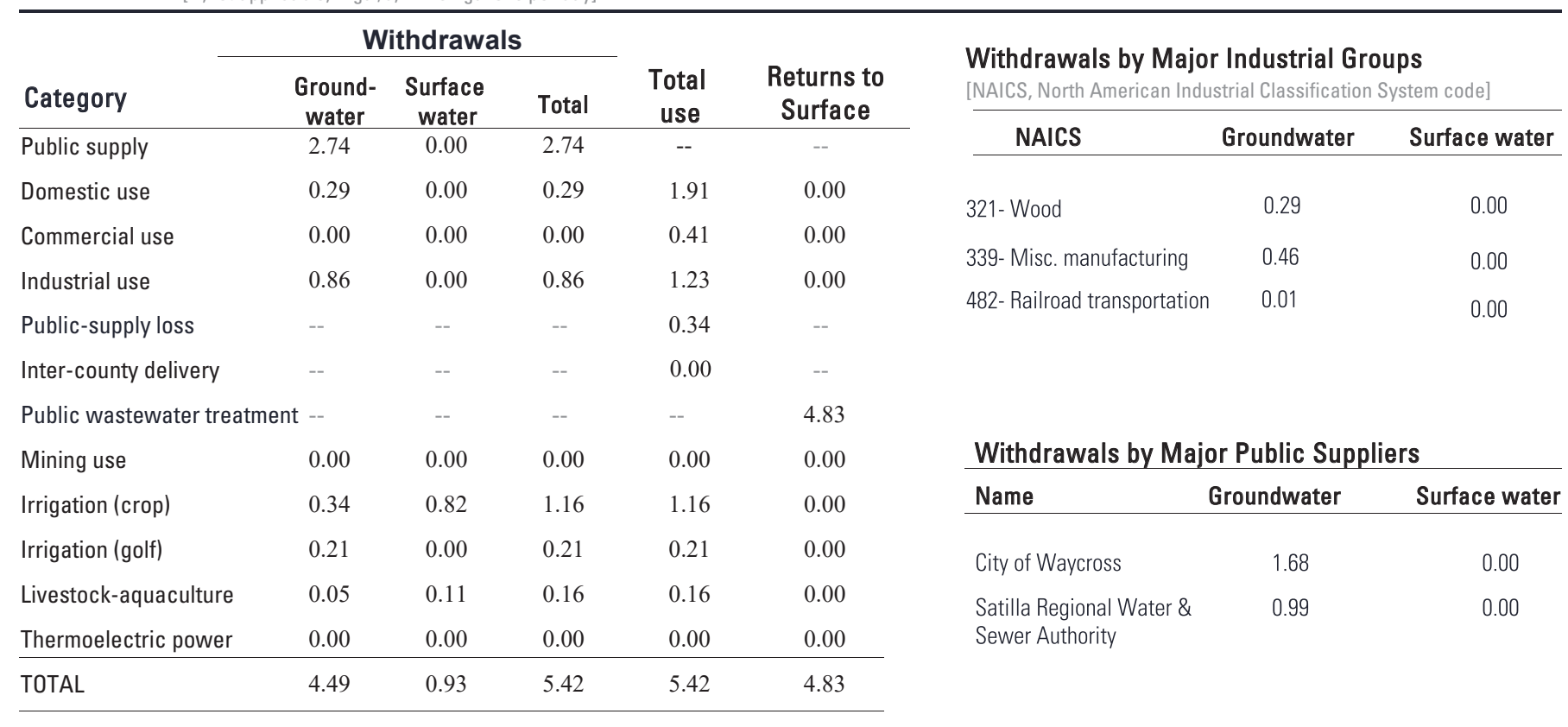

Total use is total withdrawal plus public supply deliveries and losses.

Withdrawals by water source

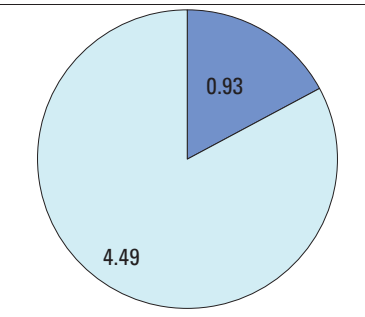

Withdrawals, in million

gallons per day

Surface water

Groundwater

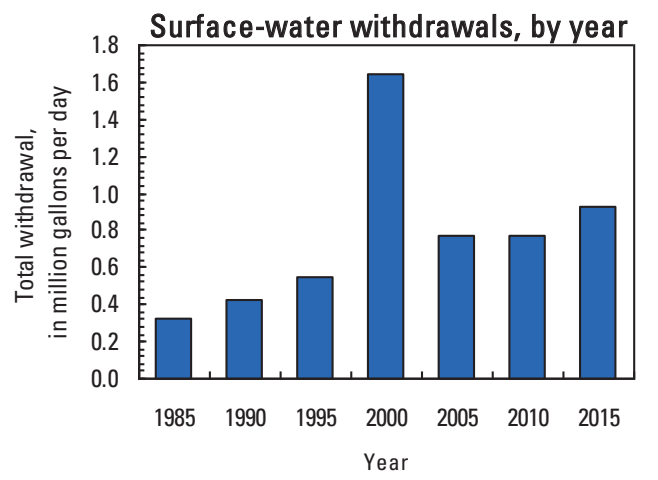

Groundwater withdrawals by aquifer

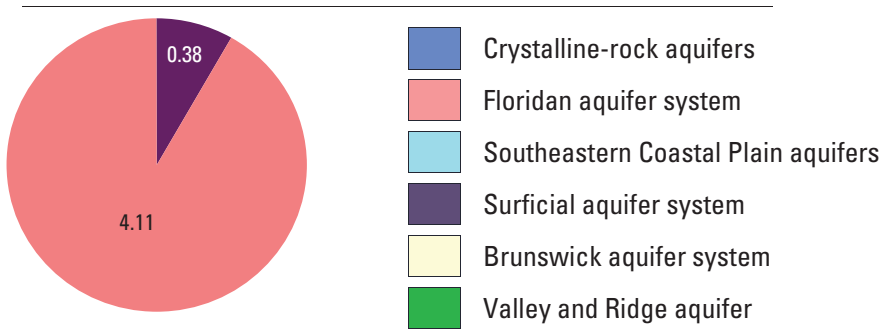




\section{WARREN COUNTY}

Population

5,460

Population served by public supply-groundwater $\quad 364$

Population served by public supply-surface water $\quad 3,271$

Acres irrigated

2015 WATER WITHDRAWALS AND ESTIMATED USE, IN MILLION GALLONS PER DAY

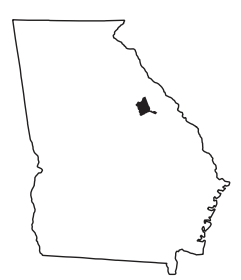

[--,not applicable; Mgal/d, million gallons per day]

\begin{tabular}{|c|c|c|c|c|c|c|c|c|}
\hline \multirow{3}{*}{$\begin{array}{l}\text { Category } \\
\text { Public supply }\end{array}$} & \multicolumn{3}{|c|}{ Withdrawals } & \multirow{2}{*}{$\begin{array}{c}\text { Total } \\
\text { use }\end{array}$} & \multirow{2}{*}{$\begin{array}{l}\text { Returns to } \\
\text { Surface }\end{array}$} & \multirow{2}{*}{\multicolumn{3}{|c|}{$\begin{array}{l}\text { Withdrawals by Major Industrial Groups } \\
\text { [NAICS, North American Industrial Classification System code] }\end{array}$}} \\
\hline & \multirow{2}{*}{$\begin{array}{c}\begin{array}{c}\text { Ground } \\
\text { water }\end{array} \\
0.02\end{array}$} & \multirow{2}{*}{$\begin{array}{c}\text { Surface } \\
\text { water }\end{array}$} & \multirow{2}{*}{$\begin{array}{c}\text { Total } \\
0.32\end{array}$} & & & & & \\
\hline & & & & & surtace & NAICS & Groundwater & Surface water \\
\hline Domestic use & 0.14 & 0.00 & 0.14 & 0.36 & 0.00 & \multirow{3}{*}{ 212- Mining } & \multirow{3}{*}{0.14} & \multirow{3}{*}{0.39} \\
\hline Commercial use & 0.00 & 0.00 & 0.00 & 0.04 & 0.00 & & & \\
\hline Industrial use & 0.14 & 0.00 & 0.14 & 0.16 & 0.00 & & & \\
\hline Public-supply loss & -- & -- & -- & 0.04 & -- & & & \\
\hline Inter-county delivery & -- & -- & -- & 0.00 & -- & & & \\
\hline Public wastewater treatment & t -- & -- & -- & -- & 6.46 & & & \\
\hline Mining use & 0.16 & 0.10 & 0.26 & 0.26 & 0.00 & \multicolumn{3}{|c|}{ Withdrawals by Major Public Suppliers } \\
\hline Irrigation (crop) & 0.00 & 0.00 & 0.00 & 0.00 & 0.00 & Name & Groundwater & Surface water \\
\hline Irrigation (golf) & 0.00 & 0.00 & 0.00 & 0.00 & 0.00 & \multirow[t]{4}{*}{ City of Warrenton } & \multirow[t]{4}{*}{0.00} & \multirow[t]{3}{*}{0.30} \\
\hline Livestock-aquaculture & 0.16 & 0.05 & 0.21 & 0.21 & 0.00 & & & \\
\hline Thermoelectric power & 0.00 & 0.00 & 0.00 & 0.00 & 0.00 & & & \\
\hline TOTAL & 0.62 & 0.45 & 1.07 & 1.07 & 6.46 & & & \\
\hline
\end{tabular}

Total use is total withdrawal plus public supply deliveries and losses.

Withdrawals by water source

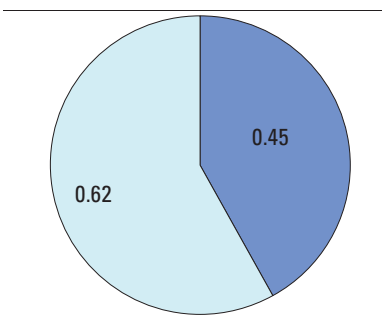

Withdrawals, in million gallons per day

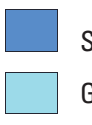

Surface water Groundwater

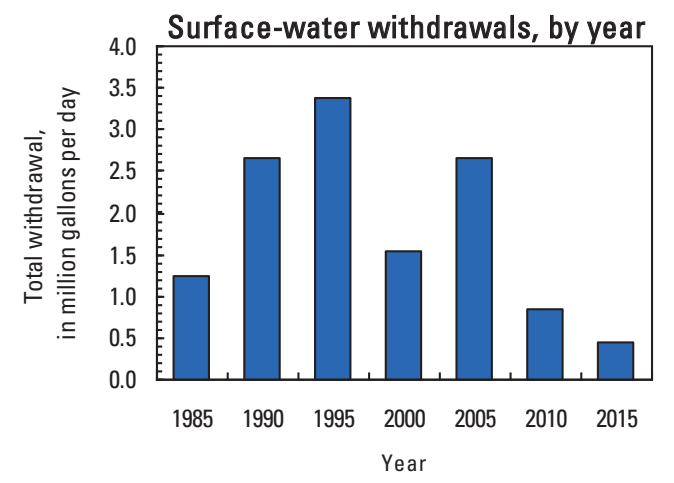

\section{Groundwater withdrawals by aquifer}
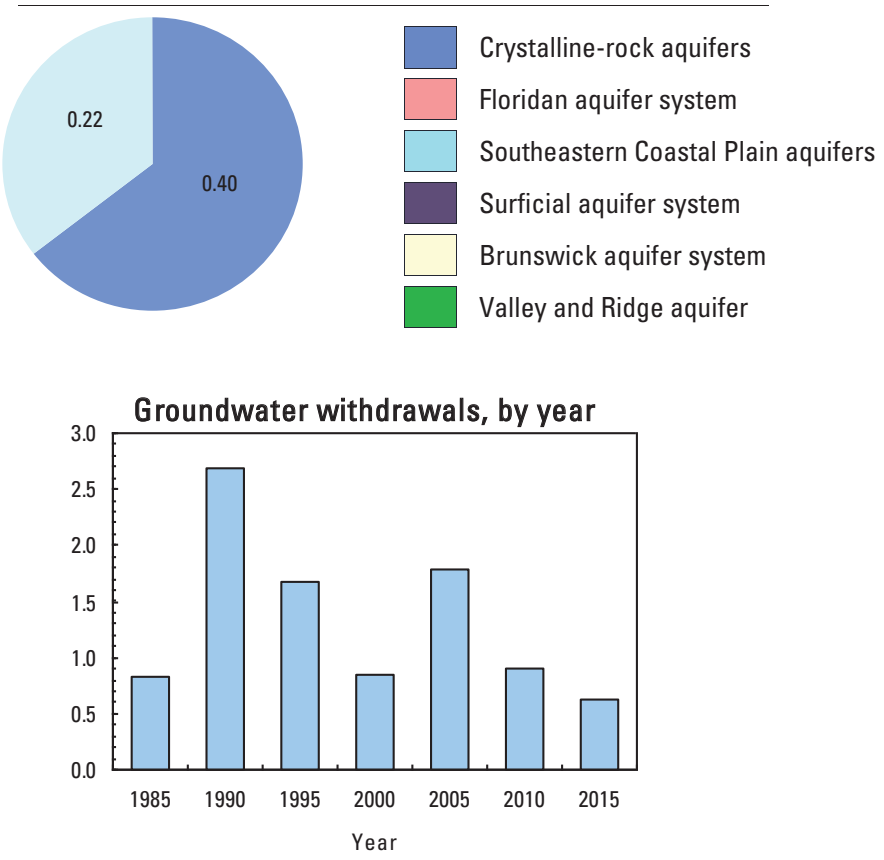


\section{WASHINGTON COUNTY}

Population

Population served by public supply-groundwater

Population served by public supply-surface water

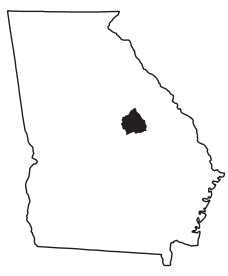

2015 WATER WITHDRAWALS AND ESTIMATED USE, IN MILLION GALLONS PER DAY

[--,not applicable; Mgal/d, million gallons per day]

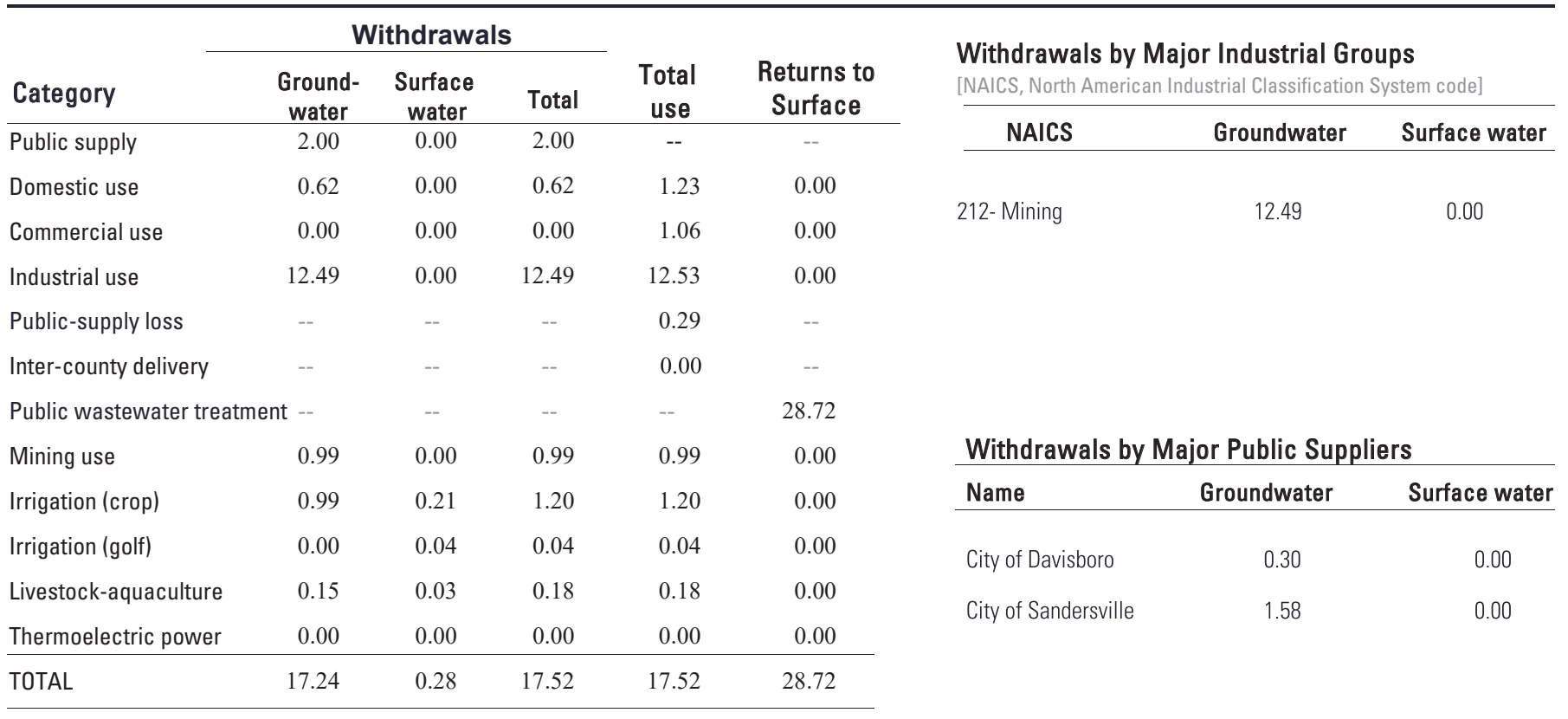

Total use is total withdrawal plus public supply deliveries and losses.

Withdrawals by water source

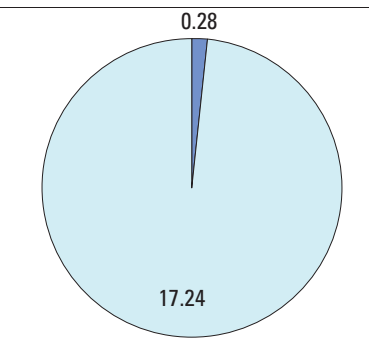

Withdrawals, in million gallons per day

Surface water

Groundwater

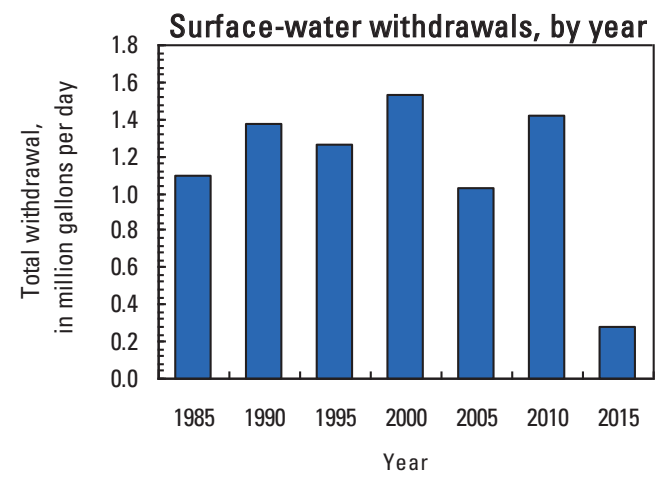

Groundwater withdrawals by aquifer

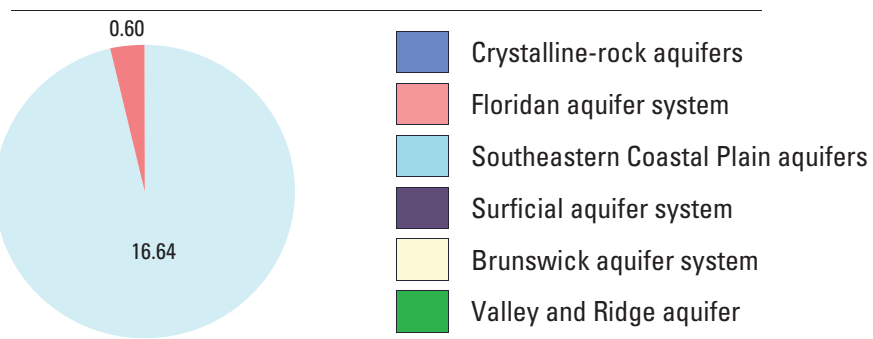

Groundwater withdrawals, by year

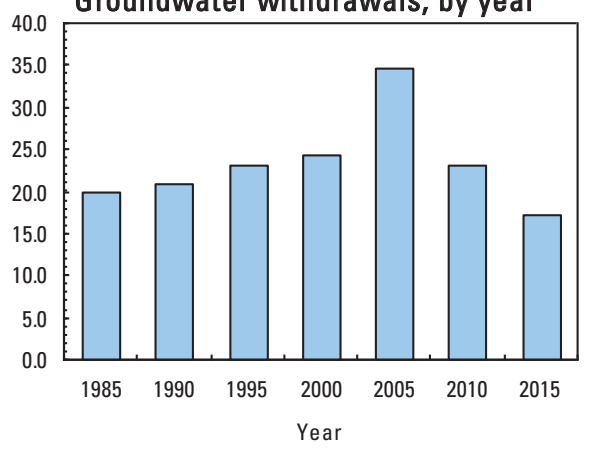




\section{WAYNE COUNTY}

Population

Population served by public supply-groundwater $\quad 13,756$

Population served by public supply-surface water $\quad 0$

Acres irrigated

2015 WATER WITHDRAWALS AND ESTIMATED USE, IN MILLION GALLONS PER DAY

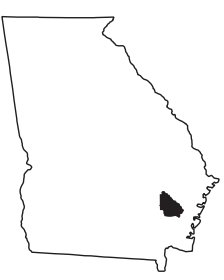

[--,not applicable; Mgal/d, million gallons per day]

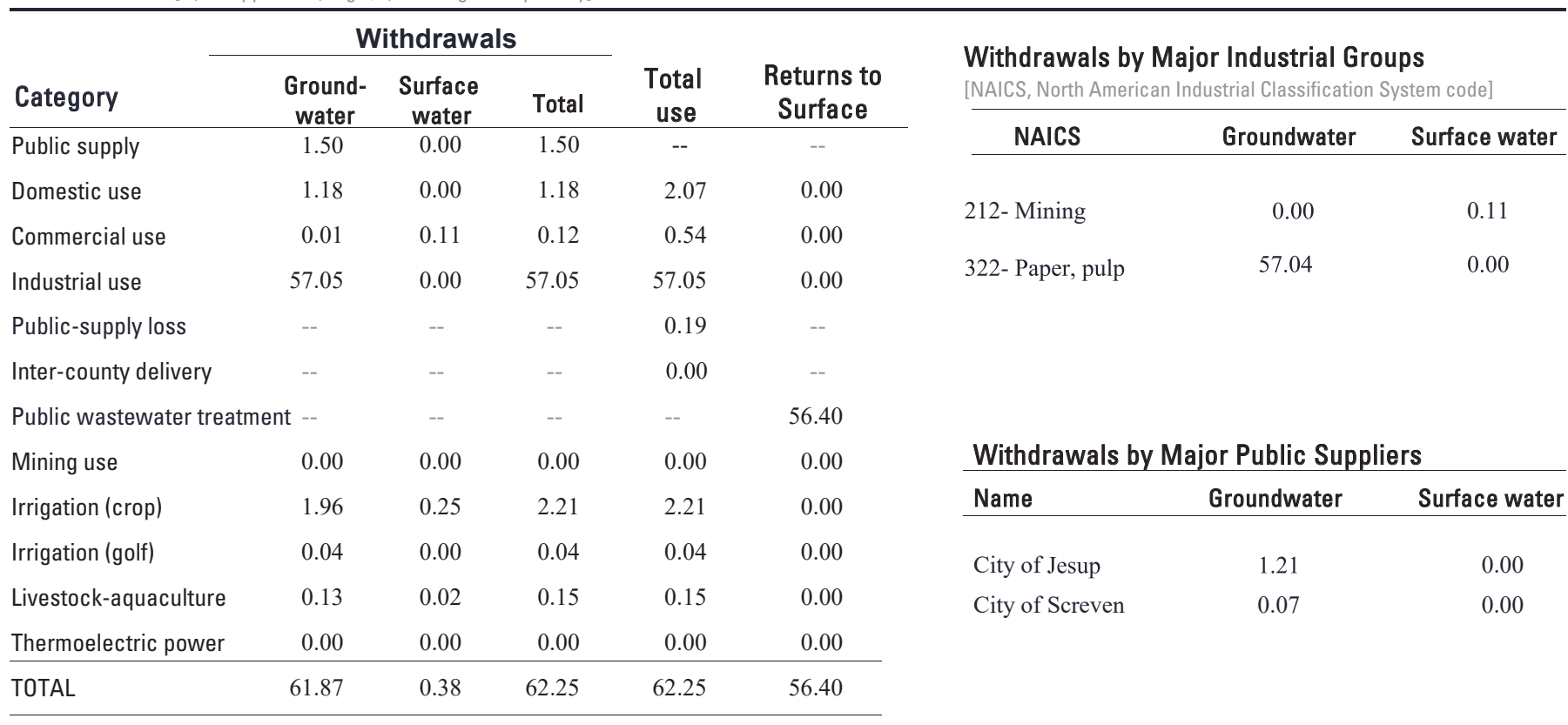

Total use is total withdrawal plus public supply deliveries and losses.

Withdrawals by water source

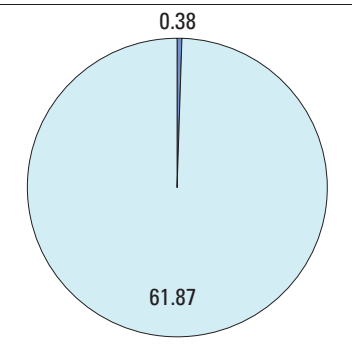

Withdrawals, in million gallons per day

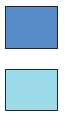

Surface water

Groundwater

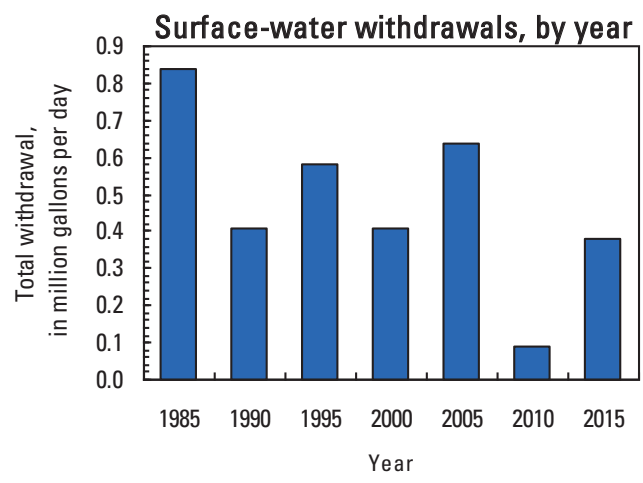

Groundwater withdrawals by aquifer
\begin{aligned} & \hline Crystalline-rock aquifers \\ & \hline 0.14 Floridan aquifer system \\ & \hline Southeastern Coastal Plain aquifers \\ & Surficial aquifer system \\ &$\square 1.73$ Brunswick aquifer system \\ & Valley and Ridge aquifer \\ &\end{aligned}

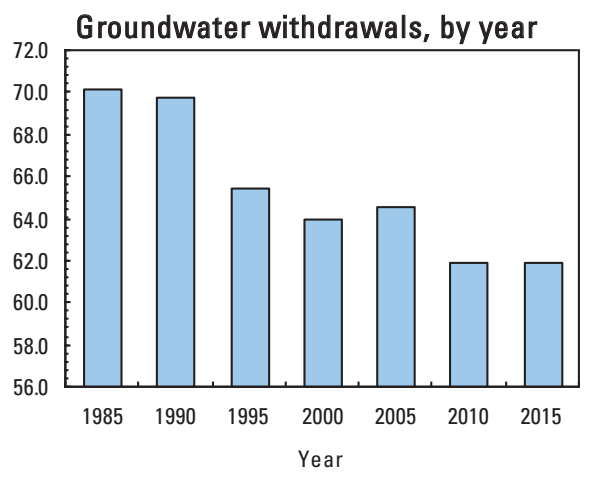




\section{WEBSTER COUNTY}

Population

Population served by public supply-groundwater

1,038

Population served by public supply-surface water

0

Acres irrigated

11,070

2015 WATER WITHDRAWALS AND ESTIMATED USE, IN MILLION GALLONS PER DAY

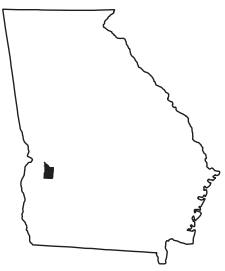

[--,not applicable; Mgal/d, million gallons per day]

\begin{tabular}{|c|c|c|c|c|c|c|c|c|}
\hline \multirow{3}{*}{ Category } & \multicolumn{3}{|c|}{ Withdrawals } & \multirow{3}{*}{$\begin{array}{c}\text { Total } \\
\text { use }\end{array}$} & \multirow{3}{*}{$\begin{array}{l}\text { Returns to } \\
\text { Surface }\end{array}$} & \multirow{2}{*}{\multicolumn{3}{|c|}{$\begin{array}{l}\text { Withdrawals by Major Industrial Groups } \\
\text { [NAICS, North American Industrial Classification System code] }\end{array}$}} \\
\hline & \multirow{2}{*}{$\begin{array}{c}\text { Ground } \\
\text { water }\end{array}$} & \multirow{2}{*}{$\begin{array}{c}\text { Surface } \\
\text { water }\end{array}$} & \multirow{2}{*}{ Total } & & & & & \\
\hline & & & & & & \multirow{2}{*}{ NAICS } & \multirow{2}{*}{ Groundwater } & \multirow{2}{*}{ Surface water } \\
\hline Public supply & 0.10 & 0.00 & 0.10 & -- & -- & & & \\
\hline Domestic use & 0.12 & 0.00 & 0.12 & 0.20 & 0.00 & \multirow{3}{*}{ None } & \multirow{2}{*}{--} & \multirow[t]{3}{*}{--} \\
\hline Commercial use & 0.00 & 0.00 & 0.00 & 0.00 & 0.00 & & & \\
\hline Industrial use & 0.00 & 0.00 & 0.00 & 0.00 & 0.00 & & & \\
\hline Public-supply loss & -- & -- & -- & 0.02 & -- & & & \\
\hline Inter-county delivery & -- & -- & -- & 0.00 & - & & & \\
\hline Public wastewater treatment & tt -- & -- & -- & -- & 0.00 & & & \\
\hline Mining use & 0.00 & 0.00 & 0.00 & 0.00 & 0.00 & \multicolumn{3}{|c|}{ Withdrawals by Major Public Suppliers } \\
\hline Irrigation (crop) & 0.95 & 3.42 & 4.37 & 4.37 & 0.00 & Name & Groundwater & Surface water \\
\hline Irrigation (golf) & 0.00 & 0.00 & 0.00 & 0.00 & 0.00 & \multirow{4}{*}{$\begin{array}{l}\text { Unified Government of } \\
\text { Webster County }\end{array}$} & \multirow[t]{4}{*}{0.10} & \multirow[t]{3}{*}{0.00} \\
\hline Livestock-aquaculture & 0.01 & 0.03 & 0.04 & 0.04 & 0.00 & & & \\
\hline Thermoelectric power & 0.00 & 0.00 & 0.00 & 0.00 & 0.00 & & & \\
\hline TOTAL & 1.18 & 3.45 & 4.63 & 4.63 & 0.00 & & & \\
\hline
\end{tabular}

Total use is total withdrawal plus public supply deliveries and losses.

Withdrawals by water source

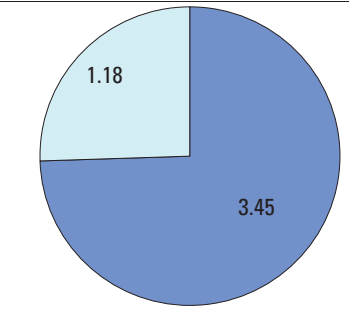

Withdrawals, in million

gallons per day

Surface water

Groundwater
Groundwater withdrawals by aquifer

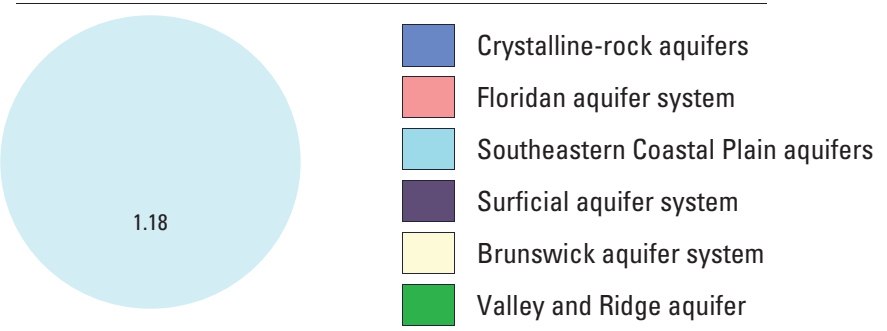

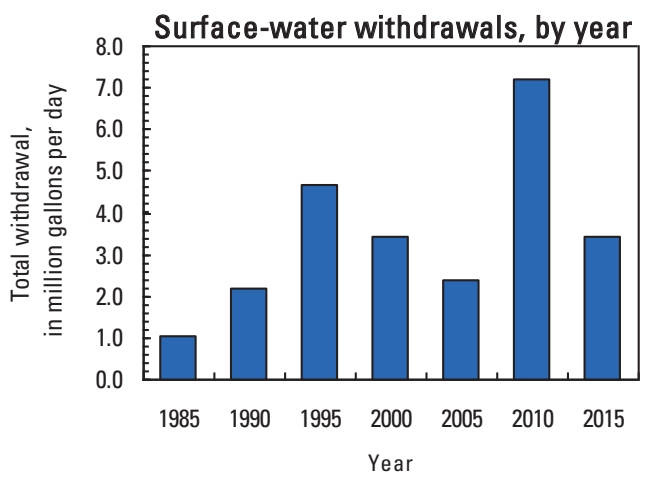

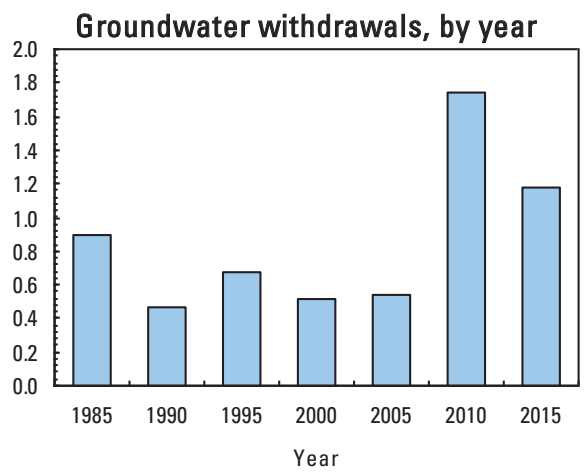




\section{WHEELER COUNTY}

Population

Population served by public supply-groundwater

Population served by public supply-surface water

Acres irrigated

2015 WATER WITHDRAWALS AND ESTIMATED USE, IN MILLION GALLONS PER DAY

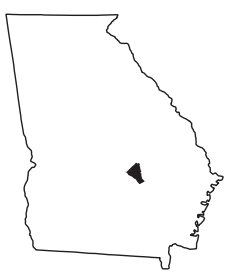

[--,not applicable; Mgal/d, million gallons per day]

\begin{tabular}{|c|c|c|c|c|c|c|c|c|}
\hline \multirow{2}{*}{ Category } & \multicolumn{3}{|c|}{ Withdrawals } & \multirow{3}{*}{$\begin{array}{c}\text { Total } \\
\text { use }\end{array}$} & \multirow{3}{*}{$\begin{array}{l}\text { Returns to } \\
\text { Surface }\end{array}$} & \multirow{2}{*}{\multicolumn{3}{|c|}{$\begin{array}{l}\text { Withdrawals by Major Industrial Groups } \\
\text { [NAICS, North American Industrial Classification System code] }\end{array}$}} \\
\hline & \multirow{2}{*}{$\begin{array}{l}\text { Ground- } \\
\text { water }\end{array}$} & $\begin{array}{c}\text { Surface } \\
\text { water }\end{array}$ & \multirow{2}{*}{ Total } & & & & & \\
\hline Public supply & & $\begin{array}{c}\text { water } \\
0.00\end{array}$ & & & & \multirow[t]{3}{*}{ NAICS } & Groundwater & Surface water \\
\hline Domestic use & 0.26 & 0.00 & 0.26 & 0.38 & 0.00 & & & \\
\hline Commercial use & 0.00 & 0.00 & 0.00 & 0.03 & 0.00 & & - & - \\
\hline Inter-county delivery & -- & -- & -- & 0.00 & -- & & & \\
\hline \multicolumn{2}{|l|}{ Public wastewater treatment -- } & -- & -- & -- & 0.47 & & & \\
\hline Mining use & 0.00 & 0.00 & 0.00 & 0.00 & 0.00 & \multicolumn{3}{|c|}{ Withdrawals by Major Public Suppliers } \\
\hline Irrigation (crop) & 1.16 & 0.64 & 1.80 & 1.80 & 0.00 & Name & Groundwater & Surface water \\
\hline TOTAL & 1.88 & 0.69 & 2.57 & 2.57 & 0.47 & & & \\
\hline
\end{tabular}

Total use is total withdrawal plus public supply deliveries and losses.

Withdrawals by water source

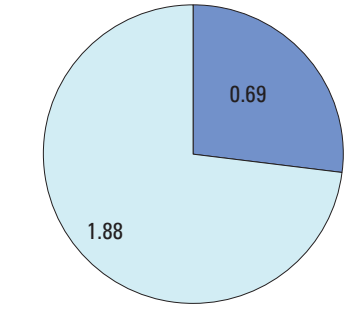

Withdrawals, in million gallons per day

Surface water Groundwater

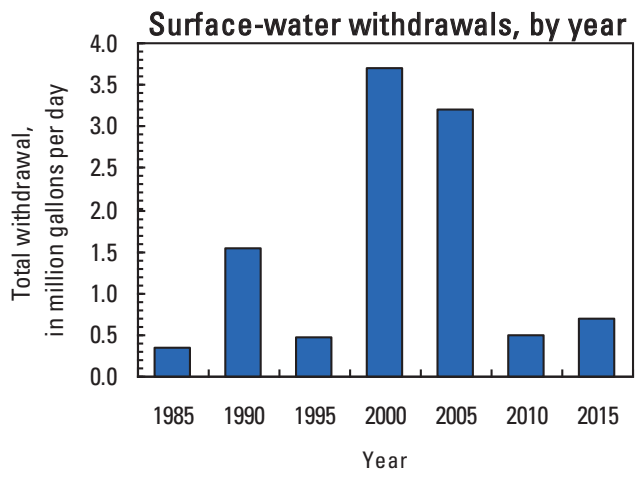

Groundwater withdrawals by aquifer
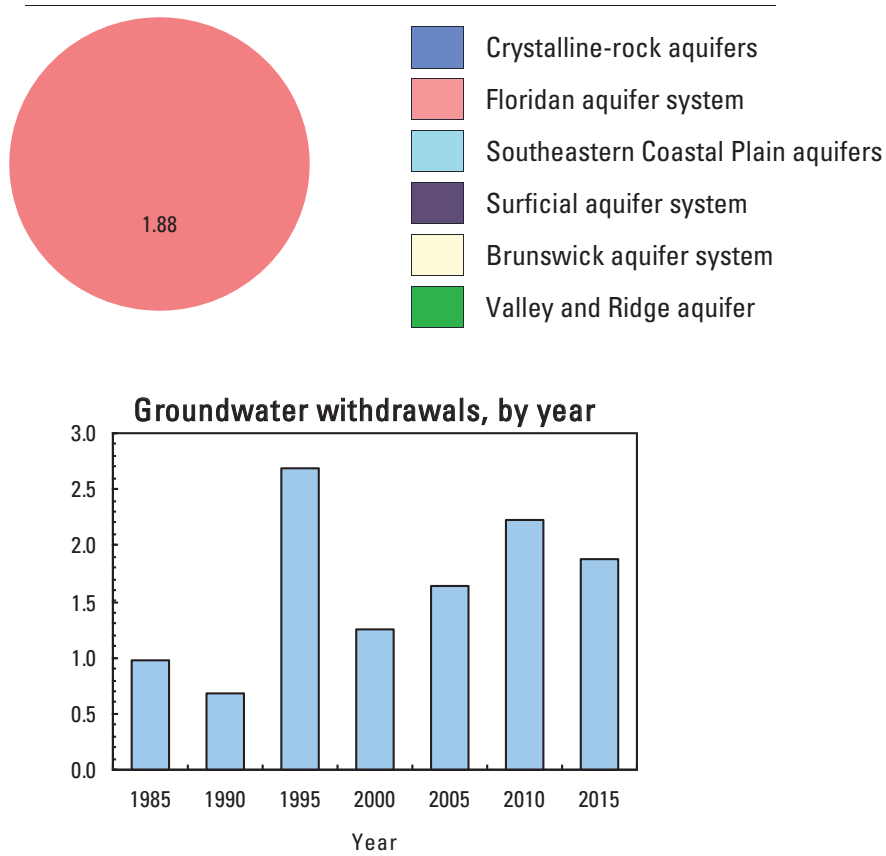


\section{WHITE COUNTY}

Population

Population served by public supply-groundwater

Population served by public supply-surface water

Acres irrigated

2015 WATER WITHDRAWALS AND ESTIMATED USE, IN MILLION GALLONS PER DAY

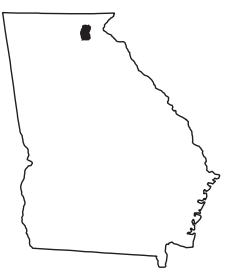

[--,not applicable; Mgal/d, million gallons per day]

\begin{tabular}{|c|c|c|c|c|c|}
\hline \multirow[b]{2}{*}{ Category } & \multicolumn{3}{|c|}{ Withdrawals } & \multirow[b]{2}{*}{$\begin{array}{c}\text { Total } \\
\text { use }\end{array}$} & \multirow[b]{2}{*}{$\begin{array}{c}\text { Returns to } \\
\text { Surface }\end{array}$} \\
\hline & $\begin{array}{c}\text { Ground - } \\
\text { water }\end{array}$ & $\begin{array}{c}\text { Surface } \\
\text { water }\end{array}$ & Total & & \\
\hline Public supply & 0.85 & 0.62 & 1.47 & -- & -- \\
\hline Domestic use & 1.05 & 0.00 & 1.05 & 1.86 & 0.00 \\
\hline Commercial use & 0.00 & 0.00 & 0.00 & 0.47 & 0.00 \\
\hline Industrial use & 0.00 & 0.00 & 0.00 & 0.03 & 0.00 \\
\hline Public-supply loss & -- & -- & -- & 0.16 & -- \\
\hline Inter-county delivery & -- & -- & -- & 0.00 & -- \\
\hline Public wastewater treatment & t -- & -- & -- & -- & 0.13 \\
\hline Mining use & 0.00 & 0.00 & 0.00 & 0.00 & 0.00 \\
\hline Irrigation (crop) & 0.00 & 0.00 & 0.00 & 0.00 & 0.00 \\
\hline Irrigation (golf) & 0.00 & 0.00 & 0.00 & 0.00 & 0.00 \\
\hline Livestock-aquaculture & 0.11 & 0.21 & 0.32 & 0.32 & 0.00 \\
\hline Thermoelectric power & 0.00 & 0.00 & 0.00 & 0.00 & 0.00 \\
\hline TOTAL & 2.01 & 0.83 & 2.84 & 2.84 & 0.13 \\
\hline
\end{tabular}

Withdrawals by Major Industrial Groups

[NAICS, North American Industrial Classification System code]

\begin{tabular}{cll}
\hline NAICS & Groundwater & Surface water \\
\hline
\end{tabular}

None

Withdrawals by Major Public Suppliers

Name

Groundwater

Surface water

White County

0.00

0.62

Water \& Sewerage

Authority

Cleveland Waterworks

0.46

0.00

City of Helen

0.27

0.00

Total use is total withdrawal plus public supply deliveries and losses.

Withdrawals by water source

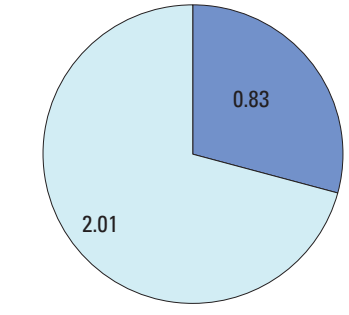

Withdrawals, in million gallons per day

Surface water

Groundwater

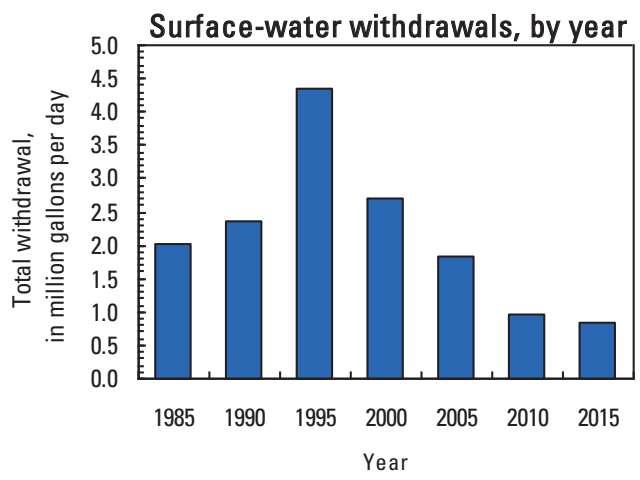

Groundwater withdrawals by aquifer

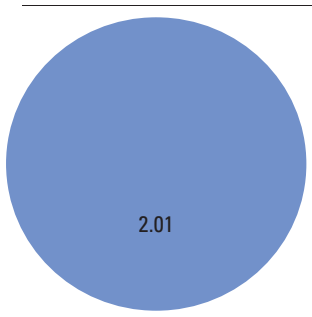

Crystalline-rock aquifers

Floridan aquifer system

Southeastern Coastal Plain aquifers

Surficial aquifer system

Brunswick aquifer system

Valley and Ridge aquifer

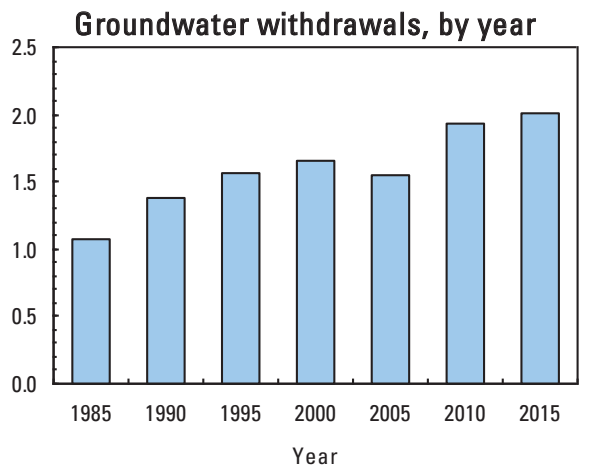




\section{WHITFIELD COUNTY}

Population

Population served by public supply-groundwater

Population served by public supply-surface water $\quad 99,315$

Acres irrigated

2015 WATER WITHDRAWALS AND ESTIMATED USE, IN MILLION GALLONS PER DAY

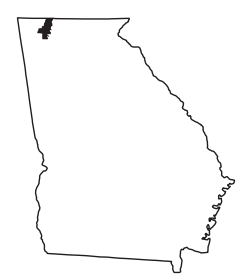

[--,not applicable; Mgal/d, million gallons per day]

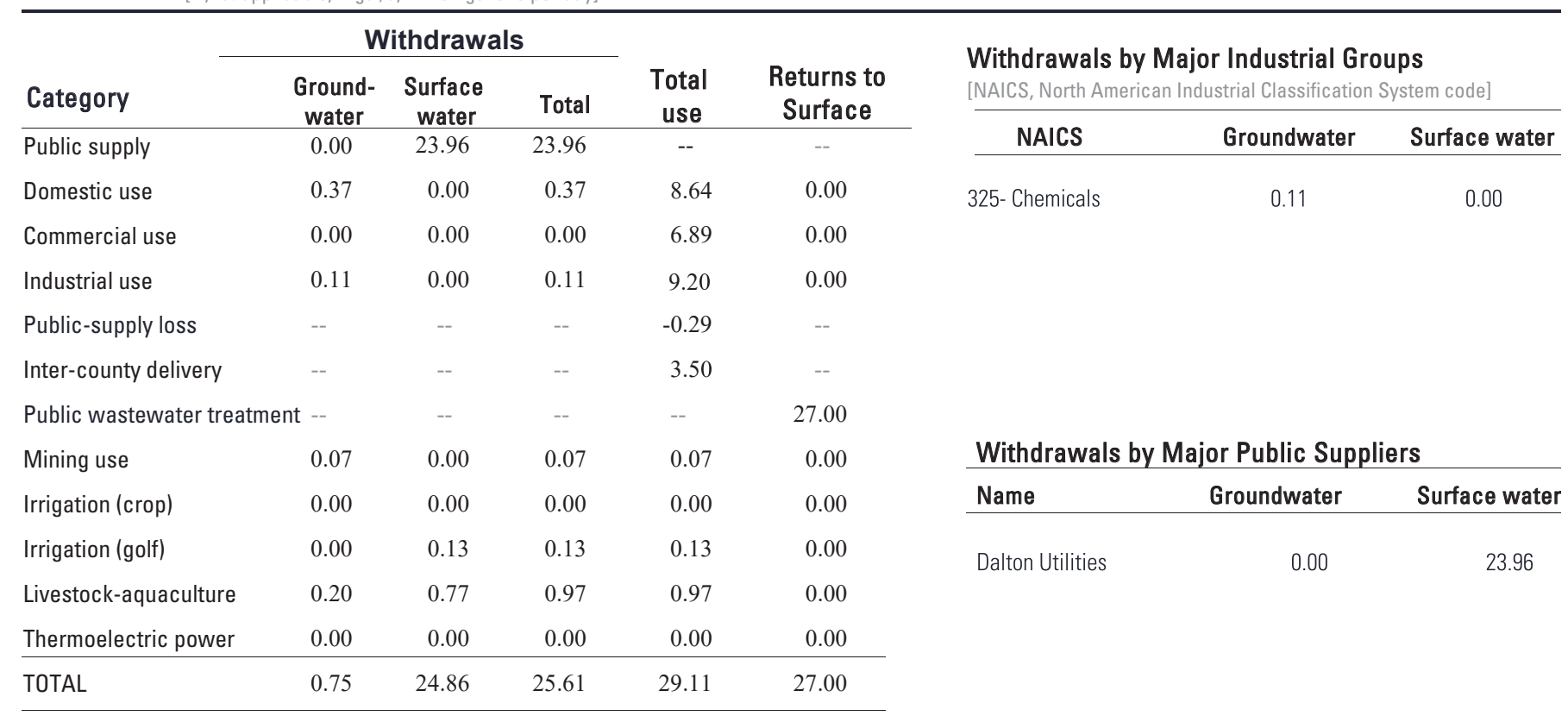

Total use is total withdrawal plus public supply deliveries and losses. An estimate of $4 \mathrm{Mgal} / \mathrm{d}$ was delivered from neighboring counties in 2015. An estimate of $0.5 \mathrm{Mgal} / \mathrm{d}$ was delivered from Whitfield County to neighboring counties in 2015.

Withdrawals by water source

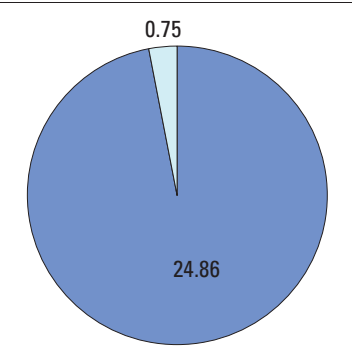

Withdrawals, in million

gallons per day

Surface water

Groundwater

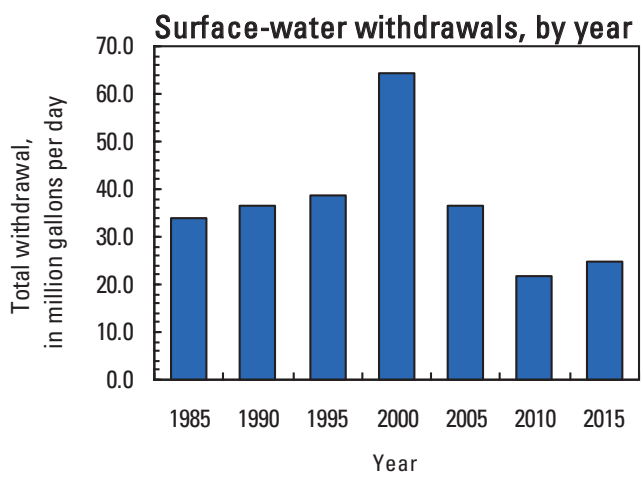

Groundwater withdrawals by aquifer
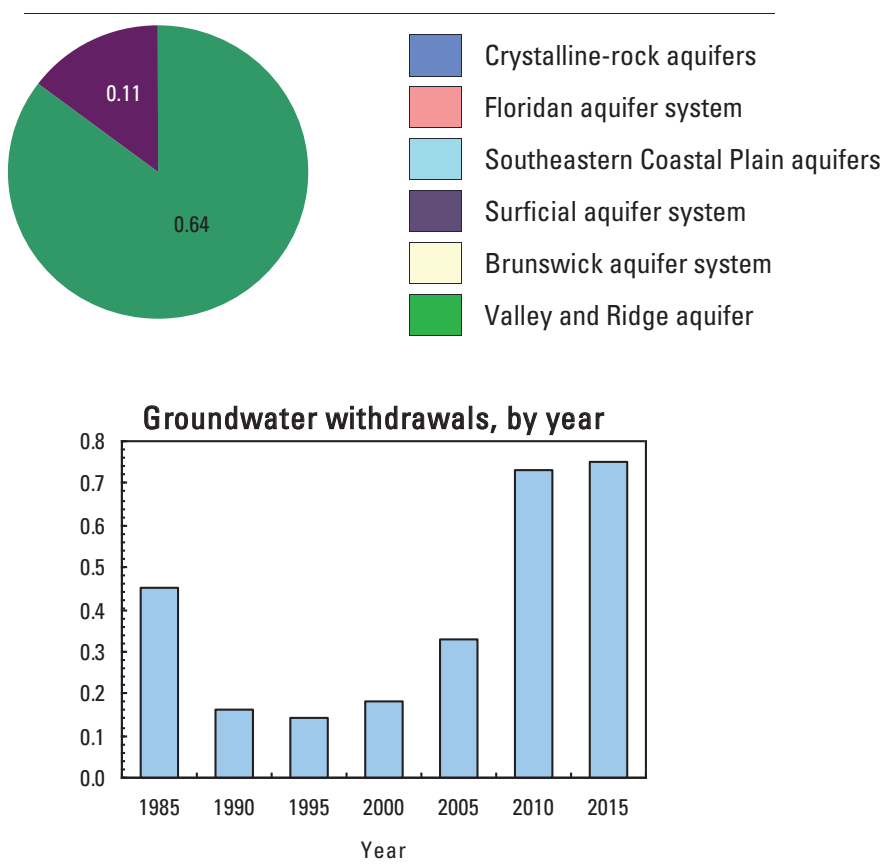


\section{WILCOX COUNTY}

Population

Population served by public supply-groundwater

Population served by public supply-surface water

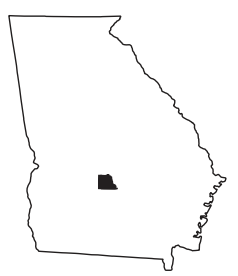

2015 WATER WITHDRAWALS AND ESTIMATED USE, IN MILLION GALLONS PER DAY

[--,not applicable; Mgal/d, million gallons per day]

\begin{tabular}{|c|c|c|c|c|c|c|c|c|}
\hline \multirow{3}{*}{ Category } & \multicolumn{3}{|c|}{ Withdrawals } & \multirow{3}{*}{$\begin{array}{c}\text { Total } \\
\text { use }\end{array}$} & \multirow{3}{*}{$\begin{array}{l}\text { Returns to } \\
\text { Surface }\end{array}$} & \multirow{2}{*}{\multicolumn{3}{|c|}{$\begin{array}{l}\text { Withdrawals by Major Industrial Groups } \\
\text { [NAICS, North American Industrial Classification System code] }\end{array}$}} \\
\hline & \multirow{2}{*}{$\begin{array}{l}\text { Ground- } \\
\text { water }\end{array}$} & \multirow{2}{*}{$\begin{array}{c}\text { Surface } \\
\text { water }\end{array}$} & \multirow{2}{*}{ Total } & & & & & \\
\hline & & & & & & \multirow[t]{4}{*}{ NAICS } & \multirow[t]{2}{*}{ Groundwater } & \multirow{2}{*}{ Surface water } \\
\hline Public supply & 0.44 & 0.00 & 0.44 & -- & -- & & & \\
\hline Domestic use & 0.23 & 0.00 & 0.23 & 0.58 & 0.00 & & -- & -- \\
\hline Commercial use & 0.02 & 0.00 & 0.02 & 0.07 & 0.00 & & & \\
\hline Industrial use & 0.00 & 0.00 & 0.00 & 0.00 & 0.00 & & & \\
\hline Public-supply loss & -- & -- & -- & 0.04 & -- & & & \\
\hline Inter-county delivery & - & - & - & 0.00 & - & & & \\
\hline Public wastewater treatment & $\mathrm{t}--$ & -- & -- & -- & 0.33 & & & \\
\hline Mining use & 0.00 & 0.00 & 0.00 & 0.00 & 0.00 & \multicolumn{3}{|c|}{ Withdrawals by Major Public Suppliers } \\
\hline Irrigation (crop) & 6.32 & 2.13 & 8.45 & 8.45 & 0.00 & Name & Groundwater & Surface water \\
\hline Irrigation (golf) & 0.00 & 0.00 & 0.00 & 0.00 & 0.00 & City of Rochelle & 0.10 & 0.00 \\
\hline Livestock-aquaculture & 0.34 & 8.93 & 9.27 & 9.27 & 0.00 & & & \\
\hline Thermoelectric power & 0.00 & 0.00 & 0.00 & 0.00 & 0.00 & & & \\
\hline TOTAL & 7.35 & 11.06 & 18.41 & 18.41 & 0.33 & & & \\
\hline
\end{tabular}

Total use is total withdrawal plus public supply deliveries and losses.

Withdrawals by water source

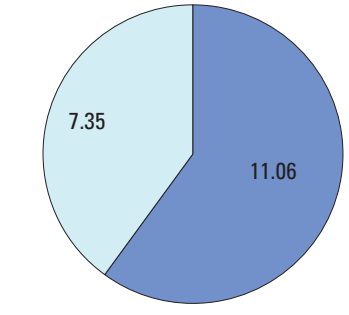

Withdrawals, in million gallons per day

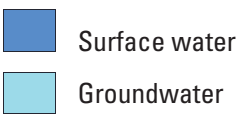

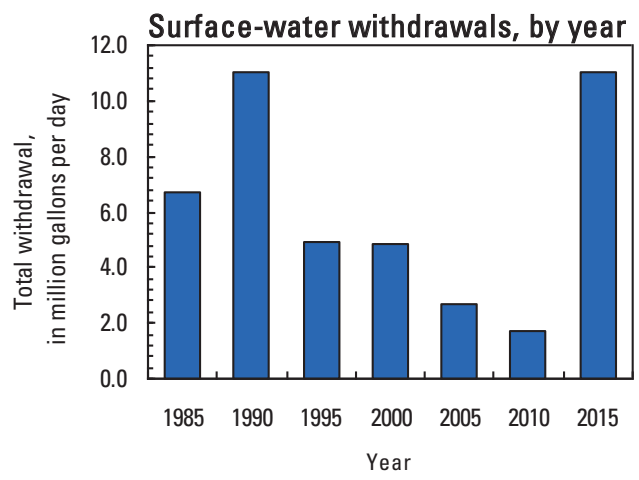

Groundwater withdrawals by aquifer

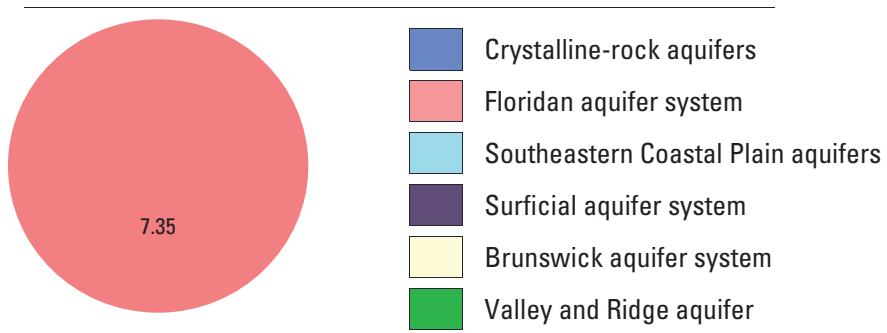




\section{WILKES COUNTY}

Population

9,867

Population served by public supply-groundwater

Population served by public supply-surface water

4,134

Acres irrigated

30

2015 WATER WITHDRAWALS AND ESTIMATED USE, IN MILLION GALLONS PER DAY

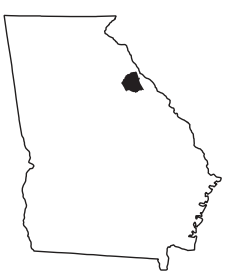

[--,not applicable; Mgal/d, million gallons per day]

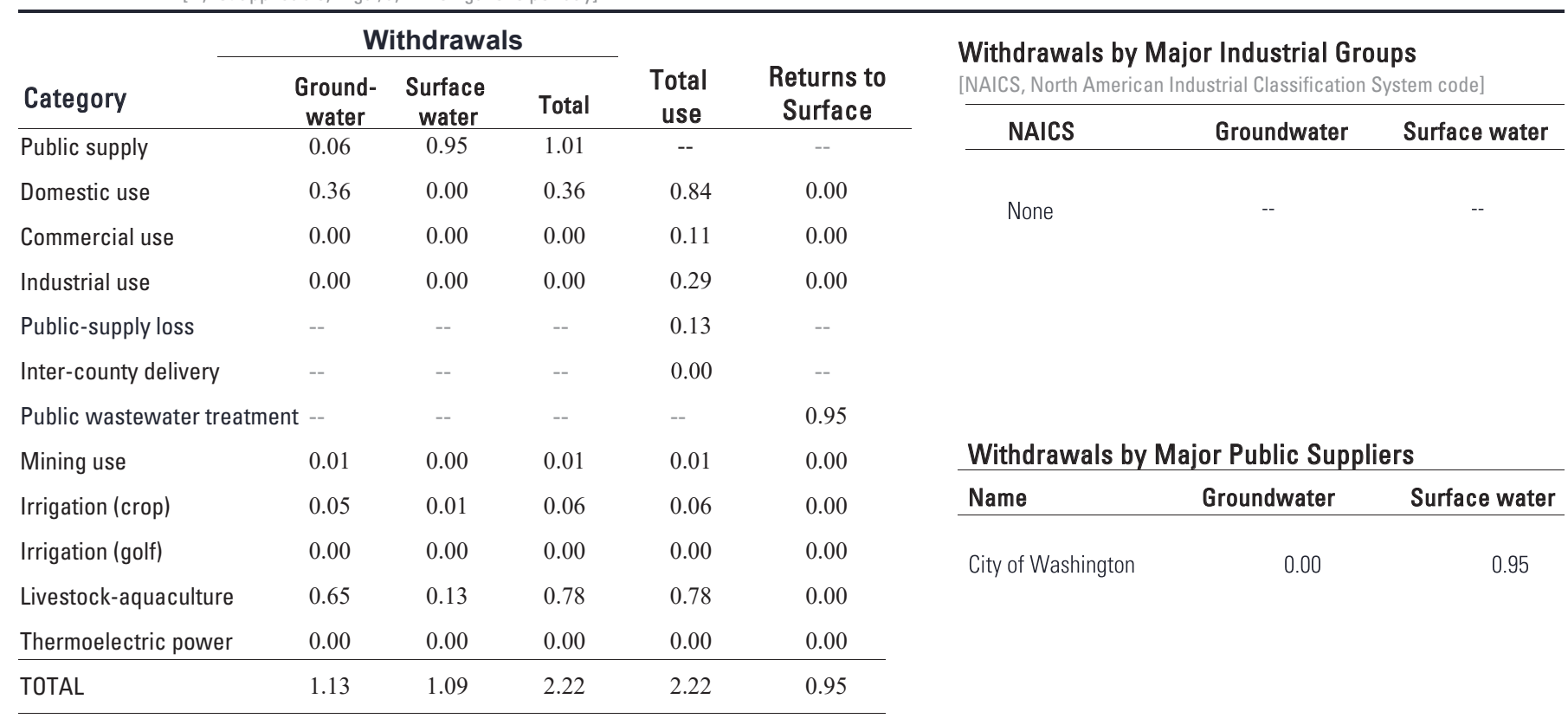

Total use is total withdrawal plus public supply deliveries and losses.

Withdrawals by water source

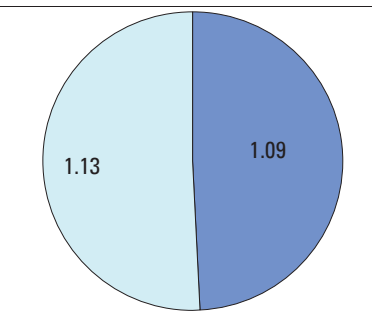

Withdrawals, in million

gallons per day

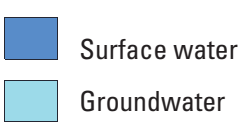

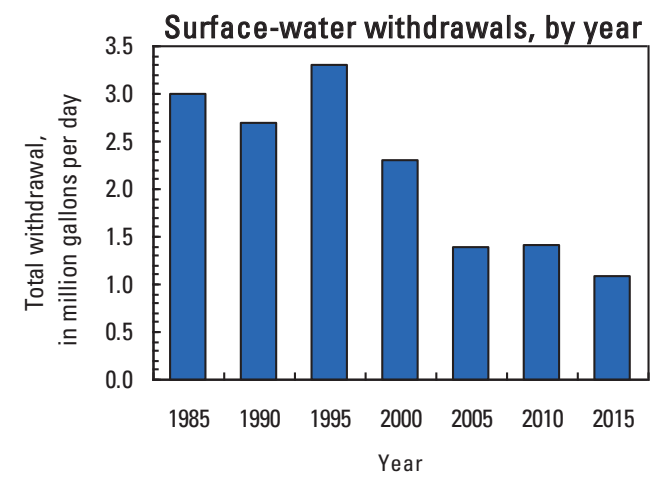

Groundwater withdrawals by aquifer

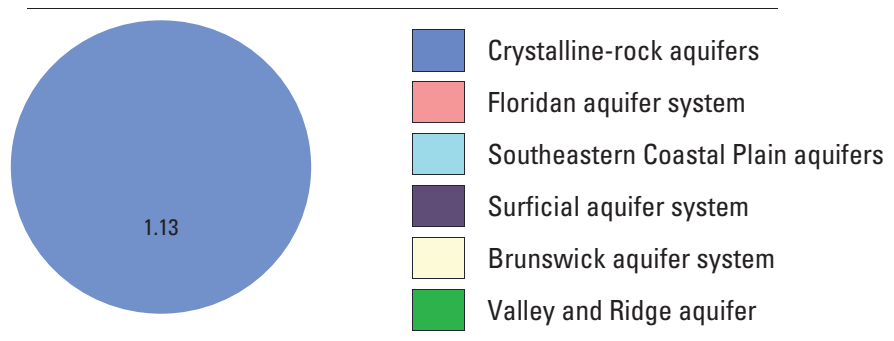

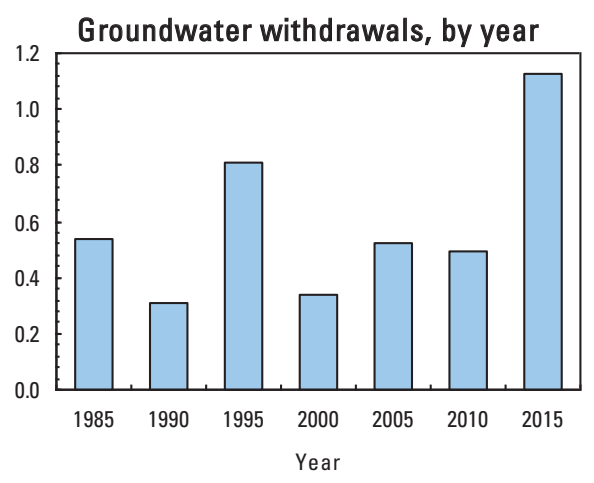




\section{WILKINSON COUNTY}

Population

Population served by public supply-groundwater

Population served by public supply-surface water

Acres irrigated

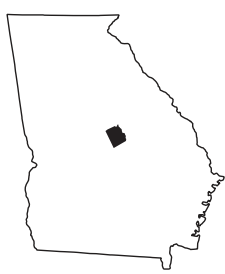

2015 WATER WITHDRAWALS AND ESTIMATED USE, IN MILLION GALLONS PER DAY

[--,not applicable; Mgal/d, million gallons per day]

\begin{tabular}{|c|c|c|c|c|c|c|c|c|}
\hline \multirow[b]{2}{*}{ Category } & \multicolumn{3}{|c|}{ Withdrawals } & \multirow{2}{*}{$\begin{array}{c}\text { Total } \\
\text { use }\end{array}$} & \multirow{2}{*}{$\begin{array}{l}\text { Returns to } \\
\text { Surface }\end{array}$} & \multirow{2}{*}{\multicolumn{3}{|c|}{$\begin{array}{l}\text { Withdrawals by Major Industrial Groups } \\
\text { [NAICS, North American Industrial Classification System code] }\end{array}$}} \\
\hline & Ground- & Surface & Total & & & & & \\
\hline Public supply & 0.70 & 0.00 & 0.70 & -- & -- & NAICS & Groundwater & Surface water \\
\hline Domestic use & 0.19 & 0.00 & 0.19 & 0.67 & 0.00 & \multirow[b]{2}{*}{ 212- Mining } & \multirow[b]{2}{*}{12.18} & \multirow[b]{2}{*}{0.25} \\
\hline Commercial use & 0.00 & 0.00 & 0.00 & 0.09 & 0.00 & & & \\
\hline Industrial use & 12.45 & 0.25 & 12.70 & 12.76 & 0.00 & 322- Paper, pulp & 0.05 & 0.00 \\
\hline Public-supply loss & -- & -- & -- & 0.07 & -- & \multirow[t]{2}{*}{ 327- Stone, clay } & \multirow[t]{2}{*}{0.22} & \multirow[t]{2}{*}{0.00} \\
\hline Inter-county delivery & -- & -- & -- & 0.00 & -- & & & \\
\hline Public wastewater treatment & it -- & -- & -- & -- & 6.44 & & & \\
\hline Mining use & 0.27 & 1.19 & 1.46 & 1.46 & 0.00 & \multicolumn{3}{|c|}{ Withdrawals by Major Public Suppliers } \\
\hline Irrigation (crop) & 0.04 & 0.00 & 0.04 & 0.04 & 0.00 & Name & Groundwater & Surface water \\
\hline Irrigation (golf) & 0.00 & 0.00 & 0.00 & 0.00 & 0.00 & City of Gordon & 0.30 & 0.00 \\
\hline Livestock-aquaculture & 0.03 & 0.00 & 0.03 & 0.03 & 0.00 & City of Irwinton & 0.20 & 0.00 \\
\hline Thermoelectric power & 0.00 & 0.00 & 0.00 & 0.00 & 0.00 & City of Mclntyre & 0.08 & 0.00 \\
\hline TOTAL & 13.68 & 1.44 & 15.12 & 15.12 & 6.44 & & & \\
\hline
\end{tabular}

Total use is total withdrawal plus public supply deliveries and losses.

Withdrawals by water source

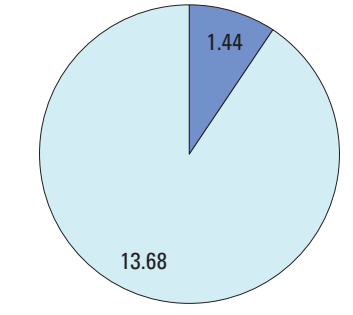

Withdrawals, in million gallons per day

Surface water

Groundwater

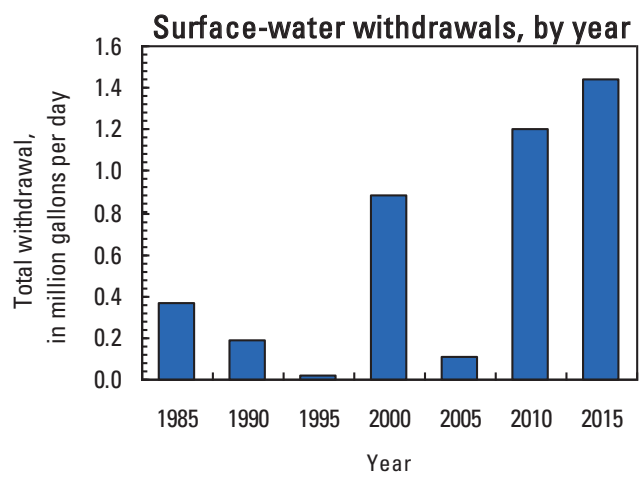

Groundwater withdrawals by aquifer

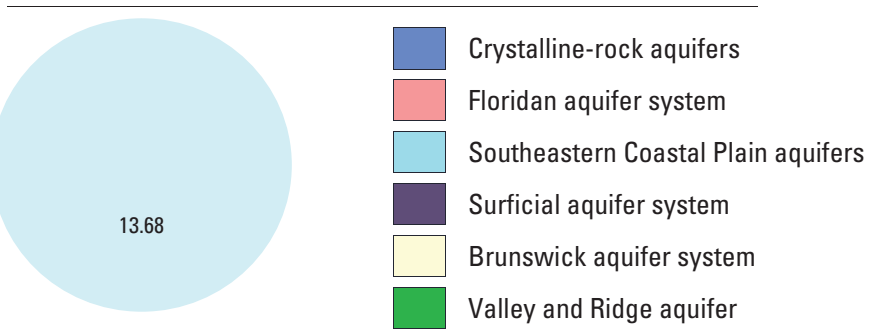

Groundwater withdrawals, by year

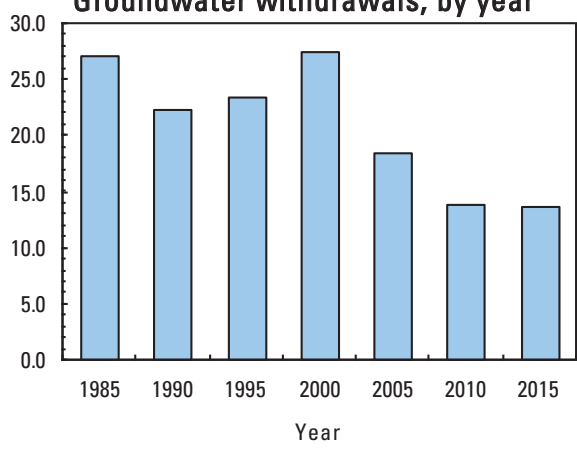




\section{WORTH COUNTY}

Population

20,699

Population served by public supply-groundwater $\quad 9,267$

Population served by public supply-surface water 0

Acres irrigated

2015 WATER WITHDRAWALS AND ESTIMATED USE, IN MILLION GALLONS PER DAY

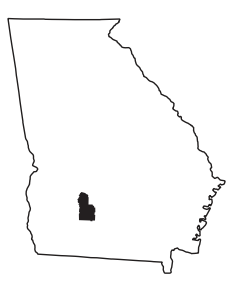

[--,not applicable; Mgal/d, million gallons per day]

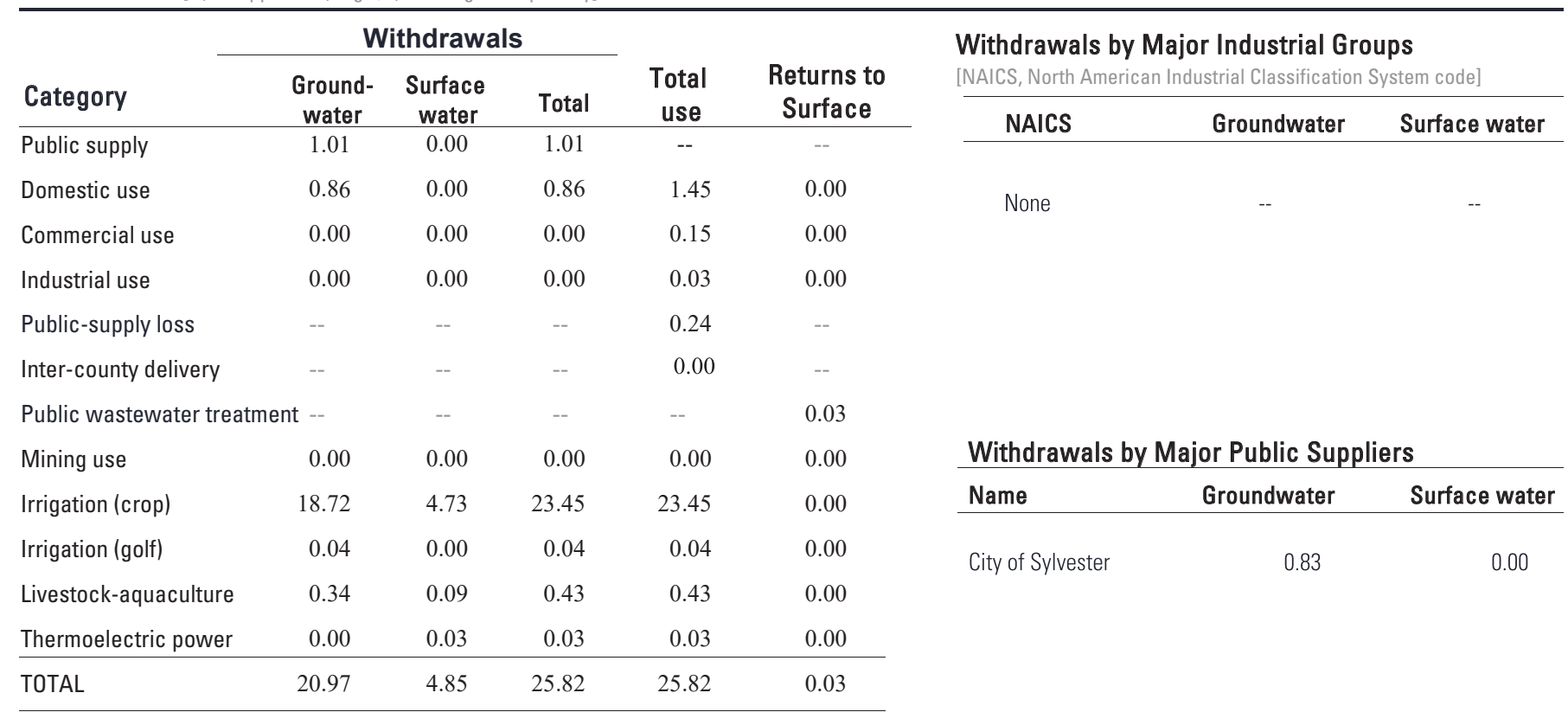

Total use is total withdrawal plus public supply deliveries and losses.

Withdrawals by water source

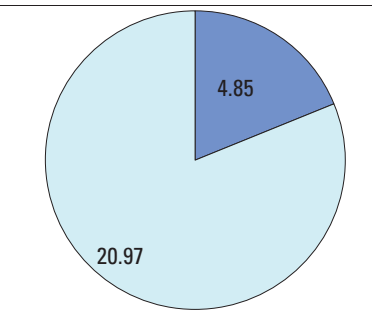

Withdrawals, in million

gallons per day

Surface water

Groundwater
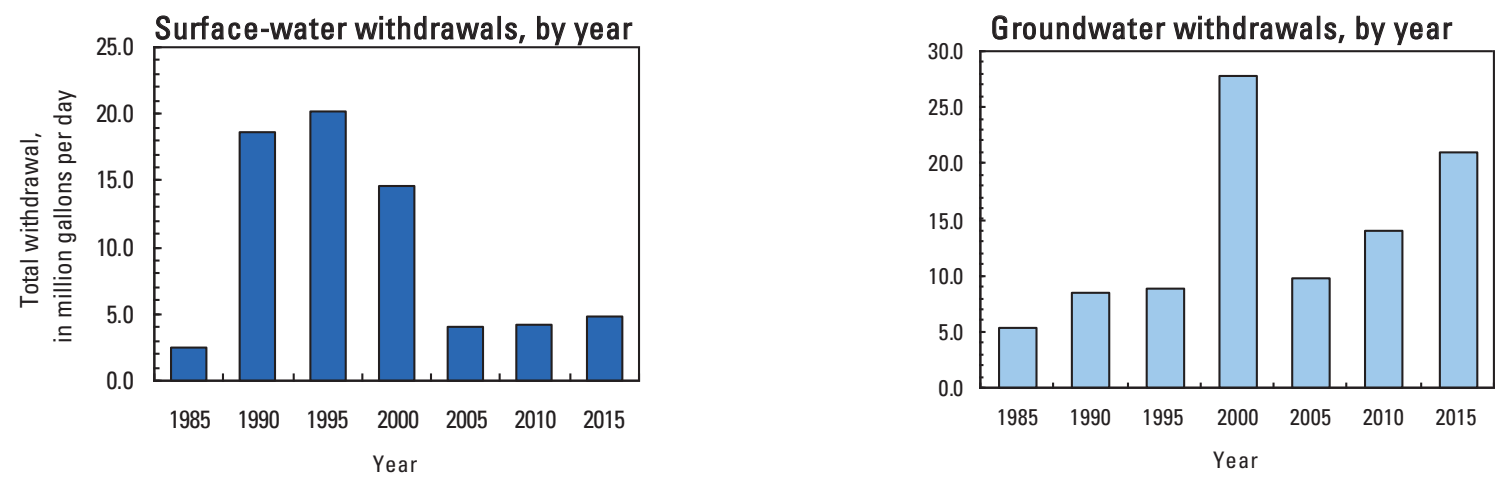



\section{$\frac{\mathbb{2}}{3}$}

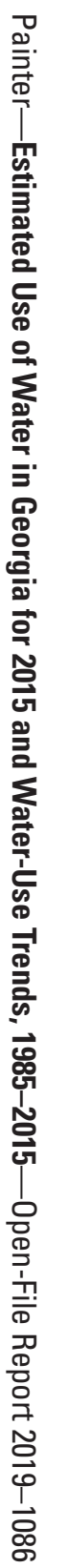

